\title{
Advanced Turbine System Conceptual Design and Product Development
}

Final Report

Prepared by: Albrecht H. Mayer

Date: July 15,2000

Contract No.: DE-AC21-95MC30245

Submitted by: ABB Power Generation Inc

5309 Commonwealth Centre Parkway

Midlothian, VA 23112 
This report was prepared as an account of work sponsored by an agency of the United States Government. Neither the United States Government nor any agency thereof, nor any of their employees, makes any warranty, express or implied, or assumes any legal liability or responsibility for the accuracy, completeness, or usefulness of any information, apparatus, product, or process disclosed, or represents that its use would not infringe privately owned rights. Reference herein to any specific commercial product, process, or service by trade name, trademark, manufacturer, or otherwise does not necessarily constitute or imply its endorsement, recommendation, or favoring by the United States Government or any agency thereof. The views and opinions of authors expressed herein do not necessarily state or reflect those of the United States Government or any agency thereof.

Available to the public from the National Technical Information Service, U.S. Department of Commerce, 5285 Port Royal Road, Springfield, VA 22161; phone orders accepted at (703) 487-4650. 


\section{Table of Contents}

0. Executive Summary

4

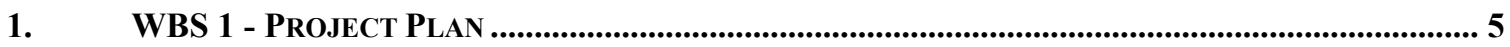

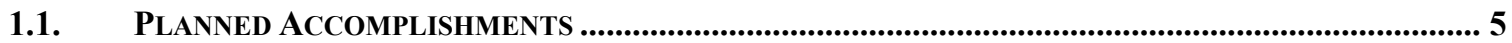

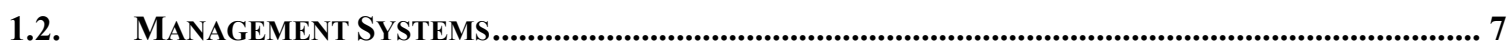

1.3. TECHNICAL SUPPORT SYSTEMS ......................................................................................... 10

1.4. ADMINISTRATIVE SUPPORT SYSTEMS ............................................................................ 10

1.5. Labor, Cost and Milestone/Schedule Plans..................................................................... 11

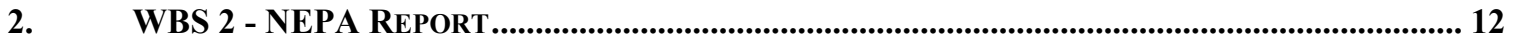

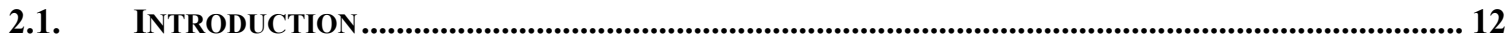

2.2. CRITICAL TESTING................................................................................................................. 12

2.2.1. Thermal Barrier Coating (TBC)/Material Testing in a Hot Gas Environment (WBS 8.2).. 13

2.2.2. Specimen Heat Transfer Tests WBS 8.3 ................................................................................... 14

2.2.3. Film Cooled Turbine Testing WBS 8.5 ...................................................................................... 14

2.3 ENVIRONMENTAL INFORMATION FOR CRITICAL TESTING ........................................................ 14

2.3.1 WBS 8.2 - Thermal Barrier Coating (TBC)/Material Testing in a Hot Gas Environment. 15

2.3.2 WBS 8.3 - Specimen Heat Transfer Tests ...................................................................... 16

2.3.3 WBS 8.5 - Film Cooled Turbine Testing .............................................................................. 16

3 WBS 3 - GFATS SELECTION ............................................................................................. 18

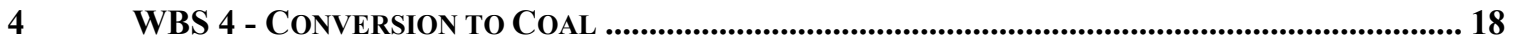

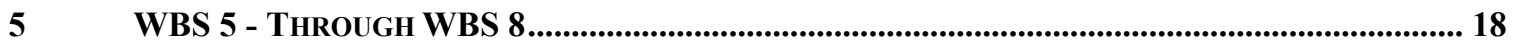

5.1 TASK 8.2.4.1 EVAluation OF TBC CoATING SYSTEMS IN High Thermal GradientS ........ 18

5.2 TASK 8.2.4.2 MONITORING THE DEGRADATION OF TBC COATING SYSTEMS ........................... 19

5.3 TASK 8.2.4.3 THERMO-MECHANICAL FATIGUE OF TBC SYSTEMS......................................... 19

5.4 TASK 8.2.4.4 KINETICS OF OXIDATION IN STEAM ................................................................... 21

5.5 TASK 8.3 AdVANCED AIR COOLED BLAdING ...................................................................... 21

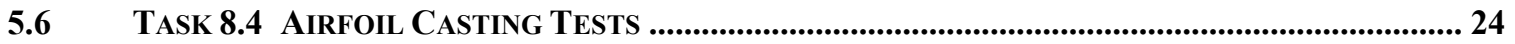

5.7 TASK 8.5 TURBINE AERODYNAMiCS \& AERO-THERMAL INTEGRATION................................. 24

5.7.1 Experimental Investigation of Cooled Turbine Performance ................................................... 25

5.7.2 Testing in Short Duration Facilities .................................................................................. 25

5.7.3 Test Section Modifications and Airfoil Design and Manufacture ........................................... 26

5.7.4 Film-Cooled Turbine Airfoils........................................................................................... 27

5.7.5 Turbine Efficiency Measurement Instrumentation ............................................................... 28

5.8 TASK 8.6 AdVANCED STEAM COOLED VANES ................................................................ 32

5.9 Advanced Air CoOled Turbine Engine Demonstration At Midland Cogeneration

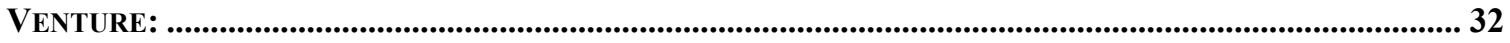

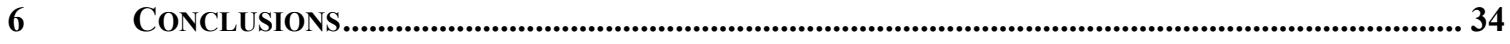

7 APPENDIX

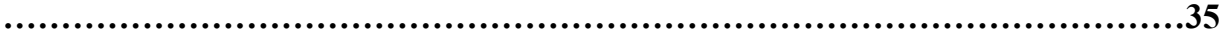

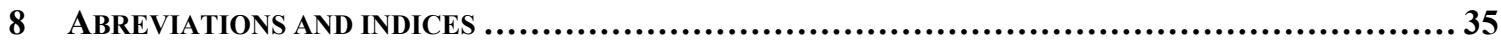




\section{EXECUTIVE SUMMARY}

ABB Power Generation Inc has been awarded with its contract in April 1995, during phase II of the Department of Energy Advanced Turbine Systems Program (ATS). The contract has been modified several times to a technology readiness program. ABB provided under the contract research and development with focus on Material, Cooling and Aerodynamic design integration to support the program goals of:

$>60 \%$ power plant efficiency

$>$ Single digit NOx

$>$ Decrease cost of electricity to the consumers

$>$ reliability

The work packages haven been carried out in a partnership between $A B B$ Power Generation Inc. and its subcontractors ABB Power Plant Laboratories in Windsor CT, ABB Power Generation Ltd. in Baden Switzerland and the Massachusetts Institute of Technology under the guidance of the Federal Energy Technology Center.

Under the material program various advanced coating systems have been evaluated for their reliability under conditions similar to the degradation modes in an advanced turbine design. Non-destructive coating degradation methods have been compared. The oxidation behavior of super alloys in a steam environment was analyzed.

Pressure loss and heat transfer correlations for non surface air cooling have derived under the cooling technology program. In addition basic correlations for steam cooling were developed.

A test rig to measure heat transfer and the influence of surface cooling on the aerodynamic performance of a turbine stage has been developed. The test rid allowed to measure the aerodynamic performance and the heat transfer under full engine conditions.

The technology readiness has been demonstrated in an turbine upgrade design that is in operation since 1997. 


\section{WBS 1 - Project Plan}

Asea Brown Boveri (ABB) has continually focused its efforts on improving gas turbine cycles since ABB's first industrial gas turbine began commercial operation in 1939. Those efforts have included significant improvements in gas turbine performance in simple and combined cycle operation; increased temperature capability; reduced emissions; and enhanced reliability, availability, and maintainability (RAM). To achieve these improvements, significant R\&D investments were required. To achieve the goals of the Advanced Turbine System (ATS), substantial R\&D efforts are similarly required.

While the performance, emission, RAM, and cost goals for the Advanced Turbine System are aggressive, they are considered achievable and they do reflect industry input, including ABB's. ABB has participated in the DOEsponsored pre-ATS workshops from the very beginning starting with the initial Gas Turbine Research Needs workshop at Clemson University in April, 1991, continuing with the follow-up workshop (Gas Turbine Research Needs II) at Clemson in January, 1992, and continuing with subsequent DOE-sponsored ATS workshops soliciting industry input.

The barrier issues and risks associated with meeting the ATS goals are understood by ABB. These areas are typical whenever significant improvements in performance are required and when rapid commercialization of the product is desired. Efforts to minimize barrier issues and risks require an integrated, concurrent engineering development approach to be successful. $A B B$ has extensive concurrent engineering development experience and has consistently commercialized new products with advanced technologies in time to meet market demands. Such experience is being utilized to plan and execute this ATS program phase on time and within budget while minimizing the technical risks.

\subsection{Planned Accomplishments}

Subsequent to ABB's initial proposal submittal in November, 1992, for the Conceptual Design and Product Development phase of the ATS program, 
ABB introduced an Advanced Cycle System ( $A C S^{T M}$ ) gas turbine designated as the ABB Model GT24. This gas turbine incorporates innovative cycle improvements and advanced materials to achieve high levels (58\% net efficiency on LHV basis) of combined cycle performance through the use of a reheat turbine stage, higher pressure ratio compressor, and sequential, annular combustors.

ABB's complete work breakdown structure (WBS) which defines all of the planned accomplishments includes:

- Complete tradeoff studies to determine the optimum changes to the baseline system which makes it most attractive from performance, emissions, cost, RAM, fuel flexibility, and marketability aspects. These studies and changes will address additional innovative cycle changes and high temperature turbine developments which will be used to define the basic configuration of ABB's natural gas-fired advanced turbine system (GFATS)

- Complete design, testing, evaluation, and/or analysis of the following critical system components.

1. Complete initial performance testing of base material and thermal barrier coating (TBC) systems in a hot gas environment (burner rig) for potential materials/TBC systems. Also complete initial testing of various thickness TBC's with simulated airfoil thermal gradients to determine TBC performance.

2. Complete heat transfer tests of stylized specimens to measure the heat transfer effectiveness of conceptual ATS turbine airfoil advanced cooling configurations.

3. Complete heat transfer/aerodynamic testing of a film-cooled rotating blade stage to determine heat transfer coefficients and establish aerodynamic performance levels to use for subsequent design work.

These test programs reflect ABB's concurrent engineering approach by addressing development needs through obtaining essential heat 
transfer/aerodynamic data at the appropriate levels, assessing candidate material and coating performance, evaluating manufacturing feasibility of possible cooling schemes, and demonstrating the feasibility of an advanced measurement system to determine hot gas part temperatures in-situ as a potential development measurement tool for subsequent designs.

\subsection{Management Systems}

\section{Organization Structure}

The ABB organizations and team members to achieve the planned accomplishments described below. The prime contractor is ABB Power Generation, Inc. (PGI) and the work was accomplished and/or guided by ABB $\mathrm{PGI}$ and the following team members:

- $\quad$ ABB Power Generation, Ltd. (Inter-divisional Partner)

- $\quad$ ABB Power Plant Laboratories (Inter-divisional Partner)

- $\quad$ Massachusetts Institute of Technology (MIT) Gas Turbine Laboratory (Subcontractor)

- $\quad$ ABB Commercial Power Plant Customer (Subcontractor)

The ABB Program Manager is responsible for all interfaces with the DOE and for coordinating the ATS efforts with the ABB organizations and subcontractors which are part of ABB's ATS team. The Program Manager is the single point contact to the DOE on all ATS matters. He has been delegated the responsibility by $A B B$ to ensure that the technical, administrative, cost, and schedule requirements of the program contract are being satisfied by the ATS team. In this role he is expected to have frequent communications with DOE program and subcontract personnel. He will be assisted by ABB PGI internal organizations (Supply Management, Financial, Quality Assurance, Document Control) in administering the program's contract, administrative, and cost/schedule requirements.

To maintain a strong focus on the technical requirements of the program, $A B B$ has established a Technical Director to specifically oversee the technical work. $\mathrm{He}$ is responsible to the Program Manager for coordinating the technical 
efforts and serves as the ABB Deputy Program Manager. To ensure effective management and communications between team members, $A B B$ is requiring each organization to establish a program manager who will be the single point of contact for all ATS matters (technical, schedule, cost, and contractual) for that organization. The names of the currently established program managers by organization are also indicated in Figure III-1.

The work planned for accomplishment by the various partners and subcontractors includes the following:

ABB Power Generation, Inc.: Overall project coordination, supporting gas turbine, power plant and BOP equipment system integration, marketing, and overseeing domestic gas turbine testing.

ABB Power Generation, Ltd.: Conducting core gas turbine design and analysis efforts and specimen heat transfer tests.

ABB Power Plant Laboratories: Conducting heat recovery steam generator (HRSG) studies and coating/material tests.

MIT Gas Turbine Laboratory: Conducting film-cooled turbine heat transfer and aerodynamic tests

ABB Power Plant Customer: Making power plant and gas turbine (GT11N1) available and providing support for conducting the demonstration test.

To ensure that the ATS development best serves strategic and changing market business issues, a Steering Committee consisting of key ABB power plant executives is being established. The role of the ABB Gas Turbine Power Plant Steering Committee is to provide senior management oversight of the program. This program oversight will be accomplished through periodic reviews scheduled by the ABB Program Manager. The reviews provide the forum to advise the Steering Committee of program progress and to identify critical issues which may require action by the Steering Committee to achieve the program's technical and commercialization objectives. 


\section{Project Control System}

The cost control system to be utilized as part of the overall management of this contract incorporates the concepts of planning the total efforts, assigning resource budgets to elements of the plan, and measuring progress against the plan in terms of actuals, estimates-to-complete, and budgeted accomplishments.

The proposal WBS and schedule are similarly updated, time-phased budgets are assigned by task and organization, and final calculations are made based on the rates which were agreed upon in the contract. From this data the baseline budgets, against which all cost all schedule variances will be measured, are established and any difference between the planning budgets and baseline budgets will be accounted for as part of a Management Reserve Log.

Monthly actuals are received from the official accounting records. The actuals are reviewed and summarized at the proper level, reviewed/approved by the ABB Program Manager, and the monthly reports prepared and submitted to the DOE. All data utilized in the preparation of Cost and Labor Management Reports are reconcilable to internal books of account, customer billings, and program management reports. With regards to the Labor Management, Cost Management, and Labor Plan Reports:

- Reporting elements are at the second or third level of the WBS depending on the dollar value of the task and the number of organizations (and dollar value) accomplishing the task.

- Subcontractor labor is not recorded internally by $A B B P G I$ for reporting purposes and are not included in the PGI plans and reports.

- All costs reported are based on the actual recording of costs in our internal books of account.

- The cost share incurred by various team members is not included in the Cost Management Report since that cost is not charged to our books. 
- Subcontractor costs and estimates reflect the month in which the billings are received (actuals) or anticipated to be received (estimates) at ABB PGI, not the month in which the cost was incurred by the subcontractor.

Establishment and operation of the program schedules are accomplished within the Program Manager's organization

All schedules are reviewed and revised on a monthly basis. The relationships between time-phased budgets, estimates-to-complete, and progress is reviewed by both the Program Manager and Financial Department to ensure that both timely and accurate information is being reported. The input for the monthly Milestone/Schedule Status Report is from the Project schedule.

\subsection{Technical Support Systems}

The primary technical efforts for this Conceptual Design and Product Development phase of the ATS program are directed towards meeting performance, emissions, RAM, and cost goals of ABB's proposed ATS system. Satisfying these requirements will be accomplished utilizing ABB's well-established gas turbine component design criteria, analytical tools, and design databases which reflect ABB's extensive gas turbine design and development experience.

All current $A B B$ design products are designed and reviewed from a total system standpoint. These efforts include considerations for analytical model validation, RAM, cost benefits analyses, systems engineering, safety and codes, environmental effects, manufacturing feasibility, configuration control, and quality assurance of the design and end-product.

\subsection{Administrative Support Systems}

Two administrative systems are used for this contract: Procurement and Accounting. These will be discussed in the following sections.

\section{Procurement System}


ABB's PGI procurement efforts are handled by the Supply Management Department. Procurement policies and procedures are contained in ABB PGI's Supply Management Operating Procedures Manual.

With regards to this ATS contract, applicable prime contract provisions are included in purchase orders to subcontractors.

\section{Accounting System}

The segregation and accumulation of all direct costs associated with a particular project or program is tracked by assigning a unique six-digit alphanumeric identifier called a project number. Within each project direct costs are further segregated by general ledger account number and work detail code (or Work Breakdown Structure (WBS) number). All direct labor is recorded based on actual hours worked via a network PC system. The associated labor costs are calculated based on actuals and/or approved rates in accordance with contract requirements.

Material, travel, subcontract costs, and the like are recorded against the project number and work detail code account (WBS) based on purchase orders, shop orders, travel expense reports, and other miscellaneous documents which authorize the expenditure of direct costs. All direct costs are accumulated and identified in monthly reporting by work detail code (WBS) for cost control purposes. These reports are distributed to the respective program managers. These reports will be the basis for the Cost Management Report.

The accounting system provides the ability to identify, monitor, and report program costs in an integrated, uniform manner.

\subsection{Labor, Cost and Milestone/Schedule Plans}

The Labor Plan, Cost Plan, and Milestone/Schedule Plan for the ATS Conceptual Design and Product Development Program presented to the DOE. These initial plans are based on reporting to the second or third WBS level, depending on task dollar value and number of organizations (and dollar value) performing work under that task. These plans are based on the forthcoming 
submittal of the Project Plan. As previously discussed in Section III, subcontractor labor is not included in the ABB PGI labor plan.

\section{WBS 2 - NEPA Report}

\subsection{Introduction}

ABB planned to accomplish the following major tasks:

- Complete trade-off studies to determine which optimum changes to ABB's baseline gas turbine system make it most attractive from performance, emissions, cost, RAM, fuel flexibility, and marketability aspects. These studies and changes address additional innovative cycle changes and high temperature turbine developments which will be used to define the basic configuration of ABB's natural gas-fired advanced turbine system (GFATS).

- Complete design, testing, evaluation, and/or analysis of the following critical system components:

a) Complete laboratory testing of turbine airfoil materials and coatings in a combustion test rig to test the performance materials and thermal barrier coating (TBC) systems in a hot gas environment.

b) Complete laboratory heat transfer tests of stylized specimens to measure the heat transfer effectiveness of conceptual ATS turbine airfoil advanced cooling configurations.

c) Complete heat transfer/aerodynamic testing of a film-cooled rotating blade stage in a blowdown test rig to determine heat transfer coefficients and establish aerodynamic performance levels to use for subsequent design work

\subsection{Critical Testing}


As previously discussed, ABB planned to conduct five critical component test programs. The technical content of these programs are discussed in the following sections by ABB WBS number.

\subsubsection{Thermal Barrier Coating (TBC)/Material Testing in a Hot Gas Environment (WBS 8.2)}

A three-phase program has been defined to evaluate TBC/base material heat transfer performance, oxidation resistance, and degradation. This testing will be conducted at ABB Power Plant Laboratories in Windsor, Connecticut. In particular:

\section{Testing of TBC Coated Tubular Specimens (WBS 8.2.4.1)}

The purpose of this phase is to characterize the temperature profiles in TBC coated parts as a function of certain operational parameters and to evaluate the performance of coating systems in thermal cyclic conditions and in the presence of cooling air. Tubular specimens will be coated with commercially available EB-PVD and Air Plasma TBC's and tested in a small burner rig under thermal cycling conditions. Internal cooling air will be provided to the inside of the tubes to represent airfoil temperature gradients. Temperature sensors will be attached to each test piece to enable critical temperatures to be measured during the course of testing.

\section{Testing of TBC Coated Cooled Airfoils (WBS 8.2.4.2)}

The purpose of this phase is to evaluate the cyclic oxidation performance of TBC coated, internally cooled airfoil specimens. Airfoil shapes will be produced by directional solidification and by single crystals. These airfoils will be processed with and without laser drilled cooling holes and subsequently coated with state-of-the-art bond coats and TBC's. Critical temperatures on the surface and in the cooling passages will be measured, and cyclic oxidation testing will be performed to determine TBC life under various conditions.

\section{Degradation Monitoring of TBC Coated Airfoils (WBS 8.2.4.3)}


The purpose of this phase is to determine the condition of TBC's and bond coats by evaluating NDE techniques. During this task both eddy current and ultrasonic techniques will be used to characterize coatings. Vane specimens will be coated with ABB TBC's plus bond coats. Thickness measurements will be made using the eddy current system. These parts will be sectioned, and wall/coating thicknesses will be verified by metallography. In addition, samples will be subjected to cyclic oxidation testing to obtain oxide growth and coating depletion similar to that which occurs in a gas turbine. Metallography and SEM analysis will be performed to quantify coating degradation.

\subsubsection{Specimen Heat Transfer Tests WBS 8.3}

Heat transfer testing of stylized airfoil specimens will be conducted to measure the pressure drop and heat transfer effectiveness of conceptual ATS turbine airfoil advanced cooling configurations. This work was accomplished within ABB's Corporate Research Facility in Dattwil, Switzerland.

\subsubsection{Film Cooled Turbine Testing WBS 8.5}

The turbine power output and efficiency, airfoil and endwall heat transfer, and stage inlet and exit aerodynamics of a conceptual ATS cooled turbine stage will be measured. A cooled turbine stage will be designed and manufactured out of aluminum which is compatible with the planned test section ( maximum tip diameter of about 0.5 meter) at the Massachusetts Institute of Technology (MIT) Gas Turbine Laboratory blowdown test facility. Complete stage inlet and exit aerodynamic surveys and airfoil heat flux measurements will be made under fully scaled operating conditions.

\subsection{Environmental Information for Critical Testing}

The environmental information for the critical test programs are described below: 


\subsubsection{WBS 8.2 - Thermal Barrier Coating (TBC)/Material Testing in a Hot Gas Environment}

The work proposed under this task includes laboratory testing of ceramic based Thermal Barrier Coatings (TBC) at the ABB Power Plant Laboratories (PPL) Hot Gas Corrosion Test Facility in Windsor, Connecticut. The testing primarily involves exposing TBC coated alloy specimens to a high temperature gas environment. The hot gas environment is produced using an LCS-4 Laboratory Combustor System manufactured by Becon, Inc. (South Windsor, Connecticut). The basic elements of this natural gas fired system include the combustor, combustor stand, computer control console, and rotating sample fixture. In its most basic configuration, the system is capable of exposing eight $3 / 8$ inch diameter samples approximately $3-4$ inches long to a hot gas stream. The combustor is capable of producing gas temperatures up to $3000^{\circ} \mathrm{F}$ with velocities approaching 1500 feet per second. This existing equipment is housed in a $10 \times 16$ foot enclosed room and is equipped with automated safety features for automated operation (i.e., combustible gas detectors, flame scanners, etc.).

The hot gas conditions anticipated for testing will require the burner to operate at $3000^{\circ} \mathrm{F}$ at $250-300$ feet per second. At these conditions, the burner consumes $8.8 \mathrm{ft} 3 /$ minute of pipeline supplied natural gas and utilizes approximately 12 pounds of air per minute. This translates into a burn rate of 535,000 BTU per hour and an air/fuel ratio of approximately 32:1. The hot gases from the combustor will exit through an exhaust stack primarily in the form of $\mathrm{CO}_{2}, \mathrm{CO}$ and $\mathrm{H}_{2} \mathrm{O}$. During this test program the combustor is expected to operate approximately 5500 hours.

The regulations concerning the abatement of air pollution by the Connecticut Department of Environmental Protection (DEP) require natural gas fuel burning equipment rated more than 11 million BTUs per hour to be permitted (DEP Section 22a-174-3(a)(1)(F)). The laboratory combustor described above (0.535 million BTUs per hour) is far below the threshold requiring a permit.

The potential for air emissions is less than 5 tons per year for individual pollutants such as $\mathrm{NO}_{\mathbf{x}}, \mathrm{CO}$, and $\mathrm{SO}_{\mathbf{x}}$; therefore, this equipment does not 
require a permit under Connecticut Department of Environmental Protection (DEP) regulations (DEP Section 22a-174-3(a)(1)(K)).

The anticipated environmental impact of operating this laboratory test equipment described above is minimal. The facility does not use any water (for cooling, etc.) and does not discharge any water or solid waste. No state permits are required for the operation of this equipment.

\subsubsection{WBS 8.3 - Specimen Heat Transfer Tests}

Internal cooling configurations for turbine airfoils will be tested for heat transfer and pressure loss. Cooling configurations are evaluated for heat transfer characteristics using well-known liquid crystal techniques. The configurations are tested for pressure drop characteristics by flowing dry air through the model and measuring pressure drop and flowrate.

The heat transfer test involves flowing warm air through a scale model of proposed designs and recording data using video cameras. A scale model of proposed designs is machined from plexiglas or cast from commercially available liquid resin. Selected surfaces of the model are painted with a special-purpose paint containing temperature-sensitive liquid crystals, also commercially available. Room air is heated electrically using resistance heaters and ducted through insulated piping to the model. Tests are shortterm, generally lasting only a matter of minutes. Pressure loss testing is conducted on the same models as used for liquid crystal testing.

Tests will be conducted using facilities already in place and functioning for related development work. This testing will have no impact on air quality, water resources, land use, or waste management. There are no ecological, socioeconomic, archaeological, cultural or historical resources, noise, or occupational safety and health impacts as a result of this testing.

\subsubsection{WBS 8.5 - Film Cooled Turbine Testing}

This testing will be conducted in the MIT blowdown test facility (BDT) located in the MIT Gas Turbine Laboratory in Building 31 on the main MIT campus in 
Cambridge, Massachusetts. This facility has been in operation since 1982 and requires only minor modification for this project. The BDT is a closed, short duration ( 0.5 second) turbine test facility. It is electrically heated and driven. The only effluents are inert gases carbon dioxide $\left(\mathrm{CO}_{2}\right)$, nitrogen $\left(\mathrm{N}_{2}\right)$, or argon, approximately 100 pounds per test. No solid or liquid waste is produced.

The blowdown facility produces insignificant environmental impact. Specifically:

- Only carbon dioxide, nitrogen, and argon will be exhausted from the facility, approximately 100 pounds per test.

- A few gallons of water per minute (<25 GPM) of city water are used to cool the facility during test preparation (4 hours). The water is discharged to the city drains.

- No additional land is needed for the testing.

- No liquid or solid waste is produced.

- There are no ecological impacts as the testing is within a building on an urban campus.

- No new hires are planned other than use of a graduate student.

- There are no archeological, cultural, or historical resources involved.

- Noise is not an issue because the facility is within a building and is not audible from the exterior (also it operates for only 0.5 second per day).

- MIT has a rigorous program of occupational safety and health and is closely monitored by local federal and government agencies.

- There are no cumulative impacts. 
- No federal, state, or local permits or licenses are needed for this testing.

\section{WBS 3 - GFATS Selection}

The system study has been reported in April 1996. The technical information provided to the DOE is accurate, however due to changes in market and priorities in ABB's development strategy the conclusions are somewhat modified. The proposed integrated steam cooling into ABB's Advanced Cycle System is currently not of highest priority. The results of the system study are therefore not further discussed in this Final Report

\section{WBS 4 - Conversion to Coal}

Deleted from Technology based program efforts.

\section{$5 \quad$ WBS 5 - Through WBS 8}

In the replanned program WBS 5 through WBS 7 have been canceled. The main work has been focused on the following items

\subsection{Task 8.2.4.1 Evaluation of TBC Coating Systems in High Thermal Gradients}

An extensive program of testing has been carried out in a burner rig firing natural gas to evaluate the performance of various overlay coatings and combinations of bond-coat thermal barrier coatings that are used in gas turbines for power generation. The aim was to evaluate performance in conditions similar to those experienced in service so that cycling to low temperature from the flame temperature of $1100^{\circ} \mathrm{C}$ occurred at $22 \mathrm{~h}$ intervals and the testpieces with TBC's were cooled to generate a thermal gradient through the coating. 
The results show that the coatings overlaid with a thin diffusion coating (i.e., an aluminide or similar) showed the good performance based on weight loss and the remaining aluminum content in the coatings. SV-20 showed consistently good results with SV-23 exhibiting the greatest gain in weight. The results for SV-34 were difficult to interpret due to the large difference in weight loss between the two SV-34 samples.

Of the test pieces coated with TBC the standard $300 \mu \mathrm{m}$ thickness was superior and at least for these conditions of heat transfer there appeared to be no advantage with a $1 \mathrm{~mm}$ thick ceramic layer. A model to predict the build-up of stresses in the TBC gave results which were broadly in agreement with practical experience. More detailed results can be found in Appendix 1

\subsection{Task 8.2.4.2 Monitoring the Degradation of TBC Coating Systems}

There is currently no satisfactory non-destructive method for measuring the condition of overlay coatings applied on new or engine-run engine parts in gas turbines. The purpose of this program has been to evaluate the potential of various methods e.g. eddy-current and ultrasonic, for the non-destructive evaluation of overlay coatings in thermal barrier coatings systems and the results are described in this report. It is shown that a variant of the eddycurrent method will give good correlations with metallographic measurements for determining the thickness of new coatings but neither eddy-current nor ultrasonic techniques gave a good measure of the extent of degradation of engine-run parts. There is however scope for improvement and some recommendations are made.

\subsection{Task 8.2.4.3 Thermo-Mechanical Fatigue of TBC systems}

Since components coated with TBC's are subjected to severe mechanical and thermal loads which vary during the operation of the engine the response of thermal barrier coated material to TMF is an important aspect of design.

"Out-of-Phase" thermal mechanical fatigue (TMF) testing of CMSX-4 and coated specimens was performed. Metallic coatings reduced TMF lives of CMSX-4, with CMSX-4 + SV-34 showing the least reduction. TBC coated 
specimens showed lives comparable to baseline tests. This may be attributed to the test conditions. Life prediction of baseline TMF testing of CMSX-4 could be made within a $2 x$ life.

The results are summarized as:

1. Coated testpieces of CMSX-4 showed reduced TMF resistance compared with the uncoated alloy in "out-of-phase" testing at various strain ranges.

2. Testpieces coated with SV-34 showed the smallest reduction in TMF resistance and aluminide overcoats also performed well, particularly at the higher strain ranges.

3. Increased maximum temperatures and the introduction of hold times both reduced TMF resistance.

4. The TMF resistance of testpieces coated with thermal barriers was comparable to that of the uncoated alloy presumably because of the effect of the TBC in reducing metal temperatures.

5. In most cases failure occurred as a result of the propogation of cracks that initiated in the coating and in testpieces with TBC's oxide growth made little contribution to the failure process. Perhaps a longer dwell time at the maximum temperature would have changed this.

6. The results for the uncoated testpieces were reasonably consistent with the predictions of a simple model.

7. This type of test probably represent the behavior in cyclic or peaking conditions but does not reproduce failure mechanisms in base loaded unit. 


\subsection{Task 8.2.4.4 Kinetics of Oxidation in Steam}

Results of test exposure of candidate superalloy materials to steam for 5,000 hours at temperatures up to $950 \mathrm{C}[1,742 \mathrm{~F}]$ do not identify any major oxidation problems which might preclude the use of steam cooling in an advanced technology gas turbine. While only limited data were found for comparison with air, there do not appear to be any great differences in the two environments. For alloys which form a protective chromia scale (i.e. IN 617 and IN 738LC), vaporization loss of chromium at high gas velocity is of similar concern in both environments. While the specific test bars of CM 247LC used in these tests did not perform well, this is attributed to compositional or casting related issues and should not be generalized. The alloys IN 738LC and CM 247LC experienced spallation of the oxide scale similar to that observed for many alloys undergoing cyclic oxidation testing. CMSX-4, which forms a protective alumina scale, experienced the least oxidation of the alloys tested. More detailed results can be found in Appendix 2

\subsection{Task 8.3 Advanced Air Cooled Blading}

The Advanced Air Cooled-Blading task includes the subtasks: (a) the development of wall cooling technology, (b) the development of alternate air transfer systems and (c) the development of airfoil manufacturing technology. The subject of the present report is subtask (a) the development of impingement wall cooling technology. This subtask included the evaluation of heat transfer from several enhanced target geometries and comparisons with current impingement target plate heat transfer technology.

Impingement cooling produces relatively high heat transfer coefficients and high heat exchanger effectivenesses. These heat transfer benefits are obtained at the cost of relatively high pressure drops in the cooling system, compared to pressure drops in smooth coolant passages. However, there are tradeoffs that allow high heat transfer with greater amounts of coolant flow or with the use of enhanced heat transfer surfaces. These trade-off relationships are less well defined and the impingement target plate is often limited by the 
pressure drop available or the subsequent use of the cooling flow. Prior to the start of the DOE contract task at ABB, the impingement cooling heat transfer open literature was reviewed with respect to available design correlations and to the range of flow and geometric parameters used to develop the correlations. Unfortunately the open literature does not contain data obtained by the rocket and aircraft turbine engine manufacturers for specific high performance cooling applications, often with enhanced surfaces. The conclusions from this literature review included: (a) the range of test conditions were usually less than required for large, electricity-producing turbine engines, (b) the various correlations produce a 10 to 50 percent variation in heat transfer for the same geometric and flow conditions, depending on the experimental base used to derive the correlation, and (c) a large fraction of the data base available comes from evaluation of configurations proposed for particular cooling applications.

Impingement cooling has a complex flow associated with the heat transfer process. For flat smooth target plates, the flow under the impingement point has laminar-like flow with the heat transfer proportional to the Reynolds number to the 0.5 power. At locations where the jets interact, the flow is turbulent and the heat transfer increases as the Reynolds number to 0.7 to 0.8 power. The area applicable to each region varies with geometry and Reynolds number and probably accounts for the variations in the correlations, especially when used outside of the data range for each correlation. As pins or other surface enhancement geometries are employed, the flow and heat transfer become more complex with flow blockages, turbulence producing mechanisms, thermal conduction through nonuniform-thickness surfaces and locally varying heat transfer coefficients.

The approach for the present ATS Impingement Cooling Heat Transfer subtask of the Advanced Air-Cooled Blading Task was to conduct a parametric experimental heat transfer study with a limited number of enhanced surface configurations over the range of dimensionless parameters envisioned for ATS airfoil cooling applications. The acquisition of data with the appropriate flow parameters with enhanced surfaces allows the use of the data for design applications without extrapolation of results or the use of design correlations outside the range of applicable correlations. When feasible with the available 
air supplies, the range was extended to that required for combustor cooling applications. The heat transfer results from the enhanced surfaces were compared with results from a baseline target plate configuration and with results from other experiments published in the open literature.

The test section and data acquisition equipment used in these experiments were assembled and checked out on previous ABB sponsored impingement cooling heat transfer experimental projects. The design, fabrication and testing of the impingement heat transfer characteristics of three different enhanced target plate configurations and a baseline target plate configuration were conducted under this subtask.

For the DOE sponsored task and the subject of this report, heat transfer experiments were conducted with five (5) different target plate configurations:

- Baseline Target Plate (Copper flat plate)

- Target Plate A1 (Copper plate model with copper pins)

- Target Plate A2 (Copper plate model with Teflon pins)

- Target Plate B (Copper plate model with curvilinear pins)

- Target Plate C (Aluminum model with recessed dimples) For each target plate surface configuration, the heat transfer experiments were conducted with selected impingement orifice plate configurations and with selected spacings between the orifice plate and the heat transfer target plate.

The heat transfer results for the copper flat plate (Baseline) were in good agreement with a well recognized correlation for the flow regions used in the correlation but deviated from the correlation at higher Reynolds numbers. The results with the copper pins (Target Plate A1) showed modest increases of approximately 35 percent in heat transfer at lower Reynolds numbers, decreasing with increased Reynolds number. An analytical model and preliminary ABB-sponsored experimental study with a lower thermal conductivity test plate and pins showed that effective heat transfer coefficients less than the flat plate values could occur with pin surfaces at high Reynolds numbers. This decrease in heat transfer is attributed to the high heat transfer coefficients and the use of materials with lower thermal conductivity in turbine engines. Consequently, a pin geometry that has excellent pin effectiveness was selected for the fourth model (Target Plate B). The heat transfer performance from Target Plate B was approximately the same as from Target 
Plate A1 with less material required and improved castability. Consequently, this target plate configuration is expected to perform better with engine materials. Review of the results from the previous target plate configurations and the insight that additional surface area with high "pin" efficiencies are required for improved impingement cooling performance led to the design of a target surface with recessed dimples, i.e., hemispherical cavities, with a target surface area ratio of 1.8 for the impingement target surface. Although the effective heat transfer coefficients were 20 to 90 percent greater for Target Plate $\mathrm{C}$ compared to a flat plate depending upon the impingement target plate configuration, this configuration will require additional tests to determine optimum geometrical ratios. The Target Plate $B$ will be employed in new versions of $A B B$ turbine cooling systems.

\subsection{Task 8.4 Airfoil Casting Tests}

The ABB funded program elements in terms of Advanced air cooled castings have been suspended in February 1997.

\subsection{Task 8.5 Turbine Aerodynamics \& Aero-Thermal Integration}

The objective of this research was to quantitatively establish the impact of film cooling upon turbine stage performance through detailed experimental measurements. The work addressed the fundamental issue, resulting from the application of film cooling to high performance gas turbines, of how the interaction of the cooling stream with the main turbine through flow quantitatively impacts turbine aerodynamic performance. Extensive testing of a $1 / 4$-scale model a first low pressure turbine stage was conducted in the MIT Blowdown Turbine Facility on both the cooled stage and the equivalent uncooled stage. The goals of the effort were to make absolute performance measurements at $0.5 \%$ accuracy and relative measurements to $0.25 \%$. These goals were achieved, and for the first time on any high performance turbine, data was obtained at the detail and precision necessary to resolve the effects of configuration and operating conditions upon turbine stage efficiency. 


\subsubsection{Experimental Investigation of Cooled Turbine Performance}

Most previous investigations on the interaction of the coolant flow with the turbine main flow have been focused on the heat transfer problem. This is understandable given that small changes in the inlet temperature produce dramatic changes not only on the cycle performance but also on the structural integrity and life of the highly stressed turbine components. Achieving the desired balance between these competing factors is probably the most challenging task in the design process.

Comparatively little attention, however, has been focused in the past experimental investigations on how the coolant flow impacts the aerodynamic performance of the turbine and the consequent effects upon overall gas turbine system design. The reasons lay not in the fact that the problem is unimportant but in the level of complexity and cost of an experimental approach to the problem. Detailed experimental study of coolant flow interactions is impossible in the operational environment as a result of the extreme high temperatures and oxidizing conditions present in engine turbines. The technology to perform measurements at the required level of detail within this environment is well beyond our current capability.

This situation has changed dramatically over the past decade with the development of improved experimental techniques. The experimental investigations preformed under the present program were enabled by the introduction of short duration testing, and improved instrumentation technologies developed at MIT (the Blowdown Turbine Facility) and at other institutions located in the U.S. and other countries.

\subsubsection{Testing in Short Duration Facilities}

In the MIT facility, tests are performed on geometrically scaled models of actual engine turbine stages. Test conditions, (pressures, temperatures, shaft speed, etc.), are held at experimentally 'benign' levels for the measurements, but are set at the appropriate ratios to match the important non-dimensional parameters which govern the fluid physics, (e.g. stage pressure ratio, 
corrected speed, specific heat ratio, Reynolds number, gas to wall and coolant to gas temperature ratios, etc.). Thus set, these conditions provide an environment that accurately simulates the engine turbine operational environment. Through our knowledge of the fundamental physical scaling laws, the measured values of the dependent non-dimensional parameters (e.g., corrected through-flow, efficiency, heat transfer coefficients) will then match those occurring in the operational machine.

Short duration facilities also make use of the fact that the time scales of the flow through the turbine are of the order of the blade passage and rotor revolution times. These are typically in the range of 0.01 to 10 milliseconds. Thus any instrumentation developed to measure phenomena at these scales (e.g. shock, wake, and hot streak interactions), will only need a short time window to perform them. This is very important in testing high performance turbine stages, where, even under the scaled test conditions, the model turbine can produce substantial power (up to $\sim 2 \mathrm{MW}$ ). The overall energy consumption of a blowdown test is thus very low and leads to the advantage of performing flexible, low relative cost tests. The MIT facility can provide fully scaled conditions for 0.25 to 0.75 seconds, which is more than adequate for turbine heat transfer and aerodynamic performance investigations. In the past, these techniques have greatly added to our experimental knowledge of the fluid dynamic processes that govern heat transfer in cooled turbines and, under the present program, we have now obtained a first look into the detailed aerodynamic behavior of the cooled turbine.

This program offered several significant technical challenges including the construction of an accurately scaled, cooled test turbine stage and the development of instrumentation required to measure performance efficiency on a cooled stage in the short duration environment.

\subsubsection{Test Section Modifications and Airfoil Design and Manufacture}

To fit within the test section of the existing facility, the two-meter diameter ABB stage had to be reduced in scale by a factor of 4 . The existing shaft assembly and eddy current power absorber were retained but a new disk was required 
for the rotor. A new nozzle vane mount assembly and coolant manifolds were also required. The manifold provided three independent coolant feeds to the vanes, rotor and tip shroud respectively. ABB supplied the coordinates for the full-scale machine from which MIT designed the scaled airfoils using a stateof-the-art 3-D solid modeling package. Allowances for centrifugal and thermal growth were factored into the final design drawings and coordinate files for airfoil production. The operating conditions of the facility allowed the airfoils to be fabricated from an aluminum alloy, and the blade and vanes were produced using a 5-axis milling machine. Solid airfoils were produced for use in the initial series of un-cooled tests. After these test, the airfoils were modified to the cooled configuration.

\subsubsection{Film-Cooled Turbine Airfoils}

Construction of the cooling holes for $1 / 4$ scaled airfoils proved challenging. As a result of the requirement to maintain exact geometric similarity, the hole diameters were on the order of 0.008 inch. The film-cooled airfoils used in the test stage are shown in the figure below. The blade cooling configuration consists of five rows of coolant injection holes: two on the leading edge, one on the suction surface, one on the pressure surface, and one directly out the trailing edge. The vane has twelve rows of holes along the airfoils surface as well as holes arrayed on the hub and tip platforms. The internal passages were not directly replicated in the model blades and vanes. The blade cooling manifold channels proved to be challenging to fabricate. The 'bowed' leading edge of the blade required that the manifold be produced with two plunge EDM cuts, starting at the tip and hub respectively and properly aligning at midspan. The rear manifold employed a wire EDM process. Tolerances had to be maintained to several thousandths of an inch to avoid breaking through the blade surface. The laser drilling of the airfoil film-cooling holes also proved challenging because (at $1 / 4$-scale) their diameters were 0.008 ". The leading edge holes were especially difficult to fabricate due to their compound angles and close spacing. After consultation with $A B B$, it was decided not attempt the fabrication of the blade trailing edge row because the small trailing edge thickness at model scale. 


\subsubsection{Turbine Efficiency Measurement Instrumentation}

Turbine stage efficiency is commonly inferred by employing 'rake' or 'brake' measurements. In the 'rake' approach, total temperatures and total pressure measurement surveys, performed across the inlet and outlet of the stage, are used to infer the stage pressure ratio and the total enthalpy drop (i.e., actual specific work) across the stage. In the 'brake' approach, measurements of shaft power and turbine through flow are used to infer the actual work produced. For both techniques, the stage pressure ratio is used to infer the 'ideal' specific performance against which the actual performance is compared to estimate stage efficiency.

Although the rake approach has been extensively developed for short duration testing of un-cooled stages, the estimated uncertainties of this method were too high for use in film cooled turbine investigations and pointed to the need for an alternative approach. Further analysis showed that the brake method would satisfy the accuracy requirements. This led to an assessment and consequently the development of short duration torque and mass flow instrumentation for use in the present program. Torque was measured by modifying mounting assembly of the existing eddy current brake power so that load cells calibrated to $0.05 \%$ could establish the resultant torque. Mass flow was measured by the installation of an ANSI standard critical venturi flow nozzle, calibrated to $0.1 \%$, mounted downstream of the test turbine. The estimated measurement uncertainty of the overall system, including corrections for the non-adiabatic process inherent with short duration tests, was determined to be $0.45 \%$ for absolute efficiency measurements and $0.25 \%$ for relative measurements.

The MIT Blowdown Test Facility consists of a heated supply tank separated from the test section by a large diameter, fast-acting valve. The test section contains the 0.52-meter (20.4 inch) diameter test turbine stage and rotating assembly, including the eddy current brake power absorber and a 10KW drive motor. The test section throttle, used to set the test pressure ratio, then discharges through a critical flow venturi to a dump tank. The flow path 
upstream of the stage includes a turbulence generating screen, boundary layer bleeds, and a combustor like contraction.

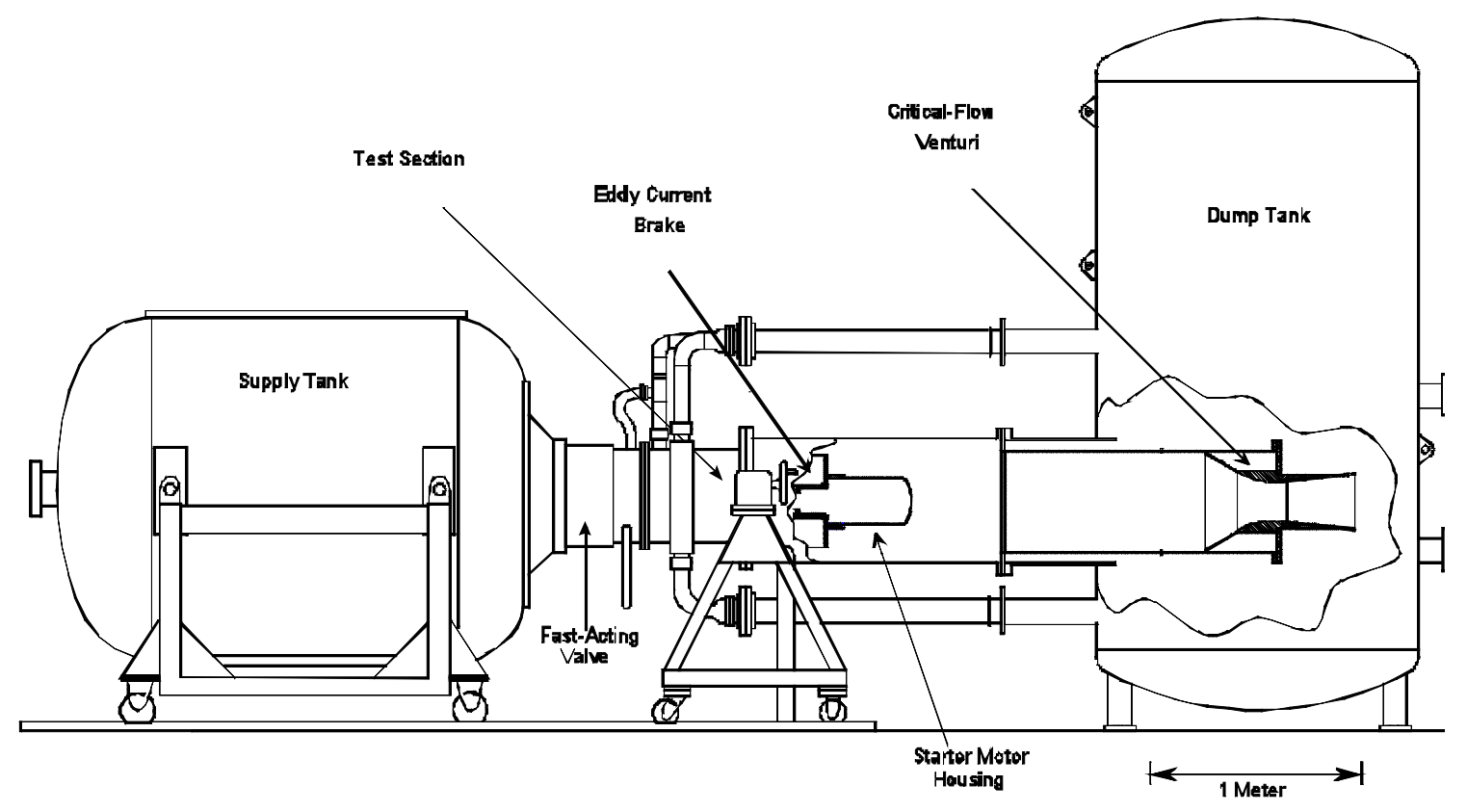

When operating, the entire facility is initially evacuated and the supply tank and fast-acting valve are heated to the initial run temperature. The valve is 
then closed and the supply tank is filled with either pure $\mathrm{CO}_{2}$ or a $\mathrm{CO}_{2} / \mathrm{Argon}$ mixture (used to reproduce the ratio of specific heats at the combustor exit of the engine). The turbine rotor is then brought up to the desired operating speed in vacuum. To start the test, the main valve is opened and the eddy current brake is energized simultaneously. After a startup transient of 300-400 milliseconds, the corrected speed and pressure ratio can be held to approximately $0.05 \%$ and $0.1 \%$ per rotor revolution over a duration of $400-500$ milliseconds. During this interval, the corrected speed and pressure ratio may be allowed to slowly vary by several per-cent to provide data over a range turbine operating points from a single test.

The facility has been designed to closely simulate all the non-dimensional parameters important to turbine fluid mechanics and heat transfer, including Reynolds number, Mach number, Prandtl number, Rossby number, the ratio of specific heats, corrected speed and mass flow, and coolant-to-gas and gas-tometal temperature ratios.

Experiments were first run on the un-cooled stage configuration to provide both a baseline database for the follow-on cooled tests and to confirm the accuracy and repeatability of the efficiency measurement technique under more easily modeled conditions. After fabrication of the cooled airfoils a second set of experiment were conducted. The test conditions covered a range pressure ratio, corrected speed, rotor tip gap spacing, gas to wall temperature ratio, coolant mass flow rate, and coolant to inlet temperature and momentum ratios. These test have provided the most detailed and accurate measurements to date characterizing the influence of film cooling upon turbine aerodynamic performance.

The challenges of making high accuracy turbine aerodynamic performance measurements in a short duration test facility have been met. Gas turbine research engineers and engine developers now have the ability to perform fully scaled tests on model turbines at a fraction of the cost of conventional steady state engine test rigs. The short duration test environment allows the use of instrumentation that can provide the spatial and temporal resolutions and measurement coverage necessary to enable a detailed probing of the flow field, which is unavailable in the harsh environment of operational engine turbines. The development, under this program, of the turbine torque and mass flow measurement techniques, along with the previously developed 
instrumentation for measuring turbine inlet temperature and pressure ratio, have enabled this capability. The program has provided the technology necessary to support a better understanding of the physics of cooled turbine flows and the capability to obtain much needed data for the validation of CFD codes and the development of improved design methodologies. More detailed results can be found in Appendix 3 


\subsection{Task 8.6 Advanced Steam Cooled Vanes}

The favorable heat transfer properties of steam admit the possibility of closed loop, fully internally cooled turbine components in environments which have traditionally required extensive internal and surface film cooling in air-cooled systems. This would include operation with the turbine inlet temperature in the $1750 \mathrm{~K}$ range. The favorable heat transfer to flow resistance ratios of steam should permit considerable enhancement of cooling passage internal heat transfer effectiveness in components, previously developed for convective cooling with compressor bleed air. The concept of closed loop cooling at the firing temperatures of interest here poses a number of formidable challenges from heat transfer, flow management/sealing, and structural/materials perspectives. The objectives of this effort are to generate a data base for steam cooled components and to conduct sub-component bench tests of critical cooling features and seals.

More detailed results can be found in Appendix 4.

\subsection{Advanced Air Cooled Turbine Engine Demonstration at Midland Cogeneration Venture:}

- The gas turbine performance in terms of power output and efficiency have been realized and in fact exceeded

- The performance and durability testing of the new turbine module has proved successful with all metal temperatures and pressures being within the design limits.

- Operating costs of Combined Cycle Power Plants can be diminished significantly by GT-Upratings and contribute to higher competitiveness, even if some additional investment is needed. 
ABB Power Generation Inc.

July 15, 2000

- The environmental impact of the uprating is favorable with reductions in Nox 


\section{Conclusions}

ABB has completed its technology based program. The results developed under WBS 8, concentrated on technology development and demonstration have been partially implemented in newer turbine designs. A significant improvement in heat rate and power output has been demonstrated.

$A B B$ will use the knowledge gained to further improve the efficiency of its Advanced Cycle System, which has been developed and introduced into the marked out side ABB's ATS activities. The technology will lead to a power plant design that meets the ATS performance goals of over $60 \%$ plant efficiency, decreased electricity costs to consumers and lowest emissions. 
ABB Power Generation Inc.

July 15,2000

\section{Appendix}

Appendix 1 TMF results

Appendix 2 Oxidation in a steam environment results

Appendix 3 Aero Thermal integration results

Appendix 4 Steam cooling 


\section{List of Abbreviations and Indices}

$\begin{array}{ll}\text { WBS } & \text { Work Break down Structure } \\ \text { TBC } & \text { Thermal Barrier Coating } \\ \text { RAM } & \text { Reliability, Availability and Maintainability } \\ \text { MIT } & \text { Massachusetts Institute of Technology } \\ \text { NOX } & \text { Nitrogenoxide } \\ \text { CO2 } & \text { Carbondioxide } \\ \text { CO } & \text { Carbonmonoxide } \\ \text { SOX } & \text { Sulfuroxide } \\ \text { TMF } & \text { Thermo Mechanical Fatigue } \\ \text { CFD } & \text { Computer Fluid Dynamics } \\ \text { BTU } & \text { British Thermal Unit }\end{array}$

Note: The list of used abbreviations and indices in the appendix are listed in the appendix itself. 
Appendix 1

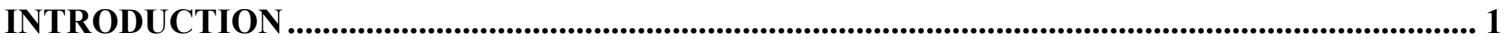

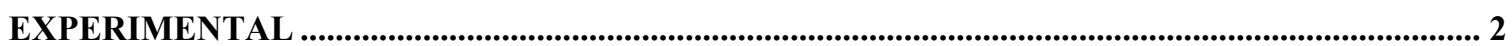

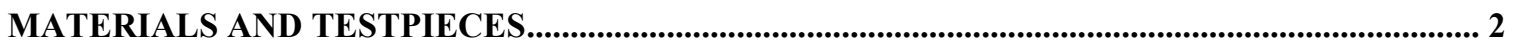

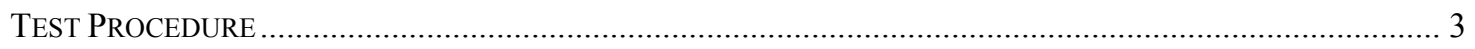

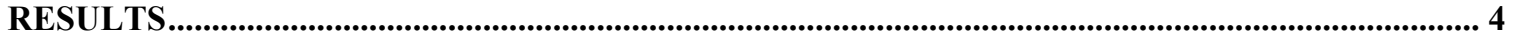

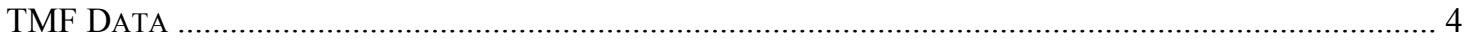

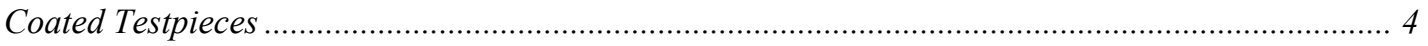

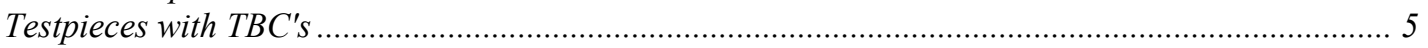

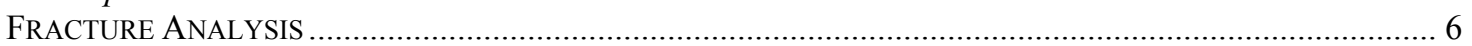

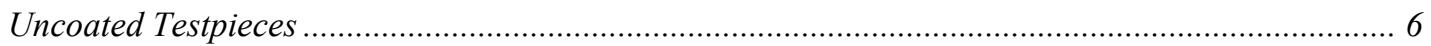

Testpieces with Coatings......................................................................................................... 7

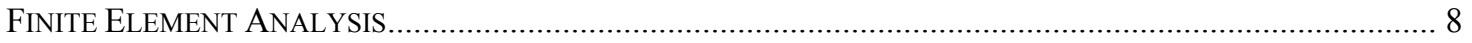

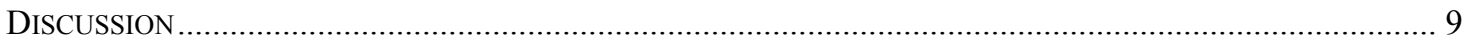

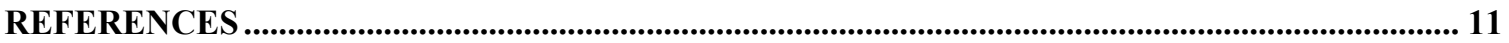




\section{Introduction}

When gas turbines for power generation operate in either cyclic or peaking modes, the cyclic stresses and temperatures involved can give rise to fatigue conditions in critical components such as blades and vanes. The most damaging form of fatigue is that due to the superimposed effects of variations in mechanical and thermal stresses i.e., thermal-mechanical-fatigue (TMF) and some effort has been made to quantify the effect of TMF on component performance (ref. G. Leverant). ${ }^{1}$

A further complication arises with coated components since overlay or ceramic thermal barrier coatings can be brittle at the lower temperatures and this will further reduce resistance to TMF. However, data on the effects of coating systems on TMF performance are limited and tend to be specific to certain combinations of substrate and coatings.

Accordingly a program of work has been carried out to evaluate the effects of various types of coatings on the TMF behavior of a single crystal alloy used for blades and vanes in the latest versions of $A B B$ engines and the results are presented in this report. The effects of overlay, diffusion and thermal barrier coatings have been determined and base-line data on the uncoated alloy has been obtained in similar conditions for comparison.

\section{Experimental}

\section{Materials and Testpieces}

The CMSX-4 alloy used in the test program was cast by Howmet Corporation as single crystal testpiece blanks with a crystal orientation nominally in the [001] direction along the axis of the bar. All testpiece blanks were heat treated in the standard "step-wise" procedure up to $1140 \mathrm{C}$ and in all cases a "post-coating" heat treatment was performed at $1080 \mathrm{C}$ for $4 \mathrm{~h}$. Subsequently a precipitation heat treatment was carried out in vacuum at $871 \mathrm{C}$ for $20 \mathrm{~h}$. Details of the compositions of the alloy and coatings are given in Table 1.

The hollow testpieces were machined to the dimensions showed in Figure 1 using an NC machine and according to the procedure recommended by DECRC, Heidelberg and which included a low-stress grinding procedure. 


\section{Test Procedure}

Testing was carried out by MarTest, Cincinnati, Ohio, a specialist testing organization. Servo-hydraulic machines were used Figures 2 and 3 in an "out of phase" fatigue cycle, i.e., one in which the maximum compressive load occurs at the maximum temperature and the maximum tensile load at the lowest temperature was applied to individual testpieces. Induction heating was used but testpieces with a ceramic thermal barrier coating were heated using a silicon carbide igniter method.

Strains were measured by linear-variable differential transducers attached to extensometers, which "touched on" to the testpiece gauge section and temperatures were measured by a thermocouple attached to the center of the testpiece by spring loading.

In order to calibrate the equipment testpieces were heated to $400 \mathrm{C}$ and to $1000 \mathrm{C}$ and a small load was applied at each temperature to enable the elastic modulus to be determined. Similarly a full thermal cycle was applied and the strain due to thermal expansion was determined. This information was then used as input data for the computer controlled test program to compensate for thermal strains so that only mechanical strains were applied to the testpieces.

The procedure for carrying out a TMF test involved heating from $50 \mathrm{C}$ to $400 \mathrm{C}$ under zero strain and from $400 \mathrm{C}$ to $1000 \mathrm{C}$ under compressive strains; on reaching $1000 \mathrm{C}$ the testpiece was cooled to $400 \mathrm{C}$ and subsequently to $50 \mathrm{C}$, again at zero strain. Both heating and cooling rates were linear at $10 \mathrm{C} /$ second for a metallic system (i.e. non-TBC coated). The cooling cycle involved passing high pressure air through the hollow testpiece until the set temperature was reached and for testpieces coated with TBC the furnace opened automatically on cooling to enable heat to flow from the coated surface. Typical hysteresis loops are shown in Figure 4.

A schematic representation of a typical strain-temperature-time profile is shown in Figure 5. The plastic strain, which occurred at the maximum temperature, resulted in a tensile stress being developed during cooling and returning to zero strains at $400 \mathrm{C}$. Upon further cooling from $400 \mathrm{C}$ to $50 \mathrm{C}$ the tensile stress normally increased, depending on test condition, due to the increased strength of the material at the lower temperatures. 


\section{Results}

\section{TMF Data}

The results obtained for uncoated testpieces of CMSX-4 with maximum cycle temperature of $1000 \mathrm{C}$ are shown in Figure 6 and cycles to failure increased by about two orders of magnitude for a decrease in total mechanical strain range of about $50 \%$ i.e., from $1.2 \%$ to $0.5 \%$. The data (one test was terminated before fracture) can be described by a relationship of the form:

$$
N_{f}=A \cdot \Delta \varepsilon^{n}
$$

where:

$$
\begin{gathered}
=\text { the mechanical strain range, } \\
A=\text { a constant }(=458.55 \text { in this case }), \\
n=\text { constant }(=-6.15)
\end{gathered}
$$

and although the data are not extensive the variability is small and comparable to that observed in low-cycle fatigue testing ${ }^{2}$.

The effects of increased maximum cycle temperature and of hold times of five minutes at $1000 \mathrm{C}$ and $1050 \mathrm{C}$ are shown in Figure 7 and due to the restricted nature of the test program only two testpieces were available for each condition. However, it is evident that, with the exception of one result, increased maximum temperature and a hold-time of five minutes in the cycle reduced the number of cycles to failure. The maximum effect was for a hold time of five minutes at $1050 \mathrm{C}$ when the number of cycles to failure was reduced by about an order of magnitude compared with the baseline data. In Figure 8, results from DECRC, Heidelberg on the comparable alloy MK4 are shown for comparison with data from this investigation using the $2 \%$ load drop criterion. It can be seen that for the two tests carried out on materials cast in the <001> direction, the TMF performance was slightly better than that for CMSX-4 but the cooling rate was slower for the MK4 test pieces (3.7 C/second) so that greater TMF life would be expected. The lower modulus material showed reduced TMF resistance.

\section{Coated Testpieces}

The results for testpieces with either MCrAlY type overlay coatings or with aluminide-type diffusion coatings are shown in Figure 9, where it is evident that in every case the coated testpieces show reduced TMF-life for a given strain range compared to the uncoated alloy and of the various coatings SV-20 showed the greatest reduction, i.e., about an order of magnitude less than for the baseline 
data at a strain range of $0.6 \%$. SV-34 coatings showed the best results for and overlay (LPPS) coating.

Since both coatings have similarly low levels of ductility at lower temperatures the superior performance of SV-34 is presumably due to the higher strength of this Re-containing coating.

The surprising feature of these results is the relatively good performance of the testpieces with aluminide coatings including those with SV-20 over-aluminided with SiPdAl, particularly at the higher strain ranges. The aluminide coatings are thinner so that when the cracks form during testing they are shorter than the cracks in the overlay coatings and the stress intensity at the crack tip is presumably lower. Thus a higher stress may be needed to propagate the crack into the substrate in this case. Another point is that the aluminide coatings may have a closer thermal expansion mismatch with the substrate, and it has been claimed $^{3}$ that the practice favored by GE of overcoating MCrAIY's with aluminide has improved TMF life.

The number of cycles to a $2 \%$ load drop is used as a failure criterion and may be correlated with the number of cycles to crack initiation and data based on this failure criterion are shown in comparison to cycles to failure in Figure 10 and trends similar to those mentioned above were observed in this case also. Significantly the data for both failure criteria were almost coincident for the uncoated alloy indicating perhaps that crack growth was an insignificant component of the failure process.

\section{Testpieces with TBC's}

The results of the limited amount of testing carried out on testpieces with TBC coatings are shown in Figure 11 and comparison can only be made for a strain range of about $1 \%$. The surprising observation is that a 300- $m$ TBC applied by plasma spray showed cyclic lives close to that of the uncoated baseline alloy. This was also true for one of the testpieces with an EB-PVD ceramic coating although two other testpieces with this type of coating showed cycles to failure reduced by a factor of about five compared with the uncoated alloy. The thicker, layer, i.e., 1-mm of TBC resulted in a slightly reduced TMF life in comparison with the baseline data. Possible explanations for the behavior observed are indicated below, viz.

- the heating and cooling rates were slower for the TBC coated testpieces than for the other testpieces so that the thermal fatigue loading would be reduced; 
- the test was controlled by temperature measurement at the surface of the testpiece but the presence of the TBC would reduce the temperature of the underlying metal and this may have been the dominant factor. This has been confirmed at DECRC Heidelberg where thermocouples have been attached to the metallic bond coat and sprayed with TBC. The lower bond coat surface temperature results in longer lives.

Service experience shows that the presence of a TBC on turbine components will improve TMF life so that the data reported here are consistent with engine behavior. However, data on service performance is for the most part limited to base-loaded units and the type of TMF test carried out in the present program is more relevant to peaking or cyclic behavior and this was reflected in the fracture mechanism. Failure of components with TBC's in base-loaded units involves spalling of the TBC prior to cracking. As the components become available from peaking and cycling units comparison will be made with the fracture behavior observed here to determine the relevance of the TMF test to service performance. However, in at least one case components from a peaking unit show cracking in the bond coat below the TBC, i.e. consistent with the fracture mode reported here. The failure mechanism will be discussed further in a later section of the report.

\section{Fracture Analysis}

\section{Uncoated Testpieces}

Multiple cracking was observed in the uncoated testpieces at the various strain ranges tested, and generally, cracks initiated from both outside and inside surfaces of the testpiece and subsequently propagated into the substrate perpendicular to the surface. Extensive secondary cracking was observed, as can be seen in Figure 12 and was greatest on the internal surface. Occasionally a secondary crack oriented 45 to the specimen surface was observed (Figure 13), and small multiple cracks were often present on the surface. Figure 14 shows a typical fracture surface of a broken testpiece, and a surface SEM image of cracking in a testpiece fractured at a strain range of $1.0 \%$ is shown in Figure 15 and multiple surface cracking is evident. These cracks appear to initiate at discrete points on the surface. Secondary cracks to a depth of 595- $\mathrm{m}$ were observed on a testpiece fractured at a maximum temperature of $1000 \mathrm{C}$ and a mechanical strain of $0.85 \%$.

The number of secondary cracks did not appear to be a function of the strain range, but at the lower strain ranges, the surface cracks appeared more open. 
This may be related to the fact that the mean stress associated with a small strain amplitude is higher than that for larger strain ranges, although the stress amplitude is smaller for lower strain ranges. Figure 16 shows a cross section of a specimen tested at $0.45 \%$ strain and it can be seen that the surface crack is open and becomes narrower at the base. Figure 17 shows a SEM image of the surface crack tested at a strain of $0.65 \%$.

\section{Testpieces with Coatings}

For testpieces coated with SV-20, much larger circumferential cracking was present than for uncoated materials, as shown in Figure 18 and generally cracks initiated in the coating. A depleted zone was observed around these circumferential cracks indicating that oxidation had occurred even though dwell time at the maximum temperature was short. Cracks became narrower and more perpendicular to the axis of the bar after propagating into the substrate (Figure 19). Generally very few cracks were observed on the inside surface of the testpieces.

The number of secondary cracks observed was greater at the lower strain ranges and the degree of penetration into the substrate was greater. For example, at $0.45 \%$ strain a total of 95 secondary cracks was observed and the deepest crack penetrated $660 \mathrm{~m}$ into the substrate. At a strain of $0.65 \%$, a total of 17 cracks was observed at the outside surface with the deepest crack penetrating to a depth of $210 \mathrm{~m}$. In addition, the morphology of cracking was different at the lower strain ranges. A somewhat larger coating depleted zone was observed with some crack branching in the coating, and a higher incidence of cracks penetrating into the substrate at an angle to the surface. In addition other small cracks were observed that did not penetrate and were blunted in the coating.

\section{Pt-Al}

In this case many of the secondary cracks did not penetrate through the coating but were blunted at the diffusion zone (Figure 20). For equivalent test conditions there were considerably fewer secondary cracks in the platinum aluminide system than in testpieces coated with SV-20 and several of these had penetrated much further into the substrate. Surface cracking was not extensive except close to the fracture surface as shown in Figure 21. 


\section{SV-20 + SiPdAl}

Many more secondary cracks were observed at higher strains in these testpieces than in the comparable testpieces coated with SV-20. This may have resulted in increased lives in that cracks did not reach critical lengths until later stages.

\section{TBC}

As mentioned in section 3.3 these testpieces did not fail in the manner typical of that normally seen in base-loaded gas turbines i.e. oxidation of the bond coat and cracking in the TBC just above the oxide leading to spallation of the TBC. Some of the cracking observed was in the bond coat below the TBC, which was intact. This type of cracking has been observed on occasion in engines, which had operated in peaking mode and had accumulated many cycles, but there is generally insufficient information on the fracture mechanism of engine-run parts in these conditions. In the testpieces examined here some of the cracks appeared to be emanating from the "rough surface" of the bond coat (Figure 22).

\section{Finite Element Analysis}

A finite element (FE) analysis was performed as part of a "Small Business R\&D Project sponsored by NASA by DCT Technologies, a consulting engineering company located in Cleveland, and Figure 23 shows a schematic representation of the FE model. The model shows that the crack will initiate at the interface under the influence of a radial tensile stress. The computational results also showed that the maximum tension in the coating was concentrated at the peak region near the interface, and Figure 24 presents the axial strain profiles as a function of time for the bulk of the testpiece. Figure 25 shows the corresponding radial stress at a peak region in the TBC for a $1.0 \%$ mechanical strain range and it can be seen that the radial stress is negative or compressive in nature at the early stage of testing. As the test continues there is a gradual build up of tensile radial stress, and it is evident that for the testpiece fractured at a mechanical strain range of $1.0 \%$ the maximum radial stress changed from zero to approximately $150 \mathrm{MPa}$ in less than 2,000 seconds. The analysis also revealed that the radial stress reached a maximum value at the end of each cycle as the axial compression was released and the specimen was cooled. It should be noted that in this analysis no specific failure criterion was established for the TBC, and the process of crack formation and subsequent propagation was not analyzed. However, some indications of the failure mechanism can be 
determined from analysis of the behavior of testpieces coated with TBC which were exposed in a burner rig and which have also been analyzed by the FE method. In this case cracks form above the peaks in the bondcoat $t$ and the FE calculations predict that the stresses in the valleys change from compression to tension during exposure in the burner rig and cracks then propagate from peak to peak through regions of tensile stress until fracture occurs.

It should be noted that the overall radial stress distribution in the TMF tests is somewhat different from that produced during exposure in the burner rig. First, there is little oxidation in the TMF test so that oxide growth stresses are small. Second, the maximum radial stress above the peaks is predicted to be lower in the TMF test (200 MPa) than in the burner rig $(275 \mathrm{MPa})$. Therefore it is likely that the compression band in the valleys is much larger in the TMF tests and hence the likelihood of failure in the TBC is lower. Figures 26 and 27 show that the valleys remain in compression between the peaks and the crack when formed above the peak is not likely to propagate into the valley regions leading to failure of the TBC. This behavior is shown in Figure 22 where failure occurred in the bond coat by cracking below the ceramic in the bond coat and subsequent propagation into the substrate.

\section{Discussion}

The ability to predict the effect of TMF on the life of turbine components is an important requirement for the efficient design of blades and vanes in modern engines. However the complexity of the interactions between the deformation and fracture processes and the difficulty of quantifying the effects of operating parameters thereon has limited the extent to which appropriate predictive schemes have been developed. In the present investigation the main objective was to evaluate the effects of various coatings systems on TMF behavior and the principal variable was strain range and the influence of variations in temperature and hold time were not investigated, except to a small extent on uncoated test bars.

However, a limited number of tests at various temperatures and with hold times included has been carried out on the uncoated material and these results were used to evaluate a simple model proposed by Bernstein ${ }^{4}$. The model can be described by the following relationship

$$
N_{f}=A \Delta \varepsilon_{m}{ }^{\alpha} \sigma_{\text {max }}^{\beta}
$$

where: 


$$
\begin{aligned}
& \Delta \varepsilon_{\mathrm{m}}=\text { Mechanical strain range applied } \\
& \sigma_{\max }=\text { Maximum tensile stress at saturation }
\end{aligned}
$$

We have modified the equation slightly to reflect the difference in definition of $\mathrm{N}_{\mathrm{f}}$, which represents the number of cycles to failure in the present investigation whereas $N_{f}$ represented cycles to crack initiation in Bernstein's original equation.

The results of this analysis are shown in Fig 28, and it can be seen that the lives predicted by the Bernstein equation are in reasonable agreement with the experimental data. Within the strain range tested the predicted lives were within a factor of two of the experimental data and the level of agreement is consistent with that normally observed for low-cycle fatigue data which is considered more amenable to analysis.

A slightly modified version of this equation has been used at DECRC Heidelberg and in this case the relationship is of the form:

$$
N_{f}=A \Delta \varepsilon_{m}{ }^{\alpha} \Delta \sigma^{\beta}
$$

where is the stress range.

The results for the uncoated alloy have also been plotted in accordance with equation (3) in Figure 28, and again reasonable agreement is obtained. The advantage of this approach is that modulus changes due to variation in orientation of the single crystal, which affect the stress range, are incorporated.

\section{Conclusions}

1. Coated testpieces of CMSX-4 showed reduced TMF resistance compared with the uncoated alloy in "out-of-phase" testing at various strain ranges.

2. Testpieces coated with SV-34 showed the smallest reduction in TMF resistance and aluminide overcoats also performed well, particularly at the higher strain ranges.

3. Increased maximum temperatures and the introduction of hold times both reduced TMF resistance.

4. The TMF resistance of testpieces coated with thermal barriers was comparable to that of the uncoated alloy presumably because of the effect of the TBC in reducing metal temperatures. 
5. In most cases failure occurred as a result of the propogation of cracks that initiated in the coating and in testpieces with TBC's oxide growth made little contribution to the failure process. Perhaps a longer dwell time at the maximum temperature would have changed this.

6. The results for the uncoated testpieces were reasonably consistent with the predictions of a simple model.

7. This type of test probably represent the behavior in cyclic or peaking conditions but does not reproduce failure mechanisms in base loaded unit.

\section{References}

1. G. R. Leverant, T.E. Strangman, and B.S. Langer, Superalloys: Metallurgy and Manufacture, $3^{\text {rd }}$ Internation 'Symposium Seven Springs Pa. 1976 285295

2. G. B. Thomas and R. K. Varma, Review of BCR-VAMAS low cycle fatigue intercomparison program, in "Harmonisation of Testing Practice for High Temperature Materials", M.S. Loveday and T. B. Gibbons, Eds., 1992, Chapter 8, Elsevier Science Publishers, London.

3. G. R. Leverant, Private communication

4. H. L. Bernstein, Life Management System for General Electric Frame 7E Gas Turbine, R. Viswanathan and J. M. Allen., eds Life Assessment and Repair Technology for Combustion Turbine Hot Section Components, ASM International, Materials Park, Ohio, 1990, 111-118

5. W. Hartnagel, Private communication 
Appendix 1

Table 1: Composition of coatings tested (wt.\%)

\begin{tabular}{|c|c|c|c|c|c|c|c|c|}
\hline Coating & $\mathrm{Cr}$ & $\mathrm{Co}$ & $\mathrm{Al}$ & $\mathrm{Y}$ & $\mathrm{Si}$ & $\mathrm{Ta}$ & $\mathrm{Re}$ & $\mathrm{Ni}$ \\
\hline SV-20 & 25 & - & 5.5 & 0.5 & 3.0 & 1.0 & - & Bal. \\
\hline SV-23 & 16 & 18 & 8.0 & 0.5 & 2.0 & 0.5 & - & Bal. \\
\hline SV-34 & 13 & 24 & 11.5 & 0.3 & 1.2 & 0.5 & 3.0 & Bal. \\
\hline
\end{tabular}


Appendix 1
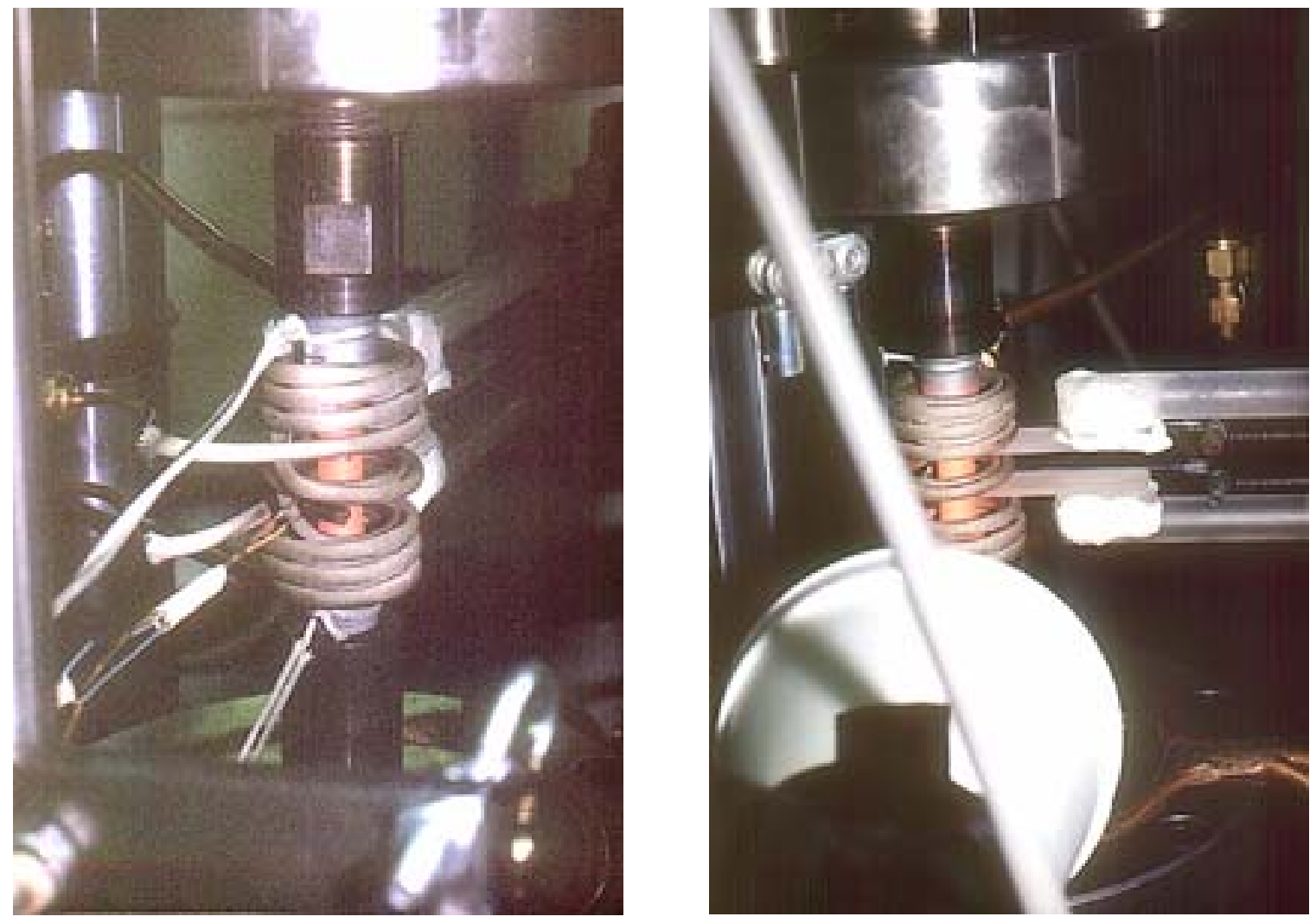

Figure 1: Picture of CMSX-4 single crystal bars undergoing TMF testing. 
Appendix 1

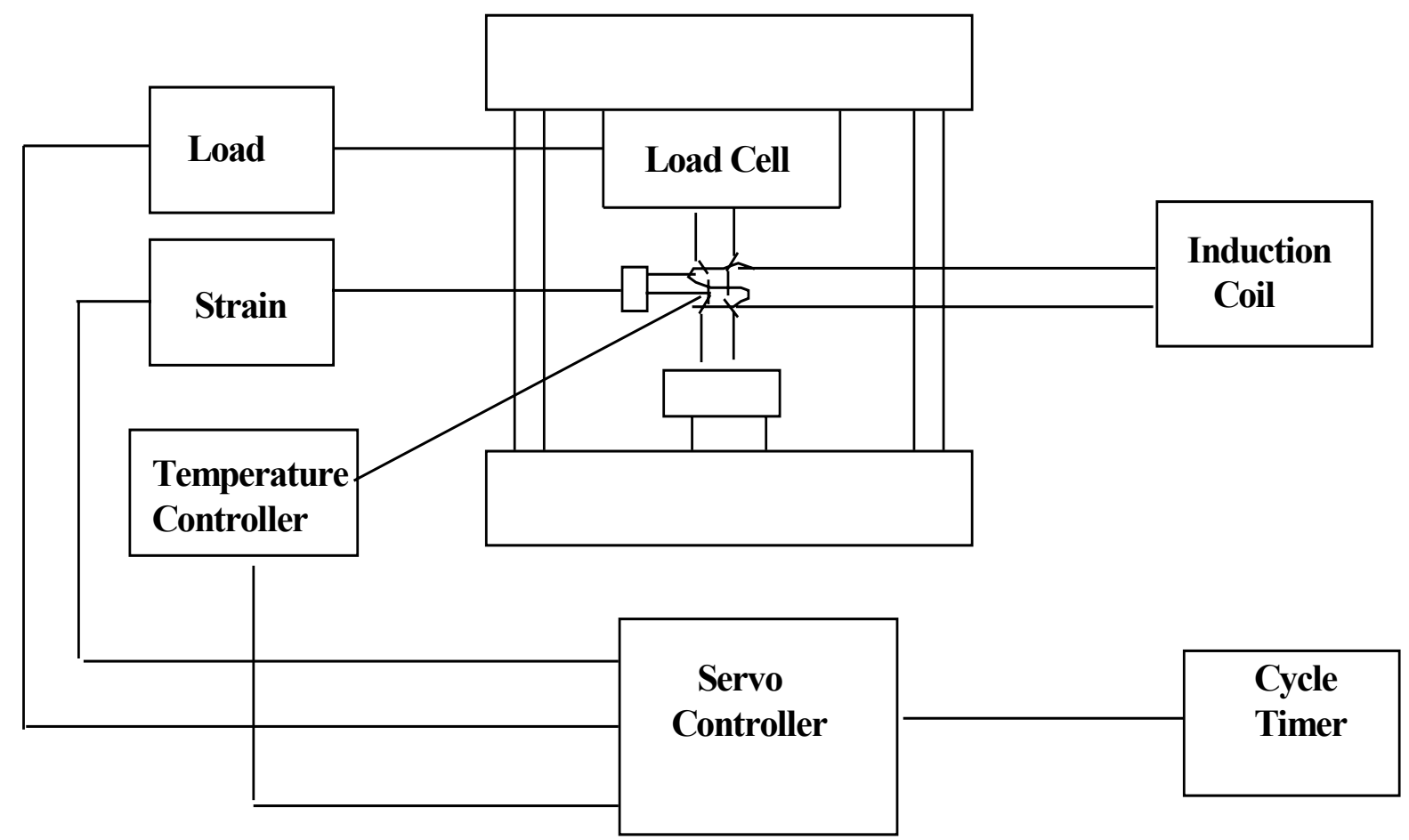

Figure 2: Control system used for TMF testing of metallic systems. Testing of ceramics employed a resistance furnace, which heated the test bar by radiation. 
Appendix 1

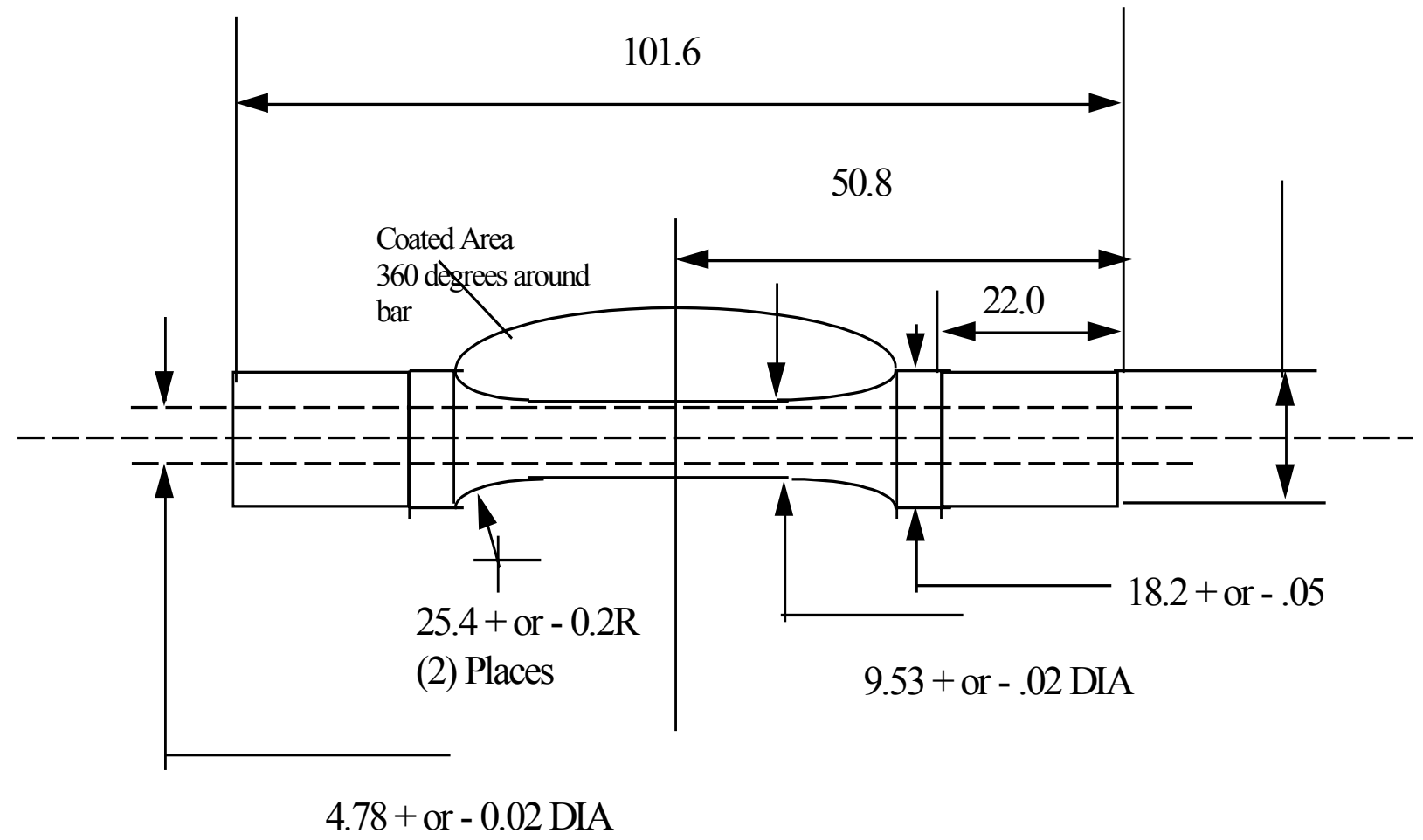

All dimensions in $\mathrm{mm}$

Not Drawn to Scale

Figure 3: Thermal mechanical fatigue test bar configuration. 
Appendix 1

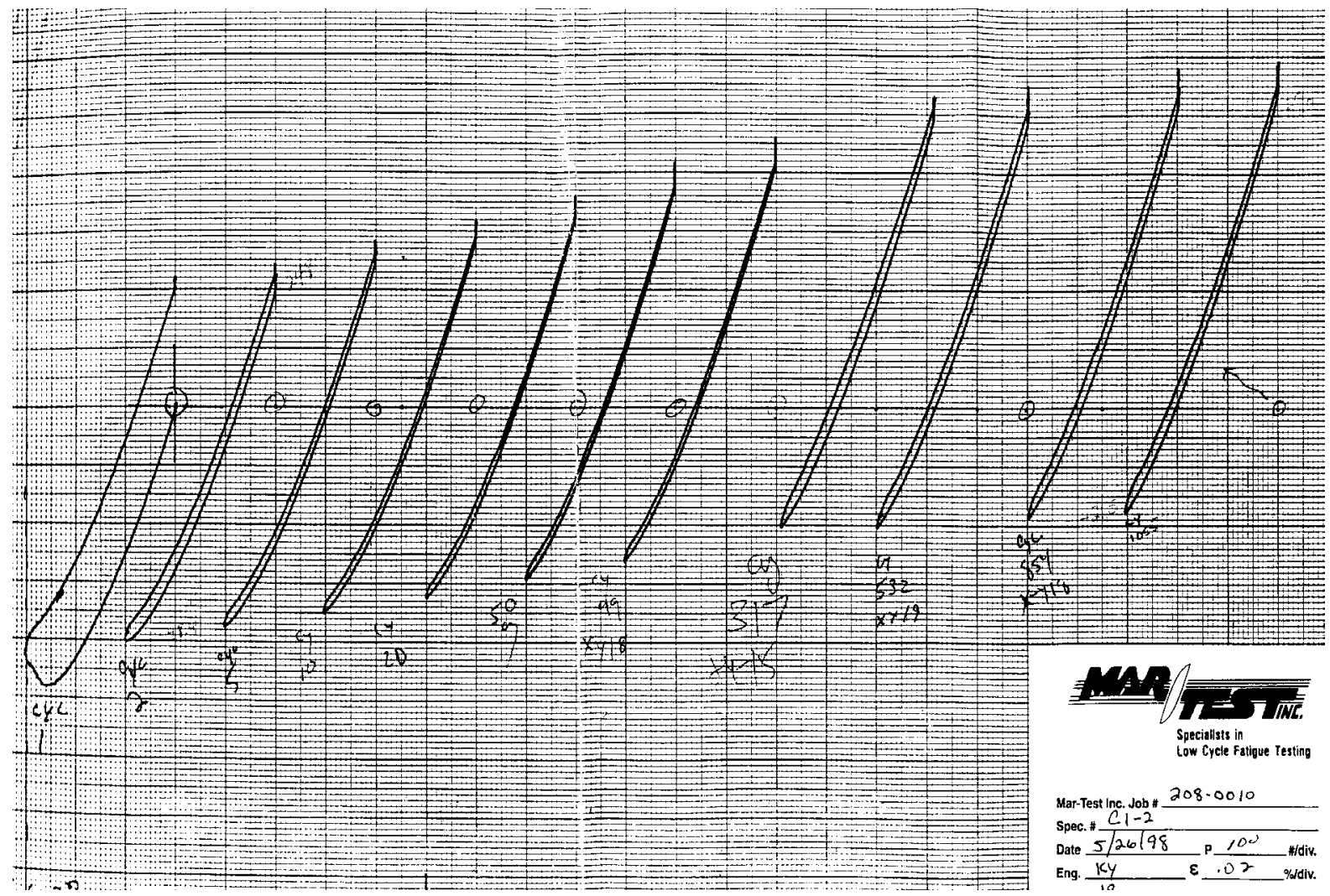

Figure 4: Typical hysteresis curves for TMF testing. (Note increasing tensile load with cycles) 
Appendix 1
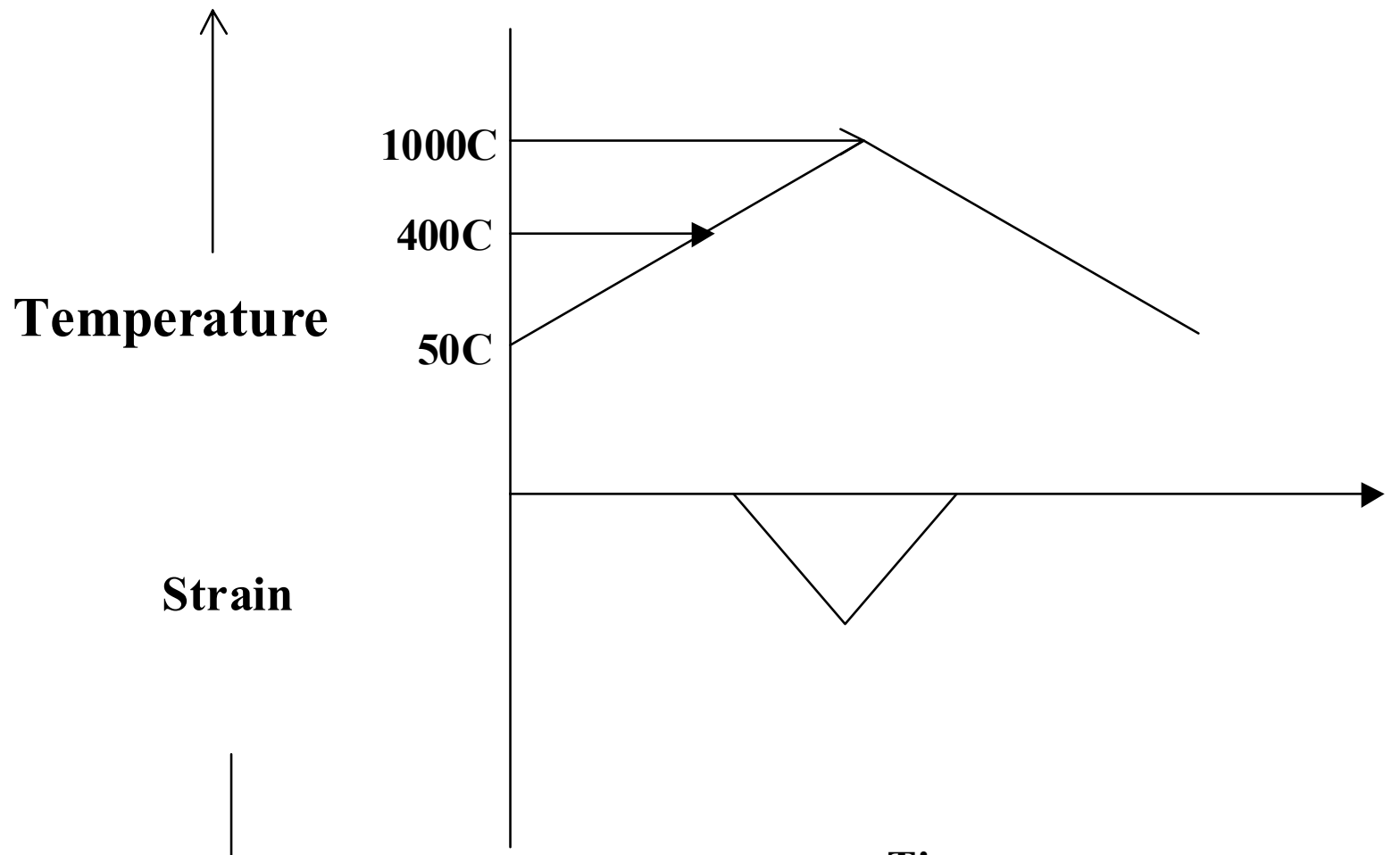

Time

\section{Strain}

$$
\downarrow
$$

Figure 5: Typical temperature strain cycle for "Out of Phase" TMF test, with a compressive hold at the maximum temperature. 
Appendix 1

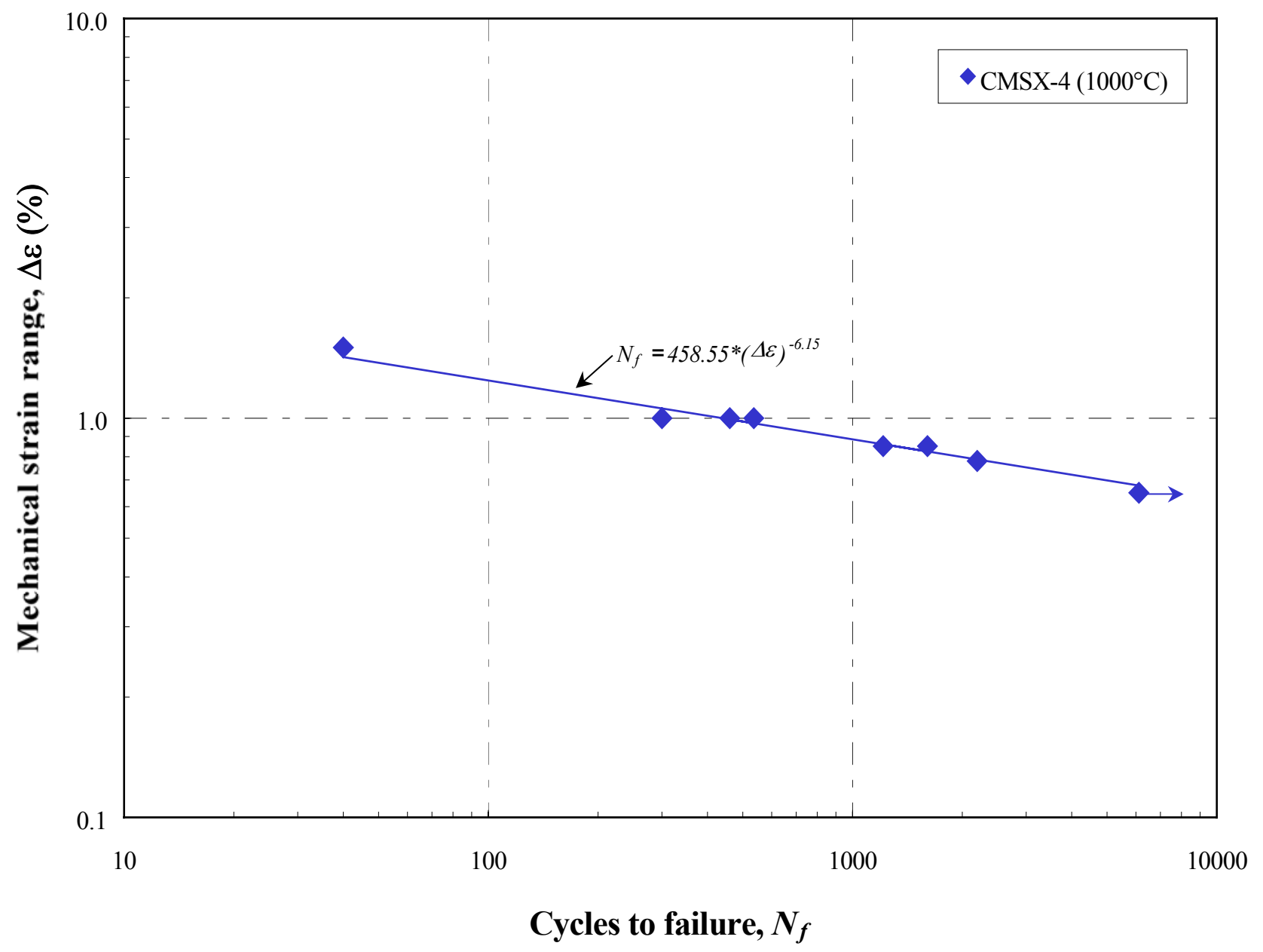

Figure 6: CMSX-4 baseline TMF data. 
Appendix 1

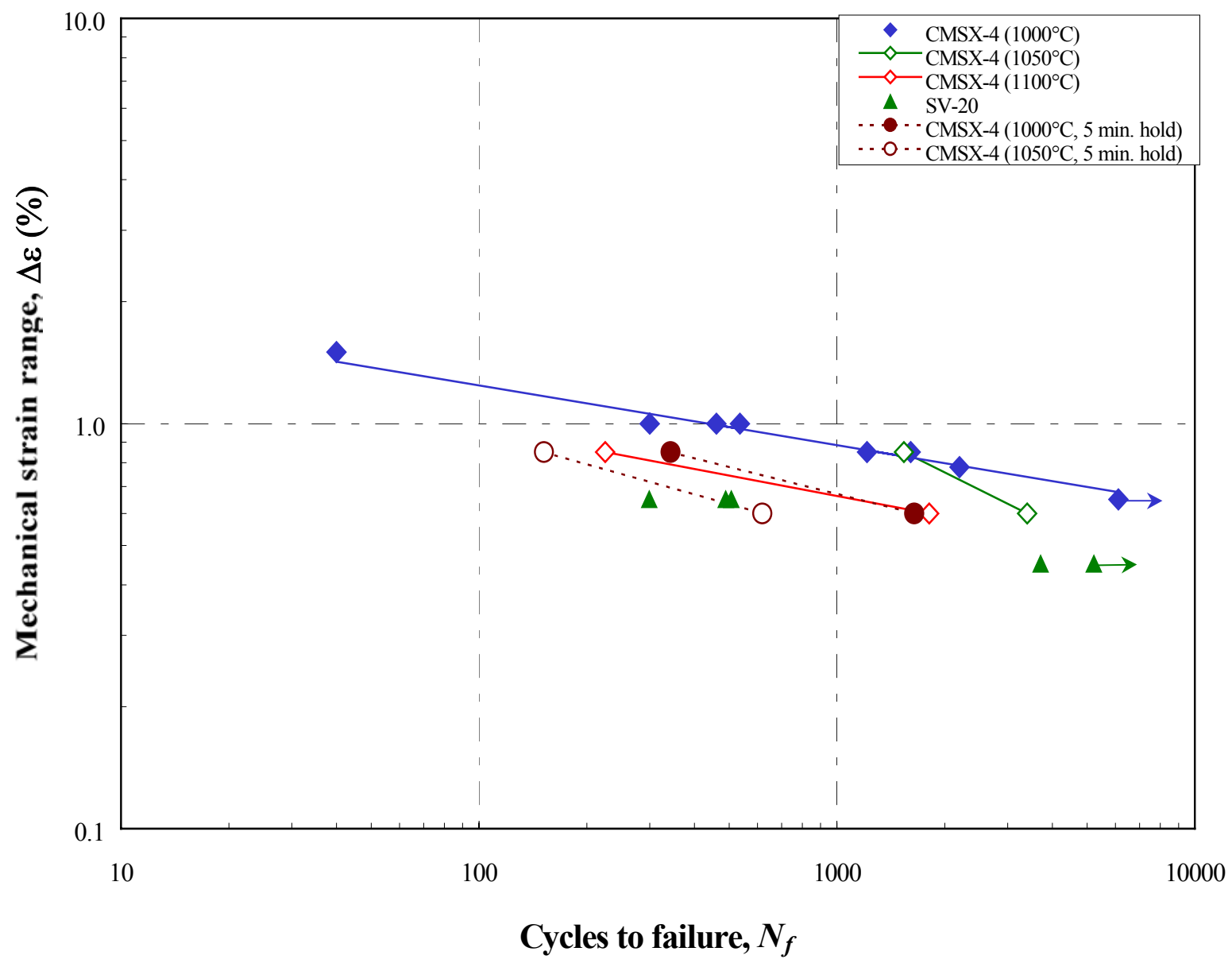

Figure 7: TMF data showing cycles to failure as a function of mechanical strain, temperature, and hold time. 
Appendix 1

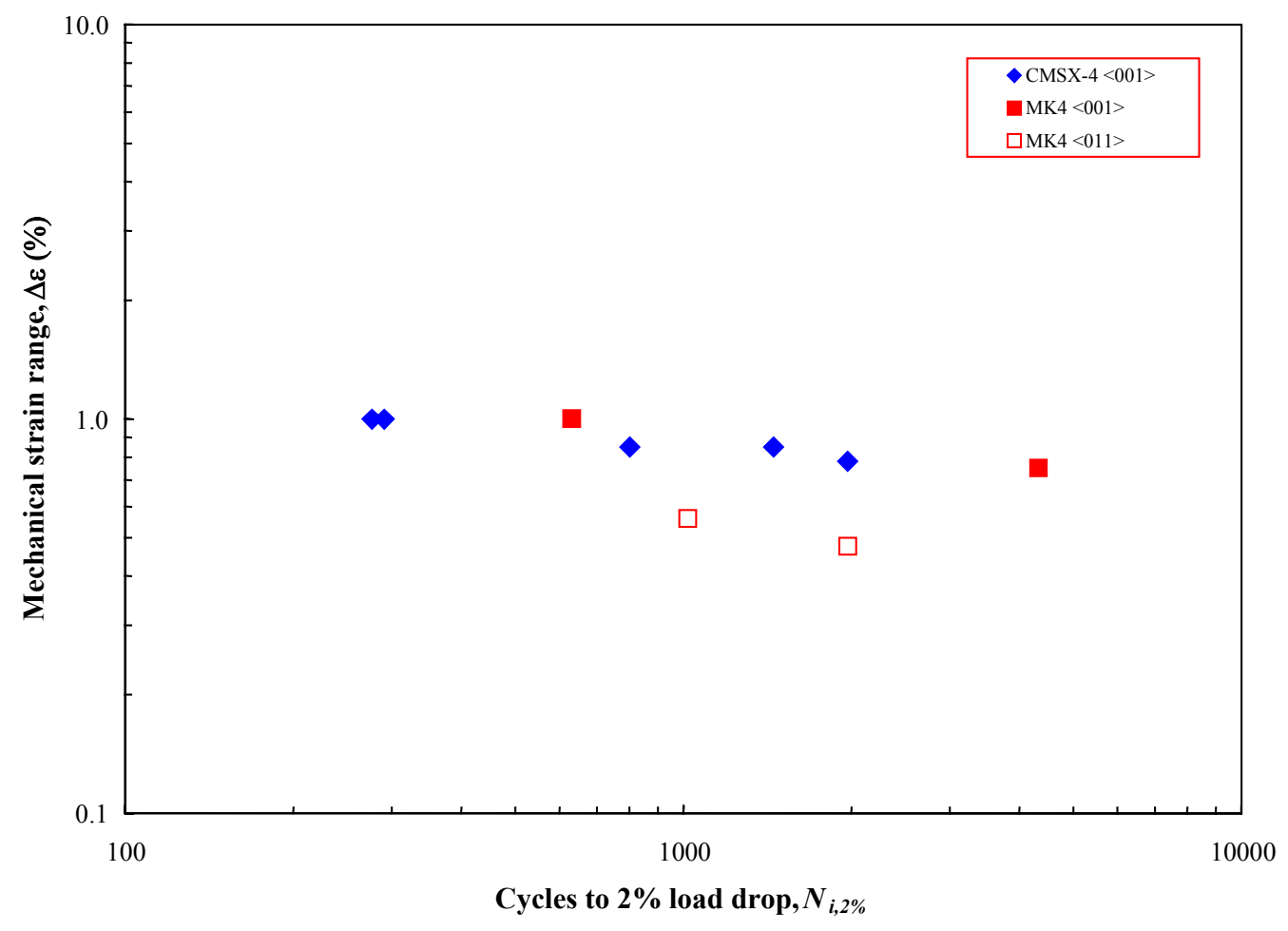

Figure 8: Cycles to $2 \%$ load drop as a function of mechanical strain for CMSX-4 and MK4. 
Appendix 1

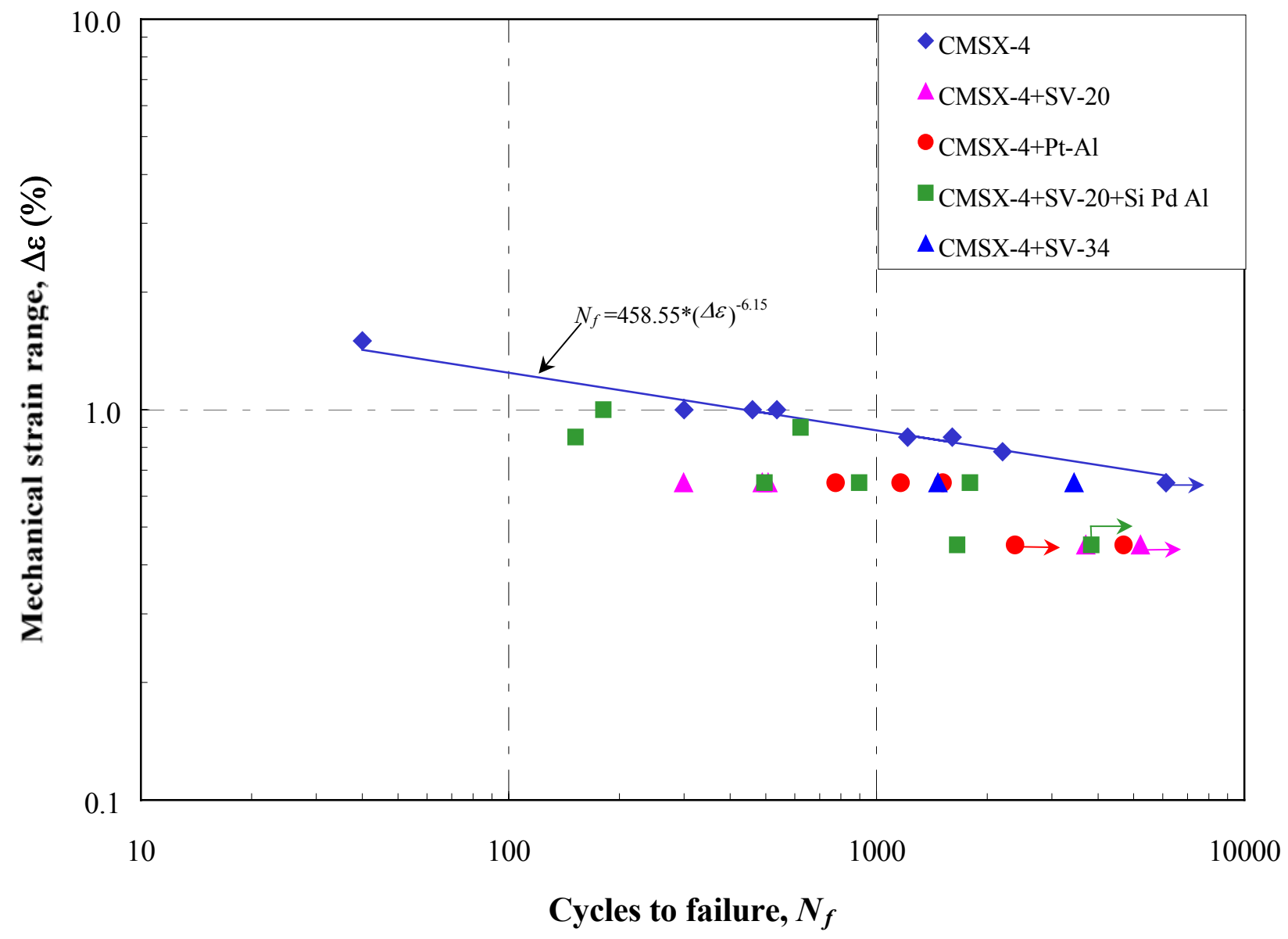

Figure 9: TMF life of metallic coating systems compared to CMSX-4 uncoated baseline. 
Appendix 1

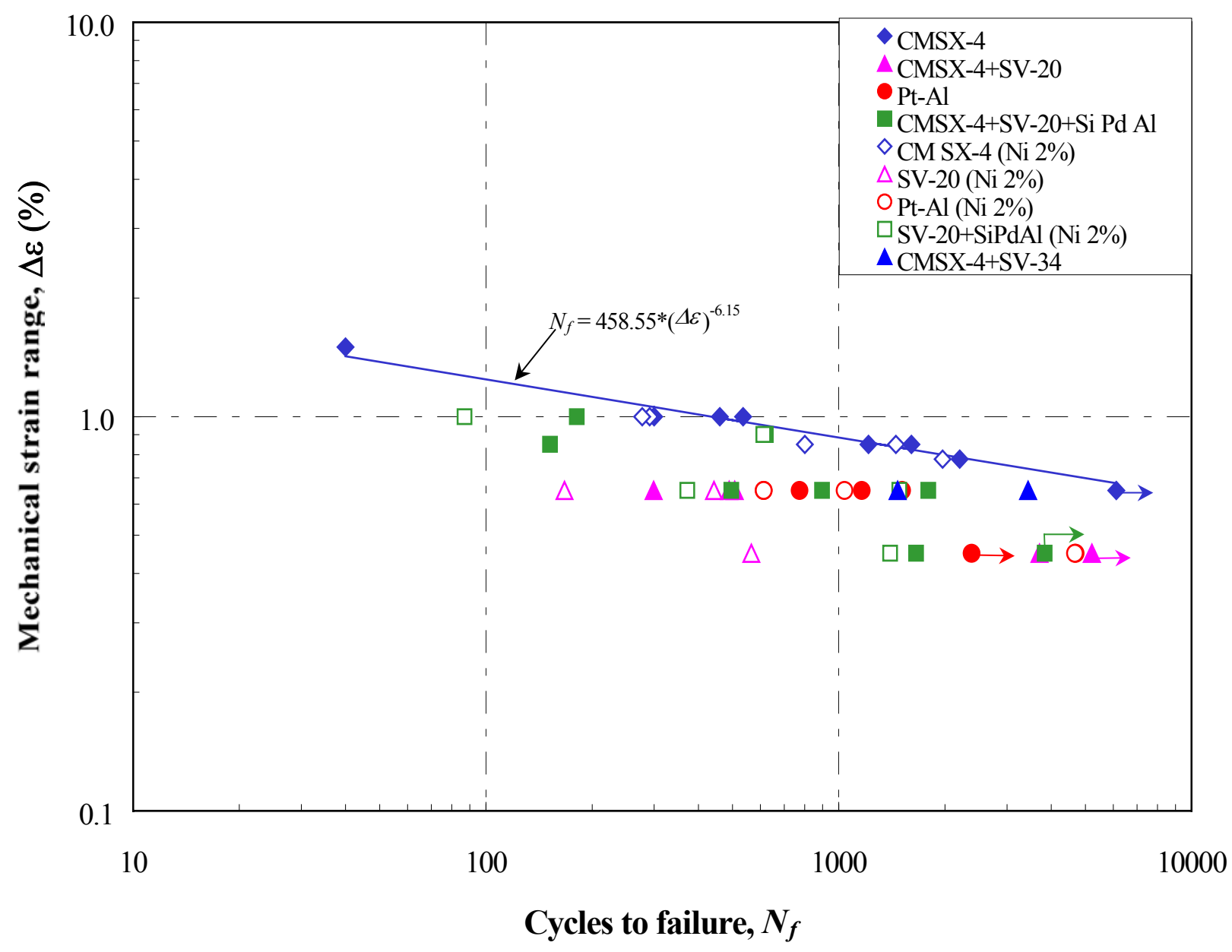

Figure 10: Cycles to failure as a function of mechanical strain. Comparison between cycles to failure and cycles to $2 \%$ load drop. 
Appendix 1

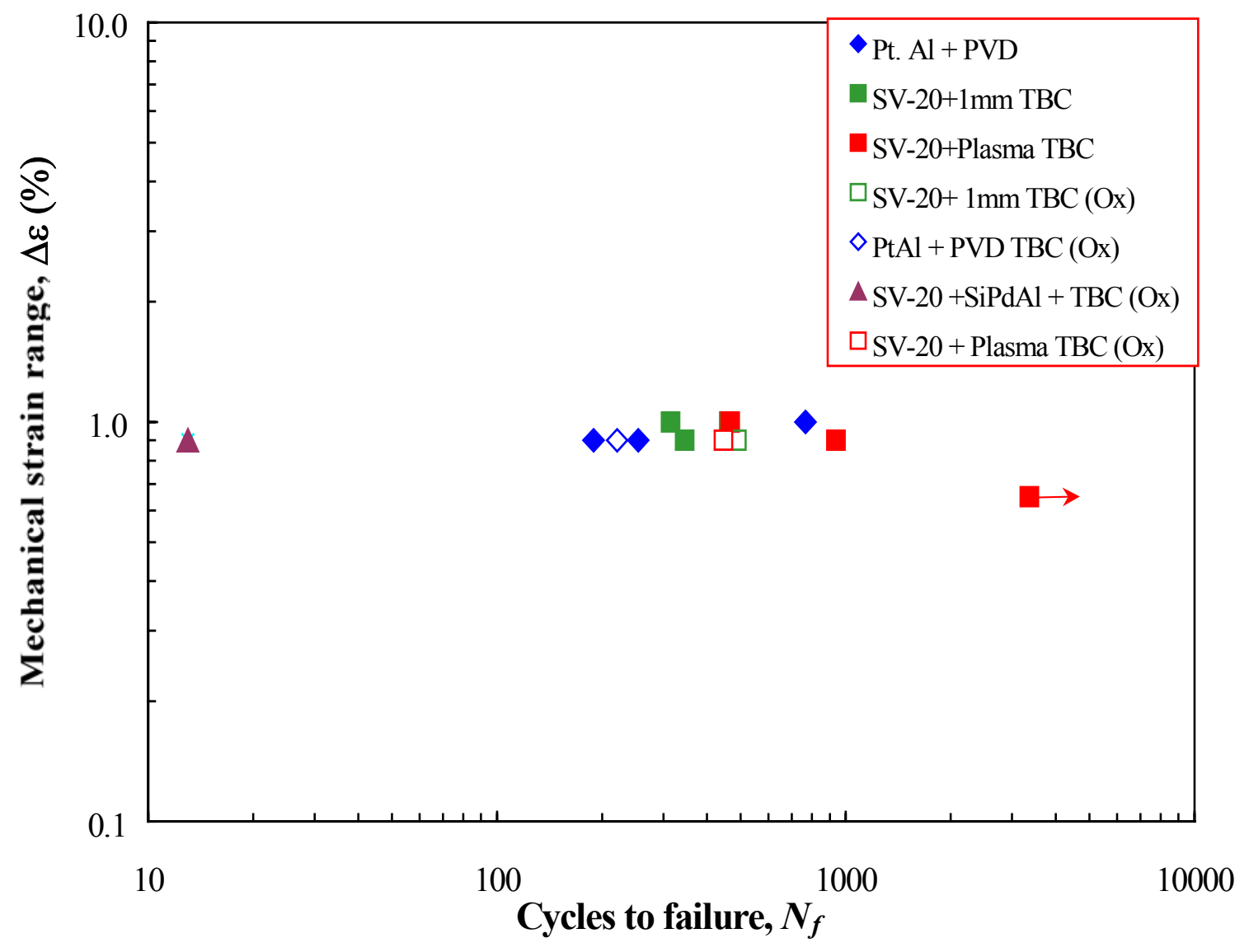

Figure 11: TMF data for TBC coated systems. 

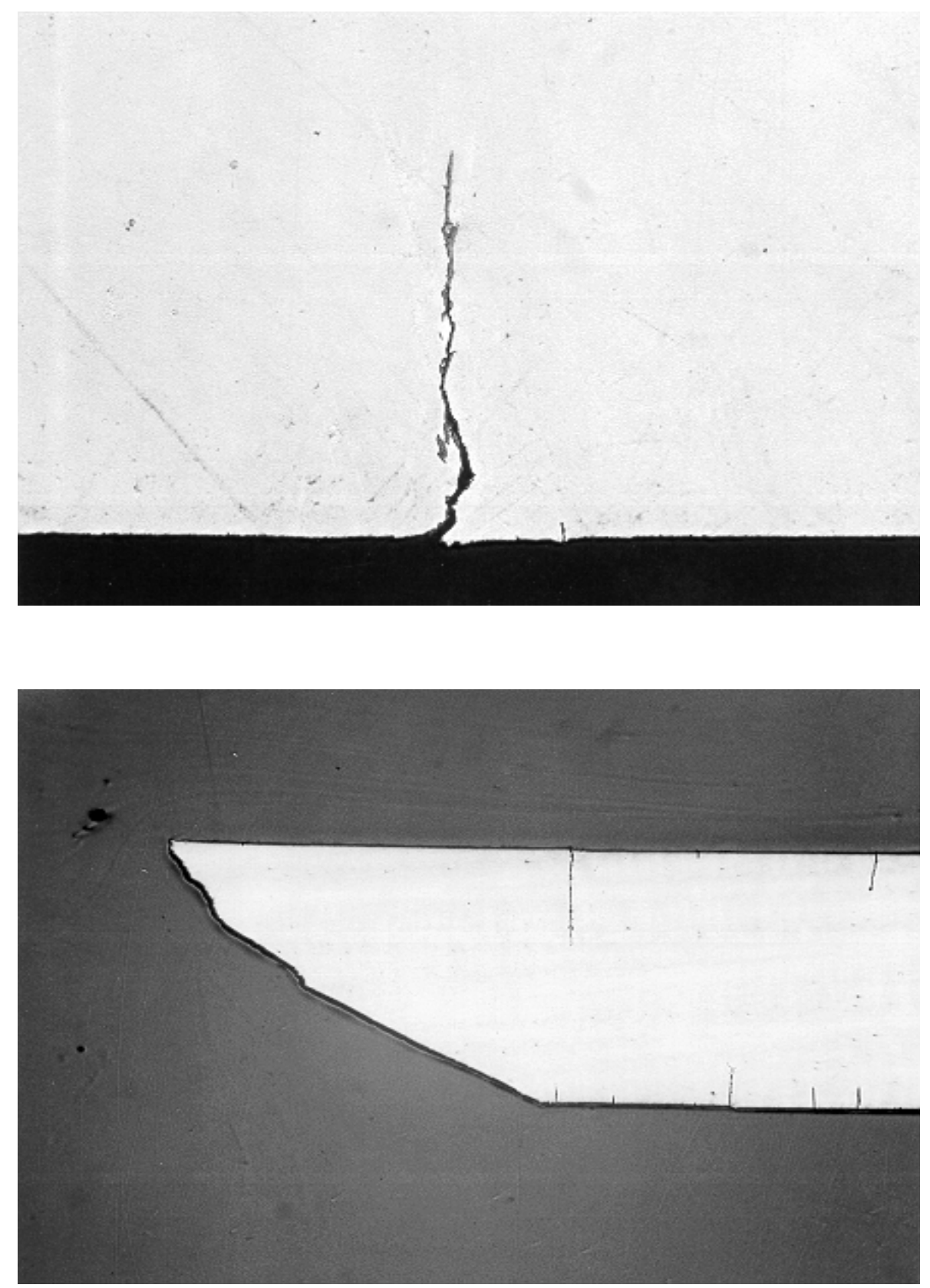

Figure 12: Secondary cracks on baseline CMSX-4 specimen. Note multiple secondary cracks on outer diameter (OD) and inner diameter (ID) of specimen. ( $T_{\max }=1000^{\circ} \mathrm{C}, \Delta \varepsilon=0.85 \%, N_{f}=1,756$ cycles) 
Appendix 1

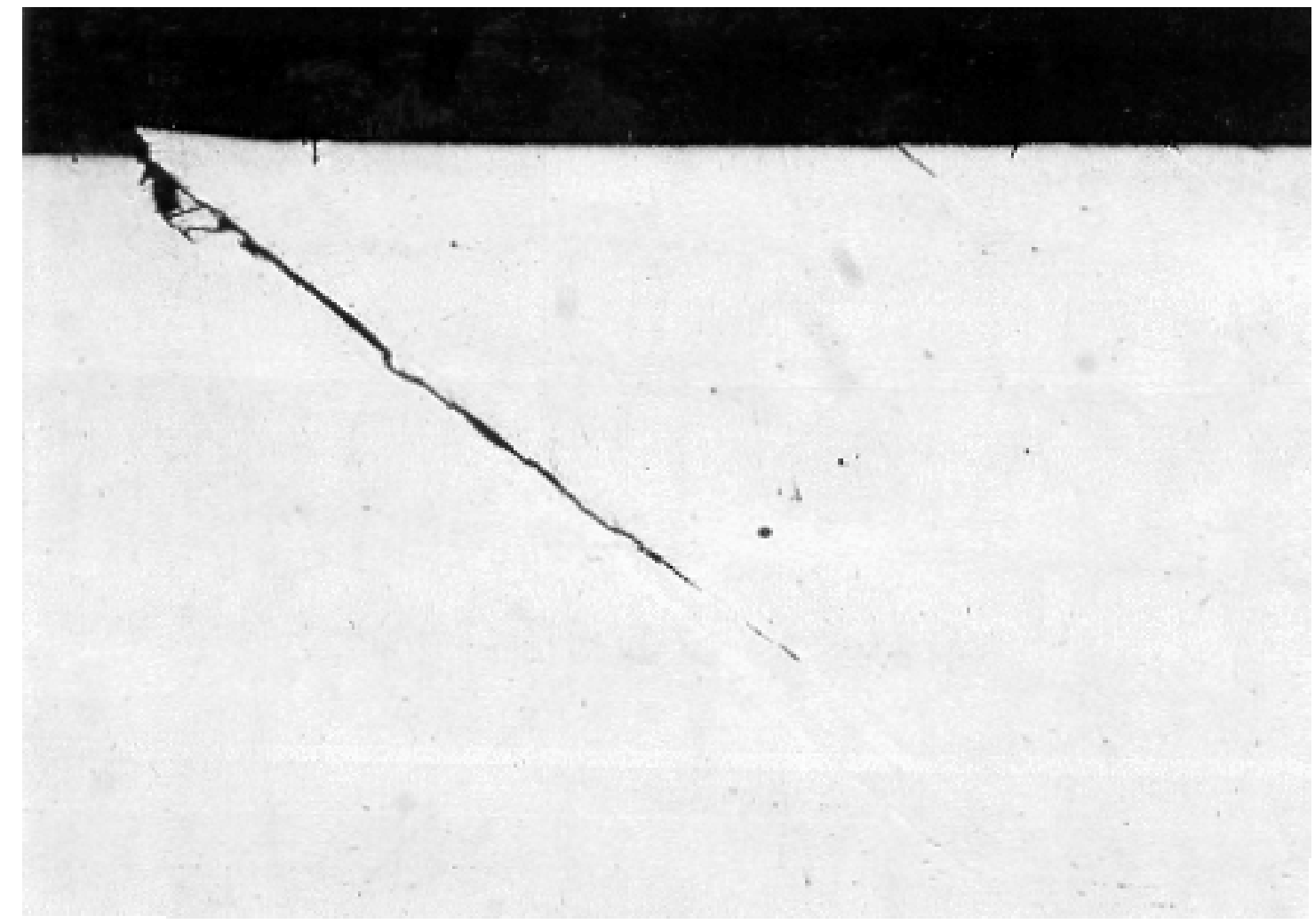

Figure 13: CMSX baseline TMF specimen. $\left(T_{\max }=1000^{\circ} \mathrm{C}, \Delta \varepsilon=1.0 \%, N_{f}=\right.$ 536 cycles, 50x Magnification) 
Appendix 1
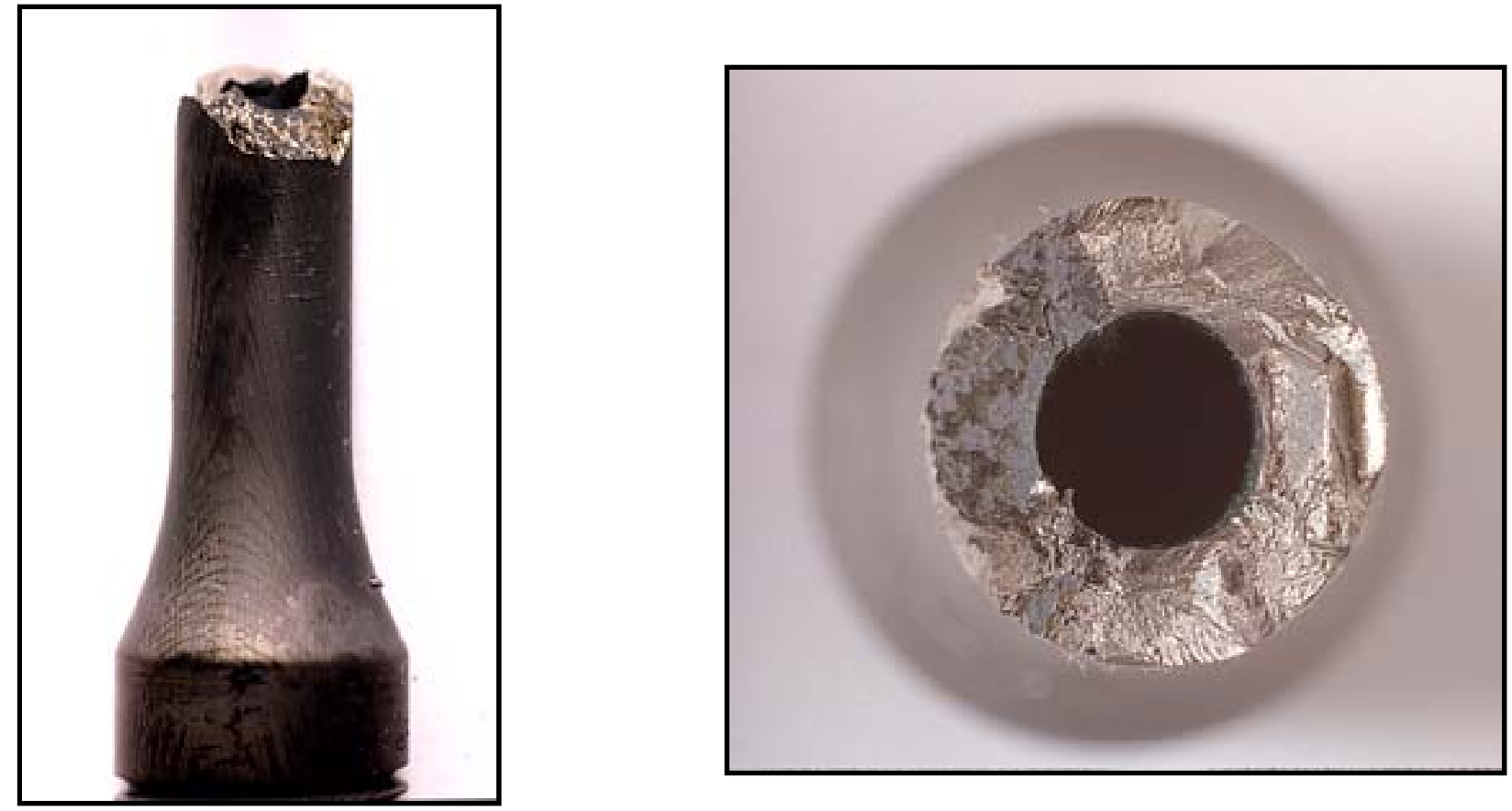

Figure 14: Fracture surface for TMF specimen of CMSX-4. $\left(T_{\max }=1000^{\circ} \mathrm{C}, \Delta \varepsilon\right.$ $=1.0 \%, N_{f}=300$ cycles) 
Appendix 1
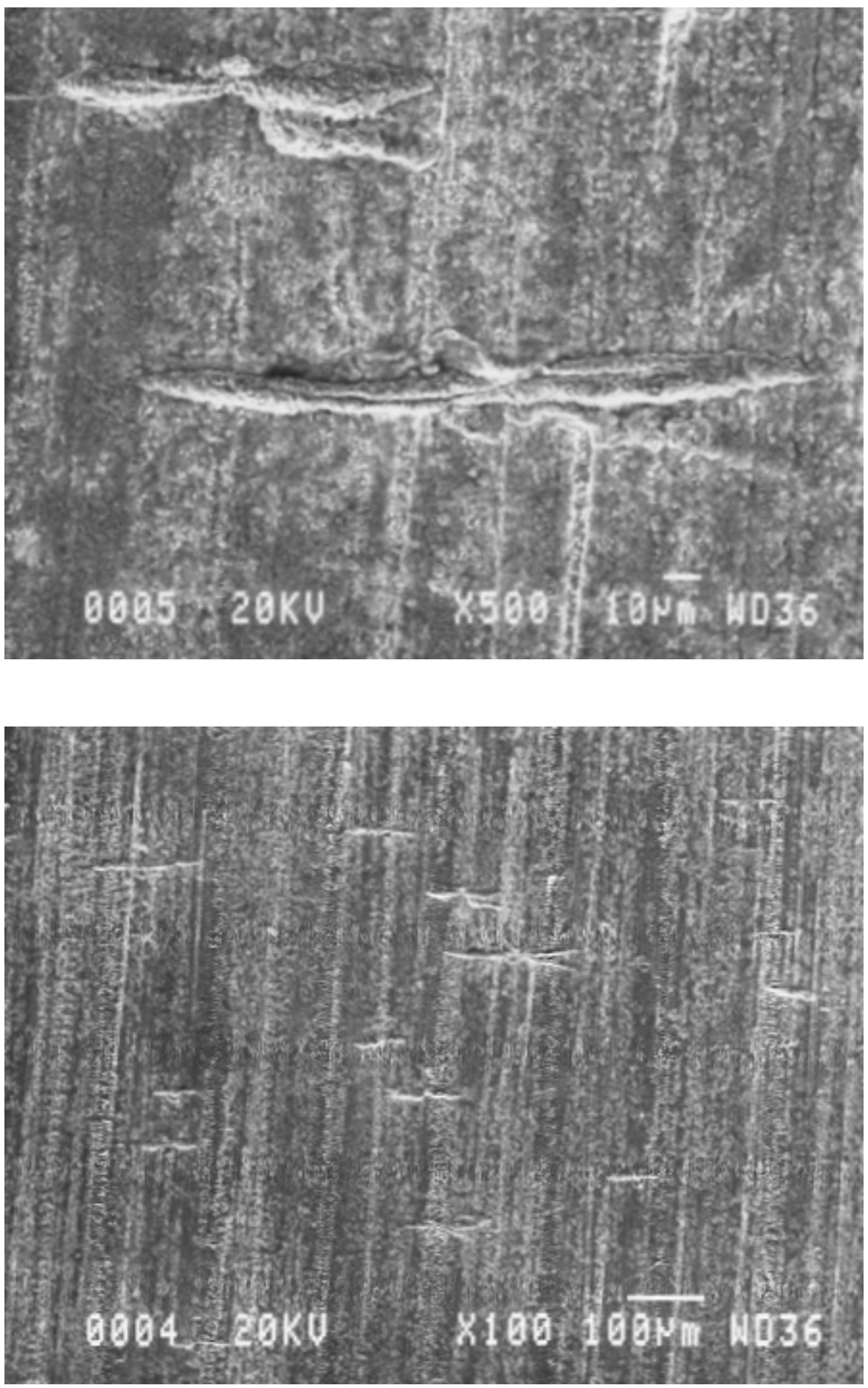

Figure 15: Point initiated multiple surface cracks for specimen. $\left(T_{\max }=\right.$ $\left.1000^{\circ} \mathrm{C}, \Delta \varepsilon=1.0 \%\right)$ 
Appendix 1

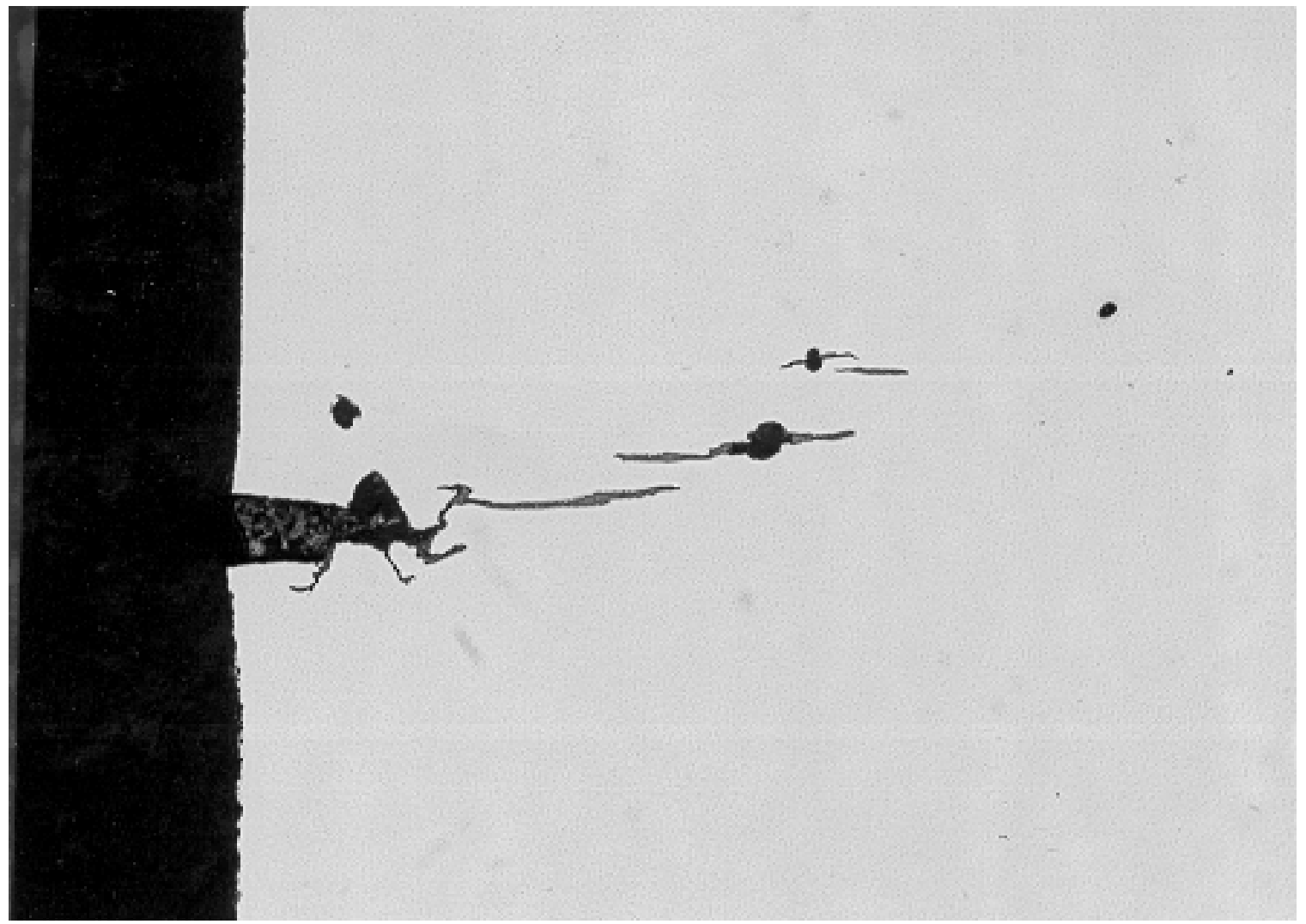

Figure 16: Open surface crack on CMSX-4 baseline sample. $\left(T_{\max }=1000^{\circ} \mathrm{C}\right.$, $\Delta \varepsilon=0.65 \%, N_{f}=6,116$ cycles) 
Appendix 1
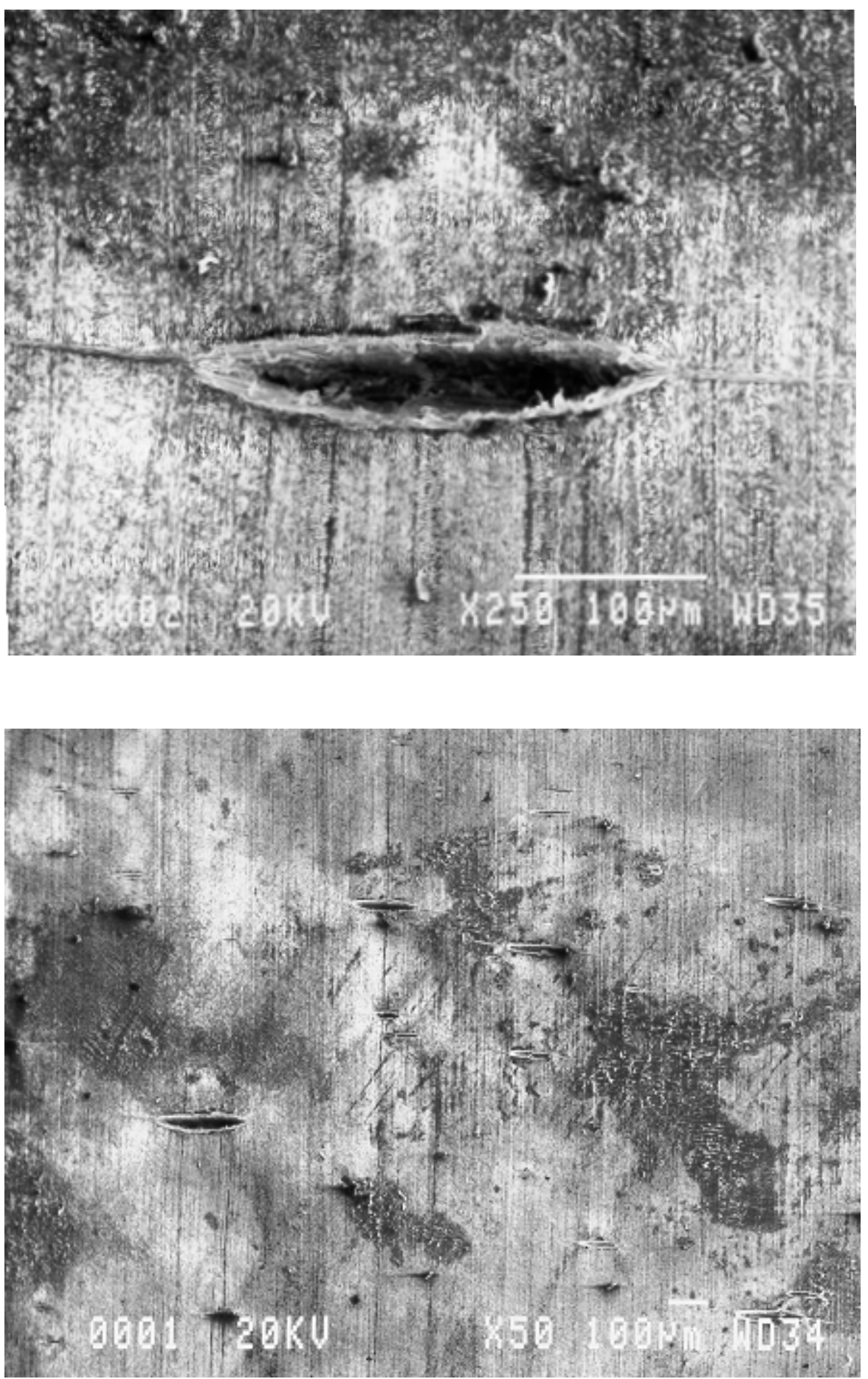

Figure 17: Point initiated multiple surface cracking for CMSX-4 sample.

(Note cracks appear more open at smaller strain range. $T_{\max }=1000^{\circ} \mathrm{C}, \Delta \varepsilon=$ $0.65 \%)$ 
Appendix 1
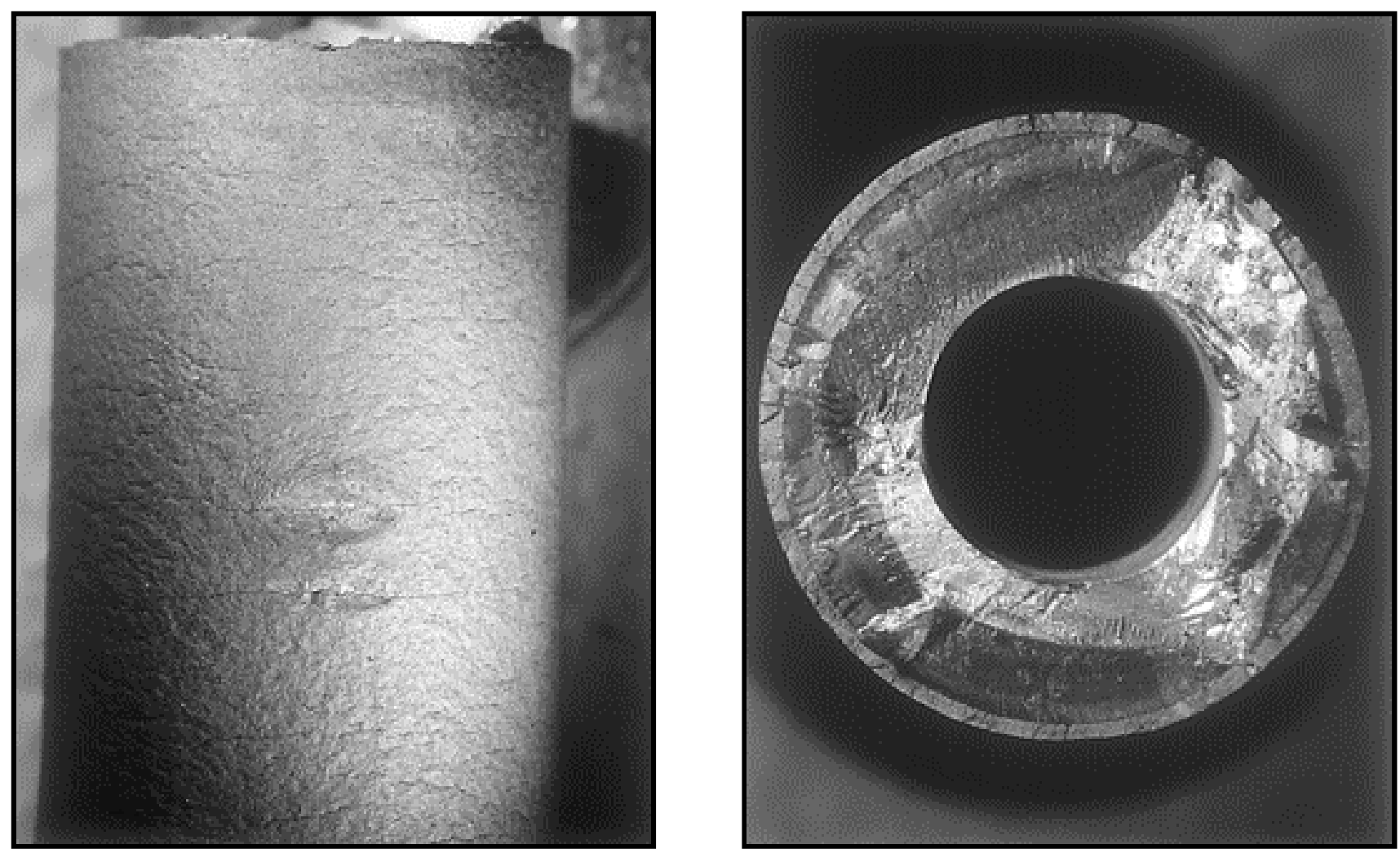

Figure 18: Fracture surface of a CMSX-4 testpiece with SV-20 coating. ( $\Delta \varepsilon=$ $0.65 \%, N_{f}=1,513$ cycles) 

Appendix 1

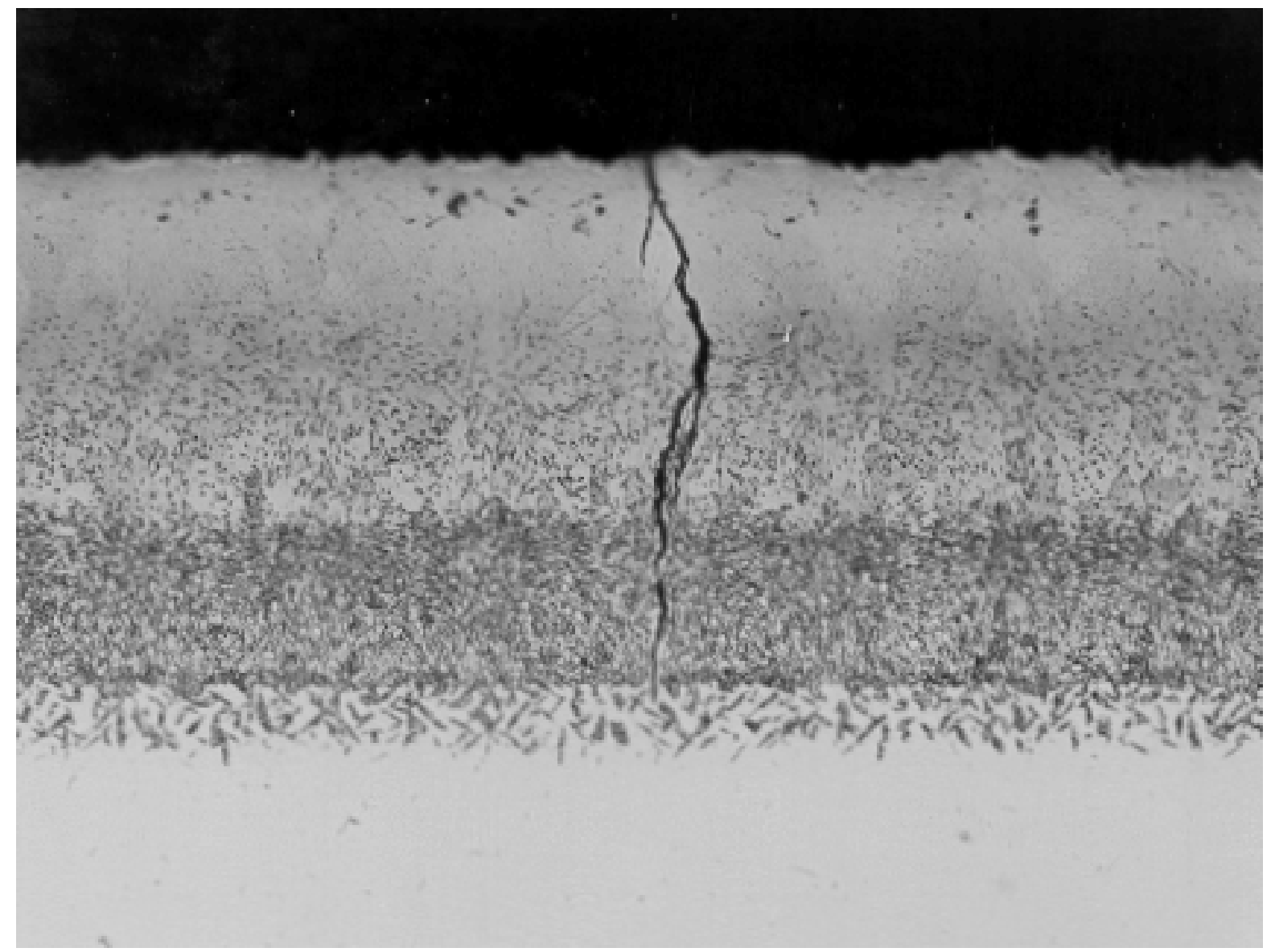

Figure 20: Irregular crack propagation in CMSX-4 + Pt Al coated sample. (Note crack is confined to coating and diffusion zone. $T_{\max }=1000^{\circ} \mathrm{C}, \Delta \varepsilon=$ $0.45 \%)$ 
Appendix 1
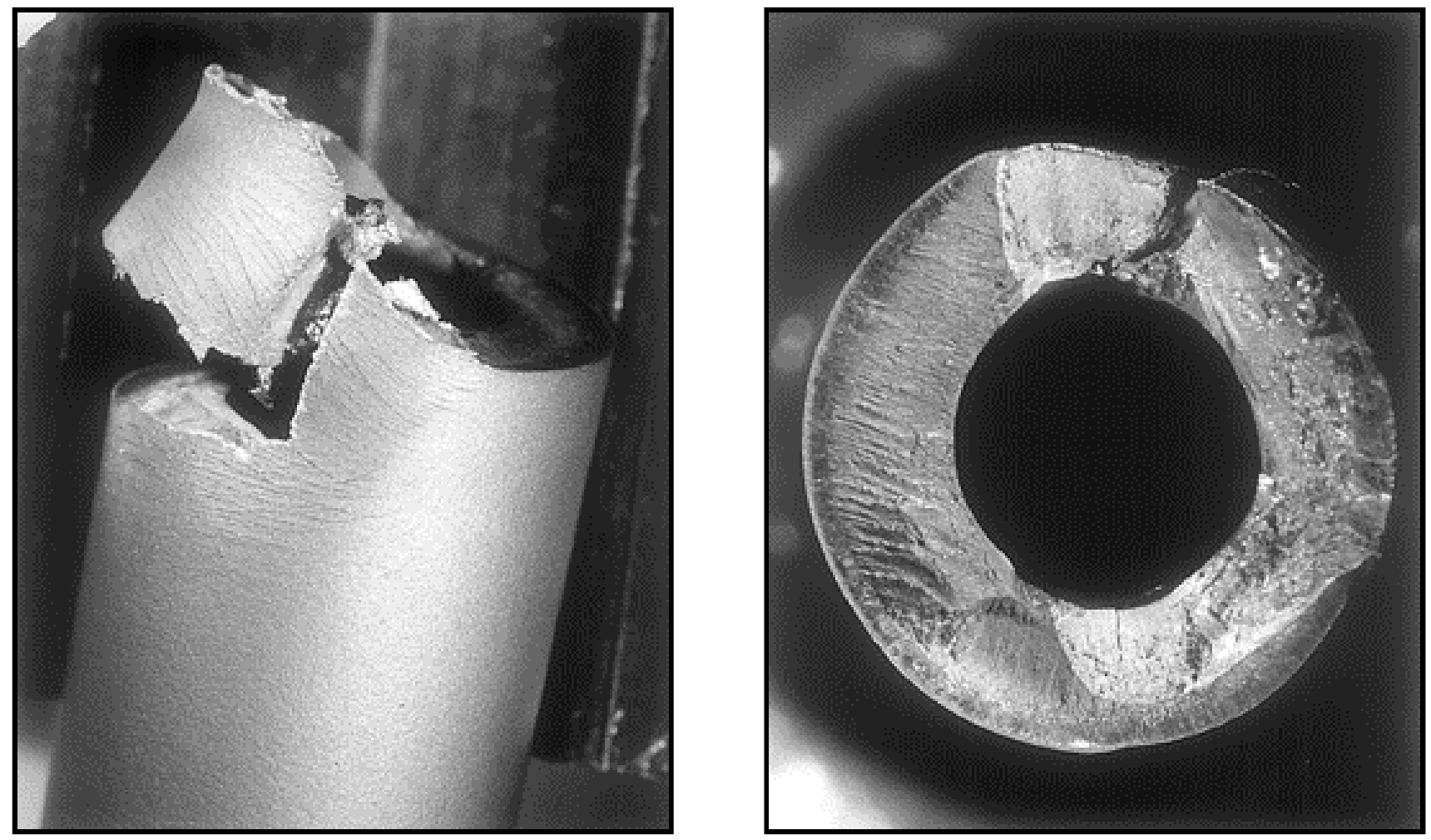

Figure 21: Fractured bar from "Out of Phase" TMF test of CMSX-4 + Pt Al coating. ( $\Delta \varepsilon=0.65 \%, N_{f}=1,513$ cycles) 
Appendix 1
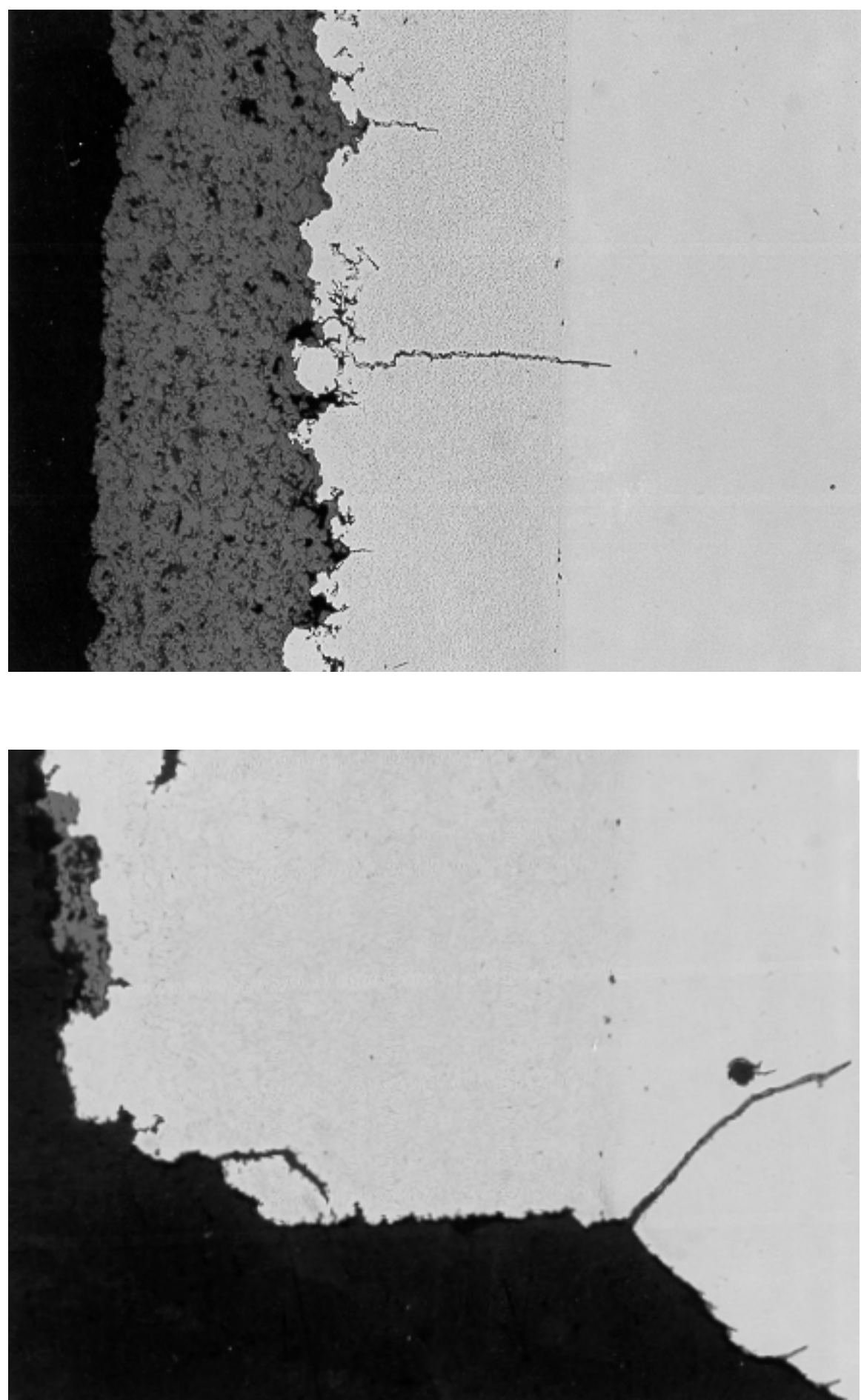

Figure 22: SV-20 + SL-20 + TBC TMF testpiece tested at $1000^{\circ} \mathrm{C}$. 
Appendix 1

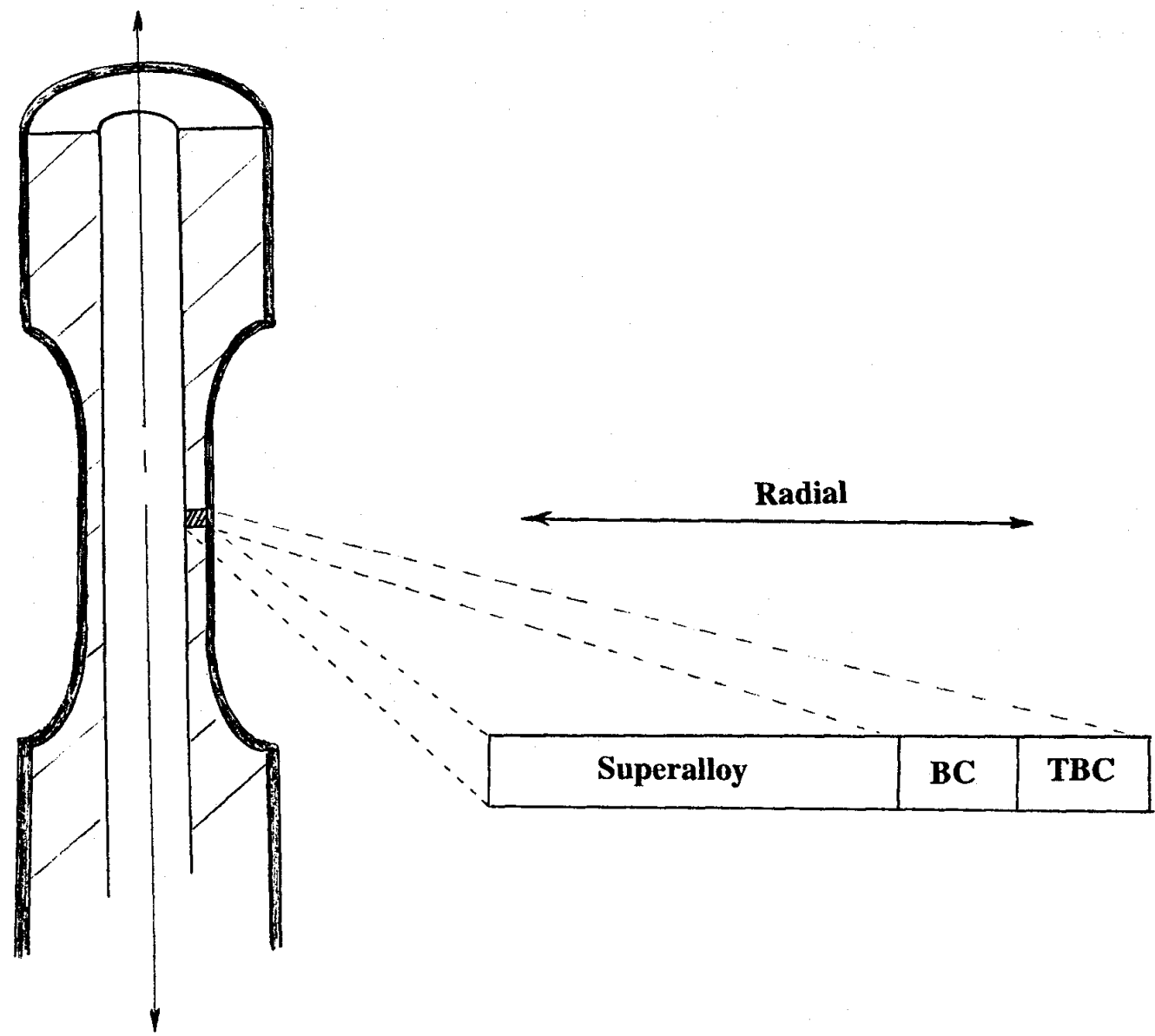

Figure 23: Schematic illustration of TMF specimen finite element model configuration 
Appendix 1

\section{ABB TMF Test - Strain $=1.00$ Axial Strain Bulk TBC}

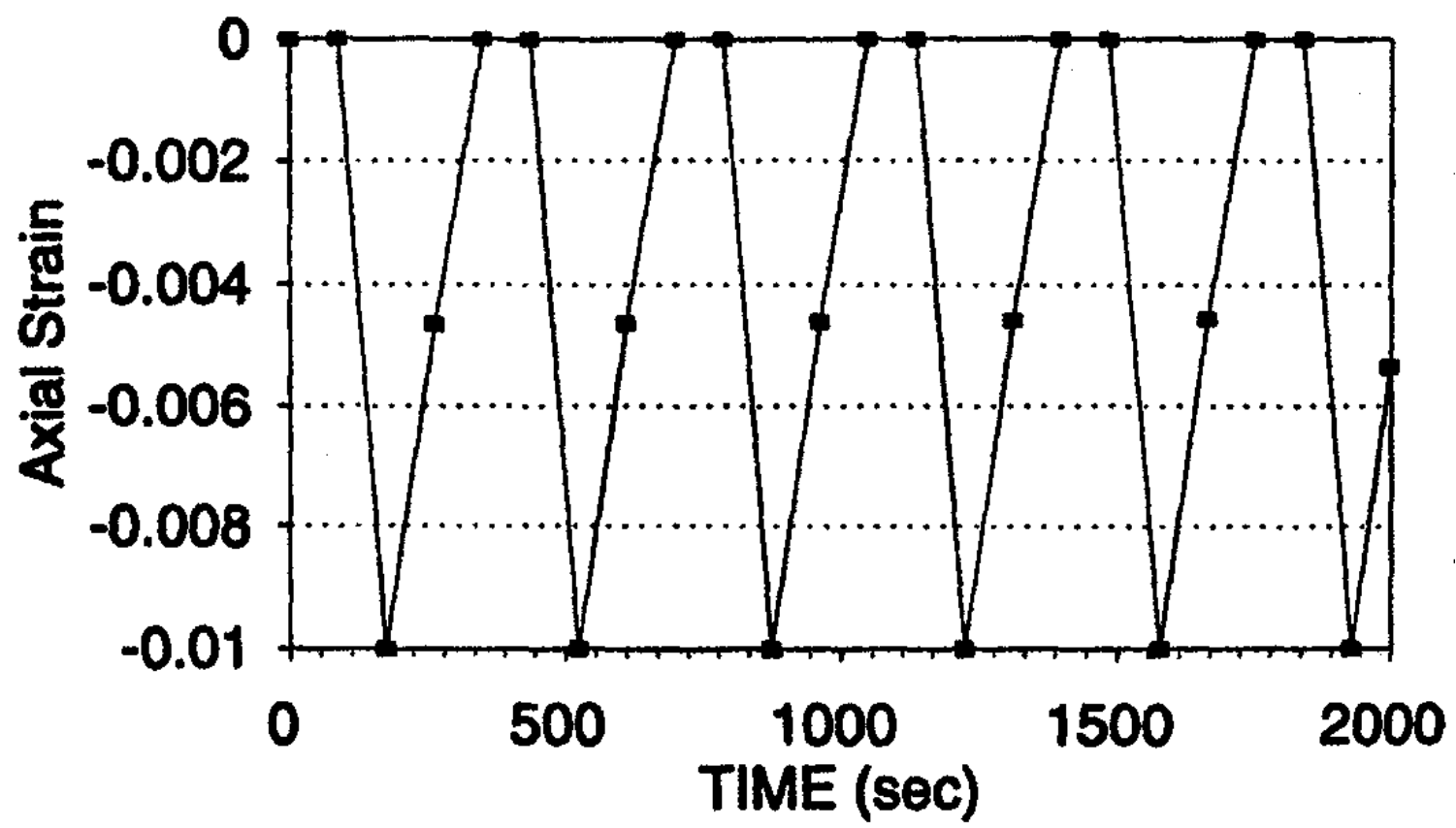

ABB TMF Test - Strain $=0.85$
Axial Strain Bulk TBC

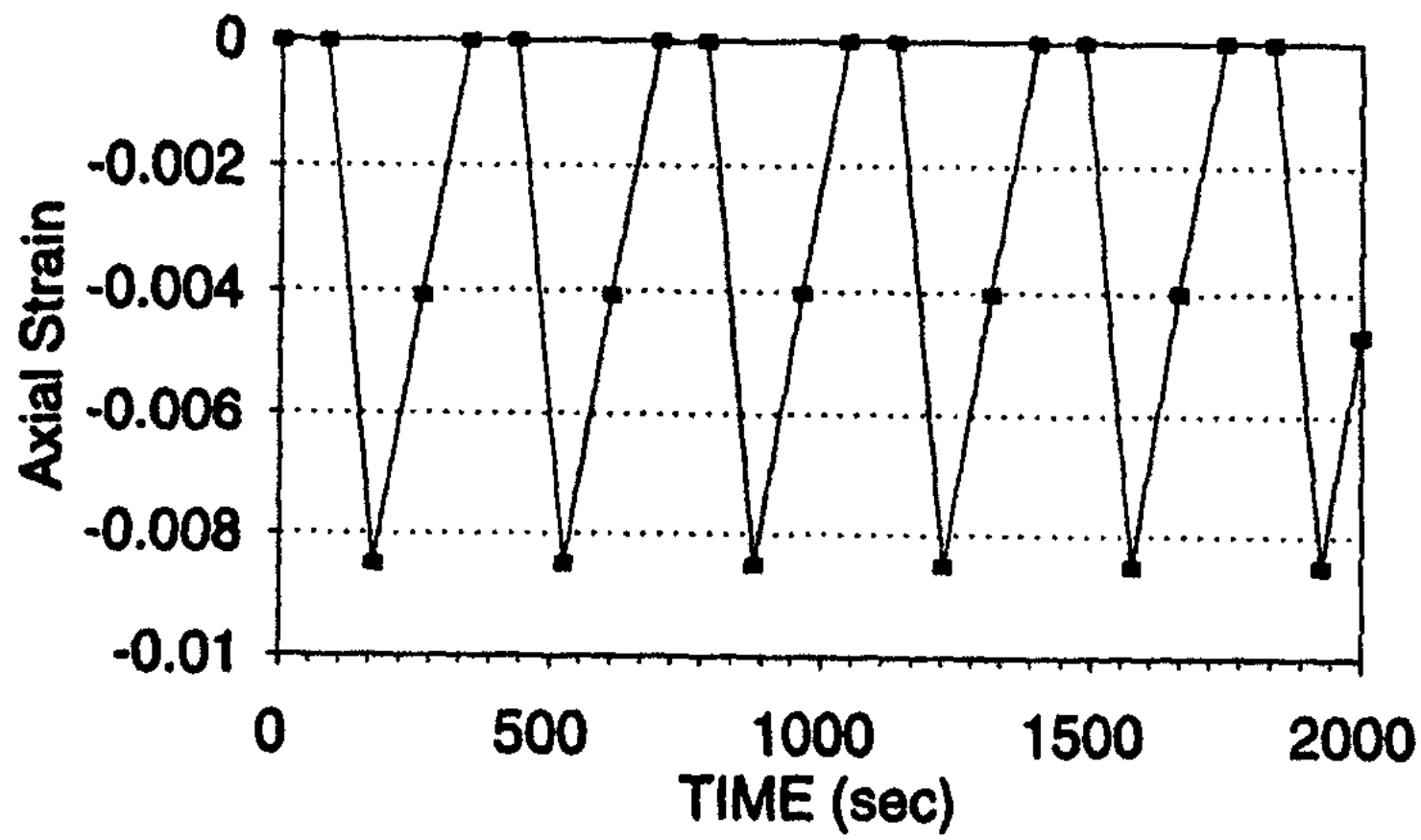




\section{ABB TMF Test - Strain $=0.65$ Axlal Strain Bulk TBC}

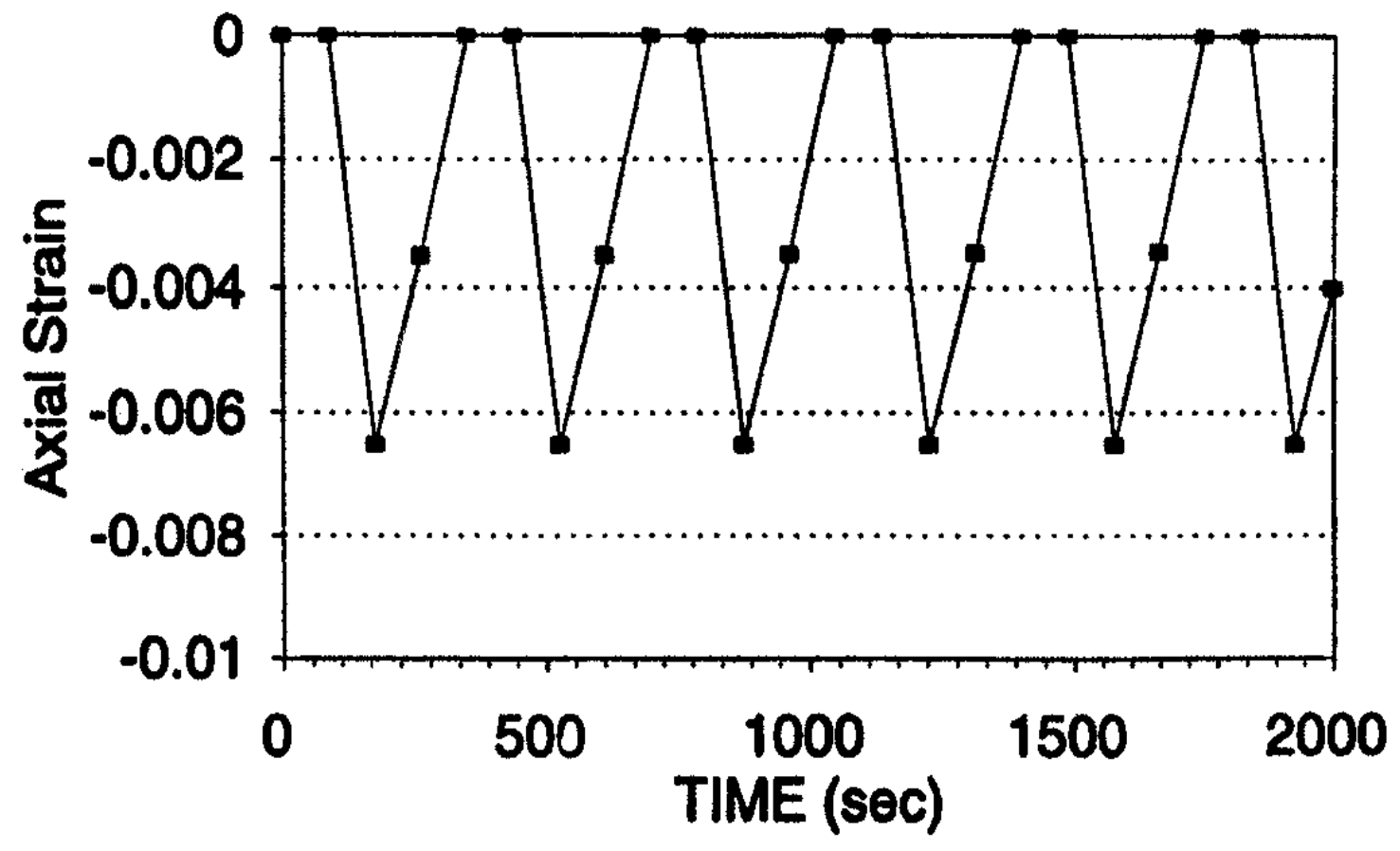

Figure 24: Axial strain time history of TBC coated testpiece for different strain ranges. 


\section{ABB TMF Test - Strain $=1.0$ Radial Stress Bulk TBC}

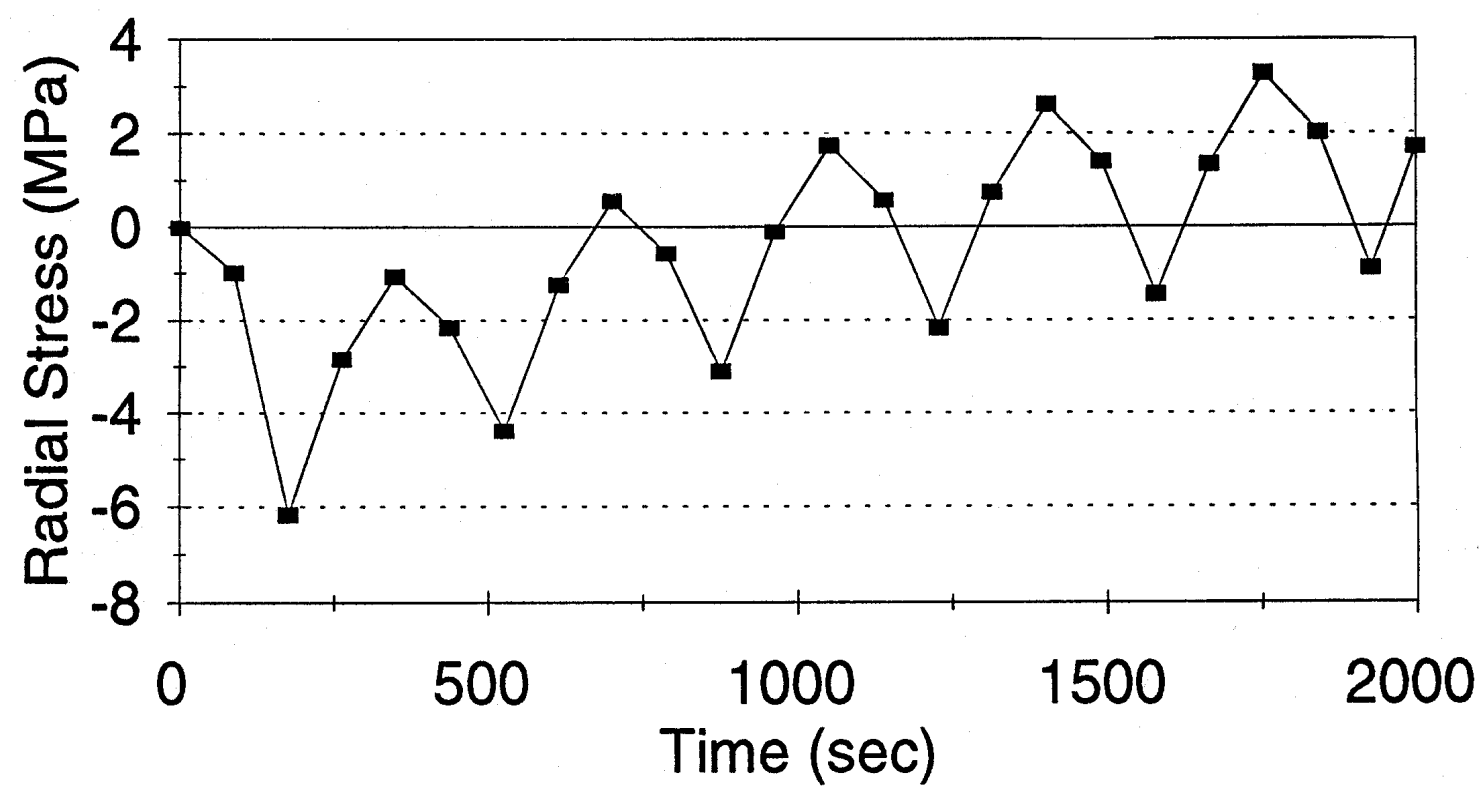

ABB TMF Test - Strain $=1.0$ Radial Stress at Peak (TBC)

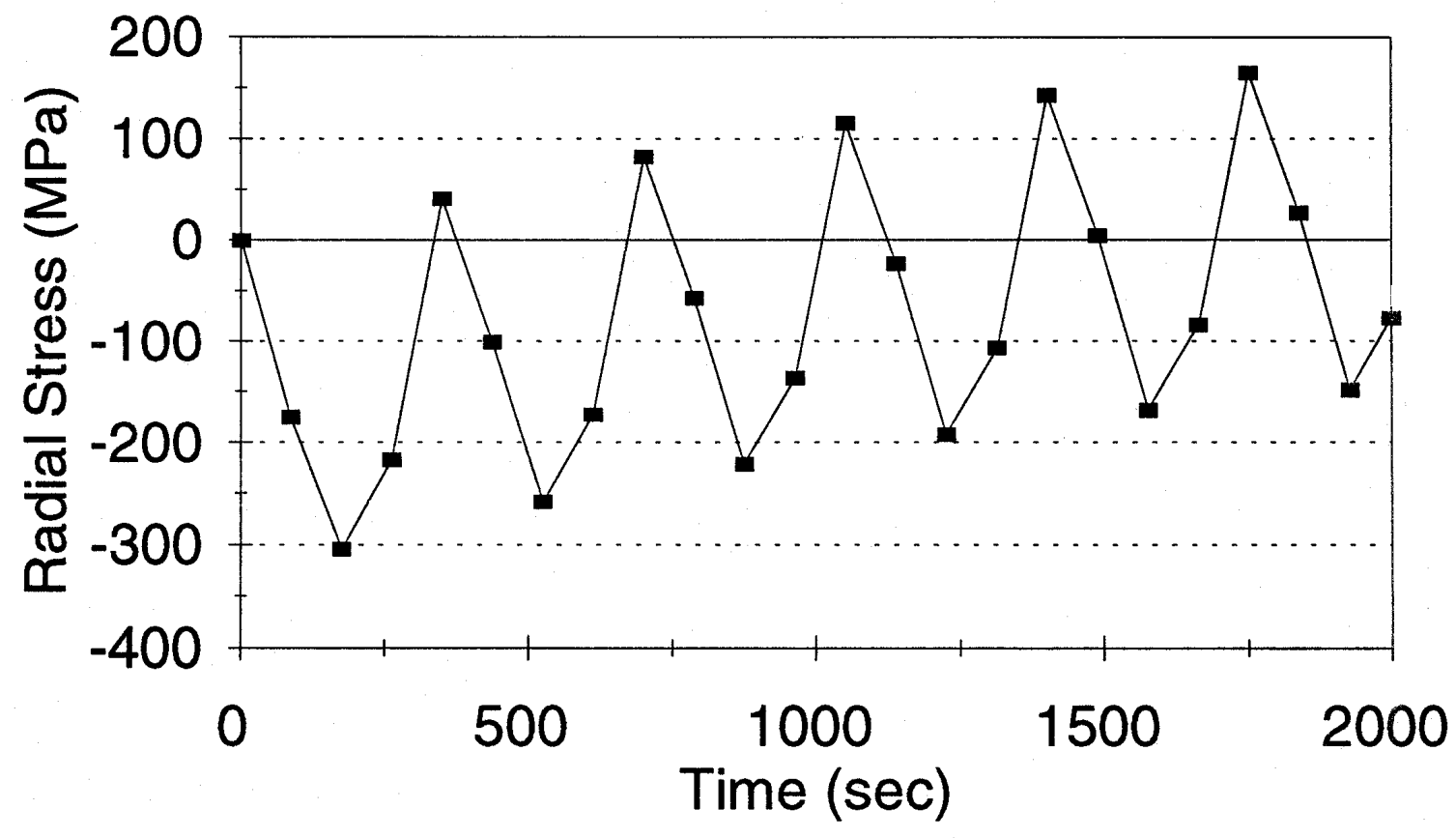

Figure 25: Radial residual stresses at different locations for TBC coated 
Appendix 1

samples. $\left(T_{\max }=1000^{\circ} \mathrm{C}, \Delta \varepsilon=1.0 \%\right)$

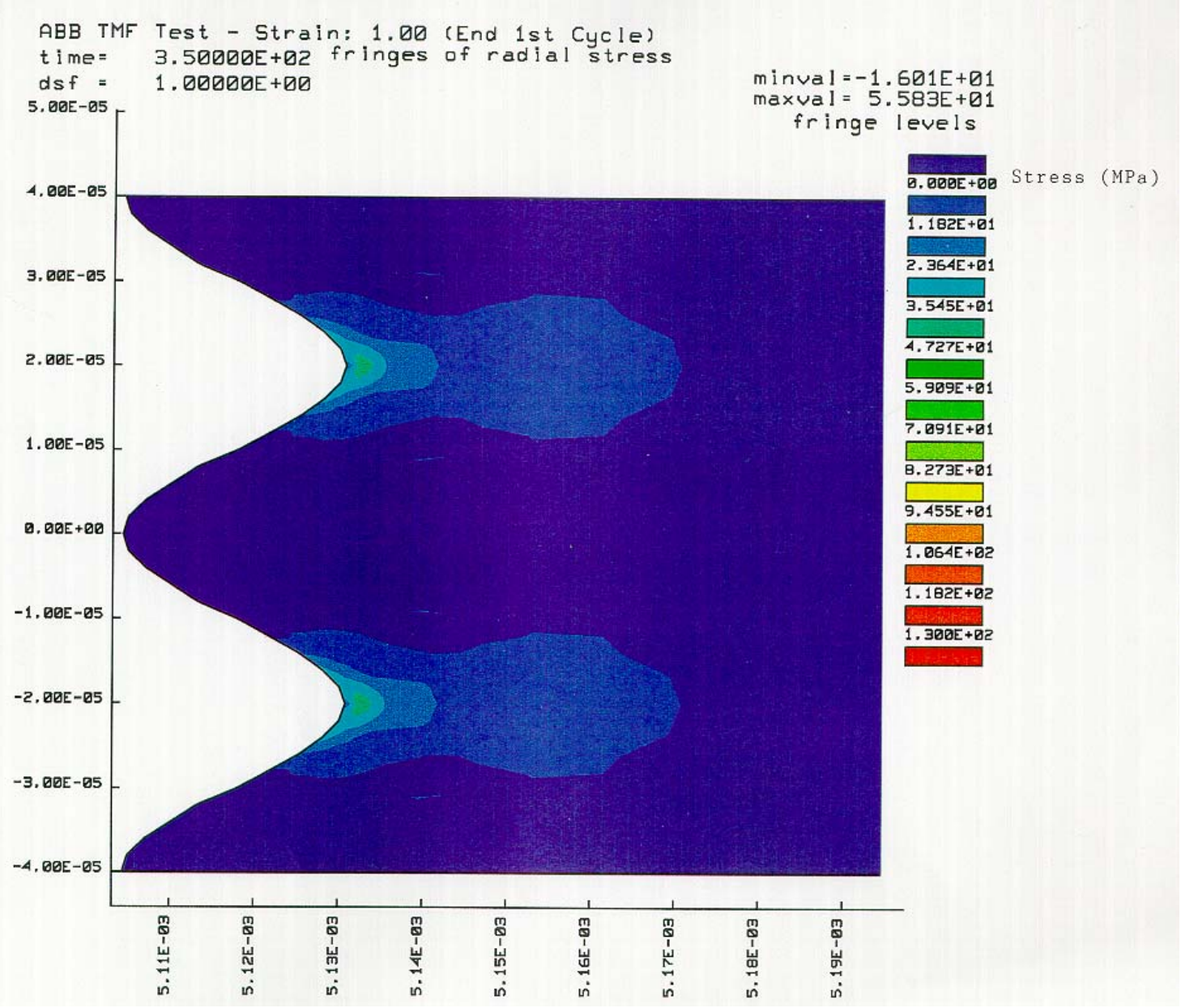

Figure 26: Strain profile of TBC coated TMF specimen at the end of the first cycle. $(\Delta \varepsilon=1.0 \%)$ 
Appendix 1

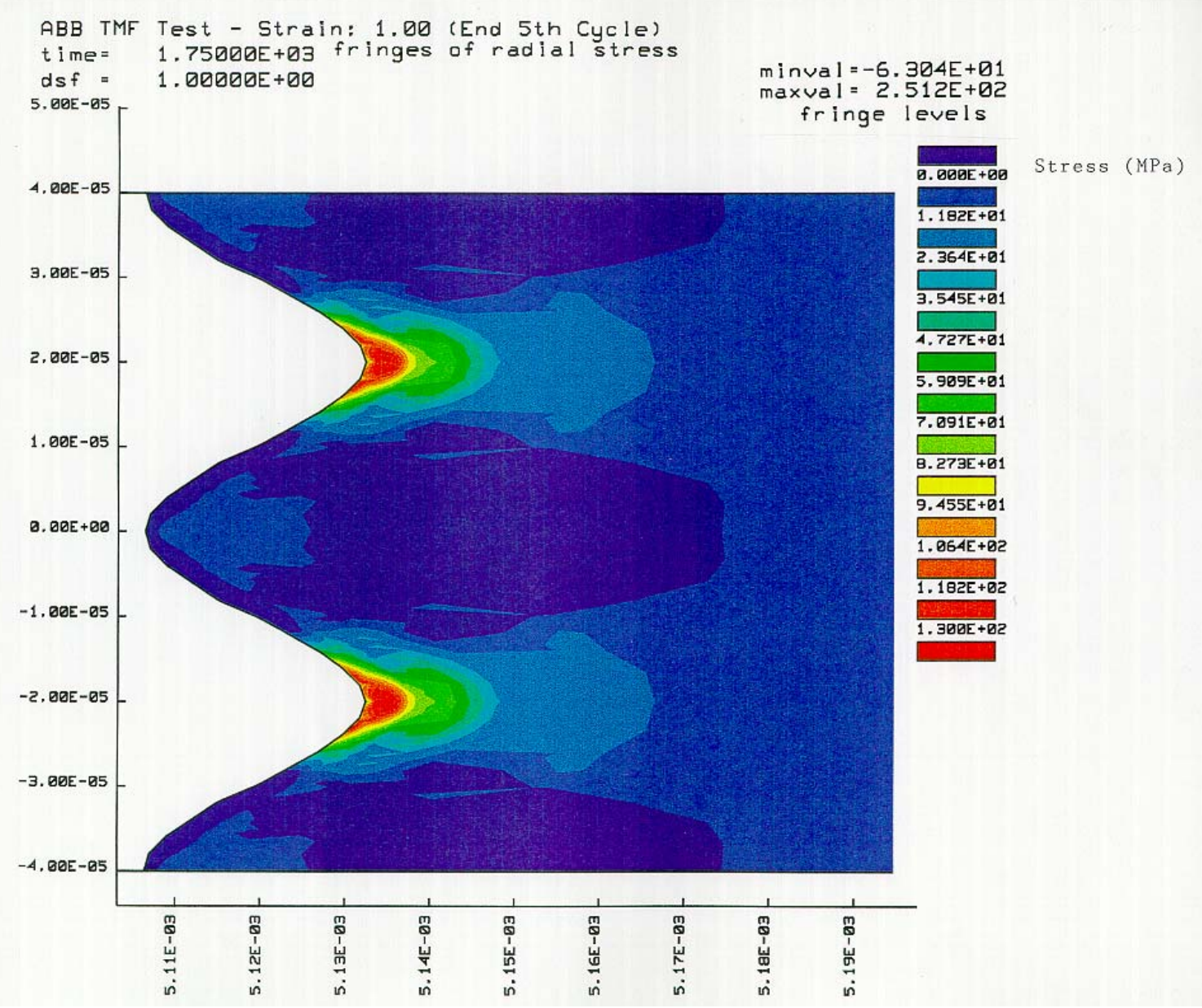

Figure 27: Strain profile of TBC coated TMF specimen at the end of the fifth cycle. $(\Delta \varepsilon=1.0 \%)$ 
Appendix 1

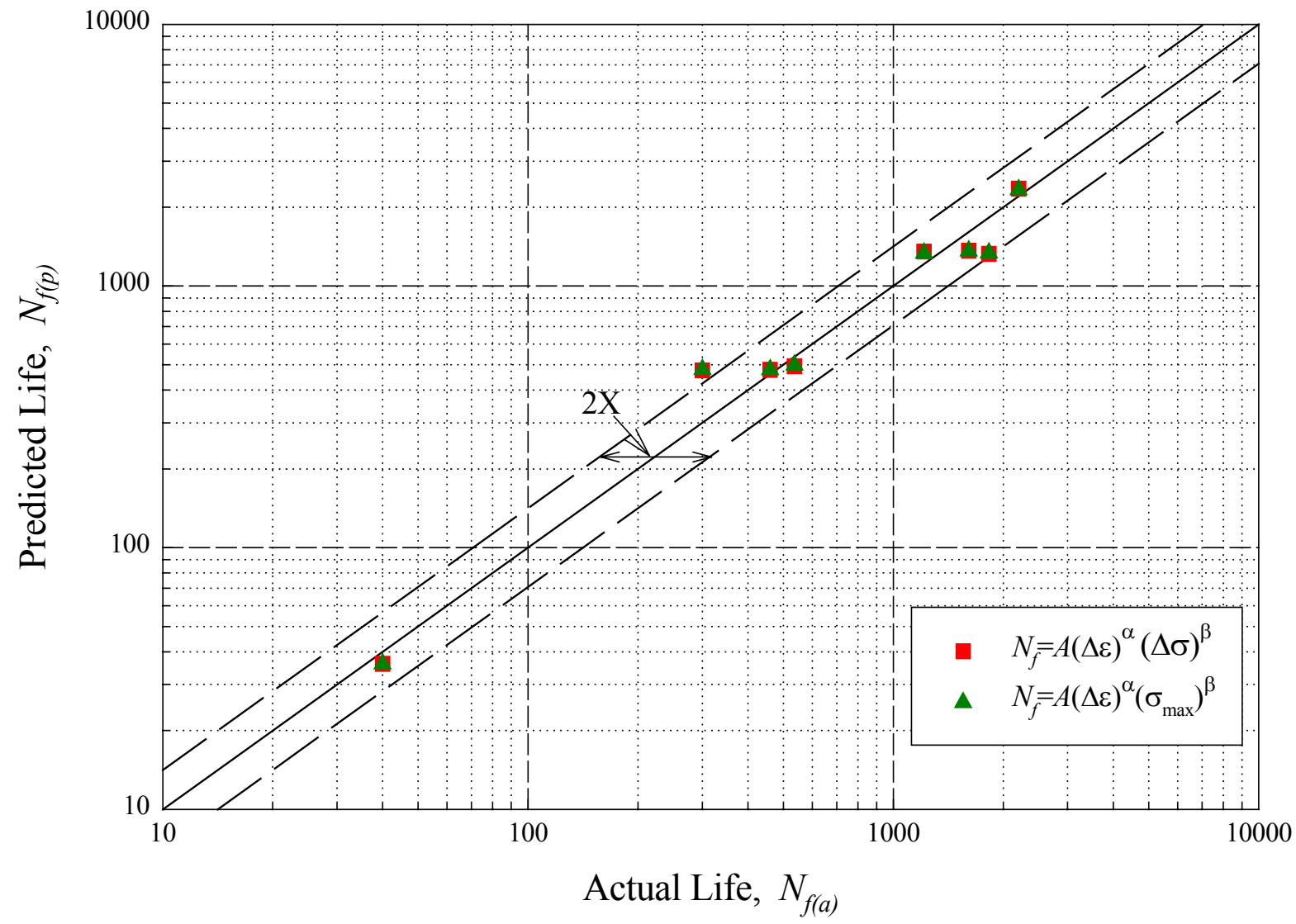

Figure 28: Actual TMF life vs. predicted one for baseline CMSX-4 using Bernstein model. 
INTRODUCTION

RESULTS

Overview of Test

IN 617 Results

Parabolic Growth Model

Vaporization

Effect of Pressure

Weight Gain Results $\quad 10$

IN 738LC

CM 247LC

CMSX-4 11

$\begin{array}{ll}\text { Air Oxidation Results } & 12\end{array}$

$\begin{array}{ll}\text { Metallographic Examination Results } & 13\end{array}$

$\begin{array}{lc}\text { REFERENCES } & 14\end{array}$

$\begin{array}{ll}\text { APPENDIX } & 15\end{array}$

List of Figures

Figure 1: Overall view of the steam exposure test facility. 2

Figure 2: Temperature profile along the length of one furnace. 2

Figure 3: View of test pins in sample tray. $\quad 3$

Figure 4: Processes active during the exposure period. $\quad 4$

Figure 5: Weight gain of IN 617 during exposure.

Figure 6: Fitting of parabolic growth model to IN 617 results. $\quad 6$

Figure 7: Arrhenius plot for IN 617 steam oxidation. $\quad 7$

Figure 8: Vapor pressure of volatile chromium oxides in air. $\quad 8$

Figure 9: Vapor pressure of volatile chromium oxides/oxyhydroxides in steam. 8

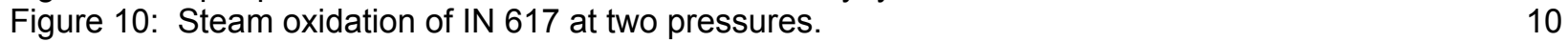

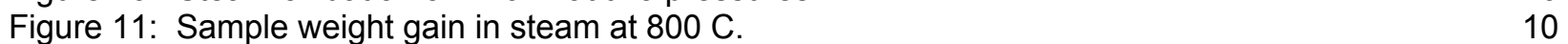

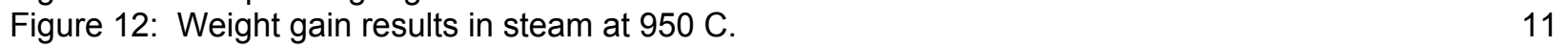

Figure 13: Arrhenius plot for CMSX-4. 12

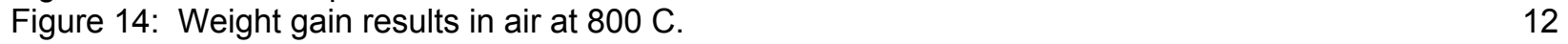

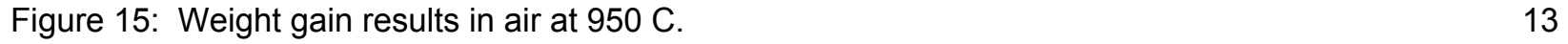




\section{Introduction}

Increased performance of gas turbines is achieved with increasing turbine inlet temperature. A limit on turbine inlet temperature is imposed by materials capabilities, in particular strength and oxidation resistance. Technically sophisticated techniques are utilized to control metal temperature in the face of increasing inlet gas temperature. Thermal barrier coatings are used to insulate the components and air is used to provide a cooling medium. Advanced gas turbine system designs are now being considered which use steam as the cooling medium. The flow of heated steam can then be integrated into the Rankine cycle portion of a combined cycle power plant with a net gain in overall energy efficiency.

The use of steam as a cooling medium is not without potential problems. Steam provides an oxidizing environment due to its thermal dissociation into hydrogen and oxygen and its direct action as an oxidizing agent. The steam acts as a reactant to generate metal oxyhydroxides which may have significant volatility in the temperature range under consideration. Formation of an oxide scale layer on the internal vane cooling passages places an additional thermal resistance in the heat flow path which will increase metal temperature. Spallation of the oxide layer may generate particulate large enough to restrict or block smaller cooling channels. An internal ABB literature review (Ref. 1) highlighted a lack of information on the steam oxidation of directionally solidified (DS) and single crystal (SX) materials which are of greatest interest for the advanced gas turbine. This report contains the results of an experimental study of the oxidation resistance of superalloys when exposed to an isothermal steam environment at 800 $\mathrm{C}$ and $950 \mathrm{C}$ and $0.1 \mathrm{MPa}$ pressure. Superalloys tested were IN 617, IN 738LC (CC), CM 247LC (DS), and CMSX-4 (SX). Sample exposure time ranged up to 5,000 hours.

\section{Conclusions and Recommendations}

Results of test exposure of candidate superalloy materials to steam for 5,000 hours at temperatures up to $950 \mathrm{C}[1,742 \mathrm{~F}]$ do not identify any major oxidation problems which might preclude the use of steam cooling in an advanced technology gas turbine. While only limited data were found for comparison with air, there do not appear to be any great differences in the two environments. For alloys which form a protective chromia scale (i.e. IN 617 and IN 738LC), vaporization loss of chromium at high gas velocity is of similar concern in both environments. While the specific test bars of CM 247LC used in these tests did not perform well, this is attributed to compositional or casting related issues and should not be generalized. The alloys IN 738LC and CM 247LC experienced spallation of the oxide scale similar to that observed for many alloys undergoing cyclic oxidation testing. CMSX-4, which forms a protective alumina scale, experienced the least oxidation of the alloys tested.

\section{Test Description}

Testing was carried out utilizing the equipment shown in Figure 1. This facility includes three independently controlled chambers for exposing samples to a specific environment. Each chamber consists of a $10 \mathrm{~cm}$ [4 in] diameter quartz tube, $152.4 \mathrm{~cm}[5 \mathrm{ft}]$ long. The tube is concentrically located within a set of electric heaters which provide for a center temperature control zone and two end zones for adjustment of the temperature profile. Temperature is controlled by a Type $R$ thermocouple input to a PID controller and monitored by four Type $\mathrm{K}$ thermocouples along the heated length. The temperature profile typically provided a zone of $\pm 5 \mathrm{C}[ \pm 9 \mathrm{~F}]$ for sample exposure (see Figure 2 ). 


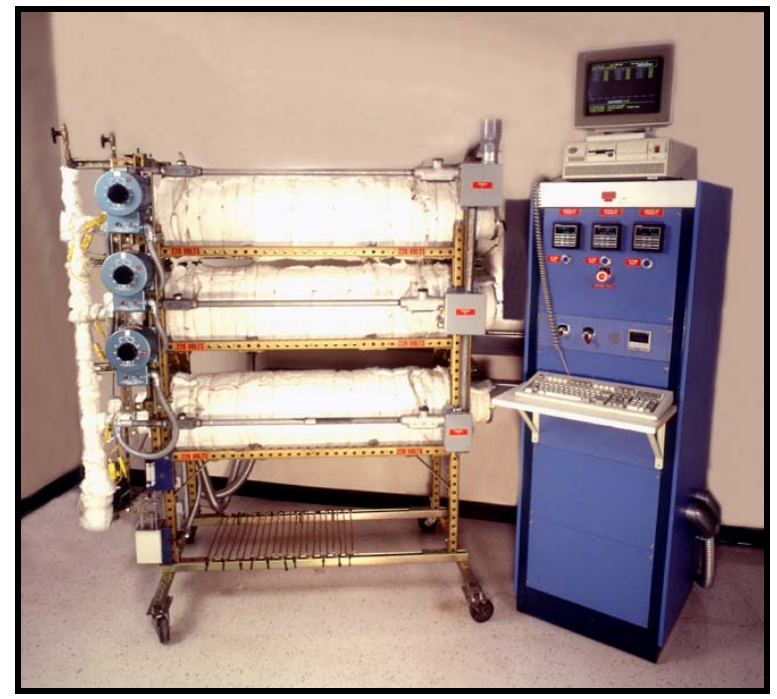

Figure 1: Overall view of the steam exposure test facility.

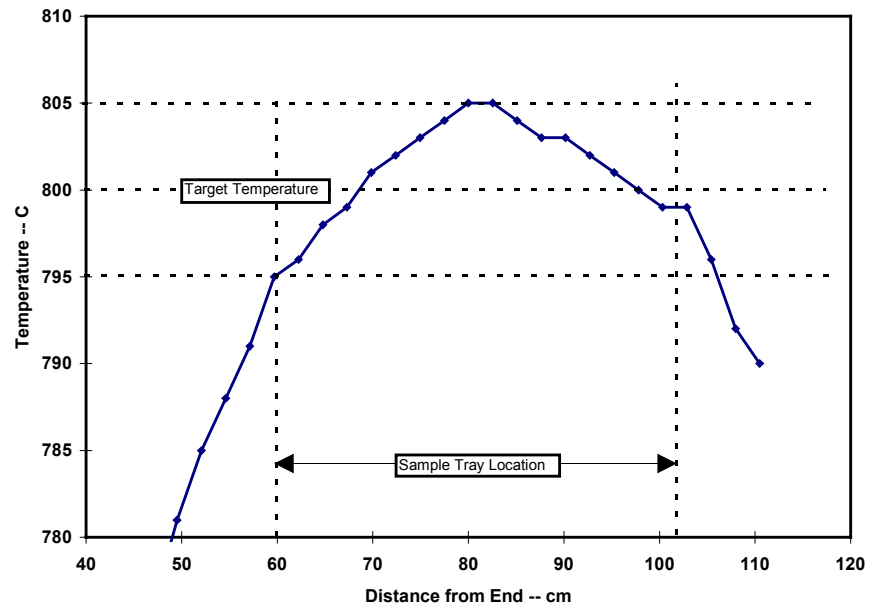

Figure 2: Temperature profile along the length of one furnace.

The facility included a steam supply system. Demineralized water was contained in a nitrogen purged reservoir. A peristaltic pump transferred water from this reservoir to an electrically heated block which vaporized the water. The resulting steam was then piped via heat-traced lines to the sample exposure chambers. Within the chamber, the steam flowed through a ceramic-tubed heat exchanger which preheated the incoming steam and cooled the exiting steam. The preheated steam then flowed through the lower portion of the chamber, gaining additional heat. At the end of the chamber, the steam then progressed to the upper portion, reversed direction, and flowed over the test samples at approximately 30 $\mathrm{cm} / \mathrm{min}[1 \mathrm{ft} / \mathrm{min}$ ]. After passing through the heat exchanger, the steam was collected and flowed into an air-cooled condenser and then back to the reservoir.

As noted above, the chamber was divided into an upper side and a lower side; the division was accomplished with an alumina fiber board located at the centerline of the quartz tube. The lower portion was reserved for preheating the flowing steam. In the upper portion resided cast ceramic trays containing the test specimens. A typical tray is shown in Figure 3. 


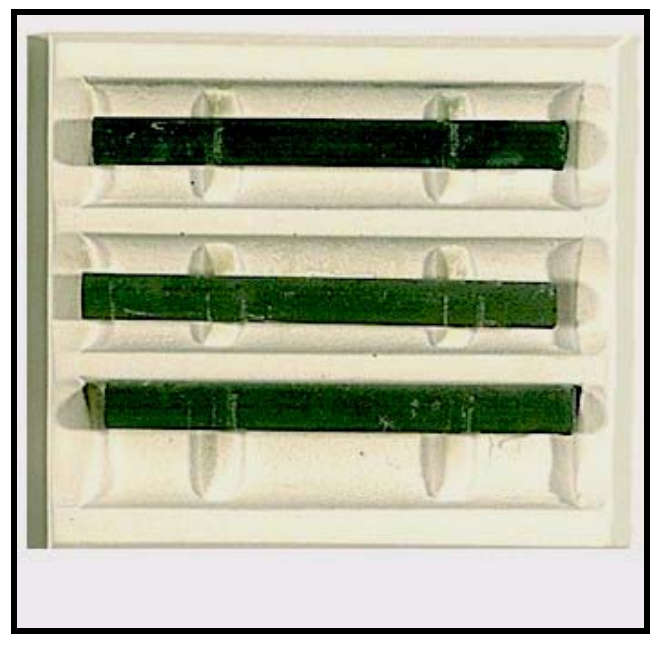

Figure 3: View of test pins in sample tray.

Test specimens consisted of bars approximately $7.5 \mathrm{~cm}$ [3 in] long and $0.95 \mathrm{~cm} \mathrm{[3/8} \mathrm{in]} \mathrm{in} \mathrm{diameter.}$ Certified analyses of the materials are listed in the Appendix, Table 1. The IN 617 was a wrought bar with a machined surface; the other samples were castings. The IN 738LC sample was in equiaxed, conventionally cast (CC) form. The CM 247LC was directionally solidified (DS) and the CMSX-4 was single crystal (SC). The three cast materials were given typical production specification heat treatment and a caustic leaching to remove residual mold material from the surface and rinsed in deionized water. The surfaces were not otherwise altered to maintain simulation of the conditions expected of internal blade/vane passages.

The test procedure consisted of loading the samples into their designated tray and slot, loading the trays into the furnaces and commencing heatup. The steam generator was started and steam vented to atmosphere to warm up the supply lines. When the furnaces reached a temperature of $300 \mathrm{C}$ [572 F] (approximately $1.5 \mathrm{~h}$ ), steam was diverted into the furnaces to create the desired steam environment for the samples. Samples reached test temperature after approximately another $2.5 \mathrm{~h}$, providing an opportunity for thorough purging of the exposure chamber. After an appropriate period of exposure, the procedure was reversed for cooldown. At $300 \mathrm{C}[572 \mathrm{~F}]$, the steam supply was discontinued and substituted by a flow of nitrogen gas. The nitrogen flow permitted purging of the residual steam and prevented condensation of moisture on the samples when cooling to room temperature. Samples were removed from the furnaces and weighted on an analytical balance. If exfoliated material was observed in the trays, it was collected, slot by slot, and weighted also. The procedure was then repeated for another exposure cycle.

\section{Results}

\section{Overview of Test}

The basic principles of oxidation applicable to these test is reviewed in Ref. 2. When exposed to the steam environment, several processes are active. These processes are illustrated in the following figure. 


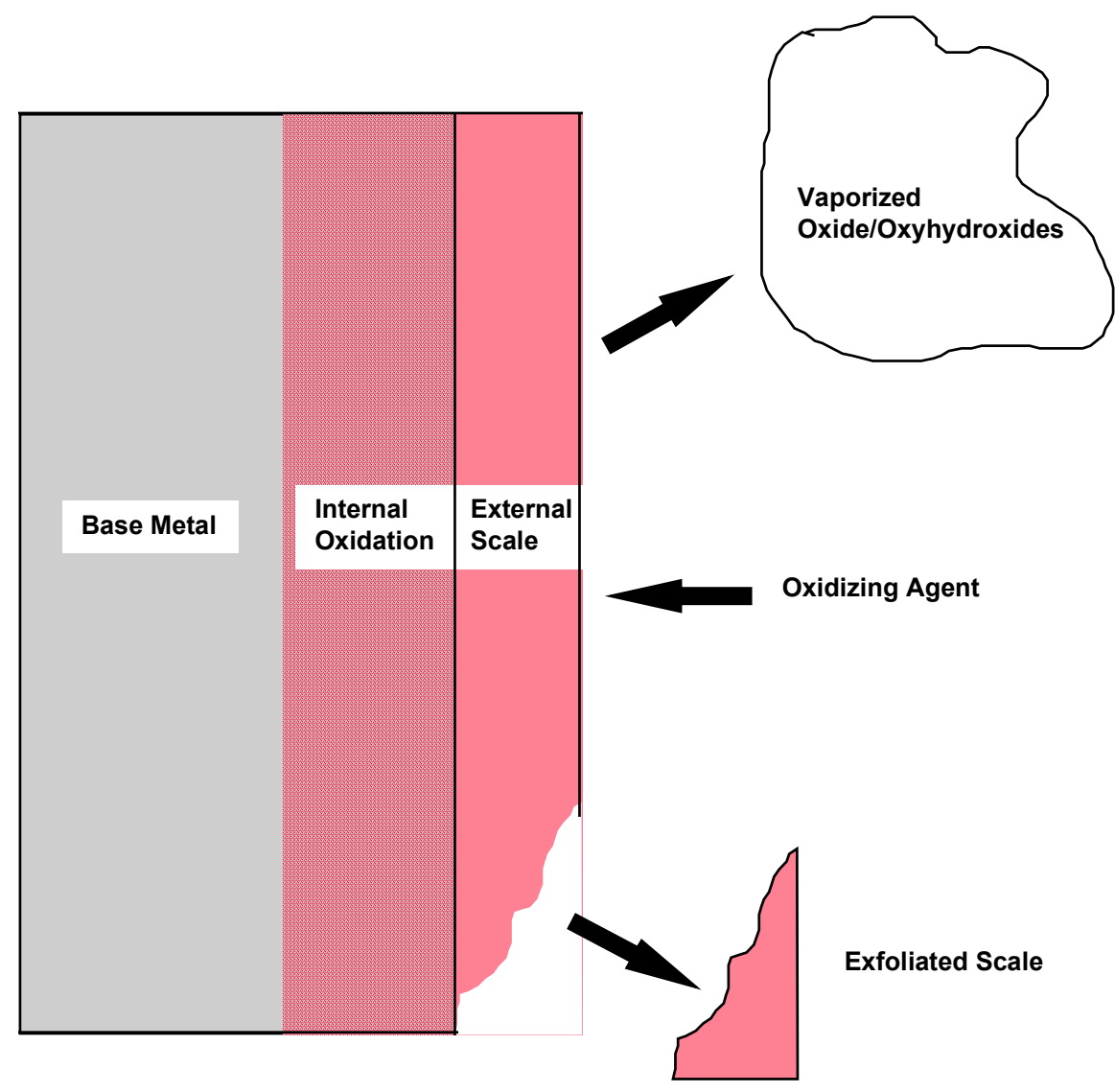

Figure 4: Processes active during the exposure period.

The environment provides a source of oxidizing agent at the surface of the sample. The oxidizing agent initiates a process that results in the formation of metal oxides. The external oxide scale may adhere to the surface or may crack and exfoliate. Additionally, material may be removed from the sample via vaporization of the oxidation products, either directly as oxides or as oxyhydroxides. Based on the description of the test methodology previously given, weight gain data may be described as follows:

$$
W_{t 1}=W_{t 0}+O-E-V
$$

where:

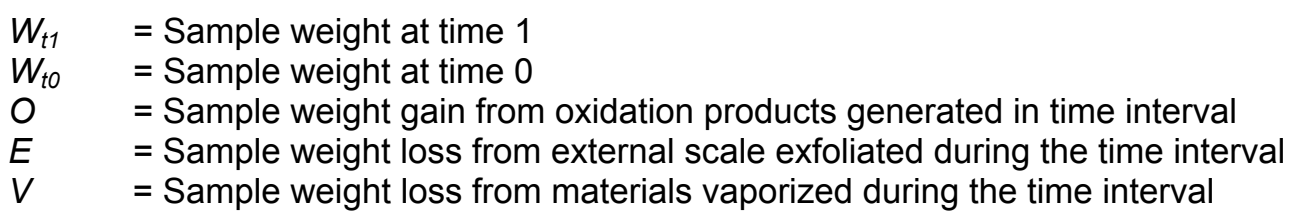

The sample weight gain from oxidation products generated in the time interval, $O$, represents the weight of oxygen reacting with the alloy metallic elements and remaining attached to the sample. It thus represents either internal oxidation products or non-exfoliated external scale which is formed during the time interval. The sample weight loss from external scale (assumed to be metal oxides) exfoliated during the time interval, $E$, represents either a loss of metal or both metal and oxygen, since external scale may or may not have been formed during the time period. It is typical for external scale to grow to some thickness before sufficient strains build up to cause scale cracking and subsequent exfoliation. Finally, 
the sample weight loss from materials evaporated during the time interval, $\mathrm{V}$, represents the loss of metal as long as the vaporized oxide/oxyhydroxide was formed during the time period, which is assumed to be the case. Test results will be interpreted within the context of this overall model.

\section{IN 617 Results}

The basic weight gain data for IN 617 specimens is presented in Figure 5.

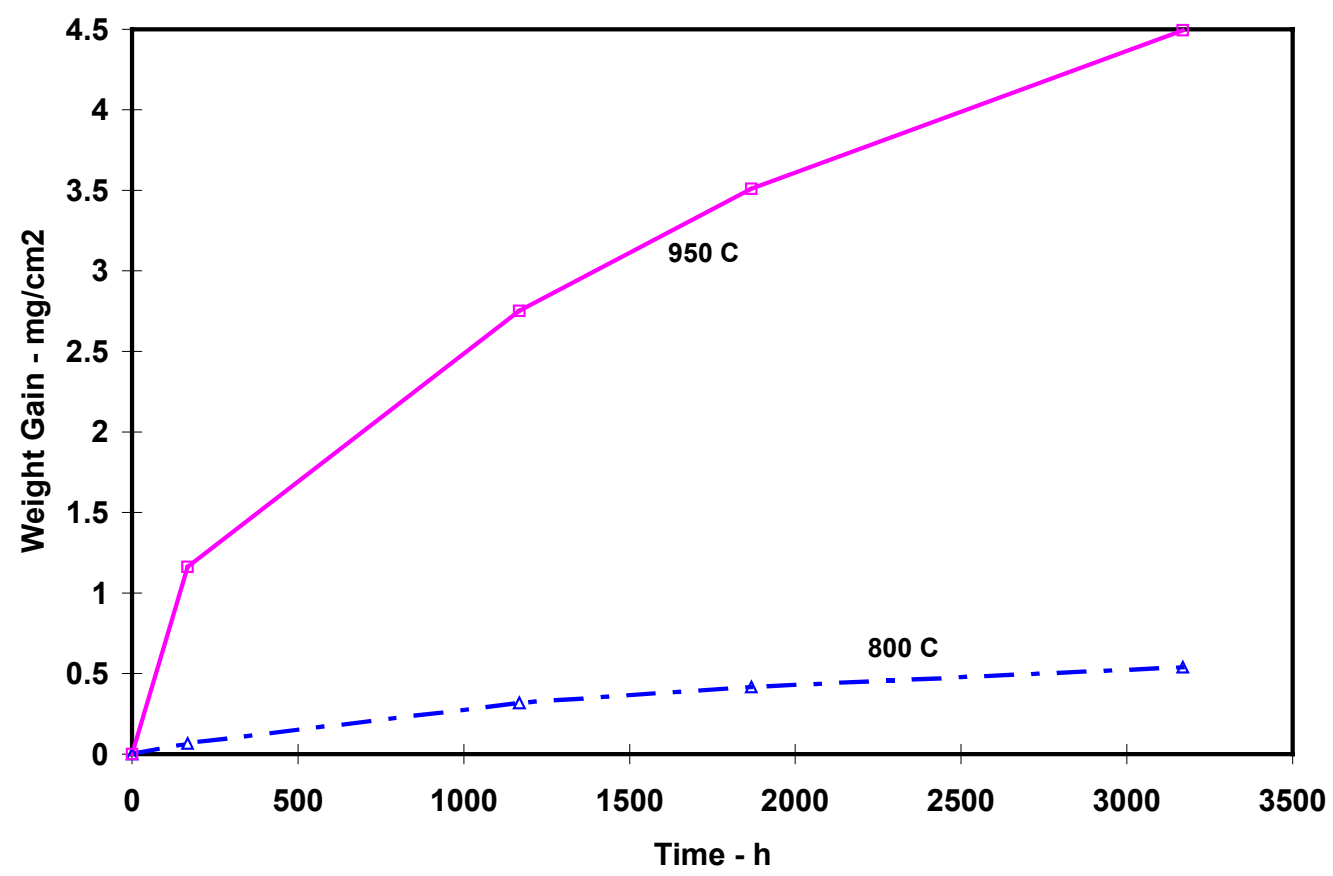

Figure 5: Weight gain of IN 617 during exposure.

\section{Parabolic Growth Model}

If the scale growth rate is limited by diffusion through the scale layer, a parabolic model should describe the weight gain versus time, i.e.:

$$
\left(\frac{M}{A}\right)^{2}=k_{p} t
$$

where $M / A$ is the total mass gain per unit area, $k_{p}$ is the parabolic rate constant, and $t$ is time.

These data may be fitted to a parabolic oxidation model by plotting weight gain versus the square root of time, as shown in the following figure. 


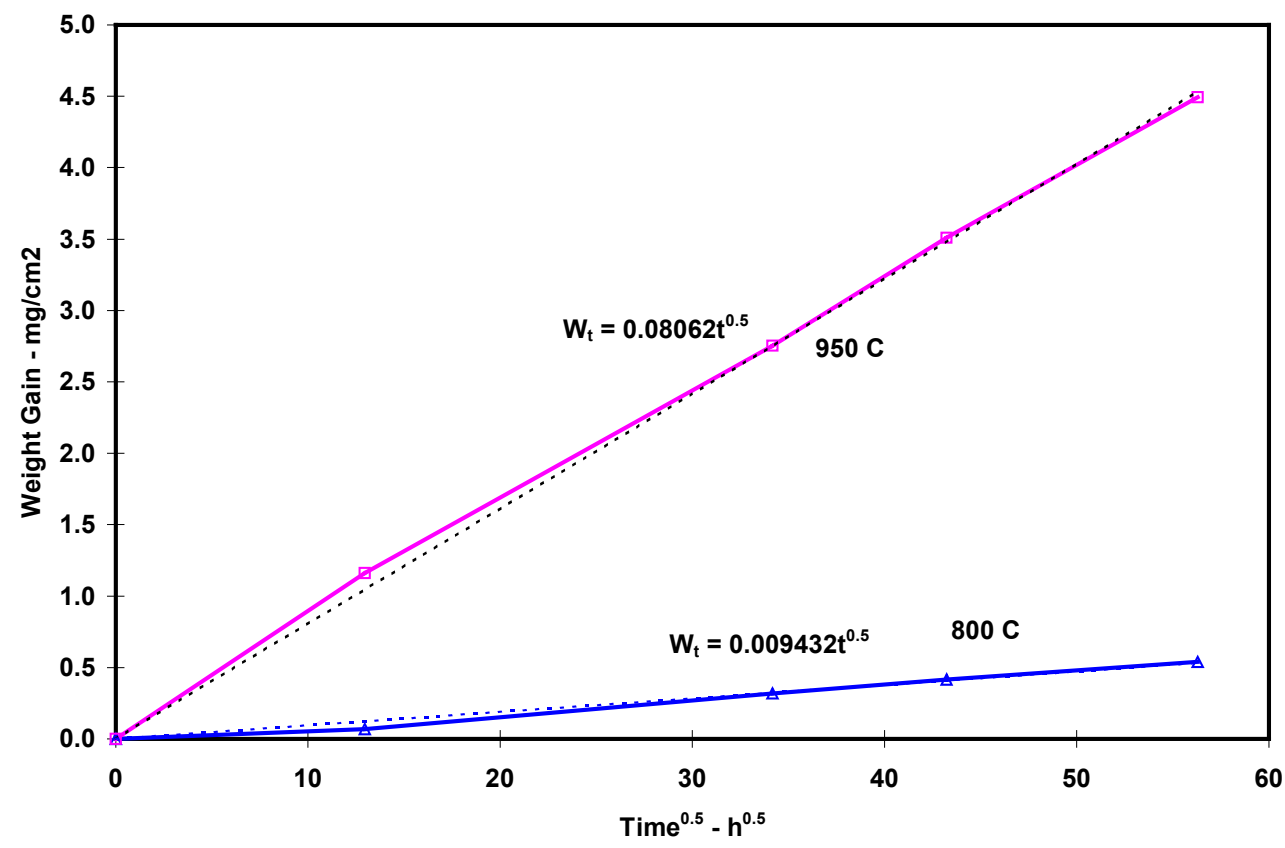

Figure 6: Fitting of parabolic growth model to IN 617 results.

The slope of the fitted line is the square root of the parabolic rate constant, $\mathrm{k}_{\mathrm{p}}$. In conventional units, the rate constants for steam oxidation of IN 617 are calculated to be $2.47 \times 10^{-14} \mathrm{~g}^{2} / \mathrm{cm}^{4}$.s at $800 \mathrm{C}$ and $1.81 \mathrm{x}$ $10^{-12} \mathrm{~g}^{2} / \mathrm{cm}^{4}$.s at $950 \mathrm{C}$. These rate constants can be interpreted in terms of an Arrhenius model where:

$$
k_{p}=A e^{-E_{a} / R T}
$$

with $E_{a}$ being the activation energy of the reaction. The value of $E_{a}$ can be determined from the slope of the line in a plot of log $k_{p}$ versus $1 / T$, Figure 7 . In this case, the activation energy is determined to be 312 $\mathrm{kJ} / \mathrm{mol}$. This compares with the activation energy for growth of $\mathrm{Cr}_{2} \mathrm{O}_{3}$ of $\cong 250 \mathrm{~kJ} / \mathrm{mol}$ and that for $\mathrm{Al}_{2} \mathrm{O}_{3}$ of $\cong 500 \mathrm{~kJ} / \mathrm{mol}$, values which bracket the activation energies normally observed for superalloy oxidation in air/oxygen. 


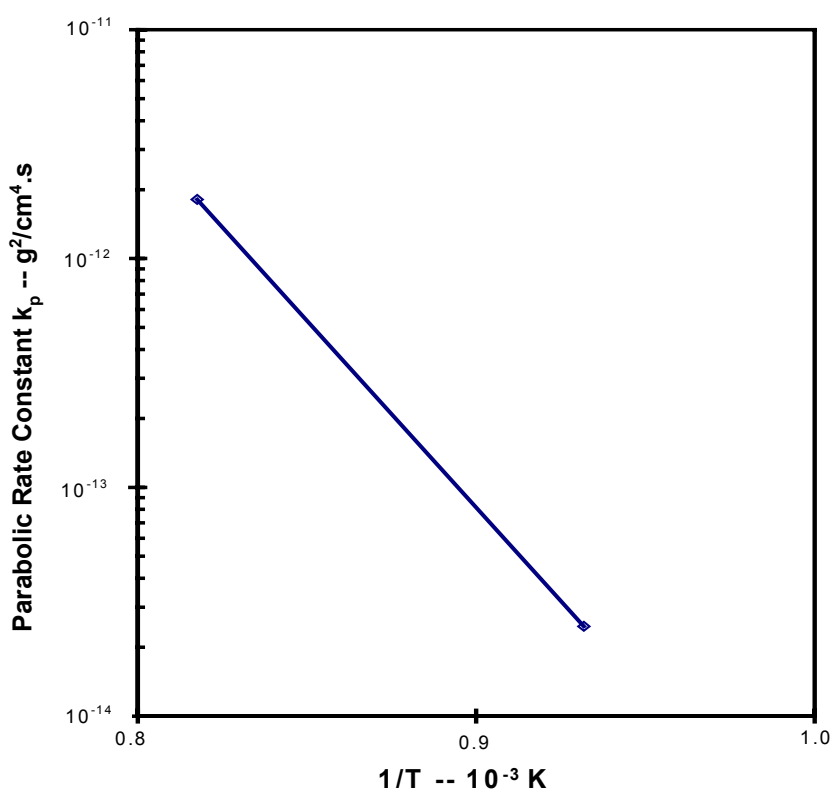

Figure 7: Arrhenius plot for IN 617 steam oxidation.

As discussed above, the steam oxidation of IN 617 seems to offer little to differentiate from air oxidation.

\section{Vaporization}

The upper temperature limit for use of alloys which form protective chromia scales, $\mathrm{Cr}_{2} \mathrm{O}_{3}$, is set by the loss of volatile $\mathrm{CrO}_{3}$ which forms in oxidizing atmospheres. When $\mathrm{Cr}_{2} \mathrm{O}_{3}$ is heated above 1,000 C in an oxidizing atmosphere, a loss in weight is noted which has been attributed to the formation and subsequent vaporization of $\mathrm{CrO}_{3}$. As the vapor pressure of chromium trioxide rises above approximately $10^{-5} \mathrm{~mol}-\%$ (i.e. $\sim 10^{-7}$ bar), the loss of chromium become significant in relation to the weight gain of the sample, as noted by Tedmon (Ref. 3). Thermodynamic calculations for the vapor pressure in air of various chromium oxides in equilibrium with solid $\mathrm{Cr}_{2} \mathrm{O}_{3}$ are shown in Figure 8 . In quantitative terms, the weight gain of the sample via oxygen pickup, as given by Eq. (2), is countered by a mass loss of chromium at a constant rate.

$$
\left(\frac{M}{A}\right)=-k_{v} t
$$

where $k_{v}$ is a vaporization rate constant. Consideration of these two processes shows that a point in time will be reached when the scale maintains a constant thickness and the sample experiences a linear mass loss with time. 


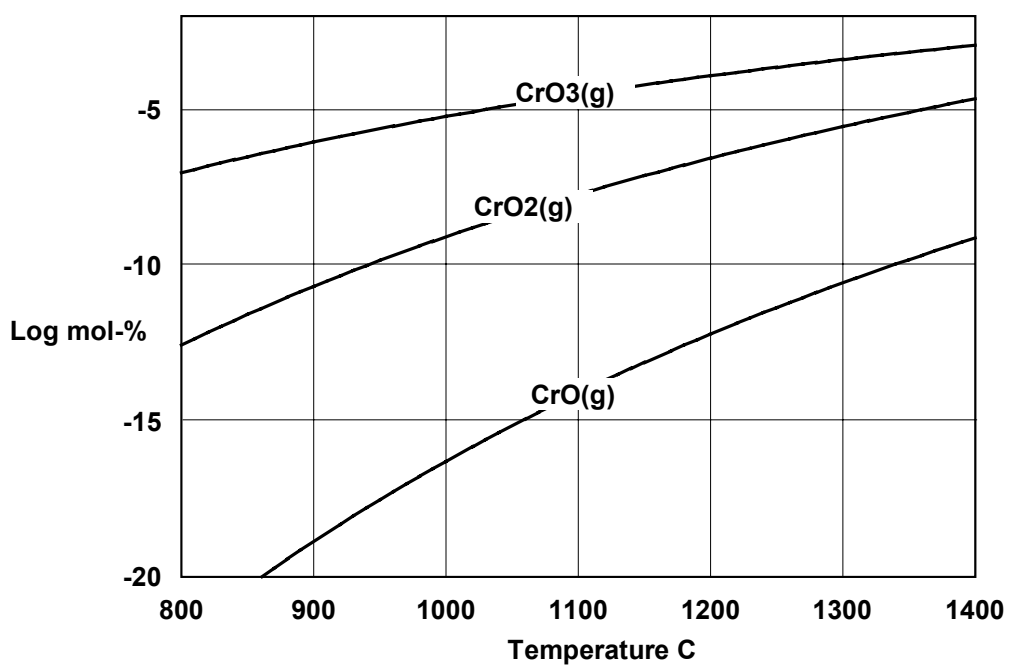

Figure 8: Vapor pressure of volatile chromium oxides in air.

A similar situation is to be expected for the steam oxidation of chromia-forming alloys such as IN 617, A range of additional volatile chromium oxyhydroxides must now be considered. The major volatile species are shown in Figure 9.

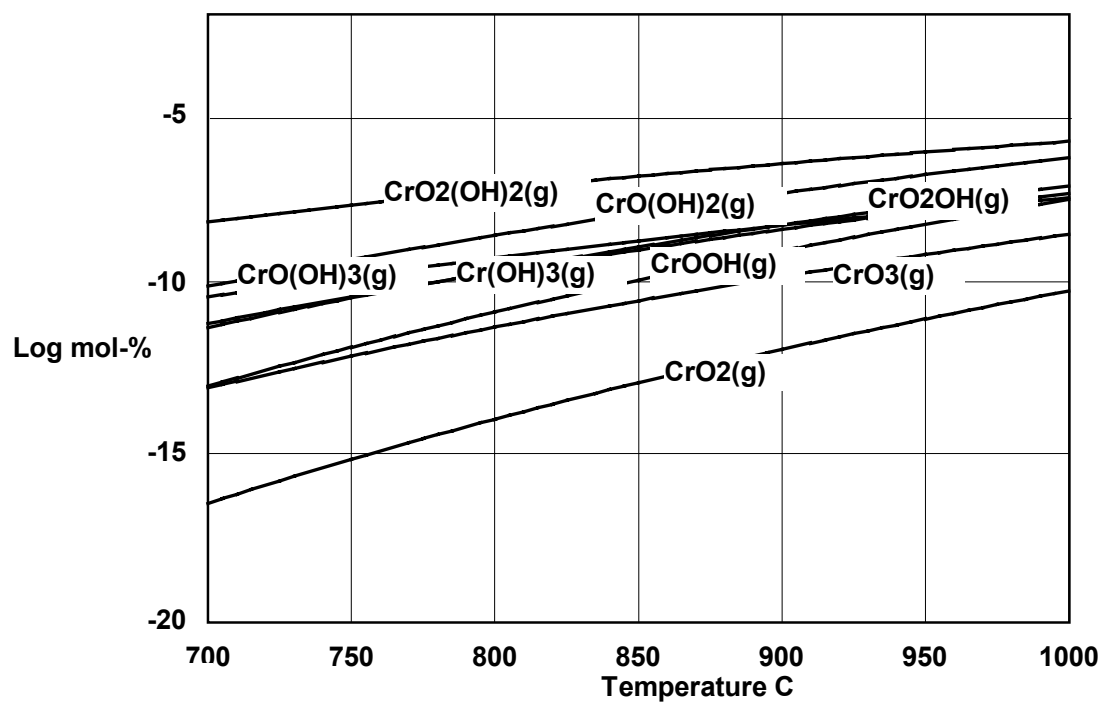

Figure 9: Vapor pressure of volatile chromium oxides/oxyhydroxides in steam.

While the vapor pressure of $\mathrm{CrO}_{3}$ is reduced from the value calculated for an air atmosphere, the overall vapor pressure of all volatile chromium species is approximately the same. The rate of loss of chromium via vaporization would thus be expected to be similar for air and steam atmospheres.

The maximum possible rate of vaporization of a species may be calculated via the Hertz-Langmuir equation: 


$$
J_{i}=\frac{P_{i}}{\sqrt{2 \pi m_{i} k T}}
$$

where $J_{i}$ is the flux rate in molecules per $\mathrm{cm}^{2}$ per sec, $P_{i}$ is the vapor pressure, $m_{i}$ is the mass of a molecule, $k$ is Boltzmann's constant, and $T$ is the temperature in K. Following Rapp (Ref. 4), the gravimetric weight loss for a sample may be determined by reformatting and restating with more convenient units to:

$$
G_{i}=\frac{P_{i}}{2.256 \times 10^{-2}}\left(\frac{M_{i}}{T}\right)^{0.5}
$$

where $G_{i}$ is the maximum possible vaporization weight loss in grams per $\mathrm{cm}^{2}$ per second, $P_{i}$ is the vapor pressure in atmospheres, and $M_{i}$ is the molecular weight of the species. At $950 \mathrm{C}$, the maximum rate of chromium loss is thus estimated to be $0.91 \times 10^{-3} \mathrm{mg} / \mathrm{cm}^{2}$.s. The kinetic expression used to model the actual weight loss, Eq. 2, may be differentiated with respect to time to allow calculation of the rate of weight gain at any time $t$ :

$$
\frac{d\left(\frac{M}{A}\right)}{d t}=0.5 k_{p} t^{-0.5}
$$

At $5,000 \mathrm{~h}$, the rate of weight gain at $950 \mathrm{C}$ is calculated by Eq. 7 to be $1.58 \times 10^{-7} \mathrm{~g} / \mathrm{cm}^{2}$.s with no obvious departure from parabolic behavior. However, it is clear that some volatilization is occurring based on observed staining of the alumina sample tray during the exposure time. The rate of loss in weight due to vaporization must therefore be only a minor fraction of this value, i.e. $<<1.58 \times 10^{-7} \mathrm{~g} / \mathrm{cm}^{2}$.s. While this may seem inconsistent with the Hertz-Langmuir calculation, results presented by Graham and Davis (Ref. 5 ) show that the actual vaporization rate may be only a very small fraction of the maximum rate when low gas velocity is used in experiments. The influence of chromia vaporization is likely to be greater in the high velocity environment of a gas turbine blade cooling passage. It must be emphasized, however, that this analysis does not indicate that the situation will be much different between steam and air cooling.

Aluminum oxides and oxyhydroxides do not exhibit significant vapor pressure in the temperature range expected in gas turbine service. Vaporization of the protective oxide layer is thus not an issue for the alumina-forming alloys tested, CM 247LC and CMSX-4, for either air or steam cooling.

\section{Effect of Pressure}

The tests reported here were performed at atmospheric pressure. However, the actual gas turbine application will employ steam at an elevated pressure. Abe and Yoshida (Ref. 6) report the results of exposure of IN 617 to steam at $800 \mathrm{C}$ and $4 \mathrm{MPa}$ pressure. A comparison of results is presented in Figure 10. The high pressure results exhibit non-parabolic (closer to cubic) kinetics but tend toward similar kinetics to those observed in these tests as the 1,000 hour point is reached. On further exposure, the high pressure sample exhibited breakaway corrosion attributed to scale cracking and subsequent oxidation of a chromium depleted zone beneath the scale. A similar effect was not observed in these tests. The noted differences in these results are most likely due to differences in alloy composition and preparation rather than due to any significant influence of steam pressure. 
Appendix 2

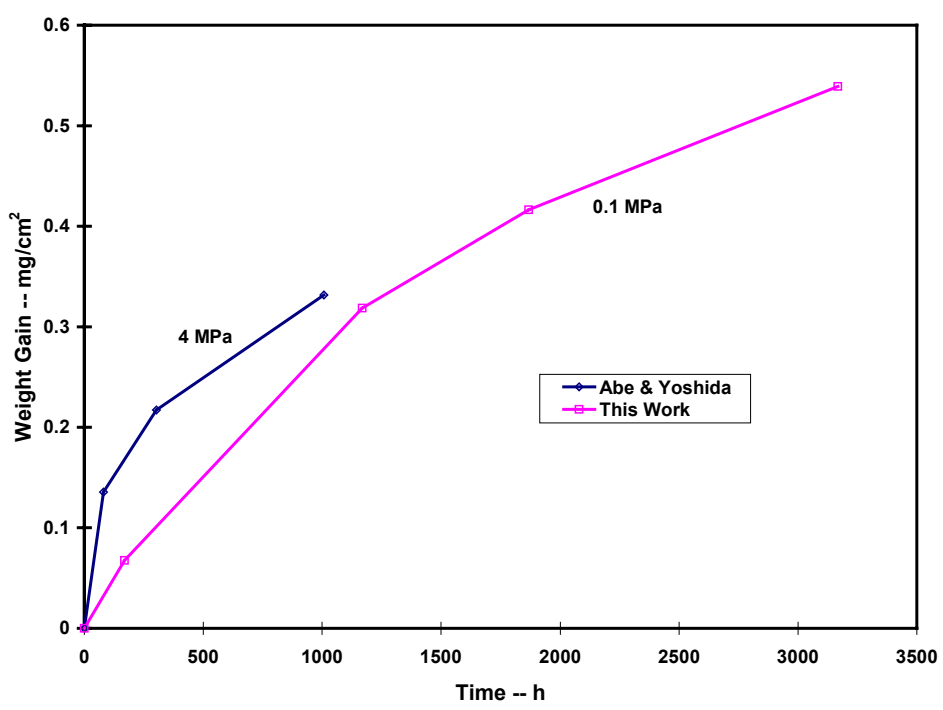

Figure 10: Steam oxidation of IN 617 at two pressures.

\section{Weight Gain Results}

Weight gain data for the alloys IN 738LC, CM 247LC, and CMSX-4 are presented in Figures 11 and 12 for $800 \mathrm{C}$ and $950 \mathrm{C}$ exposure temperatures.

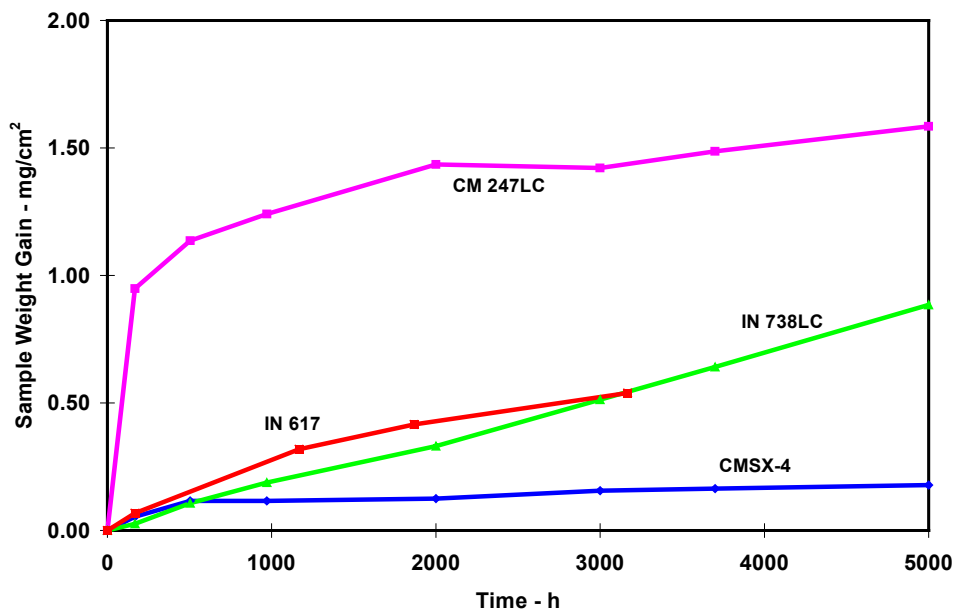

Figure 11: Sample weight gain in steam at $800 \mathrm{C}$. 


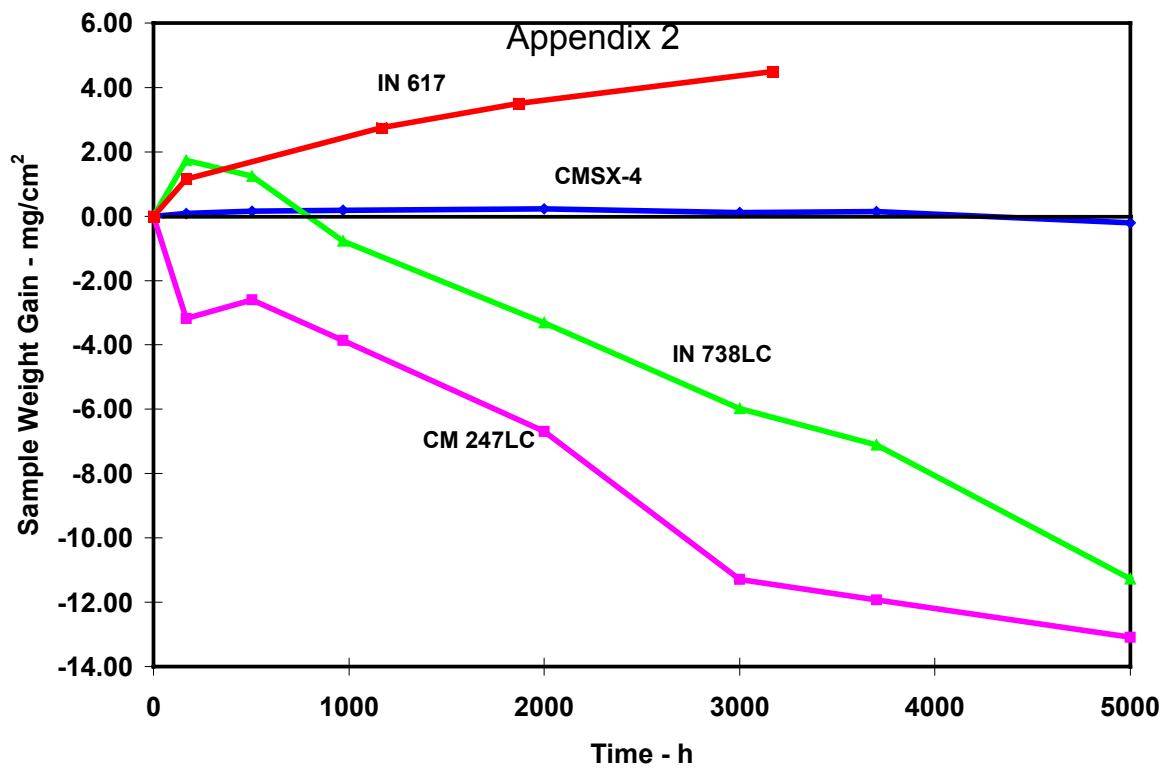

Figure 12: Weight gain results in steam at $950 \mathrm{C}$.

As previously discussed, IN 617 exhibits weight gain over time which closely matches a parabolic growth model. This is not generally the case for the other alloys tested.

\section{IN 738LC}

This alloy exhibits weight gain versus time behavior which is typical for alloys which experience scale cracking and exfoliation with cyclic temperature exposure. At $800 \mathrm{C}$, this leads to the apparent linear oxidation behavior while at $950 \mathrm{C}$, exfoliation dominates the weight gain data after an initial period for the scale to grow to a thickness which can no longer tolerate the thermal strain of the heat-up/cool-down cycle.

\section{247LC}

The samples of CM 247LC exhibit somewhat unusual behavior in these tests. At $800 \mathrm{C}$, oxidation is initially much more rapid than anticipated, followed by a period of more controlled growth. At $950 \mathrm{C}$, the samples severely exfoliated throughout the entire test period. Examination of some samples from the same batch of test pins revealed segregation of hafnium at the surface of the pin. It is suspected that the oxidation behavior shown here may not be typical of the performance that this alloy would generally show.

\section{CMSX-4}

This alloy exhibited the best oxidation performance of the alloys tested. Oxidation kinetics were nearly parabolic in the early stages of exposure. Fitting of the early data, through 1,000 hours, to a parabolic growth model yielded values of $k_{p}$ of $6.7 \times 10^{-15}$ at $800 \mathrm{C}$ and $1.2 \times 10^{-13} \mathrm{~g}^{2} / \mathrm{cm}^{4} . \mathrm{s}$ at $950 \mathrm{C}$. Figure 13 compares these values with literature values (Ref. 7) for a similar single crystal superalloy, CMSX-2, oxidized in air. As was noted for IN 617, there appears to be little difference between air and steam oxidation. At exposures longer than 1,000 hours, the samples at $800 \mathrm{C}$ exhibited insignificant additional weight gain while at $950 \mathrm{C}$, slight exfoliation resulted in minor weight loss. 


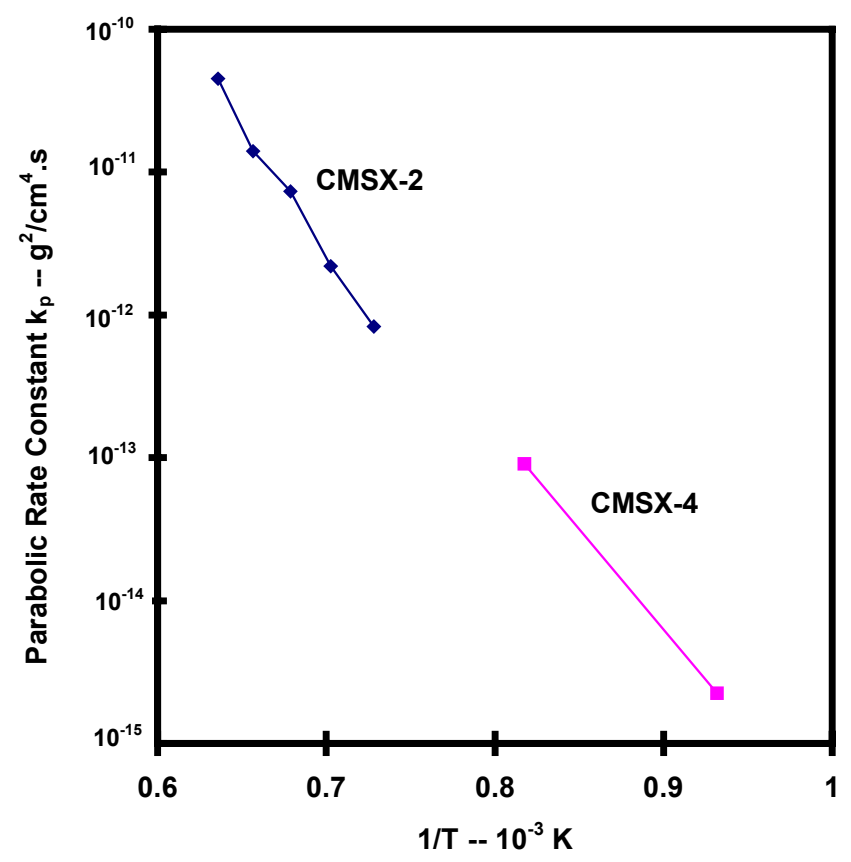

Figure 13: Arrhenius plot for CMSX-4.

\section{Air Oxidation Results}

In order to further understand possible differences between steam and air oxidation of the test alloys, samples of IN 738LC, CM 247LC, and CMSX-4 were exposed to air in the test facility for approximately 1,200 hours. Weight gain results are provided in Figures 14 and 15.

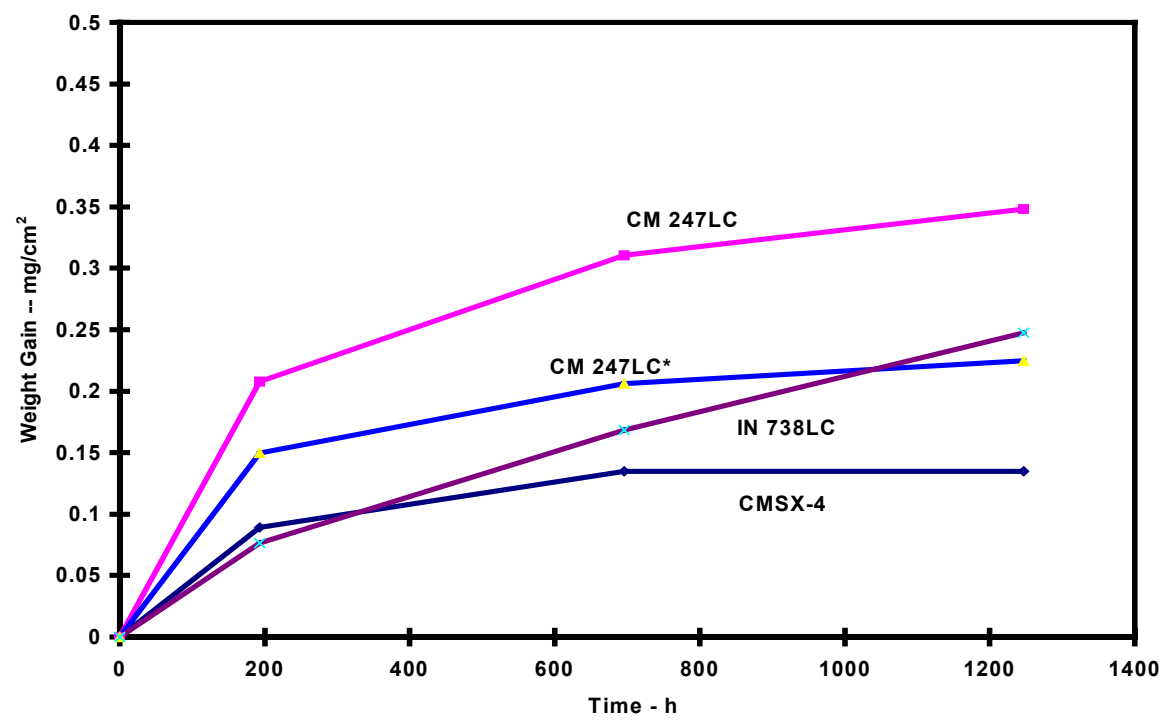

Figure 14: Weight gain results in air at $800 \mathrm{C}$. 


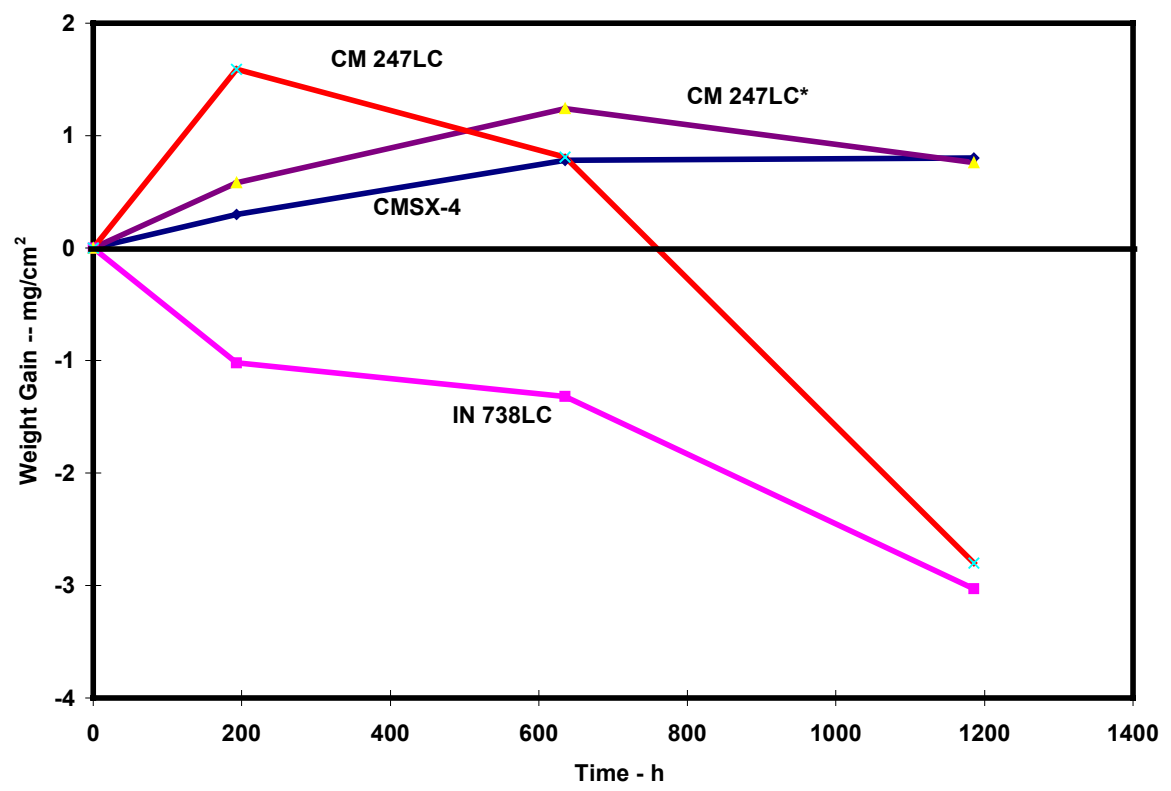

Figure 15: Weight gain results in air at $950 \mathrm{C}$.

Since the performance of the CM 247LC was suspect in the steam oxidation testing, samples of this alloy (in the form of $12 \mathrm{~mm}$ OD bars with ground surface finish and identified in the figures as CM 247LC*) from a different source were exposed in parallel in these tests in air. This second source sample of CM 247LC did perform better, adding further confirmation of possible deficiencies with the samples tested in steam.

In general, the pattern of weight gain and oxide spallation in air exposure for the various alloys followed the same pattern observed in steam.

\section{Metallographic Examination Results}

A the conclusion of the 5,000 hour exposure period, crossections of selected pins were metallographically examined by optical and scanning electron microscopy. Results are documented in the Appendix in the form of optical micrographs with magnification of $100 \mathrm{X}$ and $500 \mathrm{X}$ for each alloy at $800 \mathrm{C}$ and $950 \mathrm{C}$. Additionally, backscatter electron images of the samples exposed at $950 \mathrm{C}$ are presented in Photo 1 . In Photo 2, these images are overlayed by elemental dot maps color-keyed as follows: nickel -- blue, chromium -- green, aluminum -- red, and oxygen -- yellow. Results are generally as expected. IN 617 and IN 738LC samples have developed an external scale of chromia with an internal oxidation product of alumina. The CM 247LC and CMSX-4 samples have developed an alumina protective oxide layer with no gross internal oxidation. 


\section{References}

1. Nava Paz, J. C., Goodstine, S. L., and Gibbons, T. B., "Oxidation of Ni-Based Superalloys in Superheated Steam - Review of Literature and Preliminary Thermochemical Analysis", Internal ABB Report MCW TR-9422, July 1994.

2. Smialek, J. L., and Meier, G. H., Chapter 11: High Temperature Oxidation, in Superalloys II, Ed. Sims, Stoloff, and Hagel, John Wiley \& Sons Inc., New York, 1987, p. 293-326.

3. Tedmon, C. S. Jr, "The Effect of Oxide Volatilization on the Oxidation Kinetics of $\mathrm{Cr}$ and Fe-Cr Alloys", Journal of the Electrochemical Society, Vol 113 No. 8, August 1966, p.766-768.

4. Rapp, R. A., "Vaporization Losses from $\mathrm{Cr}_{2} \mathrm{O}_{3}$ Protective Scales", AGARD Conference on HighTemperature Corrosion of Structural Materials, February 1972.

5. Graham, H. C., and Davis, H. H., "Oxidation/Vaporization Kinetics of $\mathrm{Cr}_{2} \mathrm{O}_{3}$ ", Journal of the American Ceramic Society, Vol 54 No. 2, February 1971, p. 89-93.

6. Abe, F., and Yoshida, H., "Corrosion Behaviors of Heat Resisting Alloys in Steam at $800{ }^{\circ} \mathrm{C}$ and 40 atm Pressure", Z. Metallkde., Bd. 76 (1985) H. 3, p. 219-225.

7. Pieraggi, B., and Dabosi, F., "High-Temperature Oxidation of a Single Crystal Ni-based Superalloy", Werkstoffe und Korrosion 38, p. 548-590, 1987. 
Appendix 2

\section{Appendix}


Table 1: Certified Analyses for Test Bars

$\begin{array}{lllll}\text { Source } & \text { Metal Samples } & \text { PCC } & \text { PCC } & \text { PCC } \\ \text { Alloy } & \text { IN } 617 & \text { IN 738LC } & \text { CM 247LC } & \text { CMSX-4 }\end{array}$

\section{Element Amount (wt\% or ppm)}

\begin{tabular}{|c|c|c|c|c|}
\hline C & 0.08 & 0.11 & 0.11 & $21 \mathrm{ppm}$ \\
\hline$S$ & $<.001$ & 0.0005 & 0.0003 & 3 ppm \\
\hline $\mathrm{Si}$ & 0.12 & 0.02 & 0.03 & 0.011 \\
\hline $\mathrm{Mn}$ & 0.02 & $<.01$ & $<0.01$ & $<0.001$ \\
\hline$P$ & & $<.010$ & $<0.010$ & $<0.005$ \\
\hline $\mathrm{Cr}$ & 21.65 & 15.88 & 8.12 & 6.4 \\
\hline Mo & 9.6 & 1.76 & 0.48 & 0.6 \\
\hline $\mathrm{Fe}$ & 0.55 & 0.04 & 0.02 & 0.027 \\
\hline $\mathrm{Ti}$ & 0.25 & 3.37 & 0.68 & 1.03 \\
\hline $\mathrm{Al}$ & 1.21 & 3.55 & 5.57 & 5.62 \\
\hline Co & 12.57 & 8.15 & 9.28 & 9.6 \\
\hline $\mathrm{Ni}$ & 53.94 & Balance & Balance & Balance \\
\hline W & & 2.48 & 9.41 & 6.4 \\
\hline V & & $<0.05$ & $<0.01$ & $<0.005$ \\
\hline $\mathrm{Cu}$ & 0.01 & $<0.02$ & 0.02 & $<0.001$ \\
\hline $\mathrm{Zr}$ & & 0.037 & 0.011 & $<10$ ppm \\
\hline$B$ & & 0.009 & 0.017 & $<20$ ppm \\
\hline $\mathrm{Cb}$ & & 0.89 & $<0.01$ & $<0.05$ \\
\hline $\mathrm{Ta}$ & & 1.78 & 1.19 & 6.5 \\
\hline $\mathrm{Re}$ & & $<0.05$ & & 2.9 \\
\hline $\mathrm{Hf}$ & & $<0.05$ & 1.36 & 0.1 \\
\hline $\mathrm{N}$ & & 11 ppm & $5 \mathrm{ppm}$ & $2 \mathrm{ppm}$ \\
\hline $\mathrm{O}$ & & $6 \mathrm{ppm}$ & $5 \mathrm{ppm}$ & $2 \mathrm{ppm}$ \\
\hline $\mathrm{Mg}$ & & & & 37 ppm \\
\hline Sn & & & & $<0.5 \mathrm{ppm}$ \\
\hline $\mathrm{Ag}$ & & $<3$ ppm & & $<1$ \\
\hline $\mathrm{Se}$ & & $<0.5 \mathrm{ppm}$ & & $<0.5$ ppm \\
\hline $\mathrm{Te}$ & & $<0.5 \mathrm{ppm}$ & & $<0.2 \mathrm{ppm}$ \\
\hline $\mathrm{TI}$ & & $<0.5$ ppm & & $<0.2$ ppm \\
\hline $\mathrm{Pb}$ & & $<1.0$ ppm & & $<0.5 \mathrm{ppm}$ \\
\hline $\mathrm{Bi}$ & & $<0.3$ ppm & & $<0.2$ ppm \\
\hline
\end{tabular}




\section{IN 617950 C}
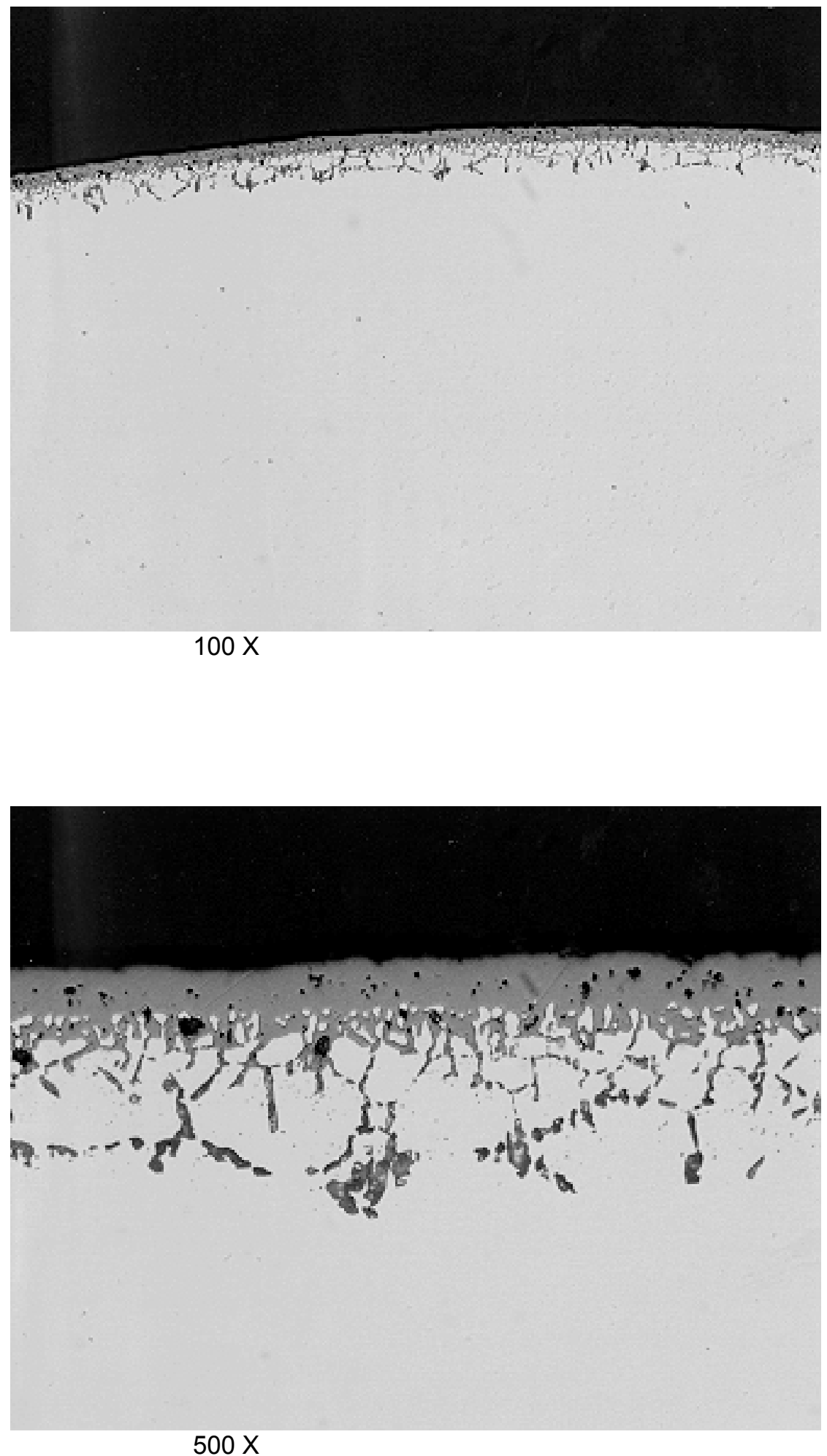


\section{IN 617800 C}
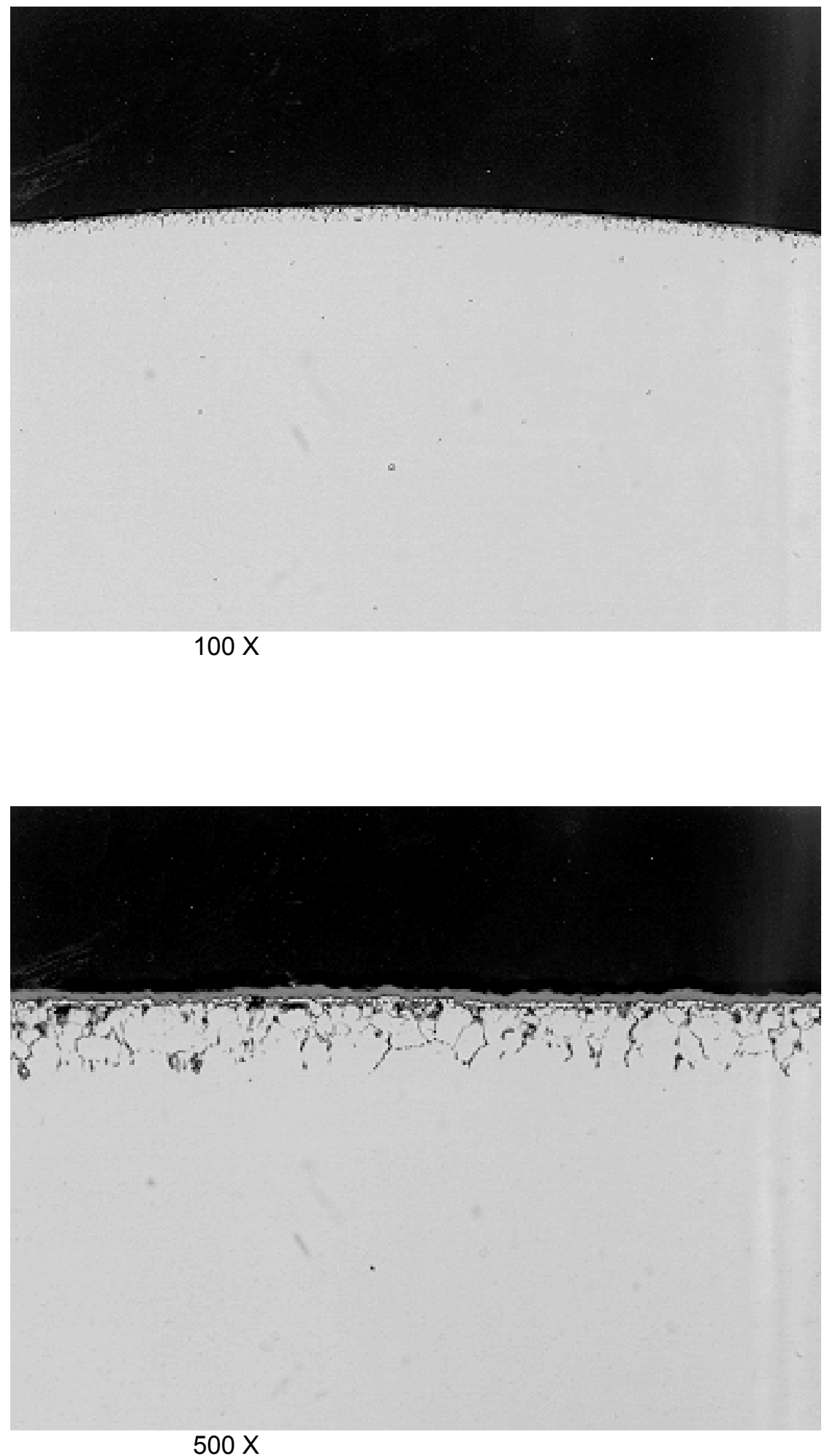

Page 18 
IN 738LC 800 C
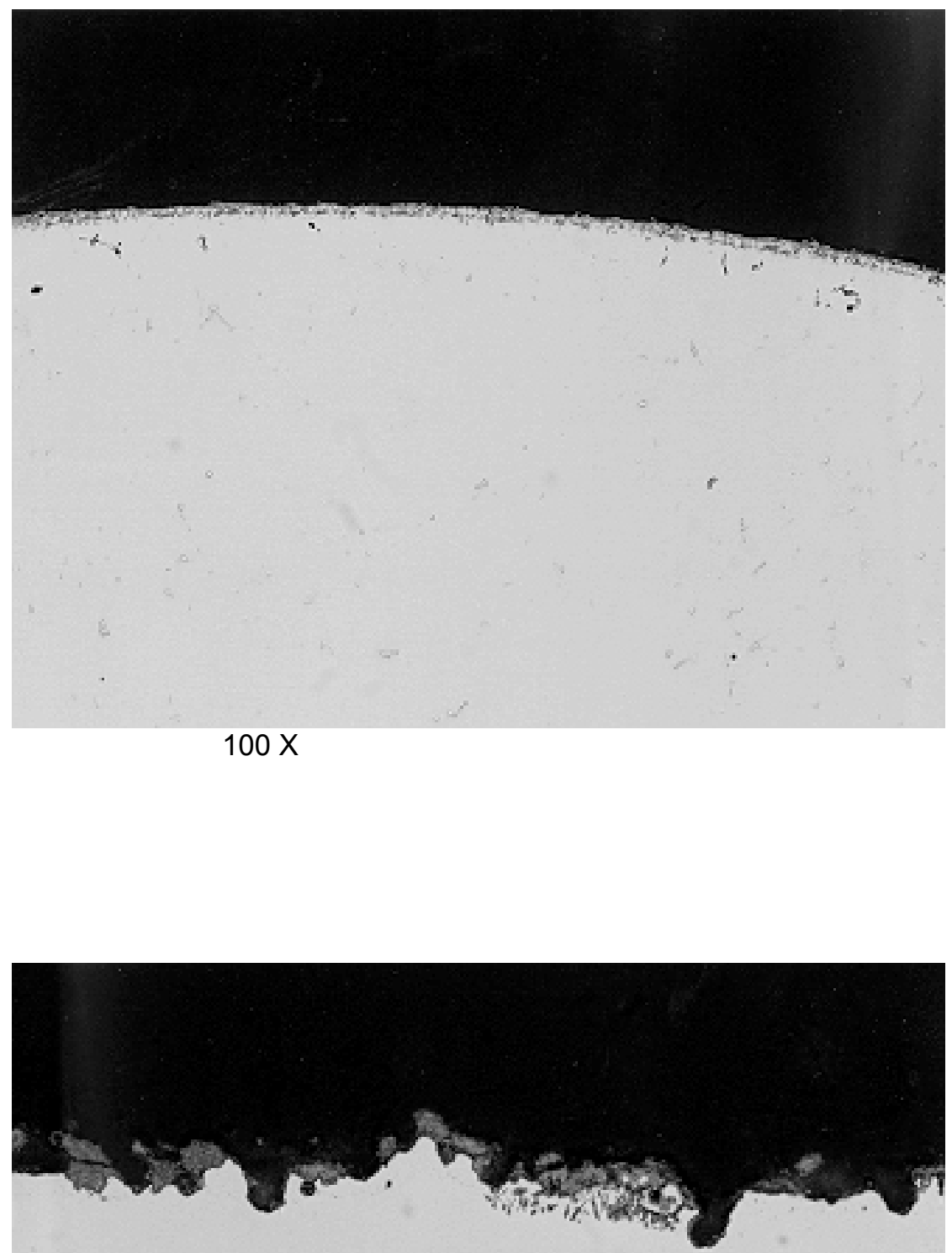

$500 x$

Page 19 
IN 738LC 950 C
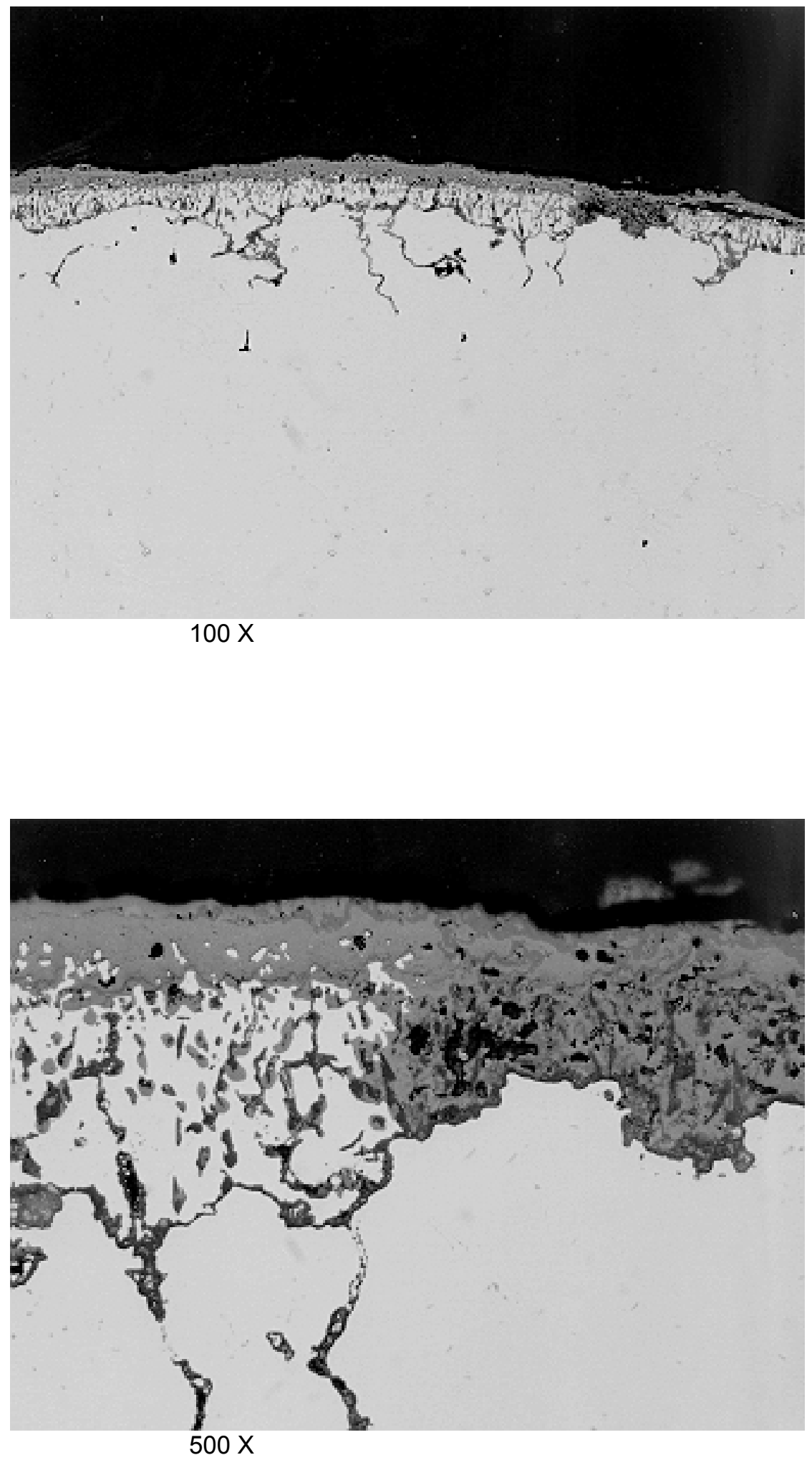
CM 247LC 800 C

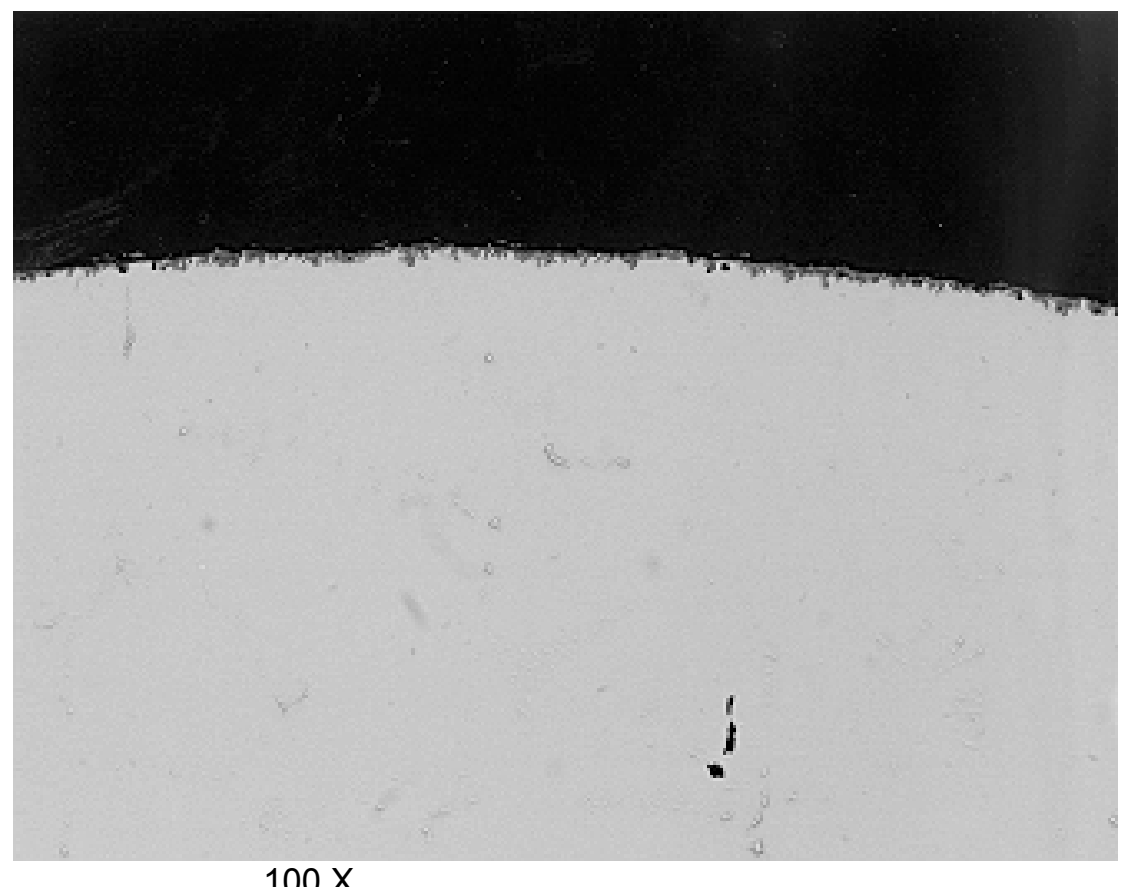

$100 x$

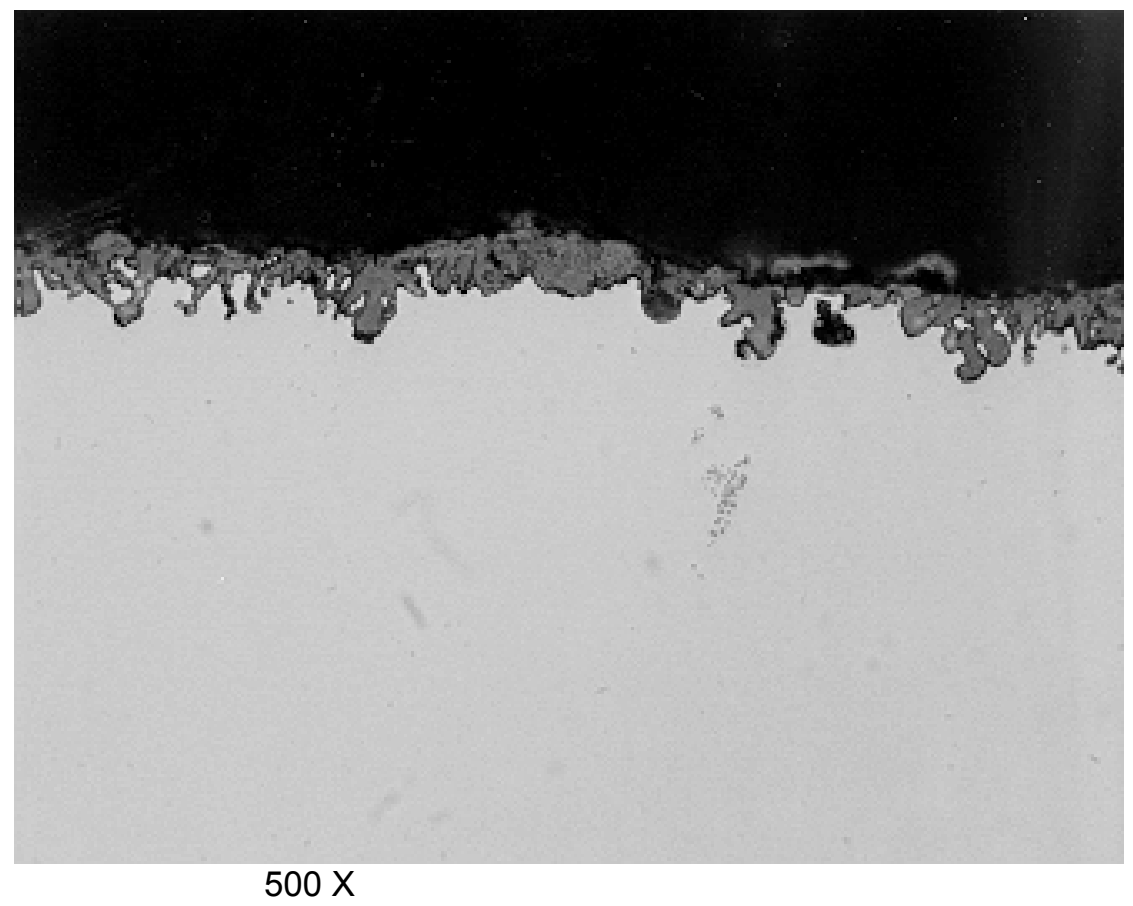

Page 21 
CM 247LC 950 C
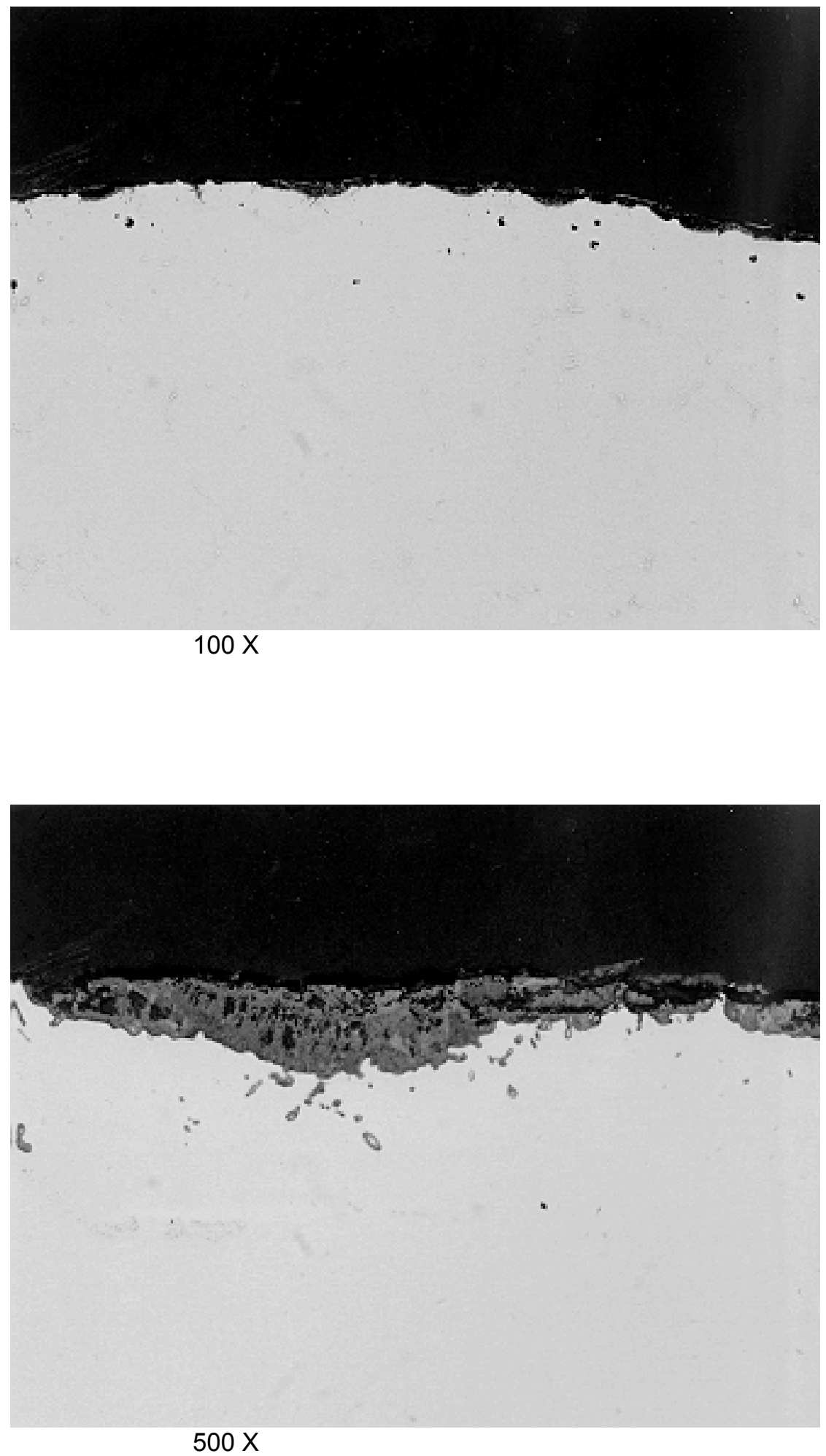

Page 22 
CMSX-4 $800 \mathrm{C}$
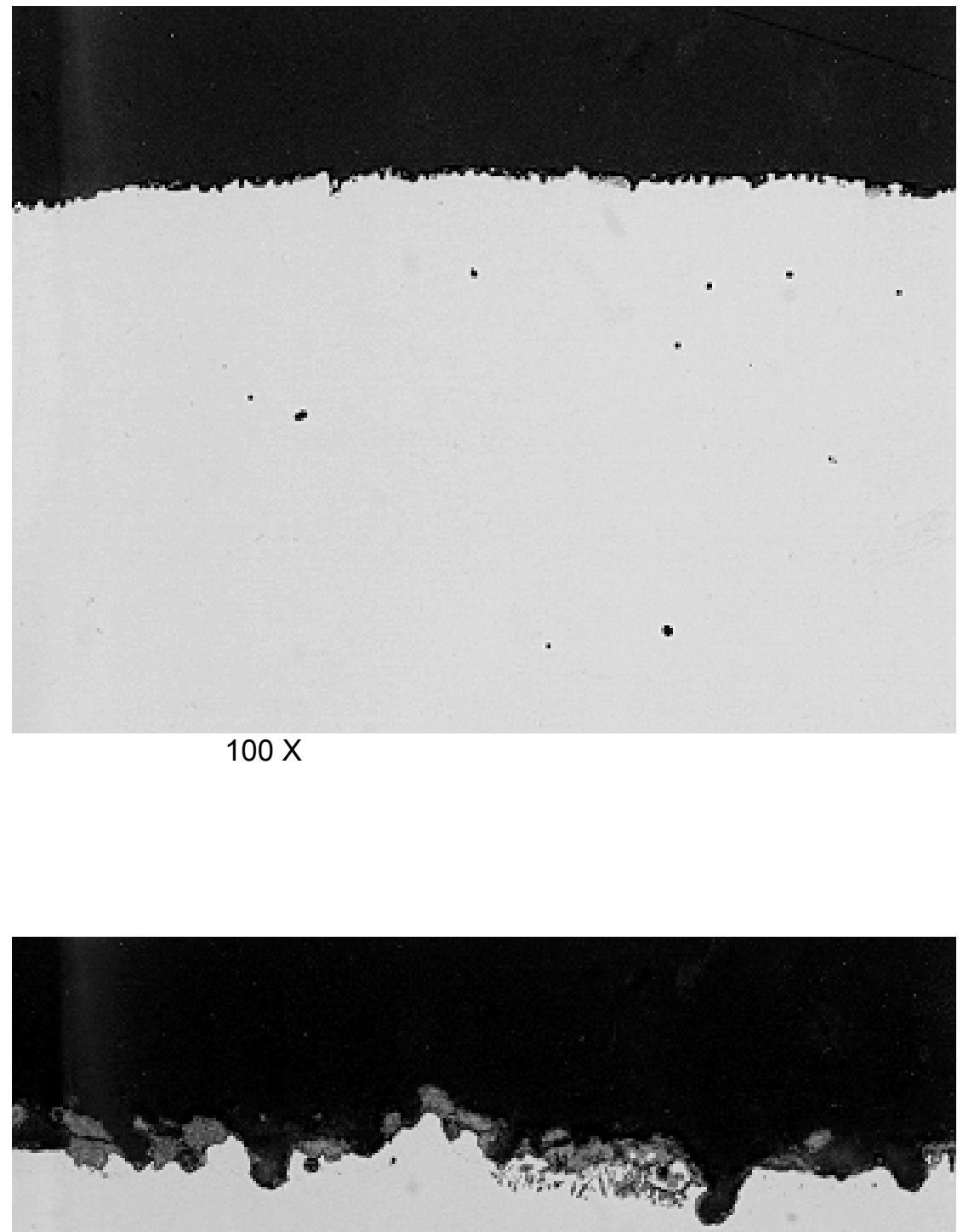

$500 x$ 
CMSX-4 950 C
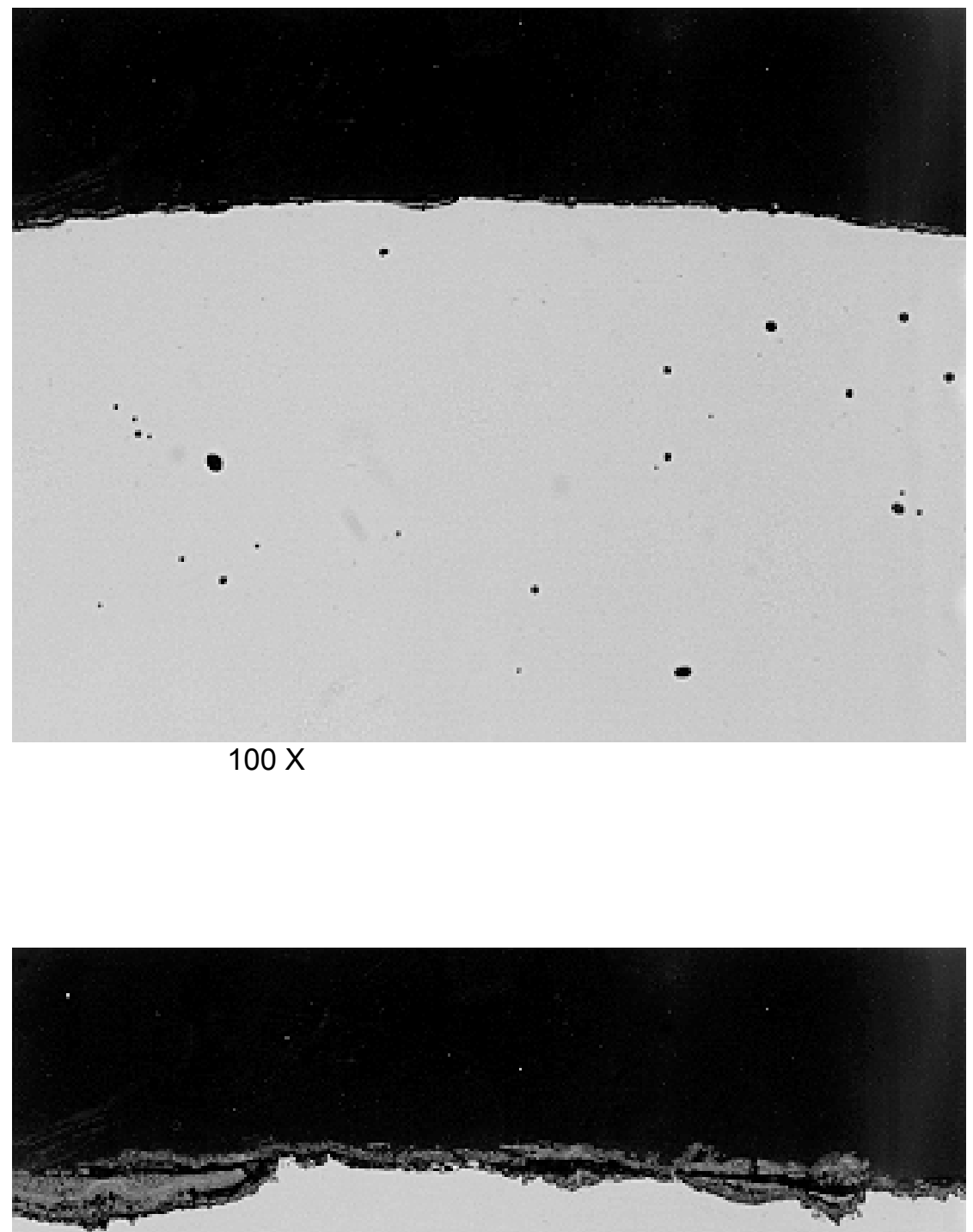

$500 x$ 


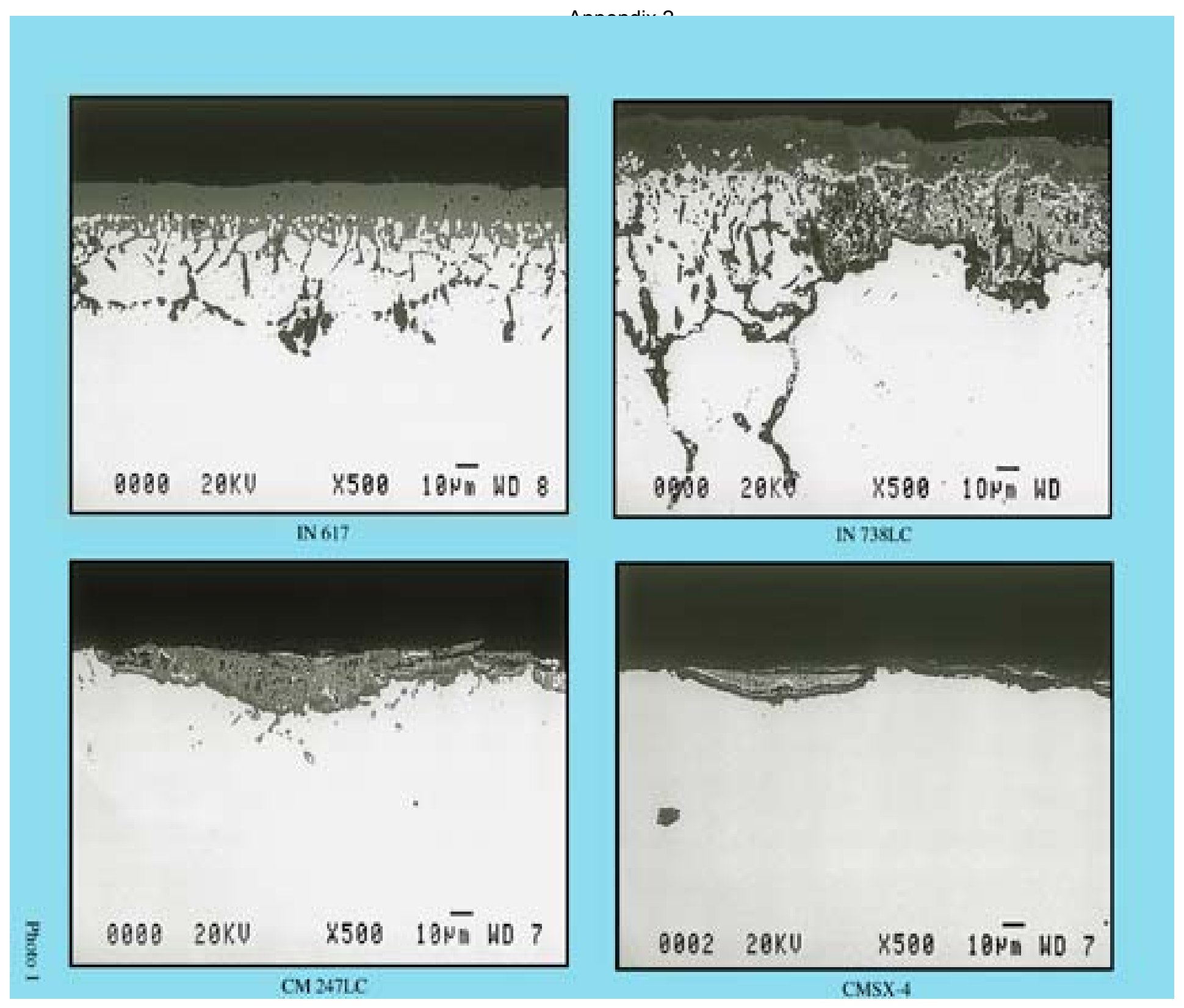




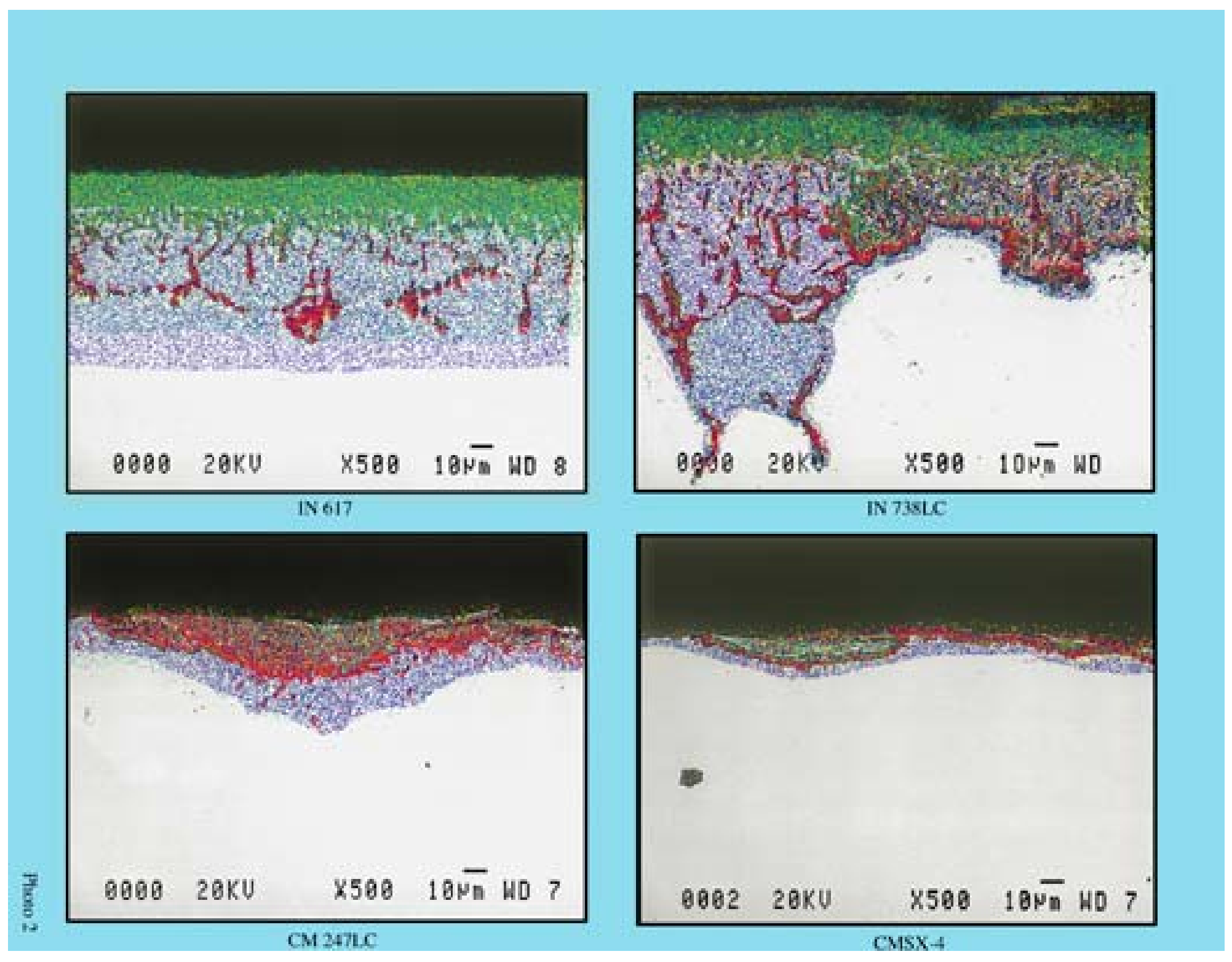


Appendix 3

\section{Appendix 3}

Aero Thermal integration results 
Gas Turbine Laboratory

Department of Aeronautics and Astronautics

Massachusetts Institute of Technology

Cambridge, MA 02139

Final Technical Report

entitled

\title{
THE MEASUREMENT OF COOLED TURBINE PERFORMANCE
}

\author{
prepared for \\ ABB ALSTOM Power, Inc. \\ 5309 Commonwealth Centre Parkway \\ Midlothian, VA 23112 \\ Mr. Albrecht Mayer \\ Project Manager \\ (804)-763 2127
}

Investigator: $\quad$ Gerald R. Guenette, Jr.

Principal Research Engineer

Gas Turbine Laboratory

Research Students: Rory Keogh

Yi Cai

Jason J. Jacobs

Léo M. Grépin

Christopher M. Spadaccini

March 2000 
1. Executive Summary

2. Program Overview

3. Blowdown Turbine Test Facility

4. Fabrication of the Model Film-Cooled Turbine Stage

5. Turbine Torque Measurement

6. Mass Flow Measurement

7. Aerodynamic Performance Measurements

8. Experimental Program

9. Aerodynamic ('Rake') Measurement of Adiabatic Efficiency

10. Mechanical ('Brake') Measurement of Aerodynamic Efficiency

11. Un-Cooled Turbine Performance Testing

12. Film-Cooled Turbine Performance Testing

13. Experimental Data: Un-Cooled Tests

14. Efficiency Calculations: Un-Cooled Tests

15. Experimental Data: Film-Cooled Tests 


\section{Program Overview}

\subsection{Introduction}

To meet the demand for higher power densities from gas turbine systems, designers have devoted much effort over the past half-century toward increasing turbine inlet temperature. This has been accomplished through the development of both extraordinary turbine and disk materials and sophisticated internal and external cooling arrangements. The aerodynamic performance of turbines has likewise increased enormously, leading to polytropic efficiencies in the low $90 \%$ range. This efficiency increase has come through improved design techniques based on a better understanding of the fluid mechanics of turbines and the application of increasingly more powerful computational tools.

Supporting these improvements are empirical observations acquired through many years of testing on engines, component test rigs, and sub-scale experiments. Each of these test options have their advantages and limitations. For an engine test, the measurement uncertainty for individual component efficiencies is high, while sub-scale experiments may not model all relevant physical phenomena. An attractive option may be the full-scale test rig, however the high cost of running such facilities has limited turbine research at this level.

Over the past two decades a technology based on short-duration testing techniques has been developed. Short duration tests can provide highly accurate, detailed turbine measurements at relatively low cost. The methodology was made possible by the rapid advancement of high frequency response instrumentation and data acquisition technologies and by a realization that the physically relevant time scales of the processes within a turbine are on the order of hundreds of microseconds. Under suitable conditions, test durations of less than a second can provide tens of thousands of characteristic flow times over which to conduct an experimental investigation. Test facilities based on these methods can provide significant benefits to measurement resolution and flexibility while reducing the costs of the test hardware and operation.

Short duration techniques have subsequently been developed and successfully applied by a number of investigators $[2.1,2.2,2.3]$ to the detailed study of cooled turbine heat transfer and cooling. A goal of the present work was to extend these capabilities to the investigation of turbine aerodynamic performance. The work reported in Sections 5, 6, and 7 covers the development and demonstration of the resulting aerodynamic performance measurement technique. This effort was part of the overall program to provide a detailed study on the influence of film cooling upon the aerodynamic performance of a fully scaled, film cooled turbine stage.

\subsection{Turbine Aerodynamic Performance Measurements}

The benefits to be achieved from the employment of progressively more elaborate external film cooling schemes must be weighed against a variety of design issues. One critical issue is the impact of main flow/coolant flow interactions on turbine aerodynamic performance. The increase in turbine inlet temperature has come at the expense of turbine aerodynamic 
efficiency. The ability to quantitatively measure such penalties, however, has always been in question.

Several areas of active turbine research would benefit significantly from the ability to perform full stage tests economically. For example, given that approximately $20 \%$ of the loss from the nozzle guide vane results from wake mixing losses downstream of the vane trailing edge, the conditions under which mixing occurs will strongly influence the total loss, as discussed by Denton, [2.4]. The nozzle guide vane performance is commonly assessed from cascade tests where the vane wake mixes out at constant static pressure. However, in an engine (and fully scaled test rigs) the nozzle wake will mix out in the downstream rotor passage, and as discussed by Rose, [2.5], the resulting losses will differ significantly. This is especially significant for cooled nozzle guide vanes where up to $7 \%$ of the total mass flow is used for cooling and mixing losses increase significantly. Another area of active research that would benefit from full stage testing is the influence of unsteady rotor-stator interactions on the performance of blade film cooling schemes, Abhari [2.6].

\subsection{Experimental Investigation of Cooled Turbine Performance}

Many investigations on the interaction of the coolant flow with the turbine main flow have focused on the heat transfer problem. This is understandable given that small changes in the inlet temperature produce dramatic changes on the cycle performance and on the structural integrity and life of the highly stressed turbine components. Achieving the desired balance between these competing factors is a challenging task in the design process.

Comparatively little attention has been focused in past experimental investigations on how the coolant flow impacts the aerodynamic performance of the turbine and the consequent effects upon overall gas turbine system design. The reasons lay not in the fact that the problem is unimportant but in the level of complexity and cost of an experimental approach to the problem. Detailed experimental study of coolant flow interactions is impossible in the operational environment as a result of the extreme high temperatures and oxidizing conditions present in engine turbines. The technology to perform measurements within this environment at the required level of detail and accuracy is well beyond our current capability.

This situation has changed dramatically over the past two decade with the development of improved experimental techniques. The investigations preformed under the present program were enabled by the introduction of short duration testing, and improved instrumentation technologies developed at MIT and at other institutions located in the U.S. and other countries.

\subsection{Testing in Short Duration Facilities}

In the MIT facility, tests are performed on geometrically scaled models of actual engine turbine stages. Test conditions, (pressures, temperatures, shaft speed, etc.), are held at experimentally 'benign' levels for the measurements, but are set at the appropriate ratios to match the important independent non-dimensional parameters which govern the fluid physics, (e.g. stage pressure ratio, corrected speed, specific heat ratio, Reynolds number, gas to wall and coolant to gas temperature ratios, etc.). Thus set, these conditions provide an environment that accurately simulates the engine turbine operational environment. Through our knowledge of the 
fundamental physical scaling laws, the measured values of the dependent non-dimensional parameters (e.g., corrected through-flow, efficiency, heat transfer coefficients) will then match those occurring in the operational machine.

Short duration facilities also make use of the fact that the time scales of the flow through the turbine are of the order of the blade passage and rotor revolution times. These are typically in the range of 0.1 to 10 milliseconds. Thus any instrumentation developed to measure phenomena at these scales, (e.g. shock, wake, and hot streak interactions), will only need a short time window $(\sim 100 \mathrm{~ms})$ to perform them. This is very important in testing high performance turbine stages, where, even the scaled test environment will generate substantial power (up to $\sim 2 \mathrm{MW}$ ) from a model turbine. However, the overall energy consumption of a short duration test is very low and leads to the advantage of performing flexible, low relative cost tests. The MIT facility can provide fully scaled conditions durations of 300 to $800 \mathrm{~ms}$, which is more than adequate for turbine heat transfer and aerodynamic performance investigations. Our experience has shown that these techniques have greatly added to our experimental knowledge of the heat transfer and (under the current program) the aerodynamic loss processes in film cooled turbines.

\subsection{Turbine Efficiency Measurement Instrumentation}

Turbine stage efficiency is commonly determined by employing 'rake' and/or 'brake' based measurements. In the 'rake' approach, total temperatures and total pressure measurement surveys, performed across the inlet and outlet of the stage, are used to measure the stage pressure ratio and the total enthalpy drop (i.e., actual specific work) across the stage. In the 'brake' approach, measurements of shaft power and turbine through flow are used to determine the actual work produced. For both techniques, the stage pressure ratio is used to establish the 'ideal' specific performance against which the actual performance is compared to estimate stage efficiency.

Although the rake approach has been extensively developed for short duration testing of un-cooled stages, the estimated uncertainties of this method were too high for use in film cooled turbine investigations and pointed to the need for an alternative approach. Further analysis showed that the brake method would satisfy the accuracy requirements. This led to an assessment and consequently the development of short duration torque and mass flow instrumentation for use in the present program. Torque was measured by modifying mounting assembly of the existing eddy current brake power so that load cells could establish the resultant torque. Mass flow was measured by the installation of an ANSI standard critical venturi flow nozzle mounted downstream of the test turbine. The estimated measurement uncertainty of the overall system, including corrections for the non-adiabatic process inherent with short duration tests, was determined to be $0.45 \%$ for absolute efficiency measurements and $0.25 \%$ for relative measurements.

\subsection{Test Section Modifications and Un-Cooled Airfoil Fabrication}

To fit within the test section of the existing facility, the two-meter diameter ABB stage had to be reduced in scale by a factor of 4 . The existing shaft assembly and eddy current power absorber were retained but a new disk was required for the GT-24 rotor blade count. A new nozzle vane mount assembly and coolant feed manifolds were also required. The manifolds 
were designed to provided three independent coolant feeds to the vanes, rotor and tip shroud respectively.

ABB supplied the coordinates for the full-scale machine from which MIT designed the scaled airfoils using a state-of-the-art 3-D solid modeling package. Allowances for centrifugal and thermal growth were factored into the final design drawings and coordinate files for airfoil production. The operating conditions of the facility allowed the airfoils to be fabricated from an aluminum alloy, and the blade and vanes were produced using a 5-axis milling machine. Solid airfoils were produced for use in the initial series of un-cooled tests. After these test, the airfoils were modified to the cooled configuration.

\subsection{Film-Cooled Turbine Airfoils}

Construction of the cooling holes for $1 / 4$ scaled GT-24 model airfoils proved challenging. As a result of the requirement to maintain exact geometric similarity, the hole diameters were on the order of 0.008 inch. The film-cooled airfoils used in the test stage are shown in the figure below. The GT-24 blade cooling configuration consists of five rows of coolant injection holes: two on the leading edge, one on the suction surface, one on the pressure surface, and one directly out the trailing edge. The vane has twelve rows of holes along the airfoils surface as well as holes arrayed on the hub and tip platforms. The internal passages were not directly replicated in the model blades and vanes. The blade cooling manifold channels proved to be challenging to fabricate. The 'bowed' leading edge of the blade required that the manifold be produced with two plunge EDM cuts, starting at the tip and hub respectively and properly aligning at mid-span. The rear manifold employed a wire EDM process. Tolerances had to be maintained to several thousandths of an inch to avoid breaking through the blade surface. The laser drilling of the airfoil film-cooling holes also proved challenging because (at 1/4-scale) their diameters were 0.008". The leading edge holes were especially difficult to fabricate due to their compound angles and close spacing. After consultation with $\mathrm{ABB}$, it was decided not attempt the fabrication of the blade trailing edge row because the small trailing edge thickness at model scale.

\subsection{Experimental Program}

Experiments were first run on the un-cooled stage configuration to provide both a baseline database for the follow-on cooled tests and to confirm the accuracy and repeatability of the efficiency measurement technique under more easily modeled conditions. After fabrication of the cooled airfoils a second set of experiment were conducted. The test conditions covered a range pressure ratio, corrected speed, rotor tip gap spacing, gas to wall temperature ratio, coolant mass flow rate, and coolant to inlet temperature and momentum ratios. These test have provided the most detailed and accurate measurements to date characterizing the influence of film cooling upon turbine aerodynamic performance. 


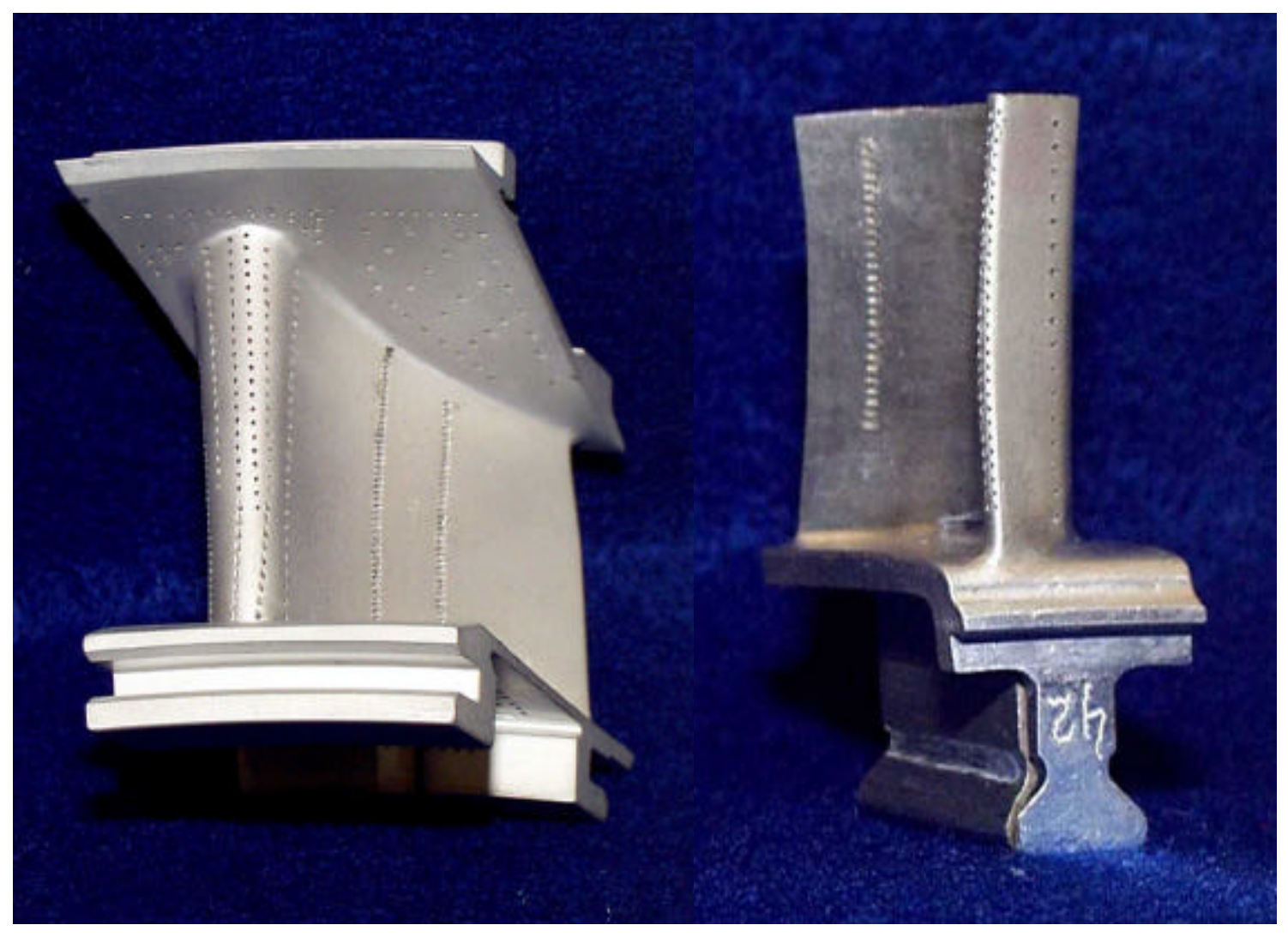




\subsection{References}

[2.1] Guenette, G.R., "A Fully Scaled Short Duration Turbine Experiment," Sc.D. Thesis, Massachusetts Institute of Technology, 1985

[2.2] Dunn, M.G., Martin, H.L., and Stanek, M.J., "Heat-Flux and Pressure Measurements and Comparison With Prediction for a Low Aspect Ratio Turbine Stage," ASME Paper No. 86-GT-79, 1986

[2.3] Jones, T.V., “Gas Turbine Studies at Oxford 1969-1987,” ASME Paper No. 88-GT-112, 1988.

[2.4] Denton, J., "Loss Mechanisms in Turbomachines." ASME J. of Turbomachinery, Vol. 115 pp. 621-656, 1993.

[2.5] Rose, M., and Harvey, N., "Turbomachinery Wakes: Differential Work and Mixing Loss." ASME Paper, 99-GT-25, 1999

[2.6] Abhari, R., "An Experimental Study of Film Cooling in a Rotating Transonic Turbine." ASME J. of Turbomachinery, Vol. 116 pp. 63-70, 1994. 


\section{Blowdown Turbine Test Facility}

\subsection{Introduction}

The MIT Blowdown Turbine Facility is a short-duration, blowdown wind tunnel capable of testing a complete, rotating stage in an environment which rigorously simulates the fluid physics and heat transfer phenomena which occur in a turbine. This chapter reviews the MIT Blowdown Turbine facility. Overall configuration and operational procedures are described. Scaling of the turbine operating point for achieving full scale similarity is discussed. Individual components are examined as well as instrumentation and the recently modified coolant feed system. The data acquisition system is also briefly presented.

\subsection{Facility Configuration}

The MIT Blowdown Turbine test facility is shown in Figure 3.1. The facility consists of seven primary components: the supply tank, fast acting valve, test section, eddy current brake torque meter, critical flow venturi nozzle, coolant feed system (not shown), and dump tank. A detailed review of the overall design of the facility can be found in Guenette [3.1].

\subsubsection{Supply Tank and Main Valve}

The supply tank is a 364 cubic foot cylindrical pressure vessel rated at 150 psi. It is surrounded by an external jacket through which heat transfer oil is circulated to heat the tank to the desired test temperature. A stirring fan is employed to ensure a uniform test gas temperature distribution.

The test section is separated from the supply tank by a fast-acting, axially traversing, plug valve. This valve is designed to fully open in approximately $50 \mathrm{~ms}$ and introduce disturbancefree flow into the test section. Valve dynamics are controlled by damping chambers which produce a force on the plug whose magnitude and direction depend on its position and velocity. In its fully closed position, this force acts to seal the plug. After the seal is broken, the valve plug is accelerated open. As it nears its fully open position the plug is decelerated to rest. The force used to break the seal is provided by an internal pneumatic piston assembly actuated by a small compressed gas cylinder. 


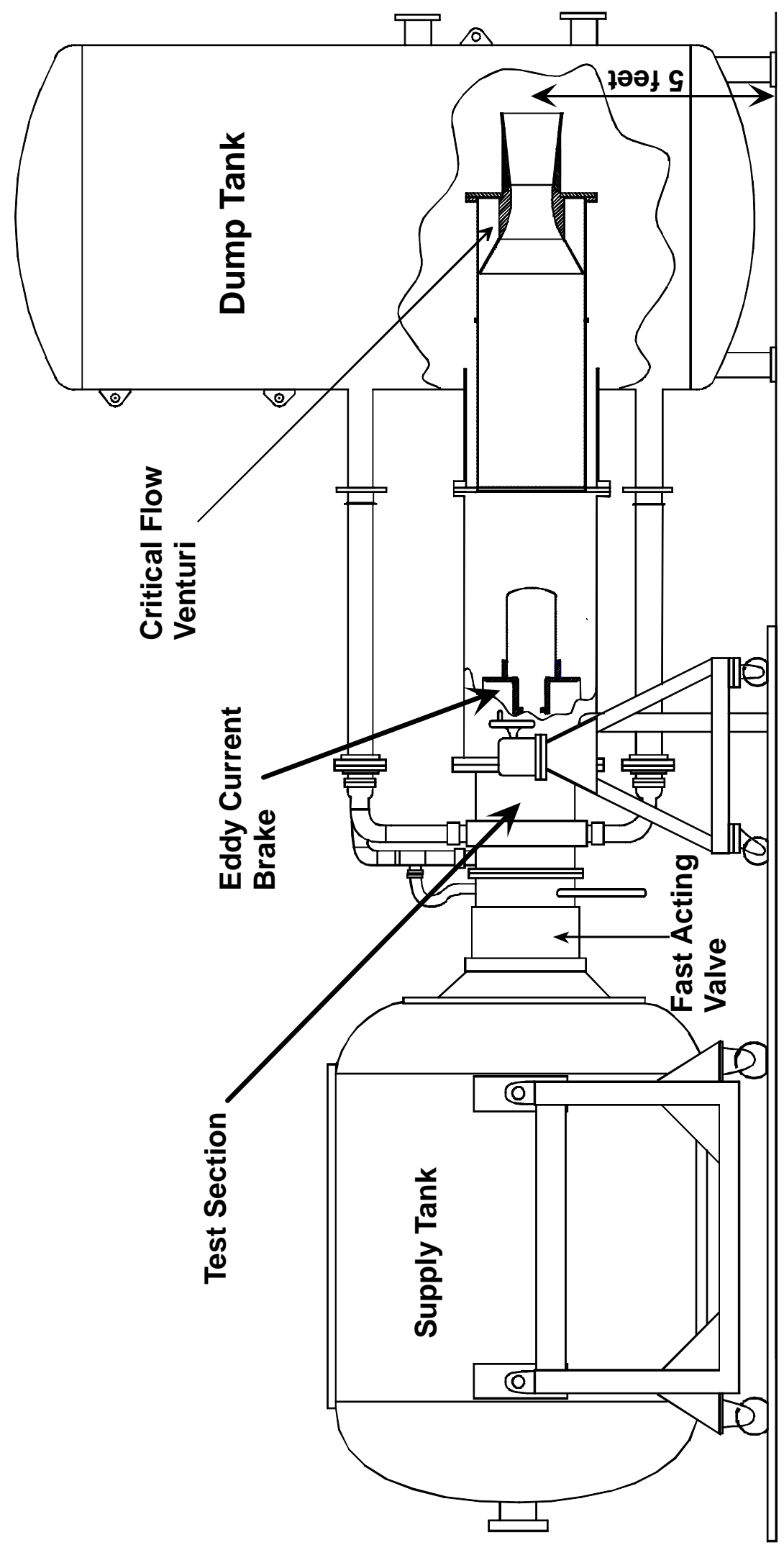

Figure 3.1: Blowdown Turbine Experimental Facility 


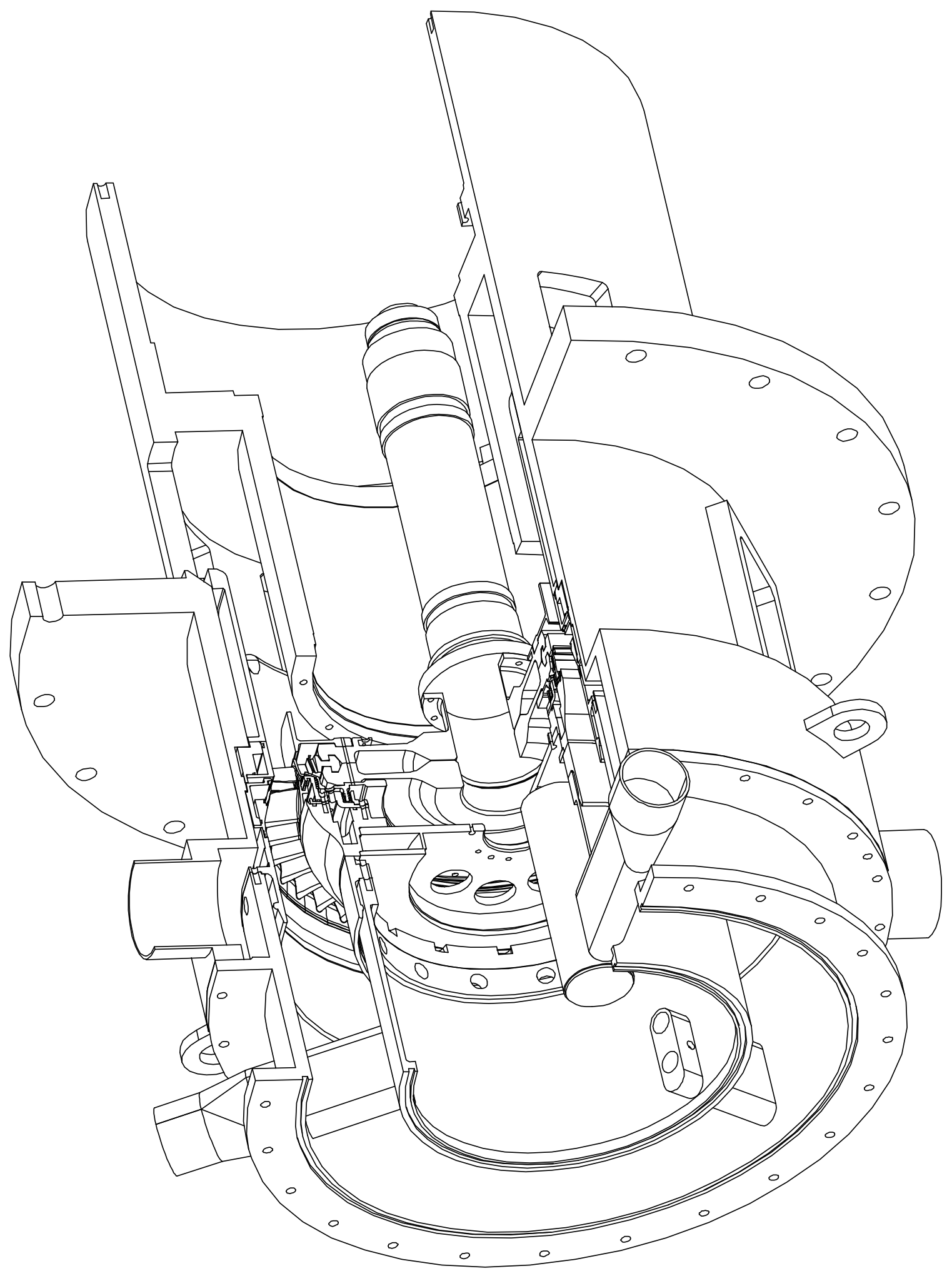

Figure 3.2: Test Section Detail 


\subsubsection{Test Section}

A cut-away of the test section assembly is shown in Figures 3.2 and 3.3. The test section is composed of the forward frame, main frame, and the rotor unit The forward frame contains the nozzle guide vanes, the inlet boundary layer bleeds, and the rotor heat shield and mates to the main valve. The forward frame is followed by the main frame which houses the turbine rotor assembly, the downstream probe translator package, the eddy current brake magnet assembly, the turbine throttle and drive motor. The rotor unit contains the rotor discs, blades, bearings, shaft, and the eddy current brake 'loss' drum (not shown). This drum is inserted into the eddy current brake magnet assembly which will be discussed in below and in more detail in Section 4.2.

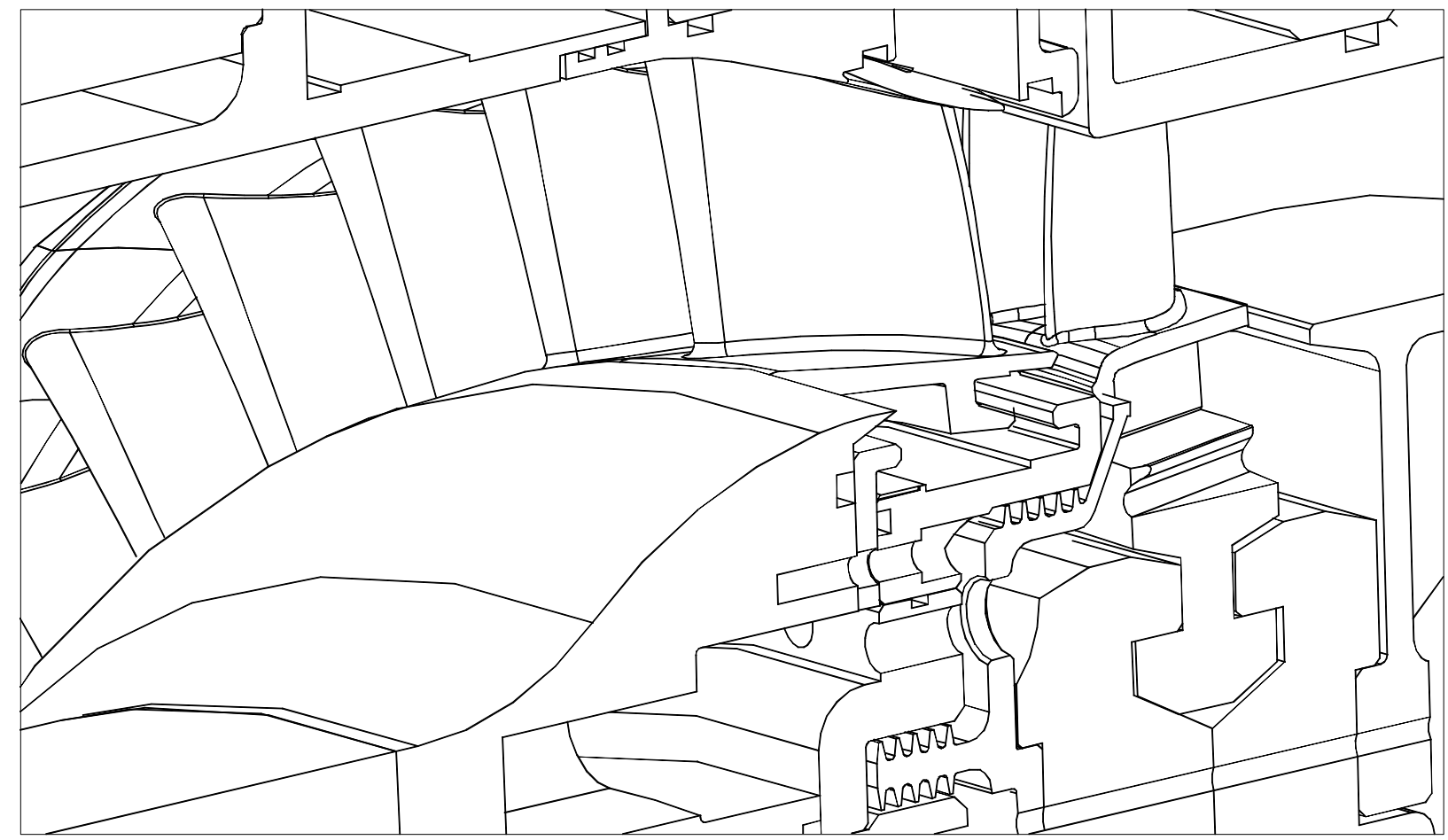

Figure 3.3: Turbine Installation Detail

A cross-sectional view of the test section flow path is shown in Figure 3.4. Upstream of the turbine stage a boundary layer bleed provides clean inlet flow. The rotor exit flow is exhausted through an adjustable throttling area to provide the desired pressure ratio across the turbine. During the usable portion of the test, this area is choked. Also shown in this figure are the upstream and downstream measurement locations. Downstream, the total temperature and pressure probes are mounted on a circumferential translator. 


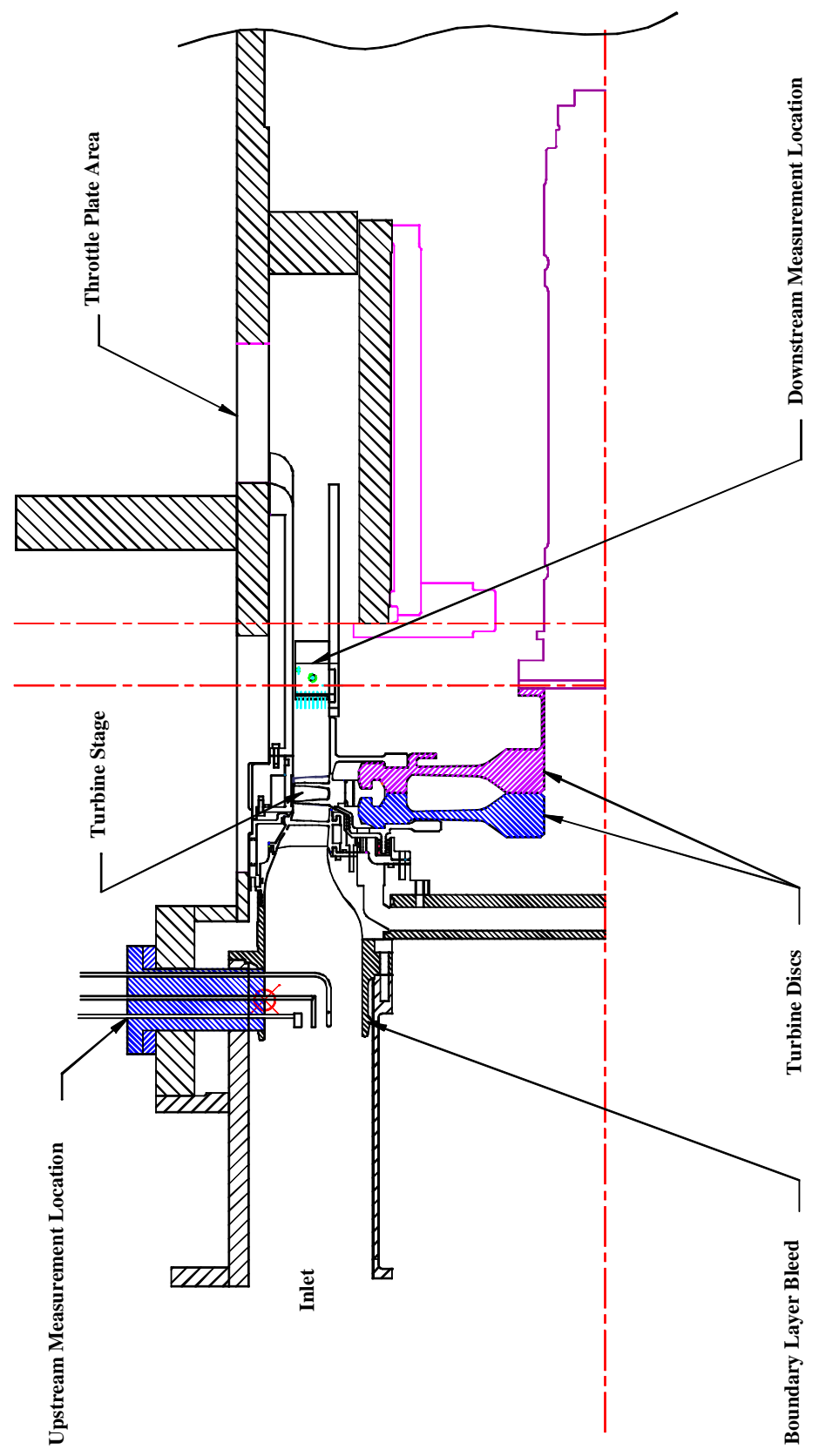

Figure 3.4: Test Section Flow Path 


\subsubsection{Circumferential Probe Translators}

Both upstream and downstream circumferential translators are available to provide measurement of non-axisymmetric features of the turbine flow field. Both are composed of drums forming the inner annulus of the turbine entrance and exit and are mounted on thin-line bearings. For the experiments reported herein only the downstream translator was employed.

The downstream translator houses three 'canisters', spaced $20^{\circ}$ apart, which contain the appropriate electrical and pneumatic connections to support either total temperature or pressure rakes. Connections are also provide for several wall static pressure taps. The translator is restricted to a $350^{\circ}$ rotation angle, by a spring loaded hard stop, to prevent the translator from freely spinning and destroying the canisters' electrical wiring. .The translator is driven by a Baldor ME-4090-BLBCE servomotor delivering approximately 3(in-lb) of torque through an equivalent 13.5:1 gear ratio. This motor is controlled by a Galil DMC-400-10 card and powered by a Copley MB6-10 amplifier. In addition to the hard stop, an aluminum shear pin is installed on the shaft coupling exiting the servomotor to prevent translator runaway.

\subsubsection{Eddy Current Brake}

The eddy current brake serves as both the turbine load and the turbine torque meter. It consists of two components, a cylindrical, Inconel drum directly attached to the rotor shaft and a stationary array of DC excited electromagnets arranged circumferentially around the drum. Electric currents are induced in the drum as it rotates through the applied magnetic field. The flow of this current through electrical resistance of the drum dissipates the power produced by the turbine which appears as resistive heating of the drum. Turbine speed is controlled by setting the magnitude of the applied magnetic field.

The braking system was modified by Keogh, [3.2], to serve as a shaft torque meter. The electromagnet assembly was mounted on bearings and reacting the braking torque measured through two load cells. A detailed description of the brake and its modification for torque measurement will be presented in Section 4 of this report.

\subsubsection{Critical Flow Venturi Nozzle}

Under this program, a critical flow venturi was developed and installed in line with the exit flow path to measure the mass flow rate through the turbine. The nozzle design and upstream duct requirements are based the ANSI standard, [3.3], for torroidal throat critical flow venturi. Modifications to the facility were required to meet installation requirements for the nozzle

The main design challenge was to incorporate the required upstream duct length into the facility given the space restrictions. This was accomplished be installing the critical flow venturi and the upstream duct inside the dump tank. A 66 inch extension was added between the dump tank and main frame in order to relocate the eddy current brake and starter motor. Extensions were also required connect the boundary layer bleeds to the dump tank, and to connect the fill system to the supply tank.

A 50\% open area screen is installed at the entrance to upstream duct. This reduces the total pressure non-uniformity caused by the stepped transition from annular to circular cross section around the starter motor. The nozzle was calibrated with the upstream duct and a simulated blockage in place. 
The nozzle was designed and built by Flow Systems Inc. of Boulder Colorado and calibrated by Colorado EESI.. This calibration is traceable to the National Institute for Standards and Technology (NIST). A cross section of the critical flow venturi nozzle is shown in Figure 3.1 and a more detailed review of its design and usage can be found in Section 4 and in [3.2].

\subsubsection{Coolant Feed System}

The coolant system consists of a coolant supply tank, a fast-acting pneumatic ball valve, a pipe network, and several orifice plates for metering the flow. Like the facility itself, the cooling system operates in a blowdown mode. The existing facility coolant system was modified to provide three independent and separately metered streams to the rotor blades, vanes, and tip casing.

The coolant supply tank is $3.97 \mathrm{ft}^{3}$ and has a pressure rating of 450 psia. Like the main supply tank, it is surrounded by a metal jacket and insulation for controlling tank temperature. A flow of liquid nitrogen mixed with compressed air is used to cool the tank lining and the test gas to the desired temperature. The fast-acting ball valve at the tank exit is actuated by a $1000 \mathrm{psi}$ argon bottle. It is triggered simultaneously with the main valve and closes approximately 1.2 seconds later. The piping network feeds the coolant from the supply tank into the test section and to the turbine.

An overhead view of this network can be seen in Figure 3.5. Three independent feeds are employed. One each to the rotor blades, nozzles, and tip casing. The flow is split in a manifold downstream of the ball-valve. Each split is metered by a thick, square edged, choked orifice with known discharge coefficient which accurately sets the mass flow ratios to each turbine component.

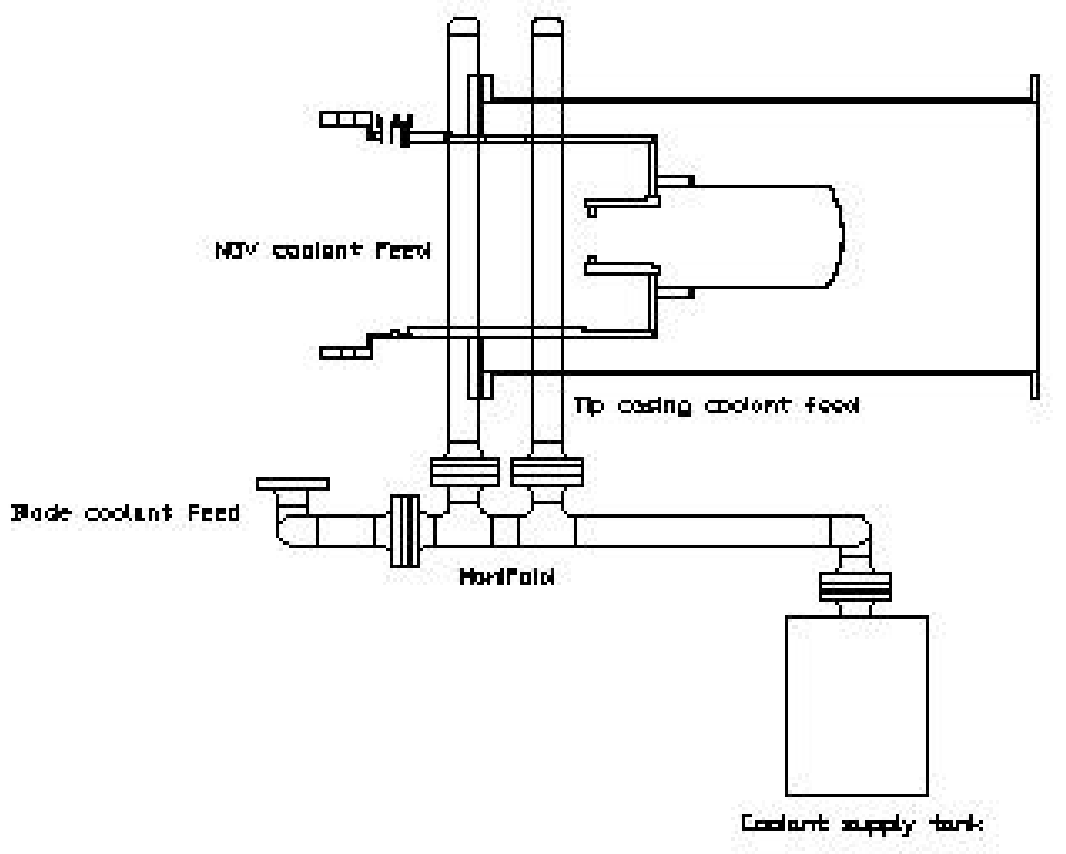

Figure 3.5: Coolant Feed System 


\subsection{Scaling of Test Conditions}

Validity of scaled wind tunnel experiments depends on similarity between the test flow and the actual flow being simulated. In dimensionless form, the equations for mass continuity, momentum and energy transfer produce non-dimensional parameters which completely characterize the physics of flow field. Similarity between experiment and reality only requires that these non-dimensional parameters be reproduced.

For an un-cooled turbine stage operating under adiabatic conditions, corrected mass flow and total temperature ratio (or efficiency) depend on four dimensionless parameters: total pressure ratio, corrected speed, Reynolds number, and specific heat ratio, [3.1]. Two additional parameters are required to simulate the heat flux distribution: the gas to wall temperature ratio and the Prandtl number. For a film-cooled turbine test, the coolant mass and momentum flux ratios must also be reproduced. These parameters, which govern test similarity, can be matched by properly setting the facility initial operating conditions.

The operating conditions consist of the test gas composition, upstream total temperature and pressure, coolant total temperature and pressure, mechanical speed, throttle area, and brake excitation. The supply tank temperature is set by matching the gas to wall temperature ratio. Specific heat ratio is matched by setting the test gas mixture ratio, typically Argon and $\mathrm{CO}_{2}$. Shaft mechanical speed is set by matching the corrected speeds. Initial supply pressure is set by the Reynolds number, and pressure ratio by the downstream throttle position. Table 3.1 summarizes the conditions of a typical blowdown experiment and compares them to that of a full scale engine.

Table 3.1: MIT Blowdown Turbine Scaling

\begin{tabular}{|l|l|l|}
\hline Parameters & Full Scale Engine & MIT Test Facility \\
\hline Working Fluid & Air & Argon $-\mathrm{CO}_{2}$ \\
Ratio of Specific Heats & 1.28 & 1.28 \\
Mean Metal Temperature & $1100 \mathrm{~K}$ & $300 \mathrm{~K}$ \\
Metal to Gas Temp Ratio & 0.647 & 0.647 \\
Inlet Total Temperature & $1700 \mathrm{~K}$ & $464 \mathrm{~K}$ \\
Reference Length* & $865.16 \mathrm{~mm}$ & $225.933 \mathrm{~mm}$ \\
Reynolds Number & $5.6 \cdot 10^{6}$ & $5.6 \cdot 10^{6}$ \\
Inlet Total Pressure & $15 \mathrm{~atm}$ & $7 \mathrm{~atm}$ \\
Exit Total Pressure & $7.4 \mathrm{~atm}$ & 3.47 \\
Exit Total Temperature & $1470 \mathrm{~K}$ & $401 \mathrm{~K}$ \\
Prandtl Number & 0.928 & 0.742 \\
Design Rotor Speed & $3600 \mathrm{rpm}$ & $5954 \mathrm{rpm}$ \\
Design Mass Flow & $312 \mathrm{~kg} / \mathrm{s}$ & $23.3 \mathrm{~kg} / \mathrm{s}$ \\
Coolant/Inlet Flow & $9.8 \%$ & $9.8 \%$ \\
Stage Power Output & $91 \mathrm{MW}$ & $1.26 \mathrm{MW}$ \\
Test Time & Continuous & $\approx 0.800 \mathrm{~s}$ \\
\hline
\end{tabular}

* Basic Radius BA on ABB GT24 LPT Stage 1 Blade 


\subsection{Test Procedure}

Preparations for a blowdown experiment begin by determining the required conditions for achieving full-scale turbine operating point. These consist of the upstream total temperature and pressure, coolant total temperature and pressure, test gas composition, mechanical speed, throttle area, and brake excitation. The experiment then proceeds as follows:

1. The entire facility is evacuated and the throttle plate is positioned for a predetermined area based on operational experience.

2. The main supply tank is heated to the desired upstream total temperature and is brought to thermal equilibrium.

3. The main valve is sealed and the supply tank is filled with the required test gas mixture to the desired upstream total pressure.

4. Simultaneously, the coolant supply tank is filled to the desired pressure and cooled to the predetermined temperature.

5. At this post-fill state, all differential pressure transducers are calibrated by cycling their back-pressure ports between vacuum and atmosphere. This provides a scale factor for each transducer. Instrumentation and calibration are discussed in further detail in section 3.5.

6. The brake excitation is set.

7. The data acquisition system and translator servo-motor controller are set to stand-by mode and are waiting to be triggered.

8. The turbine rotor is then accelerated to the desired mechanical speed by a starter motor.

9. Once this speed is exceeded, the motor is powered down and the rotor spins freely in the vacuum. It is slowly decelerated by bearing friction and the applied back EMF of the drive motor .

10. When the decelerating rotor reaches the preset speed, a trigger occurs causing the main valve and coolant ball valve to open. Simultaneously, the data acquisition system begins collecting data, the downstream translator begins it traverse, and the eddy current brake torque meter is energized.

11. The test gases then flow through the test section and quasi-steady state operation is reached after a $300 \mathrm{~ms}$ transient. The useful test window is approximately $500 \mathrm{~ms}$.

12. After approximately 1.2 seconds the coolant ball-valve closes and the brake is turned off.

13. The rotor decelerates and comes to a stop.

14. Once the gas inside the tunnel stabilizes, all differential pressure transducers are recalibrated to check for drift.

15. The tunnel is then re-evacuated and cooled to room temperature. 


\subsection{Instrumentation}

\subsubsection{Introduction}

The facility was designed to provide a rather benign experimental environment for flow measurement. The nature of the short duration experiment casts a strict requirement on the time response of instrumentation used to sample its flow field. Performance calculations based on these measurements also require a high level of instrumentation accuracy. These considerations led to the development, under this program, of improved total temperature and pressure instrumentation with the capability of accurately characterizing turbine performance. The design of this new instrumentation is detailed in reference [3.4]. Also presented the estimates of the overall uncertainty in total temperature and pressure measurements. Following this is a brief explanation of other instrumentation used in the Blowdown Turbine. Finally, the standard differential pressure transducer calibration procedure is discussed followed by a description of the data acquisition system.

\subsubsection{Total Temperature Instrumentation}

The MIT Gas Turbine Laboratory has engaged, over the past 10 years, in the development of total temperature probes for use in turbine aero-performance measurements in the Blowdown Test Facility. With each new design, time response and instrument accuracy generally improved. Work under this program focused on reducing the transient errors resulting from the heat transfer processes within the thermocouple junction support wires and led to a design with much faster time response than previously achieved. Attention was also paid to the probe head design to reduce probe sensitivity to flow angle, which is important for downstream wake measurements.

Details of the temperature probes are shown in Figures 3.6 and 3.7. The measurement heads consist of 0.0005 inch diameter type $\mathrm{K}$ thermocouple junctions mounted in vented stagnation tubes. The rake probe shown in figure 3.7 is mounted on the downstream translator and contains an internal temperature reference junction. An identical probe with four heads is also mounted on the downstream translator and a stationary six head version is mounted upstream of the turbine. The radial rake of 6 heads is positioned roughly mid-way between two of the facility's inner annulus support struts. The inlet rake can employ either an internally mounted or external reference junction.

Three additional single head probes, spaced $120^{\circ}$ apart, are mounted upstream at midspan on the inlet annulus. The thermocouple reference junctions for these probes are external. Three total temperature probes are dedicated to monitoring the flow through the critical flow venturi nozzle. These probes are also 0.0005" diameter type $\mathrm{K}$ thermocouples and have similar time response and accuracy to the upstream probes.

The cooling system is instrumented with seven type $\mathrm{K}$ thermocouples. These probes do not require the time response of the turbine inlet and outlet sensors and employ 0.003" junctions. These probes are located at points upstream of the choked metering orifices and as close as possible to where the coolant enters the test section.

All thermocouples, with the exception of the turbine inlet and outlet rakes, are referenced to Omega TRCIII ice point cells. The signals are processed using high stability DC amplifiers and filters prior to being recorded by the data acquisition system. The long term static accuracy 
of the system, (consisting of the measurement head, reference junction and signal conditioning electronics), is $0.13 \mathrm{~K}$.

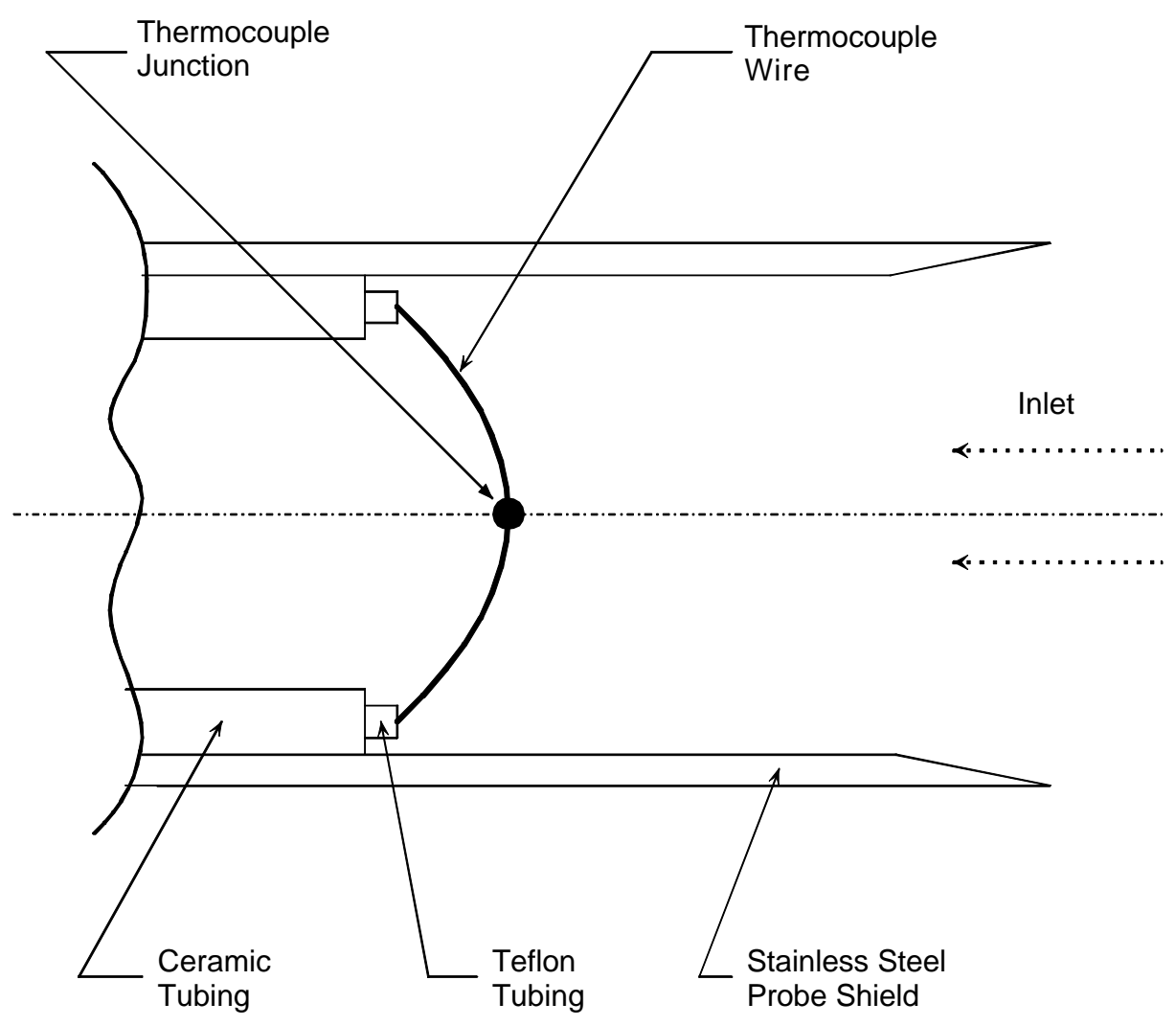

Figure 3.6: Temperature Probe Head Detail 


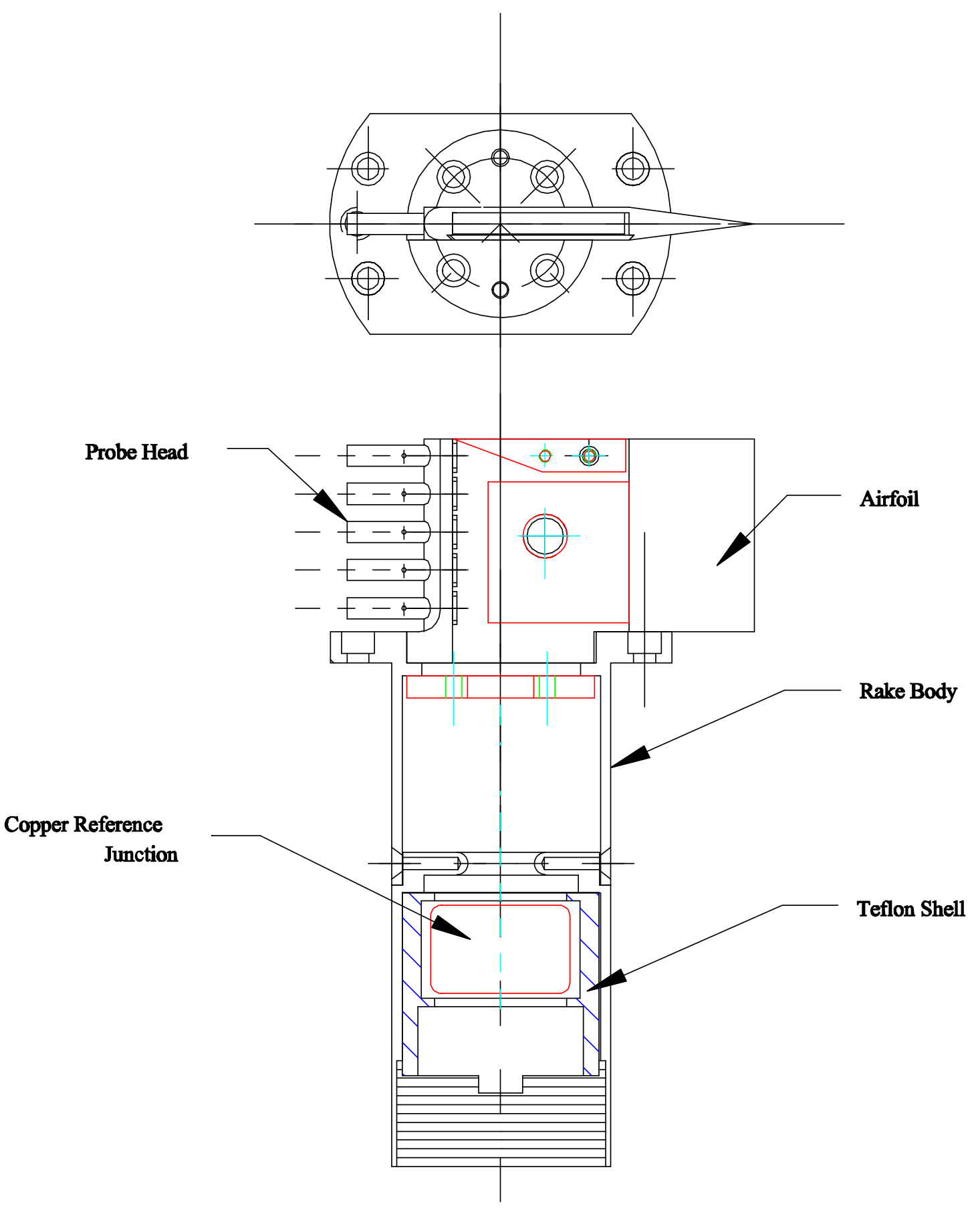

Figure 3.7: Downstream Total Temperature Rake 


\subsubsection{Total Pressure Instrumentation}

The primary concerns in the design of the total pressure sensors for this facility are fast time response and flow angle insensitivity. The second criteria is particularly important for downstream measurements where the probes are circumferentially translated through the NGV wakes. To achieve the desired time response, semiconductor strain gauge type pressure transducers, manufactured by Kulite, were used. A disadvantage of these transducers is their tendency tend to drift with changes in temperature. As a consequence, extensive run-time calibration routines were developed and used during every experiment. To allow a larger range of inlet flow angle to the probe head, either vented Keil type or $15^{\circ}$ beveled impact heads were employed. Acceptable flow angles are estimated to be $\pm 27.5^{\circ}$.

Downstream of the turbine stage a rake type pressure probe, with eight $15^{\circ}$ beveled impact heads, is employed to survey the pressure in the radial direction. The probe is mounted on the downstream translator to resolve flow characteristics in the circumferential direction. The pressure transducers, (100psig XCQ-063-100), are mounted inside the probe body below the heads. Transducer reference backpressure is provided via a tubing arrangement within the translator. The estimated average uncertainty in total pressure measurement from these heads is $0.25 \%$. More information on the design of this probe is available in [3.4].

In addition to this rake, there are differential sensors located upstream of the turbine and in the supply, coolant, and dump tanks. There are also several highly accurate $(0.05 \%)$ pressure sensors (manufactured by Sensotec) mounted throughout the facility for calibration reference. The coolant system is instrumented with several lower accuracy $(0.1 \%)$ Sensotec transducers.

\subsubsection{Other Instrumentation}

Turbine speed and translator position are monitored by their respective digital encoders. The optical shaft encoder consists of two rings of 366 divisions per revolution in quadrature and a once per revolution ring. It is directly mounted to the turbine shaft. Both digital and analog circuitry process the encoder signals to provide shaft position and velocity for storage by the computer data acquisition system.

Two piezoelectric sensors are mounted on the bearing housing to record shaft vibration during an experiment.

The eddy current brake torque meter is instrumented with two load cells which provide a measure of shaft torque. This will be further discussed in Section 4.3. The brake voltage and current are also recorded by the data acquisition system. A list of all instrumentation is provided in Table 3.2.

Facility reference instrumentation provides known conditions for use in the calibration procedures. For these, time response is traded for absolute accuracy and stability as all tunnel temperature and pressure measurements are based on these references. Pressure references are available in the supply tank and test section via slower, but more accurate capacitive type pressure transducers. Located on the supply tank is a Sensotec Super TJE 150psia (0.05\%) transducer. A Sensotec TJE 50psia (0.1\%) transducer is located in the critical flow venturi. The upstream Pitot probes are referenced to a Setra Model $2281 \mathrm{psid}$ capacitive type pressure transducer. Temperature calibrations are referenced to a Rosemount Standard Platinum Resistance Thermometer Model 162N100A (S/N3779) and, when possible, thermocouples are referenced to Omega TRCIII ice point calibration cells. Efforts are now being made to convert all temperature references to the ice point to simplify temperature data reduction. 
Table 3.2: Blowdown Turbine Instrumentation

\begin{tabular}{|c|c|c|}
\hline Sensor & Type & Location \\
\hline \multicolumn{3}{|c|}{ Facility Reference } \\
\hline PFEF300 & $\begin{array}{l}300 \text { psia, Sensotec: STJE/1835/15 } \\
\text { S/N: } 589494\end{array}$ & $\begin{array}{l}\text { Calibration: Coolant Tank / Facility } \\
\text { Run Time: Coolant Tank Initial Pressure }\end{array}$ \\
\hline PREF150 & $\begin{array}{l}150 \text { psia, Sensotec: STJE/1833-12-04 } \\
\text { S/N: } 587965\end{array}$ & $\begin{array}{l}\text { Calibration: Supply Tank / Facility } \\
\text { Run Time: Supply Tank Initial Pressure }\end{array}$ \\
\hline PREF050 & $\begin{array}{l}50 \text { psia, Sensotec: TJE/0713-04TJA-12 } \\
\text { S/N: } 631656\end{array}$ & $\begin{array}{l}\text { Calibration: Facility } \\
\text { Run Time: Critical Flow Venturi, Test } \\
\text { Section Initial Pressure }\end{array}$ \\
\hline PREF001 & 1 psid, Setra: 228-1 S/N:708984 & Calibration: Inlet Pitot Tubes \\
\hline TREF3997 & $\begin{array}{l}\text { Rosemount Model 162N100A, S/N3779 } \\
\text { Standard Platinum Resistance Thermometer }\end{array}$ & Standard Calibration Reference \\
\hline TREF10 & $0-100 \stackrel{\circ}{ } \mathrm{C}$ Platinum RTD & Run Time Reference \\
\hline
\end{tabular}

\begin{tabular}{|c|c|c|}
\hline \multicolumn{3}{|c|}{ Supply Tank } \\
\hline PTOA & 150 psid, pressure, Kulite & Supply Tank, Internal \\
\hline PTOB & 150 psid, pressure, Kulite & Supply Tank, Internal \\
\hline TTOA & $600^{\circ} \mathrm{F}$ temperature, type $\mathrm{J} \mathrm{T} / \mathrm{C}$ & Supply Tank, Internal \\
\hline TTOB & $600^{\circ} \mathrm{F}$ temperature, type J T/C & Supply Tank, Internal \\
\hline TTOC & $600^{\circ} \mathrm{F}$ temperature, type J T/C & Supply Tank, Internal \\
\hline
\end{tabular}

\begin{tabular}{|c|c|c|}
\hline \multicolumn{3}{|c|}{ Shaft Monitoring } \\
\hline FTACH & Speed, Digital & Shaft/Bearing Assembly \\
\hline ATACH & Speed, Analog & Shaft/Bearing Assembly \\
\hline FBRI NG & Vibration, forward & Shaft/Bearing Assembly \\
\hline RBRI NG & Vibration, rear & Shaft/Bearing Assembly \\
\hline
\end{tabular}

\begin{tabular}{|c|c|c|}
\hline \multicolumn{3}{|c|}{ Eddy Current Brake } \\
\hline VTOT & Excitation Volt & External \\
\hline I TOT & Total Current & External \\
\hline I BRK & Excitation Current & External \\
\hline ECBF1 & Load Cell \#1 & Internal \\
\hline ECBF2 & Load Cell \#2 & Internal \\
\hline
\end{tabular}

\begin{tabular}{||l|l|l||}
\hline \hline Main Valve & \multicolumn{1}{||}{} \\
\hline PVLV & 450 psia Kulite & Valve Damping Chamber \\
\hline XVLV & $0-8$ in Linear Potentiometer & Valve Slider \\
\hline
\end{tabular}

\begin{tabular}{|c|c|c|}
\hline \multicolumn{3}{|l|}{ Turbine Inlet } \\
\hline PT2x, $(x=A, B, C)$ & Total Pressure & Main Frame, $120^{\circ}$ apart \\
\hline TT2x. $\quad(x=A, B, C)$ & Total Temperature & Main Frame, $120^{\circ}$ apart \\
\hline PP2x, $(x=A, B, C)$ & Dynamic Pressure & Main Frame, $120^{\circ}$ apart \\
\hline PTYRn, $(n=1,2,3,4,5,6)$ & Total Pressure, 6 head radial rake & Main Frame, fixed location \\
\hline TTR101- n, ( n=1, 2, 3, 4, 5, 6) & Total Temperature, 6 head radial rake & Main Frame, fixed location \\
\hline
\end{tabular}


Table 3.2: Blowdown Turbine Instrumentation, continued

\begin{tabular}{|c|c|c|}
\hline Sensor & Type & Location \\
\hline \multicolumn{3}{|l|}{ Turbine Outlet } \\
\hline PT45Rn, n=1, 2, 3, 4, 5, 6, 7, 8 & Total Pressure, 8 head radial rake & Downstream Translator \\
\hline TTR103- n, n=1, 2, 3, 4, 5 & Total Temperature, 5 head radial rake & Downstream Translator \\
\hline RTD103- $n, n=1,2$ & T/C Reference RTD's, 2 locations & Downstream Translator \\
\hline TTR104- $n, \quad n=1,2,3,4$ & Total Temperature, 4 head radial rake & Downstream Translator \\
\hline RDT104- $n, n=1,2$ & T/C Reference RTD's, 2 locations & Downstream Translator \\
\hline P45HUB & 50 psid wall static pressure & Downstream Translator Hub \\
\hline P45A & 50 psid wall static pressure & Main Frame Window \\
\hline
\end{tabular}

\begin{tabular}{||l|l|l||}
\hline \multicolumn{2}{||l||}{ Critical Flow Venturi } & Venturi Inlet \\
\hline PREF050 & Static Pressure & Flow Conditioning Nozzle \\
\hline PNOZ & Static Pressure & Venturi Inlet \\
\hline TTMFM & Total Temperature & Venturi Inlet \\
\hline TTMFMR & Total Temperature & Flow Conditioning Nozzle \\
\hline TTNOZ1 & Total Temperature & Flow Conditioning Nozzle \\
\hline TTNOZ2 & Total Temperature & \\
\hline
\end{tabular}

\begin{tabular}{|c|c|c|}
\hline Dump Tank & & \\
\hline PDMP & Static Pressure & Dump Tank \\
\hline
\end{tabular}

\begin{tabular}{||l|l|l||}
\hline \hline Cooling System & $\begin{array}{l}\text { Coolant Supply Tank } \\
\text { S/N: } 589494\end{array}$ \\
\hline PREF300 & 300 psia Kulite & Coolant Supply Tank \\
\hline PTC0 & Total Pressure, 300psig Sensotek & Coolant Supply Manifold \\
\hline PTC1 & Total Pressure, 100psig Sensotek & Vane Feed Manifold \\
\hline PTC2V & Total Pressure, 100psig Sensotek & Blade Feed Manifold \\
\hline PTC2B & Static Pressure, 100psig Sensotek & Vane Feed Entrance \\
\hline PCCV & Static Pressure, 100psig Sensotek & Tip Casing Entrance \\
\hline PCCC & Total Temperature & Coolant Tank Exit \\
\hline TTC0 & Total Temperature & Coolant Supply Manifold \\
\hline TTC1 & Total Temperature & Blade Feed Manifold \\
\hline TTC2B & Total Temperature & Vane Feed Entrance, B window \\
\hline TTCBV & Total Temperature & Casing Feed Entrance, B window \\
\hline TTCBC & Total Temperature & Vane Feed Entrance, C window \\
\hline TTCCV & Total Temperature & Casing Feed Entrance, C window \\
\hline TTCCC & & \\
\hline
\end{tabular}




\subsection{Pressure Transducer Calibration}

A standard calibration procedure is responsible for the calibration of all differential pressure transducers. It is performed immediately after the supply tank is filled and at the conclusion of each experiment. Calibration data just before and after each experiment allows transducer drift and non-linearity to be quantified. Figure 3.8 shows a typical calibration trace of a differential pressure transducer. This figure and the calibration sequence are described below.

1. All transducers are provided a vacuum back-pressure reference by an external vacuum pump. For a post-fill calibration this produces a zero pressure differential across the transducers which are located inside the tunnel. For those in the supply or coolant tanks, a pressure differential equal to the initial tank pressure is recorded.

2. Data is taken at this condition for approximately two minutes as shown by the first segment of the trace in Figure 3.8.

3. After two minutes, the back-pressure reference is exposed to atmospheric pressure. Data is acquired for two minutes as shown in Figure 3.8.

4. After four minutes, the back-pressure reference is returned to vacuum where it remains for the experiment.

5. This procedure is repeated after the blowdown experiment has been completed.

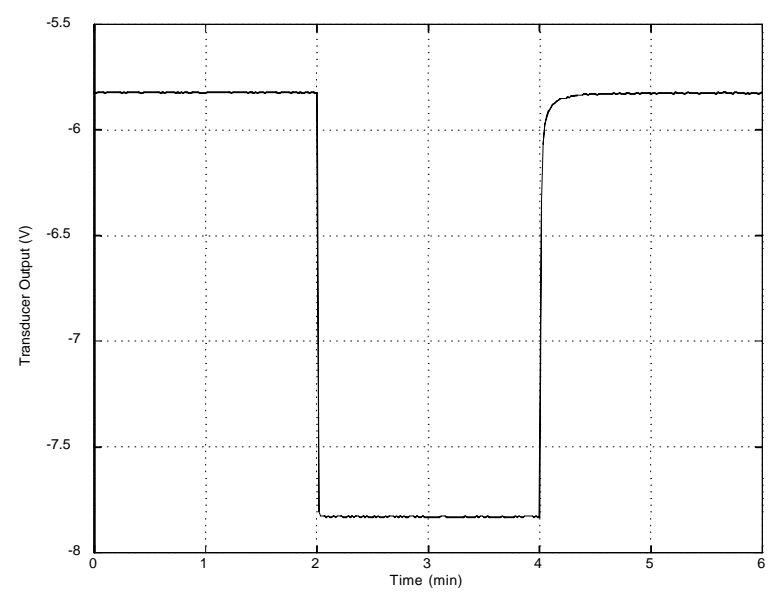

Figure 3.8: Typical Differential Pressure Calibration Trace

These data provide a voltage change for each differential transducer which corresponds to the local atmospheric pressure. A transducer scale factor can then be computed. Transducer zeros are taken to be the average output prior to the opening of the main valve. The scale and zero can then be used in the data reduction process via the linear calibration relation:

\subsection{Data Acquisition}

The short duration nature of the blowdown experiment not only places special requirements on instrumentation, but on data acquisition (DAQ) as well. The DAQ systems must monitor all instrumented channels at a sampling rate sufficient to capture all time scales of interest for the duration of the experiment. For aerodynamic performance measurements, which is the focus of this work, $5 \mathrm{kHz}$ data sampling is sufficient to resolve the necessary time scales. 
Although to accurately monitor turbine speed, the $200 \mathrm{kHz}$ high-speed system is often utilized. The DAQ envelope typically spans 2-4 seconds to record useful data and monitor tunnel 'spindown.' The precise clocking sequence can be modified to capture the desired window. In addition, the posttest calibration lends further insight into the posttest tunnel state.

The data acquisition system monitors all instrumented channels at a sampling rate sufficient for capturing time scales of interest during the experiment. For aerodynamic performance measurements, $5 \mathrm{kHz}$ is adequate. The system typically acquires data for $2-4$ seconds depending on the experimental conditions being monitored. The current hardware includes a Pentium II $450 \mathrm{MHz}$ computer which is programmed with LabVIEW lab automation software.

The current DAQ system consists of three computers. Two low-speed systems, each of which is capable of monitoring up to 64 channels, sample at $2.5 \mathrm{kHz}$ and $5 \mathrm{kHz}$. The high-speed system monitors eight channel at up to $200 \mathrm{kHz}$. Table 3.3 summarizes the Blowdown Turbine DAQ equipment.

Table 3.3: Blowdown Turbine Data Acquisition

\begin{tabular}{|l|l|l|l|}
\hline Specification & Dell Omniplex 590 & Dell 486D/50 & Dell 466T \\
\hline System & 1 & 2 & 3 \\
\hline Speed & High & Low & Low \\
\hline Operating System & DOS & DOS & Win95 \\
\hline DAQ Platform & In-house & In-House & LabView \\
\hline DAQ Board & ADTEK AD-830 & Analogic HSDAS-16 & NI MIO-64E3 \\
\hline Resolution & 12 -bit & 16 -bit & 12 -bit \\
\hline Board Channels & 8 & $4 x 16$ & 64 \\
\hline Number of Boards & 4 & N/A & 1 \\
\hline Total Channels & 32 simultaneous & 64 multiplexed & 64 multiplexed \\
\hline Sampling Rate per Channel & $200 \mathrm{kHz}$ & $2.5 \mathrm{kHz}$ & $5 \mathrm{kHz}$ \\
\hline
\end{tabular}

\subsection{Summary}

This section has discussed the MIT Blowdown Turbine short duration experimental facility used to acquire turbine data for calculation of adiabatic efficiency. It has covered runtime preparations including determination of blowdown operating conditions to achieve fullscale turbine similarity. The design of new total temperature and pressure instrumentation for highly accurate flow field measurements was briefly discussed along with other instrumentation used to characterize turbine performance. This was followed by descriptions of the standard differential pressure transducer calibration procedure and the downstream translator, a device for circumferentially surveying the downstream flow field. This section concludes with information on Blowdown Turbine data acquisition system. 


\subsection{References}

[3.1] Guenette. G.R., "A Fully Scaled Short Duration Turbine Experiment," Sc.D. Thesis, Massachusetts Institute of Technology, August 1985.

[3.2] Keogh, R., "Shaft efficiency Measurements of a Fully Scaled Turbine in a Short Duration Experiment," Masters Thesis, Massachusetts Institute of Technology, February 1998.

[3.3] ASME/ANSI MFC-7M-1987, "Measurement of Gas Flow by Means of Critical Flow Venturi Nozzles," An American National Standard, The American Society of Mechanical Engineers, 1987.

[3.4] Cai. Y., "Aerodynamic Performance Measurements in a Fully Scaled Turbine," Masters Thesis, Massachusetts Institute of Technology, February, 1998. 


\section{$4 \quad$ Fabrication of the Model Film-Cooled Turbine Stage}

By Christopher M. Spadaccini

\subsection{Introduction}

This Chapter presents a detailed description of the fabrication of the film-cooled turbine stage. In order to quantify the losses due to coolant flow injection, the $1 / 4$-sacle film-cooled model turbine was required to have similar external geometry, including the film hole quantity, distribution, diameters, and injection orientations over the airfoil surfaces. The internal passages were not directly replicated since the focus the program was on external aerodynamic and heat transfer. After an initial series of tests were performed, in the spring of 1998, on the un-cooled model stage, work then proceeded on the development and testing of the film-cooled version.

As a result of programmatic considerations, it was decided, with the concurrence of $\mathrm{ABB}$, to modify the existing solid un-cooled turbine airfoils. This was accomplished by the use of sophisticated machining techniques, such as electro-discharge machining (EDM) and laser machining. The un-cooled turbine blade and vane are shown in Figure 4.1. Fabrication of the film-cooled turbine proved to be difficult, costly, and time consuming. The complex geometry of the holes and passages pushed existing manufacturing technologies to their limits. The cooling hole geometries and machining techniques will be reviewed here.
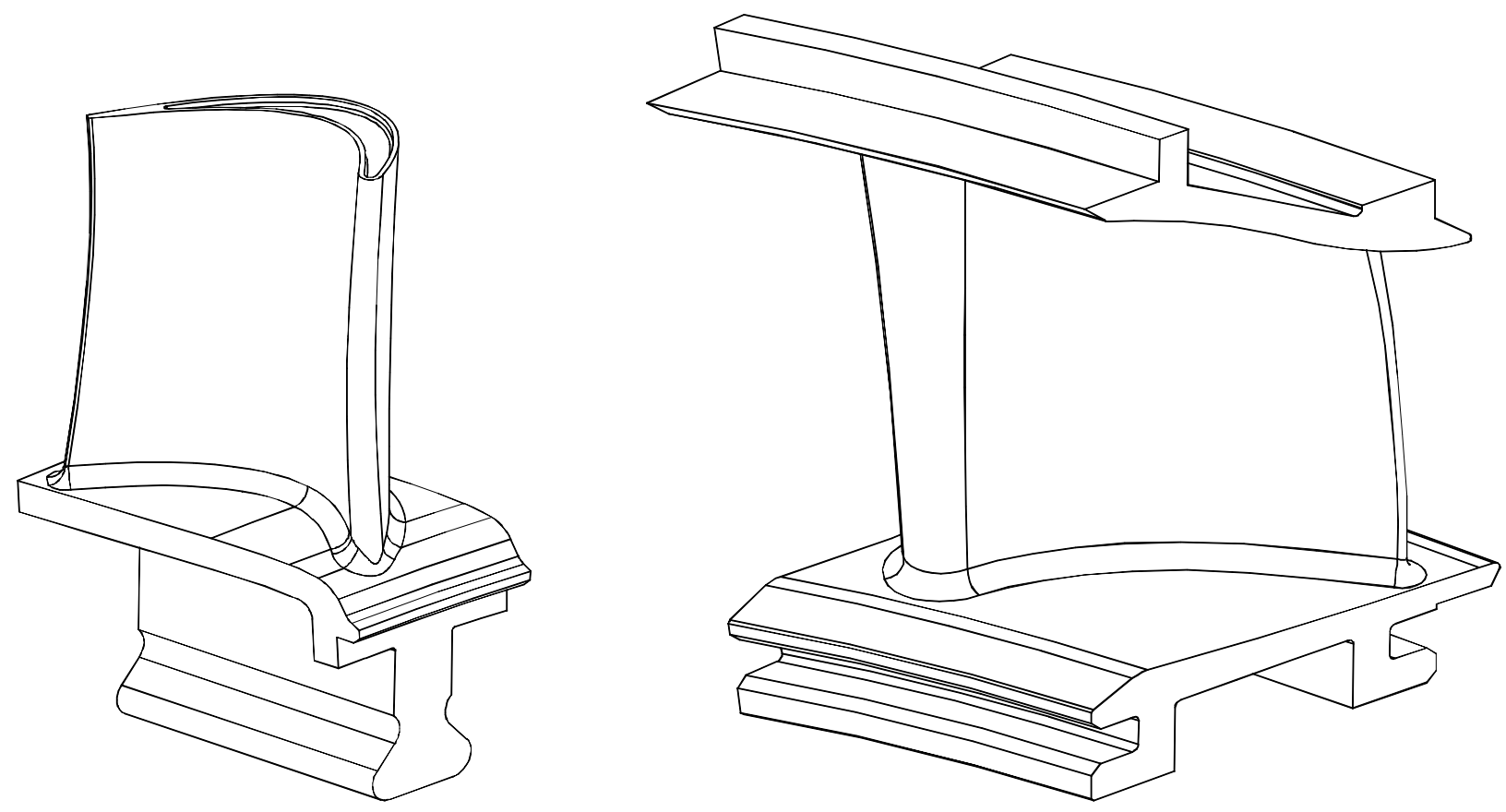

Figure 4.1: Un-cooled Blade and Vane 


\subsection{Film-Cooled Turbine Geometry}

\subsubsection{Film-Cooled Turbine Blade}

The scaled ABB film-cooled turbine blade can be seen in Figure 4.2. The blade cooling configuration consists of five rows of coolant injection holes: two on the leading edge, one on the suction surface, one on the pressure surface, and one directly out of the trailing edge. Each hole is also oriented at a compound angle. The numbers and sizes of the holes are summarized in Table 4.1.

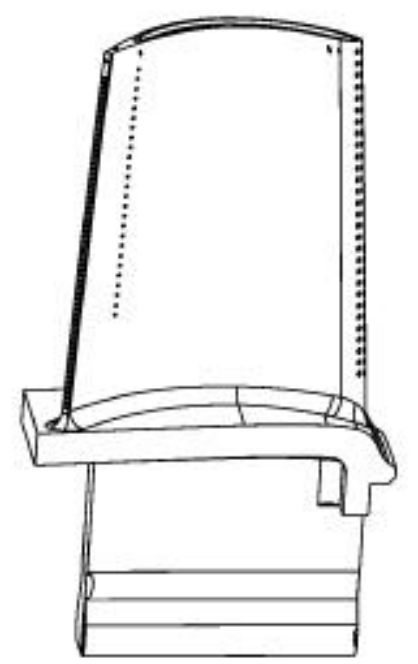

Figure 4.2: Filmed Cooled Turbine Blade

\begin{tabular}{|c|c|c|c|}
\hline Row & Number of Holes & Diameter & Location \\
\hline 1 & 17 & $0.008 ”$ & Suction Surface \\
\hline 2 & 29 & $0.008 ”$ & Leading Edge \\
\hline 3 & 29 & $0.008 ”$ & Leading Edge \\
\hline 4 & 26 & $0.008 ”$ & Suction Surface \\
\hline
\end{tabular}

Table 4.1: Blade Cooling Hole Arrangement

The internal passages of the actual ABB turbine have not been replicated. The purpose of this work is to study the effects of film-coolant injection; therefore, only simple manifold channels through the blade are needed. Two channels will be used to feed the blade cooling holes; one for the leading edge and suction surface rows, and one for the pressure surface and trailing edge rows. These passages should provide a relatively constant wall thickness. 


\subsubsection{Film-Cooled Turbine Nozzle Guide Vane}

The scaled ABB film-cooled nozzle guide vane can be seen in Figure 4.3. The vane cooling configuration consists of 12 rows of coolant injection holes on the airfoil surface, as well as several holes on the upper and lower platforms. Like the blade, each hole is oriented at a compound angle. Table 3.2 summarizes the important features of this layout.

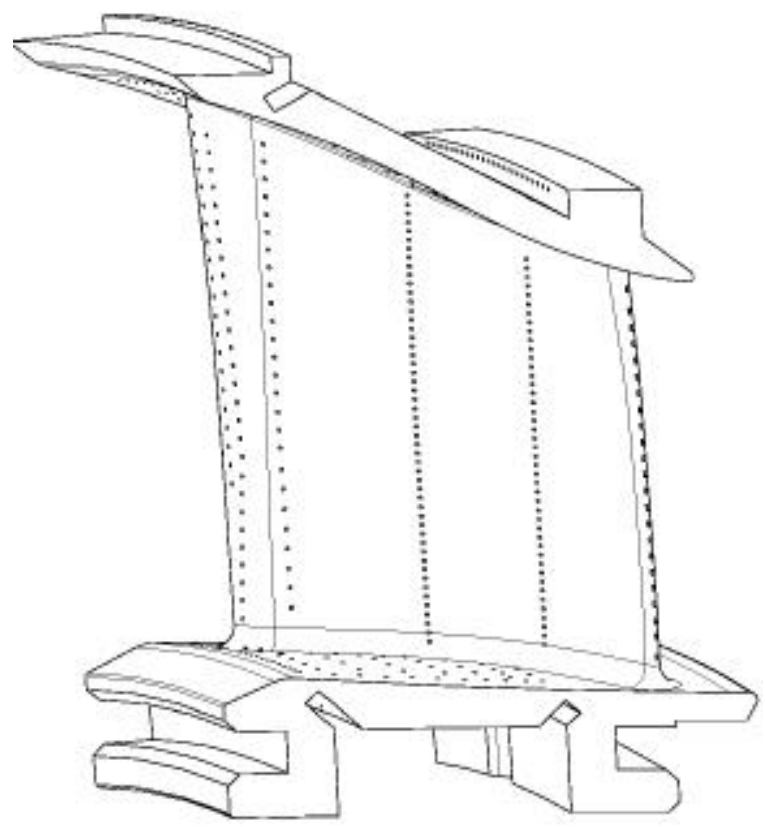

Figure 4.3: Cooled Turbine Vane

\begin{tabular}{|c|c|c|c|}
\hline Row/Group & Number of Holes & Diameter & Location \\
\hline 1 & 25 & $0.021 ”$ & Trailing Edge \\
\hline 2 & 44 & $0.008 "$ & Pressure Surface \\
\hline 3 & 51 & $0.008 "$ & Pressure Surface \\
\hline 4 & 26 & $0.008 "$ & Pressure Surface \\
\hline 5 & 28 & $0.008^{\prime \prime}$ & Leading Edge \\
\hline 6 & 21 & $0.008 ”$ & Leading Edge \\
\hline 7 & 29 & $0.008 "$ & Leading Edge \\
\hline 8 & 19 & $0.008 "$ & Leading Edge \\
\hline 9 & 45 & $0.008 "$ & Suction Surface \\
\hline 0 & 42 & $0.008 ”$ & Suction Surface \\
\hline 11 & 40 & $0.008 "$ & Suction Surface \\
\hline 12 & 22 & $0.008 "$ & Suction Surface \\
\hline $\mathrm{A}$ & 107 & $0.008 "$ & Outer Platform \\
\hline $\mathrm{B}$ & 91 & $0.008 "$ & Inner Platform \\
\hline
\end{tabular}

Table 4.2: Vane Cooling Holes

Like the cooled turbine blade, the internal passages of the actual ABB vanes have not been replicated. Two vertical channels were required to feed all of the airfoil surface cooling 
holes. Coolant will be supplied to the platform holes via manifolds above and below the locations where the vanes mate to the facility.

\subsubsection{Film-Cooled Turbine Tip Casing}

The rotor tip casing (the outer annulus wall surrounding the blade tips) also requires film cooling holes. The actual ABB geometry could not be directly scaled and accommodated in the MIT Blowdown Turbine facility. As a result, a geometry which provided the same coolant flow, but could be fabricated for use in the Blowdown Turbine, was devised. This configuration consists of two rows of film cooling holes located at the leading and trailing edges of the tip casing ring. There are 1544 leading edge holes with a 0.012" diameter and 1200 trailing edge holes with a $0.015 "$ diameter.

\subsection{Electro-Discharge Machining}

\subsubsection{Introduction}

Electro-discharge machining (EDM) is a process by which an electrode, usually made of a copper-tungsten alloy, is used to "burn" a detail into a metal piece. The piece which is to be machined is submerged in a water and oil solution while the energized electrode cuts the metal. This is required to keep the part cool and to flush away debris as it is burned away. Different types of metals respond differently to the EDM process and various burn rates can be achieved. The power used for burning a piece is set based on the type of material being cut, the desired rate of burn, and the desired finish. The quality of the finish is determined by the amount of 'overburn' provided by the electrode. The electrode does not actually come into contact with the part and does not apply any load. It is the field created by the electrode that actually burns the metal. The power of this field, or over-burn, can be adjusted to improve the finish of the cut. Overburns of 0.002-0.020" are common.

There are two types of EDM processes; wire EDM and plunge, or vertical, EDM. Wire EDM is the simplest and fastest process. The electrode consists of a wire which passes through the material in the same fashion as conventional band saw. The disadvantage of this method is the physical constraint of the wire itself. This limits the work to two-dimensional details that pass through the entire part.

Plunge EDM is a slower and more complicated process, but can produce a more complex detail. The electrode is actually machined to the shape of the desired cut and then plunged vertically into the material. The detail of the cut is limited only by the ability to machine the electrode. Plunge EDM has a slower burn rate than a wire cut simply because there is significantly more material being removed at one time. Another difficulty with the vertical EDM process is the ability to remove debris as it burns away. With a wire cut there is a hole passing through the entire part allowing EDM fluid to flow through and remove debris. This is not always the case with plunge EDM causing it to be a slower process. If debris is not properly removed, the particles can become charged and cause additional burning.

Both the plunge and wire EDM processes were used to manufacture the cooling manifold passages in the blades and vanes. All EDM work was performed on two-axis Computer Numerical Control (CNC) machines by Johnson Precision Inc. 


\subsubsection{Blade Manifold Channels}

The blade cooling manifold channels proved to be challenging to fabricate. Figure 4.4 shows a cross section of these passages. The forward manifold was cut using plunge EDM while the rear was a wire cut.

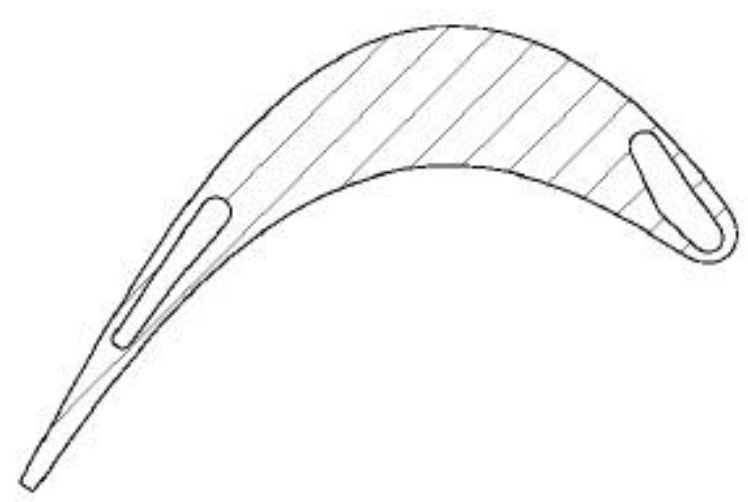

Figure 4.4: Blade Cooling Manifold Passages

The forward passage required two vertical EDM plunges; one cut at an angle from the top of the part and another from the bottom. This was done to follow the contour of the leading edge which can be seen in Figure 4.1. An approximately constant wall thickness of 0.010 " was maintained. However, this process required a high degree of accuracy so that the two plunges would mate correctly inside the blade. To achieve this, a complex fixture was designed to hold each blade in the identical position. The fixture and blade can be seen in Figure 4.5.

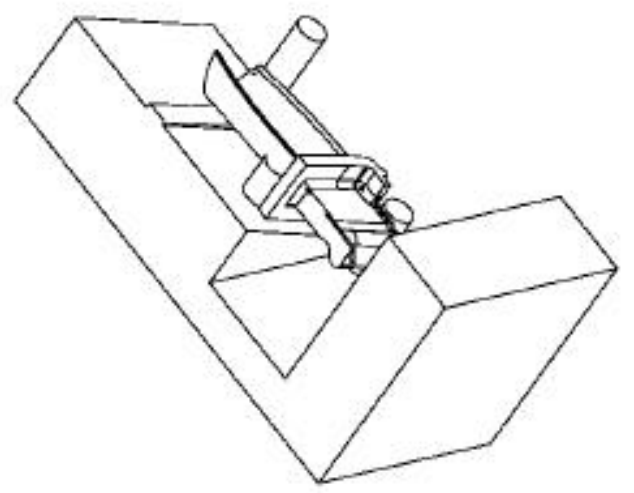

Figure 4.5: Blade Fixture for EDM 
The rear channel was produced with a simple wire cut directly through the blade. A vertical plunge was not possible for this channel due to the slender geometry. An electrode with this geometry would actually burn itself away before passing through the part. The tolerances of this channel also required a high degree of precision, therefore the same fixture was used.

The blade material also caused a problem with the EDM process. The parts were originally fabricated out of an aluminum casting alloy; AL-2618. This particular alloy contained sporadic impurities, or inclusions. When the electrode encountered these inclusions, the impurity would burn away creating a hole through the piece. As a result, several blades were destroyed in the EDM process.

\subsubsection{Nozzle Guide Vane Manifold Channels}

The NGV manifold passages were not as difficult to machine as the blade. A crosssection of these passages is shown in Figure 4.6. The relative ease of machining these details was a result of using only the wire EDM process. Again, precision cuts were required to maintain a relatively constant wall thickness and a custom designed fixture was required. This is shown in Figure 4.7.

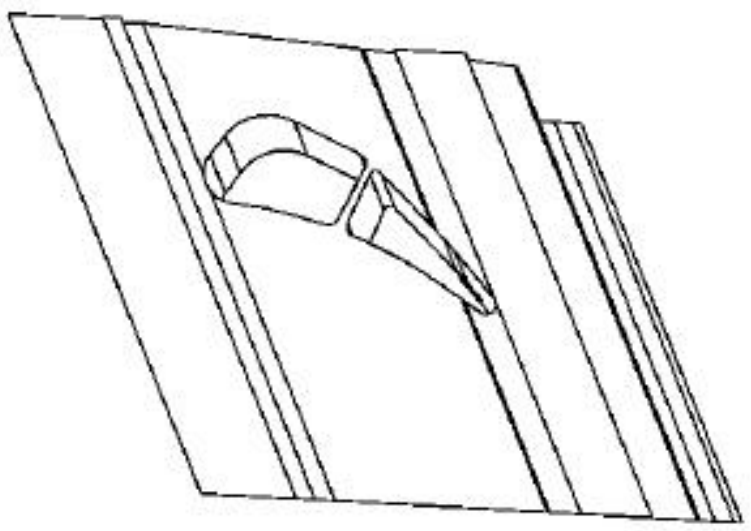

Figure 4.6: Vane Cooling Manifold Passages
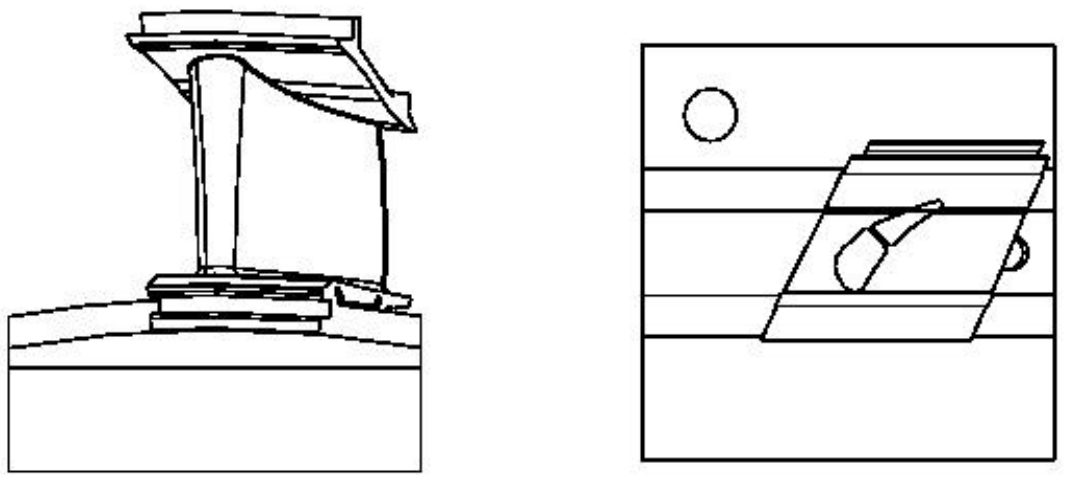

Figure 4.7: Vane Fixture for EDM 


\subsection{Laser Drilling}

\subsubsection{Introduction}

Laser drilling was chosen to fabricate the film cooling holes due to its speed, precision, and ability to drill extremely small, deep holes. No other method was found to fabricate the $1 / 4$ scale (0.008" diameter) ABB cooling holes. However, even the laser was not capable of drilling some of the holes in the scaled geometry. This was due to their large length-to-diameter ratios (L/D) and shallow angles. The laser was able to handle an L/D up to approximately 15 . The limitations of the laser and contingency procedures for difficult holes will be discussed in later sections. The machines used for this work were of the 5-axis CNC type and all work was performed by Laser Services Inc.

Laser drilling consists of using a high power, finely focused, beam of light to cut holes in various materials. There are several types of lasers used for cutting and drilling material. For this application a YAg (Yttrium-Silver) laser was used. There are several parameters which can affect the size, shape, and depth of the holes being drilled. These include:

1. Power: The power output (Watts) by the laser significantly affects the holes being drilled. The higher the power, the deeper and larger the hole.

2. Pulse: The laser is not run continuously, but instead is pulsed. The duration of each pulse and the number of pulses can be controlled to achieve the desired hole geometry. By pulsing the laser, the material is effectively "chipped" away. If the laser were to run continuously, the material would begin to melt.

3. Focus: By changing the focal point of the laser, the point of highest power density can be moved. Moving the focus can help to create a sharper, smaller hole.

4. Assist Gas: A nozzle flowing gas (oxygen for this application) onto the point were the laser is cutting is used as a kind of catalyst. The gas aids in the cutting of the material. By adjusting the type and flow rate of assist gas, a more precise cut can be made.

There are also several typical problems which are encountered when using a laser for small hole drilling. Some of these problems are:

1. Flare: The holes tend to be larger where the beam enters the material than where it exits. As the material is chipped away, its only path is back out through the initial opening.

2. Re-cast layer: As the molten material is chipped away from the part it tends to spray out onto the surface. It can re-adhere itself to the piece and even plug nearby holes. This recast layer must be removed. This problem varies with material. The harder the material, the less re-cast and the more clean the hole. The AL-2618 alloy used in this application was very soft and poor for laser drilling. The re-cast layer became a serious problem and its removal will be discussed.

3. Back-striking: This problem occurs when a high powered beam breaks through a part and strikes another region of the piece. This occurred when drilling holes into the cooling manifold channels. The beam would break through the first wall where it was intended and then proceed to strike the wall on the opposite side of the channel. 
4. Bouncing: If the incidence angle of the beam is to shallow, it will scratch the piece and bounce off without actually drilling a hole. For this application the smallest allowable angle between the beam and the material was approximately $40^{\circ}$.

A cross section of a typical laser drilled hole is shown in Figure 4.8. The flare and re-cast layer can be seen. The severity of the flare and re-cast layer increased with increasing L/D and decreasing incident beam angle.

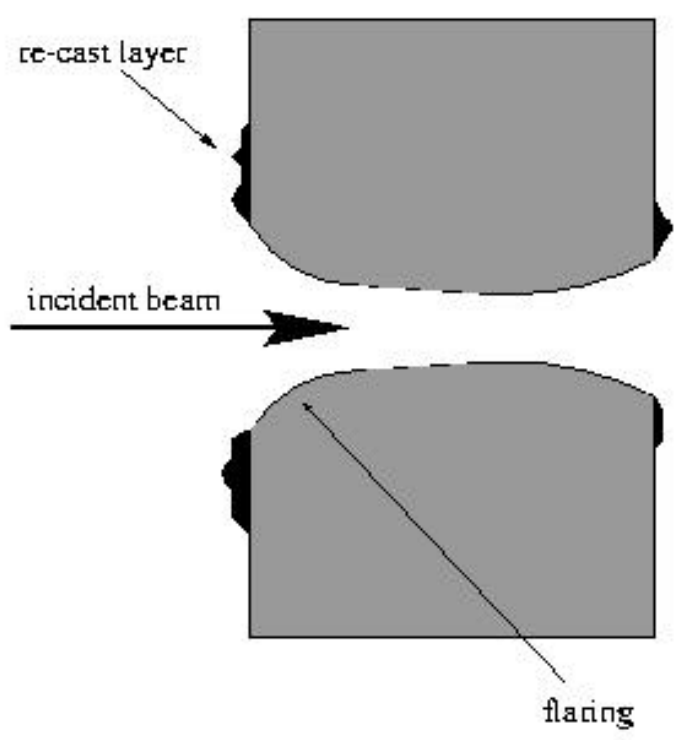

Figure 4.8: Typical Laser Drilled Cross Section

\subsubsection{Blade Cooling Holes}

The laser drilling of the blade film-cooling holes proved to be challenging. The hole geometry is shown in Figure 3.3. The two leading edge rows of holes were difficult to fabricate due to their compound angles and close spacing. Small, yet complex movements of the 5-axis machinery were required to drill at these difficult angles. The close spacing of the holes caused problems with the re-cast layer. The debris from the drilling tended to fill nearby holes. This problem was overcome by a thorough cleaning post-process.

The suction surface row did not cause any difficulties due to the relatively large hole spacing and reasonable incidence angles. A back-striking problem was encountered when drilling the pressure surface holes. Due to the thin geometry of the rear manifold passage, the laser beam tended to cut through the entire blade rather than just the one desired wall. To overcome this, the inside of the channel was lined with copper tape which reflects the beam.

The trailing edge holes could not be manufactured. The design called for 69 holes with 0.008 " diameter and a spacing of approximately 0.012" center-to-center. In addition to this, the 
$\mathrm{L} / \mathrm{D}$ of these holes was on the order of 30. Due to these constraints, the trailing edge holes were eliminated from the design, with the concurrence of ABB.

A new fixture was designed to provide better access to the blade for drilling. It can be seen in Figure 4.9.

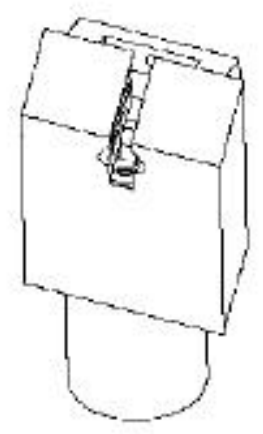

Figure 9: Blade Fixture for Laser Drilling

\subsubsection{Nozzle Guide Vane Cooling Holes}

The laser machining of the NGV film cooling holes was more difficult than those drilled into the blade. The large number of holes and variety of compound angles made this an arduous task. Figure 4.3 shows the complexity of the geometry. Every possible problem described in the above sections was encountered when drilling the NGV cooling holes.

The leading edge holes were numerous and closely spaced. This led to the development of a significant re-cast layer which later had to be removed. Difficulties were encountered with the incidence angles of the suction surface and pressure surface holes. Many of the original angles were shallow enough to cause the beam to bounce off of the NGV without burning a hole. As a result, these angles were simply changed to the smallest allowable angle for a quality hole. Back-striking was also common but overcome with the use of copper lining.

Like the blade, the trailing edge holes proved to be the most difficult. These holes required an L/D of approximately 25 which was not possible with the YAg laser technology. However, these holes could not be eliminated as on the blade trailing edge. They accounted for a substantial portion of the total NGV cooling flow and without them, the part could not be considered a reasonable scaled model of the ABB NGV. As a result, the holes were scaled up to 0.028 " and reduced in number to 15 . This allowed for the same coolant flow but simplified their manufacturability. With this change, the trailing edge holes were conventionally drilled.

The upper and lower platform holes called for both difficult angles and large L/D ratios. Angles which caused the beam to bounce off of the surface were altered to eliminate this problem. To reduce L/D ratios, the non-flow sides of the platforms were machined to reduce the thickness of the material. In addition to this, channels were cut in certain locations to aid in this same fashion. Figure 4.10 shows these modifications. 


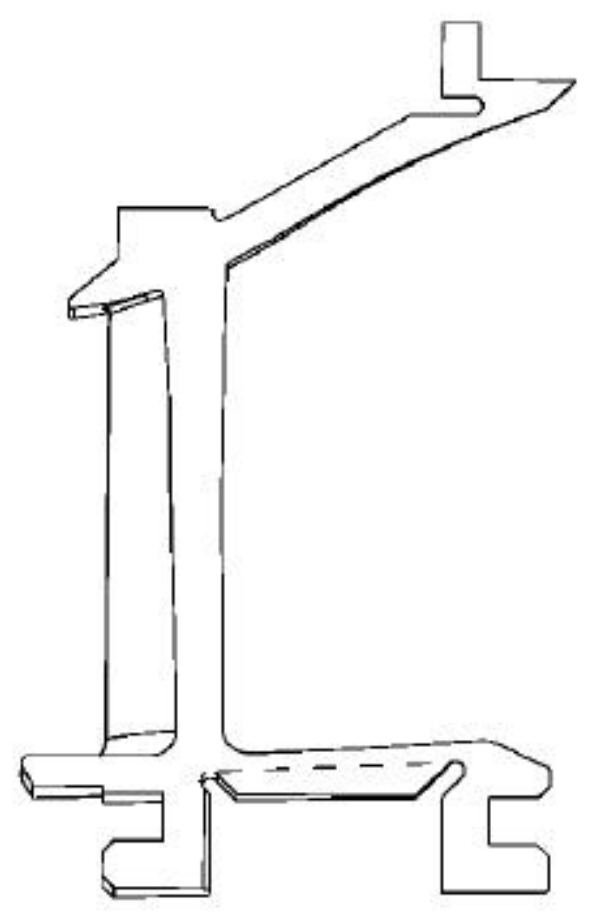

Figure 4.10: Vane Platform Modifications for Laser Drilling

\subsubsection{Tip Casing Cooling Holes}

The tip casing cooling holes were not as difficult to fabricate. The tip casing ring was fixed so that the laser head lay on its interior. The ring was then rotated to drill two rows of cooling holes. A significant re-cast layer developed and was later removed by cleaning procedures which will be described in the next section.

\subsubsection{Cleaning Procedures}

The laser drilled cooling holes required a substantial amount of cleaning. A significant re-cast layer developed on many of the parts and there were deposits of debris within the holes themselves. Ultrasonic cleaning was used to remove all loose debris within the holes or on the surface of the parts. This consists of submerging the blades and vanes in ultrasonic cleaning fluid (a type of oil) which is inside the cleaning mechanism itself. A high frequency sound wave propagates through the fluid causing any loose particles to be flushed away. After this procedure was completed, the holes were cleaned by hand with $0.006 "$ wire.

These procedures adequately cleaned the blades, however, a re-cast layer still remained on the vanes. These parts were then cleaned with an aluminum oxide blasting process. Small aluminum oxide particles were mixed with water and sprayed over the vanes. This was successful in removing the remaining re-cast layer without causing a significant change in the surface finish of the part.

\subsection{Laser Welding}

The blade manifold channels pass through the entire part and must be capped off on the blade's free end. The process of laser welding was selected to perform this task. Laser welding 
utilizes a beam similar to that used in the drilling and cutting processes. However, the beam melts the metal and is capable of welding two pieces together rather than cutting the material.

Small aluminum end-caps were cut to the approximate cross sectional shape of the blade cooling passages. These pieces were then placed over the ends of the passages and laser welded to the blade. After some trial-and-error, the process worked well and all of the blades were fitted with laser welded end-caps.

To ensure that these end-caps could withstand the stresses induced by rotation and the pressure forces of the coolant, a simple experiment was performed. Sample pieces were welded and then pressurized to 450 psi to simulate operational loads. All of the samples remained intact under these conditions. The blade with end cap is shown in Figure 4.11

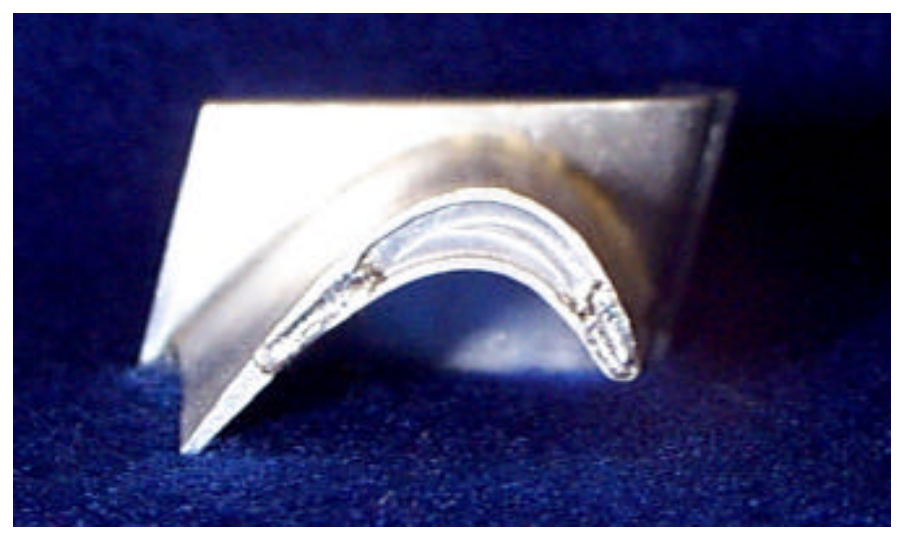

Figure 4.11: Blade Manifold End Caps

\subsection{Summary}

This chapter has presented the film-cooled turbine geometry and its fabrication. The conversion from the un-cooled turbine was explained and the machining techniques used were reviewed. The advantages and problems associated with electro-discharge machining and laser drilling were discussed. Modifications to the scaled turbine design due to machining limitations were presented. Finally, the laser welding process was briefly described. 


\title{
$5 \quad$ Turbine Torque Measurement
}

\author{
By Rory Keogh
}

\subsection{Introduction}

A measurement of the shaft power is required to estimate the real work generated by the turbine stage. The eddy current brake, which is used to dissipate the power generated by the turbine, was modified so that the torque transmitted from the brake to the main frame of the facility can be measured directly. The power produced by the turbine can then be expressed as,

$$
\mathrm{P}=\mathrm{T} \omega+I \frac{d \omega}{d t} \omega
$$

where $\mathrm{P}$ is the shaft power, $\mathrm{T}$ is the torque measured at the brake, $\omega$ is the angular speed, and $I$ is the moment of inertia of the rotating components (the blades, disks, shaft, and the brake drum). The frictional losses will be shown to be negligible compared to the other terms. This chapter will first review the design of the old eddy current brake and then outline how it was modified so that the torque can be measured. The calibration of the brake torque meter and rotor inertia will then be described in detail. The shaft speed measurement and the data acquisition system will also be reviewed.

\subsection{Eddy Current Brake Design}

The turbine requires a load to maintain and control its speed, even within the time intervals typical of tests conducted in this short duration facility. Otherwise the turbine would rapidly accelerate away from the desired test point and, under many test conditions, could even reach its design limit speed. This places strict requirements upon the operational consistency and reliability of the turbine load. The theory, development, and design of the eddy current brake load is described in detail by Guenette [5.1].

The eddy current brake is simple in concept; it is basically an electrical generator. It rotor, attached directly to the turbine shaft, is a cylindrical, electrically resistive 'loss' drum made of INCO 718. Its stator, attached to the main facility housing, is an alternating array of DC-excited electromagnets which provide a magnetic field which passes through the drum perpendicular to its axis of rotation. The motion of the drum through this applied magnetic field induces electrical currents within the drum parallel to the axis of rotation. The flow of this current produces an induced magnetic field whose interaction with the applied field results in a Lorentz force that opposes the motion of the drum. This force (or torque in rotating geometry) provides the braking. The power absorbed by this process appears as 'resistive heating' of the loss drum, (hence the choice of INCONEL). A cross sectional view of the brake assembly is shown in Figure 5.1 


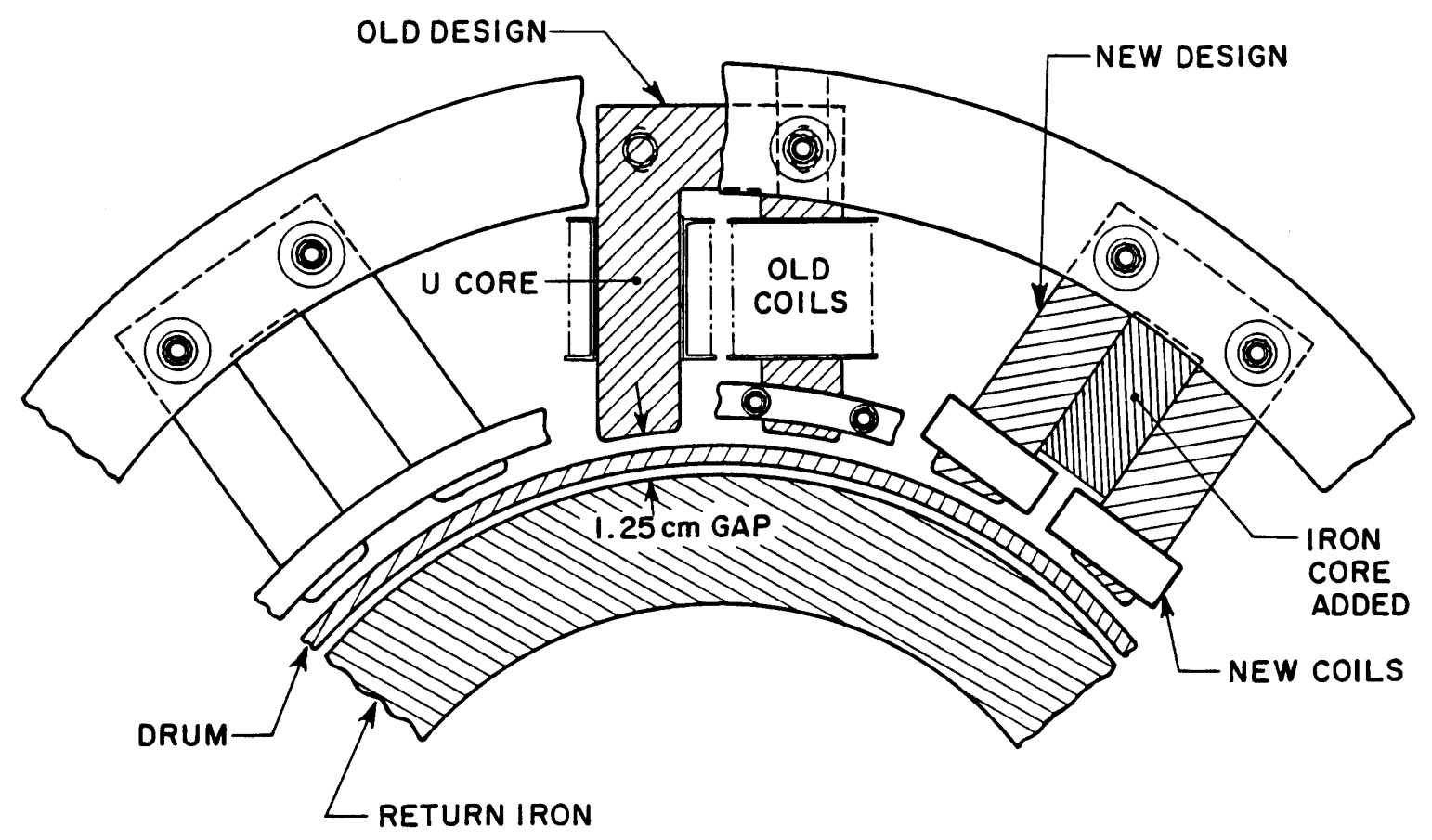

Figure 5.1: Eddy Brake Cross Section - Showing rotating drum and excitation magnets. The flow current in the loss drum alternates in-to and out-from the page.

When the magnetic field generated by the induced current is small compared to the applied magnetic field, the braking force is linearly proportional to the velocity past the magnetic poles. As the velocity is increased, the induced field strength grows relative to that of the applied field. This reduces the incremental rise in braking force with speed (i.e., reduces the slope of the torque versus speed curve) until a critical speed, $\omega_{o}$ is reached. At this point the induced field strength equals that of the applied field and the braking force begins to decrease with increasing speed. A detailed analysis of the brake can be found in Appendix B of [5.1]. A simple model of the basic eddy current brake torque versus speed characteristic is the induction motor model which closely approximates the brake behavior up to the critical speed, $\omega_{o}$,

$$
\mathrm{T}=k \omega_{o} B_{0}^{2} \frac{\left(\frac{\omega}{\omega_{o}}\right)}{\left[1+\left(\frac{\omega}{\omega_{o}}\right)^{2}\right]}
$$

where $\mathrm{T}$ is the torque, $k$ is a constant established by the geometry and material properties, $B_{0}$ is the applied magnetic field strength, $\omega$ is the angular velocity, and $\omega_{o}$ is the critical velocity at which the induced field strength equals the applied field. Figure 5.2 shows the shape of this 
characteristic. The brake was designed so that the critical speed $\omega_{o}$ is above the turbine operating speed.

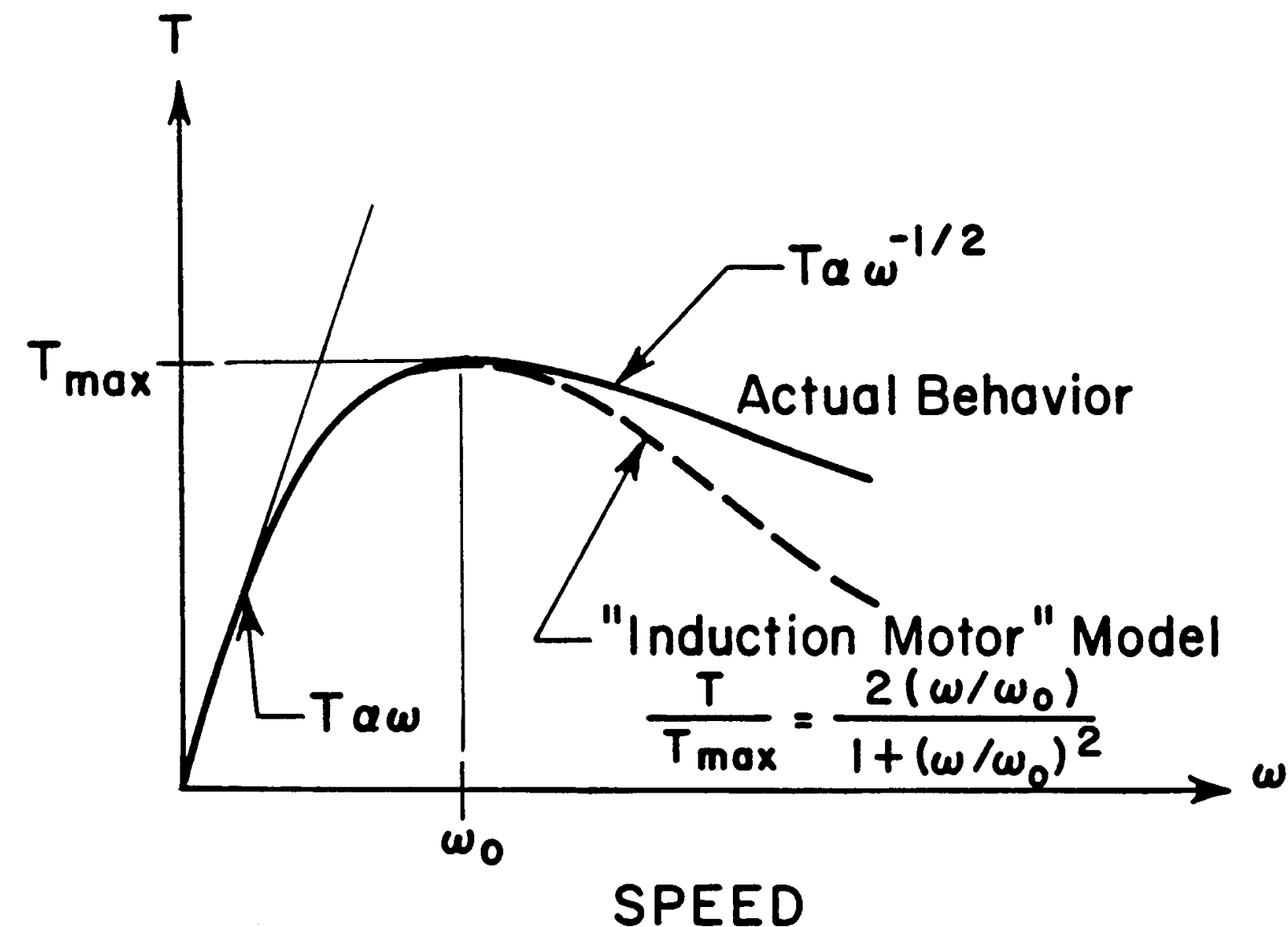

Figure 5.2: Brake Torque vs. Speed Characteristic and the Induction Motor Model - Showing the reduction in torque as the critical speed is approached.

The critical speed is a function of geometry and material properties,

$$
\omega_{o}=\frac{\pi\left(\frac{2 g}{\tau}\right)}{\mu \sigma \Delta r}
$$

where $\mu$ is the permeability of free space, $\sigma$ is the electrical conductivity of the loss drum, $2 g$ is the gap between adjacent poles of the magnet, $\tau$ is the pole pitch of the magnets, $r$ is the center of rotation, and $\Delta$ is the thickness of the drum. The geometric design parameter, $k$, can be written as,

$$
k=N A_{p} \sigma_{e f f} \Delta r^{2} M
$$


where $A_{p}$ is the cross sectional area of the magnetic pole face normal to the direction of the applied field, $N$ is the number of poles, and $M$ is an empirical field fringing parameter approximately 1.2 to 1.7. $\left(M \approx A_{p} / A_{g}\right.$, where $A_{g}$ is the mean area, per pole, of the applied field passing through the drum.)

The eddy current brake configuration is summarized in Table 5.1. A cantilever drum mounted directly to the turbine shaft was chosen for the rotating conductor for reasons of mechanical simplicity. The drum serves as a heat sink as the power is absorbed by resistive dissipation. The drum is un-cooled and during the test its temperature rises as the power is absorbed. The eddy brake magnets provide a 0.7 Tesla magnetic field across the $1.25 \mathrm{~cm}$ gap, rise from zero to full field strength in 50 milliseconds, and can maintain a constant field strength for a period of up to two seconds. The brake turn-on time must be compatible with the 50 milliseconds turn-on time of the main valve. The brake must also turn off automatically in order to prevent overheating of the coils and more importantly, the loss drum which is limited to 1200 ${ }^{\circ} \mathrm{F}$. The switching circuit shown in Figure 5.3 is used to turn on the current.

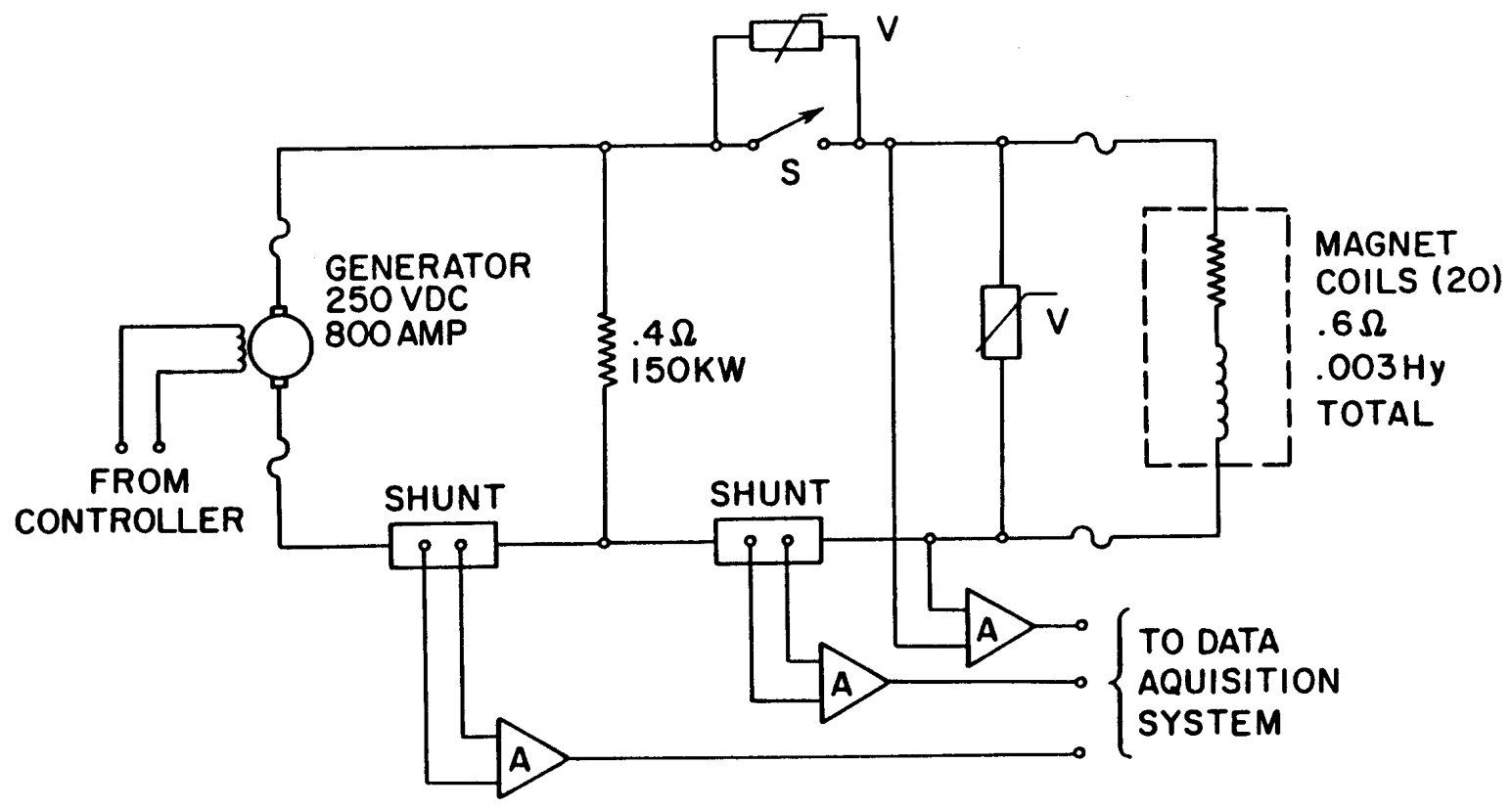

A- ISOLATION AMPLIFIERS (AD 289J)

S - VACUUM CONTACTOR (ITT RP9OOK)

V - VARISTOR (GE VSIIBA6O)

Figure 5.3: Brake Magnet Switching Circuit

The power source for the magnets is a $250 \mathrm{hp} \mathrm{D.C.} \mathrm{motor-generator} \mathrm{set} \mathrm{rated} \mathrm{at} 250 \mathrm{~V}$ and $600 \mathrm{~A}$ at continuous service. This generator was never intended to come up to load in 50 milliseconds, therefore a water cooled $0.4 \mathrm{ohm}$ ballast resistor is used to initially establish the operating point on the generator load line. A vacuum contractor rated at $50 \mathrm{kV}$ and $150 \mathrm{~A}$ 
continuous service is used to switch the magnet coils in parallel with the ballast resistor. Starting from a high load condition, the generator is then able to handle the additional load of the coils with negligible transients.

During brake operation, 800 amps at $200 \mathrm{~V}$ is drawn from the generator, which has sufficient inertia to provide this overload of current for two seconds. The magnets are deenergized by switching off the shunt field excitation to the generator and allowing the current to decay. This prevents the high voltage arcing problem associated with the opening of a large inductive circuit. Both fuses and a fast acting DC circuit breaker protect the generator. The magnet coils are also fused with a 2 to 4 second time constant. One kilojoule varistors are included to protect the switch and magnetic coils. Isolation amplifiers are used to protect the acquisition system channels monitoring the brake currents and voltages.

\section{Table 5.1: Eddy Current Brake Configuration Summary}

\begin{tabular}{|ll|}
\hline Design Point & \\
Power & $1,000,000$ watts \\
Speed & 6,190 RPM \\
\hline
\end{tabular}

\begin{tabular}{|c|c|}
\hline \multicolumn{2}{|l|}{ Loss Drum } \\
\hline \multicolumn{2}{|l|}{ Physical Properties } \\
\hline Material & INCO 718 \\
\hline Magnetic Permeability & $\mu=4 \pi 10^{-7} \mathrm{H} / \mathrm{m}$ \\
\hline Electrical Conductivity & $\sigma=0.801 \cdot 10^{6}(\Omega \cdot \mathrm{m})^{-1}$ \\
\hline Density & $\rho=8.19 \mathrm{~g} / \mathrm{cc}$ \\
\hline Specific Heat Capacity & $\mathrm{C}_{\mathrm{p}}=427 \mathrm{~J} /(\mathrm{kg} \mathrm{K})$ \\
\hline \multicolumn{2}{|l|}{ Configuration } \\
\hline Mean Radius & $\mathrm{r}=0.1619 \mathrm{~m}$ \\
\hline Thickness & $\Delta=6.35 \cdot 10^{-3} \mathrm{~m}$ \\
\hline Axial Length & $\mathrm{w}=0.1524 \mathrm{~m}$ \\
\hline
\end{tabular}

\section{Excitation Magnets}

Cores

Material

Saturation Limit

Number of Poles

Pole Width, (circumferential)

Pole Length, (axial)

Pole Pitch

Mechanical Air Gap

\section{Coils}

Turns

Resistance

Inductance

Power Dissipation
Grade M-6 transformer stock and ingot iron

Approx. 20,000 Gauss

$\mathrm{N}=20$

$2 \mathrm{a}=0.0254 \mathrm{~m}$

$2 \mathrm{~b}=0.1524 \mathrm{~m}$

$\mathrm{T}=0.0509 \mathrm{~m}$

$\mathrm{Lg}=0.0127 \mathrm{~m}$

$N=444$ turns per coil, \#23 AWG magnet wire

$R_{c}=12 \mathrm{~W}$ per coil

$L_{c}=0.12 \mathrm{H}$ per coil

$P_{c}=2700$ watts per coil; @ $15 \mathrm{~A}$

\section{Machine Characteristics \\ Magnet Time Constant \\ Drum Effective Conductivity \\ Drum Axial Resistance \\ Total Induced Current \\ Drum Heating}

$$
\begin{aligned}
& \tau_{c}=0.01 \mathrm{~s} \\
& \sigma_{e f f}=0.801 \cdot 10^{6}(\Omega \cdot \mathrm{m})^{-1} \\
& R_{D}=3.27 \cdot 10^{-5} \Omega \\
& i_{e q}=175,000 \mathrm{~A} @ 1 \mathrm{MW} \text { dissipation } \\
& \mathrm{DT}=290^{\circ} \mathrm{C} \text { per MJ absorbed }
\end{aligned}
$$




\subsection{Torque Meter Design}

The Eddy Current Brake was redesigned so that the torque transmitted to the brake could be measured during the test. In the old design the magnet assembly was attached to the main frame and the torque was transmitted directly from the brake. The new design assembled the magnets and return-iron together and then mounted the unit on bearings to the main frame. The brake was then restrained using two load cells that measured the forces transmitted to main frame. The load cells were then calibrated as outlined in the following section.

The redesign proved to be mostly a matter of mechanical design. The original eddy brake was designed at the same time as the rest of the facility, so the main frame was specifically designed to accommodate the brake. The original design proved to be very successful and operated flawlessly for fifteen years. The challenge was to redesign the brake economically while maintaining its reliability and robustness.

The redesigned eddy current brake is shown in Figure 5.4. The new parts consist of the bearing housing and the support plate. The bearing housing both supports the bearings so that a preload can be applied, and also acts as a shaft that allows the assembly to rotate. The bearing housing is then bolted to the support plate and the magnets and the return iron are then assembled to it. The motor support was reworked in order to accommodate the bearings. The assembly is then free to rotate about the motor support. The preload on the bearings can be adjusted by means of a shim plate between the bearing retainer and the motor support. Two brackets that are mounted to rear side of support plate to restrain the brake, these brackets extend through the aft wall of the motor support. The brackets are attached to the motor support through two S-beam load cells, as shown in Figure 5.5.

The brake was designed for two diametrically opposed load cells. For the first build, however, only one was installed. This proved to be unsuitable as was shown from the brake static calibration tests. With one load cell, a reaction load must be transmitted through the bearings. The starting torque of the bearings is proportional to this reaction load. This starting torque resulted in hysterisis during the brake calibration. The addition of the second load cell reduced the reaction load on the bearings and the hysterisis problem. This is discussed further in the following section. 


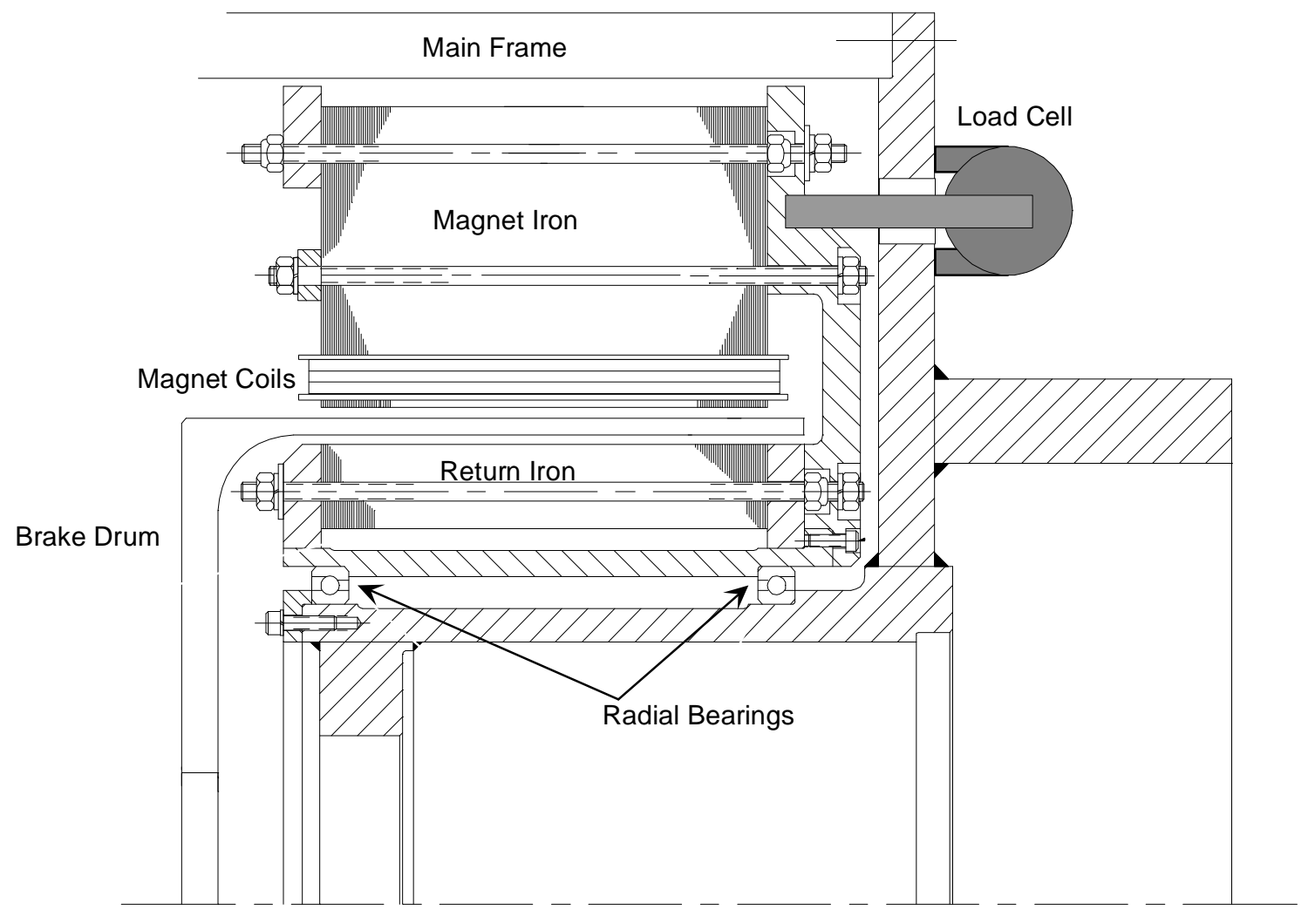

Figure 5.4: Eddy Current Brake Torque Meter - Showing the modifications for the bearing mounts and the load cell restraints.

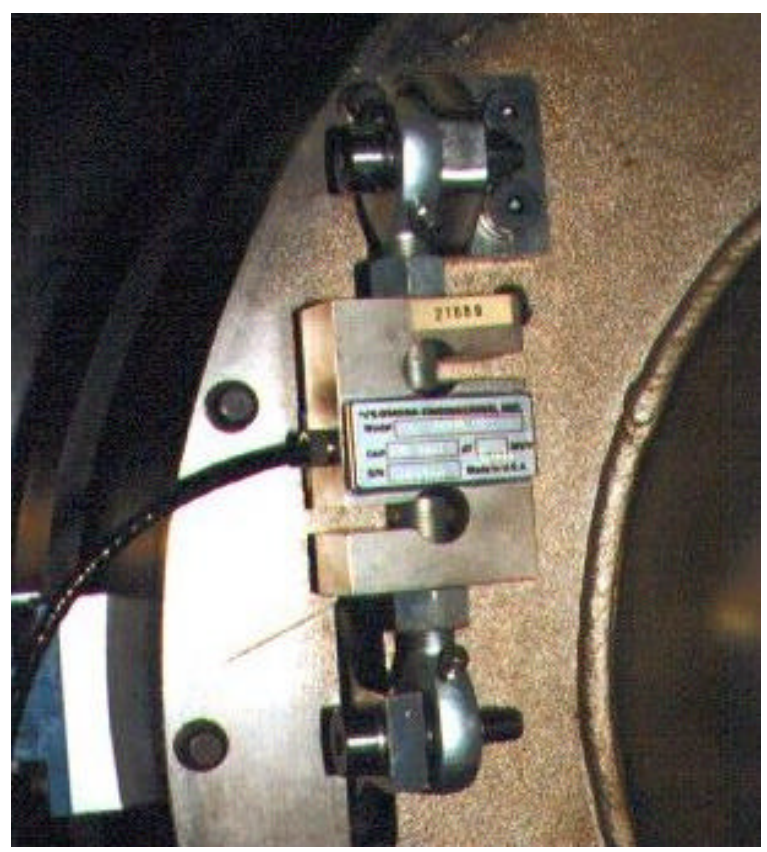

Figure 5.5: Load Cell Mounting Arrangement 


\subsection{Torque Meter Calibration}

Two approaches were used to calibrate the eddy current brake torque meter. Firstly, a static calibration performed on the brake using a precision torque sensor that mounted in series with the brake load cells. Secondly, a series of spin-down tests verified that brake performance was independent of the applied magnetic field.

\subsubsection{Static Calibration}

\subsubsection{Calibration Setup}

The torque meter is calibrated statically by applying a load to it through a precision torque sensor. The torque sensor is a commercial unit that has a calibration record traceable to NIST. The calibration setup is designed to minimize any bias errors that are introduced.

Figure 5.6 shows the mechanical setup for the calibration. The mounting plate shown in this figure transmits the calibration load from the torque sensor, through the brake assemble, to the load cells.

The design of this mounting plate ensures that the axis of the torque sensor is concentric with the axis of rotation of the brake. This important as any side loads applied to the torque sensor would place an additional torque to the brake (and therefore the load cells being calibrated) that would not be measured by the torque sensor. The resulting error would be the $\vec{\varepsilon} \cdot \vec{F}_{s}$, where $\vec{\varepsilon}$, is the eccentricity and $\vec{F}_{s}$, is the side load. The potential errors can be reduced by minimizing the both the eccentricity and the side load.

The calibration setup shown in Figure 5.6 will introduce a side load on the torque sensor. This is a direct result of the way that the torque for the calibration is generated. The maximum error will occur if,

$$
\vec{\varepsilon} \cdot \vec{F}_{s}=\varepsilon F_{s}
$$

From Figure 5.6 the side load can be estimated as,

$$
F_{s}=\frac{\mathrm{T}}{d} \frac{L_{1}}{L_{2}}
$$

The bias introduced by the calibration setup can be estimated as,

$$
\frac{B_{\mathrm{T}}}{\mathrm{T}}=\frac{\varepsilon}{d} \frac{L_{1}}{L_{2}}
$$

The maximum limit of the eccentricity $\varepsilon$, can be estimated by considering the design of the mounting plate. This plate is designed to ensure that the torque sensor is concentric and perpendicular with respect to the brake. A male alignment flange on the plate is inserted into the precision bearing-race. Another male alignment flange on the plate is aligned with the torque sensor. The alignment flanges are concentric to the plate to within 0.001 inches. The flanges 


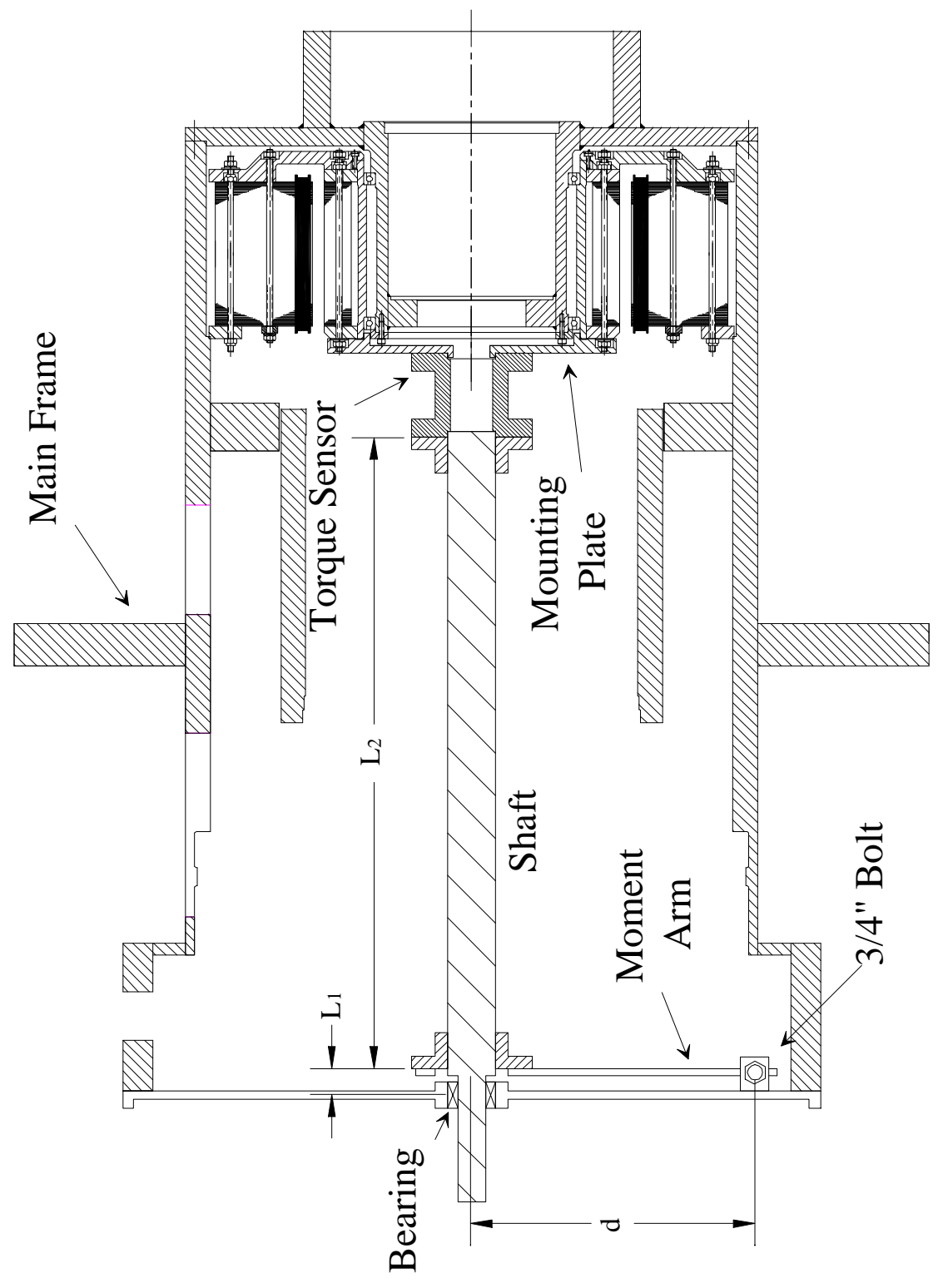

Figure 5.6: Static Calibration Setup 
were fit to their respective mates with clearances of less than 0.001 inches. The plate is bolted to return iron assemble using ten 0.25 inch bolts, through which the calibration load is transmitted.

A shaft extends from the torque sensor to the front of the main frame where the torque can be applied. A bearing, mounted to an I-beam spanning the mouth of the main frame, supports the shaft. A lever arm is attached to the shaft in order to apply the torque. A 0.75 -inch bolt, which is tightened through a nut welded to the I-beam, forces the lever arm and I-beam apart. This generates the required torque. A summary of the results is contained in Table 5.2. A more complex calibration setup would have been required to eliminate the side load.

Another potential source of bias error in the calibration setup is the perpendicularity of the torque sensor with respect to the brake. This is a second order contribution, as the error will be proportional to the sine of the angle between the axis of the torque sensor and the brake. The load cells are calibrated for a maximum torque of 20,000 inch-pounds. This is a considerable load and it required the calibration hardware to pretty beefy.

\subsubsection{Data Acquisition System}

This section describes the data acquisition setup for the load cells and torque sensor used to calibrate them. Precision strain gage amplifiers (Analog Devices 2B31) provide the excitation and amplify the output signal from the load cells. The amplifier gain and offset adjustment were replaced by precision resistors to ensure that the amplifier settings could not be adjusted. The idea being to calibrate the load cells and amplifiers as unit. The amplifier output range was set at 0-4 volts to match the input range of the data acquisition cards. The output range for the amplifier dropped to approximately 0-2 volts when the second load cell was added. The amplifiers were originally calibrated for use with only one load cell.

A voltmeter (Fluke Digital Multimeter 8520A) was used to record the output from the torque sensor. A 6-wire arrangement was used so that the bridge excitation could by measured at the connector provided by the manufacturer.

For the calibration, the data was recorded manually. The gain and offset for the calibration were determined by performing a linear least squares fit of the recorded data.

\subsubsection{Static Calibration Results}

This section discusses the results of the eddy current brake torque meter static calibration. As discussed earlier the torque meter was originally designed. Figure 5.7 shows the results for the calibration tests using only one load cell. For each of the calibration runs, the deviation of the data points from the mean of the calibrations is plotted. Calibration tests 5 and 7 were performed as the torque was increased whereas for calibration 6 and 8 the torque was being reduced. The data shows a significant and consistent trend difference between the loading and unloading calibrations. The data shows a large discrepancy at low torque levels and more consistent results at higher torque levels. 


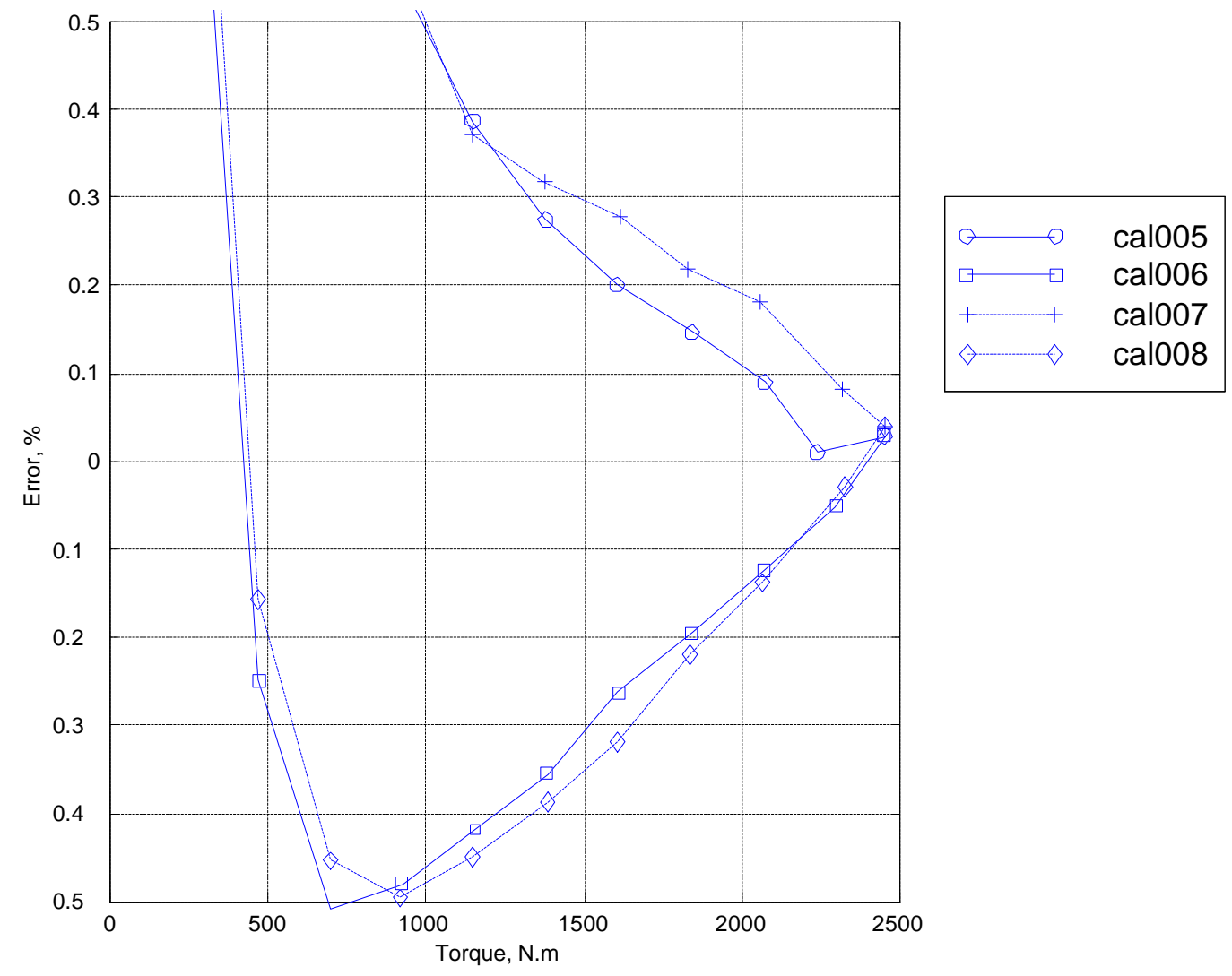

Figure 5.7: Static Calibration - One Load Cell

If the starting friction of the bearings caused the problem, one would expect to see the opposite trend in the data. The torque measured should be lower than the applied torque (assuming that the average represents the actual torque). If there is a normal load on the bearing, the ball is depressed into the bearing race. For the ball to move it must effectively climb out of a depression that has been created by the normal load. In this case the normal load is caused by the reaction to the load cell. The slope of the starting friction versus normal load curve will decrease as the load is increased. The starting friction to torque ratio will be smaller at higher torque levels.

A description of other observations can help explain the data. As the torque is increased from one test point to the next, the torque level will slowly relax by approximately $0-5 \%$. This takes about 20-30 seconds for the output voltages to stabilize so that a reading can be taken (three voltages must be recorded). If the torque is being decreased during the calibration the opposite trend in observed. The torque level will increase $0-5 \%$ until the reading stabilizes.

The torque is transmitted from the mounting plate through the return iron assembly and on to the load cells. The return iron is made up of a laminated transformer material that is held together by 10 quarter inch rods. This laminated material probably relaxes somewhat after the load has been increased. This can explain the observations seen above. Bearing starting friction can actually explain the irregularities in the test data. Because of the relaxation in the return iron, the torque is actually decreasing before the reading is taken, even though the torque is being increased from one data point to the next. The reverse trend is seen when the as the torque is 
reduced. As the load is being increased, the torque is overestimated at low torque levels. The torque is underestimated at low torque levels as the load is reduced.

These problems can be attributed to the reaction on the bearings. To solve this problem a second load cell was added, the idea being to eliminate the reaction load on the bearings.

However, adding a second load cell makes the system statically indeterminate, so the bearing load can be reduced, but it cannot be easily eliminated.

Figure 5.8 shows the calibration results with the addition of the second load cell. The trend in the data is similar to the previous results, except that the magnitudes have been reduced. Table 5.2 contains data for two separate sets of calibration test that were performed 3 months apart. All of the calibration hardware was disassembled in between the two calibration tests. The calibration proved to be very repeatable.

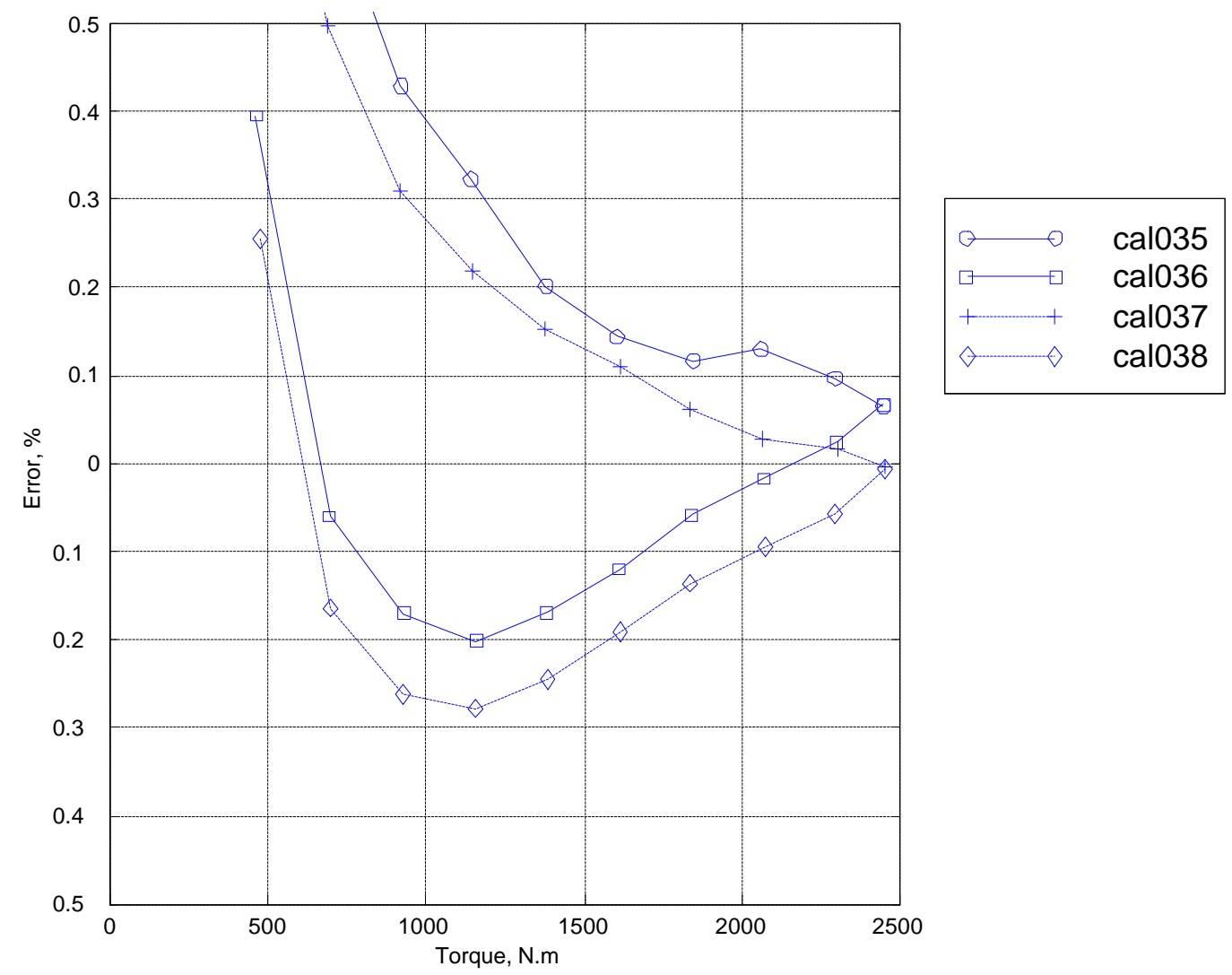

Figure 5.8: Static Calibration - Two Load Cells 


\begin{tabular}{|l|l|l|l|l|l|}
\hline \multicolumn{6}{|l|}{ Table 5.2: Torque Meter Static Calibration Summary } \\
\hline \multirow{2}{*}{ Calibration \# } & Scale & Zero & dT/dt & Mean & Deviation \\
\cline { 2 - 6 } & $\mathrm{N} \mathrm{m} /$ volt & $\mathrm{N} \mathrm{m} /$ volt & & $\mathrm{N} \mathrm{m} /$ volt & $\%$ \\
\hline 35 & 682.43 & -17.07 & + & & \\
\hline 36 & 684.41 & -26.17 & - & 683.42 & $-0.037 \%$ \\
\hline 37 & 682.18 & -17.74 & + & & \\
\hline 38 & 684.74 & -28.27 & - & 683.46 & $-0.031 \%$ \\
\hline 39 & 682.15 & -17.30 & + & & \\
\hline 40 & 685.03 & -29.76 & - & 683.59 & $-0.012 \%$ \\
\hline 41 & 682.82 & -21.04 & + & & \\
\hline 42 & 684.52 & -29.18 & - & 683.67 & $0.00 \%$ \\
\hline 43 & 682.17 & -17.32 & + & & \\
\hline 44 & 685.81 & -31.97 & - & 683.99 & $0.046 \%$ \\
\hline 45 & 683.45 & -21.82 & + & & \\
\hline 46 & 684.36 & -28.14 & - & 683.90 & $0.034 \%$ \\
\hline & & Mean and Standard Deviation & $\mathbf{8 6 3 . 6 7}$ & $\mathbf{0 . 0 3 4 \%}$ \\
\hline
\end{tabular}

The calibration results at the higher torque levels are excellent, they are repeatable to within $\pm 0.1 \%$. However, the results are disappointing at low torque levels. During a typical blowdown test the torque measured at the brake will not be steady. A small imbalance in the rotor causes the brake to vibrate at the shaft frequency. Because of this vibration, the torque reading fluctuates by few percent about its mean value. The hysterisis seen in this static calibration should not have an affect on the torque measured in the real experiment. The torque reading will effectively jump back and fourth between the loading and unloading curves in Figure 5.8. The result can be seen as a problem with the static calibration and not as an actual problem with the operation of the eddy current brake torque meter.

\subsubsection{Spin-Down Calibration}

This section describes the spin-down calibration. There are three objectives for the spindown test. Firstly, to estimate the moment inertia of the rotating components. As shown in Equation 5.1, the inertia is required to estimate the shaft power. The second objective of the test is to show that the repeatability the static calibration for high torque levels, is true for the all torque levels measured by the brake. Measuring the inertia at several different brake settings, and determining its repeatability, can do this. The final objective this test is to show that the strength of the magnetic field has no effect on the measurement.

This test was pretty straight forward, the experiment was setup as in the real blowdown experiment. The only difference being that the supply tank was not charged and the fast acting valve was not armed. Without the test gas, no power is generated so from Equation 5.1 the rotor inertia can be estimated,

$$
I=-\frac{\mathrm{T}}{\dot{\omega}}
$$


Bearing friction was estimated from the rotor deceleration prior to the firing of the brake and was found to be negligible.

Three different brake settings were tested. Table 5.3 contains the important parameters for the test. Figure-5.9 shows the rotor speed and Figure 5.10 the unfiltered data for the torque measurement, for the three runs.

An FFT analysis of the of the torque signal showed peeks between $70-100 \mathrm{~Hz}$, this is due to shaft vibration. A 5 pole Butterworth filter with a cutoff at $50 \mathrm{~Hz}$, and phase shifting was applied to smooth the raw data.

Equation 5.8 requires that the derivative of the speed signal be taken. This is not practical, as differentiation will amplify the noise in the already noisy signal. Instead a linear least squares curve was fit to the data and its derivative was taken to get the angular deceleration. The curve was fit to 80 milliseconds segments of data, the torque is taken as the mean torque over the same range.

Figure 5.11 shows the calculated inertia for the four tests. The inertia is plotted as a function of the torque level at which it is estimated. The data shows that the calculated inertia is constant over the entire range of tested. Figure 5.12 shows the deviation from the mean torque over the entire range. The mean inertia is $1.8085 \mathrm{~kg} \mathrm{~m}^{2}$ and the standard deviation is $0.13 \%$.

\begin{tabular}{|l|l|l|l|l|l|}
\hline Table 5.3: Torquemeter Spin-Down Calibration Summary \\
\hline Calibration \# & Brake Excitation & Total Current & Rotor Speed & Measured Inertia & Deviation \\
\hline & volts & amps & rps & $\mathrm{kg} \mathrm{m}^{2}$ & $\%$ \\
\hline 1 & 133.7 & 254.0 & 100.16 & 1.8049 & -0.20 \\
\hline 2 & 213.5 & 386.6 & 100.16 & 1.8095 & +0.06 \\
\hline 3 & 214.0 & 386.7 & 100.16 & 1.8086 & +0.01 \\
\hline 4 & 243.5 & 432.5 & 100.16 & 1.8109 & +0.13 \\
\hline \multicolumn{7}{|l|}{ Mean and Standard Deviation } & $\mathbf{1 . 8 0 8 5}$ & $\mathbf{+ 0 . 1 3}$ \\
\hline
\end{tabular}

\subsubsection{Uncertainty Analysis}

The bias estimate for the torque measurement was determined by combining the quoted uncertainty for the torque sensor $(0.05 \%)$ and the precision index for the static calibration (from Table-5.2). This gives a bias limit of $0.084 \%$. The precision index for the torque measurement is taken from the spin-down calibration (Table-5.3). The combined $95 \%$ uncertainty level is $0.27 \%$.

\subsection{Summary}

A static calibration has been performed to determine the scale for the eddy current brake. At high torque levels the measurement are very repeatable. The result was less conclusive at lower torque levels. The mean of the scales for the loading and unloading of each calibration cycle repeats with a standard deviation of $0.034 \%$.

A spin-down test was performed to estimate the inertia of the rotating components. This calibration showed that the inertia measurement repeated with a standard deviation of $0.13 \%$ over the entire torque range of interest. This shows that the scale determined from the static calibration at the high torque levels is valid over the entire range of operation of the brake. The spin-down test also verified that the brake current does not adversely affect the torque measurement. The estimated uncertainty $\left(\mathrm{U}_{95}\right)$ for the eddy current brake torque measurement is $0.27 \%$. 


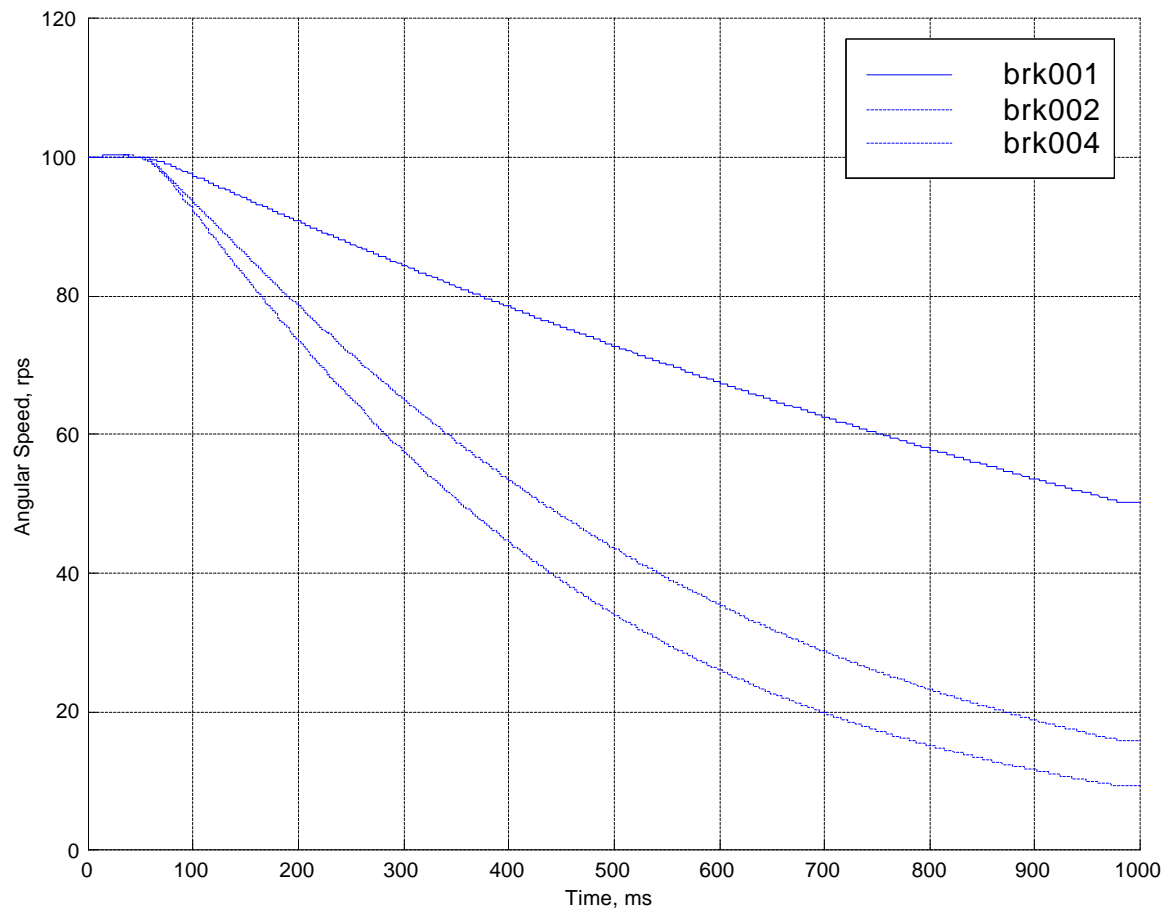

Figure 5.9: Spin-Down Test, Rotational Speed

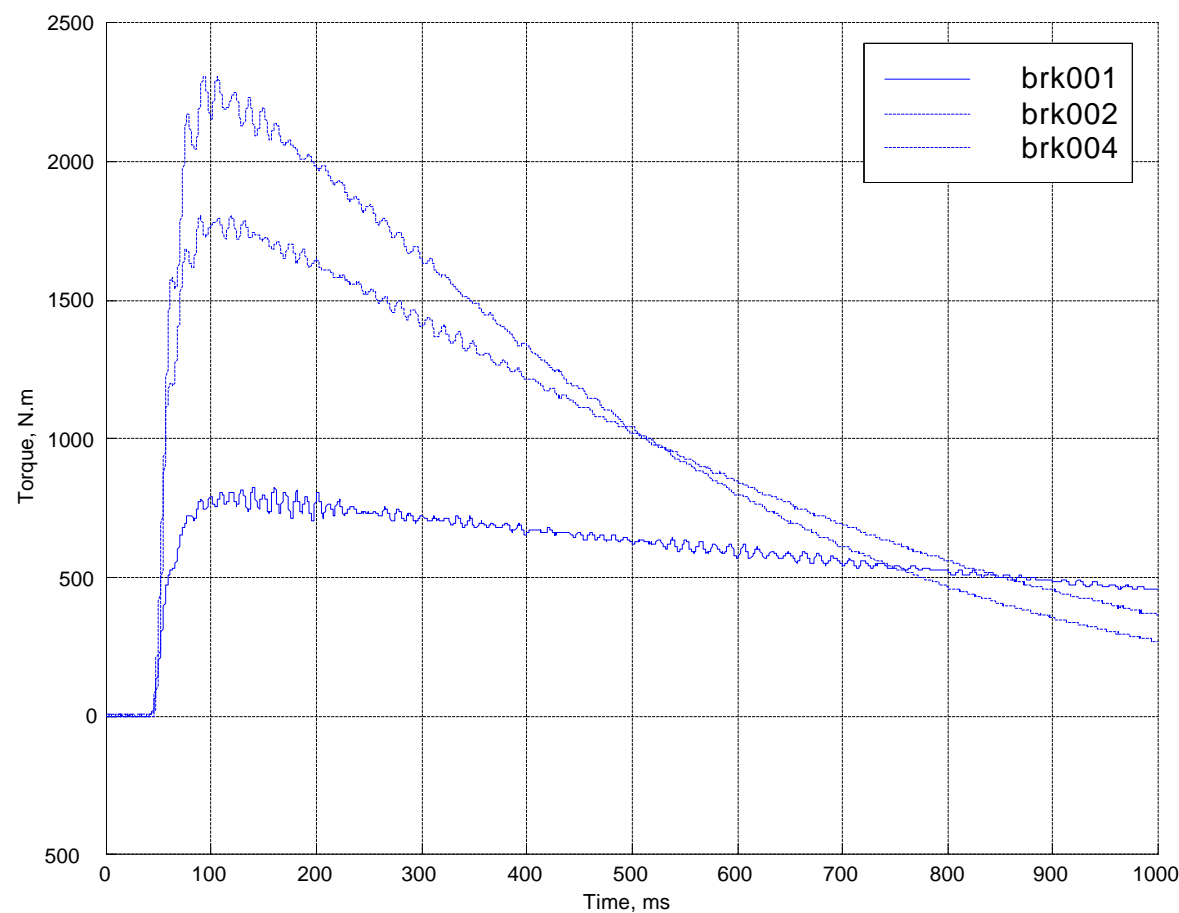

Figure 5.10: Spin-Down Test, Brake Torque 


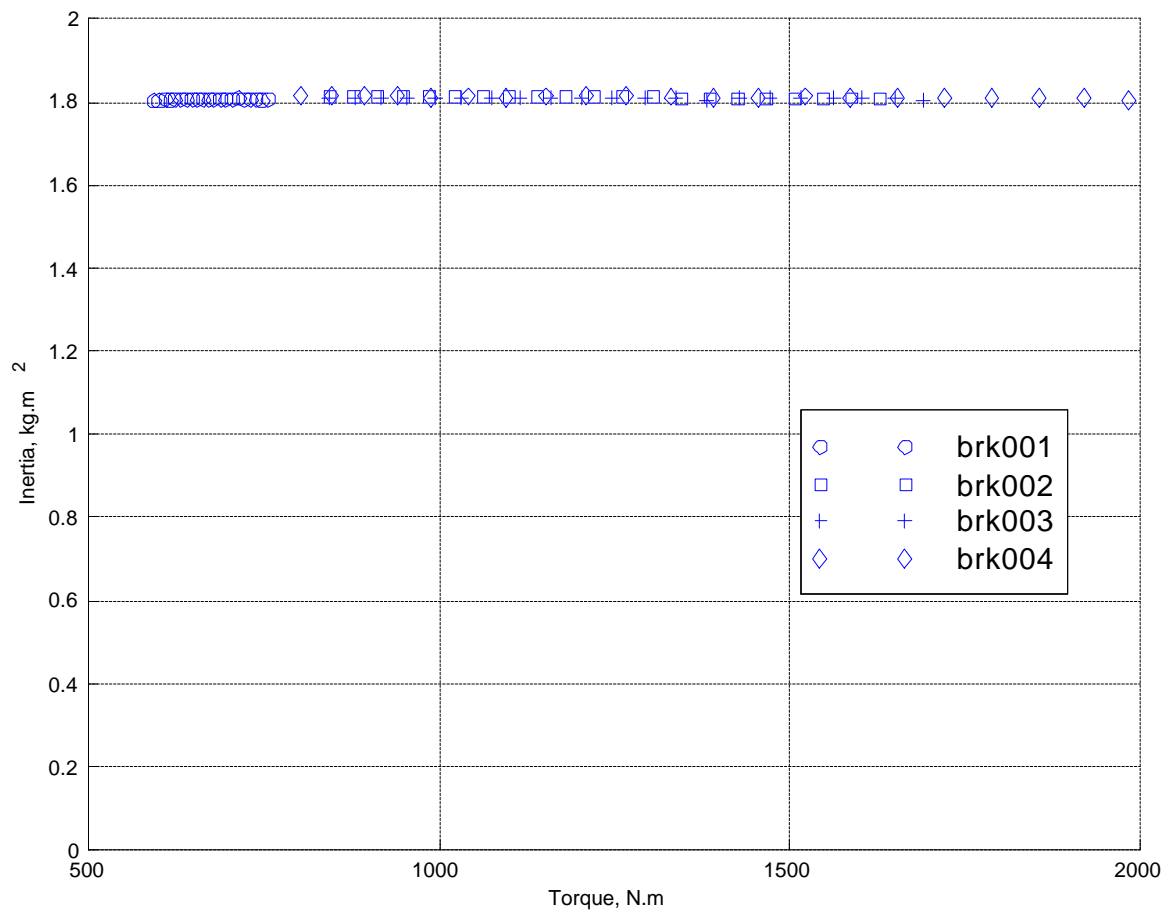

Figure 5.11: Spin-Down Tests, Calculated Inertia vs. Torque

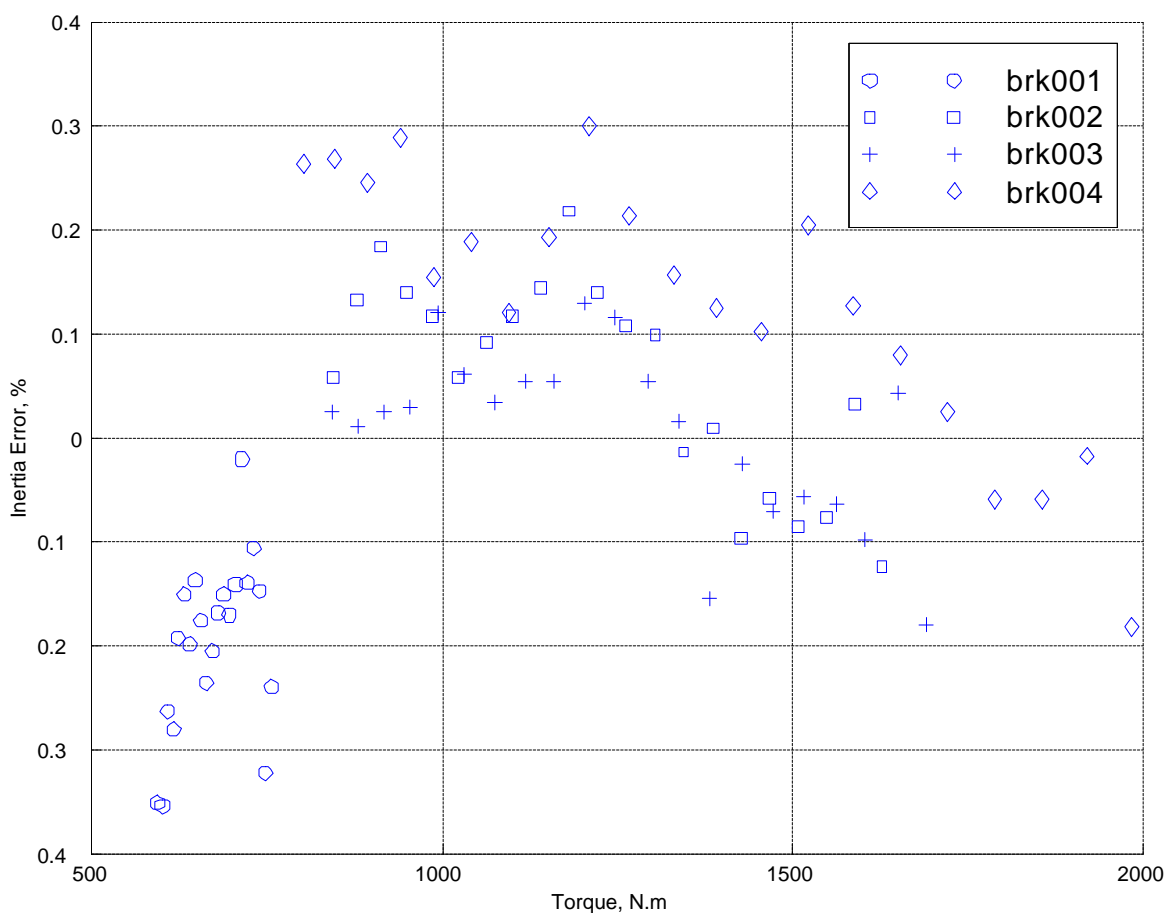

Figure 5.12: Spin-Down Tests, Inertia Error vs. Torque 


\subsection{References}

[5.1] Guenette, G.R., "A Fully Scaled Short Duration Turbine Experiment”, Sc.D. Thesis, Massachusetts Institute of Technology, August 1985. 


\title{
6 Mass Flow Measurement
}

\author{
By Rory Keogh
}

\subsection{Introduction}

Accurate turbine mass flow measurement is required in order to evaluate stage shaft efficiency and turbine through-flow capacity. In this study a critical flow venturi is used to measure the turbine mass flow. This method was chosen, as it is an industry standard for measuring large volumetric flow rates [6.1]. A nationally recognized laboratory calibrated the discharge coefficient of the nozzle with tractability to the National Institute for Standards and Technology. The calibrated venturi is used as a transfer standard for the measurement of mass flow in the blowdown turbine facility. A well-established method for dealing with the real gas behavior of test gases is another benefit of the critical flow venturi.

Sections 6.2, 6.3, and 6.4 review the ANSI standard [6.2] for mass flow measurement, and how it was applied to the blowdown turbine facility. Section 6.5 outlines a correction that must be applied to the measured flow rate to account for the transient effects of the short duration test. Section 6.6 reviews the error analysis for the mass flow measurement and the transient correction.

\subsection{The Sonic Nozzle Standard}

A sonic nozzle is a venturi nozzle through which the mass flow rate is the maximum possible for given upstream conditions. At critical flow or choked conditions, the average gas velocity at the nozzle throat is the local sonic velocity. A sonic nozzle was chosen as a mass flow meter because of its well defined fluid dynamic characteristics. Because of its smooth convergent divergent section the discharge coefficient of the venturi nozzle is known to be very lose to unity. If the nozzle geometry and installation conform to ANSI standard, the calculated discharge coefficient will have a stated uncertainty of $\pm 0.5 \%$. This uncertainty can be reduced to about $\pm 0.25 \%$ by calibrating the nozzle against a calibration laboratory primary standard.

This second option was chosen for two reasons, firstly, the maximum accuracy was desired, and secondly, the upstream duct requirement of the standard would not be practical for the MIT Blowdown turbine facility. If placed downstream of the turbine, 20 feet of straight ducting would be required to satisfy the standard. Colorado Engineering Experiment Inc. (CEESI) calibrated the nozzle with the upstream duct and flow conditioning as shown in Figure 6.5 .

\subsection{Basic Equations}

Ideal critical flow rates require three main conditions: (a) the flow is one-dimensional; (b) the flow is isentropic; and (c) the gas is perfect. Under these conditions, the value of critical flow rate is,

$$
\dot{m}=A C_{*_{i}} \frac{P_{o}}{\sqrt{R T_{o}}}
$$


where,

$$
C_{* i}=\sqrt{\gamma\left(\frac{2}{\gamma+1}\right)^{\sqrt{\gamma+1}}}
$$

\subsubsection{Flow Rate in Real Conditions}

\subsubsection{Discharge Coefficient}

However, the real gas flow rate will differ in two respects, firstly the flow is not quite one dimensional. Blockage due to boundary layer growth along the walls of the nozzle will reduce the mass flow. Also, there is a small static pressure non-uniformity at the throat of the nozzle due to streamline curvature effects. To account for the multi-dimensional nature of the flow, $(A)$ in Equation 6.1 is replaced by $\left(A C_{d}\right.$, ) where $C_{d}$ is discharge coefficient of the nozzle. Secondly, the test gas is not quite perfect and real gas thermodynamic effects must be considered. To account for real gas effects $C_{* i}$ in equation 6.1 is replaced by $C_{R}$. These changes yield,

$$
\dot{m}=A C_{d} C_{R} \frac{P_{o}}{\sqrt{R T_{o}}}
$$

The nozzle was calibrated at an independent, nationally recognized laboratory that specializes in the calibration of critical flow venturi nozzles for the natural gas and aerospace industries. The calibration is traceable to the National Institute of Science and Technology. The nozzle discharge coefficient is dependent on Reynolds number and is generally expressed in the form,

$$
C_{d}=a-b \operatorname{Re}_{d}^{-n}
$$

where $a, b$, and $n$ are obtained by calibration. Table 6.1 contains the calibration results for the nozzle. Brain [6.3] contains a boundary layer calculation of nozzle discharge coefficient, which is also shown in Table 6.1 for comparison. Many investigators favor this type of nozzle design because of the close agreement between the experimental and the theoretical results.

\begin{tabular}{|l|l|l|}
\hline \multicolumn{3}{|c|}{ Table 6.1: Nozzle discharge Coefficients } \\
\hline Parameter & ANSI Standard & MIT Nozzle \\
\hline $\mathrm{a}$ & 0.9885 & 0.9832 \\
\hline $\mathrm{b}$ & 0.445 & 12.798 \\
\hline $\mathrm{n}$ & -0.5 & -0.5 \\
\hline $\mathrm{Cd} @ \mathrm{Re}=6,000,000$ & 0.9929 & 0.9885 \\
\hline
\end{tabular}

Figure 6.1 shows the installation of the nozzle in the blowdown turbine facility. The flow field upstream of the nozzle is not the ideal flow field that is called for in the ANSI standard. The flow exits the turbine in an annulus and the recombines downstream of the starter motor to a 
cylindrical cross section. This will result in a significant blockage at the flow centerline. The flow conditioning upstream if the nozzle will reduce the non-uniformity before it enters the nozzle.

In order to determine the sensitivity of the nozzle to the upstream blockage, the nozzle was calibrated with and without a simulated upstream blockage in place. Figure 6.1 shows that the effect of the blockage is not significant, and Figure 6.5 shows the nozzle location relative to the simulated blockage.

\subsubsection{Critical Flow Coefficient}

When critical flow nozzles meter gasses, errors frequently arise when conventional onedimensional isentropic flow relations are used to compute the mass flow rate. The assumption usually made is that the gas is ideal. The ideal gas being defined as one that a constant specific heat capacity and a compressibility factor of unity. In many engineering applications these are valid assumptions. However, the test gas $\left(\mathrm{CO}_{2}\right)$ used in this experiment exhibits significant nonideal behavior in the range of pressures and temperatures under which the nozzle operates. The difference between $C_{* i}$ and $C_{R}$ under typical test conditions for this application is $1.0 \%$. If unaccounted for, this would be a significant source of error.

In order to estimate $C_{R}$, the pressure-density-temperature and the differential enthalpy and entropy relations must be examined. The pressure-density-temperature relation is given by,

$$
Z=\frac{P}{\rho R T}=Z(\rho, T)
$$

Alternatively $\mathrm{Z}$ can be expressed as a function of density and temperature. The expressions for the differential entropy and enthalpy are,

$$
\begin{gathered}
d S=C_{p} \frac{d T}{T}+\frac{1}{\rho^{2}}\left(\frac{\partial \rho}{\partial T}\right)_{P} d P \\
d H=T d S+\frac{1}{\rho} d P
\end{gathered}
$$

Since it is assumed that the flow is isentropic,

$$
d S \equiv 0
$$

and from the relation,

$$
d H=-V d V
$$

substitution of Equations 6.5, 6.8, and 6.9 into Equation 6.7 yields, 


$$
\begin{gathered}
\frac{d S}{R}=\frac{C_{P}}{R} \frac{d T}{T}-\left[Z+T\left(\frac{\partial Z}{\partial T}\right)_{P}\right] \frac{d P}{P}=0 \\
-\frac{V d V}{R}=\frac{C_{P}}{R} d T-T^{2}\left(\frac{\partial Z}{\partial T}\right)_{P} \frac{d P}{P}
\end{gathered}
$$

Equations 6.10 and 6.11 are integrated along the path indicated in figure 6.2.

The pressure end point of the path is $P_{1}$ satisfies the integral of Equation 6.10; that is $S_{1}=S_{0}$. At this point, Equation 6.11 can be integrated along the same path. The result of this integration permits the evaluation of the nozzle throat velocity $V_{l}$. The speed of sound in the nozzle throat can then be evaluated from the expression,

$$
\frac{1}{a^{2}}=\left(\frac{\partial \rho}{\partial P}\right)_{S}=\left(\frac{\partial \rho}{\partial P}\right)_{T}+\left(\frac{\partial \rho}{\partial T}\right)_{P}\left(\frac{\partial T}{\partial P}\right)_{S}
$$

where $\left(\frac{\partial \rho}{\partial P}\right)_{T}$ and $\left(\frac{\partial \rho}{\partial T}\right)_{P}$ are derived from the state equation 6.5, and $\left(\frac{\partial T}{\partial P}\right)_{S}$ is derived from the integrated form of Equation 6.11. The nozzle throat Mach number is then given by,

$$
M^{\prime}=\frac{V_{1}}{a_{1}}
$$

Since the Mach number in general will not be unity, a new throat temperature is estimated. The temperature correction to be added to previous temperature estimate is,

$$
\Delta T=\left[\left(\frac{\partial T}{\partial M}\right)_{S}\right]_{i} \Delta M
$$

where $\left[\left(\frac{\partial T}{\partial M}\right)_{S}\right]_{i}$ is estimated from the isentropic ideal-gas relation, and $\Delta M$ is the difference between the desired and calculated Mach number. This process is repeated until $\Delta M$ is less than $10^{-5}$. At this point the state and the velocity of the gas at the nozzle throat are considered known. The real gas critical flow coefficient is then defined as,

$$
C_{R}=\frac{P_{1}}{P_{0}} \frac{\sqrt{T_{0}}}{Z_{1} T_{1}} \sqrt{\frac{V_{1}^{2}}{R}}
$$

Two different methods were used to determine $C_{R}$. Firstly the above method was implemented using Johnson's algorithm [6.4]. The equation of state for $\mathrm{CO}_{2}$ was taken from 
Bender [6.5]. As a second check, NIST14, a code developed by the National Institute for Standards and Technology [6.6] was modified to calculate $C_{R}$. NIST14 generates the thermophysical properties of gas mixtures and accounts for the real gas interaction between the mixture components. Both of these methods gave the same result for $C_{R}$. The calculated values agreed with those reported in the ANSI standard to within $\pm 0.1 \%$. Although not recommended precise work, the standard gives sample values of $C_{R}$ for different gases which are intended as general information on the magnitude and variation of $C_{R}$.

\subsection{Nozzle Design and Installation}

\subsubsection{The ANSI Standard}

The critical flow venturi nozzle was designed to closely match the ANSI standard toroidal throat nozzle. However, because of the design constraints of modifying an existing facility, some of requirements of the standard could not be satisfied. For this reason it was decided to have the nozzle calibrated at an independent laboratory that specializes in calibrating critical flow venturi nozzles. This approach also offered the opportunity to reduce the estimated uncertainty for the nozzle discharge coefficient.

Two common nozzle designs are controlled by the standard; 1) the toroidal throat venturi nozzle and 2) the cylindrical throat nozzle. The main difference between the two is that the latter has a cylindrical section between the throat and the exit cone, whereas the toroidal throat nozzle transitions directly from the inlet contour to a divergent cone, as shown in Figure 6.6. Discharge coefficients for the toroidal throat nozzle design may be determined by theoretical calculation. The coefficients so obtained agree well with experimental data of Brain [6.3]. Because of the relative ease of calculation of the theoretical coefficient and its agreement with experimental data, some investigators favor this design over the cylindrical throat design, [6.2].

The following excerpts from the ANSI standard will paraphrase the design requirements for standard toroidal throat venturi nozzle and compare how the MIT blowdown turbine nozzle differs.

\subsection{General Requirements}

5.1.1 The venturi nozzle shall be inspected to determine conformance to this Standard.

5.1.2 The venturi nozzle shall be manufactured from a material suitable for its intended application. The following are some considerations.

(a) The material should be capable of being finished to the required condition. Some materials are unsuitable because of pits, voids, and other non-homogenates.

(b) The material, together with any surface treatment used, shall not be subjected to corrosion in the intended service.

(c) The material should be dimensionally stable and should have known and repeatable thermal expansion characteristics (if it is to be used at a temperature other than that at which the throat diameter has been measured), so that an appropriate throat diameter correction can be made.

5.1.3 The throat and toroidal inlet up to the conical divergent section of the venturi nozzle shall be smoothly finished so that the arithmetic average roughness height does not exceed $15 \cdot 10^{-6} \mathrm{~d}$. \{d is the throat diameter of the nozzle.

5.1.4 The throat and toroidal inlet up to the conical divergent section shall be free from dirt, films, or other contamination.

5.1.5 The form of the conical divergent portion of the venturi nozzle shall be controlled such that any steps, discontinuities, irregularities, and lack of concentricity shall not exceed 1pc of the local diameter. The arithmetic average roughness of the conical divergent section shall not exceed $10^{-4} \mathrm{~d}$. 


\subsection{Standard Venturi Nozzles}

\subsubsection{Toroidal Throat Venturi Nozzle}

5.2.1.1 The venturi nozzle shall conform to figure ( see [6.2] ).

5.2.1.2 For the purposes of locating other elements of the venturi nozzle critical flow metering system, the inlet plane of the venturi nozzle shall be defined as that plane perpendicular to the axis of symmetry which intersects the inlet at a diameter equal to $2.5 \mathrm{~d} \pm 0.1 \mathrm{~d}$.

5.2.1.3 The convergent part of the venturi nozzle (inlet) shall be a portion of the torus that shall extend through the minimum area section (throat) and shall be tangent to the divergent section. The contour of the inlet upstream of a diameter equal to $2.5 \mathrm{~d}$ is not specified, except that the surface at each axial location shall have a diameter equal to or greater than the extension of the toroidal contour.

5.2.1.4 The inlet toroidal surface of the venturi nozzle beginning at a diameter of $2.5 \mathrm{~d}$ perpendicular to the axis of symmetry and extending to the point of tangency shall not deviate form the shape of a torus by more than $\pm 0.001 \mathrm{~d}$. The radius of curvature of the toroidal surface in the plane of symmetry shall be $1.8 \mathrm{~d}$ to $2.2 \mathrm{~d}$.

5.2.1.5 The divergent portion of the venturi nozzle shall form a frustrum of a cone with a halfangle of $2.5^{\circ}$ to $6^{\circ}$. The length of the conical section shall not be less than the throat diameter.

\section{Installation Requirements}

\subsection{General}

This standard covers installation when either:

(a) the pipeline upstream of the nozzle is of circular cross section; or

(b) it can be assumed that there is a large space upstream of the venturi nozzle.

For case (a), the primary device shall be installed in a system meeting the requirements of para.

6.2. For case (b), the primary device shall be installed in a system meeting the requirements of para. 6.3. In both cases swirl must not exist upstream of the venturi nozzle. Where a pipeline exists upstream of the nozzle, swirl-free conditions can be ensured by installing a flow straightener of the design in figure ( see [6.2] )at a distance greater than 5D upstream of the nozzle inlet plane. $\{\mathrm{D}$ is the upstream duct diameter. $\}$

\subsection{Upstream Pipeline}

The primary device may be installed in a straight circular conduit, which shall be concentric within $0.02 \mathrm{D}$ with the centerline of the venturi nozzle. The inlet conduit up to $3 \mathrm{D}$ upstream of the venturi nozzle shall not deviate form circularity by more than $0.01 \mathrm{D}$ and shall have an arithmetic average roughness height which shall not exceed $10^{-4} \mathrm{D}$. In order to meet the coefficient specifications of this Standard the diameter of the inlet conduit shall be a minimum of $4 \mathrm{~d}$. It should be noted that the use of beta ratios $\{\mathrm{d} / \mathrm{D}\}$, larger than 0.25 increases the effect of upstream disturbances, and moreover, makes corrections necessary to the measured pressure and temperature.

\subsubsection{MIT Venturi Nozzle}

The nozzle was manufactured from Stainless Steel 304 and designed to meet the general requirements 5.1.1 to 5.1.5. The inlet contour does not meet requirements 5.2.1.3 and 5.2.1.4 of the standard. For ease of manufacture the inlet contour transitions from a toroidal to a conical section at approximately $1 \mathrm{~d}$ from the inlet plane. The divergent portion meets requirement

\subsubsection{5.}

\subsubsection{MIT Nozzle Installation}

The nozzle installation is shown in Figure 6.5. The upstream pipeline meets the requirements in 6.2 except; (a) the duct extends $2.5 \mathrm{D}$ upstream of the nozzle not $3 \mathrm{D}$, (b) a beta ratio of 0.385 was used rather than the 0.25 recommended in the standard. 


\subsection{Transient Correction}

\subsubsection{Introduction}

Critical flow venturi nozzles are intended for steady flow applications. For this application the flow is steady with respect to the time scales of the flow through the nozzle, as it is for the turbine stage itself. However, as this is a transient test, the conditions upstream of the nozzle will change somewhat over the duration of the test. As the temperatures and pressures change in the volumes connecting the turbine and the venturi nozzle, this will introduce a capacitive effect where mass is stored. The mass flow rate through the nozzle may not match that through the turbine at a given time. An estimate must be made of this effect, as if it is unaccounted for it could constitute a significant error source. The relationship between the turbine and nozzle mass flows can be described by equation,

$$
\dot{m}_{\text {turbine }}=\dot{m}_{\text {nozzle }}+\dot{m}_{\text {stroed }}
$$

The mass flow due to storage can be estimated by manipulating the state equation as follows,

$$
\begin{aligned}
& P V=m R Z T \\
& m=\left(\frac{P}{T}\right) \frac{V}{R Z}
\end{aligned}
$$

and then taking its derivative with respect to time,

$$
\dot{m}=\frac{V}{R Z}\left(\frac{1}{T}\right) \frac{d P}{d t}-\frac{V}{R Z}\left(\frac{P}{T^{2}}\right) \frac{d T}{d t}
$$

Section 6.5.4 will outline how the pressure and temperature dependent terms in Equation 6.19 are either measured or approximated.

\subsubsection{Compressional Heating}

Compressional heating is an interesting phenomenon observed in transient testing that is not seen in steady state test rigs. When a throttling process (flow through a valve) is followed by an isentropic compression (chamber filling from vacuum), the temperature of the gas in the filled compartment can exceed the initial temperature of the gas. This is seen in the MIT Blowdown Turbine facility during the start up transients of the test. In the past this phenomenon was a merely a novelty that was interesting to note on the fast response thermocouples.

Compressional heating is significantly more important to the current task of estimating the transient correction to the mass flow measurement. The gas that is heated is convected through the primary flow path in 0-100 ms and does not affect the transient correction for the primary flow path. However, there also exists a secondary flow path in parallel with the primary, as shown in Figure 6.7. The time scale for the flow through the secondary flow paths is significantly longer. The temperatures in the secondary compartments will be higher than the 
primary flow path, because of compressional heating. The compartment temperatures are required to estimate $\dot{m}_{\text {stored }}$, as shown in Equation 6.16 .

The increase in temperature due to compressional heating for a simple compartment filled through an orifice, from a constant temperature reservoir, can be estimated as follows. For isentropic compression a compartment, pressure in related to its volume by,

$$
V P^{\frac{1}{\gamma}}=k
$$

or,

$$
V=P^{-\frac{1}{\gamma}} k
$$

The fractional change in volume with pressure is,

$$
d V=-\frac{1}{\gamma} P^{-\left(\frac{1+\gamma}{\gamma}\right)} k d P
$$

The fractional change in mass can then be estimated using the state equation,

$$
d m=\rho d V=\frac{P}{R T_{0}}\left(-\frac{1}{\gamma} P^{-\frac{1+\gamma}{\gamma}}\right) P^{\frac{1}{\gamma}} d P=-\frac{1}{\gamma} \frac{V}{R T_{0}} d P
$$

The total mass in the compartment can be found by integrating $d m$ over the pressure range from vacuum to the supply pressure,

$$
m=\int_{0}^{P_{0}}-\frac{1}{\gamma} \frac{V}{R T_{0}} d P=-\frac{1}{\gamma} \frac{V P_{0}}{R T_{0}}
$$

The final compartment temperature can calculated from the mass, the final pressure, and the state equation,

$$
T=\frac{P_{0} V}{R m}=\frac{P_{0} V}{R\left(-\frac{1}{\gamma}\left(\frac{P_{0} V}{R T_{0}}\right)\right)}=\gamma T_{0}
$$

This is an important result as it shows that the final compartment temperature is only a function of the supply temperature and $\gamma$. Compressional heating is an important concept and will be referred to in the following sections. 


\subsubsection{The Model}

A fairly detailed mathematical model of the blowdown dynamics of the facility was constructed for the conceptual design of this experiment and also to determine if the correction in Equation 6.16 could be made, and with what level of confidence.

Adding another choke point in the flow path significantly affects the dynamics of the system. It was difficult to design a nozzle that was feasible to manufacture, and would remain choked for the duration of the test, and while still maintaining choked flow at the throttle plate downstream of the turbine. It is necessary to maintain choked flow across the throttle plate in order to keep the pressure ratio across the turbine stage constant. Also, it is necessary to maintain choked flow across the venturi nozzle, otherwise it wouldn't be a critical flow venturi.

In order to extend the useful test time a extended diffuser is added downstream of the nozzle. The area ratio of the diffuser is $1: 2$, this allowed a maximum permissible back pressure ratio of 0.87 . For the nozzle throat to exit area ratio and $\gamma$, this is the maximum pressure ratio that the standard [6.2] recommends without verifying that the nozzle remains choked.

The model inputs include; compartment connections, connection areas, and compartment volumes. The model assumes one-dimensional adiabatic flow between the volumes, where a simple throttling process was assumed between each compartment. At each iteration the compartment pressures determine the mass flow magnitude and direction. The compartment pressures are then updated based on changes in the internal energy of each compartment. A simple backward Euler iteration scheme was used.

Tables 6.2 and 6.3 contain sample input data for the model. Figure 6.7 shows a schematic of the model of the blowdown turbine facility. The transient mass flow correction is driven primarily by volumes 3 and 4 . These represent the dump tank extension duct and the nozzle upstream plenum. The size of volume 3 is driven by the need to relocate the eddy current brake and the starter motor out of the dump tank. The size of volume 4 is set by the need to provide an adequate length of ducting upstream of the nozzle. Volumes 3 and 4 are the dominant source of the correction and their magnitude can be estimated with a high degree of confidence. Volumes 2, 6, and 7 can be easily estimated. (Volumes 2 and 6, were also calculated with the aid of a 3D solid modeling program). Volume 8 houses the eddy current brake and is more difficult to accurately measure.

\begin{tabular}{|l|c|c|c|c|c|c|c|c|c|}
\hline \multicolumn{10}{|c|}{ Table 6.2: Compartment Volumes, $\mathbf{f t}^{\mathbf{3}}$} \\
\hline Compartment & $\mathrm{V}_{0}$ & $\mathrm{~V}_{1}$ & $\mathrm{~V}_{2}$ & $\mathrm{~V}_{3}$ & $\mathrm{~V}_{4}$ & $\mathrm{~V}_{5}$ & $\mathrm{~V}_{6}$ & $\mathrm{~V}_{7}$ & $\mathrm{~V}_{8}$ \\
\hline Volume & 364 & 5.0 & 1.45 & 23.43 & 21.45 & 523 & 0.485 & 0.619 & 1.320 \\
\hline Volume Bias Limit, \% & - & - & $2 \%$ & $1 \%$ & $1 \%-$ & & $5 \%$ & $1 \%$ & $20 \%$ \\
\hline
\end{tabular}

Table 6.3: Compartment Interconnection Areas, in ${ }^{2}$

\begin{tabular}{|l|c|c|c|c|c|c|c|c|c|c|}
\hline Compartment & A01 & A12 & A23 & A34 & A45 & A15 & A26 & A67 & A78 & A83 \\
\hline Area & - & 22.0 & 42.0 & 150 & 78 & 10.62 & 1.2 & 1.1 & 2.52 & 2.51 \\
\hline
\end{tabular}




\subsubsection{Mass Flow Correction}

In estimating the mass flow due to storage Volumes 6,7 , and 8 will be considered separately from Volumes 2, 3, and 4. Volumes 2, 3, and 4 constitute the main flow path between the turbine and the nozzle. Volumes 6,7 , and 8 are secondary compartments that cannot be feasibly be sealed and must also be considered.

The compartment pressures and temperatures for the primary flow path are measured directly. Compartments 2 and 4 are already instrumented to determine the turbine exit conditions and the conditions upstream of the critical flow venturi respectively. Total pressure and temperature probes were also installed in compartment 3.

For the secondary compartments the pressures and temperatures are a little more difficult to determine. The temperatures are very difficult to measure, both because of their location and because significant temperature gradients may exist within the compartments. The model is used to determine if any simplifying assumptions can be made about the conditions in these compartments.

Figures 6.8 and 6.9 show the predicted pressure and temperature histories during the experiment. In the secondary compartments, the temperature initially rise because of compressional heating as discussed in Section 6.5.2, and then falls as the hot gas is convected downstream. The pressures in the secondary flow path are primarily a function of the secondary mass flow and areas connecting the compartments. Both the pressures and temperatures in the secondary compartments are strongly dependent on areas connecting the compartments. The discharge coefficients for these areas are not known, and their effect can only be estimated by considering reasonable values to bound their influence.

In order to simplify the task of making this correction another approach was considered. Area $A_{67}$ is used to provide an access for instrumentation, and can be sealed without too much effort. This modification eliminates the secondary flow path that bypasses the main flow. Instead, the secondary compartments empty into the main flow path compartments from which they are filled.

Figures 6.10 and 6.11 show the predicted pressure and temperature histories with this modification. For the correction, $\mathrm{V}_{7}$ and $\mathrm{V}_{8}$ are simply lumped in with $\mathrm{V}_{3}$, and $\mathrm{V}_{6}$ is added to $\mathrm{V}_{2}$. From Figures 6.10 and 6.11 this is clearly reasonable. Figure 6.12 shows the mass flow through the turbine and nozzle. Figure 6.13 shows the error in mass flow, or the magnitude of the correction required to estimate $\dot{m}_{\text {turbine }}$ from $\dot{m}_{\text {stored }}$ and $\dot{m}_{\text {nozzle }}$. Figure 6.13 also shows the error in mass flow using this same simplification, without the modification to the facility.

The window in the test where the correction to the mass flow can be made is approximately be made is between 350 to $800 \mathrm{~ms}$. Below $350 \mathrm{~ms}$ the correction is too large, and above $800 \mathrm{~ms}$ the nozzle may not be choked. At $500 \mathrm{~ms} \dot{m}_{\text {stored }}$ is zero, the turbine mass flow equals the nozzle mass flow. As can be seen from Figure 6.10, the slope of the pressure curve is approximately zero.

In summary, a simple one-dimensional model of the blowdown turbine facility has been used to show that the difference between $\dot{m}_{\text {turbine }}$ and $\dot{m}_{\text {nozzle }}$ over the range of interest is on the order of $\pm 4 \%$. The magnitude of the correction can be estimated from pressure and temperature measurements between the nozzle and turbine. A minor change to the facility will reduce the uncertainty of this correction. 


\subsection{Mass Flow Measurement Error Analysis}

The precision index for the mass flow measurement can be estimated from,

$$
S_{\dot{m}}=\sqrt{\left(\frac{\partial \dot{m}}{\partial C_{d}} S_{C_{d}}\right)^{2}+\left(\frac{\partial \dot{m}}{\partial C_{R}} S_{C_{R}}\right)^{2}+\left(\frac{\partial \dot{m}}{\partial P_{0}} S_{P_{0}}\right)^{2}+\left(\frac{\partial \dot{m}}{\partial T_{0}} S_{T_{0}}\right)^{2}}
$$

If we define the influence coefficient for each variable in Equation 6.26 as,

$$
C_{*}=\frac{\partial \dot{m}}{\partial(*)} \frac{(*)}{\dot{m}}
$$

then Equation 6.26 can be rewritten as,

$$
\frac{S_{\dot{m}}}{\dot{m}}=\sqrt{\left(C_{C_{d}} \frac{S_{C_{d}}}{C_{d}}\right)^{2}+\left(C_{C_{R}} \frac{S_{C_{R}}}{C_{R}}\right)^{2}+\left(C_{P_{o}} \frac{S_{P_{0}}}{P_{0}}\right)^{2}+\left(C_{T_{o}} \frac{S_{T_{0}}}{T_{0}}\right)^{2}}
$$

In this form, the influence coefficient is a non-dimensional parameter that represents how a given error source will be propagate to overall measurement error. The influence coefficient represents the relative amplification of that source error through equation 6.3. The sign of the influence coefficient is not important as the term is squared. The influence coefficients $C_{C_{d}}, C_{P_{0}}$, and $C_{T_{0}}$ are straightforward,

$$
C_{C_{R}}=1, \quad C_{C_{d}}=1, \quad C_{P_{0}}=1, \quad C_{T_{0}}=-\frac{1}{2}
$$

However, the expression $\frac{S_{C_{R}}}{C_{R}}$ is more complicated. The precision index for $C_{R}$ can be estimated from,

$$
\begin{gathered}
C_{R}=f\left(C_{p}\right) \\
\left(\frac{S_{C_{R}}}{C_{R}}\right)=\frac{\partial C_{R}}{\partial C_{P}} \frac{C_{P}}{C_{R}} \frac{S_{C_{P}}}{C_{P}}=\frac{\partial C_{R}}{\partial C_{P}} \frac{S_{C_{P}}}{C_{R}}
\end{gathered}
$$

where $\frac{\partial C_{R}}{\partial C_{P}}$ can either be estimated numerically, or by approximating $C_{R}$ as $C_{* i}$ and finding its derivative with respect to $\gamma\left(C_{P}\right)$. Both of these approaches yielded the same result for $S_{C_{R}}$. A 
summary of the influence coefficients for Equation 6.28 is given in Table 6.4 which shows the values for the influence coefficients, precision indices, bias limits and $95 \%$ uncertainty estimates.

\begin{tabular}{|l|c|c|c|c|}
\hline \multicolumn{6}{|l|}{ Table 6.4: Pretest Mass Flow Uncertainties } \\
\hline Quantity & $C_{*}$ & $\mathrm{~B}$ & $\mathrm{~S}$ & $\mathrm{U}_{95}$ \\
\hline$T_{0}$ & 0.5 & $0.1 \%$ & $0.05 \%$ & $0.14 \%$ \\
\hline$P_{0}$ & 1.0 & $0.15 \%$ & $0.07 \%$ & $0.18 \%$ \\
\hline$C d$ & 1.0 & $0.35 \%$ & $0.0 \%$ & $0.35 \%$ \\
\hline$C p$ & 0.1 & $0.2 \%$ & - & $0.02 \%$ \\
\hline$\dot{m}$ & - & $0.385 \%$ & $0.074 \%$ & $0.41 \%$ \\
\hline
\end{tabular}

Similarly the bias limits can be defined as,

$$
B_{\dot{m}}=\sqrt{\left(\frac{\partial \dot{m}}{\partial C_{d}} B_{C_{d}}\right)^{2}+\left(\frac{\partial \dot{m}}{\partial C_{R}} B_{C_{R}}\right)^{2}+\left(\frac{\partial \dot{m}}{\partial P_{0}} B_{P_{0}}\right)^{2}+\left(\frac{\partial \dot{m}}{\partial T_{0}} B_{T_{0}}\right)^{2}}
$$

In terms of the influence coefficients,

$$
\frac{B_{\dot{m}}}{\dot{m}}=\sqrt{\left(C_{C_{d}} \frac{B_{C_{d}}}{C_{d}}\right)^{2}+\left(C_{C_{R}} \frac{B_{C_{R}}}{C_{R}}\right)^{2}+\left(C_{P_{o}} \frac{B_{P_{0}}}{P_{0}}\right)^{2}+\left(C_{T_{o}} \frac{B_{T_{0}}}{T_{0}}\right)^{2}}
$$

The uncertainty due to the transient correction will be small, as the maximum value of the correction is approximately $4 \%$ over the area of interest. The bias in estimating the compartment volumes is the main source of error. Fortunately, the largest volumes are the easiest to estimate. The bias for the correction will be approximately $1.0 \%$. This is not significant source of uncertainty compared to the other discharge coefficient of the nozzle.

\subsection{Summary}

A critical flow venturi has been installed in MIT blowdown turbine facility in order to measure the turbine mass flow. An independent laboratory calibrated the discharge coefficient of the nozzle with stated uncertainty of $\pm 0.35 \%$. Two independent approaches were used to estimate the influence of real gas effects on the predicted nozzle mass flow rate, both of which produced the same result. An analytical model was used to determine the magnitude of the transient correction that relates the turbine mass flow to the nozzle flow rate. This was shown to be a small correction. An uncertainty analysis of the mass flow measurement estimates the measurement uncertainty to be $\pm 0.41 \%$. 


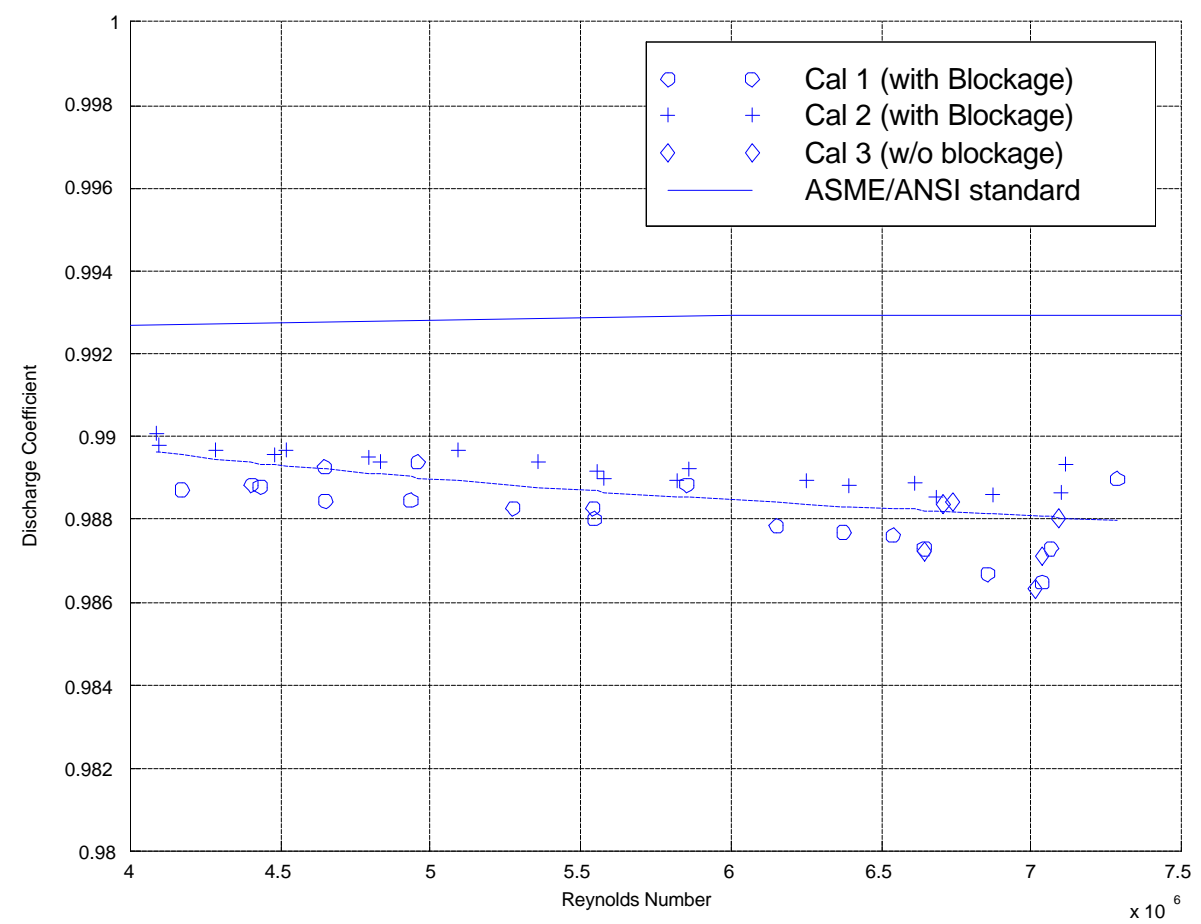

Figure 6.1: Critical Flow Venturi Calibration Results

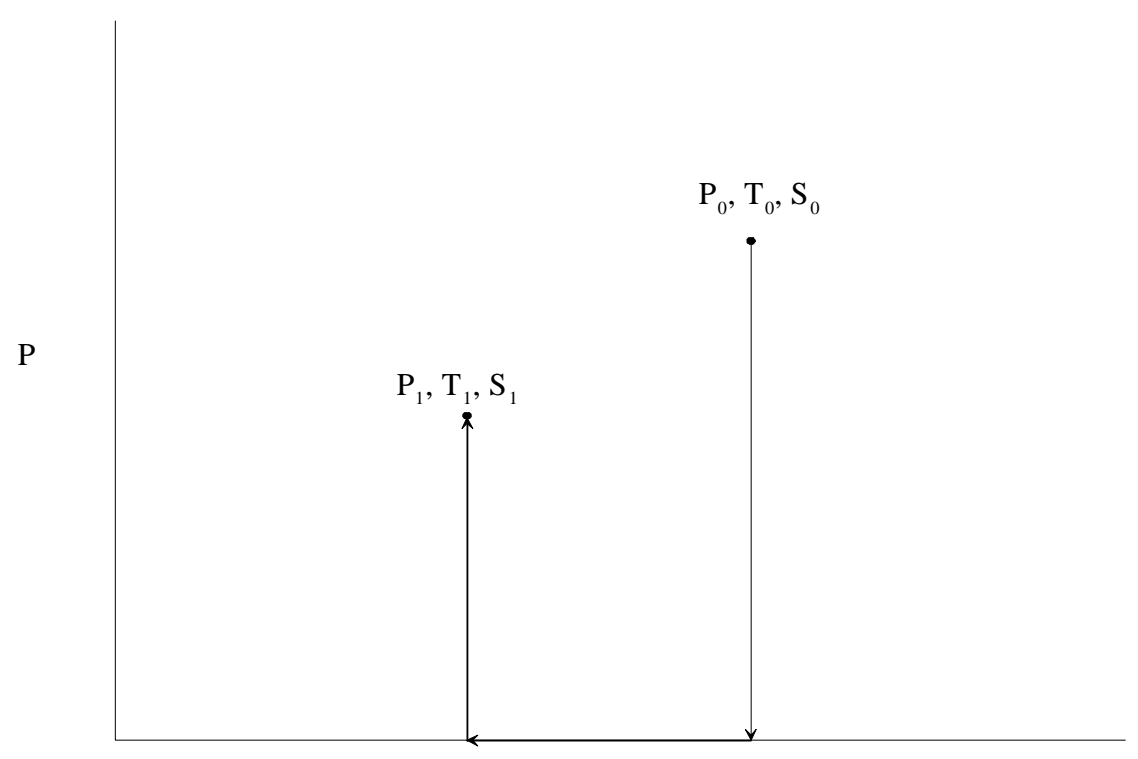

$\mathrm{T}$

Figure 6.2: Integration Path for Entropy and Enthalpy Equations 


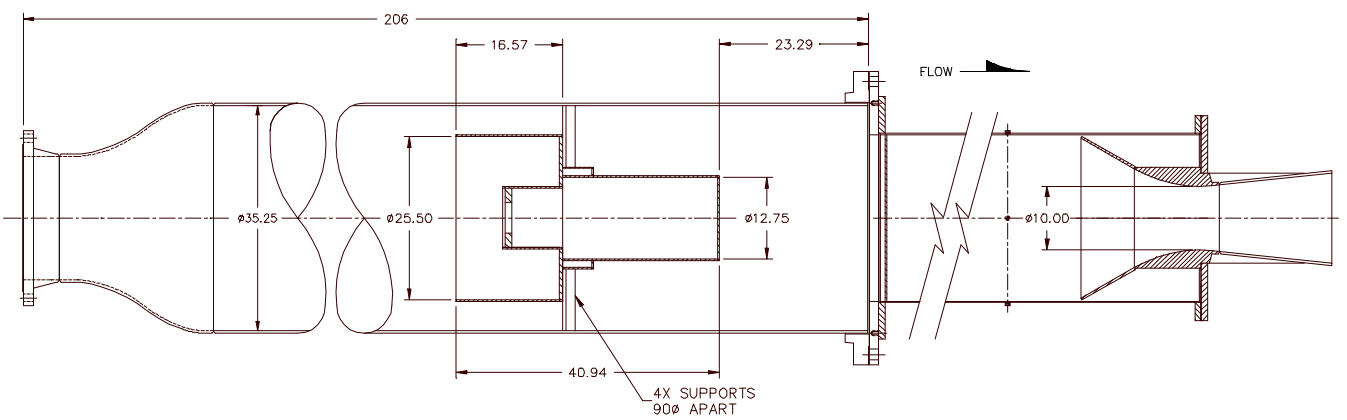

Figure 6.3: Venturi Calibration with Simulated Blockage

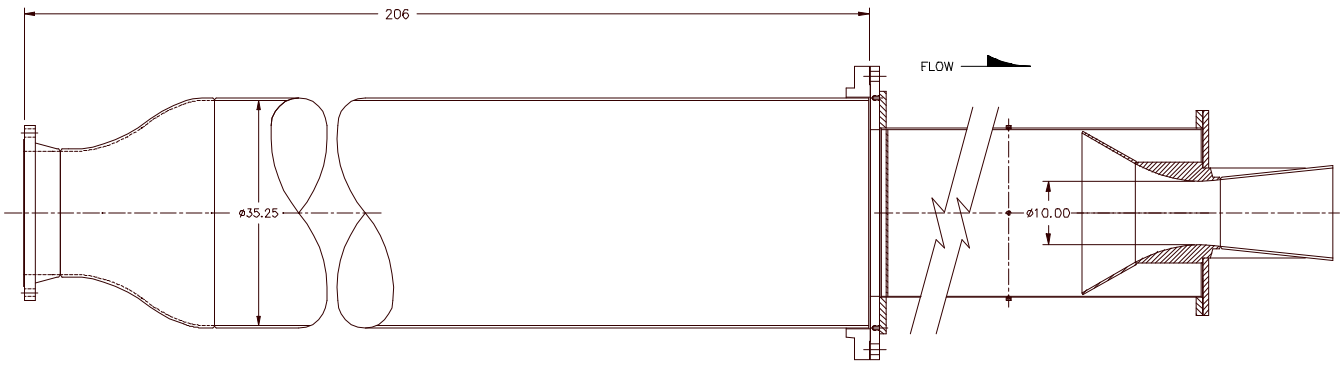

Figure 6.4: Venturi Calibration without Simulated Blockage

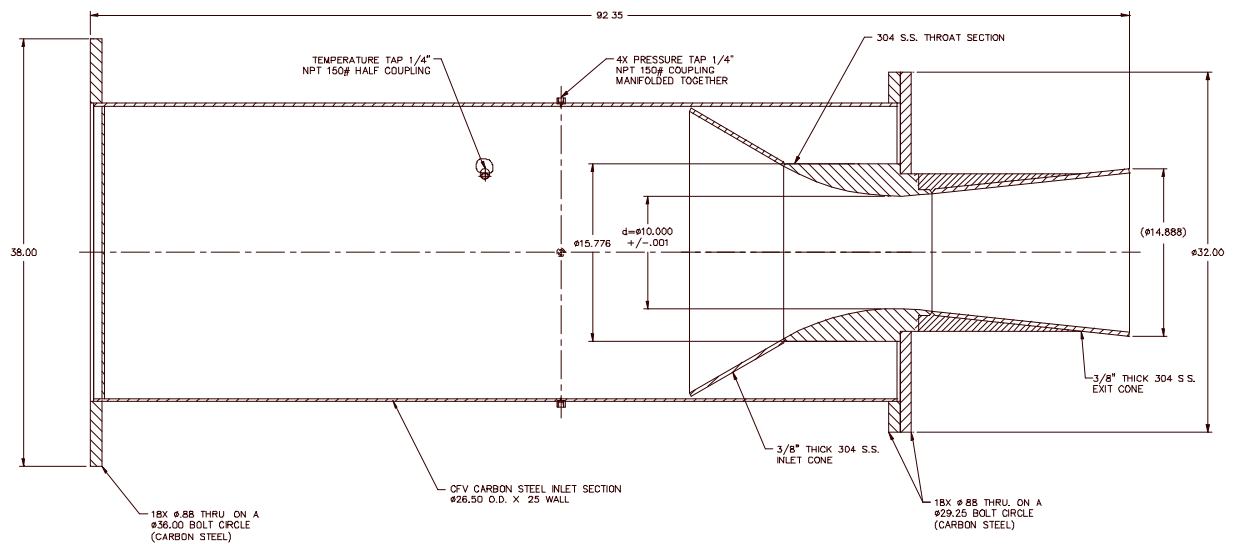

Figure 6.5: Critical Venturi Assembly 


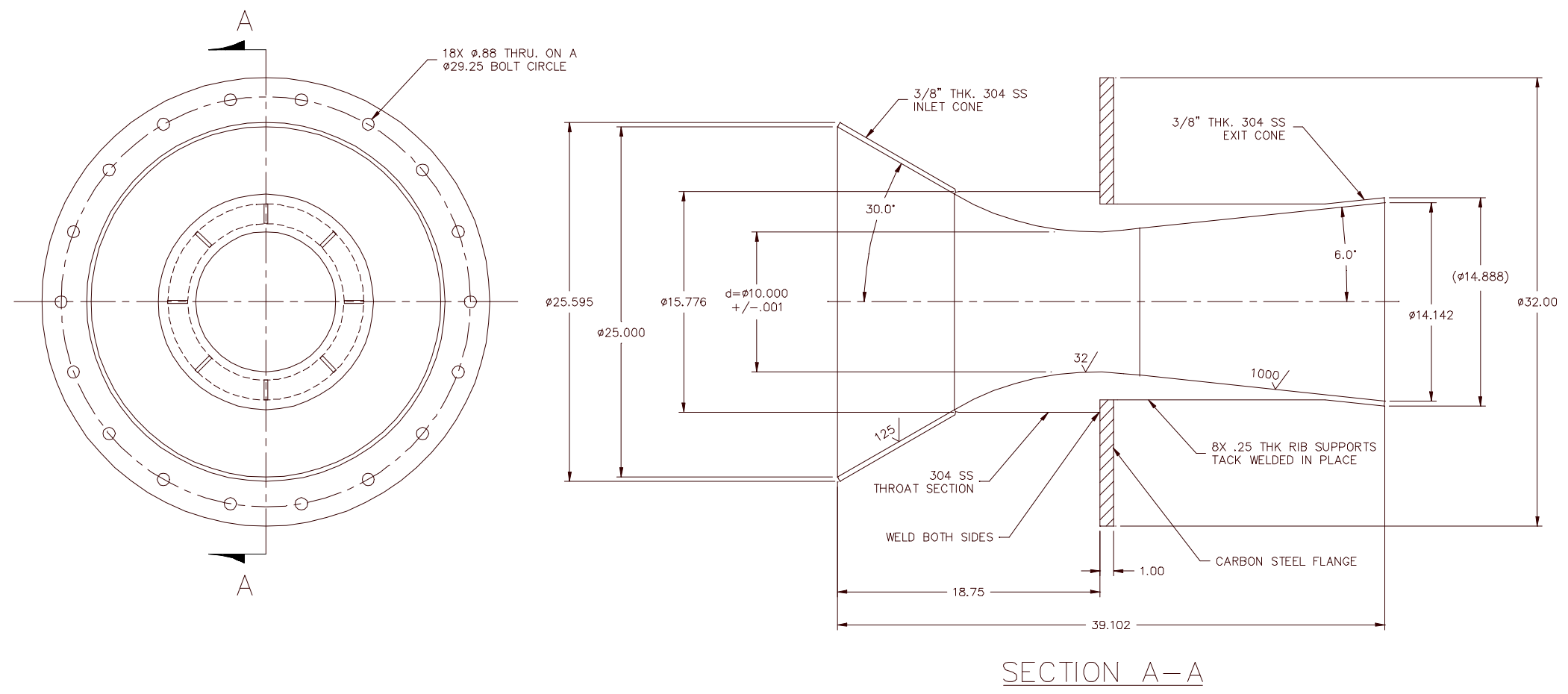

Figure 6.6: Nozzle Detail 


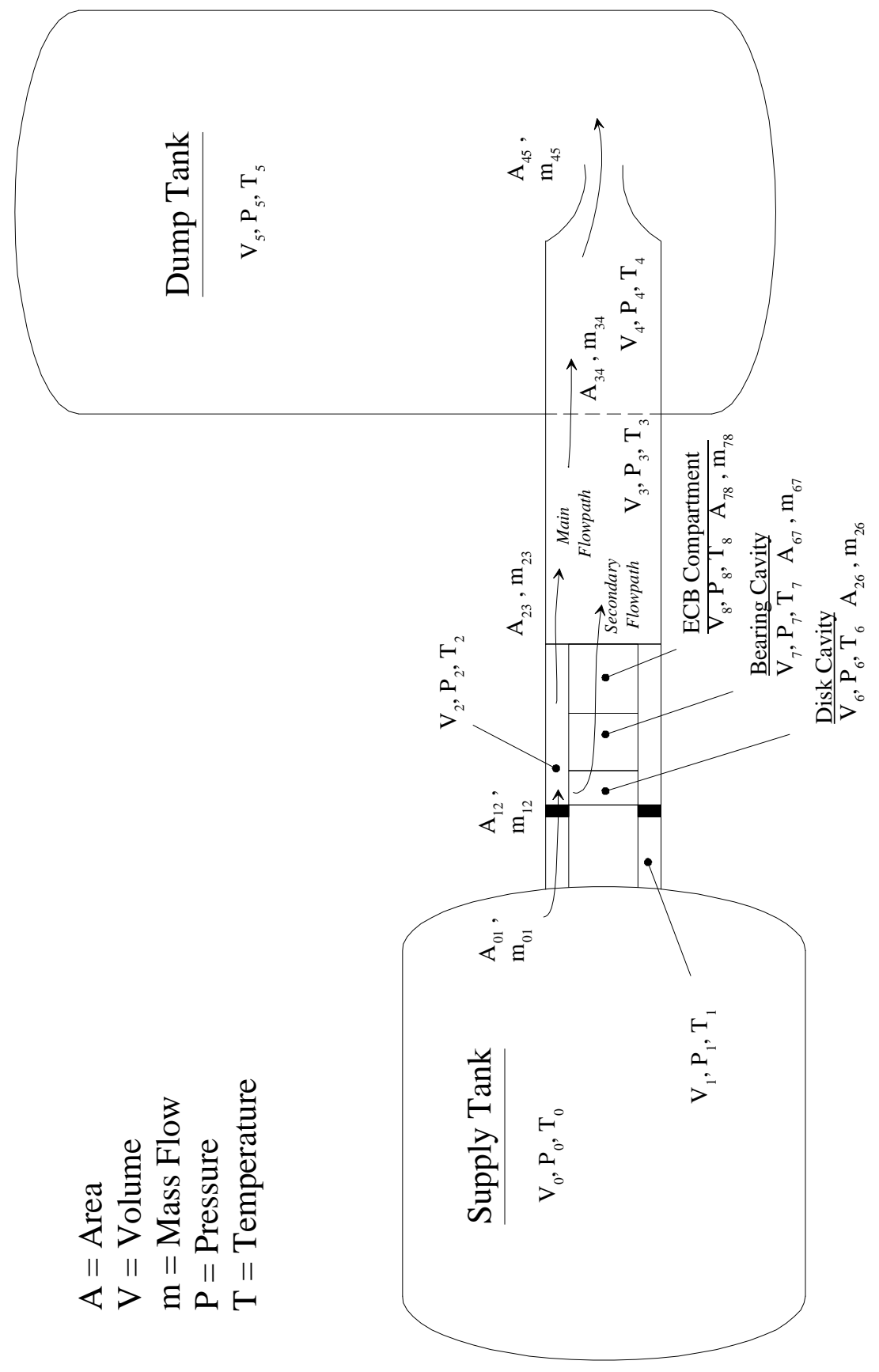

Figure 6.7: Blowdown Model Shematic 


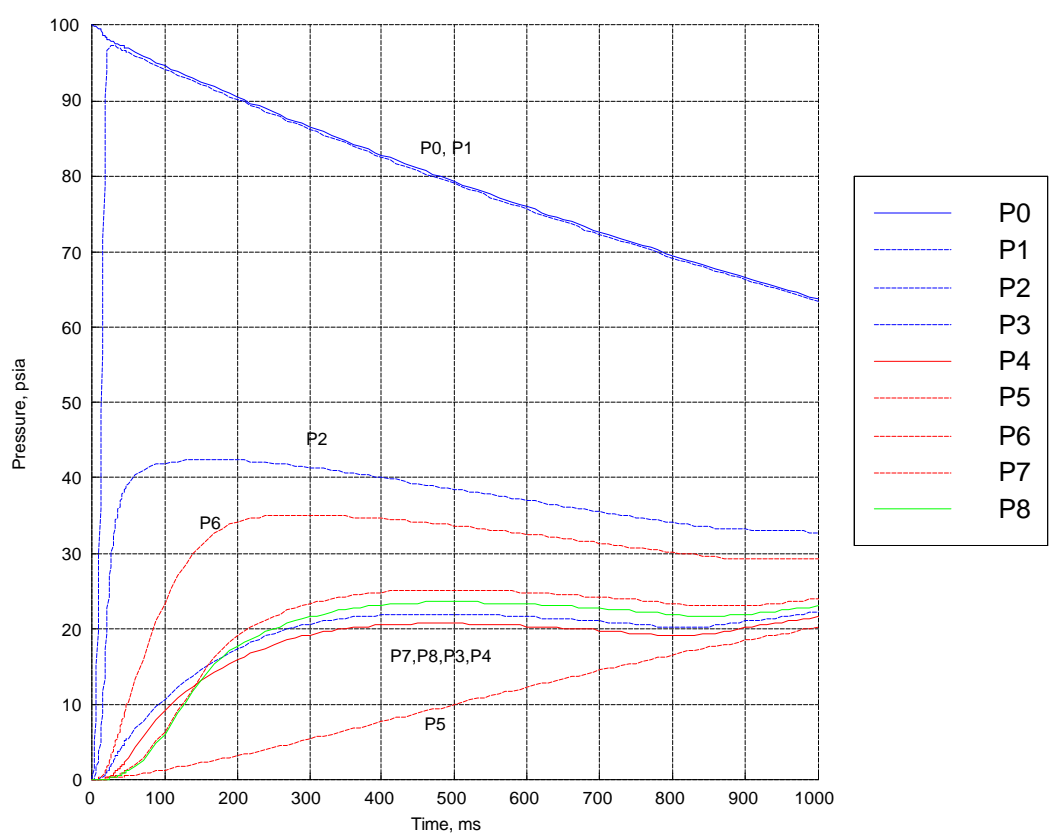

Figure 6.8: Chamber Pressures (Case 1)

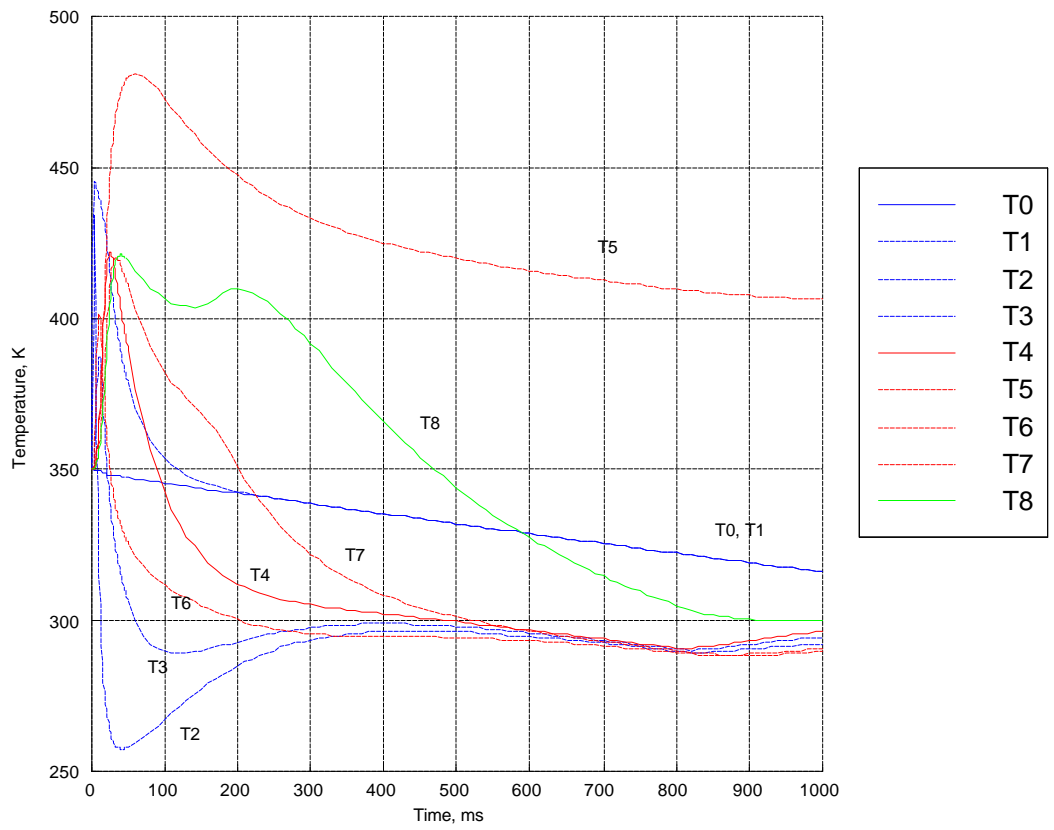

Figure 6.9: Chamber Temperatures (Case 1) 


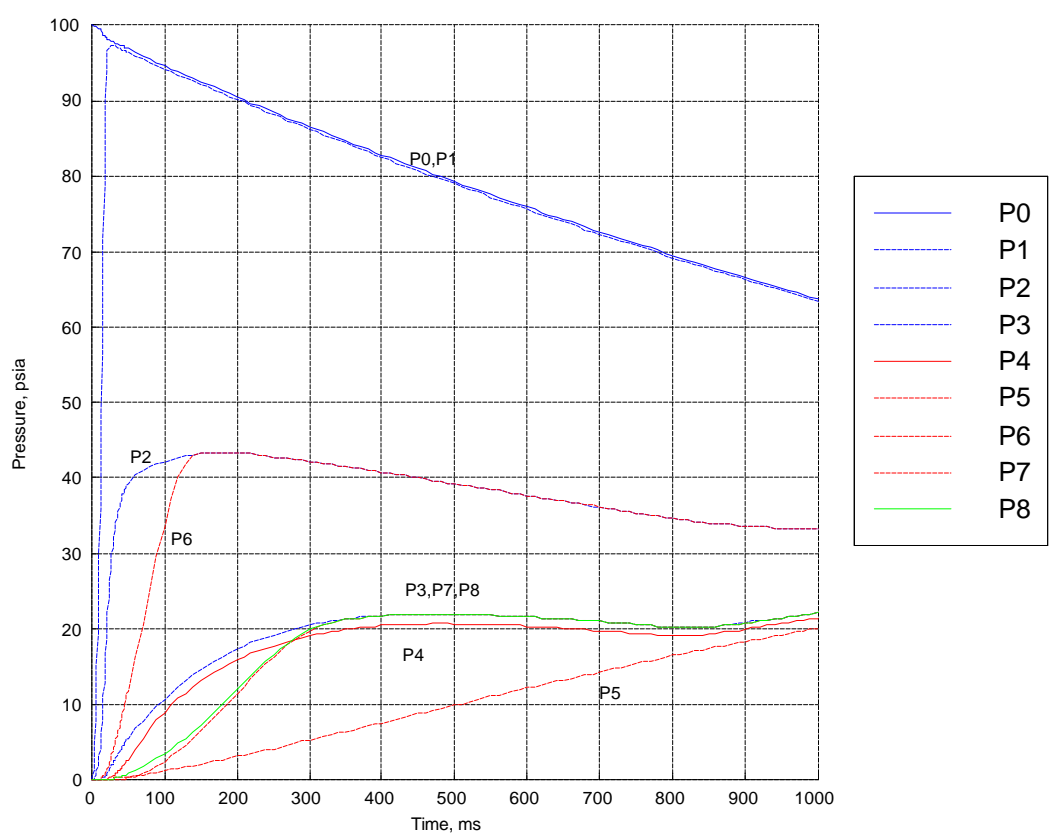

Figure 6.10: Chamber Pressures (Case 2)

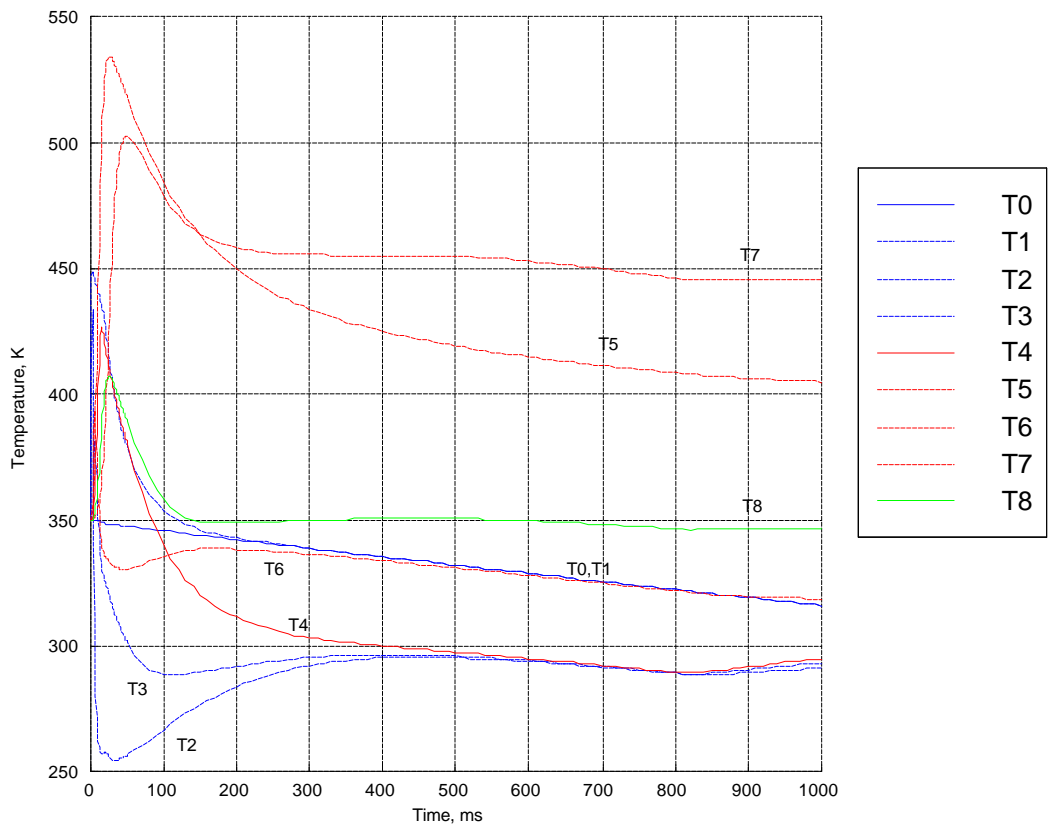

Figure 6.11: Chamber Temperatures (Case 2) 


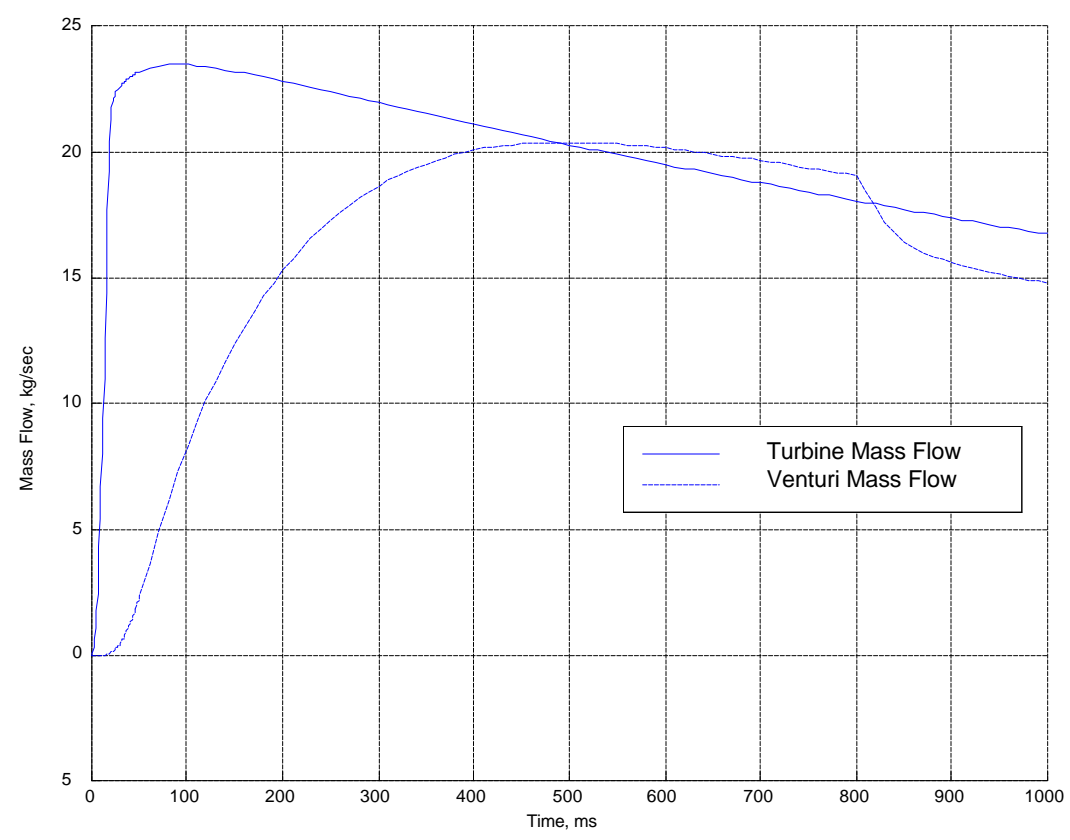

Figure 6.12: Turbine Mass Flow and Venturi Mass Flow

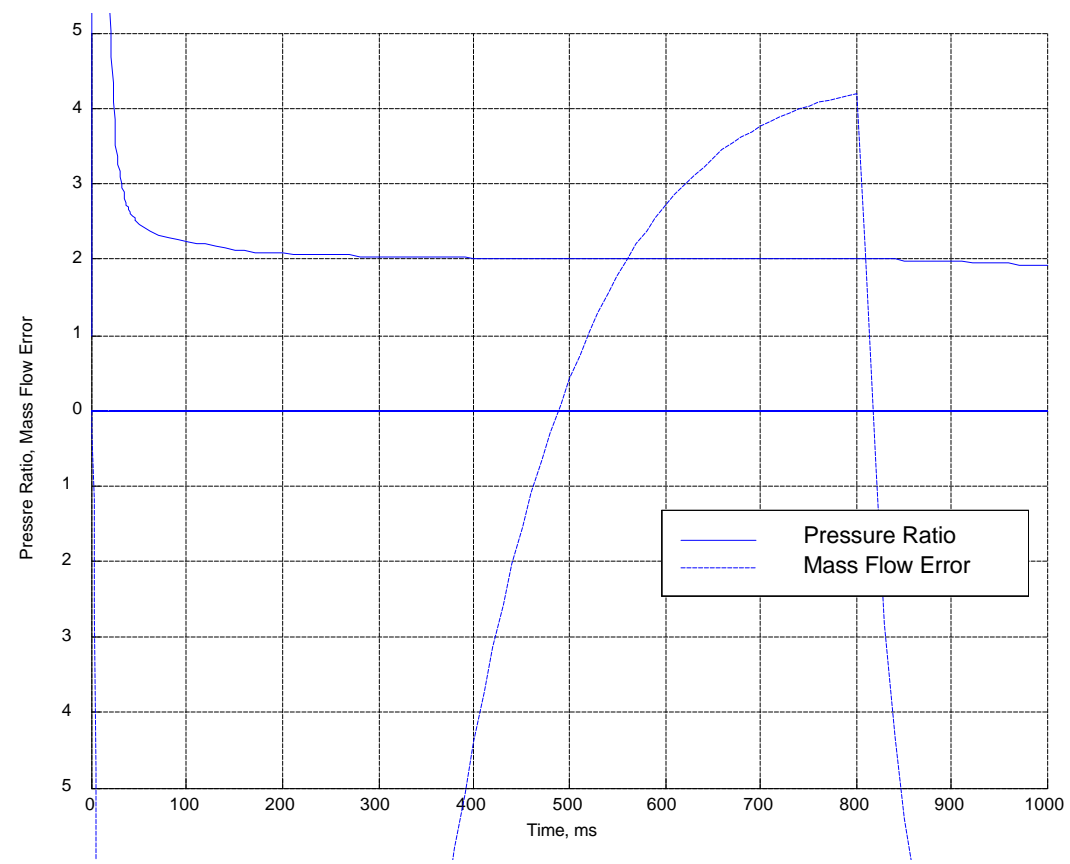

Figure 6.13: Pressure Ratio and $\dot{m}_{\text {stored }}$ 


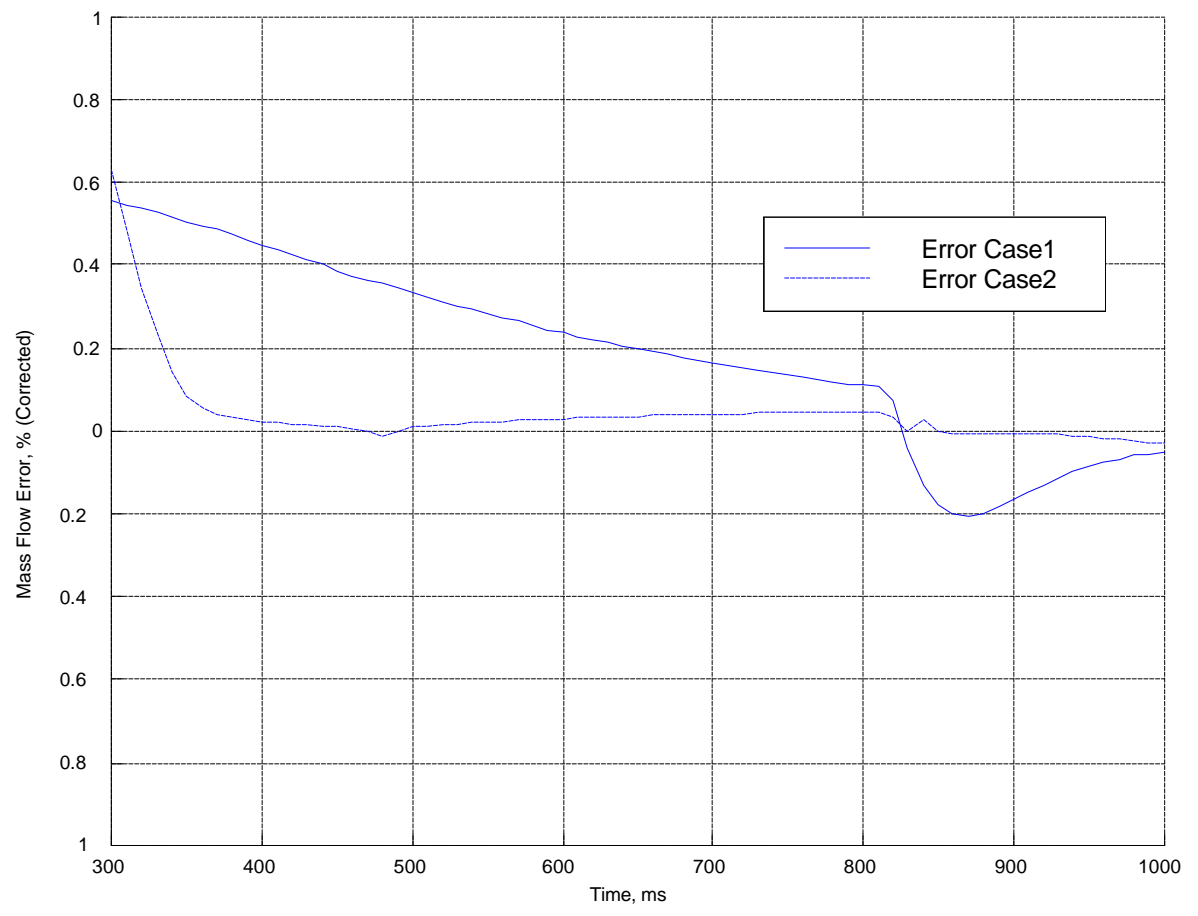

Figure 6.14: Mass Flow Error Case 1 vs Case 2 


\subsection{References}

[6.1] Doebelin, Ernest O., "Measurement Systems, Applications and Design," McGraw-Hill, Inc., fourth edition, 1990.

[6.2] ASME/ANSI MFC-7M-1987. "Measurement of Gas Flow by Means of Critical Flow Venturi Nozzles." An American National Standard, The Americal Society of Mechanical Engineers, 1987

[6.3] Brain, T.J.S., and J. Reid, "Primary Calibration of Critical Flow Venturis in HighPressure Gas," Flow Measurement of Fluids, H.H. Dijstelbergen and E.A. Spencer, eds., North Holland Publishing Co., Amsterdam, 1978: 54-64.

[6.4] Johnson R.C. "Calculation of Real Gas Effects in Flow Through Critica-Flow Nozzles," Journal of Basic Engineering, 86(3) 1964 519-526.

[6.5] Bender, E. "Equations of State Exactly Representing the Phase Behaviour of Pure Substances," Ruhr-University, Bachum, Germany.

[6.6] NIST 14. “NIST Mixture Property Data Base," Standard Reference Database 14, Version 9.08, (1992) Fluid Mixtures Data Center, NIST Thermophysics Division 838.02, Bolder, Colorado. 


\title{
7 Aerodynamic Performance Measurements
}

\author{
By Rory Keogh
}

\subsection{Introduction}

This chapter describes the measurement of turbine aerodynamic performance in the Blowdown Turbine Facility. The adiabatic turbine efficiency is defined and it is shown why it cannot be measured directly in a short duration facility. A small non-adiabatic correction must be applied to the measured efficiency. This non-adiabatic correction is discussed in detail. The uncertainty analysis for the efficiency measurement and the test results are presented.

\subsection{Adiabatic Efficiency}

The turbine being tested is operating in a fully scaled environment. Neither the full-scale nor the MIT turbines operate under adiabatic conditions. However, adiabatic efficiency is generally used as a benchmark for turbine performance as it distinguishes the influence of heat transfer from the other losses. The turbine tested can be compared with data taken from conventional adiabatic testing facilities. More importantly, the data will primarily be used to validate computational fluid dynamics codes, for which the adiabatic efficiency is more useful.

Throughout Section 7, the subscripts 1 and 2 will represent the turbine inlet and outlet stagnation conditions respectively (only stagnation conditions will be referred to here). Thus, $h_{1}$ and $h_{2}$ are the inlet and outlet stagnation enthalpies, $T_{1}$ and $T_{2}$ the stagnation temperatures, and $P_{1}$ and $P_{2}$ the stagnation pressures. This follows the notation used in [7.1].

The real gas properties for the test gas are used. One disadvantage of testing in a low temperature facility such as this is that many of the test gases available exhibit significant nonideal gas effects at low temperature (which are different than those in an engine). In the case of the turbine being studied, the difference between the change in enthalpy is approximately $0.5 \%$, when comparing assuming real versus ideal gas properties for the test conditions. This difference is significant for the efficiency measurement, but it is also small enough that ideal gas simplifications can readily be used for the purposes of uncertainty analysis. This is very useful as it simplifies the algebra while maintaining physical insight into the problem.

Real turbines generate entropy so they produce less work than the ideal. For an adiabatic turbine with losses, the outlet enthalpy is $h_{2, a d}$ with a corresponding work output of $W_{a d}$, where, $W_{a d}=h_{1}-h_{2, a d}$. The adiabatic efficiency of this turbine can be defined as:

$$
\eta_{a d}=\frac{h_{1}-h_{2, a d}}{h_{1}-h_{2, i s}}
$$

For a perfect gas with constant properties with constant properties, this reduces to the familiar form, 


$$
\eta_{a d}=\frac{1-\frac{T_{2, a d}}{T_{1}}}{1-\left(\frac{P_{2}}{P_{1}}\right)^{\frac{\gamma-1}{\gamma}}}
$$

The shaft efficiency is defined as the ratio of the actual power to the ideal power extracted from the turbine,

$$
\eta_{s}=\frac{\mathrm{T} \cdot \omega}{\dot{m}\left(h\left(T_{1}, P_{1}\right)-h\left(T_{2}, P_{2}\right)\right)}
$$

The enthalpies are found from the NIST 14 Reference Standard, [7.2], for the gas mixture at the test temperature and pressure. For the purposes of uncertainty analysis, the ideal gas form of Equation 7.3 was used,

$$
\eta_{s}=\frac{\mathrm{T} \cdot \omega}{\dot{m} C_{P} T_{1}\left(1-\left(\frac{P_{2}}{P_{1}}\right)^{\frac{\gamma-1}{\gamma}}\right)}=\frac{\mathrm{T} \cdot \omega}{\dot{m} C_{P} T_{1}\left(1-\pi_{t}^{-\frac{R}{C_{P}}}\right)}
$$

where $\mathrm{T}, \omega, \dot{m}$, and $C_{P}$ are the torque, speed, mass flow, and specific heat capacity, respectively.

In this work, we are concerned with non-ideal turbines that are non-adiabatic. If the total heat loss to the walls is $Q_{w}$, then the work output in this case is,

$$
W=h_{1}-\left(h_{2}+Q_{w}\right)
$$

where $h_{1}$ and $h_{2}$ are the enthalpies measured in the test. If the efficiency of this turbine was computed from the gas inlet and outlet enthalpies, the indicated efficiency of the turbine is,

$$
\eta_{\text {ind }}=\frac{h_{1}-h_{2}}{h_{1}-h_{2, i s}}
$$

The torque efficiency, defined as the measured work divided by the ideal change in enthalpy, is given by,

$$
\eta_{s}=\frac{W}{h_{1}-h_{2, i s}}=\frac{h_{1}-h_{2}-Q_{w}}{h_{1}-h_{2 . i s}}=\eta_{\text {ind }}-\frac{Q_{w}}{h_{1}-h_{2, i s}}
$$

The adiabatic performance is then related to the non-adiabatic test results as, 


$$
\eta_{a d}=\frac{h_{1}-h_{2}+h_{2}-h_{2, a d}}{h_{1}-h_{2, i s}}=\eta_{i n d}-\frac{h_{2, a d}-h_{2}}{h_{1}-h_{2, i s}}
$$

or,

$$
\eta_{a d}=\eta_{s}+\frac{Q_{w}}{h_{1}-h_{2, i s}}-\frac{h_{2, a d}-h_{2}}{h_{1}-h_{2, i s}}
$$

Thus, we must know both the heat transferred to the walls, $Q_{w}$, and how to form an estimate of the adiabatic exit enthalpy, $h_{2, a d}$, in order to correct the short duration, non-adiabatic rig measurement to an equivalent adiabatic efficiency.

At this point, thermodynamics alone is not sufficient to estimate $\eta_{a d}$. We must invoke some turbine fluid mechanics. First, consider how to estimate the total heat load. This can be done by one of several manners: (1) comparison of the shaft power with the measured aerodynamic rake enthalpy; (2) a one-dimensional compressible flow analysis utilizing Reynolds analogy; (3) a two-dimensional flow analysis with heat transfer; (4) by direct experimental measurement of $Q_{w}$.

Next, we must estimate the adiabatic exit enthalpy, which is not so simple. There are two sources of entropy that must be quantified: that which is due to heat transfer and that due to the influence of the heat transfer upon the turbine fluid mechanics. To calculate the entropy produced directly by heat transfer, we must know the temperature at which heat is extracted. Except for the shaft and rake power extraction case, all of the above techniques should yield the heat transfer and temperature distributions with similar accuracy. The shaft and rake case will yield a more accurate heat load but little idea of the extraction temperature. By estimating the extraction temperature to either the inlet relative total temperature for the nozzle, or the exit static temperature of the rotor, its influence can be bounded. The entropy change (positive or negative) by the modification of the flow due to cooling, we believe to be of second order compared to the direct entropy change due to cooling.

\subsection{Estimation of Adiabatic Exit Enthalpy, $h_{2, a d}$}

In a turbomachine we define the isentropic efficiency as the ratio of the actual work to the isentropic work. Thus the only factors that change this efficiency are departures form isentropic flow. These may either be heat transfer or thermodynamic irreversibility. The only rational measure of loss in a machine is entropy creation. Entropy is an unfamiliar quantity because it cannot be seen or measured directly, its values can only be inferred by the measurement of other properties. Basic thermodynamics tells us that for a single-phase fluid, entropy is a function of only two other thermodynamic properties such as temperature and pressure, (Denton [7.3]). For a perfect gas the relationship between entropy and temperature and pressure is,

$$
S-S_{r e f}=C_{P} \ln \left(\frac{T}{T_{r e f}}\right)-R \ln \left(\frac{P}{P_{r e f}}\right)
$$

Note, the pressures and temperatures in these equations may be either all static or all stagnation values because, by definition, the change from static to stagnation conditions is isentropic. 
Equation 7.10 can be rearranged to give,

$$
\left(\frac{P_{2}}{P_{1}}\right)=\left(\left(\frac{T_{1}}{T_{2}}\right) e^{-\frac{\Delta S}{C_{P}}}\right)^{\frac{\gamma}{\gamma-1}}
$$

The entropy generation due to heat transfer across a finite temperature gradient can be estimated from,

$$
\Delta S_{Q}=\int_{1}^{2}\left(\frac{\delta Q}{T}\right)_{\text {rev }}=\frac{Q_{w}}{T^{*}}
$$

where $\mathrm{T}^{*}$ is the temperature at which the heat is extracted. The adiabatic entropy change is defined as the total entropy change, less the entropy change due to heat transfer,

$$
\Delta S_{a d}=\Delta S_{T}-\Delta S_{Q}
$$

The adiabatic exit temperature can be estimated using Equation 7.11 to equate the adiabatic and non-adiabatic cases for the same pressure ratio,

$$
\left(\frac{T_{1}}{T_{a d}}\right) e^{\frac{\Delta S_{a d}}{C_{P}}}=\left(\frac{T_{1}}{T_{a d}}\right) e^{-\frac{\Delta S_{T}}{C_{P}}-\frac{\Delta S_{Q}}{C_{P}}}=\left(\frac{T_{1}}{T_{2}} e^{-\frac{\Delta S_{T}}{C_{P}}}\right)
$$

If $e^{-\frac{\Delta S_{T}}{C_{P}}}$ is replaced by its MacLaurin series expansion, with the higher order terms dropped, Equation 7.14 reduces to,

$$
T_{2, a d}=T_{2}\left(1+\frac{Q_{w}}{C_{P} T^{*}}\right)
$$

or,

$$
h_{2, a d}=h_{2}+Q_{w}\left(\frac{T_{2}}{T^{*}}\right)
$$

This can then be substituted into Equation 7.9 to yield,

$$
\eta_{a d}=\eta_{s}+\frac{Q_{w}}{h_{1}-h_{2 . i s}}-\frac{h_{2}+Q_{w}\left(\frac{T_{2}}{T^{*}}\right)-h_{2}}{h_{1}-h_{2, i s}}
$$




$$
\eta_{a d}=\eta_{s}+\frac{Q_{w}}{h_{1}-h_{2, i s}}\left(1-\frac{T_{2}}{T^{*}}\right)
$$

Equation 7.18 is very useful as it shows the effect of heat transfer on the adiabatic correction. Equation 7.19 can be derived by taking the $T^{*}$ as the mean of the turbine inlet and outlet temperatures, and substituting Equation 7.5 into 7.18,

$$
\Delta \eta_{a d}=\eta_{a d}-\eta_{s}=\left(\eta_{s}-\eta_{\text {ind }}\right)\left(\frac{1-\tau}{1+\tau}\right)
$$

Equation 7.19 is very useful for the purposes of uncertainty analysis, as data for the uncertainty of $\eta_{s}$ and $\eta_{\text {ind }}$ are already available. The uncertainty values for $\eta_{\text {ind }}$ are reported by Cai [8], and the values for $\eta_{s}$ can be found in Section 7.4, to follow. If the uncertainty of $\frac{1-\tau}{1+\tau}$ is taken to be $\pm 100 \%$, this bounds $T^{*}$ between $T_{2}$ and $T_{1}$. The results of the uncertainty analysis for $\Delta \eta_{a d}$ is contained in Table 7.1. The heat load is estimated to be $2 \%$ of the ideal enthalpy drop across the turbine (for $T_{l}=350 \mathrm{~K}$ ). The results show that the uncertainty in $\Delta \eta_{a d}$ is approximately equal to its magnitude. The actual uncertainty is small compared to the uncertainty in $\eta_{s}$ and will not significantly affect accuracy of the efficiency measurement unless $Q$ is larger. Also, assuming an uncertainty of $100 \%$ for $\frac{1-\tau}{1+\tau}$ is very conservative. For back-to-back tests $\Delta \eta_{a d}$ can be neglected.

Table 7.1: Adiabatic Correction Uncertainty

\begin{tabular}{|c|l|l|l|l|}
\hline Parameter & Value & Bias, $B$ & Precision, $S$ & Uncertainty, $U_{95}$ \\
\hline$\frac{Q}{h_{1}-h_{2, i s}}$ & 0.02 & & & \\
\hline$\tau$ & 0.87 & & & \\
\hline$\pi$ & 2.0 & & & \\
\hline$T_{1}$ & $350 \mathrm{~K}$ & & & \\
\hline$\eta_{s}$ & 0.90 & 0.0040 & 0.0020 & $0.62 \%$ \\
\hline$\eta_{\text {ind }}$ & 0.92 & 0.0041 & 0.0015 & $0.55 \%$ \\
\hline $1-\tau$ & 0.070 & 0.0700 & & $100 \%$ \\
\hline $1+\tau$ & 0.0014 & 0.0015 & 0.0002 & $107 \%$ \\
\hline$\Delta \eta_{a d}$ & 0.8986 & 0.0043 & 0.0020 & $0.65 \%$ \\
\hline$\eta_{a d}$ & & & \\
\hline
\end{tabular}




\subsection{Uncertainty Analysis}

\subsubsection{General}

All measurements have errors. These errors are the differences between the measurement and the true value. The uncertainty is an estimate of the test error, which in most cases would not be exceeded. Measurement error, $\delta$, has two components: a fixed error $\beta^{\prime}$, and a random error $\varepsilon$.

Precision (Random Error): Random error is seen in repeated measurements of the same thing. Measurements do not and are not and are not expected to agree exactly. There are numerous small effects that cause disagreements. The precision of a measurement process is determined by the variation between repeated measurements. The standard deviation $\sigma$ is used to determine the precision error $\varepsilon$. A large standard deviation means large scatter in the measurements. The statistic $S$ is calculated to estimate the standard $\sigma$ and is called the precision index.

Bias (Fixed Error): The second component of error, bias $\beta^{\prime}$, is the error that remains constant for the duration of the test. In repeated measurements, each measurement would have the same bias. The bias cannot be determined unless the measurements are compared to the true value of the quantity measured.

Measurement Uncertainty Interval: For simplicity, a single number is (some combination of bias and precision) is needed to express a reasonable limit for the total error. The single number must have a simple interpretation (like the largest error reasonably expected) and be useful without complex explanation. It is impossible to define a single rigorous statistic because the bias is an upper limit based on judgment which has unknown characteristics. Any function of these two numbers must be a hybrid combination of an unknown quantity (bias) and a statistic (precision). If both numbers were statistics, a confidence interval would be recommended. Confidence levels of $95 \%$ and $99 \%$ would be available at the discretion of the analyst. Although rigorous statistical confidence levels are not available, two uncertainty intervals are recommended by ASME/ANSI, analogous to 95\% and 99\% levels.

Where $t_{95}$ is the 95 th percentile point on the two tailed Student's $t$ distribution. The $t$ value is a function of the number of degrees of freedom (sample size), $v$, used in calculating $S$. For small samples $t$ is large and for large samples $t$ is smaller, approaching 1.96 in the lower limit. The use of $t$ inflates the limit $U$ to reduce risk of understating $\sigma$ when a small sample is used to calculate $S$. Since 30 degrees of freedom $v$ yields a $t$ of 2.04 and infinite degrees of freedom yields a $t$ of 1.96, an arbitrary selection of $t=2.0$ for values of $v$ from 30 to infinity is made, i.e.

$$
U_{95}=\sqrt{B^{2}+\left(t_{95} S\right)^{2}}
$$

\subsubsection{Pre-test versus Post-test Uncertainty Analysis}

The accuracy of the test is often part of the test requirement. Such requirements are defined by pre-test uncertainty analysis. This allows corrective action to be taken before the test to improve the uncertainties when they are too large. It is based on data and information that exists before the test, such as calibration histories, previous tests with similar instrumentation, prior measurement uncertainty analysis, and expert opinion. With complex tests, there are often 
alternatives to evaluate, including different test design configurations, instrumentation layouts, alternative calibration procedures, etc. Pre-test analysis will identify the most accurate test method. A post-test measurement uncertainty analysis is required to confirm the pre-test estimates or to identify problems. Comparison of test results with the pre-test analysis is an excellent data validity check. The precision of repeated points or redundant instrumentation should not be significantly larger than pre-test estimates. The final uncertainty interval should be based on post-test analysis.

\subsubsection{Back-To-Back Testing}

The objective of back to back testing is to determine the net effect of a design change most accurately, i.e., with the smallest measurement uncertainty. The first test is run with a standard or baseline configuration. The second test is identical to the first except that the design change is substituted in the baseline configuration. The difference between the results of the two tests is an indication of the effect of the design change.

As long as we consider only the difference or net effect between the two tests, all the fixed, constant bias errors will cancel out. The measurement error is composed of precision errors only. The efficiency uncertainty can be expressed as the following.

A Taylor series expansion of Equation 7.3 yields Equations 7.21 and 7.27, from which the measurement precision index and bias limits are calculated.

$$
S_{\eta}=\sqrt{\left(\frac{\delta \eta}{\delta \mathrm{T}} \cdot S_{\mathrm{T}}\right)^{2}+\left(\frac{\delta \eta}{\delta \dot{m}} \cdot S_{\dot{m}}\right)^{2}+\left(\frac{\delta \eta}{\delta C_{P}} \cdot S_{C_{P}}\right)^{2}+\left(\frac{\delta \eta}{\delta T_{1}} \cdot S_{T_{1}}\right)^{2}+\left(\frac{\delta \eta}{\delta \pi} \cdot S_{\pi}\right)^{2}}
$$

If we define the influence coefficient for each variable in Equation[7.21] as,

$$
C_{*}=\frac{\delta \eta}{\delta(*)} \cdot \frac{(*)}{\eta}
$$

then Equation[7.21] can be rewritten as,

$$
\frac{S_{\eta}}{\eta}=\sqrt{\left(C_{\mathrm{T}} \cdot \frac{S_{\mathrm{T}}}{\mathrm{T}}\right)^{2}+\left(C_{\dot{m}} \cdot \frac{S_{\dot{m}}}{\dot{m}}\right)^{2}+\left(C_{C_{P}} \cdot \frac{S_{C_{p}}}{C_{p}}\right)^{2}+\left(C_{\mathrm{T}} \cdot \frac{S_{T_{1}}}{T_{1}}\right)^{2}+\left(C_{\pi} \cdot \frac{S_{\pi}}{\pi}\right)^{2}}
$$

This is a more useful form than that of Equation[7.21]. The influence coefficient is a non-dimensional parameter that represents the how a given error source will be propagate to overall measurement error. The influence coefficient represents the relative amplification of that source error through Equation 7.3. The sign of the influence coefficient is not important as the term is squared. The influence coefficients $C_{\tau}, C_{\dot{m}}$, and $C_{T_{1}}$ are straightforward,

$$
C_{\mathrm{T}}=1, \quad C_{\dot{m}}=-1, C_{T_{1}}=-1
$$

However the expressions for $C_{C_{P}}$ and $C_{\pi}$ are considerably more complicated, 


$$
\begin{gathered}
C_{C_{P}}=1+\frac{\pi^{\frac{R}{C_{P}}} \cdot R \cdot \ln (\pi)}{C_{P} \cdot\left(1-\pi^{\frac{R}{C_{P}}}\right)}=1+\frac{\pi^{\frac{\gamma-1}{\gamma}} \cdot \frac{\gamma-1}{\gamma} \cdot \ln (\pi)}{1-\pi^{\frac{\gamma-1}{\gamma}}} \\
C_{\pi}=\frac{\pi^{\frac{R}{C_{P}}} \cdot R}{C_{P} \cdot\left(1-\pi^{\frac{R}{C_{P}}}\right)}=\frac{\frac{\gamma-1}{\gamma} \cdot \pi^{\frac{\gamma-1}{\gamma}}}{1-\pi^{\frac{\gamma-1}{\gamma}}}
\end{gathered}
$$

These equations were derived using a symbolic differentiation program and the results were verified numerically using sample values for $\gamma$ and $\pi$.

Similarly the Bias limits can be defined as,

$$
B_{\eta}=\sqrt{\left(\frac{\delta \eta}{\delta \mathrm{T}} \cdot B_{\mathrm{T}}\right)^{2}+\left(\frac{\delta \eta}{\delta \dot{m}} \cdot B_{\dot{m}}\right)^{2}+\left(\frac{\delta \eta}{\delta C_{P}} \cdot B_{C_{P}}\right)^{2}+\left(\frac{\delta \eta}{\delta T_{1}} \cdot B_{T}\right)^{2}+\left(\frac{\delta \eta}{\delta \pi} \cdot B_{\pi}\right)^{2}}
$$

In terms of the influence coefficients,

$$
\frac{B_{\eta}}{\eta}=\sqrt{\left(C_{\mathrm{T}} \cdot \frac{B_{\mathrm{T}}}{\mathrm{T}}\right)^{2}+\left(C_{\dot{m}} \cdot \frac{B_{\dot{m}}}{\dot{m}}\right)^{2}+\left(C_{C_{P}} \cdot \frac{B_{C_{P}}}{C_{P}}\right)^{2}+\left(C_{\mathrm{T}} \cdot \frac{B_{T_{1}}}{T_{1}}\right)^{2}+\left(C_{\pi} \cdot \frac{B_{\pi}}{\pi}\right)^{2}}
$$

Table 7.2 shows sample values for the influence coefficients. This shows that the uncertainty in pressure ratio will be amplified whereas the uncertainty in $C_{P}$ will be significantly reduced. Table 7.2 also contains a summary of the pretest uncertainties for T, $\dot{m}, T_{1}, C_{P}$, and $\pi$. The estimated uncertainty for $\eta$ will be $1.0 \%$ at a $95 \%$ confidence interval. For back-to-back test the uncertainty for changes in $\eta$ will be $0.5 \%$.

\begin{tabular}{|l|l|l|l|l|l|}
\hline Quantity & $C_{*}$ & $B_{*} / *$ & $S^{*} / *$ & $v$ & $U_{95}$ \\
\hline$T_{1}$ & 1.0 & $0.01 \%$ & $0.03 \%$ & $30+$ & $0.06 \%$ \\
\hline$\dot{m}$ & 1.0 & $0.33 \%$ & $0.13 \%$ & $30+$ & $0.41 \%$ \\
\hline $\mathrm{T}$ & 1.0 & $0.10 \%$ & $0.13 \%$ & $30+$ & $0.28 \%$ \\
\hline$\pi$ & 1.34 & $0.10 \%$ & $0.15 \%$ & $30+$ & $0.30 \%$ \\
\hline$C_{P}$ & 0.07 & $0.20 \%$ & & $30+$ & $0.20 \%$ \\
\hline$\eta$ & & $0.37 \%$ & $0.27 \%$ & $30+$ & $0.65 \%$ \\
\hline
\end{tabular}


The values for uncertainty in $\mathrm{T}$ and $\pi$ are obtained by pre test calibrations by Cai [7.4]. The uncertainties for $\mathrm{T}$ and $\dot{m}$ are discussed in Chapters 5 and 6 .

The following guideline to estimate the uncertainty in $C_{P}$ was proposed by Friend [7.5]. The bias limit for the $C_{P}$ of $\mathrm{CO}_{2}$ under ideal gas conditions is $\pm 0.1 \%$, however the bias limit is significantly higher if real gas effects are important. NIST proposed that the bias limit be increased based on the deviation of $\mathrm{CO}_{2}$ from ideal gas behavior. A limit of 5\% uncertainty for the difference from ideal gas properties was proposed as a conservative estimate of the bias limit. In our case the difference from ideal gas behavior is approx. $2 \%$, so the bias limit for the $C_{P}$ of $\mathrm{CO}_{2}$ is $\pm 0.2 \%$. In tests where the test gas is a mixture of components, the uncertainty of the composition must also be examined. 


\subsection{References}

[7.1] Guenette, G.R., Epstein, A.H., Ito, E. Turbine Aerodynamic Performance Measurements in Short Duration Facilities. AIAA/ASME/SAE/ASEE $25^{\text {th }}$ Joint Propulsion Conference. AIAA Paper, AIAA-89-2690, 1989.

[7.2] NIST 14. NIST Mixture Property Database. Standard Reference Database 14, Version 9.08 (1992). Fluid Mixtures Data Center, NIST Thermophysics Division 836.02. Bolder, $\mathrm{CO}$.

[7.3] Denton, J.D., Loss Mechanisms in Turbomachines. ASME Journal of Turbomachinery, Vol 115, pp. 621-656.

[7.4] Cai, Y., Aerodynamic Performance Measurements in a Fully Scaled Turbine. Masters Thesis, Massachusetts Institute of Technology, 1998.

[7.5] Friend, D.G., Private Correspondence about NIST 14, Summer 1997. Fluid Mixtures Data Center, NIST Thermophysics Division 838.02, Bolder, CO. 


\section{Experimental Program}

By Jason J. Jacobs

\subsection{Introduction}

The un-cooled turbine was tested using two different tip gaps over a range of operating conditions. These tests show the turbine efficiency and corrected mass flow vary with pressure ratio and corrected speed. The tests also show how a reduced tip gap influences the turbine performance over a range of operating conditions.

The cooled turbine was tested at the design tip gap over the same range operating points at the design coolant mass flow and gas-to-metal temperature ratio. These tests examine the degree to which the influence of film cooling is affected by the turbine pressure ratio and corrected speed. The turbine was then tested at its operating design point with a range of coolant mass flows. The turbine was also tested at a low gas-to-metal temperature ratio over a range of mass flows to determine if the turbine losses scaled with coolant to mainstream momentum flux ratio as has been observed in cascade tests.

\subsection{Experimental Test Conditions: Un-Cooled Turbine}

Table 8.1 summarizes the experimental conditions for the un-cooled turbine test series. The first series of runs, [024-032], tested the un-cooled turbine with a reduced tip gap, $(\sim 1.5 \%$ of span), operating over a range of operating points (pressure ratios and corrected speeds). Run [030] tested the turbine at a low Reynolds number.

The turbine with the design tip gap ( $3.0 \%$ of span), was tested over a range operating points in runs [033-038]. The influence of turbine inlet temperature (non-adiabatic effects) was studied in runs [038-040]. Run [041] tested the design tip gap at a low Reynolds number and [042] tested the same Reynolds number using air as the test gas. An off design test point was studied in run [043], where the corrected speed was approximately $133 \%$ of the design value.

\subsection{Experimental Test Conditions: Cooled Turbine}

Table 8.2 summarizes the experimental conditions for the un-cooled turbine test series. The cooled was tested at a higher inlet temperature to match the gas-to-metal temperature of the engine. Runs[118-126,128,130,131] tested the cooled turbine over a range of operating points. The design point operating conditions were repeated in order to establish the repeatability of the measurements. Runs $[127,129]$ tested the cooled turbine over a range of inlet temperatures to help characterize the non-adiabatic effects for the cooled turbine. Runs [114-116,132-133] tested the cooled turbine at the design gas-to-metal temperature ratio over a range coolant mass flows. A range of coolant mass flows was tested in runs $[110,111,113,117]$ at a lower turbine inlet temperature. A high Reynolds number case was tested in run [112] at the same low inlet temperature. 
Table 8.1: Un-Cooled Turbine Test Matrix

50\% Tip Gap - Operating Point Test Matrix

\begin{tabular}{|c|cccccc|}
\hline Test & Gas & $\mathrm{T}_{\text {in }}, \mathrm{K}$ & $\mathrm{P}_{\text {in }}, \mathrm{atm}$ & $\mathrm{PR}$ & $\mathrm{N}_{\mathrm{c}}, \%$ & $\mathrm{Re}, \%$ \\
\hline abb024 & $\mathrm{CO}_{2}$ & 350 & 5.4 & 1.93 & 95.6 & 85 \\
abb025 & $\mathrm{CO}_{2}$ & 351 & 5.4 & 1.94 & 99.8 & 84 \\
abb026 & $\mathrm{CO}_{2}$ & 350 & 5.4 & 1.94 & 99.5 & 85 \\
abb027 & $\mathrm{CO}_{2}$ & 351 & 5.4 & 2.05 & 97.8 & 84 \\
abb028 & $\mathrm{CO}_{2}$ & 350 & 5.4 & 1.92 & 92.3 & 85 \\
abb029 & $\mathrm{CO}_{2}$ & 350 & 5.4 & 1.96 & 108.9 & 84 \\
abb031 & $\mathrm{CO}_{2}$ & 350 & 5.4 & 2.13 & 97.5 & 85 \\
abb032 & $\mathrm{CO}_{2}$ & 349 & 5.4 & 1.78 & 103.9 & 84 \\
\hline
\end{tabular}

50\% Tip Gap - Low Reynolds Number

\begin{tabular}{|l|llllll|}
\hline abb030 & $\mathrm{CO}_{2}$ & 302 & 1.7 & 2.07 & 105.8 & 33 \\
\hline
\end{tabular}

Design Tip Gap - Operating Point Test Matrix

\begin{tabular}{|c|cccccc|}
\hline abb033 & $\mathrm{CO}_{2}$ & 351 & 5.4 & 1.78 & 97.4 & 83 \\
abb034 & $\mathrm{CO}_{2}$ & 350 & 5.4 & 1.95 & 101.2 & 85 \\
abb035 & $\mathrm{CO}_{2}$ & 351 & 5.4 & 2.07 & 97.7 & 84 \\
abb036 & $\mathrm{CO}_{2}$ & 351 & 5.4 & 1.94 & 98.2 & 85 \\
abb037 & $\mathrm{CO}_{2}$ & 351 & 5.4 & 1.97 & 111.6 & 84 \\
\hline
\end{tabular}

Design Tip Gap - Inlet Temperature Test Matrix

\begin{tabular}{|c|cccccc|}
\hline abb038 & $\mathrm{CO}_{2}$ & 331 & 4.9 & 1.94 & 98.8 & 84 \\
abb039 & $\mathrm{CO}_{2}$ & 342 & 5.2 & 1.95 & 104.4 & 85 \\
abb040 & $\mathrm{CO}_{2}$ & 351 & 5.4 & 1.96 & 106.5 & 85 \\
\hline
\end{tabular}

Design Tip Gap - Low Reynolds Number

\begin{tabular}{|l|llllll|}
\hline abb041 & $\mathrm{CO}_{2}$ & 326 & 2.4 & 1.95 & 101.4 & 43 \\
\hline
\end{tabular}

\section{Design Tip Gap - Alternative Test Gas}

\begin{tabular}{|l|llllll|}
\hline abb042 & Air & 318 & 3.3 & 1.98 & 104.0 & 42 \\
\hline
\end{tabular}

\section{Design Tip Gap - Off Design Operating Point}

\begin{tabular}{|l|llllll|}
\hline abb043 & $\mathrm{CO}_{2}$ & 352 & 5.4 & 2.1 & 132.6 & 83 \\
\hline
\end{tabular}


Table 8.2: Cooled Turbine Test Matrix

Low Inlet Temperature - Coolant Mass Flow Tests

\begin{tabular}{|c|cccccccc|}
\hline Test & $\mathrm{Gas}$ & $\mathrm{T}_{\text {in }}, \mathrm{K}$ & $\mathrm{P}_{\text {in, }}$ atm & $\mathrm{PR}$ & $\mathrm{N}_{\mathrm{c}}, \%$ & $\mathrm{Re}, \%$ & $\mathrm{M}_{\text {cool }}, \%$ & $\mathrm{~T}_{\text {in }} / \mathrm{T}_{\text {vane }}$ \\
\hline abb110 & $\mathrm{CO}_{2}$ & 329 & 3.6 & 2.07 & 106.3 & 63 & 4.8 & 1.11 \\
abb111 & $\mathrm{CO}_{2}$ & 329 & 3.6 & 2.04 & 106.2 & 64 & 8.4 & 1.12 \\
abb113 & $\mathrm{CO}_{2}$ & 330 & 3.6 & 2.02 & 106.0 & 64 & 10.0 & 1.13 \\
abb117 & $\mathrm{CO}_{2}$ & 331 & 3.6 & 2.01 & 105.8 & 64 & 11.7 & 1.16 \\
\hline
\end{tabular}

Low Inlet Temperature - High Reynolds Number Test

\begin{tabular}{|l|llllllll|}
\hline abb112 & $\mathrm{CO}_{2}$ & 331 & 5.4 & 2.04 & 109.6 & 95 & 6.6 & 1.13 \\
\hline
\end{tabular}

High Inlet Temperature - Operating Point Test Matrix

\begin{tabular}{|c|cccccccc|}
\hline abb118 & $\mathrm{CO}_{2}$ & 441 & 5.2 & 2.03 & 102.7 & 63 & 9.9 & 1.58 \\
abb119 & $\mathrm{CO}_{2}$ & 439 & 5.3 & 2.03 & 104.5 & 63 & 9.2 & 1.52 \\
abb120 & $\mathrm{CO}_{2}$ & 446 & 5.3 & 2.01 & 96.1 & 62 & 9.7 & 1.58 \\
abb121 & $\mathrm{CO}_{2}$ & 445 & 5.3 & 1.98 & 90.3 & 63 & 9.7 & 1.57 \\
abb122 & $\mathrm{CO}_{2}$ & 448 & 5.3 & 1.81 & 94.2 & 61 & 9.8 & 1.58 \\
abb123 & $\mathrm{CO}_{2}$ & 447 & 5.3 & 2.12 & 96.1 & 62 & 9.6 & 1.58 \\
abb124 & $\mathrm{CO}_{2}$ & 445 & 5.3 & 2.01 & 93.6 & 63 & 9.9 & 1.60 \\
abb125 & $\mathrm{CO}_{2}$ & 444 & 5.3 & 2.01 & 97.6 & 63 & 10.0 & 1.60 \\
abb126 & $\mathrm{CO}_{2}$ & 445 & 5.2 & 2.01 & 97.0 & 62 & 9.9 & 1.60 \\
abb128 & $\mathrm{CO}_{2}$ & 442 & 5.3 & 2.04 & 112.3 & 63 & 9.9 & 1.58 \\
abb130 & $\mathrm{CO}_{2}$ & 439 & 5.3 & 2.02 & 99.8 & 63 & 9.8 & 1.56 \\
abb131 & $\mathrm{CO}_{2}$ & 442 & 5.3 & 2.06 & 99.7 & 63 & 9.9 & 1.58 \\
\hline
\end{tabular}

High Inlet Temperature - Inlet Temperature Test Matrix

\begin{tabular}{|l|llllllll|}
\hline abb127 & $\mathrm{CO}_{2}$ & 420 & 4.9 & 2.02 & 98.7 & 62 & 9.6 & 1.50 \\
abb129 & $\mathrm{CO}_{2}$ & 394 & 4.5 & 2.02 & 98.8 & 62 & 9.1 & 1.39 \\
\hline
\end{tabular}

High Inlet Temperature - Coolant Mass Flow Tests

\begin{tabular}{|l|llllllll|}
\hline abb114 & $\mathrm{CO}_{2}$ & 435 & 5.3 & 2.07 & 109.1 & 63 & 7.2 & 1.53 \\
abb115 & $\mathrm{CO}_{2}$ & 440 & 5.2 & 2.05 & 104.6 & 63 & 8.7 & 1.57 \\
abb116 & $\mathrm{CO}_{2}$ & 436 & 5.2 & 2.06 & 106.9 & 63 & 7.2 & 1.53 \\
abb132 & $\mathrm{CO}_{2}$ & 439 & 5.3 & 2.03 & 98.6 & 64 & 12.0 & 1.58 \\
abb133 & $\mathrm{CO}_{2}$ & 441 & 5.3 & 2.03 & 100.4 & 64 & 12.1 & 1.59 \\
\hline
\end{tabular}




\subsection{Similarity Verification Calculations}

Every blowdown experiment produces approximately 1 million data points. What follows is a discussion on how this data is used in calculations intended to characterize turbine performance and verify that test conditions necessary for experimental similarity are met. All calculations described in this section are provided Chapter 13 for each un-cooled experiment conducted.

One of the first priorities at the conclusion of an experiment is the computation of the total pressure and temperature ratios achieved during the test. Many total pressure and temperature measurements are made both upstream and downstream, as shown in Table 3.2. These must be appropriately processed to provide the turbine test conditions. For the calculations presented herein, simple un-weighted averaging was employed. In principle, weighting based on the appropriate flux should be employed.

Upstream, the pressure and temperature measurements are available at three fixed circumferential locations between supply tank struts. Typically these measurements are identical, and are simply be averaged. Downstream, the measurements are made using circumferentially traversed, radial rakes. For the purpose of this analysis, the meaturements from each head were be weighted equally. Pressure and temperature ratio is then calculated as follows:

$$
\begin{gathered}
\pi_{t}=\frac{\tilde{P}_{T_{5}}}{\tilde{P}_{T_{4}}} \\
\tau_{t}=\frac{\tilde{T}_{T_{5}}}{\tilde{T}_{T_{4}}}
\end{gathered}
$$

where subscripts four and five represent upstream and downstream conditions respectively and (こ) indicates a simple arithmetic average.

Reynolds number is also verified proceeding an experiment,

$$
R_{e}=\frac{\dot{m}_{t}}{\mu L_{e f}}
$$

Reynolds number is proportional to turbine mass flow (calculated from critical-flow venturi calibration data), and inversely proportional to dynamic viscosity (determined from property tables).

Specific heat ratio for each test is found directly from property tables.

Corrected speed must also be determined. In terms of percent of its design value, corrected speed is written as:

$$
N_{\text {cor }, \% \text { des }}=\frac{100}{N_{\text {cor,des }}} \frac{L_{r e f} N_{\text {mech }}}{\sqrt{\gamma_{4} R T_{T_{4}}}}
$$


Speed is controlled by the eddy current brake. As described in Section 5.2, the eddy current brake provides a braking torque which is reacted through two load cells fastened to the test section. Data from these load cells enables calculation of turbine torque through the established calibration:

$$
\mathrm{T}[\mathrm{N} \cdot \mathrm{m}]=683.67 \cdot(\mathrm{ECBF} 1-\mathrm{ECBF} 2)-\mathrm{T}_{0}
$$

Turbine torque and speed are then used to calculate turbine power, (This neglects rotor acceleration accounted for in Section 10.3.2),

$$
\mathrm{P}[\mathrm{W}]=\mathrm{T} \cdot \omega
$$

As discussed in Section 3.3, experimental similarity requires that the non-dimensional similarity parameters be reproduced. These parameters were determined to be pressure ratio, corrected speed, specific heat ratio, and Reynolds number, all of which are plotted versus time for each experiment in Chapter 13. The period of time when these parameters are constant and equal to full-scale turbine design values is the time when similarity is achieved. This window varies from test to test as seen, however, it generally spans approximately $300-800 \mathrm{~ms}$. This is also the time when efficiency predictions, discussed in the next two chapters, will be representative of the full-scale turbine.

This concludes the discussion on standard calculations used in characterizing turbine performance and verifying experimental similarity. Next, through these calculations and raw experimental data, unique properties of turbomachinery flow and of the blowdown experimental technique used for this work will be demonstrated.

\subsection{Compressional Heating}

The first obvious characteristic of all blowdown temperature traces is the large spike around $50 \mathrm{~ms}$. This is the effect of what is known as compressional heating. This process is a natural characteristic of the transient tunnel start-up process and came to be understood through dynamic models of the blowdown facility put together during its development. Here, it is explained in terms of the thermodynamics of the fast-acting valve expansion process.

Figure 8.3 shows the upstream and downstream total temperature during experiment 032 , initial supply tank temperature $220^{\circ} \mathrm{F}$ or $378 \mathrm{~K}$. As seen here, the upstream total temperature, shortly after opening of the fast-acting valve, exceeds the initial supply tank total temperature. According to the first law of thermodynamics written for a calorically perfect gas,

$$
\bar{C}_{P} \cdot \delta T_{T}=\delta w-\delta q
$$

total temperature can change only as a result of work or heat transfer. This says the flow entering the upstream test section experiences one or both. However, this flow simply undergoes an isentropic expansion, as shown in the previous section, to a very low Mach number, and seemingly, to the same total temperature. 


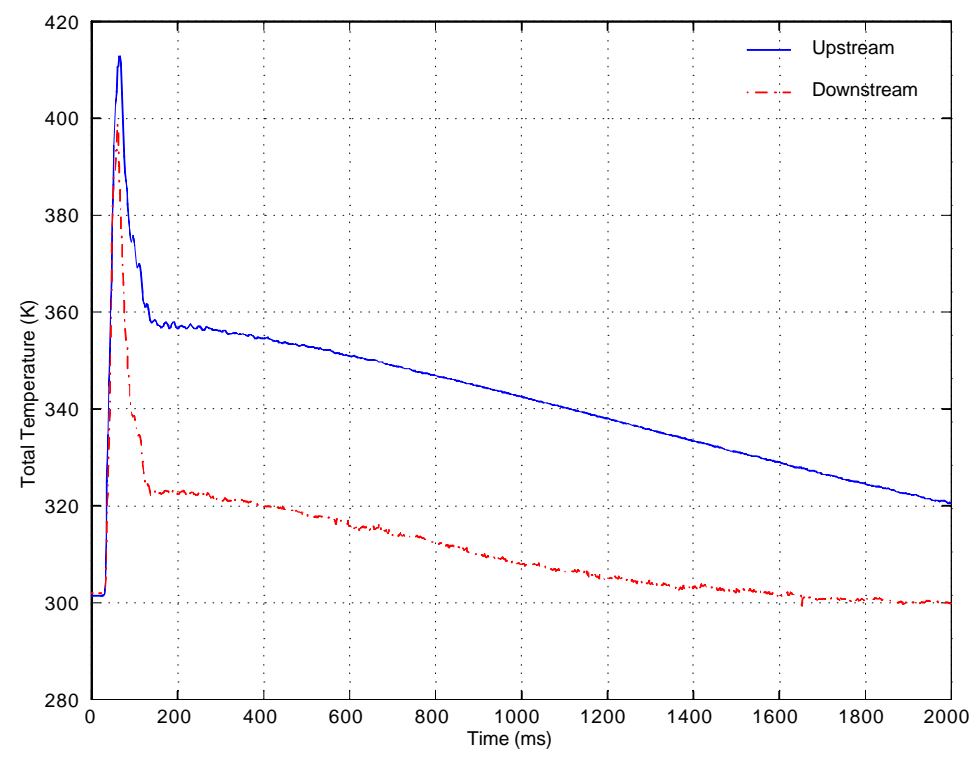

Figure 8.1: Average Upstream and Downstream Total Temperature - Experiment 032

It turns out, the expansion of gas into a large volume contributes work to and increases the total temperature of the gas inside. Reynolds calls this process "Charging of a high-pressure tank", Reynolds [8.1]. It is modeled as a valve supplying a steady one-dimensional inflow to a large adiabatic tank. Further assuming calorically perfect gas behavior and perfect mixing and neglecting kinetic energy inside the tank, the first law of thermodynamics applied to the tank from time zero to time $t$ takes the form [8.1].

$$
\Delta U_{t}=\dot{m}_{v} h_{v} t
$$

where subscript $t$ indicates a condition inside the tank and subscript $v$, at the valve. This says the internal energy inside the tank increases with the inflow of enthalpy. Applying conservation of mass yields:

$$
\dot{m}_{v} h_{v} t=\left(m_{t}(0)+\dot{m}_{v} t\right) u_{t}(t)-m_{t}(0) u_{t}(0)
$$

Perfect gas behavior is assumed to express enthalpy and internal energy in terms of temperature,

$$
\dot{m}_{v} \bar{C}_{P} T_{v} t=\left(m_{t}(0)+\dot{m}_{v} t\right) \bar{C}_{V} T_{t}(t)-m_{t}(0) \bar{C}_{V} T_{t}(0)
$$

This is an expression for the temperature inside the tank as a function of inlet conditions and time.

In applying this model to the blowdown facility, zero initial mass is assumed as the test section begins the experiment evacuated; in other words $\dot{m}_{t}(0)=0$. Equation 8.10 then becomes

$$
T_{t}=\gamma \cdot T_{v}
$$


This says immediately after the fast-acting valve opens, the (total) temperature in the upstream test section becomes the supply tank total temperature multiplied by specific heat ratio, a number always larger than unity. This increase in total temperature is due to flow work applied to the gas in the upstream test section by the gas expanding from the supply tank.

This process is known as compressional heating in the Blowdown Turbine and produces the temperature spikes shown in Figure 8.1. Just as quickly as it occurs, the volume of hot gas is convected downstream and temperature decreases along the downwind portion of these temperature spikes. In short, these spikes are simply the impulse response of the total temperature instrumentation. Since its prediction, compression heating has been recognized as a novelty and an indication of the fast time response of the instrumentation used to measure it.

\subsection{Nozzle Wake Measurements}

The downstream flow field exhibits circumferential non-uniformities due to nozzle wake shedding. Flow field measurements in this region will vary depending on the circumferential position that they are taken. The translator allows a circumferential survey capturing several of the 44 nozzle wakes during the time when similarity is achieved, depending on the rotational speed. Typically the translator operates at near 0.25 RPS to maintain all instrumentation between wind tunnel support struts. This allows measurements over approximately 5.5 wakes between 300-800 ms, a probe wake passing frequency of $11 \mathrm{~Hz}$.

Figure 8.2 shows a downstream total temperature trace taken during Experiment 032 from probe TTR104-4. A single low frequency component of amplitude near $3 \mathrm{~K}$ is clearly visible in the region of 100-1000 ms. Figure 8.3 shows the Fourier Transform of this temperature signal displaying the actual frequency content. As expected, this low frequency component is very near $11 \mathrm{~Hz}$, the frequency at which the temperature probe is traversing the nozzle wakes.

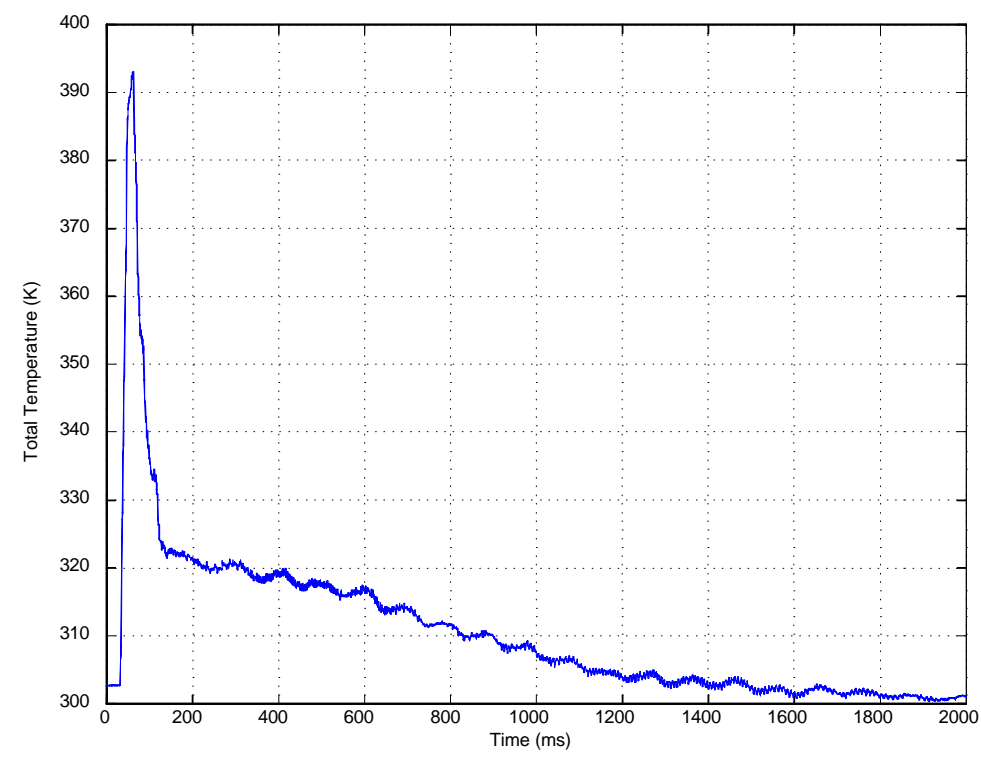

Figure 8.2: Downstream Total Temperature, (TTR104-4) - Experiment 032 


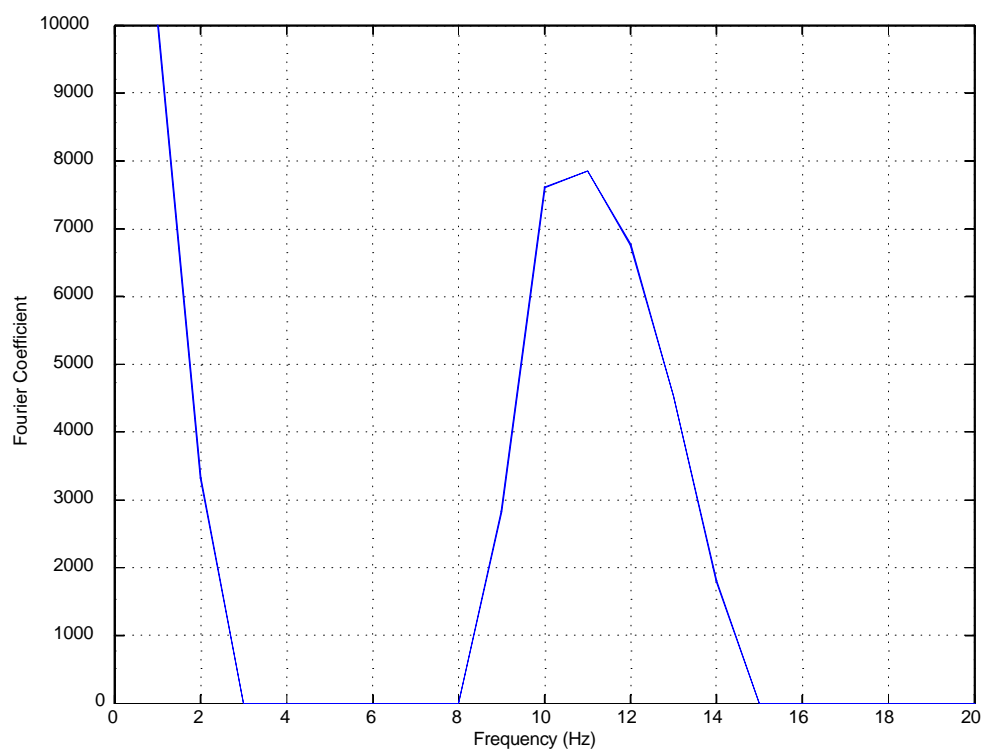

Figure 8.3: Downstream Total Temperature FFT (TTR104-4) - Experiment 032

This circumferential non-uniformity might be expected to appear in total pressure measurements as well. In fact it does. Figure 8.4 shows downstream total pressure sensor PT45R8 during the same experiment. A very similar low frequency component is observed of magnitude near $0.20 \sim$ atm or 2.94 psia. From the frequency content calculation, shown in Figure 8.5, this component is again determined to be near $11 \mathrm{~Hz}$.

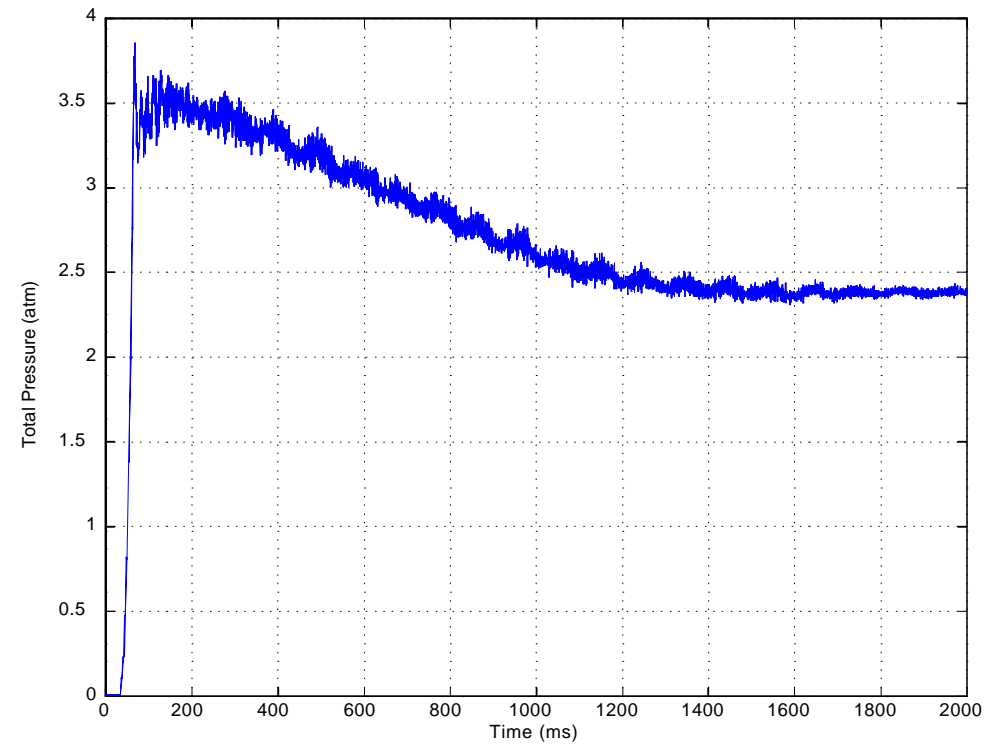

Figure 8.4: Downstream Total Pressure (PT45R8) - Experiment 032 


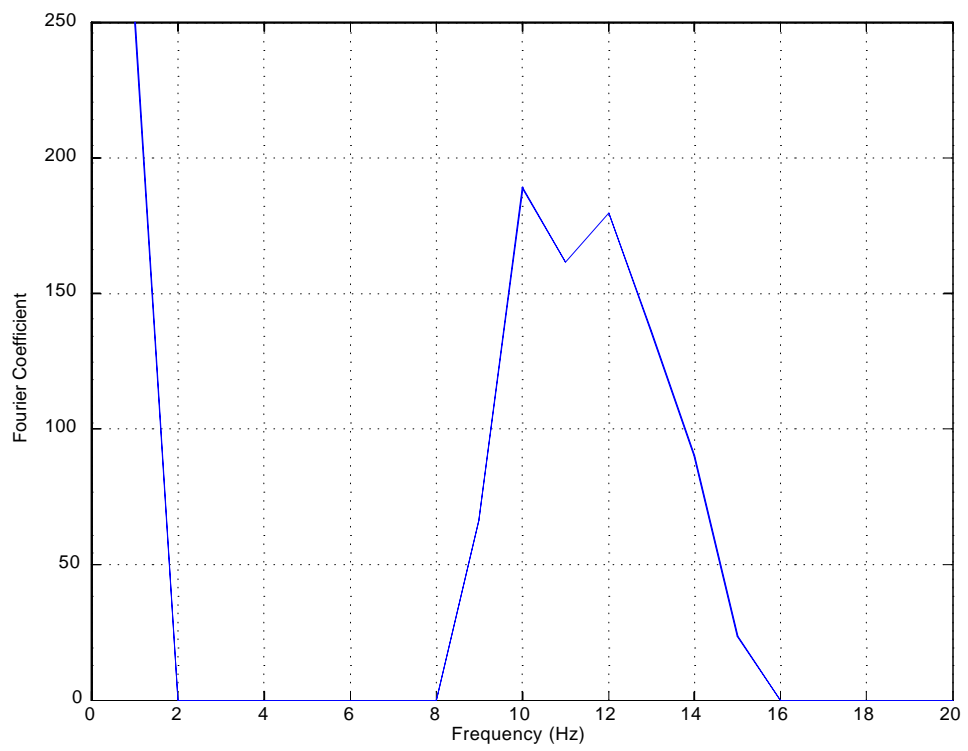

Figure 8.5: Downstream Total Pressure FFT (PT45R8) - Experiment 032

This data shows sinusoidal downstream circumferential non-uniformities in total temperature and pressure of magnitudes near $3 \mathrm{~K}$ and 3 psia. These non-uniformities are shown to be at the frequency at which the measurement probes are traversing the nozzle wakes. This clearly indicates measurement of nozzle cooling. The flow directly downstream of each nozzle, within its wake, experiences more heat transfer than the flow outside of this region reducing its temperature and pressure. This data reaffirms the need for translating downstream flow field measurements provided by the downstream translator.

\subsection{Radial Temperature Profiles}

The initial supply tank temperature required for full Reynolds number simulation (for pure $\mathrm{CO}_{2}$ test gas) at the design specific heat ratio is near $375 \mathrm{~K}$, or $215^{\circ} \mathrm{F}$. This is very low by full-scale turbine standards but significantly higher than ambient, the temperature at which the test section begins. This temperature difference will form a thermal boundary layer in the test section once flow is established through the turbine. The radial distribution of total temperature probes, labeled TTR101-1..6, TTR103-1..5, and TTR104-1..4 in Table 3.2, is specifically designed to measure this flow feature. 


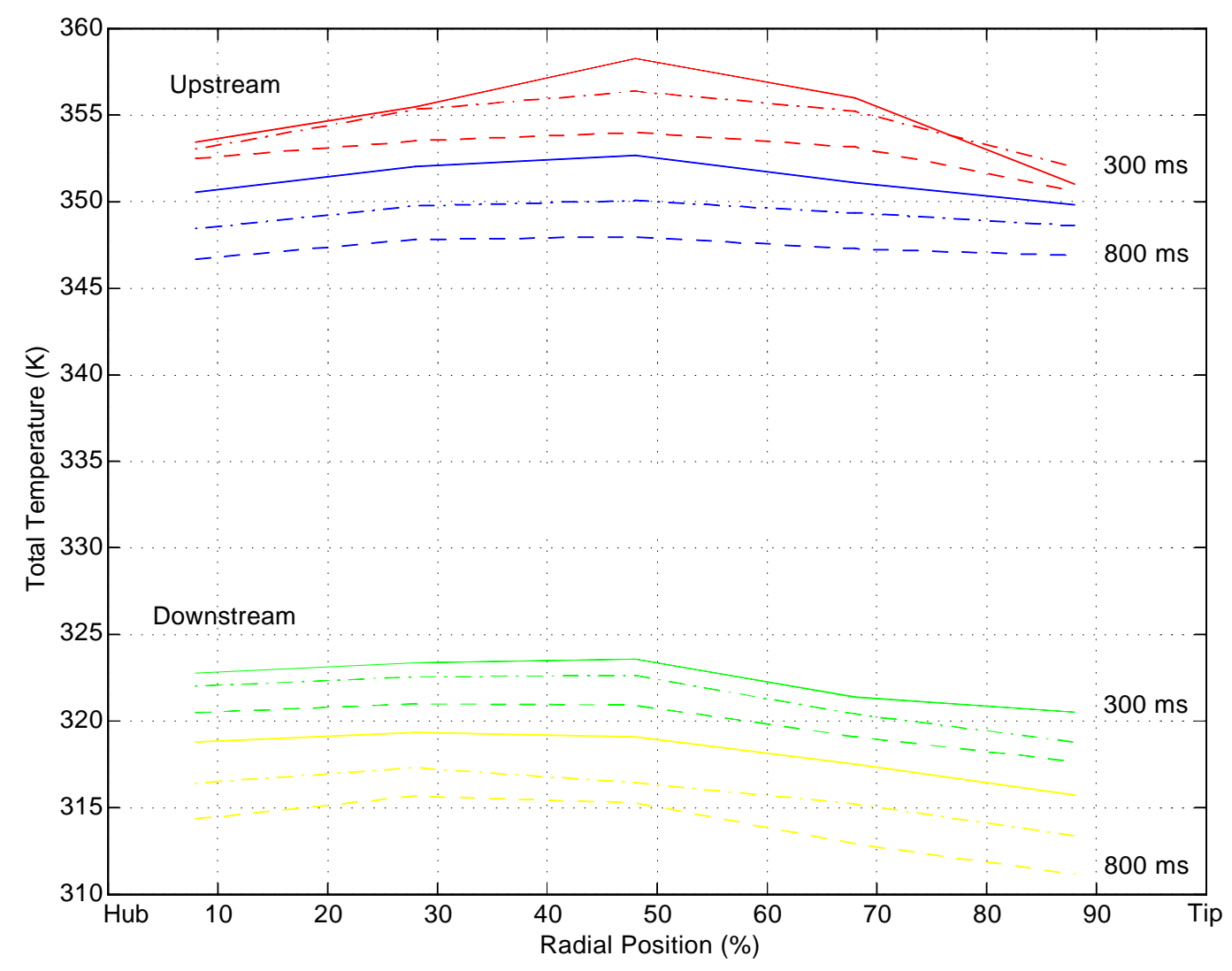

Figure 8.6: Total Temperature Radial Profiles - Experiment 032

Figure 8.6 shows upstream and downstream radial total temperature profiles observed during Experiment 032. Each curve represents the profile at a different point in time: 300, 400, $500,600,700$, and $800 \mathrm{~ms}$ profiles are shown. From this data, a thermal boundary layer of timevarying magnitude is evident. Early in the experiment, the mean-line gas temperature is near the initial supply tank temperature and the turbine metal temperature is near ambient producing a radial temperature variation of order $5 \mathrm{~K}$. As the experiment continues, the supply tank temperature decreases isentropically with supply tank pressure and the turbine metal temperature increases due to heat transfer. These effects decrease the magnitude of the thermal boundary layer in time. By $800 \mathrm{~ms}$, the upstream total temperature profile is nearly flat. It is important to note that the supply tank temperature decreases much more than the turbine metal temperature increases. This increase in metal temperature is due to heat transfer and is small due to the short duration of the experiment. Its exact magnitude, however, will be explored in Section 9.6 as it is required to correct aerodynamic efficiency predictions.

\subsection{Summary}

This chapter began by introducing the full-scale turbine operating points simulated during this work and the corresponding blowdown operating conditions required for similarity. A discussion on calculations intended to characterize turbine performance and verify matching of 
non-dimensional similarity parameters followed. Finally, unique properties of turbomachinery flow and of the short duration experimental technique used were observed through analysis of these calculations and of the experimental data. These properties included isentropic valve expansion, compressional heating, nozzle wake measurements, and radial temperature profiles.

\subsection{References}

[8.1] Reynolds, W.C., H.C. Perkins, Engineering Thermodynamics. Second Edition. McGraw-Hill, Inc. 1977. 


\section{$9 \quad$ Aerodynamic ('Rake') Measurement of Adiabatic Efficiency}

By Jason J. Jacobs

\subsection{Introduction}

This chapter will begin with a discussion of turbine adiabatic efficiency measurement using the aerodynamic approach followed by a presentation of the pre-test efficiency uncertainty analysis. This analysis is intended to estimate the level of uncertainty expected in the calculation of efficiency before the calculation takes place. This analysis will also uncover a distinction between absolute accuracy and relative precision, an important one when comparing results from back-to-back experiments. Following the uncertainty analysis, real, ideal, and perfect gas models will be explained in terms of enthalpy calculation. The goal of this discussion will be to qualify assumptions made in these models and determine how they will affect efficiency predictions. A discussion on efficiency calculation will follow. This will include the process of evaluating flow properties required for efficiency calculations as well as these calculations for Test ABB024, a baseline full-scale turbine design point simulation. This chapter concludes with a discussion on transient heat transfer correction and a post-test efficiency uncertainty analysis focusing of aerodynamic efficiency prediction repeatability.

\subsection{Approach}

Figure 9.1 shows turbine expansion processes on an enthalpy-entropy, or h-s diagram. The work extracted by the turbine during adiabatic operation is labeled $W_{a d}$. The ideal, or isentropic work, is labeled $W_{i s}$.

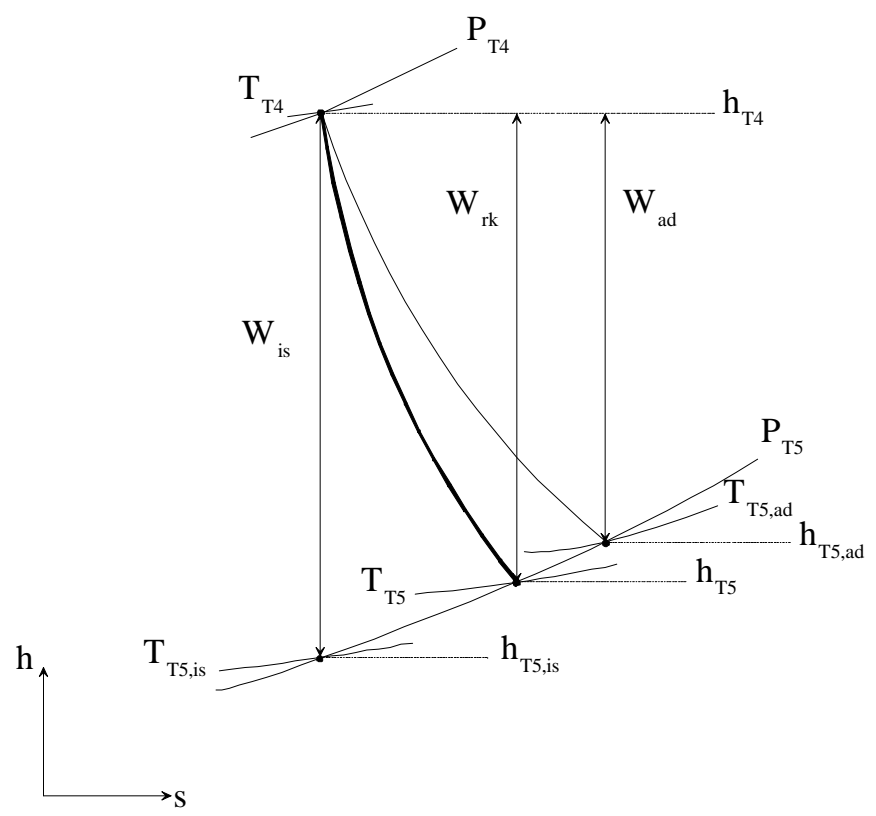

Figure 9.1: Enthalpy-Entropy Diagram Showing Turbine Expansion Process 
Turbine adiabatic efficiency is defined as the ratio of these quantities, each expressed as a change in total enthalpy:

$$
\begin{gathered}
\eta_{a d}=\frac{W_{a d}}{W_{i s}} \\
W_{a d}=h_{T_{4}}-h_{T_{5}, a d} \\
W_{i s}=h_{T_{4}}-h_{T_{5}, i s}
\end{gathered}
$$

Subscripts four and five represent upstream and downstream quantities respectively. The primary objective of this work is to determine this quantity with uncertainty less than $0.50 \%$. This is optimistic, even from steady-state data. Two approaches are taken to arrive at this goal and they differ in their calculation of actual turbine work. The first, or aerodynamic approach, determines actual turbine work from upstream and downstream temperature and pressure measurements. The second, or mechanical approach, utilizes turbine torque, speed, and mass flow for actual turbine work calculation. Other important objectives of this study are to determine by which method turbine adiabatic efficiency may be measured most accurately and most repeatable in the short duration test facility, and, quantify efficiency measurement uncertainties in each approach.

With knowledge of total and static temperature and pressure upstream and downstream, actual turbine adiabatic work may be calculated directly, as in Equation 9.2 using real gas property tables; ideal work is found similarly. (These calculations make use of a heat transfer correction due to non-adiabatic effects discussed in Section 9.6). To assess the significance of real gas effects, simplified gas models may then be employed for work evaluation. Assuming the flow through the turbine to be ideal, work may be expressed only in total temperature and pressure ratio and specific heat. Following an expression derived by Shapiro, [9.1], Equations 9.2 and 9.3 become,

$$
\begin{gathered}
W_{a d, i g}=C_{P 4} \cdot T_{T 4}-C_{P 5} \cdot T_{T 5}-\left(C_{P 4}-C_{P 5}\right) \cdot \frac{T_{4}+T_{5}}{2} \\
W_{i s, i g}=C_{P 4} \cdot T_{T 4}-C_{P 5} \cdot T_{T 4} \cdot \pi_{t}^{\frac{R}{\overline{\bar{C}_{P}}}}-\left(C_{P 4}-C_{P 5}\right) \cdot \frac{T_{4}-T_{5, i s}}{2}
\end{gathered}
$$

where

$$
\bar{C}_{P}=\frac{C_{P 4}+C_{P 5}}{2}
$$

Further assuming calorically perfect gas behavior, variation in specific heat is neglected and adiabatic and isentropic work assume a familiar form,

$$
W_{a d, c p g}=\bar{C}_{P} \cdot\left(T_{T 4}-T_{T 5}\right)
$$




$$
W_{i s, c p g}=\bar{C}_{P} \cdot\left(T_{T 4}-T_{T 5} \cdot \pi_{t}^{\frac{R}{\overline{C_{p}}}}\right)
$$

Another focus of this work will be to explore these assumptions and their validity in this situation by comparing efficiency calculations assuming ideal and calorically perfect gas behavior with real gas efficiency calculations.

Mechanically, turbine (specific) work may be calculated from measurements of turbine torque, speed, and mass flow as follows,

$$
w_{a d, b r k}=\frac{\mathrm{T} \cdot \omega}{\dot{m}_{t}}
$$

(employing corrections accounting for rotor acceleration, tunnel mass storage, and heat transfer effects discussed in Sections 10.3.2, 10.3.3, and 10.4). Efficiency may then be calculated using real gas ideal work, Equation 9.3, or by assuming ideal or calorically perfect gas behavior, Equations 9.5 or 9.8 . These calculations will also allow assessment of the validity of the ideal and calorically perfect gas assumptions.

\subsection{Pre-Test Efficiency Uncertainty Analysis}

Prior to any experiment, a pre-test uncertainty analysis is beneficial in determining how measurement uncertainties propagate through subsequent calculations. This will provide information on the consistency, or precision of the calculations. This analysis, based on techniques developed in [9.2], will estimate uncertainties in the inference of efficiency based on measurement uncertainties determined from calibration data. For algebraic convenience, this analysis will assume calorically perfect gas behavior so that efficiency may be written in terms of Equations 9.7 and 9.8 as follows:

$$
\eta_{a d, c p g}=\frac{1-\tau_{t}}{1-\pi_{t}^{\frac{R_{c c_{2}}}{\overline{\bar{c}}_{p}}}}
$$

The first component of uncertainty is random error. This error affects measurement precision and is seen through repeated measurement of the same condition. Factors contributing to random error include instrument noise, ambient conditions, data acquisition errors, usually resulting from finite resolution, and human errors. Mathematically, random error is related to the standard deviation of a set of measurements. The statistic $\mathrm{S}$, known as the precision index, approximates the standard deviation and is used to quantify random, or precision error. The adiabatic efficiency precision index is expressed in terms of a Taylor series expansion of Equation 9.10 as follows: 


$$
S_{\eta_{a d, c p g}}=\sqrt{\left.\left(\frac{\partial \eta_{a d, c p g}}{\partial \tau_{t}} \cdot S_{\tau_{t}}\right)^{2}+\left(\frac{\partial \eta_{a d, c p g}}{\partial \pi} \cdot S_{t}\right)_{\pi_{t}}\right)^{2}+\left(\frac{\partial \eta_{a d, c p g}}{\partial \bar{C}_{p}} \cdot S_{\bar{C}_{p}}\right)^{2}}
$$

where uncertainty in gas constant, $R_{\mathrm{CO} 2}$, has been neglected. This expression is nondimensionalized by introducing influence coefficients. Each measurement contributing to the calculation of efficiency has associated with it an influence coefficient. This non-dimensional coefficient represents the change in efficiency resulting from a change in that measurement. Neglecting its sign, the influence coefficient is defined as:

$$
C_{*}=\left|\frac{\partial \eta_{a d, c p g}}{\partial(*)} \cdot \frac{(*)}{\eta_{a d, c p g}}\right|
$$

By direct differentiation of Equation 9.10, the influence coefficients associated with efficiency are determined to be:

$$
\begin{gathered}
C_{\tau_{t}}=\frac{\tau_{t}}{1-\pi_{t}^{\frac{R_{c c_{2}}}{C_{p}}} \cdot \frac{1}{\eta_{a d, c p g}}} \\
C_{\pi_{t}}=\frac{\left(1-\tau_{t}\right) \pi_{t}^{\frac{R_{C O_{2}}}{C_{p}}} \cdot \frac{\left(\frac{R_{C O_{2}}}{C_{p}}\right)}{\left(1-\pi_{t}^{\frac{R_{C O_{2}}}{C_{p}}}\right)^{2}} \cdot \frac{\eta_{a d, c p g}}{C_{\bar{C}_{p}}}=\frac{\left(1-\tau_{t}\right) \pi_{t}^{\frac{R_{C O_{2}}}{\bar{C}_{p}}}}{\left(\frac{\left.R_{C O_{2}}\right)^{2}}{\bar{C}_{p}}\right)^{2}} \cdot \frac{\left(R_{C O_{2}}\right) \ln \left(\pi_{t}\right)}{\bar{C}_{p d, c p g}}}{\left(1-\pi_{t}\right)^{2}}
\end{gathered}
$$

With these definitions, Equation 9.11 may be written as,

$$
\frac{S_{\eta_{a d, p p g}}}{\eta_{a d, c p g}}=\sqrt{\left(C_{\tau_{t}} \frac{S_{\tau_{t}}}{\tau_{t}}\right)^{2}+\left(C_{\pi_{t}} \frac{S_{\pi_{t}}}{\pi_{t}}\right)^{2}+\left(C_{\bar{C}_{p}} \frac{S_{\bar{C}_{p}}}{\bar{C}_{p}}\right)^{2}}
$$

The second component of uncertainty is bias, a fixed error affecting measurement accuracy. Non-zero bias is almost always unavoidable and often due to instrumentation design and/or implementation. This error may only be determined by comparing a measured condition 
to its exact value. Because this exact value is not usually known, a reasonable limit is sought to bound this error. Just as precision index, bias is expressed in terms of influence coefficients:

$$
\frac{B_{\eta_{a d, c p g}}}{\eta_{a d, c p g}}=\sqrt{\left(C_{\tau_{t}} \cdot \frac{B_{\tau_{t}}}{\tau_{t}}\right)^{2}+\left(C_{\pi_{t}} \cdot \frac{B_{\pi_{t}}}{\pi_{t}}\right)^{2}+\left(C_{\bar{C}_{p}} \cdot \frac{B_{\bar{C}_{p}}}{\bar{C}_{p}}\right)^{2}}
$$

Precision and bias may be combined to calculate overall uncertainty. The specific calculation depends on the interpretation of uncertainty. The standard interpretation suggests that the error in a particular measurement or calculation will not exceed the calculated value of uncertainty. This statement is made with a given level of confidence, $t_{x x}$, typically $95 \%$ or $99 \%$. Assuming a confidence level, $95 \%$ in this case, the value of $t_{95}$ will depend on the number of degrees of freedom, or number of measurements used to calculate the precision index $\mathrm{S}$.

Infinitely many degrees of freedom yields a $t_{95}$ of 1.96, and 30, a value of 2.04. Following an analysis by Keogh, [9.3], a value of 2.00 is chosen for $t_{95}$ and uncertainty is expressed as:

$$
U_{95}=\sqrt{\left(t_{95} \cdot S\right)^{2}+B^{2}}
$$

Table 9.1 summarizes the pre-test efficiency uncertainty analysis. Included are nominal values of measurements contributing to efficiency, their influence coefficients, precision indices, biases, and levels of uncertainty. The nominal values listed are inferred from full-scale turbine design point calculations and initial experiments prior to those examined in this work. Precision indices and biases for temperature and pressure ratio are calculated from calibration data of Cai [9.4]. A constant bias of $0.20 \%$ is assumed for the specific heat of $\mathrm{CO}_{2}$.

Table 9.1: Pre-Test Efficiency Uncertainty Analysis Summary

\begin{tabular}{|l|l|l|l|l|l|l|l|}
\hline Measurement & Nominal & $C_{*}$ & $S_{*} / *$ & $B_{*} / *$ & $t_{95}$ & $U_{95, a b s}$ & $U_{95, \text { rel }}$ \\
\hline$\tau_{t}$ & 0.867 & 6.51 & $0.04 \%$ & $0.03 \%$ & 2.00 & $0.09 \%$ & $0.07 \%$ \\
\hline$\pi_{t}$ & 0.480 & 1.25 & $0.07 \%$ & $0.25 \%$ & 2.00 & $0.29 \%$ & $0.14 \%$ \\
\hline $\bar{C}_{p}\left(\frac{J}{k g \cdot K}\right)$ & 867.138 & 0.92 & & $0.20 \%$ & 2.00 & $0.20 \%$ & \\
\hline$\eta_{a d, c p g}$ & $90 \%$ & & $0.27 \%$ & $0.40 \%$ & 2.00 & $0.61 \%$ & $0.49 \%$ \\
\hline
\end{tabular}

As seen from this analysis, the estimated absolute uncertainty in turbine aerodynamic adiabatic efficiency is approximately $0.61 \%$. The most critical measurement in terms of uncertainty is seen to be total temperature. Because of the large total temperature ratio influence coefficient, errors in total temperature measurement will be magnified much more so than errors in total pressure measurement. This is not to say that the total pressure measurement is trivial, however, as measurement techniques and transducer behavior is not considered in this analysis.

Another important conclusion of this analysis is the distinction between absolute accuracy and relative precision. As explained previously, bias is a fixed error affecting measurement or calculation accuracy. By comparing back-to-back measurements, fixed bias 
cancels and relative uncertainty is controlled by the measurement or calculation precision index. From Table 9.1, the precision index of efficiency is approximately

$$
S_{\eta_{a d, c p g}}=\frac{S_{\eta_{a d, c p g}}}{\eta_{a d, c p g}} \cdot \eta_{a d, c p g}=0.24 \%
$$

From Equation 9.18, this gives a relative uncertainty level of approximately $0.49 \%$. This means that although any one efficiency measurement is not expected to have absolute uncertainty less than $0.50 \%$, by comparing back-to-back experiments, the change in efficiency should have relative uncertainty within this limit.

\subsection{Real Versus Ideal Gas Models for Enthalpy Evaluation}

As explained in Section 9.2, turbine adiabatic efficiency measures the percentage of ideal work extracted by the turbine during adiabatic operation; it is expressed as the ratio of adiabatic and ideal work. From the first law of thermodynamics assuming no heat transfer,

$$
\delta w=\delta h_{T}+\delta \not
$$

work is given by the change in total enthalpy. (A correction for transient heat transfer effects will be discussed in Section 9.6.) In general, this change in total enthalpy may be expressed as, [9.6],

$$
h_{T_{4}}-h_{T_{5}}=\int_{T_{5}}^{T_{4}} C_{p}(T) d T+\int_{P_{5}}^{P_{4}}\left[v-T\left(\frac{\partial v}{\partial T}\right)_{P}\right] d P+\frac{V_{4}^{2}-V_{5}^{2}}{2}
$$

This is the expression which must be evaluated in determining work. Different gas models, real, ideal, and calorically perfect, differ in their approximation of this expression. What follows is a discussion on these approximations.

The real gas model used in this work takes the form of property tables generated by NIST 14, [9.7]. This code is a compilation of experimental data on gas properties developed by the National Institute of Standards and Technology. This data allows total enthalpy to be evaluated directly from total temperature and pressure.

The next lower level of complexity is the ideal gas model. This model neglects property variation due to changes in pressure. In many cases, especially at low pressure and high temperature, pressure dependence is weak and this assumption is very reasonable. In terms of Equation 9.21, this permits neglect of the second integral. Total enthalpy change may then be expressed in terms of specific heat and total temperature:

$$
h_{T_{4}}-h_{T_{5}}=\int_{T_{5}}^{T_{4}} C_{p}(T) d T_{T}
$$

Note, however, that specific heat is a function of static temperature, thus, its variation between states four and five must be known to evaluate this integral. This turns out to be a complication not avoided by ideal gas assumptions and the integral must be approximated in some way. 
In order to approximate this integral, assumptions about the variation of specific heat with temperature are typically made. Ideal gas Equations 9.4 and 9.5 make use of expressions from Shapiro, [9.1]. Although he does not include the full derivation, he does cite one assumption of this variety:

$$
\int_{T_{T_{5}}}^{T_{T_{4}}} C_{p}(T) d T_{T} \approx \frac{C_{P_{4}}-C_{P_{5}}}{2} \cdot\left(T_{T_{4}}-T_{T_{5}}\right)
$$

This calculation only requires knowledge of specific heat at the initial and final static temperatures, not its variation over the entire temperature range. This is reasonable over a moderate temperature range as pointed out by Shapiro. Perfect gas Equations 9.7 and 9.8 make exclusive use of this assumption. This results in cancellation of specific heat from the numerator and denominator and efficiency may be expressed only in terms of total temperature and pressure ratio and specific heat.

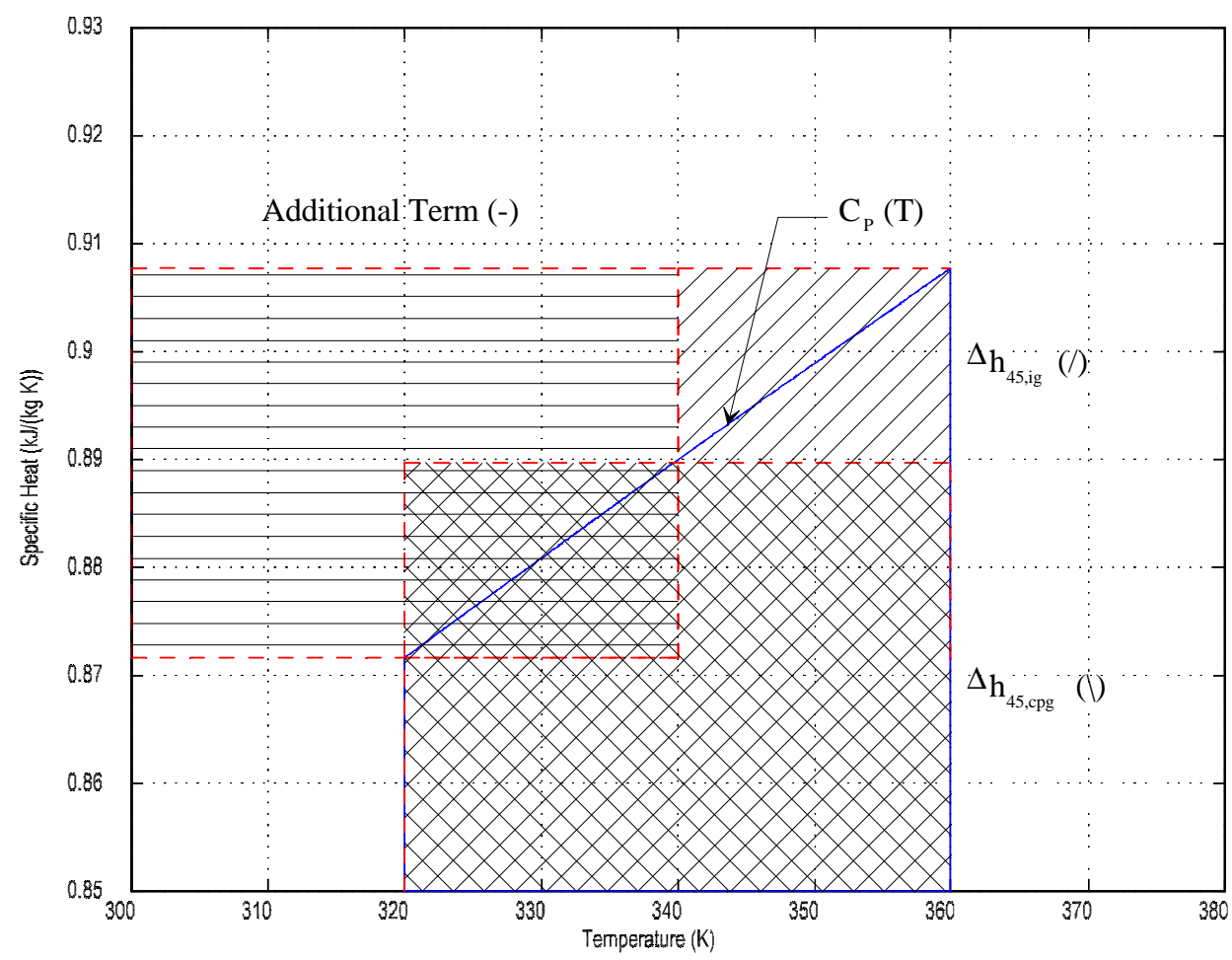

Figure 9.2: Integration of Specific Heat for Enthalpy Evaluation

Figure 9.2 graphically represents the integration of Equation 9.22 assuming ideal and calorically perfect gas behavior. Shown is the actual variation of specific heat of $\mathrm{CO}_{2}$ at constant atmospheric pressure over a range of temperatures typical of the Blowdown Turbine. This variation is calculated using NIST 14. Integration of this quantity assuming ideal gas behavior is represented by Equation 9.4 based on Shapiro's derivation. The first two terms in this equation 
produce the sum of all hatched regions in Figure 9.2. (Realize that the integrals (hatched regions) extend down and over to the $T$ and $C_{P}$ axes.) The last term subtracts the horizontally hatched region labeled Additional Term. This leaves the forward hatched region labeled $\Delta h_{45, i g}$ as the ideal gas approximation of the integral in Equation 9.22. Assuming calorically perfect gas behavior and referring to Equation 9.7, however, specific heat is held constant and the integral in Equation 9.22 is approximated by the backward hatched region in Figure 9.2 labeled $\Delta h_{5, c p g}$.

As seen from Figure 9.2, over the typical range of Blowdown Turbine operating temperatures and at constant atmospheric pressure, specific heat is very close to linear with temperature and varies approximately 5\%. If the relationship was exactly linear, the ideal and calorically perfect gas integrations of specific heat would be identical. As is, they should be very similar. This means efficiency predictions from both models should also be very similar assuming pressure variation does not significantly affect these results. This will be seen in the next section where efficiency calculations take stage.

\subsection{Efficiency Calculation}

Section 9.2 provides the framework for calculating efficiency using real gas properties

and by assuming ideal and calorically perfect gas behavior. In all cases, gas properties including total enthalpy and specific heat are required. These properties are contained in tables versus static temperature and pressure generated using NIST 14, [9.7]. This section discusses the process used to determine the required flow properties and their use in aerodynamic efficiency calculations. The results from all experiments are presented in Chapter 14.

Figure 9.3 shows, on an enthalpy-entropy diagram, where flow properties are evaluated. Quantities known from experimental data are shown in bold face and those evaluated, in normal face. Upstream total enthalpy, required to calculate efficiency, and upstream entropy, required to iterate for downstream isentropic total temperature (enthalpy), are interpolated directly from the property tables using upstream total conditions. Upstream dynamic pressure is typically $0.5 \%$ of measured upstream total pressure meaning the upstream Mach number is very low and total and static conditions are essentially interchangeable [9.6]. For this reason, upstream specific heat, for use in calculating efficiency assuming ideal and calorically perfect gas behavior, is also interpolated directly from total conditions. Downstream, the Mach number is significantly higher, however, and this approximation is not valid.

Once all upstream conditions are known, downstream total enthalpy and entropy are interpolated similarly. Interpolation of specific heat, however, requires static temperature and pressure as the Mach number in this region is significantly higher than upstream. Static pressure is known and static temperature is iterated using the following expression:

$$
T_{5}^{*}=\frac{T_{T_{5}}}{\left(\frac{P_{T_{5}}}{P_{5}}\right)^{\frac{R_{C O_{2}}}{C_{P_{5}}^{*}}}}
$$

where * indicates iteration. 


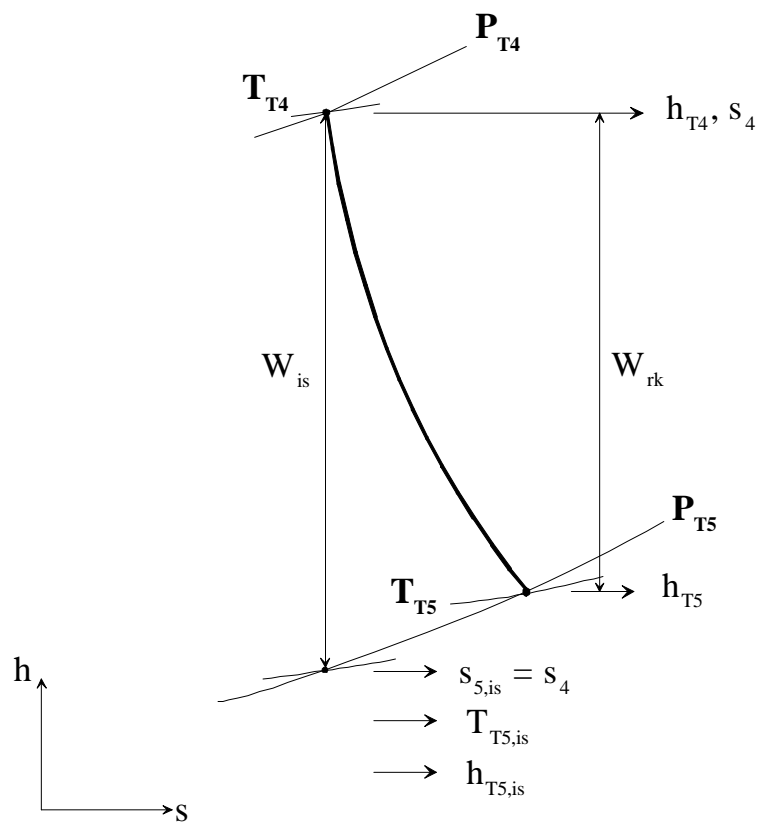

Figure 9.3: Enthalpy-Entropy Diagram Showing Evaluation of Gas Properties for Efficiency Calcuations.

With all upstream and downstream conditions known, only the ideal expansion total enthalpy is required for efficiency calculations. This enthalpy is interpolated using the downstream isentropic total temperature, or that temperature which produces an entropy equal to the upstream value.

Once all conditions are known, efficiency may be calculated as described in Section 9.2. Figure 9.4 shows these calculations for experiment 024, a simulation of the full-scale turbine design point at minimum tip clearance. Here, efficiency is referenced to the Craig and Cox empirical efficiency estimate at the smaller rotor tip clearance. presented in Jacobs, [9.8]. Numerous characteristics of this figure deserve special attention and these are where focus now shifts.

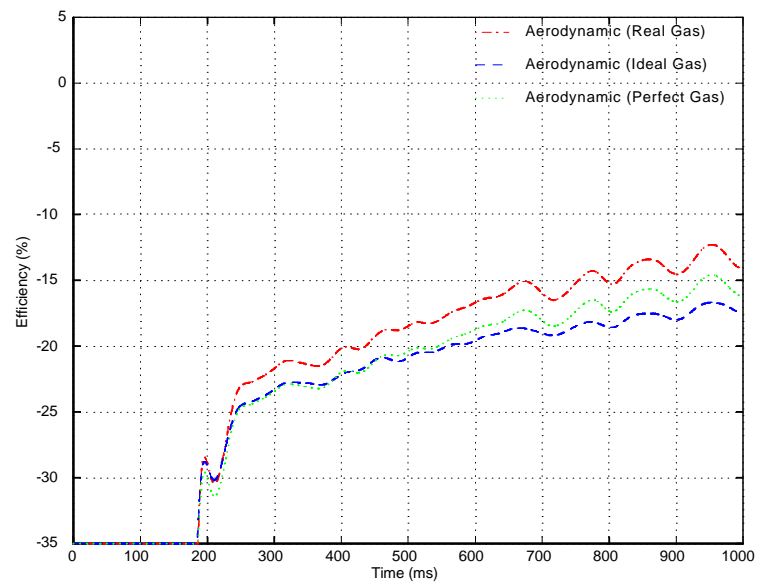

Figure 9.4: Experiment 024 Aerodynamic Rake Efficiency 
First, the efficiency predictions are very similar both in shape and magnitude. The real gas calculation, presumed to be most accurate, is consistently 2-3\% greater than the ideal gas calculation. The perfect gas calculation lies mid-way between the real and ideal gas calculations. This trend is not expected. In neglecting property variations due to pressure change, the ideal gas prediction should deviate somewhat from the real gas calculation. Further neglecting changes in specific heat, the perfect gas prediction should deviate even further. The reversal of ideal and perfect gas predictions, however, departs from this reasoning. This trend, which is reasonably consistent between experiments, is not understood.

Second, the efficiency predictions are not constant in the region of similarity. As seen in Chapter 13, Reynolds number is never constant and equal to design, however, its variation is small in terms of significantly affecting performance calculations. The trend in corrected speed is similar. It is generally constant and equal to design up to $1000 \mathrm{~ms}$ within $5-7 \%$. The remaining non-dimensional similarity parameters are relatively constant in the region of 300$1000 \mathrm{~ms}$. This suggests similarity may be reasonably assumed up to $1000 \mathrm{~ms}$ into the experiment. As seen in Figure 9.4, efficiency predictions increase up to near $800 \mathrm{~ms}$; it is only between $800-1000 \mathrm{~ms}$ that they are moderately constant. This is, however, an encouraging feature of the aerodynamic efficiency measurement as it demonstrates measurement resolution. During the experiment, upstream total temperature and pressure decrease as the supply tank empties causing the simulated operating point to "travel" slightly in time. This, in turn, causes drift in expected efficiency. On the other hand, this presents a problem in assigning a single value of efficiency for each experiment. Because similarity is feasible up to $1000 \mathrm{~ms}$, however, it is not thought irrational to concentrate on this latter period of time. Therefore, single value aerodynamic efficiencies presented in the remainder of this work will reflect this period of efficiency prediction.

Another obvious characteristic of the efficiency predictions in Figure 9.4 is the low frequency content of magnitude 2-3\%. This is reminiscent of Section 8.7 where nozzle wake measurements were discussed. A quick calculation verifies that this frequency is very near the frequency observed in the downstream total temperature and pressure traces presented in Section 8.7. Furthermore, the magnitude of these variations in percent is very near the magnitude of the total temperature variations in degrees Kelvin. These total temperature and pressure sinusoidal variations simply filter down through enthalpy calculations and appear in efficiency. If they were overlaid, it would be seen that they are $180^{\circ}$ out of phase. Inside a nozzle wake, temperature is reduced due to heat transfer to the nozzle. In terms of efficiency, this is seen as a lower temperature ratio for the same pressure ratio, or, a greater work extraction. This produces efficiency peaks over total temperature troughs. This further complicates assigning a single value of efficiency for a given experiment. The solution will be to average this sinusoidal variation.

The final observation to be made from Figure 9.4 is the label, "Experiment 024 Aerodynamic Rake Efficiency." Rake refers to the efficiency based on measured total temperature and pressure. It is not the adiabatic efficiency because of the transient nature of the experiment. The test is of sufficiently short duration that the turbine stage does not reach thermal equilibrium. A correction for the heat transfer must therefore be applied to estimate adiabatic efficiency. This correction is the topic of the next section. 


\subsection{Heat Transfer Correction}

Referring back to Figure 9.1, turbine adiabatic work is labeled $W_{a d}$. This work is required in calculating turbine adiabatic efficiency from Equation 9.1. This is not the work calculated from blowdown experimental data as described in the previous section however. This calculation, labeled $W_{r k}$ in Figure 9.1, includes heat transferred from the flow to the turbine as can be seen by rearranging the first law of thermodynamics as stated in Equation 9.11:

$$
\delta h_{T}=\delta w-\delta q
$$

In the previous section it was assumed that there was no heat transfer so that work could be expressed as a change in total enthalpy. Because of the transient nature of the blowdown experiment, however, heat is transferred from the flow to the turbine. By Equation 9.16, written as a statement of flow energy, $\delta q$ is negative and turbine work is less than the change in total enthalpy. Rake efficiency predictions uncorrected for heat transfer will thus overestimate actual turbine performance. The purpose of this section is to quantify the required heat transfer correction to rake efficiency.

According to Shang,[9.9], the difference between the adiabatic and measured exit total enthalpies is generally not equal to the heat transferred from the flow to the turbine. This is a result of the path dependent heat transfer process. To relate these enthalpies and heat transfer quantity it is assumed that the heat transfer takes place at an equivalent temperature $T^{*}$ and the entropy generated is reversible. Then, according to Cai [9.4]:

$$
h_{T_{5, a d}}-h_{T_{5}}=Q\left(\frac{T_{5}}{T^{*}}\right), \quad Q \geq 0
$$

Adiabatic efficiency is then related to rake efficiency and heat transfer by the expression:

$$
\eta_{a d}=\eta_{r k}-\frac{Q}{h_{T_{4}}-h_{T_{5, i s}}}\left(\frac{T_{5}}{T^{*}}\right)=\eta_{r k}+\Delta \eta_{r k, h t}
$$

also from Cai [9.4]. The temperature $T^{*}$ is unknown. In reality, heat is transferred from the flow to the turbine at different temperatures throughout the facility. In calculating the resulting entropy generation, however, it is convenient to assume an equivalent temperature. For the purpose of this analysis, $T^{*}$ will be assumed to equal the average of the inlet total and exit static temperatures at $400 \mathrm{~ms}$. This is the earliest time at which both aerodynamic and mechanical efficiency predictions are valid and according to Guenette [9.10], these are the most narrow bounds able to be assigned to this equivalent temperature. This provides a procedure for correcting aerodynamic rake efficiency measurements given the value of $\Delta \eta_{r k, h t}$. The task is now to determine this value.

Many methods have been proposed to estimate the heat transfer during a blowdown experiment. As explained by Guenette [9.10], measurements of turbine work by aerodynamic and mechanical approaches may be compared and heat transfer inferred. This is possible because mechanical measurements of turbine work are influenced much less by heat transfer than are aerodynamic measurements. (This will be shown in Section 10.4.) This heat transfer 
may also be determined by numerical computation. A one-dimensional compressible flow analysis utilizing Reynolds' analogy or a two-dimensional flow analysis with heat transfer may be employed [9.10]. Alternatively, this heat transfer may be determined experimentally by direct measurement [9.10]. The approach taken here, however, is to compare rake efficiency measurements between experiments simulating the same full-scale turbine operating point, but at different inlet temperatures. This will allow direct determination of $\Delta \eta_{r k, h t}$ for a given inlet temperature.

Table 9.2 summarizes the experiments compared in determining the rake efficiency correction for heat transfer. Experiments 034, 038, and 039 all simulate near full-scale turbine Reynolds number, corrected speed, and pressure ratio, each at a different inlet total temperature. The same full-scale turbine operating point is simulated in each case, thus, adiabatic efficiency should remain constant. Because the inlet temperature varies, however, different levels of heat transfer occur causing different levels of rake efficiency over-prediction by Equation 9.27. The lowest temperature experiment, 038 , should predict the lowest rake efficiency followed by 039 and 034. The result of this comparison should be $\Delta \eta_{r k, h t}$ versus inlet total temperature.

Table 9.2: Experiments Compared to Determine Rake Efficiency Heat Transfer Correction

\begin{tabular}{|l|l|l|l|}
\hline Experiment & 034 & 038 & 039 \\
\hline$\pi_{t}$ & $95.8 \%$ & $96.0 \%$ & $96.0 \%$ \\
\hline$N_{c o r}$ & $103.2 \%$ & $101.7 \%$ & $105.4 \%$ \\
\hline$R_{e}$ & $107.7 \%$ & $104.7 \%$ & $106.1 \%$ \\
\hline$\gamma_{4}$ & $99.9 \%$ & $100.8 \%$ & $100.3 \%$ \\
\hline$P_{T_{4}}, \quad(\mathrm{psia})$ & 104.5 & 94.7 & 100.4 \\
\hline$T_{T_{4}}, \quad\left({ }^{\circ} \mathrm{F}\right)$ & 220 & 175 & 200 \\
\hline
\end{tabular}

Figure 9.5 shows the difference between adiabatic and predicted rake efficiencies for these experiments; this is exactly $\Delta \eta_{r k, h t}$. As expected, all experiments over predict adiabatic efficiency as $\Delta \eta_{r k, h t}$ is less than zero for each experiment. In addition, the lowest inlet temperature experiment, 038, predicts the lowest rake efficiency, requiring the smallest correction, and as inlet temperature increases, so does the predicted rake efficiency and required absolute correction. Because these experiments simulate the same full-scale turbine operating point as shown in Table 9.2, this change in rake efficiency with inlet temperature is entirely due to increasing levels of heat transfer. This assumes, of course, the error associated with the repeatability of rake efficiency prediction is much less than the shown change in rake efficiency due to heat transfer. This is in fact the case. Unfortunately, the heat transfer correction determined from a change in efficiency will have its precision index magnified due to the limited repeatability of each efficiency prediction. The resulting uncertainty in this correction as well as in aerodynamic adiabatic efficiency will be discussed in the next section. 


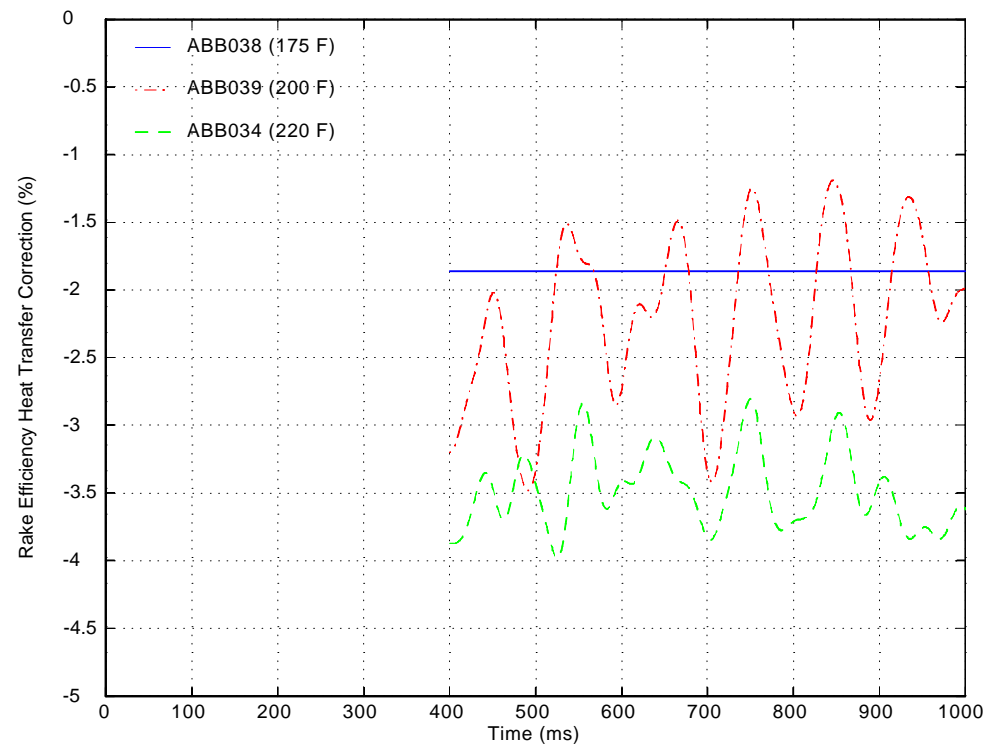

Figure 9.5: Rake Efficiency Correction for Heat Transfer

How is adiabatic efficiency determined allowing determination of these corrections? Keogh, [9.3], in a pre-test analysis of mechanical adiabatic efficiency measurement, estimates the magnitude of the heat transfer at an inlet temperature of $170{ }^{\circ} \mathrm{F}$ to be approximately $2.00 \%$ of the ideal total enthalpy drop across the turbine stage. Assuming this heat transfer takes place at a temperature $T^{*}$ as previously explained, this corresponds to a value of $\Delta \eta_{r k, h t, 170^{\circ} F}$ of approximately $-1.86 \%$. Because the conditions for this estimate are very near the inlet conditions of experiment 038 , it is assumed as a control experiment that 038 over-predicts adiabatic efficiency by exactly $1.86 \%$. Relative changes in rake efficiency between experiments 038 and 039 and experiments 038 and 034 are then determined by subtracting their rake efficiency predictions. Experiment 039, conducted at an inlet temperature of $200^{\circ} \mathrm{F}$, thus over-predicts adiabatic efficiency by approximately $2.23 \%$ and experiment 034 , at an inlet temperature of 220 ${ }^{\circ} \mathrm{F}$, by approximately $3.49 \%$. Table 9.3 summarizes the aerodynamic rake efficiency heat transfer corrections.

Table 9.3: Aerodynamic Rake Efficiency Heat Transfer Correction Summary

\begin{tabular}{|l|l|l|l|}
\hline Experiment & 034 & 038 & 039 \\
\hline $\mathrm{TT} 4,[\mathrm{~F}]$ & 220 & 175 & 200 \\
\hline $\mathrm{T}^{*},[\mathrm{~F}]$ & 134 & 104 & 120 \\
\hline$\frac{Q}{h_{T_{4}}-h_{T_{s}, i s}}$ & $3.75 \%$ & $2.00 \%$ & $2.40 \%$ \\
\hline$\Delta \eta_{r k, h t}$ & & & \\
\hline
\end{tabular}




\subsection{Post-Test Efficiency Uncertainty Analysis}

According to the pre-test uncertainty analysis presented in Section 9.3, the absolute uncertainty in aerodynamic adiabatic efficiency prediction is estimated to be $0.61 \%$ from instrument calibration data. The repeatability of this prediction is estimated to be approximately $0.49 \%$. Because bias errors remain unknown, the estimated absolute uncertainty can not be verified from experimental data. The actual repeatability of this prediction may be determined, however, and compared to the pre-test estimate. That is the purpose of this section.

Experiments 025 and 026 are near exact duplicates meant to test the repeatability of efficiency measurement. Table 9.4 summarizes these experiments and their aerodynamic rake efficiency predictions, precision indices, and repeatability. Also included are these statistics for the heat transfer correction determined in the previous section as well as for aerodynamic adiabatic efficiency.

For a small number of samples, two in this case, the precision index is defined as follows, [9.2]:

$$
S=\frac{\bar{R}}{d_{2}^{*}}
$$

where $\bar{R}$ represents the average data scatter and $d_{2}^{*}$ accounts for the number of sample degrees of freedom. A value of 1.28 is used for $d_{2}^{*}$ in this case [9.1]. This data shows that aerodynamic rake efficiency may be measured with repeatability between $0.60 \%$ and $0.65 \%$ depending on the gas model employed.

Table 9.4: Post-Test Efficiency Uncertainty Summary

\begin{tabular}{|l|l|l|l|l|l|l|}
\hline & 025 & 026 & $C_{*}$ & $S_{*} / *$ & $T_{95}$ & $U_{95, r e l}$ \\
\hline$\pi_{t}$ & $95.6 \%$ & $95.8 \%$ & & & & \\
\hline$N_{c o r}$ & $102.8 \%$ & $102.0 \%$ & & & & \\
\hline$R_{e}$ & $106.7 \%$ & $110.7 \%$ & & & & \\
\hline$\gamma_{4}$ & $99.9 \%$ & $100 * \%$ & & & & \\
\hline$\eta_{r k, r g}$ & $-13.12 \%$ & $-13.53 \%$ & & $0.38 \%$ & 2.00 & $0.65 \%$ \\
\hline$\eta_{r k, i g}$ & $-16.80 \%$ & $-17.12 \%$ & & $0.31 \%$ & 2.00 & $0.60 \%$ \\
\hline$\eta_{r k, c p g}$ & $-15.32 \%$ & $-15.71 \%$ & 1.00 .044 & $0.37 \%$ & 2.00 & $0.61 \%$ \\
\hline$\eta_{r k, h t, 220^{\circ} F}$ & $-3.49 \%$ & $-3.49 \%$ & & $29.05 \%$ & 2.00 & $2.03 \%$ \\
\hline$\eta_{a d, c p g}$ & $-18.77 \%$ & $-19.16 \%$ & & $1.34 \%$ & 2.00 & $2.12 \%$ \\
\hline
\end{tabular}

Table 9.4 also summarizes the uncertainty in correcting rake efficiency predictions for heat transfer. As explained in the previous section, the required heat transfer correction is determined by comparing experiments simulating the same full-scale turbine operating point, but at different inlet temperatures. By this method, the precision index of the resulting heat transfer correction is magnified due to the limited repeatability of each efficiency prediction used to calculate it. The result is a $2.03 \%$ relative uncertainty in the estimated $3.49 \%$ heat transfer correction. And when this correction is applied to perfect gas rake efficiency prediction, the 
relative uncertainty in adiabatic efficiency becomes $2.12 \%$. (The correction is applied to the perfect gas calculation to allow direct comparison to the pre-test uncertainty analysis.) There is also a heat transfer correction bias associated with the equivalent temperature assumption and heat load estimates. Because only relative uncertainties are being examined here, however, this bias does not affect the results.

This analysis shows that aerodynamic rake efficiency may be measured with relative uncertainty between $0.60 \%$ and $0.65 \%$. This uncertainty is near that estimated in the pre-test analysis for aerodynamic adiabatic efficiency. When these rake efficiency predictions are corrected for heat transfer, however, the relative uncertainty in adiabatic efficiency becomes approximately $2.12 \%$. This uncertainty is much higher than pre-test estimates and does not come close to meeting the uncertainty objectives of this work. The reason for this shortfall is the method used to estimate the required heat transfer correction. By comparing experiments simulating the same operating point but at different inlet temperatures, the change in rake efficiency due to heat transfer is readily available. The uncertainty of this correction, however, is subject to the repeatability of the rake efficiency predictions. The result is a correction uncertainty over $50 \%$ of the magnitude of the correction itself. This significantly increases the relative uncertainty of the adiabatic efficiency prediction determined from this correction. The solution to this problem is to determine the required heat transfer correction by analysis rather than experimental data. While this is likely to reduce precision errors in this correction it has the potential to increase bias errors and this may or may not by easily estimated with required accuracy. This analysis thus concludes that mostly due to the difficulty in estimating heat transfer, the aerodynamic approach to measuring turbine adiabatic efficiency does not produce absolute or relative uncertainties consistent with the objectives of this work. It remains to be seen in the next chapter whether or not the mechanical approach will be successful in meeting these objectives.

\subsection{Conclusions}

This chapter has discussed turbine aerodynamic efficiency measurement. The discussion began with a pre-test efficiency uncertainty analysis. The objective of this analysis was to uncover significant sources of uncertainty in the calculation of efficiency and estimate the expected absolute and relative uncertainties in efficiency calculation. It was determined that total temperature is the key measurement in calculating efficiency by the aerodynamic approach in terms of uncertainty due to the large total temperature ratio influence coefficient. Absolute and relative uncertainties were estimated to be $0.61 \%$ and $0.49 \%$ respectively.

This discussion was followed by a comparison of real and ideal gas models in terms of the approximations they use in calculating turbine work. This comparison was meant to explore the use of ideal gas assumptions in characterizing turbine performance. It was shown that over the temperature range of operation of the Blowdown Turbine, specific heat variation with temperature is near linear and ideal gas calculations should agree reasonably with real gas calculations.

The next topic of discussion was efficiency calculation from experimental data. This included efficiency predictions from experiment 024 showing an approximate $2 \%-3 \%$ deviation between real and ideal gas calculations. This baseline experiment produced near constant rake efficiencies of $-14 \%$ to $-18 \%$ between $800-1000 \mathrm{~ms}$. (Efficiency is referenced to the Craig and Cox empirical efficiency estimate at the smaller rotor tip clearance discussed by Jacobs [9.8].) 
This was followed by an analysis of efficiency prediction to determined the required aerodynamic rake efficiency heat transfer correction. It was shown that at an inlet temperature of $220^{\circ} \mathrm{F}$, aerodynamic rake efficiency must be corrected by approximately $-3.49 \%$ to account for heat transfer effects.

This chapter concludes with a post-test efficiency uncertainty analysis intended to determine adiabatic efficiency measurement relative uncertainty and verify the pre-test estimate. It was shown that rake efficiency predictions are repeatable within $0.60 \%$ to $0.65 \%$ depending on the gas model used. The relative uncertainty in the heat transfer correction was determined to be $2.03 \%$, however, as the correction was determined by comparing rake efficiency predictions. This resulted in an aerodynamic adiabatic efficiency relative uncertainty of $2.12 \%$, much higher than the $0.49 \%$ pre-test estimate. It was finally concluded that the aerodynamic approach is not capable of precise adiabatic efficiency measurement. This is primarily a result of the approach's sensitivity to non-adiabatic effects and the difficulty in estimating them. 


\subsection{References}

[9.1] Shapiro, A.H., The Dynamics and Thermodynamics of Compressible Fluid Flow, Volume 1. The Ronald Press Company, New York, NY. 1954.

[9.2] ASME/ANSI MFC-2M-1983. Measurement Uncertainty for Fluid Flow in Closed Conduits. An American National Standard, The Americal Society of Mechanical Engineers. 1988

[9.3] Keogh, R., Shaft Efficiecy Measurements of a Fully Scaled Turbine. Masters Thesis, Massachusetts Institute of Technology. 1988.

[9.4] Cai, Y., Aerodynamic Performance Measurements in a Fully Scaled Turbine., Masters Thesis, Massachusetts Institute of Technology. 1988.

[9.5] Friend, D.G., Private Correspondence About NIST14. Fluid Mixtures Data Center, NIST Thermophysics Division 838.02, Bolder, CO. Summer 1997.

[9.6] Grepin, L.M., Aerodynamic Measurement and Analysis of the FLow in an Uncooled Turbine Stage. Masters Thesis, Massachusetts Institute of Technology. 1988.

[9.7] NIST 14. NIST Mixture Property Database. Standard Reference Database 14, Version 9.08. Fluid Mixtures Data Center, NIST Thermophysics Division 838.02, Bolder, CO. 1992.

[9.8] Jacobs, J.J., Efficiency Measurements of a Single Stage Turbine in a Short Duration Facility. Masters Thesis, Massachusetts Institute of Technology, 1998.

[9.9] Shang, T., Influence of Inlet Distortion on Turbine Heat Transfer. Ph.D. Thesis, Massachusetts Institute of Technology. 1995.

[9.10] Guenette, G.R., Epstein, A.H., Ito, E. Turbine Aerodynamic Performance Measurements in Short Duration Facilities. AIAA/ASME/SAE/ASEE $25^{\text {th }}$ Joint Propulsion Conference, AIAA-89-2690. 1989. 


\section{Mechanical ('Brake') Measurement of Adiabatic Efficiency}

By Jason J. Jacobs

\subsection{Introduction}

This chapter discusses measurement of turbine adiabatic efficiency using the mechanical approach described in Section 9.2. This approach will determine turbine adiabatic efficiency from turbine torque, speed, and mass flow as well as upstream and downstream total temperature and pressure measurements.

The discussion begins with a pre-test efficiency uncertainty analysis. This analysis, intended to estimate the expected uncertainty in mechanical adiabatic efficiency measurement, follows directly from the analysis presented in Section 9.2 and is based on the uncertainty analysis method outlined in Reference [10.1]. This analysis is followed by a discussion on the actual efficiency calculation. This includes transient corrections accounting for rotor acceleration and tunnel mass storage and presentation of efficiency calculations for experiment 024, a baseline full-scale turbine design point simulation. The next section will investigate the effect of heat transfer on mechanical efficiency measurement. This is followed by a discussion on additional processes thought to affect this measurement but who are not yet accounted for and thought to be of second order. This chapter will conclude with a post-test efficiency uncertainty analysis focusing on the repeatability of mechanical efficiency measurement.

\subsection{Pre-Test Efficiency Uncertainty Analysis}

As discussed in Section 9.2, uncertainty consists of random error, represented by precision index $S$, and fixed error, represented by bias $B$. Both are expressed in terms of a Taylor series expansion and involve influence coefficients. Again, assuming perfect gas behavior for algebraic simplicity, mechanical adiabatic efficiency may be written as:

$$
\eta_{a d, b r k, p e r f}=\frac{\mathrm{T} \cdot \omega}{\dot{m}_{t} \bar{C}_{P} T_{T}\left(1-\pi_{t}{ }^{\frac{R_{C O_{2}}}{\bar{C}_{p}}}\right)}
$$

Efficiency precision index and bias then become:

$$
\begin{gathered}
\frac{S_{\eta_{a d, b r k, c p g}}}{\eta_{a d, b r k, c p g}}=\sqrt{\left(C_{\mathrm{T}} \cdot \frac{S_{\mathrm{T}}}{\mathrm{T}}\right)^{2}+\left(C_{\dot{m}_{t}} \cdot \frac{S_{\dot{m}_{t}}}{\dot{m}_{t}}\right)^{2}+\left(C_{\bar{C}_{P}} \cdot \frac{S_{\bar{C}_{P}}}{\bar{C}_{P}}\right)^{2}+\left(C_{T_{T_{4}}} \cdot \frac{S_{T_{T_{4}}}}{T_{T_{4}}}\right)^{2}+\left(C_{\pi_{t}} \cdot \frac{S_{\pi_{t}}}{\pi_{t}}\right)^{2}} \\
\frac{B_{\eta_{a d, b r k, c p s}}}{\eta_{a d, b r k, c p g}}=\sqrt{\left(C_{\mathrm{T}} \cdot \frac{B_{\mathrm{T}}}{\mathrm{T}}\right)^{2}+\left(C_{\dot{m}_{t}} \cdot \frac{B_{\dot{m}_{t}}}{\dot{m}_{t}}\right)^{2}+\left(C_{\bar{C}_{P}} \cdot \frac{B_{\bar{C}_{P}}}{\bar{C}_{P}}\right)^{2}+\left(C_{T_{T_{4}}} \cdot \frac{B_{T_{T_{4}}}}{T_{T_{4}}}\right)^{2}+\left(C_{\pi_{t}} \cdot \frac{B_{\pi_{t}}}{\pi_{t}}\right)^{2}}
\end{gathered}
$$


where uncertainties in rotor speed, $\omega$, and gas constant, $R_{\mathrm{CO} 2}$, have been neglected. The influence coefficients, $C_{*}$, come from direct differentiation of Equation 5.1. Ignoring their sign, these expressions as derived by Keogh are,

$$
\begin{gathered}
C_{\mathrm{T}}=C_{\dot{m}_{t}}=C_{T_{T_{4}}}=1 \\
C_{\bar{C}_{P}}=1+\ln \left(\pi_{t}\right) \cdot \frac{\pi_{t}^{\frac{R_{C O_{2}}}{\bar{C}_{P}}} \cdot R_{C O_{2}}}{\bar{C}_{P} \cdot\left(1-\pi_{t}^{\frac{R_{C O_{2}}}{\bar{C}_{P}}}\right)} \\
C_{\pi_{t}}=\frac{\pi_{t}^{\frac{R_{C O_{2}}}{\overline{\bar{P}}_{P}}} \cdot R_{C O_{2}}}{\bar{C}_{P} \cdot\left(1-\pi_{t}^{\frac{R_{C O_{2}}}{\overline{\bar{P}}_{P}}}\right)}
\end{gathered}
$$

Table 10.1 summarizes the pre-test mechanical efficiency uncertainty analysis. Nominal values of those measurements in Equation (10.1) contributing to efficiency are included. These are determined from design point calculations and experiments prior to those examined in this work. Precision indices and biases for torque and mass flow are taken from calibration data analyses by Keogh [2]. These uncertainty components for total temperature and pressure ratio come from similar analyses by Cai [10.3]. As in Section 9.2, a constant bias of $0.20 \%$ is assumed to account for errors in determining the specific heat of $\mathrm{CO}_{2}$ as suggested by Friend [10.4]. Finally, uncertainty is calculated as in Section 9.2, Equation 9.9, again assuming a value of 2.00 for $t_{95}$.

Table 10.1: Pre-Test Efficiency Uncertainty Analysis Summary

\begin{tabular}{|l|l|l|l|l|l|l|l|}
\hline Measurement & Nominal & $C_{*}$ & $S_{*} / *$ & $B_{*} / *$ & $t_{95}$ & $U_{95, \text { abs }}$ & $U_{95, \text { rel }}$ \\
\hline $\mathrm{T},(\mathrm{N} \cdot \mathrm{m})$ & 1992.00 & 1.00 & $0.13 \%$ & $0.10 \%$ & 2.00 & $0.28 \%$ & $0.26 \%$ \\
\hline$\omega,(\mathrm{rps})$ & 87.08 & & & & & & \\
\hline$\dot{m}_{t},(\mathrm{~kg} / \mathrm{s})$ & 25.00 & 1.00 & $0.13 \%$ & $0.33 \%$ & 2.00 & $0.42 \%$ & $0.26 \%$ \\
\hline $\bar{C}_{P},(\mathrm{~J} /(\mathrm{kg} \mathrm{K}))$ & 866.06 & 0.08 & & $0.20 \%$ & 2.00 & $0.20 \%$ & \\
\hline$T_{T_{4}},(\mathrm{~K})$ & 378.00 & 1.00 & $0.03 \%$ & $0.20 \%$ & 2.00 & $0.06 \%$ & $0.06 \%$ \\
\hline$\pi_{t}$ & 0.48 & 1.26 & $0.07 \%$ & $0.25 \%$ & 2.00 & $0.29 \%$ & $0.14 \%$ \\
\hline$R_{C O_{2}},(\mathrm{~J} /(\mathrm{kg} \mathrm{K}))$ & 188.92 & & & & & & \\
\hline$\eta_{a d, b r k, c p g}$ & $90 \%$ & & $0.21 \%$ & $0.47 \%$ & 2.00 & $0.56 \%$ & $0.37 \%$ \\
\hline
\end{tabular}


From this analysis, the estimated absolute uncertainty in mechanical adiabatic efficiency measurement is $0.56 \%$. This is very close to the $0.61 \%$ pre-test estimate of aerodynamic adiabatic efficiency absolute uncertainty from Section 9.2. In this case, however, a larger percentage of absolute uncertainty is due to fixed bias error. The precision index for mechanical efficiency measurement is approximately 0.19\%. (Refer to Equation 9.10.) By Equation 9.9, this corresponds to a relative uncertainty of approximately $0.37 \%$. This is slightly lower than the $0.49 \%$ pre-test estimate of aerodynamic adiabatic efficiency relative uncertainty and much less than the post-test $2.12 \%$ estimate. This indicates the mechanical efficiency measurement, although containing approximately the same absolute uncertainty, will be more repeatable than the aerodynamic efficiency measurement. More discussion on this conclusion will follow the post-test mechanical efficiency uncertainty analysis presented in Section 10.6.

\subsection{Efficiency Calculation}

\subsubsection{Introduction}

This section will describe the calculation of efficiency using the mechanical approach as outlined in Section 9.2. Necessary corrections to this approach accounting for rotor acceleration and tunnel mass storage will be discussed. Reference will also be made to Section 9.4 concerning determination of flow properties required for this calculation. The section will end with the presentation of efficiency calculations for experiment 024, a baseline full-scale turbine design point simulation. This is the same experiment used in Section 9.4 to first present aerodynamic rake efficiency calculations.

Section 9.3 discusses real versus ideal gas models for calculation of turbine work. It shows that over the range of temperatures encountered in the Blowdown Turbine, these models should produce similar results. This is verified by comparison of efficiency calculations from each model in Section 9.4. For this reason and because the real gas model is presumed to be most accurate, ideal turbine work for use in mechanical efficiency calculations will be determined assuming real gas behavior. Mechanical efficiency may then be expressed as:

$$
\eta_{b r k}=\frac{\mathrm{T} \cdot \omega}{\dot{m}_{t}\left(h_{T_{4}}-h_{T_{5}, i s}\right)}
$$

This expression neglects important transient effects of the Blowdown Turbine environment however. Most importantly, it assumes a constant rotor rotational speed. During a blowdown experiment, mechanical speed varies in time and this must be taken into account. Furthermore, the mass flow required for this calculation may deviate significantly from that directly measured from the venturi nozzle. This too must be accounted for. The following two subsections discuss these corrections.

\subsubsection{Power Correction}

Thus far, power has been expressed as the product of turbine torque and speed. But, this simple expression neglects rotor acceleration. To correct for this transient effect, an additional term must be added to the power expression; the result is:

$$
\mathrm{P}=\mathrm{T} \cdot \omega+I \cdot \frac{d \omega}{d t} \cdot \omega
$$


where $I$ is the moment of inertia of all rotating parts. These parts include the rotor discs and blades, the shaft, and the eddy current brake drum. This inertia was measured experimentally by so called brake tests. A brake test is identical to a blowdown experiment except the supply tank is not pressurized and the fast-acting valve is not armed. In this case, the turbine produces zero power and inertia may be found from Equation 9.8 as turbine torque and speed are measured and acceleration calculated. From numerous brake tests at different speeds and brake settings, the inertia was found to be $1.8085 \mathrm{~kg} \mathrm{~m}^{2}$ with standard deviation $0.13 \%$. This work is discussed in more detail by Keogh [10.2].

Figure 10.1 shows raw and corrected power for experiment 024 displaying the effect of this power correction. Both traces have been passed through a Butterworth lowpass filter with cutoff frequency approximately $25 \mathrm{~Hz}$. Before approximately $300 \mathrm{~ms}$, the rotor is accelerating and corrected power exceeds uncorrected. Beyond $300 \mathrm{~ms}$, the rotor is decelerated by the eddy current brake in order to hold corrected speed constant as inlet temperature decreases. This deceleration accounts for as much as a 10\% decrease in turbine power between 300-1600 ms.

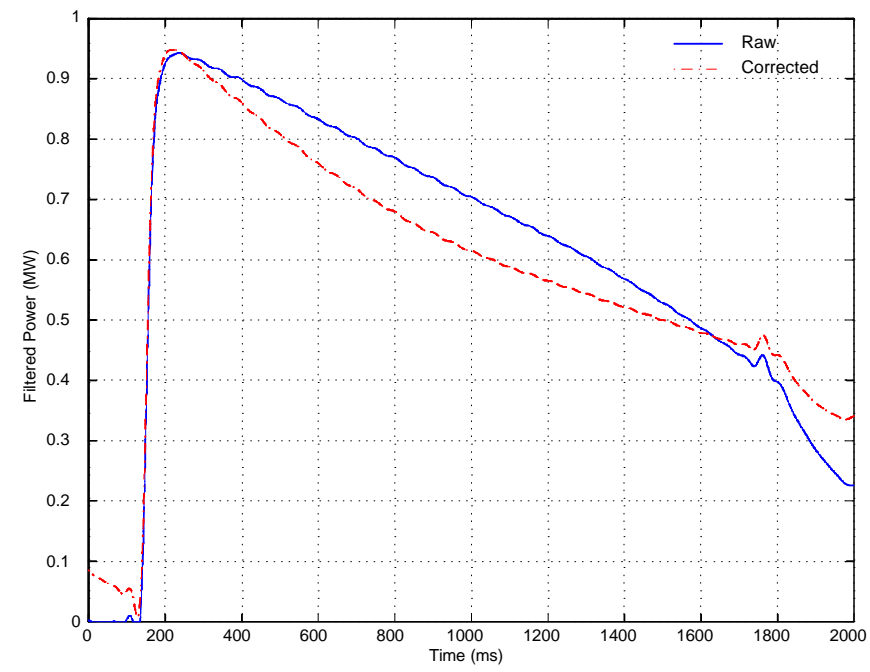

Figure 10.1: Turbine Power Correction for Rotor Acceleration

\subsubsection{Tunnel Mass Storage Correction}

The purpose of the critical-flow venturi is to measure turbine mass flow. This measurement, however, is taken at the nozzle throat located inside the dump tank whereas the measurement of interest is through the turbine stage. These measurements may differ because of the volume separating the turbine stage and nozzle throat. Density variations in these regions will result in mass storage. Turbine and nozzle mass flows are then related by the following expression:

$$
\dot{m}_{t}=\dot{m}_{n}+\frac{d M_{s}}{d t}
$$


Stored mass flow must then be estimated to determine turbine mass flow from venturi measurements.

To estimate stored mass flow, the equation of state incorporating compressibility factor is written in terms of mass:

$$
M_{s}=\frac{P}{T} \frac{V}{R_{c o_{2}} Z}
$$

This expression is then differentiated in time:

$$
\frac{d M_{s}}{d t}=\frac{V}{R_{\mathrm{CO}_{2}} Z}\left(\frac{1}{T}\right) \frac{d P}{d t}-\frac{V}{R_{\mathrm{CO}_{2}} Z}\left(\frac{P}{T^{2}}\right) \frac{d T}{d t}
$$

This expression represents stored mass flow and must be evaluated in time for each volume separating the turbine stage and nozzle throat. To assist in this task various thermocouples and pressure probes are located in the diffuser separating the test section and dump tank. Many other volumes, however, are not accessible to flow measurement. In addition, the accuracy at which many can be calculated is limited. For these reasons a transient model of the blowdown facility was used in developing this stored mass flow correction. This model allows estimation of flow properties at various tunnel locations and is the same model utilized in assessing the effect of an additional choke point downstream of the throttle exhaust. A detailed analysis of stored mass flow including results from this transient blowdown model are available in [10.2]. Here, this correction will simply be applied and its effect on nozzle mass flow presented.

Figure 10.2 shows nozzle and turbine mass flows labeled Uncorrected and Mass Correction respectively. These measurements differ by the mass flow stored in the volume separating the two measurement locations. This correction is valid between approximately 400$1000 \mathrm{~ms}$. As seen, between approximately $400-650 \mathrm{~ms}$, mass is stored in the diffuser separating the turbine and nozzle and turbine mass flow is higher than nozzle mass flow. This trend reverses at approximately $650 \mathrm{~ms}$ after which time turbine mass flow is less than that through the venturi nozzle.

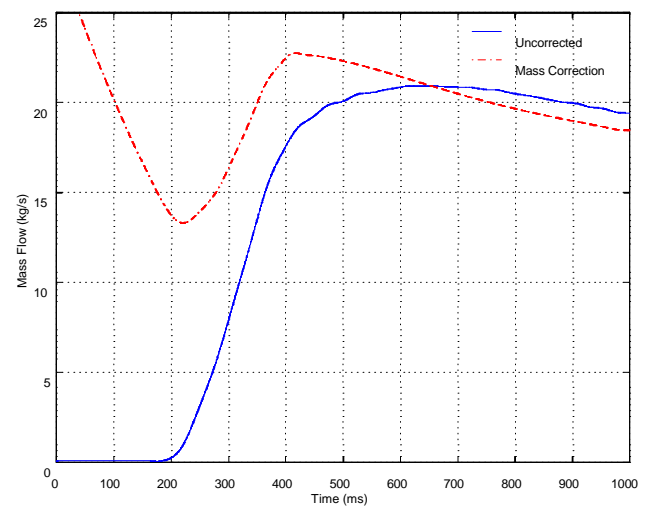

Figure 10.2 Turbime Mass Flow Correction for Tunnel Mass Storage 


\subsubsection{Corrected Efficiency Calculation}

Once turbine power and mass flow have been corrected for rotor acceleration and tunnel mass storage, mechanical efficiency may be expressed as,

$$
\eta_{b r k}=\frac{\mathrm{T} \cdot \omega+I \cdot \frac{d \omega}{d t} \cdot \omega}{\left(\dot{m}_{n}+\frac{d M_{s}}{d t}\right)\left(h_{T_{4}}-h_{T_{5}, i s}\right)}
$$

(Transient heat transfer is still to be corrected. This correction, discussed in the next section, is much smaller than that required for aerodynamic rake efficiency however.) Figure 10.3 shows this calculation for experiment 024 . Overlaid are aerodynamic rake efficiency calculations first presented in Section 9.4.

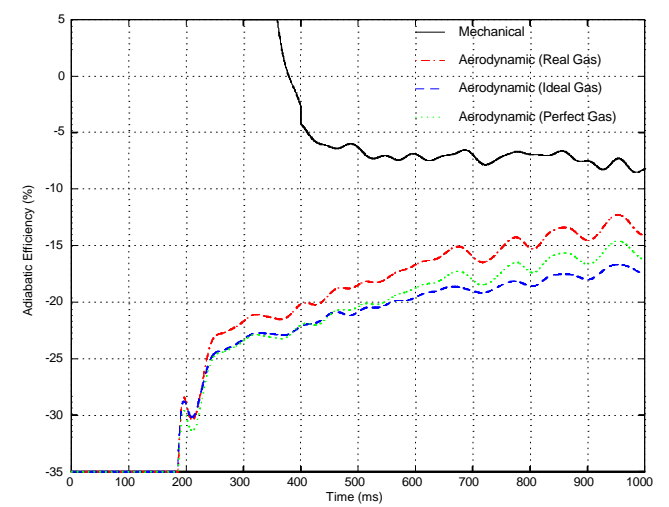

Figure 10.3: Experiment 024 Mechanical and Aerodynamic Rake Efficiencies

As seen in Figure 10.3, the mechanical approach predicts efficiency near -7\% for the baseline full-scale turbine design point simulation. (This value is referenced to the Craig and Cox empirical efficiency estimate at the smaller rotor tip clearance). This is considerably higher than the aerodynamic efficiency predictions. The mechanical prediction is also very near constant between 400-1000 ms. Aerodynamic and mechanical adiabatic efficiency predictions, those corrected for heat transfer as described in Sections 9.5 and 10.4, are presented for each experiment in Chapter 14.

\subsection{Heat Transfer Correction}

As shown in Section 9.5, heat transfer from the flow to the turbine during a blowdown experiment causes over-prediction of adiabatic efficiency using the aerodynamic approach. This is because this approach determines turbine work from the drop in total enthalpy across the turbine stage. By this calculation, work extracted from the flow can not be distinguished from heat extracted from the flow. The result is to assign turbine work a combination of work and heat transfer, a quantity larger than the actual turbine work. Thus, in order to accurately estimate adiabatic efficiency using the aerodynamic approach, the change in rake efficiency due to heat transfer had to be estimated and corrected. The purpose of this section is to similarly characterize the required heat transfer correction to mechanical efficiency. 
In Section 9.6 it was assumed that all heat transfer from the flow to the turbine occurs at an equivalent temperature $T^{*}$. Further assuming the entropy generated by this heat transfer is reversible, adiabatic and measured exit total enthalpies were related to heat transfer in Equation 9.17. This led to a relationship between adiabatic and aerodynamic rake efficiencies and heat transfer shown in Equation 9.18. Mechanical efficiency is similarly related to adiabatic efficiency and heat transfer. Referring to derivations by Keogh [10.2], this relationship can be expressed as follows,

$$
\eta_{a d, b r k}=\eta_{b r k}+\frac{Q}{h_{T_{4}}-h_{T_{5}, i s}}\left(1-\frac{T_{5}}{T^{*}}\right)=\eta_{b r k}+\Delta \eta_{b r k, h t}
$$

As seen from this equation, adiabatic efficiency is under-predicted due to heat transfer by the mechanical approach, not over-predicted as by the aerodynamic approach. In addition, the term multiplying the heat load in this correction, $\left(1-\frac{T_{5}}{T^{*}}\right)$, is much smaller than that in Equation 9.18, $\left(\frac{T_{5}}{T^{*}}\right)$. This means heat transfer will affect mechanical efficiency predictions much less than aerodynamic rake efficiency predictions. This is verified by comparing the aerodynamic corrections in Table 9.3 to the mechanical corrections explained below and summarized in Table 10.2 .

Table 10.2: Mechanical Efficiency Heat Transfer Correction Summary

\begin{tabular}{|l|l|l|l|}
\hline Experiment & 034 & 038 & 039 \\
\hline$T_{T_{4}},\left({ }^{\circ} \mathrm{F}\right)$ & 220 & 175 & 200 \\
\hline$T^{*},\left({ }^{\circ} \mathrm{F}\right)$ & 134 & 104 & 120 \\
\hline$\frac{Q}{h_{T_{4}}-h_{T_{5}, i s}}$ & $3.75 \%$ & $2.00 \%$ & $2.40 \%$ \\
\hline$\Delta \eta_{b r k, h t}$ & & & \\
\hline
\end{tabular}

As explained in Section 9.6, Keogh [10.2] estimates the heat transfer at an inlet temperature of $170{ }^{\circ} \mathrm{F}$ to be approximately $2.00 \%$ of the ideal total enthalpy drop across the turbine. The conditions for this estimate are very near those used in experiment 038 , thus, the heat transfer during this experiment is assumed to be this $2.00 \%$. This is shown in Table 9.3 along with heat loads for experiments 038 and 039 determined in Section 9.6 by comparing aerodynamic efficiency predictions. This information along with Equation 10.13 may then be used to determine the required heat transfer correction to mechanical efficiency. Again assuming an equivalent heat transfer temperature, the required correction to mechanical efficiency due to heat transfer for experiment 038 is approximately $0.14 \%$. This correction for experiments 039 and 034 , inlet temperature $200{ }^{\circ} \mathrm{F}$ and $220^{\circ} \mathrm{F}$ respectively, is approximately $0.17 \%$ and $0.27 \%$.

The reason that mechanical efficiency measurements are much less affected by heat transfer than aerodynamic rake measurements is clear. As explained above, the aerodynamic calculation of turbine work is unable to distinguish occurring heat transfer. Using the 
mechanical approach, however, turbine work is measured directly from turbine torque and speed, independent of any heat transfer taking place. This is an intuitive result that is verified by experimental data.

\subsection{Additional Corrections}

Currently, other processes thought to affect mechanical adiabatic efficiency measurement are being studied to determine appropriate corrections. These items are thought to be of second order relative to those discussed in the three previous sections, however, when combined, they could result in a significant correction to measured mechanical adiabatic efficiency.

An additional non-adiabatic effect not discussed in Section 10.4 is thought to affect mechanical adiabatic efficiency measurement. This effect is heat transfer to the supply tank struts upstream of the upstream total temperature measurement locations. Because upstream total temperature is measured between struts, the cooling behind these struts is not taken into account. For aerodynamic measurements of efficiency, the flow field is sampled between these struts upstream and downstream and this heat transfer does not alter the indicated efficiency as the circumferential temperature non-uniformity is not fully mixed-out in passing through the turbine stage. The mechanical measurement of turbine work is a global one however. A $120^{\circ}$ circumferential sector with uniform temperature can not be concentrated on and the nonuniformities outside of this sector excluded. The strut cooling effect will lower the upstream mass averaged total enthalpy below that indicated by upstream total temperature measurements. This will result in lower indicated turbine work extraction and mechanical efficiency. This correction, which will require analysis to determine the heat transferred to the supply tank struts, will likely be more significant than the non-adiabatic correction discussed in Section 10.4.

The supply tank struts similarly affect upstream total pressure measurement. Currently, the blockage resulting from these struts is not accounted for in determining upstream total pressure as these measurements are taken between struts as are total temperature measurements. The result is a lower upstream mass averaged total pressure than indicated by measurement. This will further decrease indicated mechanical efficiency.

Bearing friction absorbs turbine power and reduces that indicated by the eddy current brake torque meter. Significant levels of bearing friction would thus result in underestimation of mechanical adiabatic efficiency. Early calculations indicate, however, that this effect might contribute only on the order of $0.10 \%$ in adiabatic efficiency.

Windage of the turbine shaft and eddy current brake drum would also affect indicated turbine power. Because of low rotational speeds and small shaft and drum length to diameter ratios, this effect is thought to be very small. Calculations verifying this presumption, however, have not been completed.

This section has proposed additional effects within the Blowdown Turbine thought to affect the mechanical measurement of turbine adiabatic efficiency. These effects are currently being studied in the development of corrections that will account for them. When combined, it is expected that they may contribute several tenths of one percent to measured mechanical adiabatic efficiency.

\subsection{Post-Test Efficiency Uncertainty Analysis}

Section 10.2 estimates the absolute and relative uncertainties in mechanical adiabatic efficiency measurement to be $0.56 \%$ and $0.37 \%$ respectively. As explained in Section 9.6, efficiency bias can not be determined and absolute uncertainty can not be verified from 
experimental data. Efficiency relative uncertainty may be determined, however, allowing verification of the pre-test estimate. That is the purpose of this section. In addition, mechanical efficiency relative uncertainty will be compared to aerodynamic rake efficiency relative uncertainty determined in Section 9.6. The same comparison will be presented for mechanical and aerodynamic heat transfer corrections and the resulting mechanical and aerodynamic adiabatic efficiencies.

As in Section 9.6, experiments 025 and 026, near identical simulations of the full-scale turbine design point, will be used to determine the repeatability of the mechanical efficiency measurement. To reiterate, repeatability is determined by the precision index $\mathrm{S}$, as defined in Equation 9.19. A value of 1.28 will again be assumed for $d_{2}^{*}$.

Table 10.3 summarizes mechanical efficiency predictions from experiments 025 and 026 . As seen, they are extremely close; the relative uncertainty in this measurement is estimated to be $0.02 \%$. This is much lower than the aerodynamic rake efficiency relative uncertainty of $0.60 \%$ to $0.65 \%$ determined in Section 9.6.

Also shown in Table 10.3 are mechanical adiabatic efficiencies determined from the heat transfer correction discussed in Section 10.4. The precision index of this correction (as a percentage of the correction, $S_{*} / *$ ) is identical to that of the aerodynamic rake efficiency correction shown in Table 9.4. However, the resulting relative uncertainty in this correction is only $0.15 \%$ compared to the $2.03 \%$ relative uncertainty in the aerodynamic rake efficiency correction. This is a result of the correction magnitudes. In the mechanical case, this correction is only $0.27 \%$ where in the aerodynamic case, it is $3.49 \%$. This can be seen by the correction influence coefficients as well. The mechanical and aerodynamic correction influence coefficients are 0.003 and 0.040 respectively. In other words, the effect of the correction on aerodynamic adiabatic efficiency uncertainty will be an order of magnitude larger than the effect of the correction on mechanical adiabatic efficiency uncertainty. This is verified in Tables 9.4 and 10.3 where the aerodynamic and mechanical adiabatic efficiency relative uncertainties are seen to be $2.12 \%$ and $0.16 \%$ respectively. These uncertainties differ by more than one order of magnitude.

Table 10.3: Post-Test Uncertainty Analysis Summary

\begin{tabular}{|l|l|l|l|l|l|l|}
\hline & 025 & 026 & $C_{*}$ & $S_{*} / *$ & $t_{95}$ & $U_{95, \text { rel }}$ \\
\hline$\pi_{t}$ & $95.6 \%$ & $95.8 \%$ & & & & \\
\hline$N_{c o r}$ & $102.8 \%$ & $102.0 \%$ & & & & \\
\hline$R_{e}$ & $106.7 \%$ & $110.7 \%$ & & & & \\
\hline$\gamma_{4}$ & $99.9 \%$ & $100.8 \%$ & & & & \\
\hline$\eta_{b r k}[1]$ & $-6.8 \%$ & $-6.7 \%$ & 0.997 & $0.01 \%$ & 2.00 & $0.02 \%$ \\
\hline$\Delta \eta_{b r k, h t}$ & $0.27 \%$ & $0.27 \%$ & 0.003 & $29.05 \%$ & 2.00 & $0.15 \%$ \\
\hline$\eta_{a d, b r k}^{[1,2]}$ & $-6.5 \%$ & $-6.4 \%$ & & $0.09 \%$ & 2.00 & $0.16 \%$ \\
\hline
\end{tabular}

Table Footnotes: (Refer to Jacobs, [10.5])

[1] Efficiency is referenced to the Craig and Cox empirical efficiency estimate at the smaller rotor tip clearance presented in [10.5-Section 6.3.2]. 
[2] As previously explained, all mechanical efficiency predictions are based on real gas ideal turbine work, thus, this is where the heat transfer correction is applied. Because of the agreement between the gas models shown in Section 9.4, however, comparison of uncertainties in this prediction to pre-test estimates assuming perfect gas behavior is still valid.

This analysis thus concludes that turbine adiabatic efficiency may be measured with $0.16 \%$ relative uncertainty by the mechanical approach. This is much lower than the $0.37 \%$ pretest estimate. The absolute uncertainty of this measurement can not be verified by experimental data, however, pre-test estimates indicate a level of $0.56 \%$. This may be quite optimistic, though, given the additional corrections yet to be applied. Nonetheless, the relative uncertainty of this measurement far exceeds the goal of below $0.50 \%$. These uncertainties are also much less than those determined for the aerodynamic approach. As explained in Section 9.6, this approach does not measure adiabatic efficiency with uncertainty consistent with the objectives of this work due to the method used to determine the required heat transfer correction. However, using the same method to determine the heat transfer correction, the mechanical approach is successful. This is because of the relatively small effect heat transfer has on the mechanical measurement of efficiency. It is further concluded that for this reason alone, the mechanical approach to measuring turbine adiabatic efficiency is more effective in terms of measurement uncertainty.

\subsection{Chapter Summary}

This chapter has discussed mechanical measurement of adiabatic efficiency. The discussion began with a pre-test mechanical adiabatic efficiency uncertainty analysis. It was estimated that this measurement could be made with $0.56 \%$ absolute uncertainty, very near the pre-test aerodynamic adiabatic efficiency absolute uncertainty estimate of $0.61 \%$. In addition, mechanical adiabatic efficiency relative uncertainty was estimated to be $0.37 \%$, slightly lower than the $0.49 \%$ pre-test aerodynamic estimate.

This analysis was followed by a discussion on mechanical efficiency calculation. This concluded with the presentation of this calculation for experiment 024 , a baseline full-scale turbine design point simulation. The resulting mechanical efficiency prediction was near $-7 \%$ relative to the Craig and Cox empirical estimate and relatively constant between 400-1000 ms.

This calculation was followed by an analysis of the effect of heat transfer on mechanical efficiency measurement. It was shown, from heat transfer estimates presented in Section 9.5, that mechanical efficiency under-predicts adiabatic efficiency by approximately $0.27 \%$ at an inlet temperature of $220^{\circ} \mathrm{F}$ due to heat transfer effects.

This chapter concludes with a post-test mechanical efficiency uncertainty analysis. The objective of this analysis was to determine the actual repeatability of this measurement and compare this result to the pre-test estimate. It was shown that mechanical efficiency is repeatable within $0.02 \%$ and mechanical adiabatic efficiency, within $0.16 \%$. The additional relative uncertainty in adiabatic efficiency was due to uncertainty in the required heat transfer correction. It was still much lower than the pre-test estimate of $0.37 \%$ however. It was further concluded that because of the relative magnitudes of the heat transfer corrections required for the mechanical and aerodynamic approaches, the mechanical approach is much less sensitive to nonadiabatic effects and thus produces much more consistent efficiency predictions. 


\subsection{References}

[10.1] ASME/ANSI MFC-2M-1983. Measurement Uncertainty for Fluid Floe in Closed Conduits. An American National Standard, The American Society of Mechanical Engineers. 1988.

[10.2] Keogh, R., Shaft Efficiency Measurements of a Full Scaled Turbine in a Short Duration Facility. Masters Thesis, Massachusetts Institute of Technology. 1998.

[10.3] Cai, Y., Aerodynamic Performance Measurements in a Fully Scaled Turbine. Masters Thesis, Massachusetts Institute of Technology. 1998.

[10.4] Friend, D.G., Private Correspondence About NIST 14. Fluid Mixtures Data Center, NIST Thermophysics Division 838.02, Bolder CO. Summer 1997.

[10.5] Jacobs, J.J., Efficiency Measurements of a Single Stage Turbine in a Short Duration Facility. Masters Thesis, Massachusetts Institute of Technology. 1998. 


\section{Un-Cooled Turbine Performance Testing}

By Christopher M. Spadaccini

\subsection{Introduction}

Prior to the cooled turbine testing, an un-cooled turbine of identical blade and vane geometry was tested in the Blowdown Turbine facility. This was done to provide a baseline for comparison to the cooled tests. This test series will be reviewed here.

The efficiency measurement method developed by Keogh was used. This consists of using the eddy current brake torque meter to measure shaft torque and the downstream critical flow venturi nozzle to measure tunnel mass flow. The turbine operating design point was used as a baseline test and several off-design conditions were simulated. Variations in pressure ratio, corrected speed, and rotor tip clearance were studied. These tests were first analyzed by Jacobs where a more complete discussion of the data has been presented.

\subsection{Mechanical Efficiency}

Efficiency for a turbine can be defined as the actual power produced by the machine, normalized by the ideal power.

$$
\eta_{t}=\frac{\mathrm{P}_{\text {actual }}}{\mathrm{P}_{\text {ideal }}}
$$

The method of calculating efficiency which was utilized in these tests is referred to as the mechanical efficiency method because it requires a measurement of shaft torque. The real gas model was used for calculating enthalpy drop across the turbine because the test gas, nominally $\mathrm{CO}_{2}$, is non-ideal. Turbine mechanical efficiency can then be defined as

$$
\eta_{\text {mech }}=\frac{\mathrm{T} \cdot \omega}{\dot{m} \cdot\left(h\left(P_{4}, T_{4}\right)-\left(h\left(P_{5}, T_{5}\right)\right)\right.}
$$

where $\mathrm{T}$ is shaft torque, $\omega$ is shaft speed, $\dot{m}$ is mass flow rate, $h$ is enthalpy as a function of pressure and temperature, and subscripts 4, 5 refer to the inlet and outlet conditions, respectively. This expression does not account for the transient effects of the blowdown environment. Its assumptions include constant rotational speed, constant tunnel through flow, and no heat transfer. Corrections for these effects must be incorporated and will now be considered.

\subsection{Transient Corrections}

\subsubsection{Power Correction}

Turbine power is expressed in Equation 11.2 as the product of torque and speed, neglecting rotor acceleration. To correct for this transient effect, a second term must be added and actual turbine power becomes, 


$$
\mathrm{P}=\mathrm{T} \cdot \omega+I \cdot \frac{d \omega}{d t} \cdot \omega
$$

where $I$ is the moment of inertia of the rotating components including the turbine blades, rotor discs, shaft, and brake drum. This quantity was measured experimentally by Keogh as described in Chapter 5. The rotor was spun and the brake actuated in a vacuum. Thus, the turbine produced no power and the moment of inertia could be calculated from Equation 11.3. For the rotating system in the MIT Blowdown Turbine, the moment of inertia was found to be 1.8085 $\mathrm{kg} \cdot \mathrm{m}^{2}$ with a standard deviation of $0.13 \%$. Figure 11.1 shows both the raw and corrected power for a typical un-cooled turbine experiment.

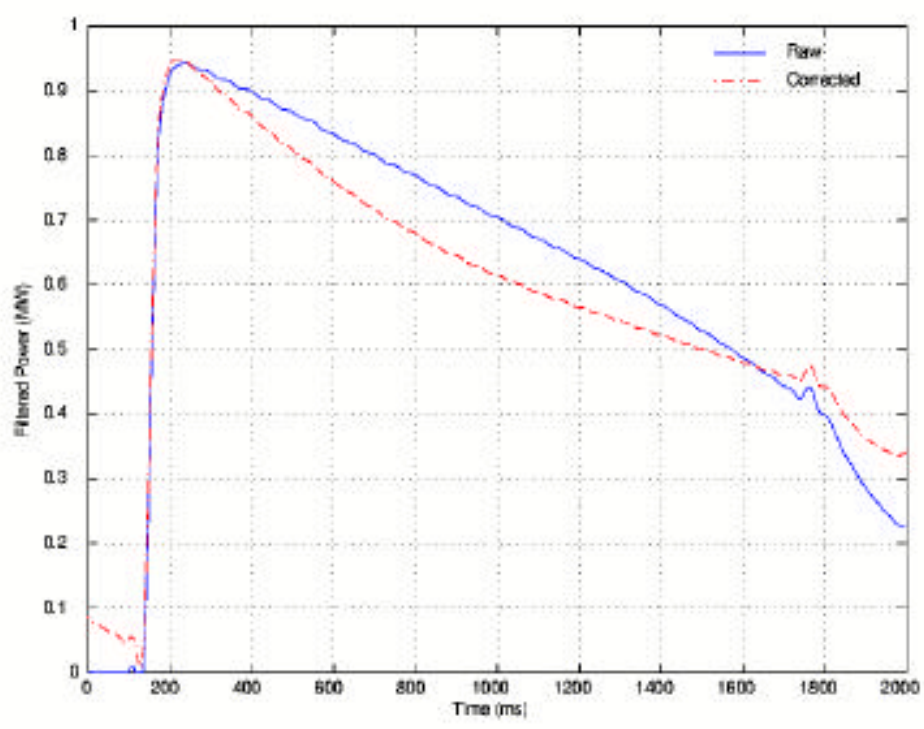

Figure 11.1: Typical Un-cooled Turbine Raw and Corrected Power

\subsubsection{Mass Storage Correction}

The critical flow venturi nozzle measures the mass flow rate through its throat. This is not necessarily the mass flow rate through the turbine, however which is located upstream of the nozzle. This quantity is required for Equation 11.2. Due to the volume separating the turbine from the venturi throat, there can be significant mass storage throughout the facility which must be accounted for to obtain an accurate measure of the turbine through-flow. Turbine and venturi nozzle mass flows can be related by,

$$
\dot{m}_{\text {turb }}=\dot{m}_{\text {noz }}+\dot{M}_{\text {stored }}
$$


where $\dot{M}_{\text {stored }}$ is mass flow stored in volumes throughout the facility. To estimate stored mass flow, the equation of state can be written in terms of mass as,

$$
M_{\text {stored }}=\frac{P V}{T R Z}
$$

with compressibility factor $Z$. This can be differentiated in time to give an expression for stored mass flow rate,

$$
\dot{M}_{\text {stored }}=\frac{V}{R Z}\left(\frac{1}{T}\right) \frac{d P}{d t}-\frac{V}{R Z}\left(\frac{P}{T^{2}}\right) \frac{d T}{d t}
$$

This expression can be evaluated in time for each volume in the facility which is properly instrumented with temperature and pressure sensors.

There are many small chambers throughout the facility that are not instrumented. To account for these volumes, a transient model of the blowdown turbine facility was developed and applied to this problem. It provides an estimate of the flow properties in regions of the tunnel which are inaccessible. A full description of the model and subsequent calculations can be found in Chapter 6 an in Keogh [11.1]. The results of this model combined with Equation 11.6 correct the mass flow measurement for tunnel mass storage. Figure 11.2 shows uncorrected and corrected turbine mass flow rate for a typical blowdown experiment.

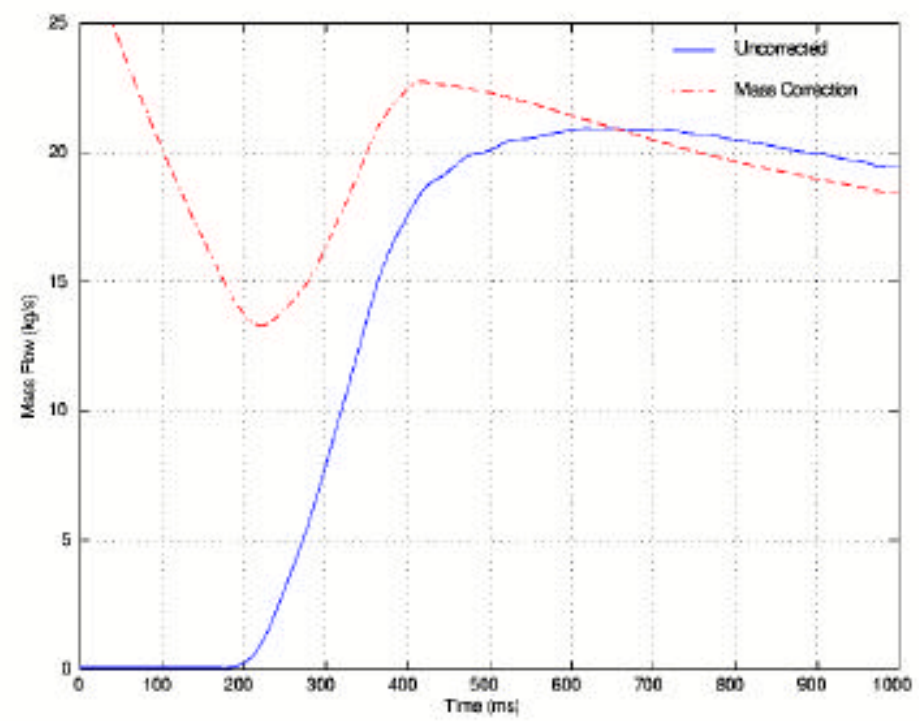

Figure 11.2: Typical Un-cooled Turbine Uncorrected and Corrected Mass Flow Rate. 


\subsubsection{Heat Transfer Correction}

The turbine does not reach thermal equilibrium during the blowdown test and the process can not be considered adiabatic. An expression to account for this effect is given by,

$$
\eta_{\text {mech, ad }}=\eta_{\text {mech }}+\frac{Q}{h\left(P_{4}, T_{4}\right)-h\left(P_{5}, T_{5}\right)}\left(1-\frac{T_{5}}{T^{*}}\right)
$$

where subscripts 4, 5 refer to the turbine inlet and outlet conditions, respectively. It is assumed that all heat transfer from the flow to the metal occurs at an equivalent temperature $T^{*}$. This correction is on the order of $0.2 \%$ for tests in the facility.

\subsection{Corrected Mechanical Efficiency}

After correcting turbine power and mass flow rate to account for transient effects, mechanical efficiency can be written as,

$$
\eta_{\text {mech }}=\frac{\mathrm{T} \cdot \omega+I \cdot \frac{d \omega}{d t} \cdot \omega}{\left(\dot{m}_{\text {noz }}+\dot{M}_{\text {stored }}\right) \cdot\left(h\left(P_{4}, T_{4}\right)-h\left(P_{5}, T_{5}\right)\right)}
$$

Heat transfer effects are small and can be accounted for in subsequent calculations. Figure 11.3 shows the mechanical efficiency calculated for a typical un-cooled turbine experiment.

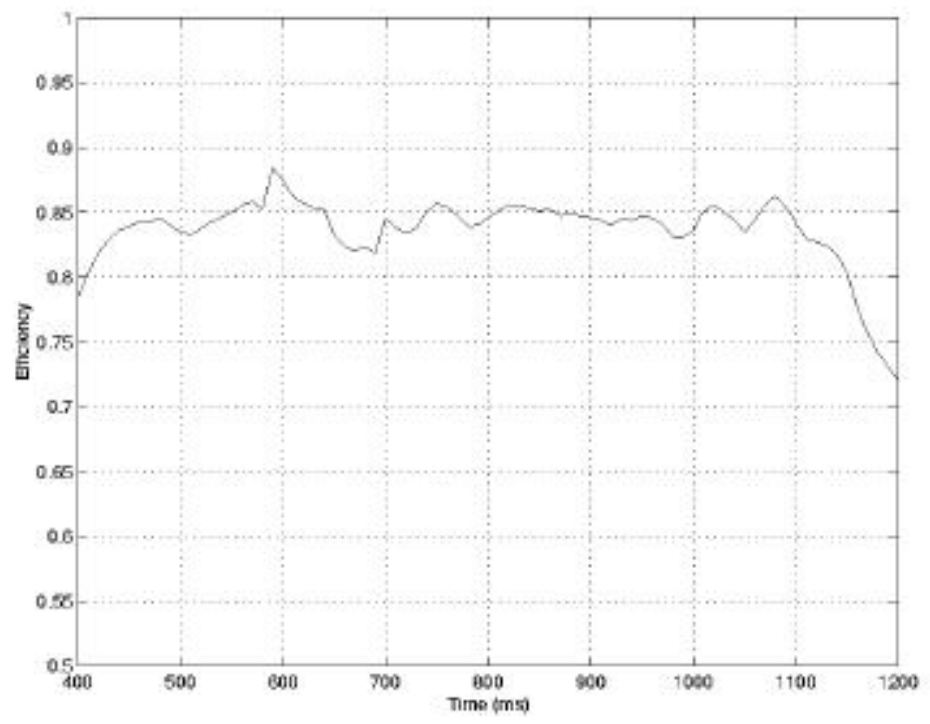

Figure 11.3: Typical Un-cooled Turbine Mechanical Efficiency 


\subsection{Test Matrix}

Several parameters were varied for the un-cooled test series. These include pressure ratio, corrected speed, and rotor tip gap. These off-design cases were compared to a baseline which is described in Table 11.1. Table 8.1 presents the entire test matrix for the un-cooled experiments.

Table 11.1: Un-cooled Turbine Baseline Test Conditions

\begin{tabular}{|l|l|}
\hline Parameter & Value \\
\hline Working Fluid & $\mathrm{CO}_{2}$ \\
\hline Specific Heat Ratio & 1.28 \\
\hline Pressure Ratio & 2.0 \\
\hline Corrected Speed & $100 \%$ \\
\hline Reynolds Number & $100 \%$ \\
\hline Rotor Tip Gap & $1.5 \%$ of blade span \\
\hline
\end{tabular}

\subsection{Results}

Figure 11.4 shows the results of the pressure ratio tests for two different rotor tip gaps. The smaller tip gap exhibited an efficiency approximately 2-3\% greater than that of the large tip gap. The larger gap causes increased secondary flows and thus, increased losses. The variation in efficiency with pressure ratio is minimal in the case of the smaller tip gap. For the larger gap, the efficiency is maximum at $\pi \approx 1.95$ which is near the baseline condition.

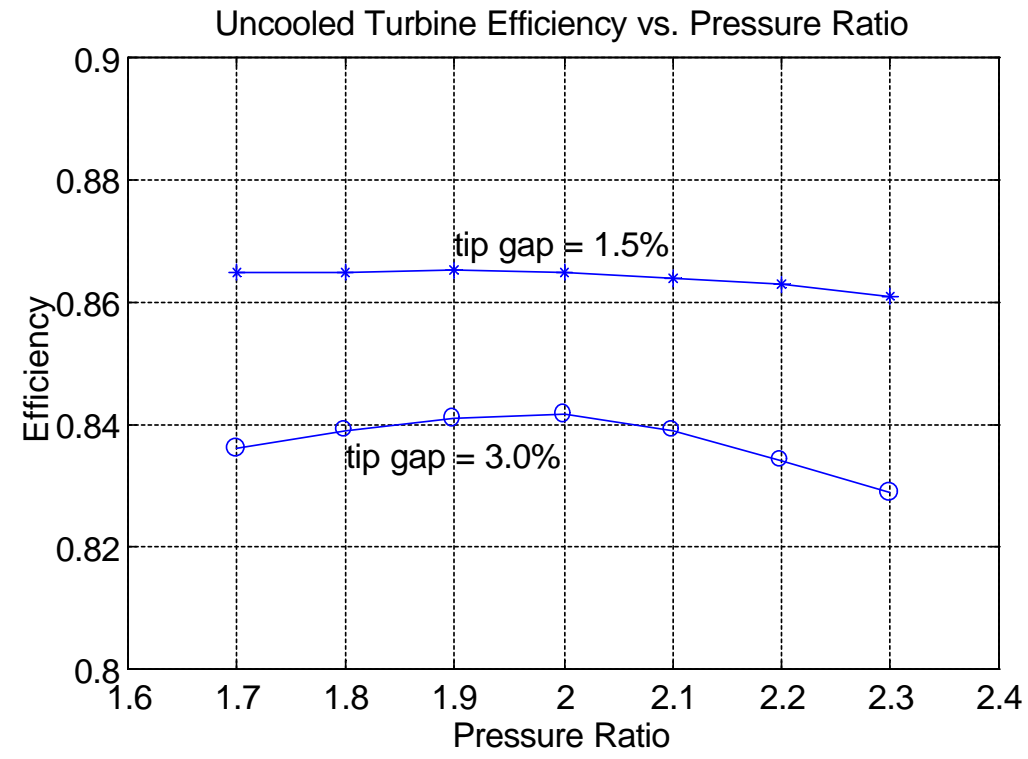

Figure 11.4: Un-cooled Turbine Efficiency vs. Pressure Ratio for Two Rotor Tip Gaps

Figure 11.5 shows the results of the corrected speed tests for the to tip gaps. Again, a difference of approximately $2-3 \%$ can be seen between the two gaps. The plot also shows that as 
speed is increased, efficiency tends to rise. However, as speed exceeds 105\% the efficiency increase slows. This efficiency rise is explained by the change in blade loading with variation in speed. As rotor speed is increased, rotor relative inlet angle is decreased. Therefore, the blade angle-of-attack is reduced and blade loading decreases. With lower loading there is higher efficiency.

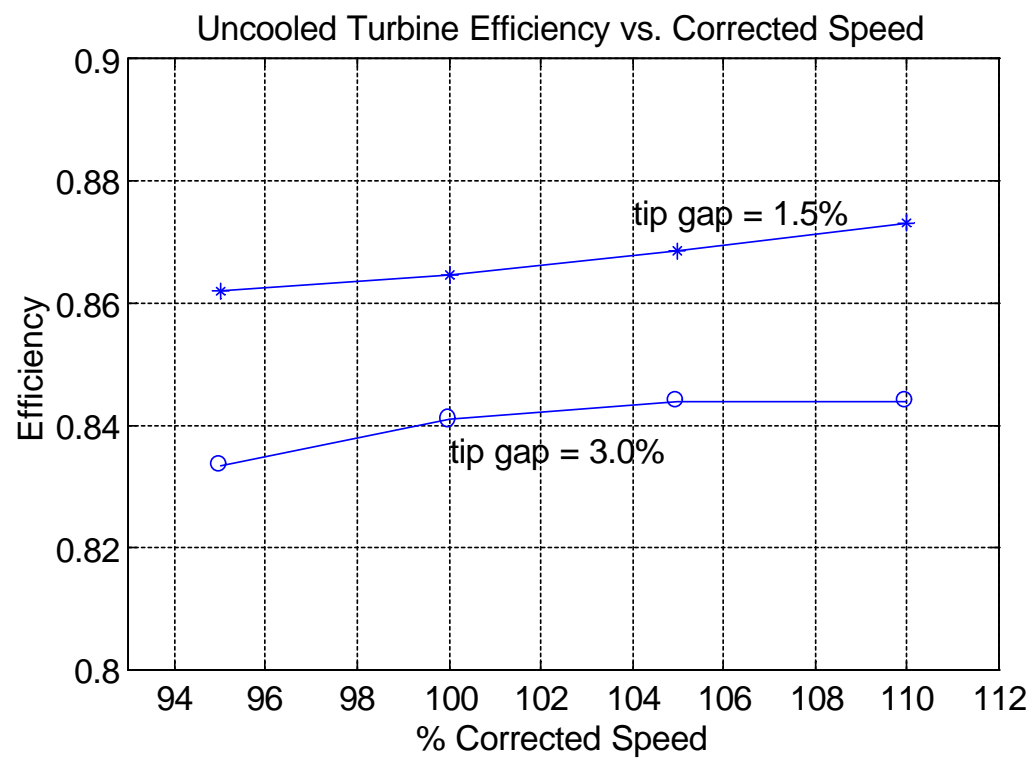

Figure 11.5: Un-cooled Turbine Efficiency vs. Corrected Speed for Two Rotor Tip Gaps

\subsection{Conclusions}

The un-cooled turbine testing has provided a unique data set over a range of operating conditions as a baseline for comparison to cooled turbine performance. A 2-3\% drop in efficiency for a two-fold increase in rotor tip gap was shown. A map of turbine performance over a significant range of pressure ratios was obtained. A corresponding map for corrected speed was also generated. The corrected speed tests confirmed that efficiency varies inversely with blade loading. In Chapter 10, the relative uncertainty of these measurements has been shown to be $0.16 \%$.

\subsection{Summary}

This chapter has reviewed the un-cooled turbine test program. The mechanical efficiency calculation and its associated transient corrections were described. The matrix of test conditions was presented. Turbine pressure ratio, corrected speed, and rotor tip gap were varied. Results from these tests were briefly discussed. The turbine exhibited changes in performance due to blade loading and increased tip gap. Finally, it was concluded that these data would be useful as a comparison to cooled turbine performance tests. 


\section{Film-Cooled Turbine Performance Testing}

By Christopher M. Spadaccini

\subsection{Introduction}

After fabrication of the film-cooled turbine and installation of the coolant feed system, a cooled performance test series was conducted. The mechanical efficiency calculation described in Chapter 11 was modified to account for the coolant flow injection. As in the un-cooled tests, a baseline condition was selected and off-design performance was simulated. Pressure ratio, corrected speed, and coolant mass flow were varied parametrically throughout the test series. In addition, a preliminary comparison to the un-cooled data will be shown.

\subsection{Cooled Turbine Mechanical Efficiency Calculation}

To compute efficiency for a film-cooled turbine, several modifications to the mechanical efficiency calculation described in the previous chapter must be made. Turbine efficiency can still be defined as the ratio of actual power produced by the machine to ideal power as shown in Equation 11.1. The actual power is measured in the Blowdown Turbine facility and is still calculated via Equation 11.3. Again, this accounts for transient effects in the blowdown environment. However, the ideal power of the turbine will not remain the same as that shown in the denominator of Equation 11.8. Ideal power for a film-cooled turbine can be estimated via several methods. For the purposes of this study, the modifications to the turbine efficiency calculation found in Kerrebrock [12.1] were used.

The injection of coolant flow into the mainstream must be factored into the ideal turbine power. The ideal power due to the coolant flow alone is

$$
\mathrm{P}_{\text {cool, ideal }}=\dot{m}_{5} \cdot\left(h\left(P_{c}, T_{c}\right)-h\left(P_{5}, T_{5}\right)\right)
$$

This definition assumes that the downstream flow is fully mixed. The ideal power due to coolant flow can be broken down further to account for the vane, blade, and tip casing flows individually.

$$
\mathrm{P}_{\text {cool, ideal }}=\mathrm{P}_{\text {vane, ideal }}+\mathrm{P}_{\text {blade, ideal }}+\mathrm{P}_{\text {casing,ideal }}
$$

Each individual component of Equation 12.2 follows the form of Equation 12.1.

The coolant mass flow which is injected is mixed with the main flow and measured as part of the total turbine through-flow by the downstream critical flow venturi nozzle. The ideal power due to the main flow is only dependent upon the turbine inlet mass flow. As a result, the turbine through-flow is over-estimated and the coolant must be factored out. Ideal power due to the turbine inlet flow then becomes,

$$
\mathrm{P}_{\text {main.ideal }}=\Delta h \cdot\left(\dot{m}_{\text {noz }}-\dot{m}_{\text {coolant }}\right)
$$


After accounting for the effects of the coolant on ideal power, the equation for mechanical efficiency is

$$
\eta_{\text {mech,cooled }}=\frac{\mathrm{T} \cdot \omega}{\left(\dot{m}_{n o z}-\dot{m}_{c o o l}\right) \cdot\left(h\left(P_{4}, T_{4}\right)-h\left(P_{5}, T_{5}\right)\right)+\mathrm{P}_{\text {cool, ideal }}}
$$

A final expression which treats transient effects such as rotor acceleration and tunnel mass storage is

$$
\eta_{\text {mech,cool }}=\frac{\mathrm{T} \cdot \omega+I \cdot \frac{d \omega}{d t} \cdot \omega}{\left(\dot{m}_{\text {noz }}+\dot{m}_{\text {stored }}-\dot{m}_{\text {cool }}\right) \cdot\left(h\left(P_{4}, T_{4}\right)-h\left(P_{5}, T_{5}\right)\right)+\mathrm{P}_{\text {cool, ideal }}}
$$

\subsection{Test Matrix}

The baseline cooled turbine performance conditions for this test series are summarized in Table 12.1. The Reynolds Number is $72 \%$ of the design value (the same that was used in the uncooled tests). To achieve the desired coolant-to-mainstream temperature ratio, the main supply tank was heated to $400{ }^{\circ} \mathrm{F}$. Tests at $100 \%$ of design Reynolds at this temperature with pure CO2 would require supply pressures greater than safety limits would permit. (Tests at full Reynolds could be conducted using a mixture of Argon and Freon-12.) The complete cooled turbine test matrix was shown in Table 8.2. Variation in coolant mass flow rate, Reynolds Number, corrected speed, and pressure ratio were tested. All tests were conducted with a tip gap of 3\% of blade span.

Table 12.1: Cooled Turbine Baseline Test Conditions

\begin{tabular}{|l|l|}
\hline Parameter & Value \\
\hline \hline Working Fluid & $\mathrm{CO} 2$ \\
\hline Reynolds Number & $72 \%$ of Design \\
\hline Pressure Ratio & 2.0 \\
\hline Corrected Speed & $100 \%$ \\
\hline Rotor Tip Gap & $3.0 \%$ of Span \\
\hline$T_{\text {inlet }} / T_{\text {coolant }}$ & 1.52 \\
\hline Coolant Mass Flow & $9.7 \%$ of Inlet \\
\hline
\end{tabular}




\subsection{Results}

\subsubsection{Baseline Conditions}

As a result of the coolant, it is more difficult to maintain constant conditions over the course of a blowdown experiment. Figures 12.1 ( $\mathrm{a}$ and $\mathrm{b}$ ) shows the pressure ratio and corrected speed for the baseline test conditions. The figures indicate a larger variation in conditions over the test window than was seen in the un-cooled experiments. Figures 12.1 ( $\mathrm{c}$ and d) are plots of coolant mass flow and efficiency. Coolant mass flow was relatively constant over the course of the test. This is expected due to the choked orifices' wide range of operating conditions. The efficiency is also relatively constant over the usable test window. Data for the entire test matrix can be found in Chapter 15.

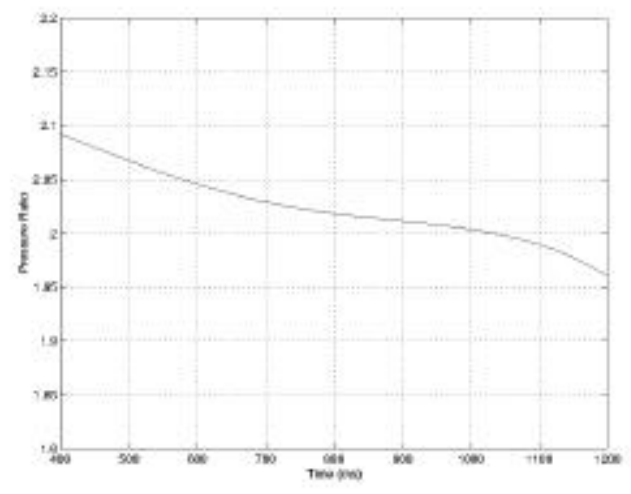

(a) Pressure Ratio

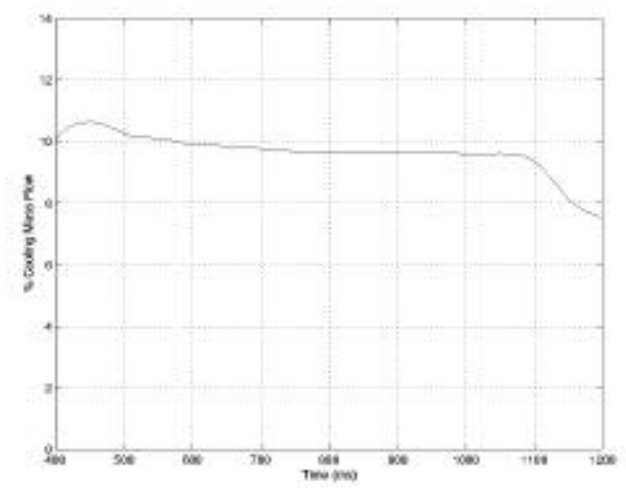

(c) Coolant Mass Flow

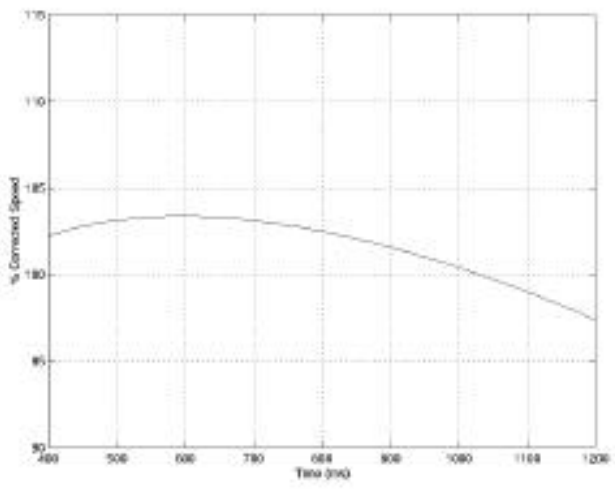

(b) Corrected Speed

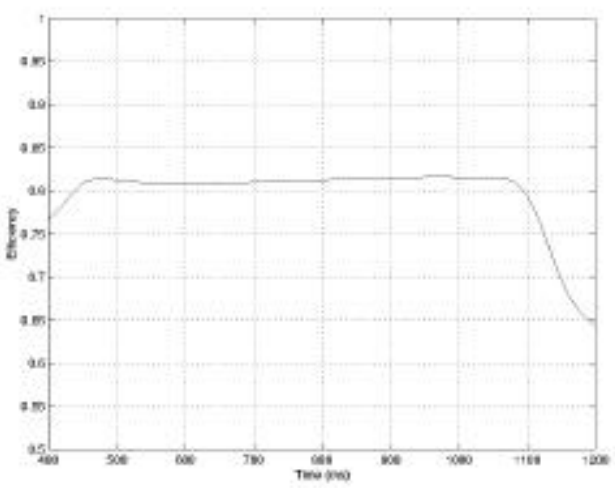

(d) Efficiency

Figure 12.1: Baseline Cooled Turbine Experiment. 


\subsubsection{Variation in Coolant Flow}

The effect of coolant mass flow on turbine performance was studied. Coolant mass flow was varied from approximately 5\% to 12\% of the main inlet flow All other parameters were held constant between tests. It was observed that as coolant mass flow increased, turbine efficiency decreased. This is due to aerodynamic losses associated with coolant flow injection. Figure 12.2 shows the coolant mass flow as a function of time for four different experiments. Figure 12.3 plots the decrease in efficiency with increasing coolant mass flow. A $2 \%$ drop in efficiency can be seen for a two-fold increase in coolant mass flow. A data point from the un-cooled turbine configuration is shown for reference. This point is not directly comparable because the cooling holes themselves may account for some aerodynamic loss.

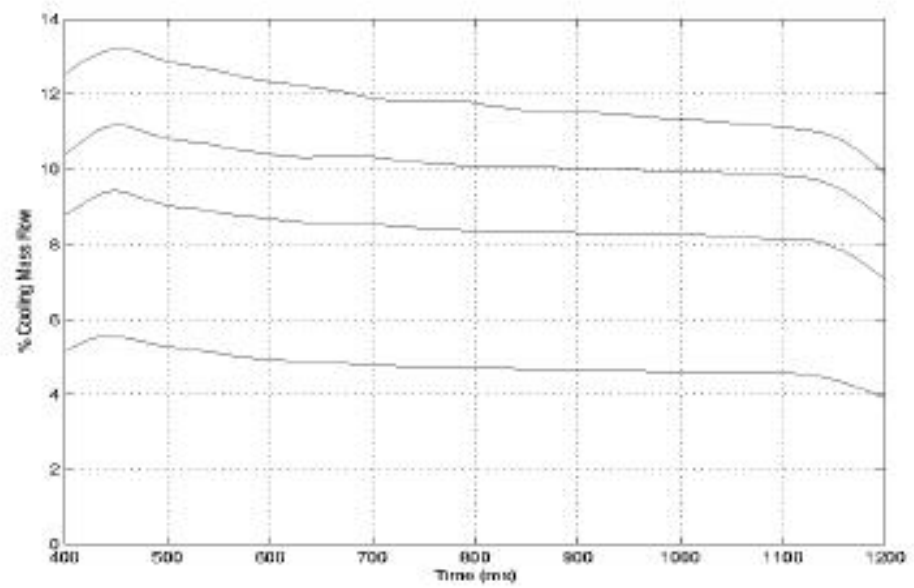

Figure 12.2: Coolant Mass Flow for Four Different Tests. 


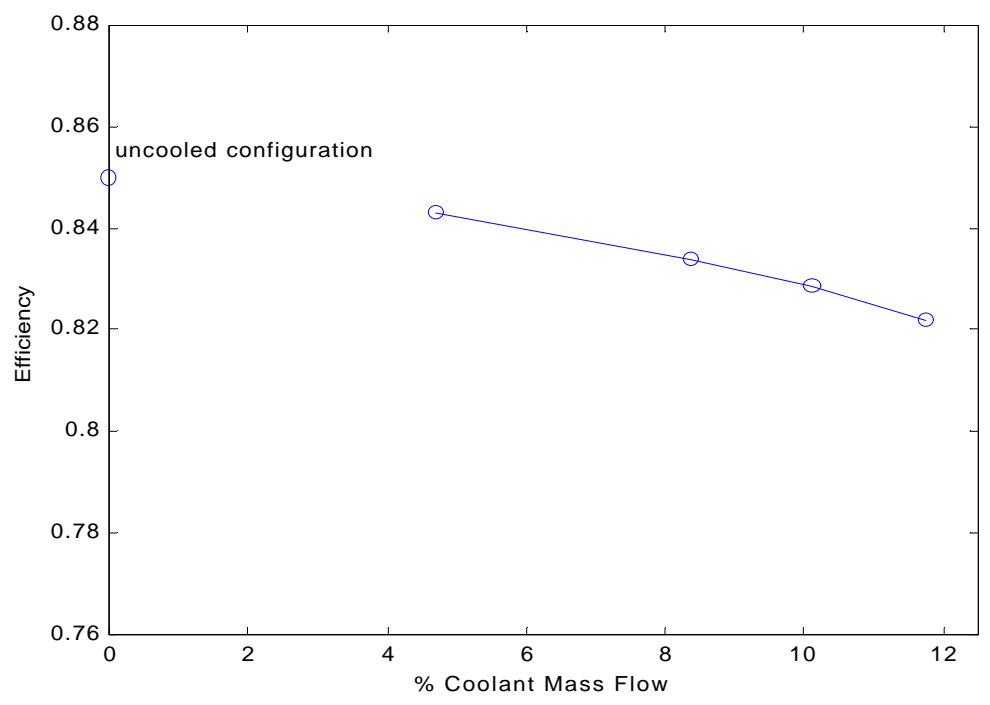

Figure 12.3: Turbine efficiency vs. Coolant Mass Flow

\subsubsection{Variation in Pressure Ratio}

As in the un-cooled experiments, pressure ratio was varied while all other conditions were held constant. Figure 12.4 shows a plot of efficiency vs. pressure ratio. This is similar to the results of the un-cooled pressure ratio tests. These data can not be directly compared to the un-cooled data because by testing at a higher temperature, the non-dimensional parameters such as Reynolds Number and gas-to-wall temperature ratio are not comparable.

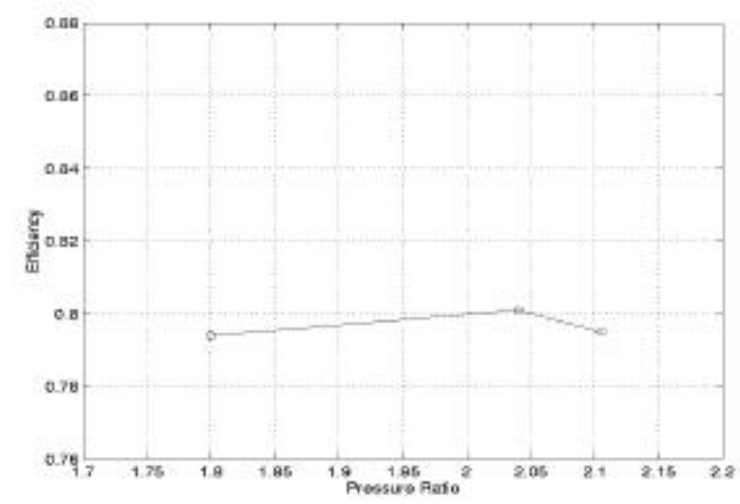

Figure 12.4: Cooled Turbine Efficiency vs. Pressure Ratio

\subsubsection{Variation in Corrected Speed}

Figure 12.5 shows a plot of efficiency vs. corrected speed. As in the pressure ratio and coolant mass flow tests, all other parameters were held constant. Like the uncooled experiments, efficiency increases with speed. Again, this is a result of a decrease in flow incidence angle and 
a decrease in blade loading. Like the pressure ratio tests, these experiments are not directly comparable to the un-cooled experiments.

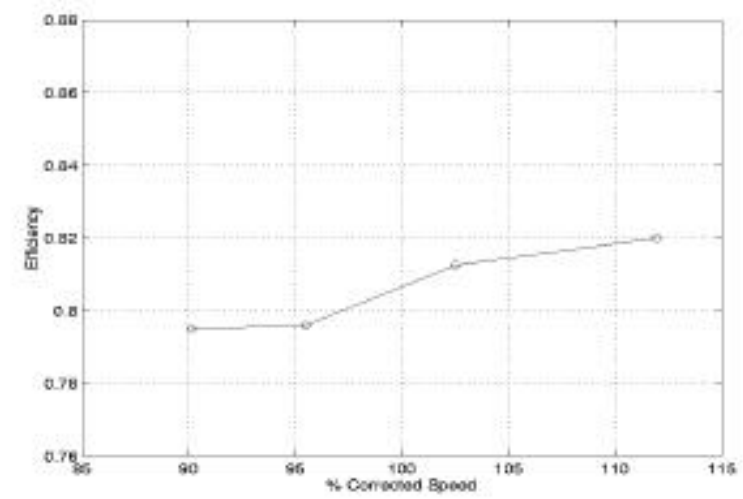

Figure 12.5: Cooled Turbine Efficiency vs. Corrected Speed

\subsection{Comparison to an Un-Cooled Turbine Test}

Cooled test ABB117 was run at conditions very similar to those used in the un-cooled test series. Figure 12.6 shows efficiency for this test plotted on the same axes as efficiency for an un-cooled test. The tip gaps in the two experiments were the same. The coolant flow in the cooled experiment was $11.75 \%$ of the main flow. An efficiency drop of approximately $2.0 \%$ is shown and can be attributed to aerodynamic losses due to coolant flow injection.

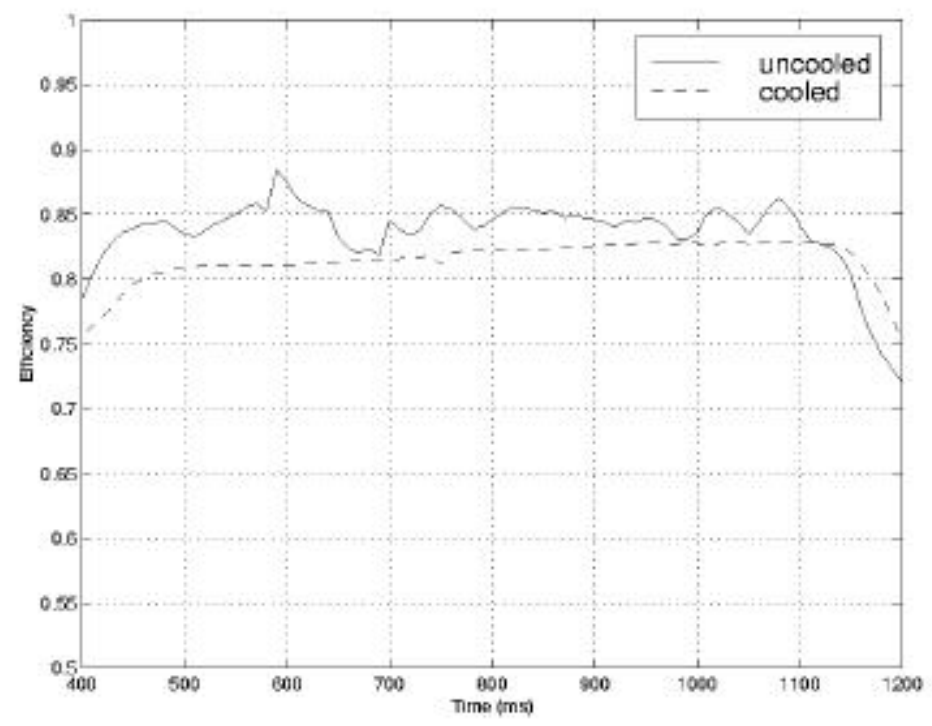

Figure 12.6: Efficiency for Comparable Un-Cooled and Film-Cooled Turbine Tests. 


\subsection{Conclusions}

Aerodynamic performance of a film-cooled turbine was measured in the Blowdown Turbine test facility. Efficiency consistently decreased with an increase in coolant mass flow rate. Trends similar to the un-cooled tests were observed when pressure ratio and corrected speed were varied. A preliminary comparison of the cooled and un-cooled data shows that turbine efficiency drops significantly with coolant flow injection. Only tests with similar nondimensional parameters were compared.

\subsection{Summary}

This chapter presents the modifications to the mechanical efficiency calculation to account for coolant flow injection. The film-cooled turbine test matrix is discussed and baseline conditions reviewed. Data for the baseline case is presented followed by the results of the coolant flow, pressure ratio, and corrected speed tests. Finally, a preliminary comparison to the un-cooled data is made. 


\subsection{Reference}

[12.1] Kerrebrock, J.L., Aircraft Engines and Gas Turbines, The MIT Press, Second Edition, 1992. 

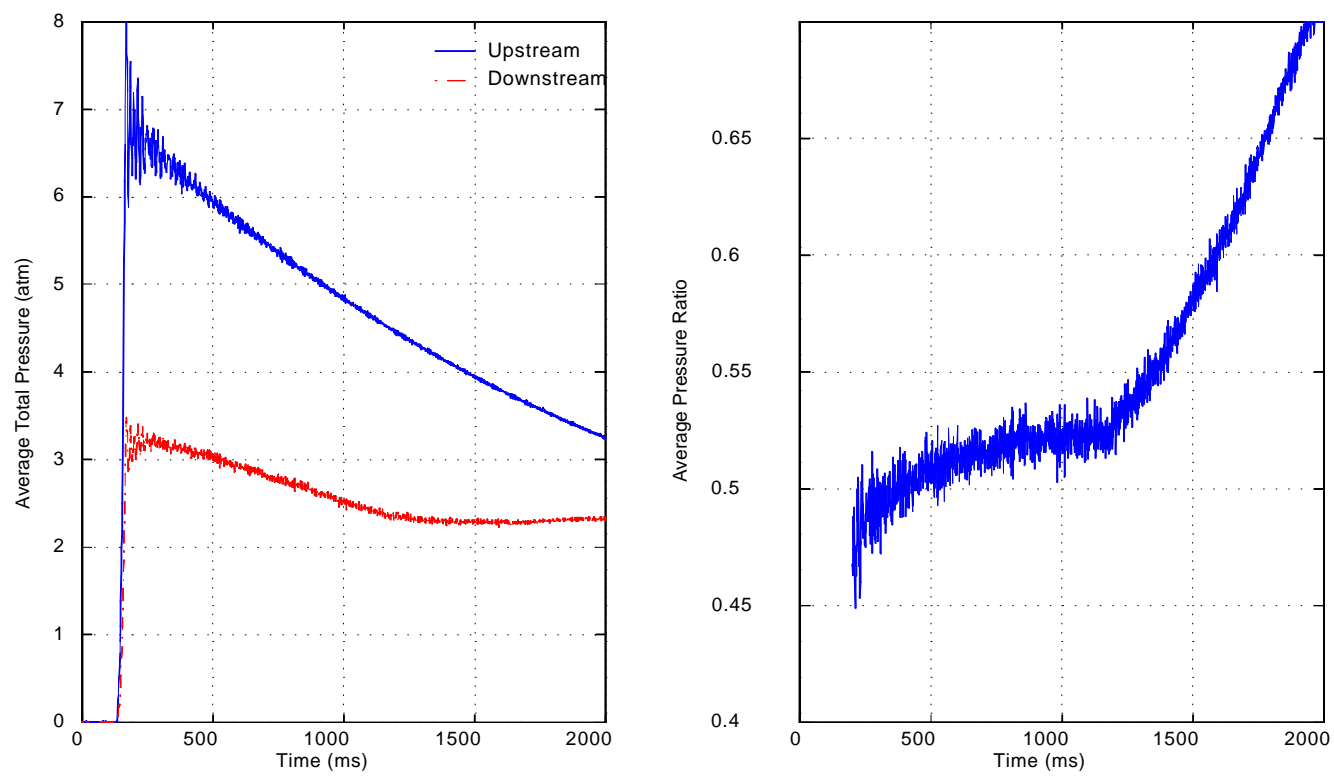

Figure 13.1: ABB024 - Average Total Pressure and Pressure Ratio
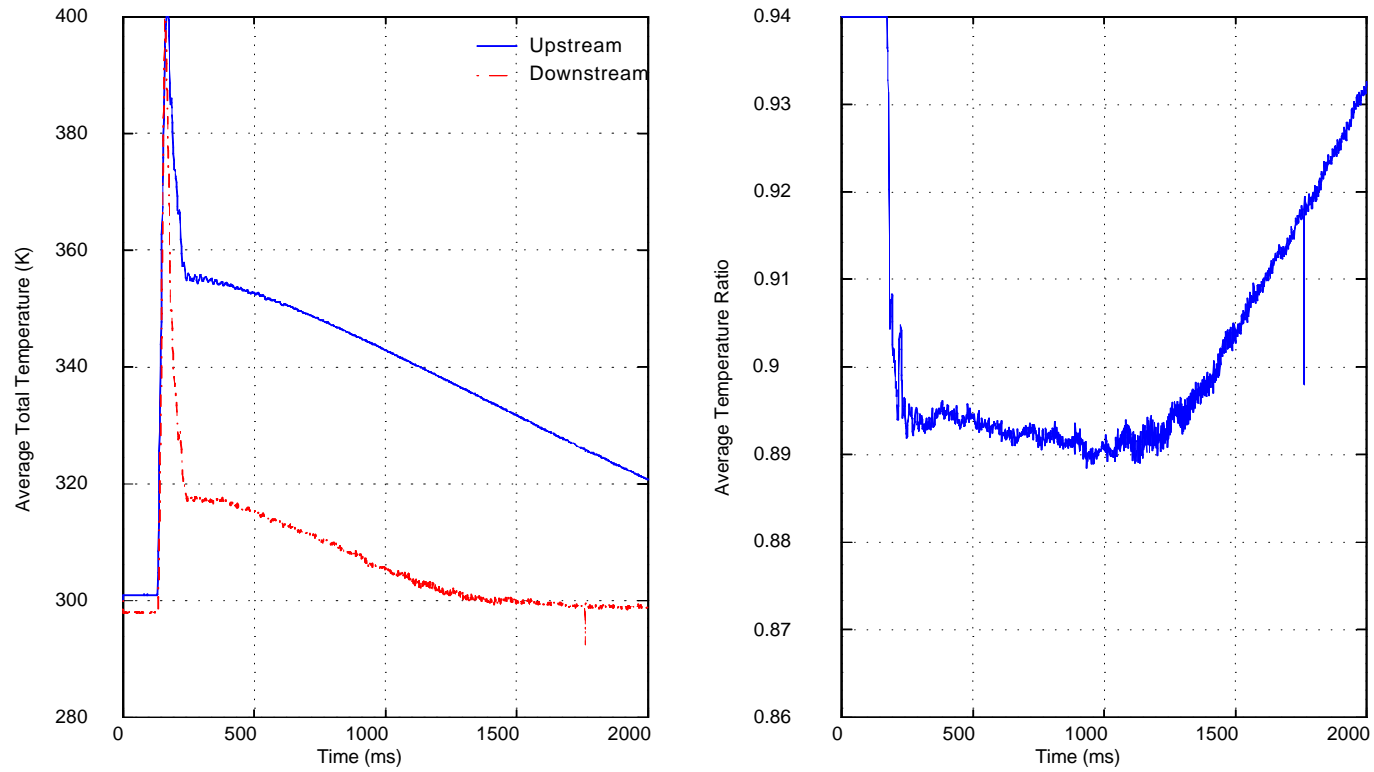

Figure 13.2: ABB024 - Average Total Temperature and Temperature Ratio 

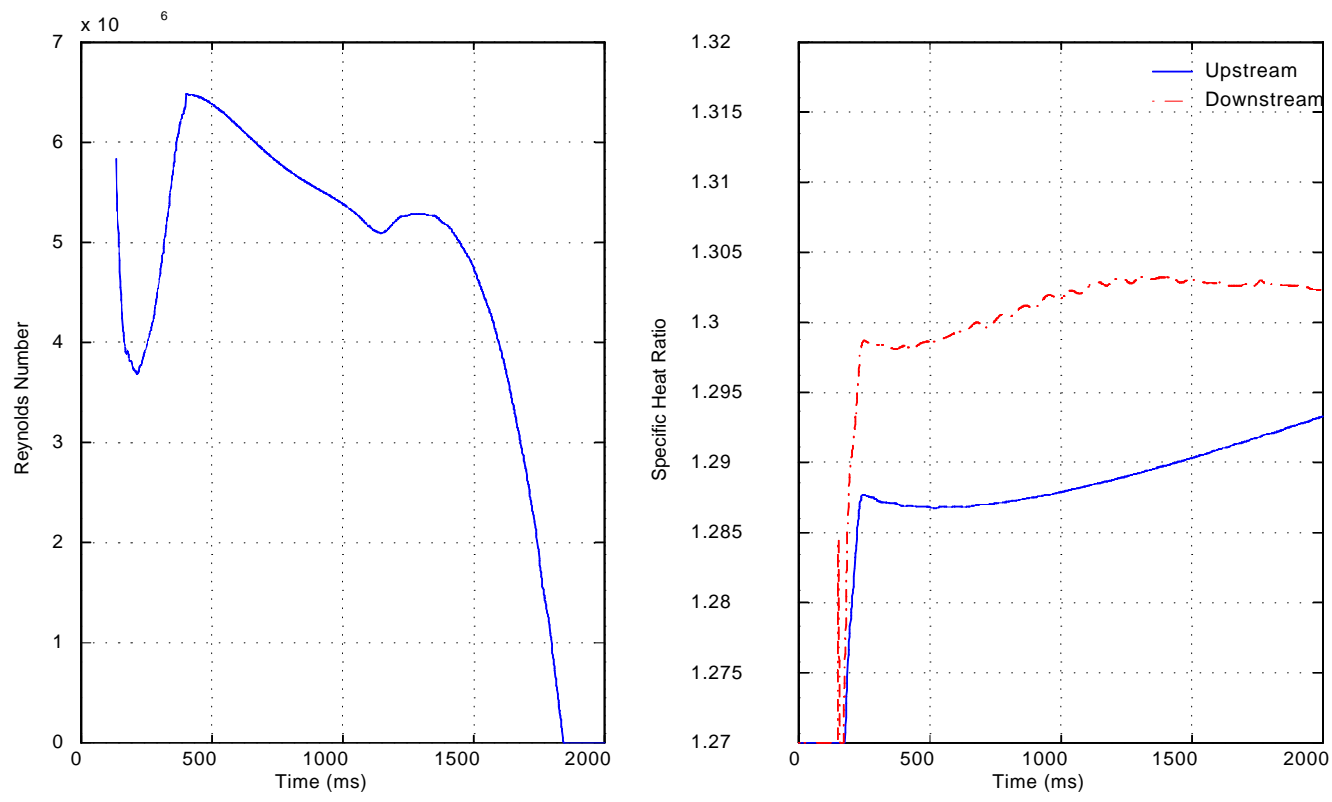

Figure 13.3: ABB024 - Reynolds Number and Specific Heat Ratio
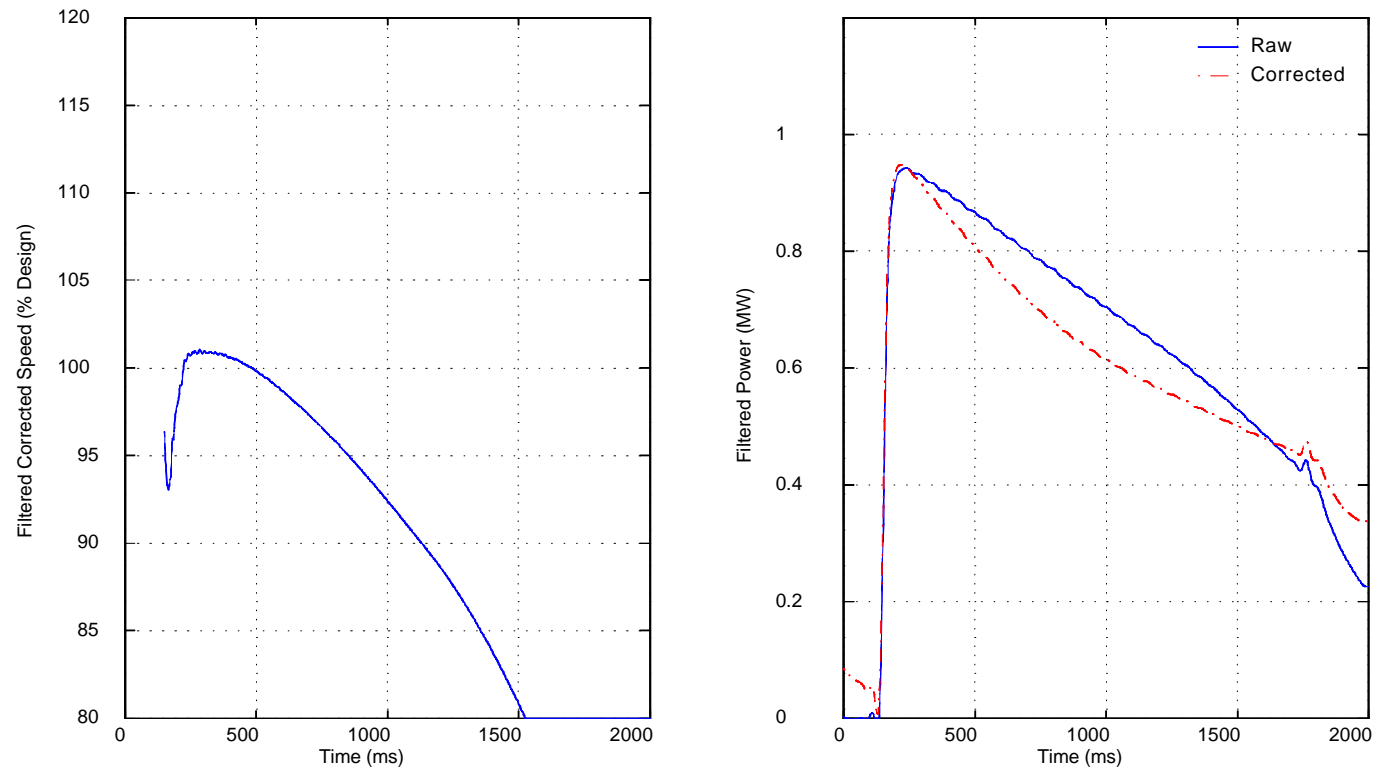

Figure 13.4: ABB024 - Corrected Speed and Power 

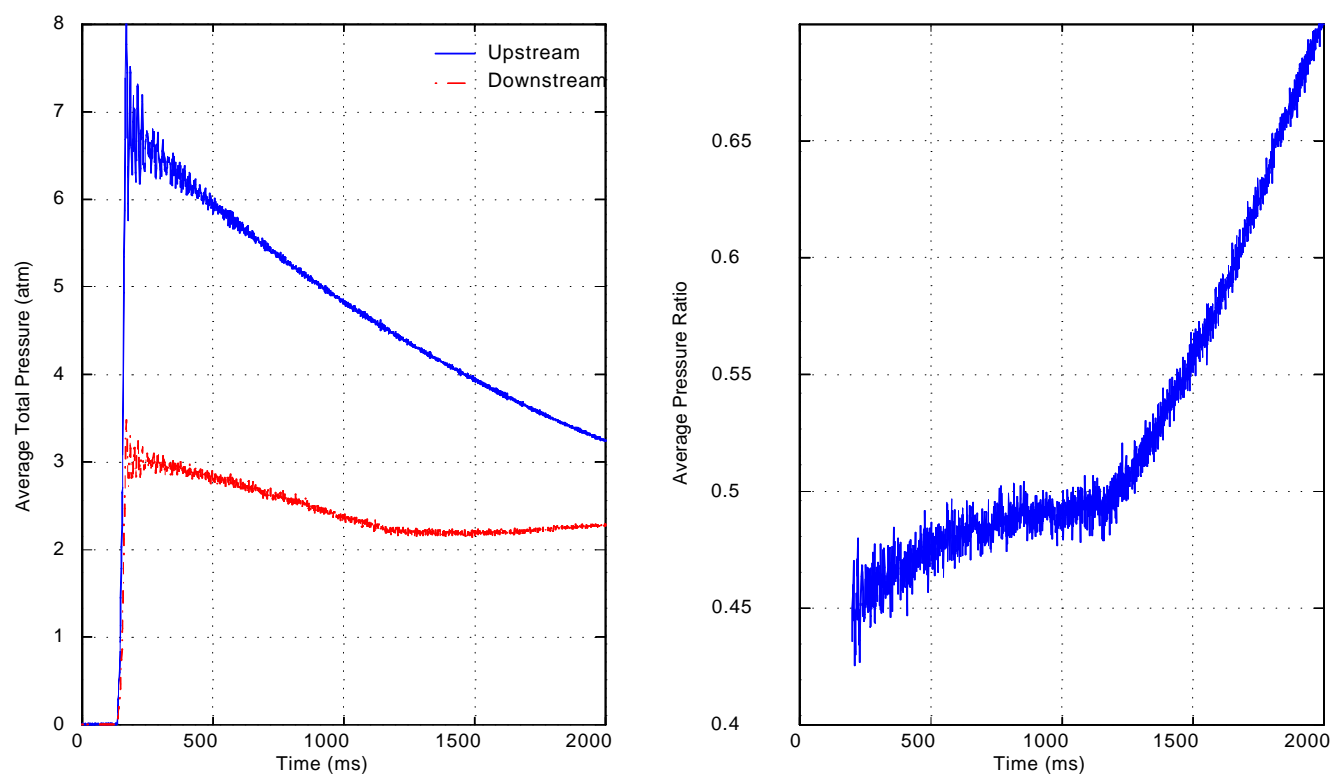

Figure 13.5: ABB027 - Average Total Pressure and Pressure Ratio
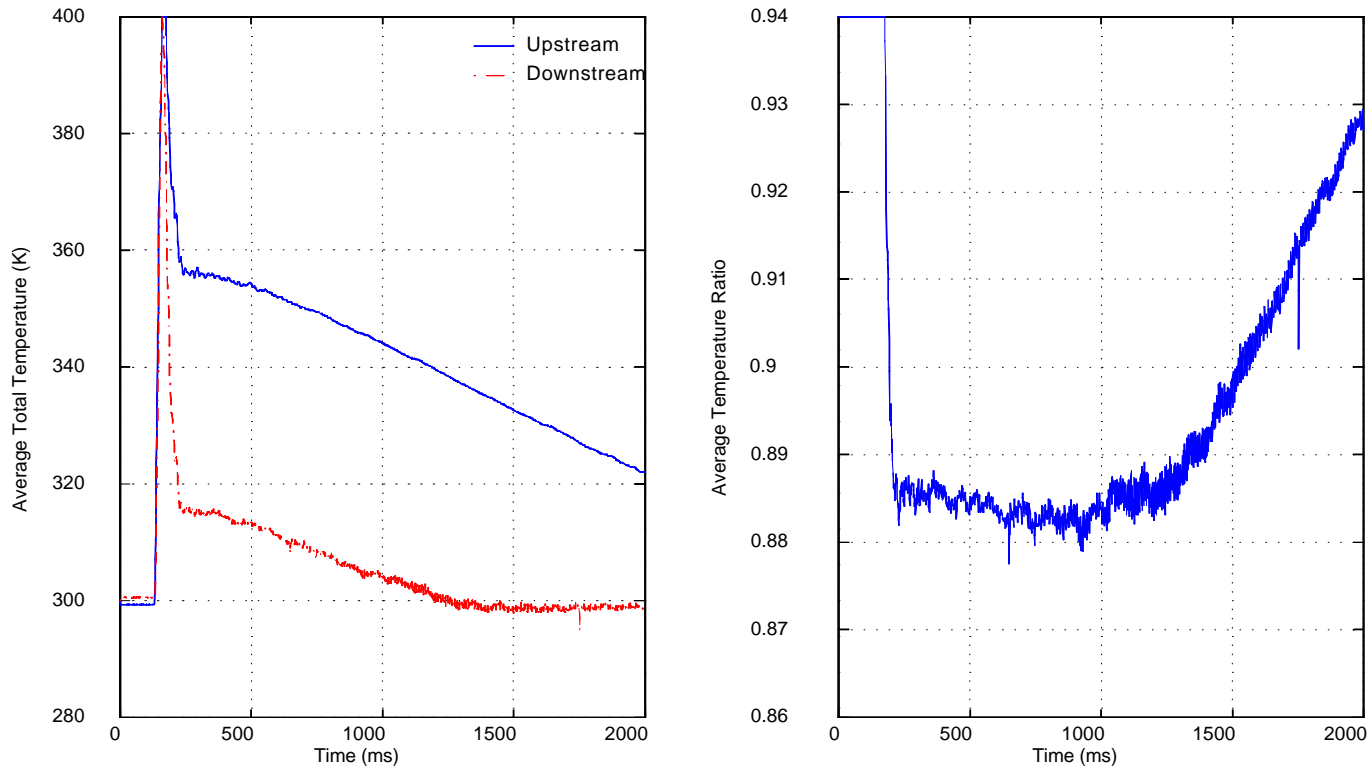

Figure 13.6: ABB027 - Average Total Temperature and Temperature Ratio 

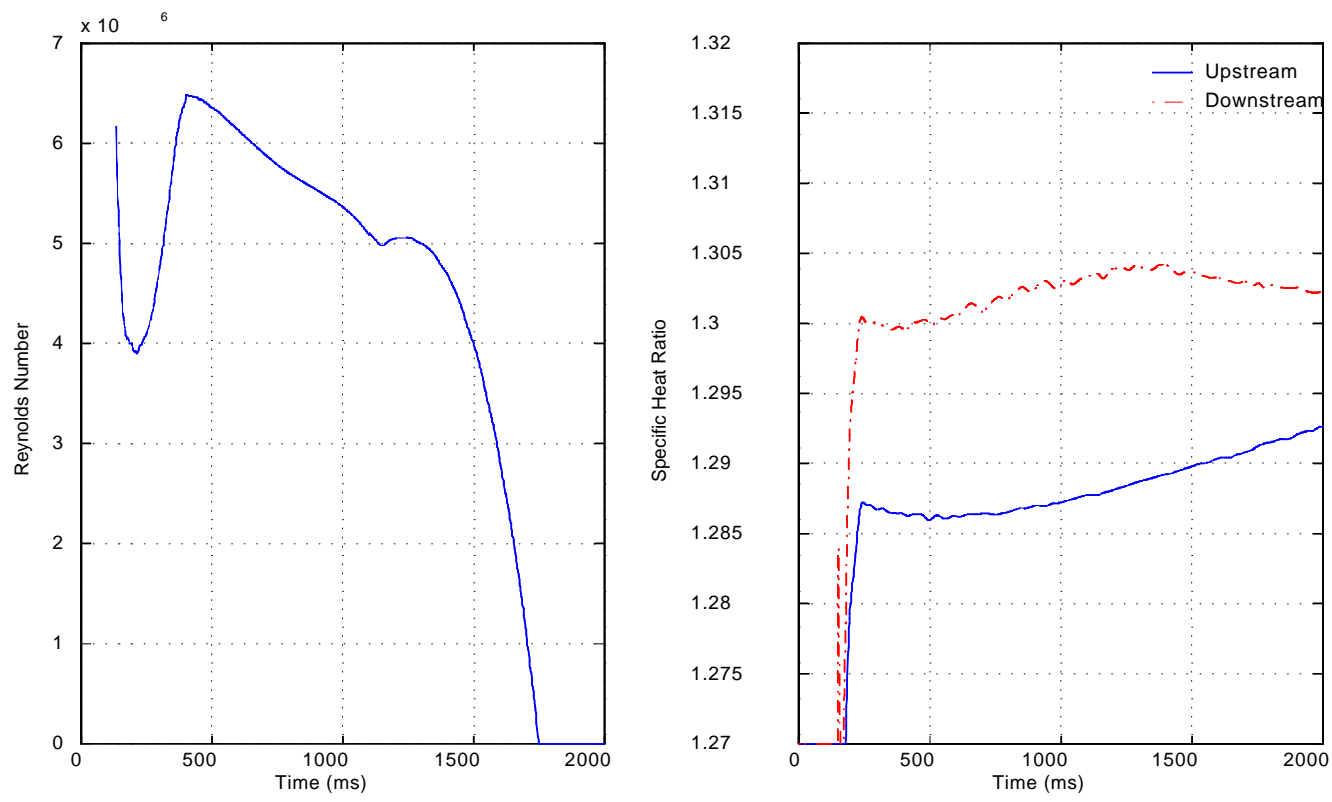

Figure 13.7: ABB027 - Reynolds Number and Specific Heat Ratio
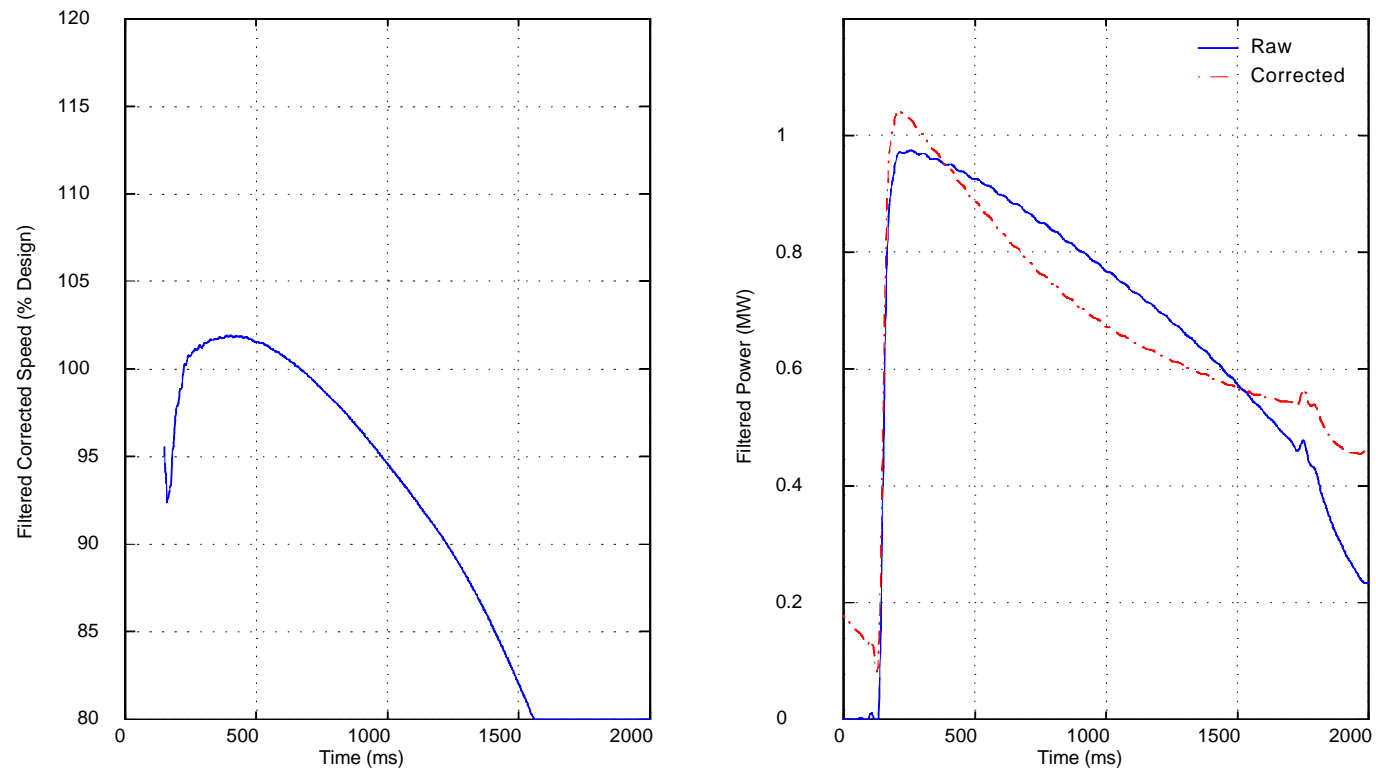

Figure 13.8: ABB027 - Corrected Speed and Power 

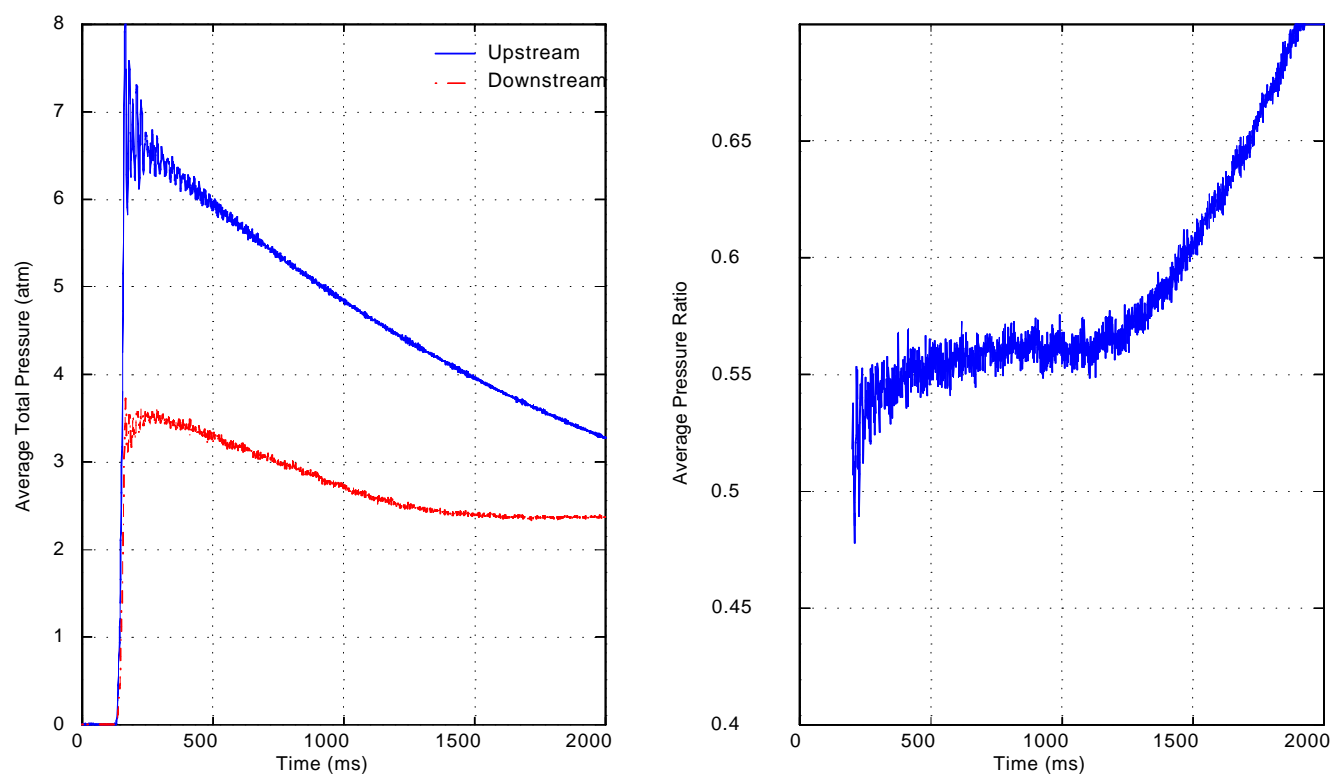

Figure 13.9: ABB032 - Average Total Pressure and Pressure Ratio
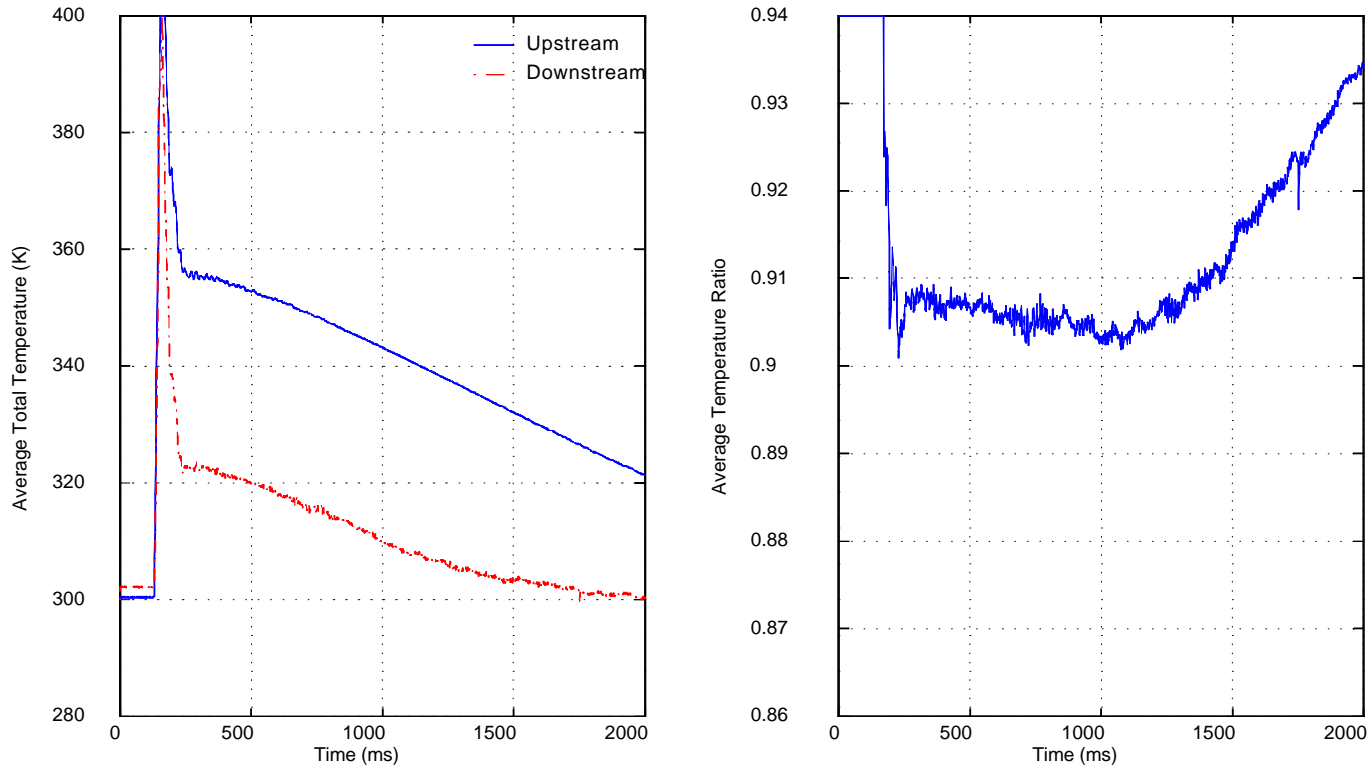

Figure 13.10: ABB032 - Average Total Temperature and Temperature Ratio 

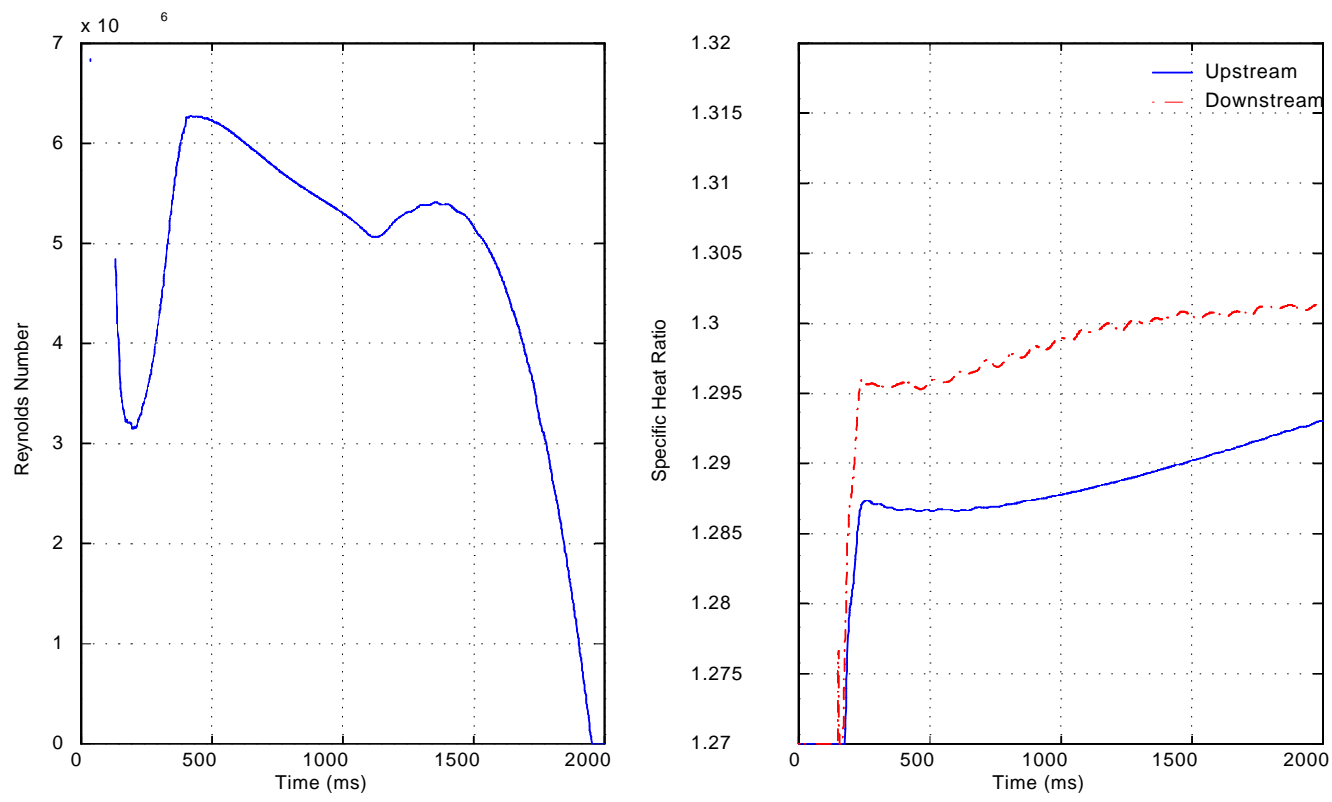

Figure 13.11: ABB032 - Reynolds Number and Specific Heat Ratio
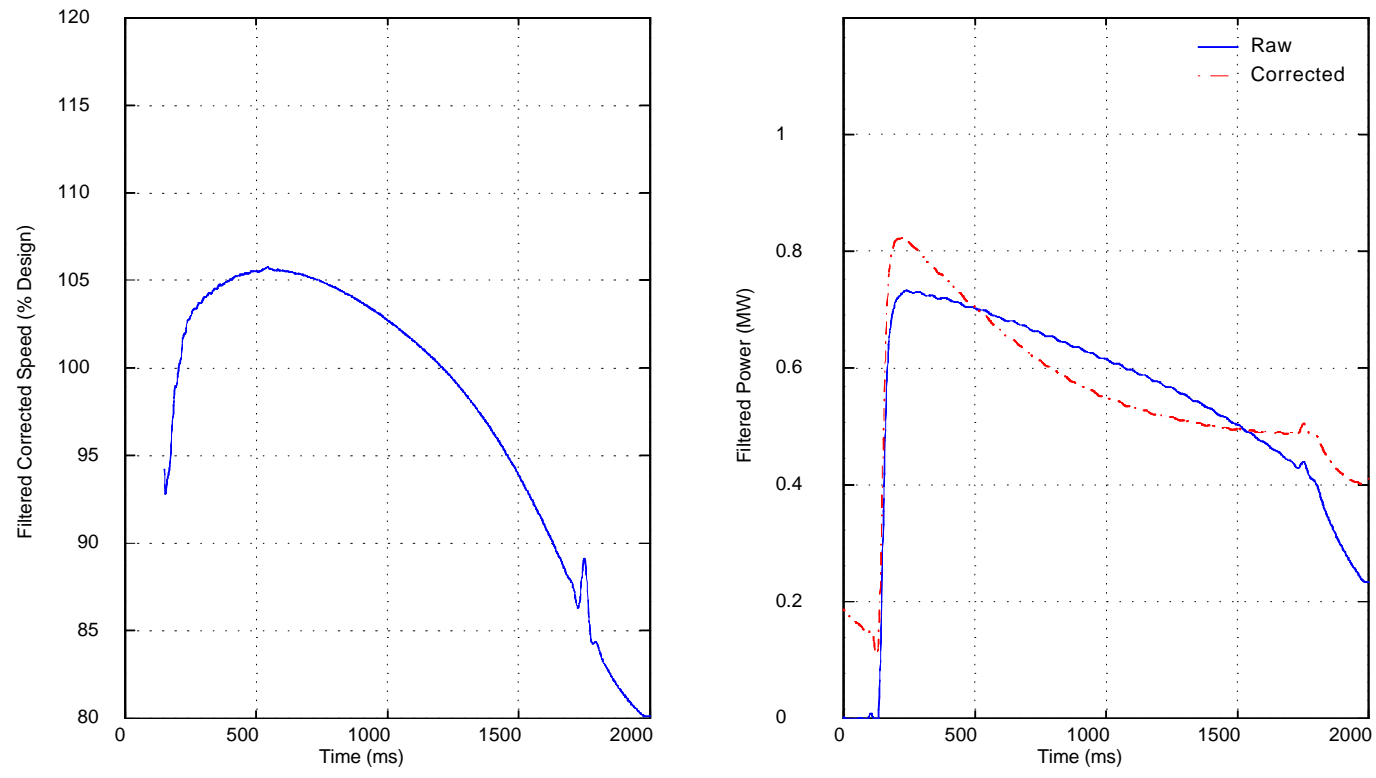

Figure 13.12: ABB032 - Corrected Speed and Power 

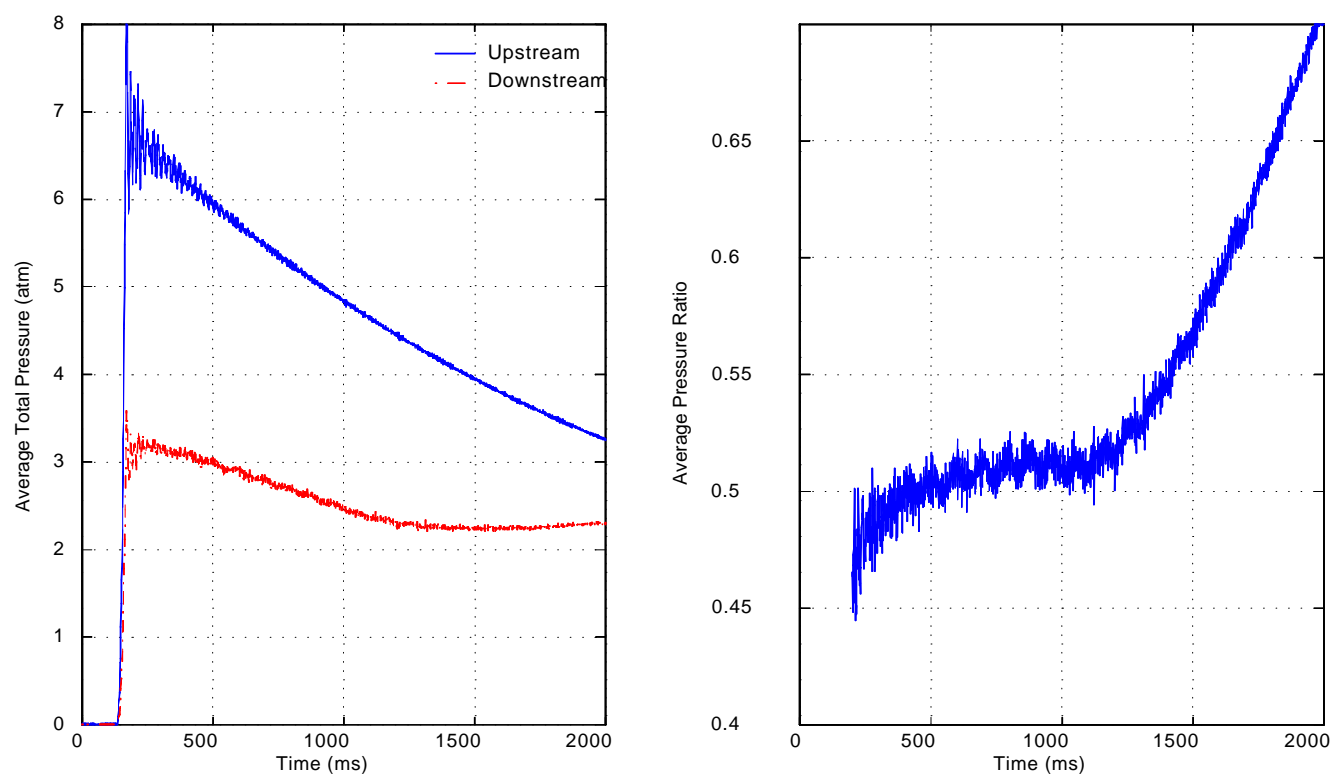

Figure 13.13: ABB029 - Average Total Pressure and Pressure Ratio
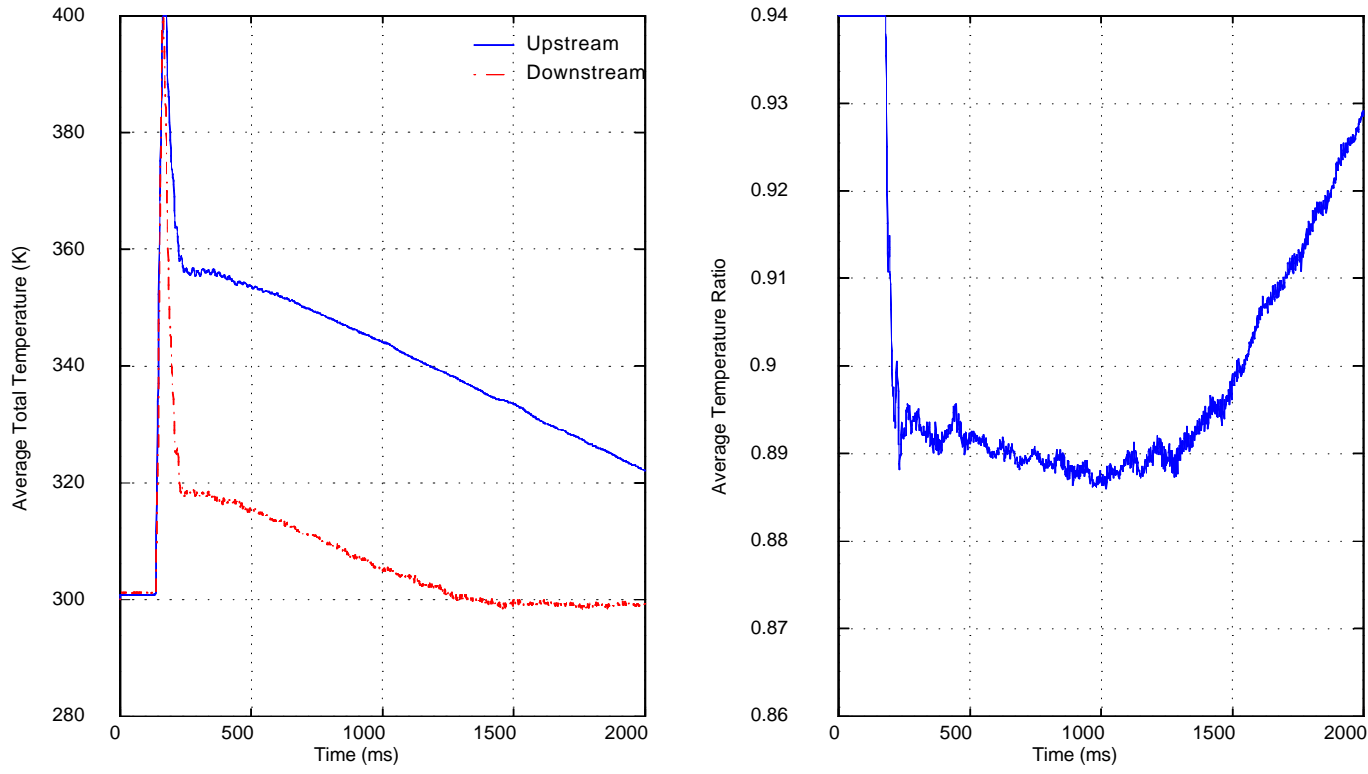

Figure 13.14: ABB029 - Average Total Temperature and Temperature Ratio 

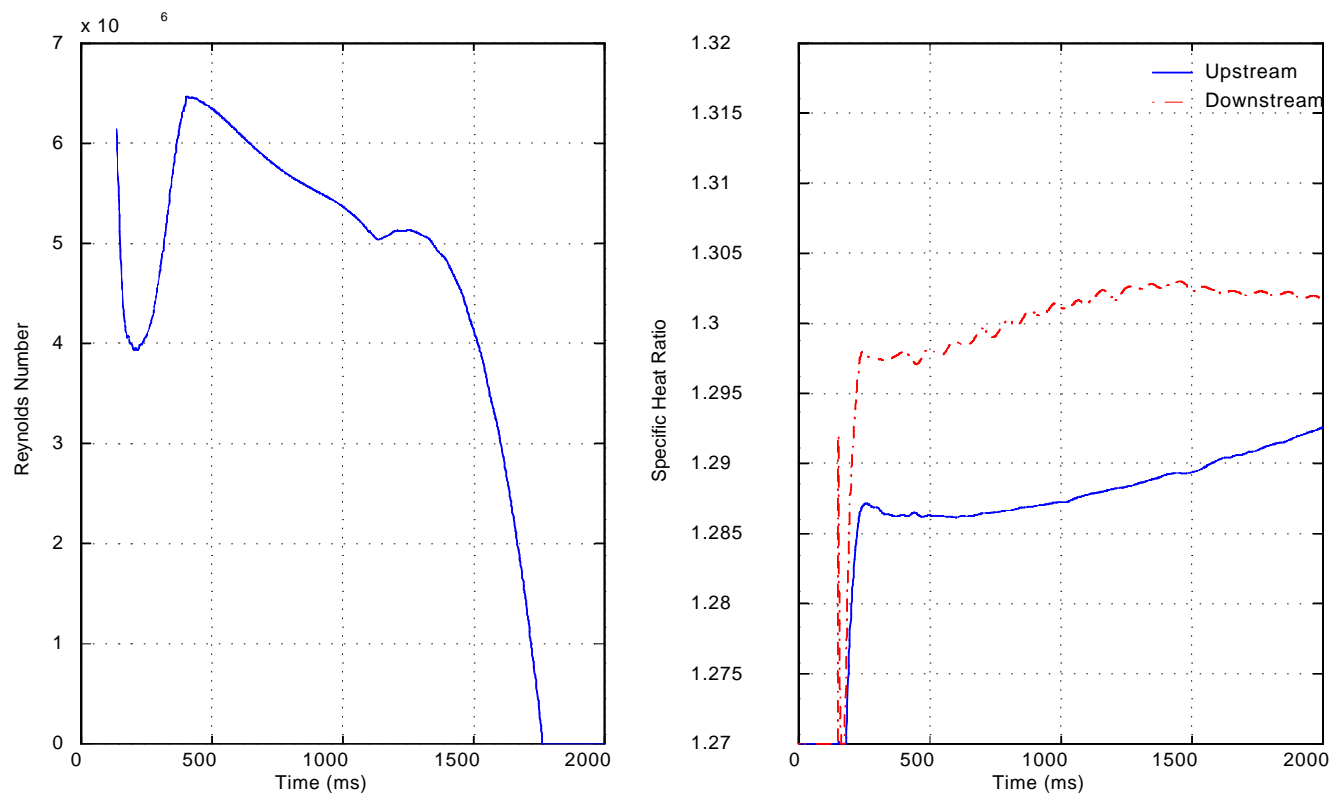

Figure 13.15: ABB029 - Reynolds Number and Specific Heat Ratio
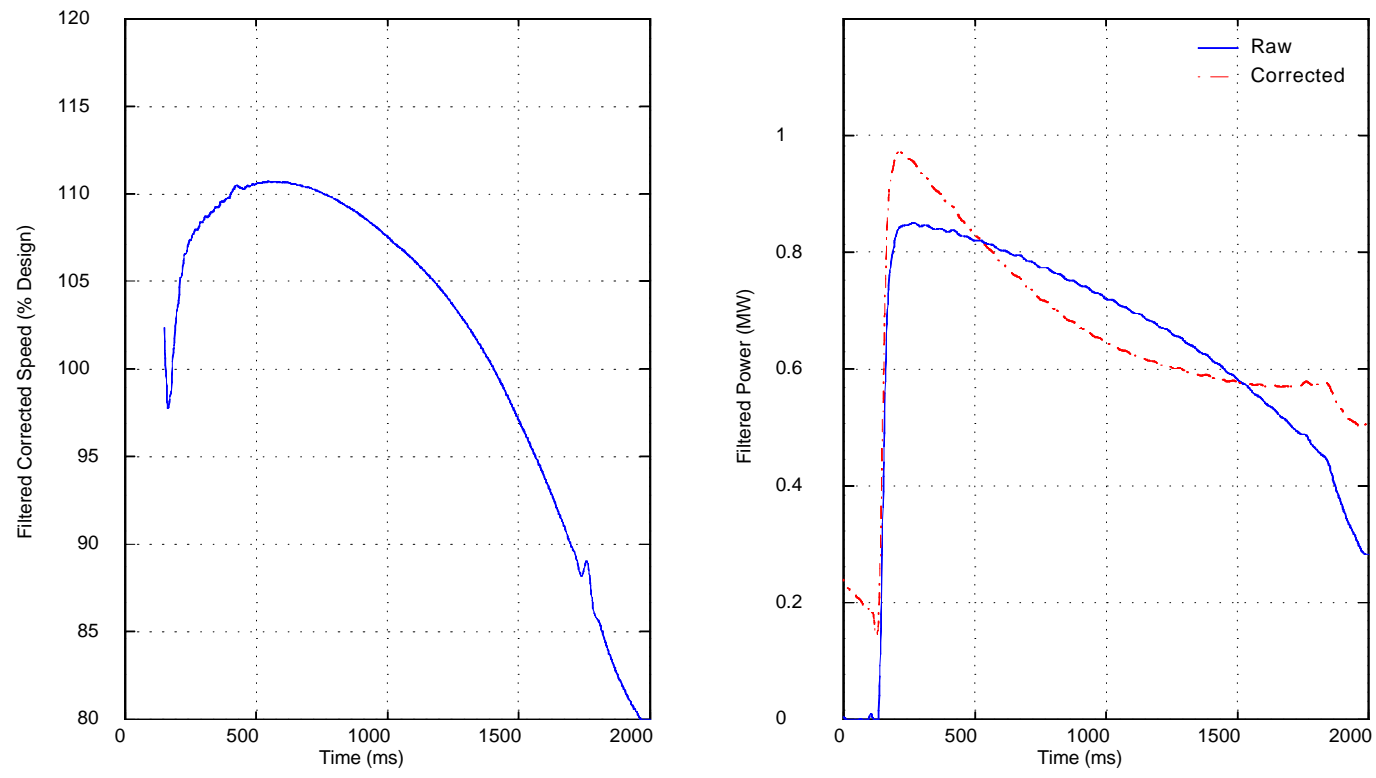

Figure 13.16: ABB029 - Corrected Speed and Power 

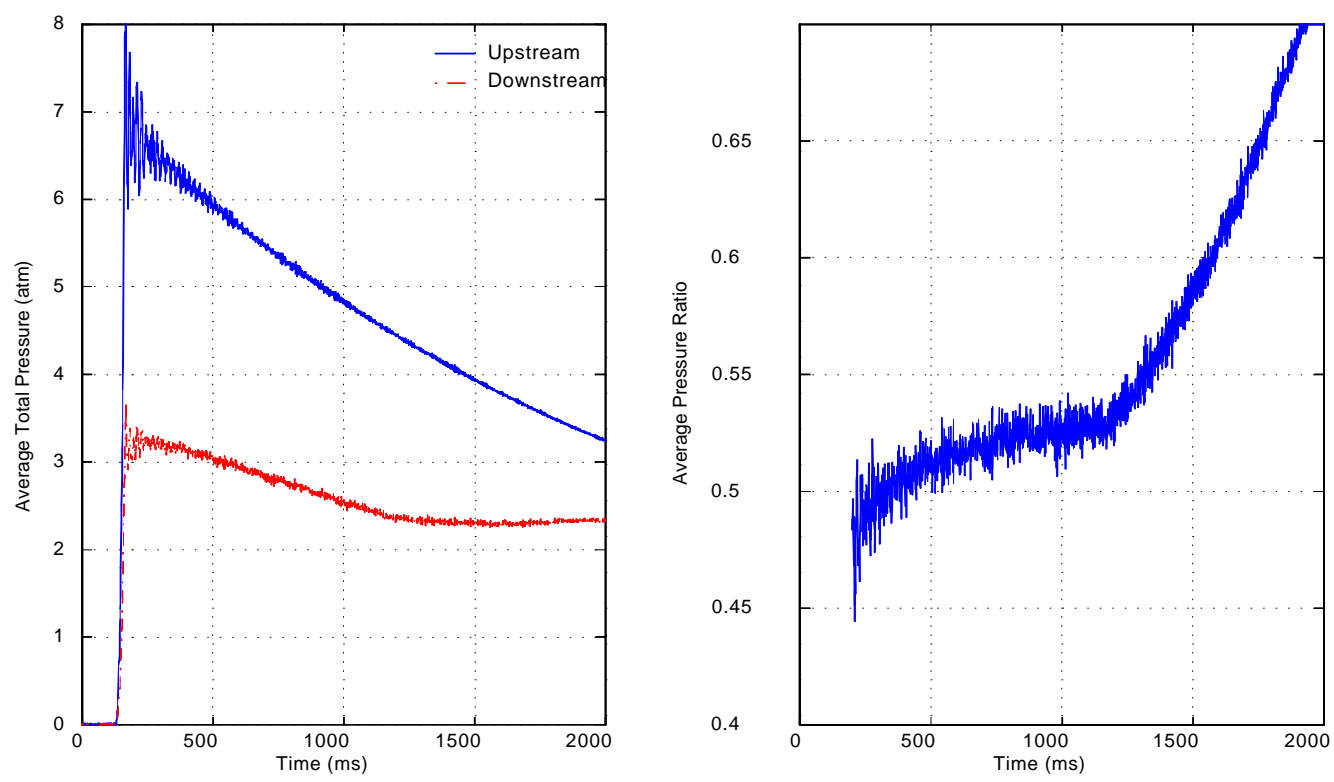

Figure 13.17: ABB028 - Average Total Pressure and Pressure Ratio
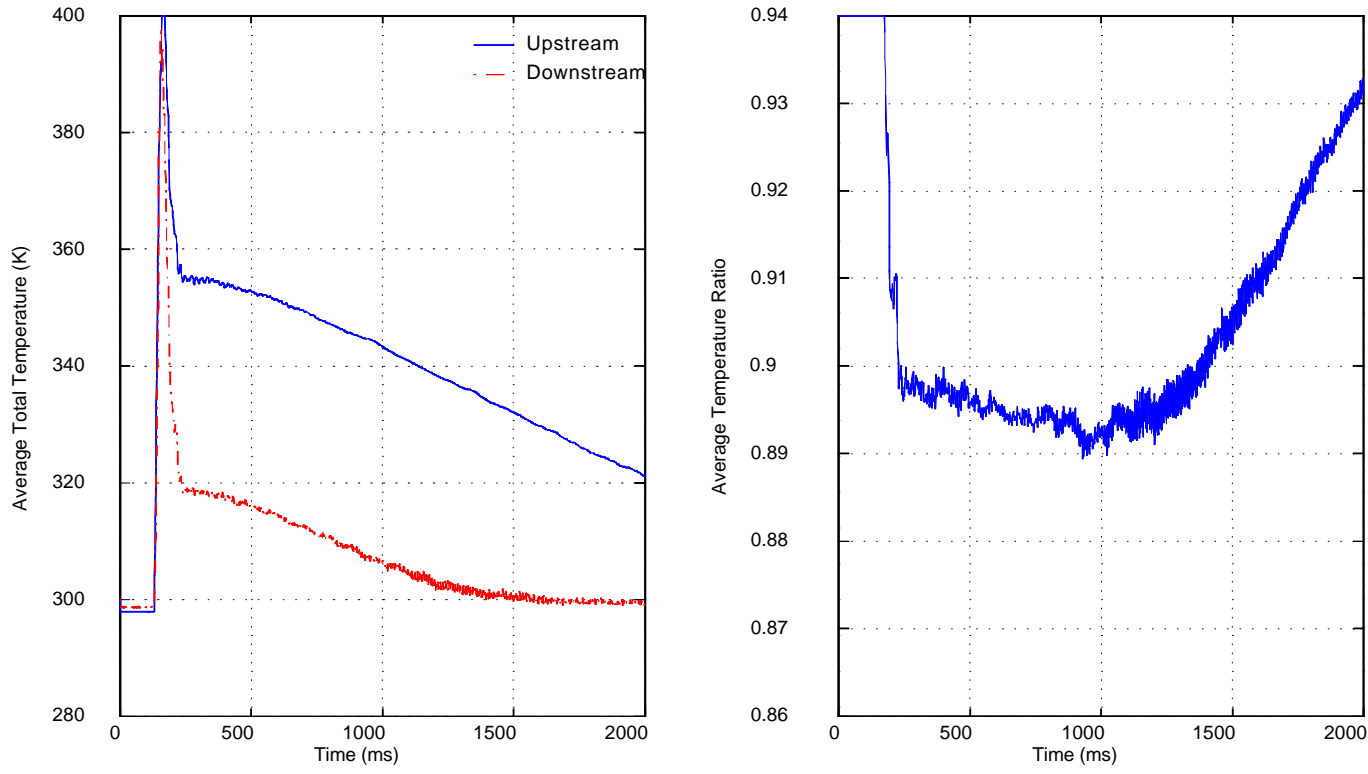

Figure 13.18: ABB028 - Average Total Temperature and Temperature Ratio 

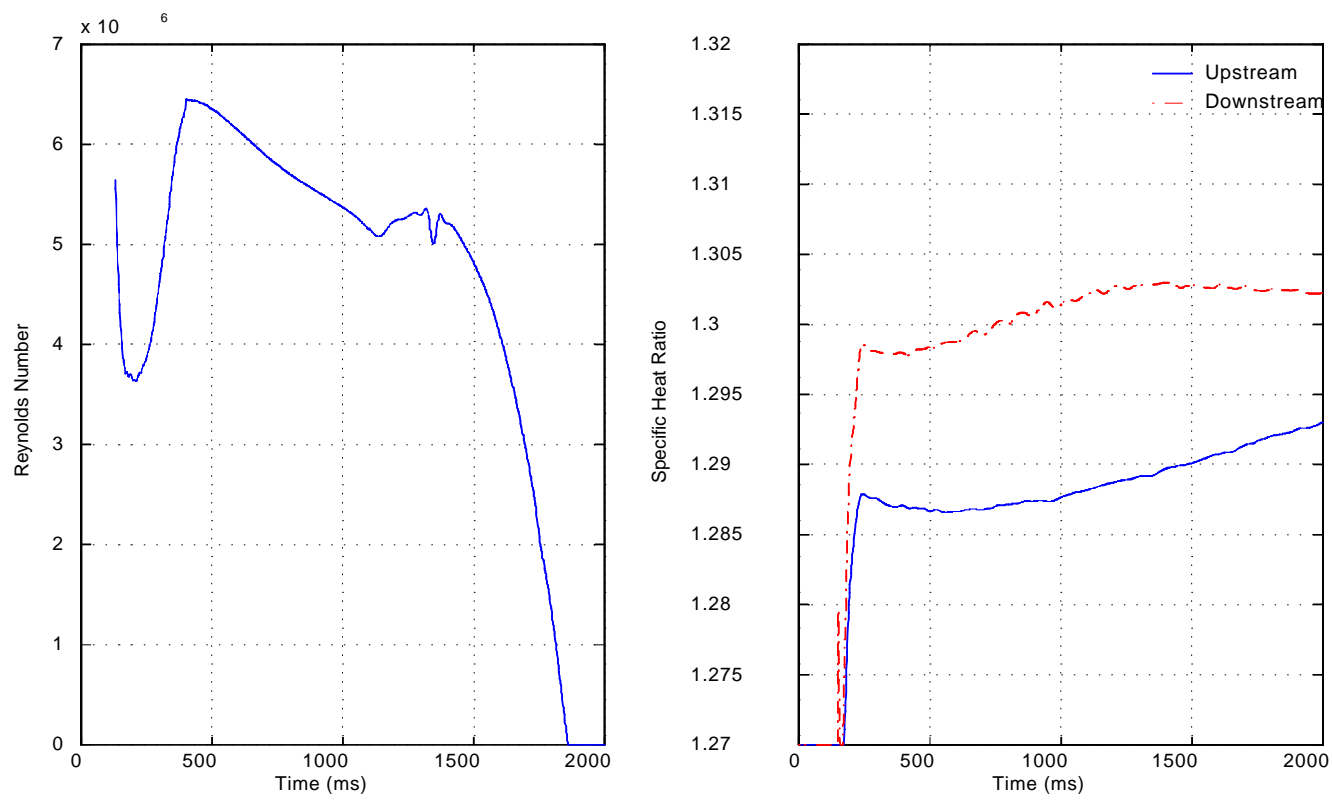

Figure 13.19: ABB028 - Reynolds Number and Specific Heat Ratio
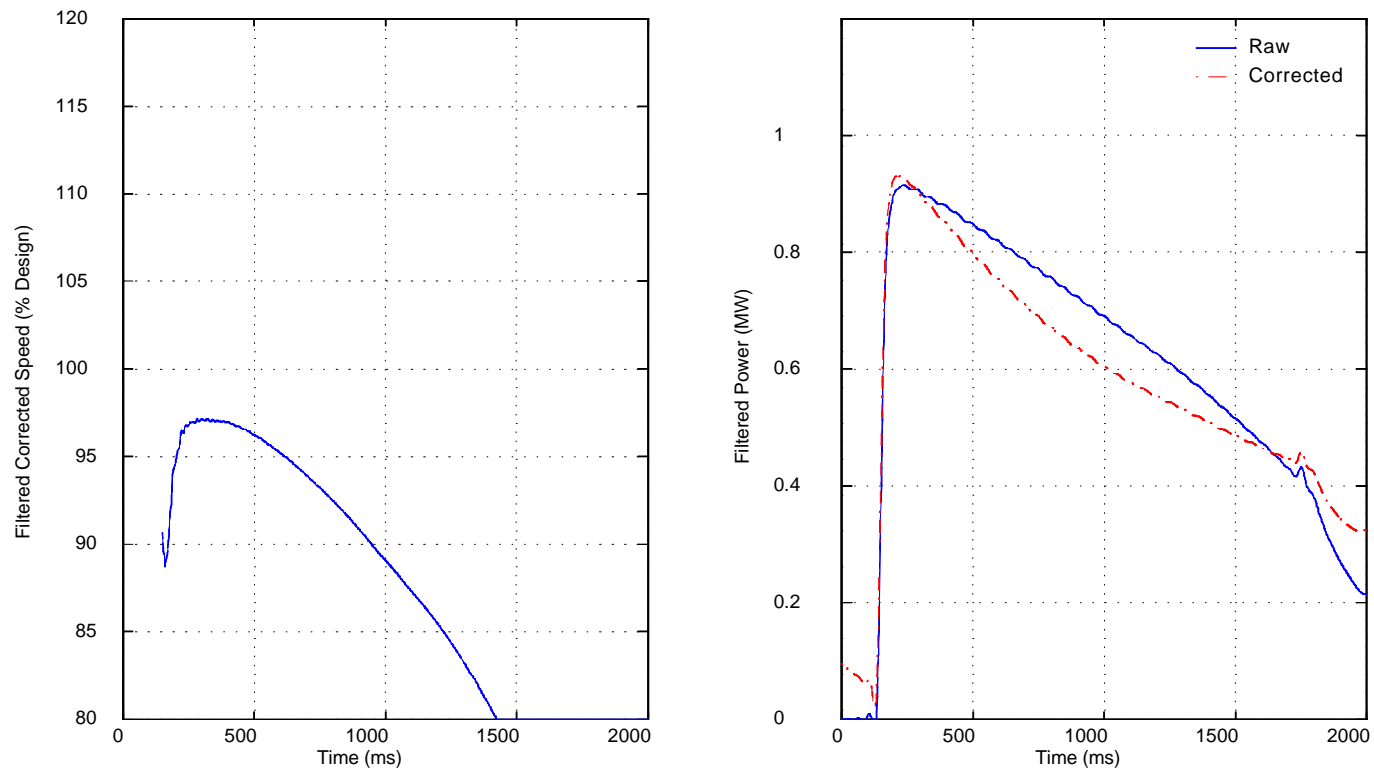

Figure 13.20: ABB028 - Corrected Speed and Power 

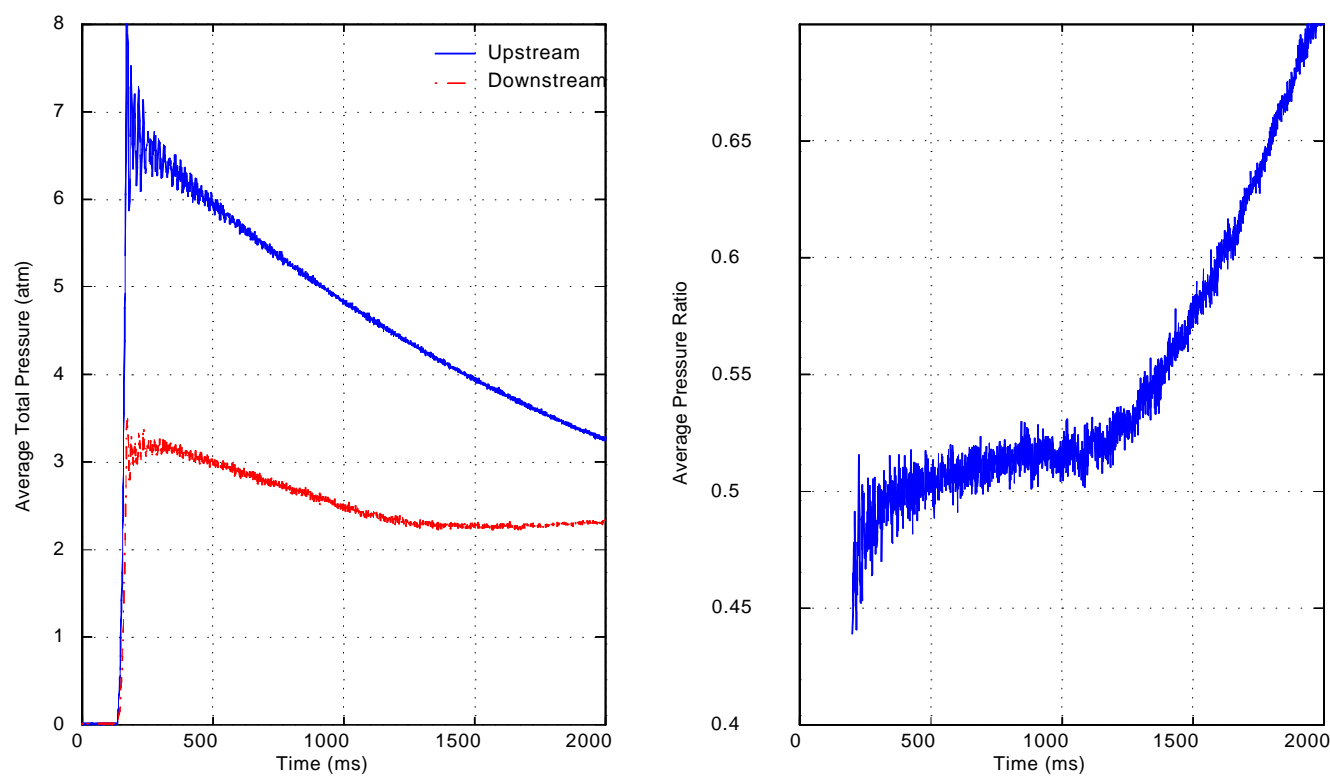

Figure 13.21: ABB034 - Average Total Pressure and Pressure Ratio
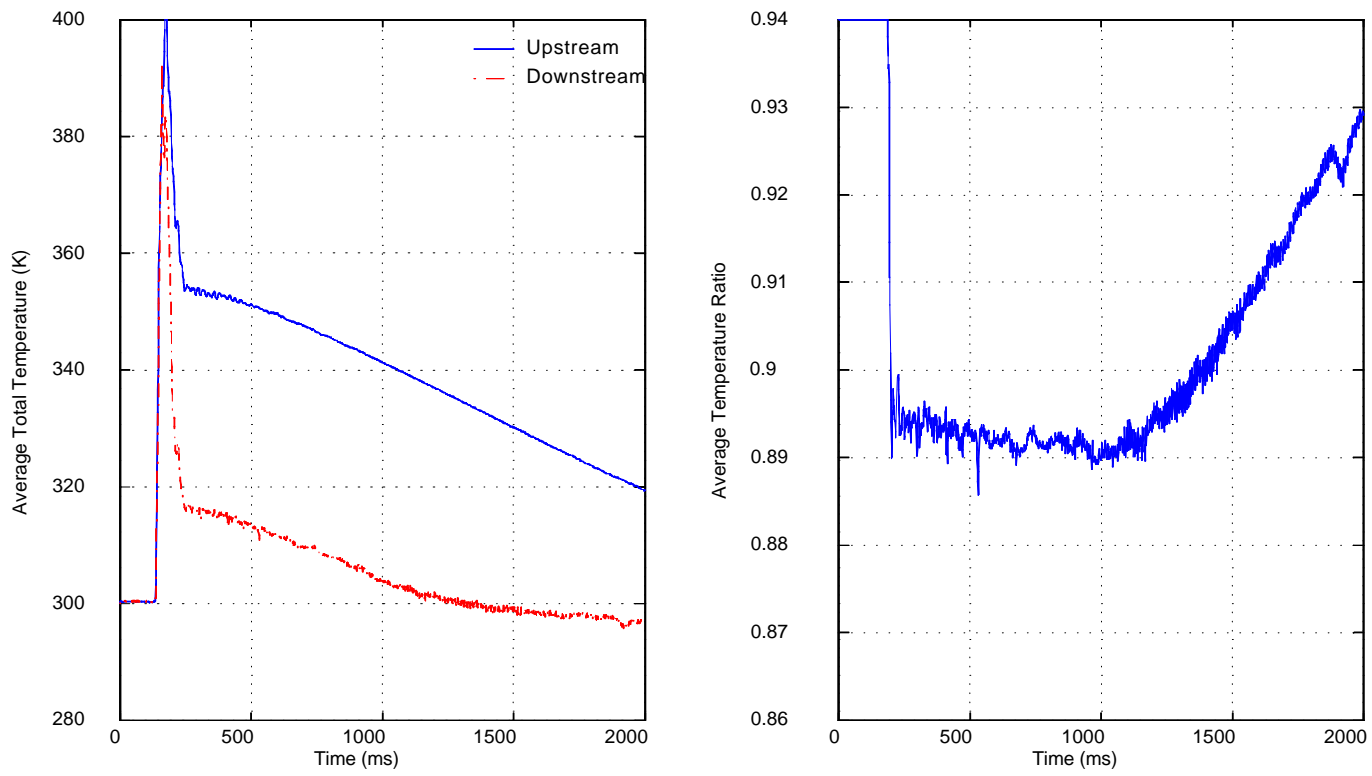

Figure 13.22: ABB034 - Average Total Temperature and Temperature Ratio 

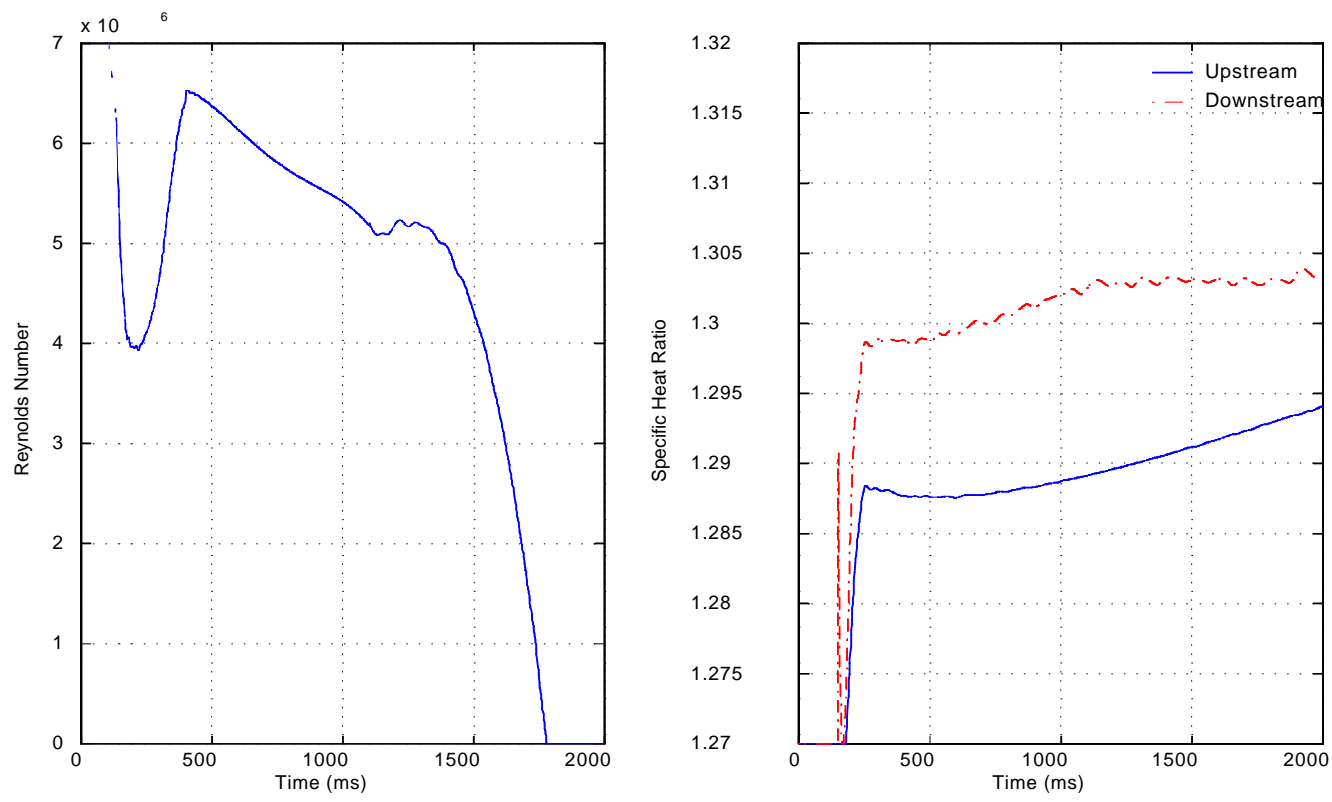

Figure 13.23: ABB034 - Reynolds Number and Specific Heat Ratio
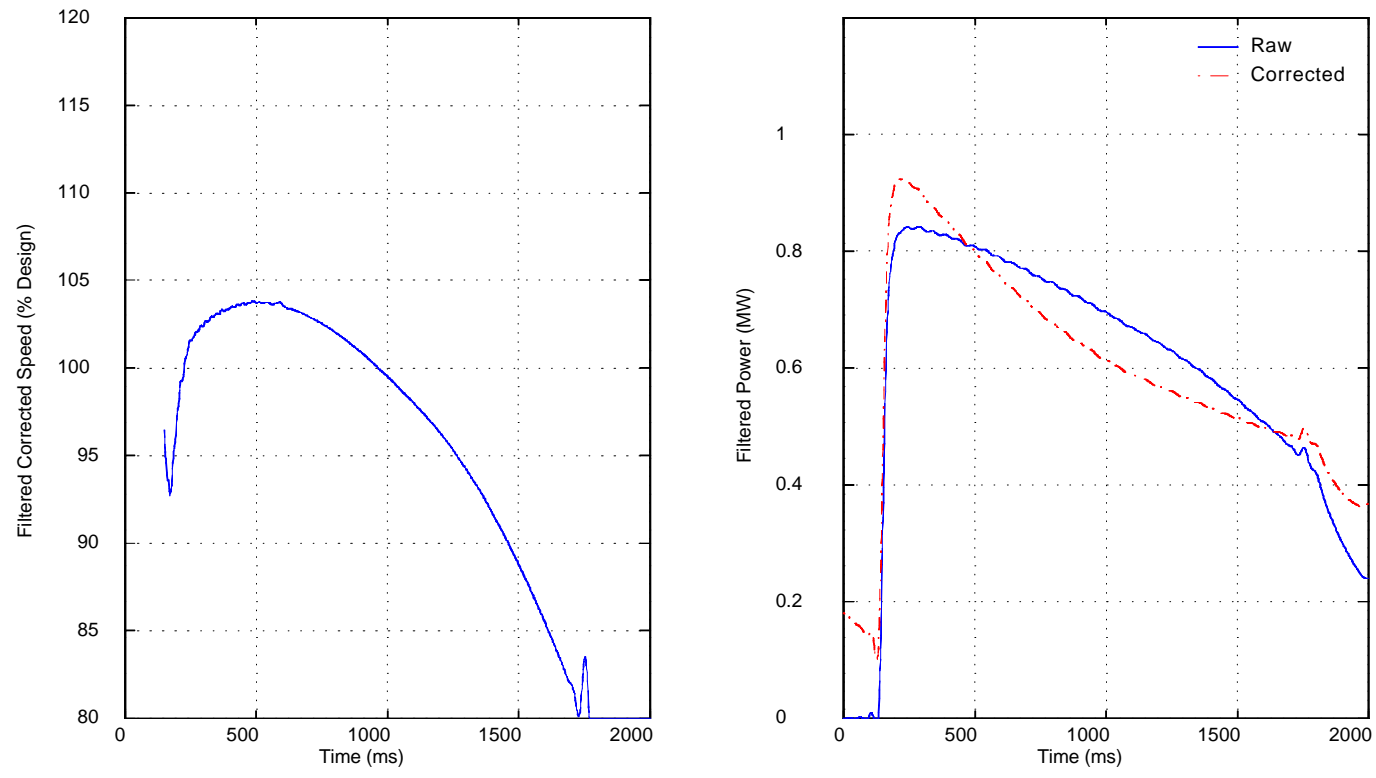

Figure 13.24: ABB034 - Corrected Speed and Power 

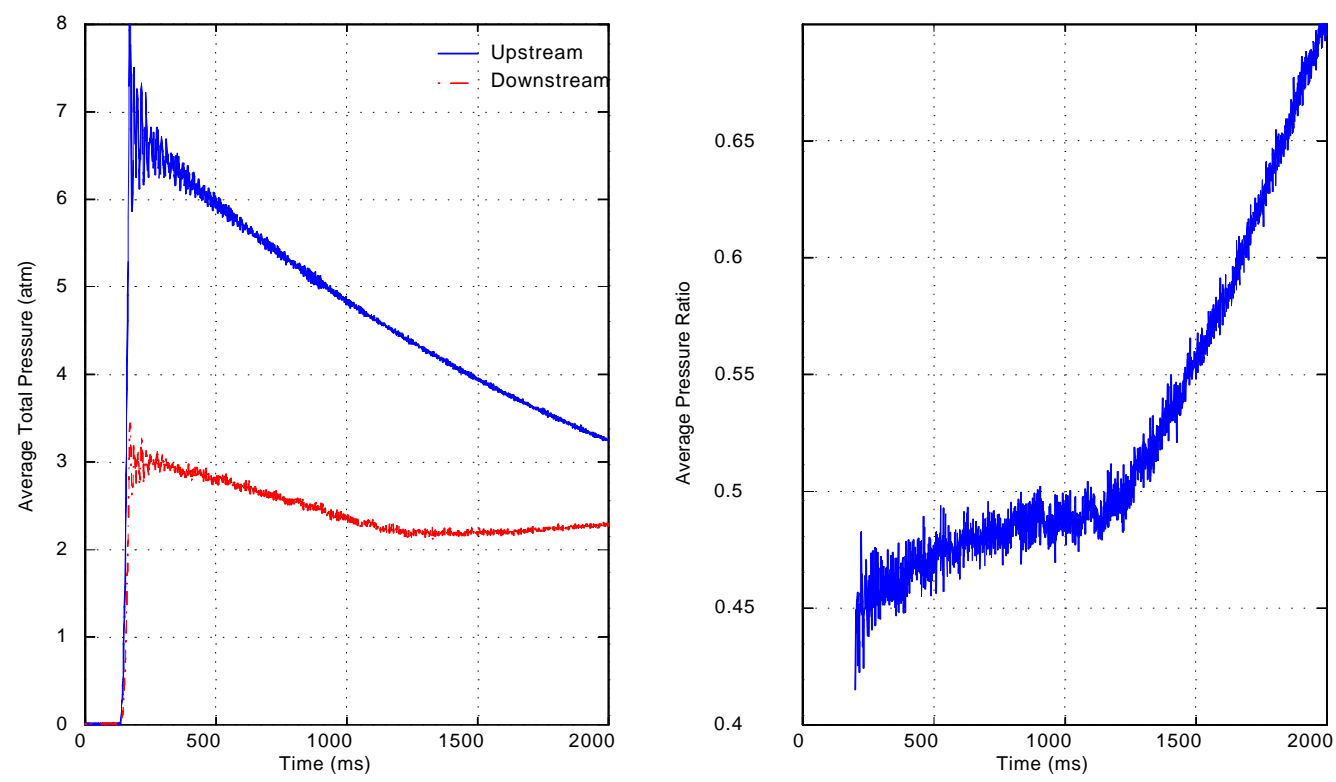

Figure 13.25: ABB035 - Average Total Pressure and Pressure Ratio
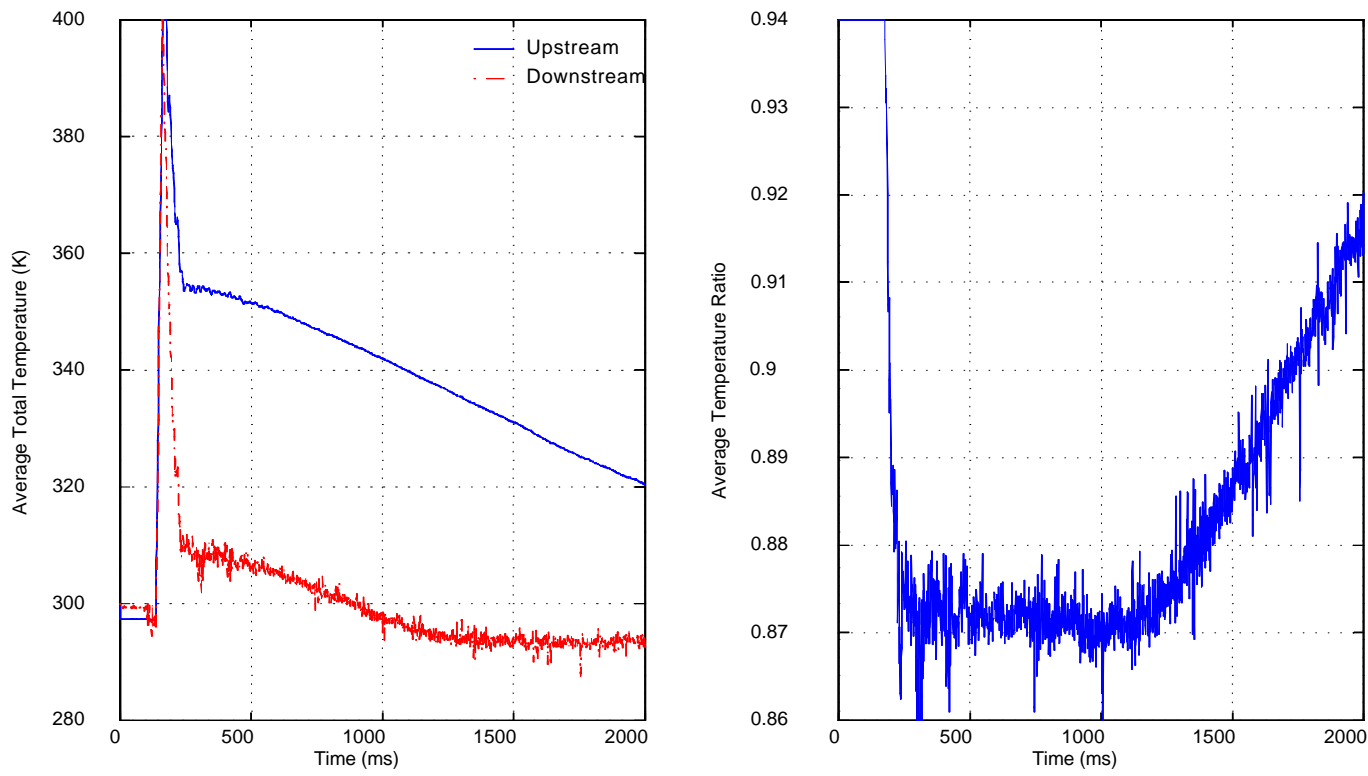

Figure 13.26: ABB035 - Average Total Temperature and Temperature Ratio 

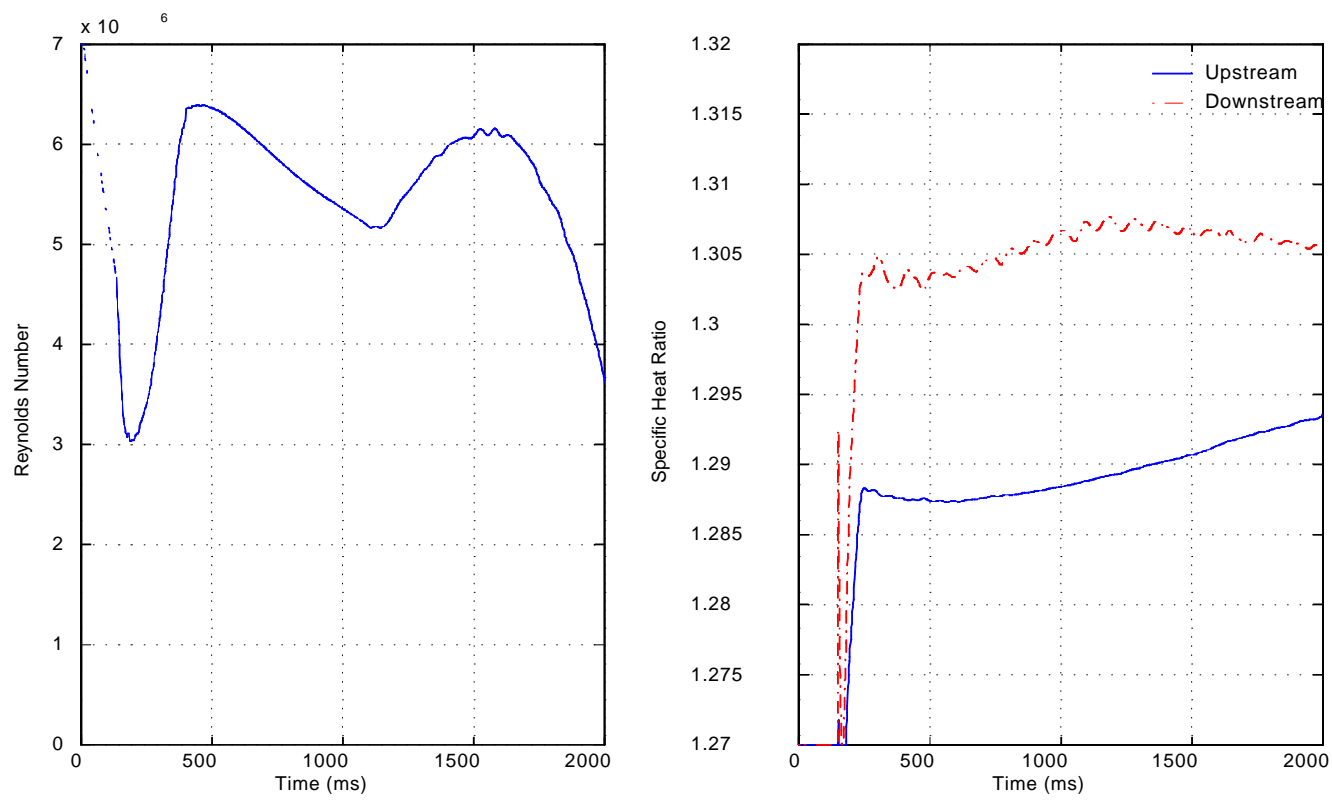

Figure 13.27: ABB035 - Reynolds Number and Specific Heat Ratio
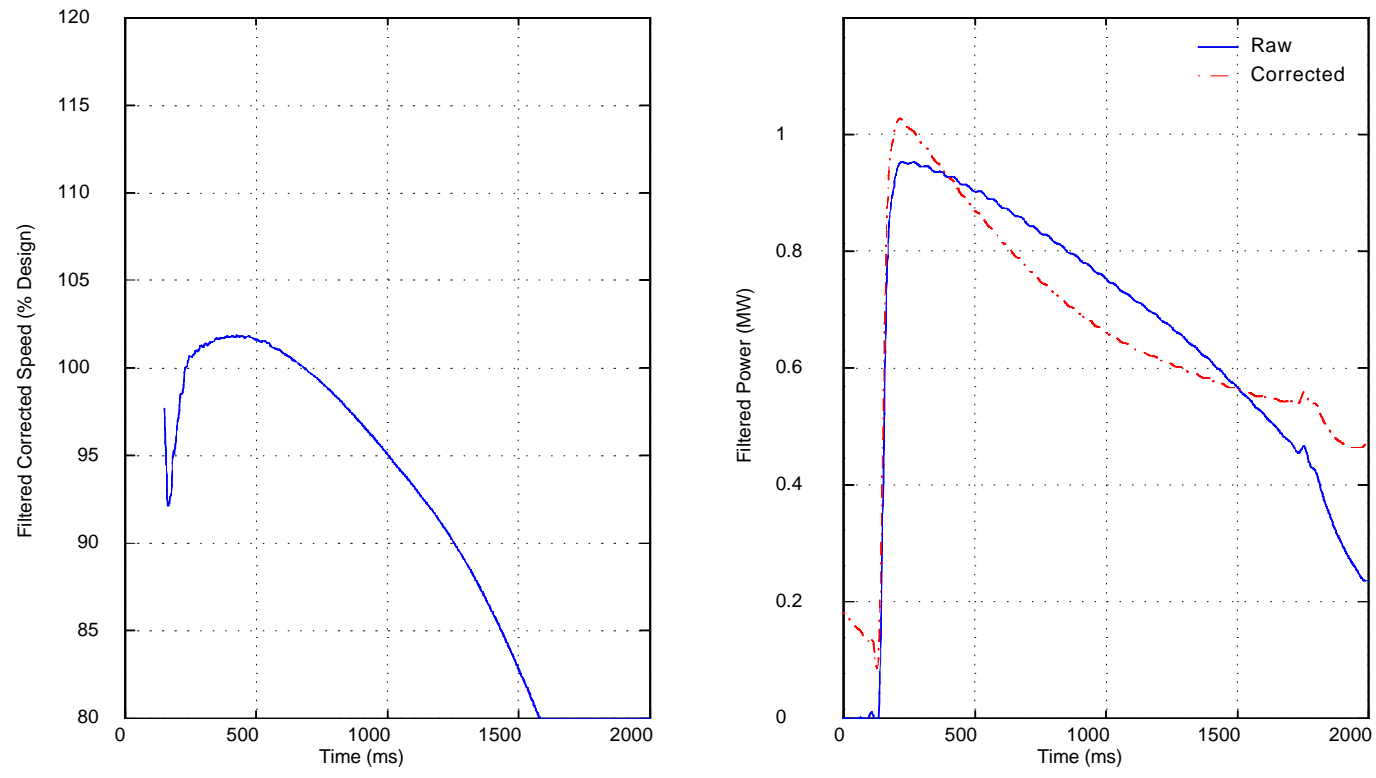

Figure 13.28: ABB035 - Corrected Speed and Power 

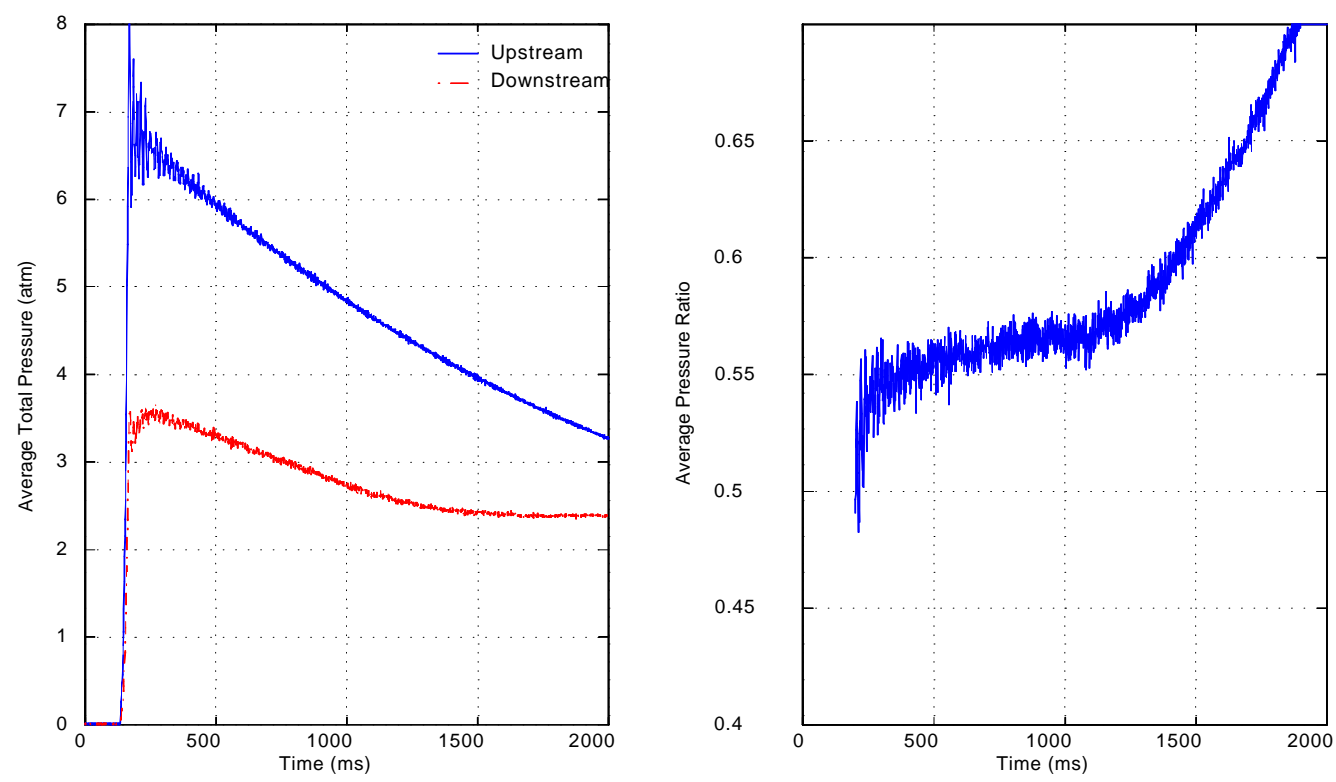

Figure 13.29: ABB033 - Average Total Pressure and Pressure Ratio
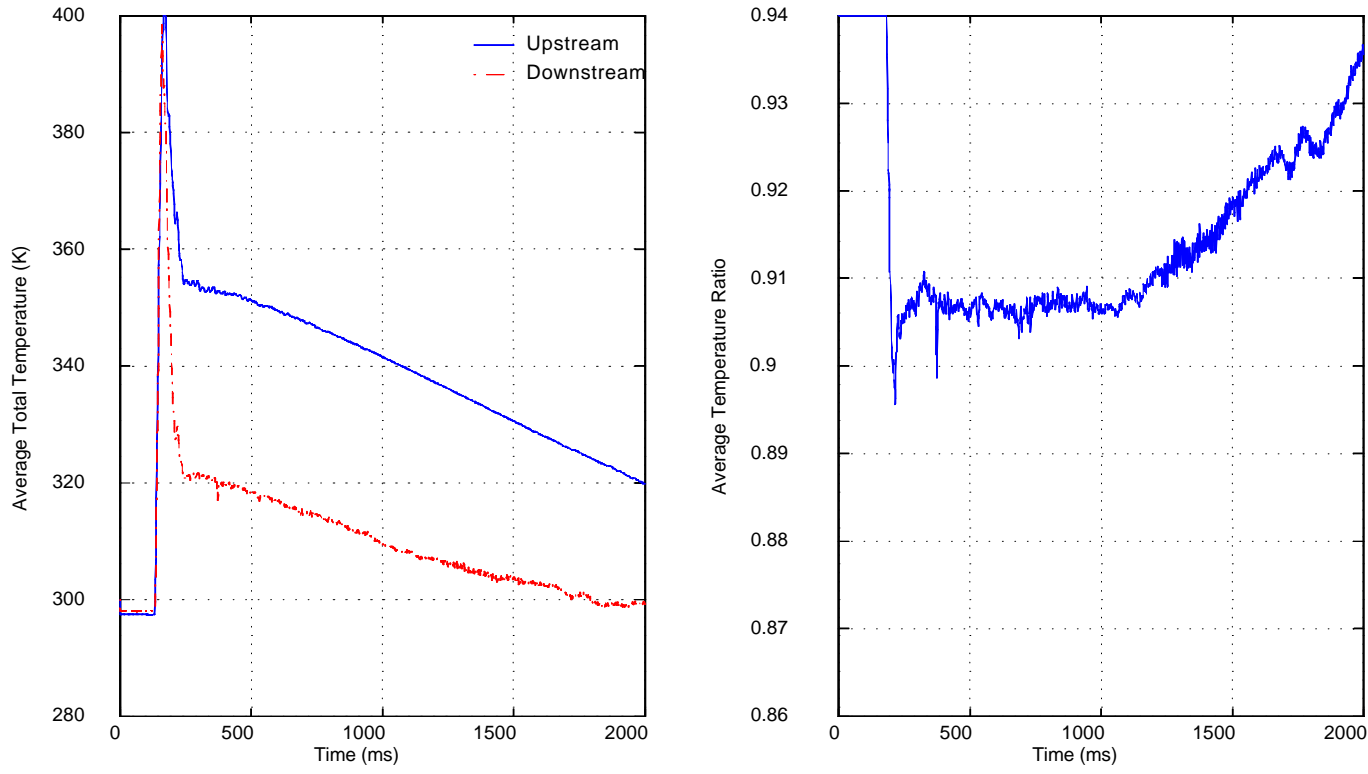

Figure 13.30: ABB033 - Average Total Temperature and Temperature Ratio 

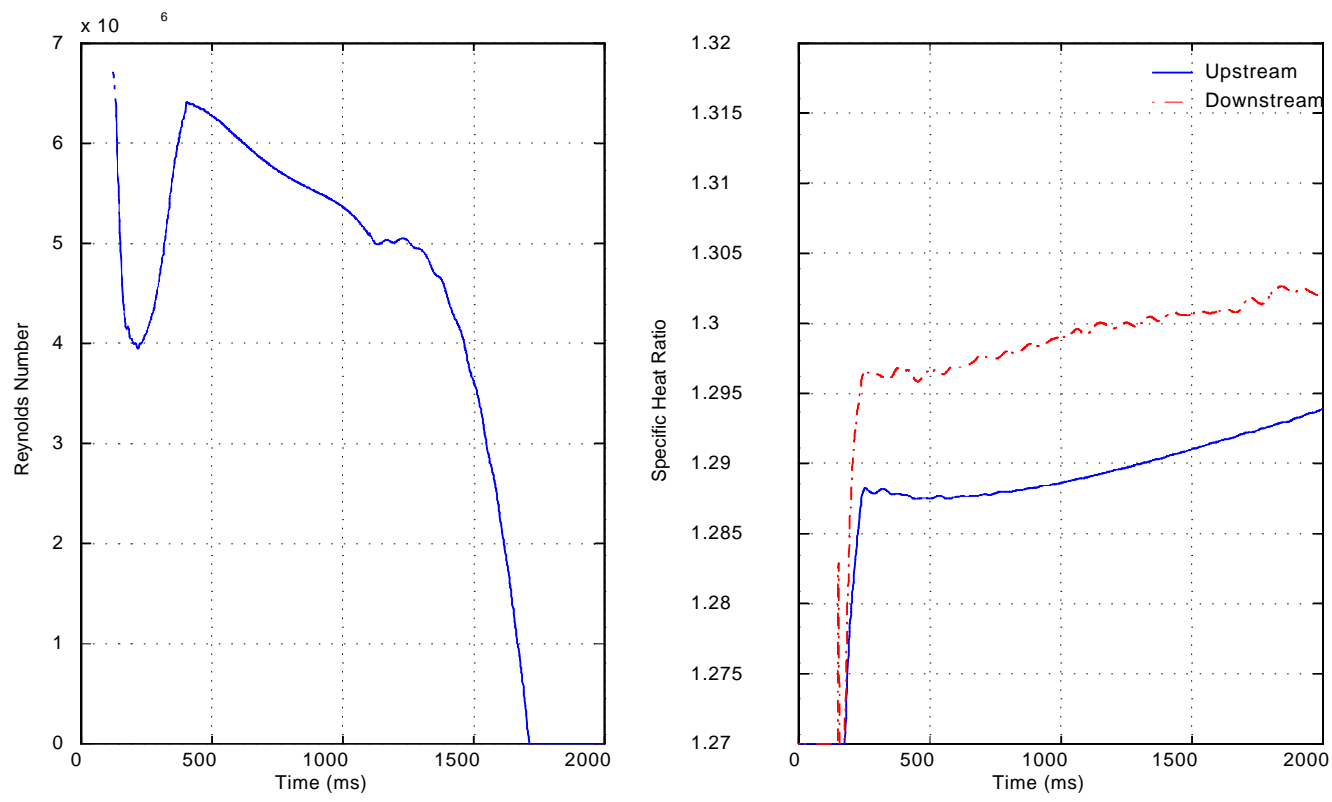

Figure 13.31: ABB033 - Reynolds Number and Specific Heat Ratio
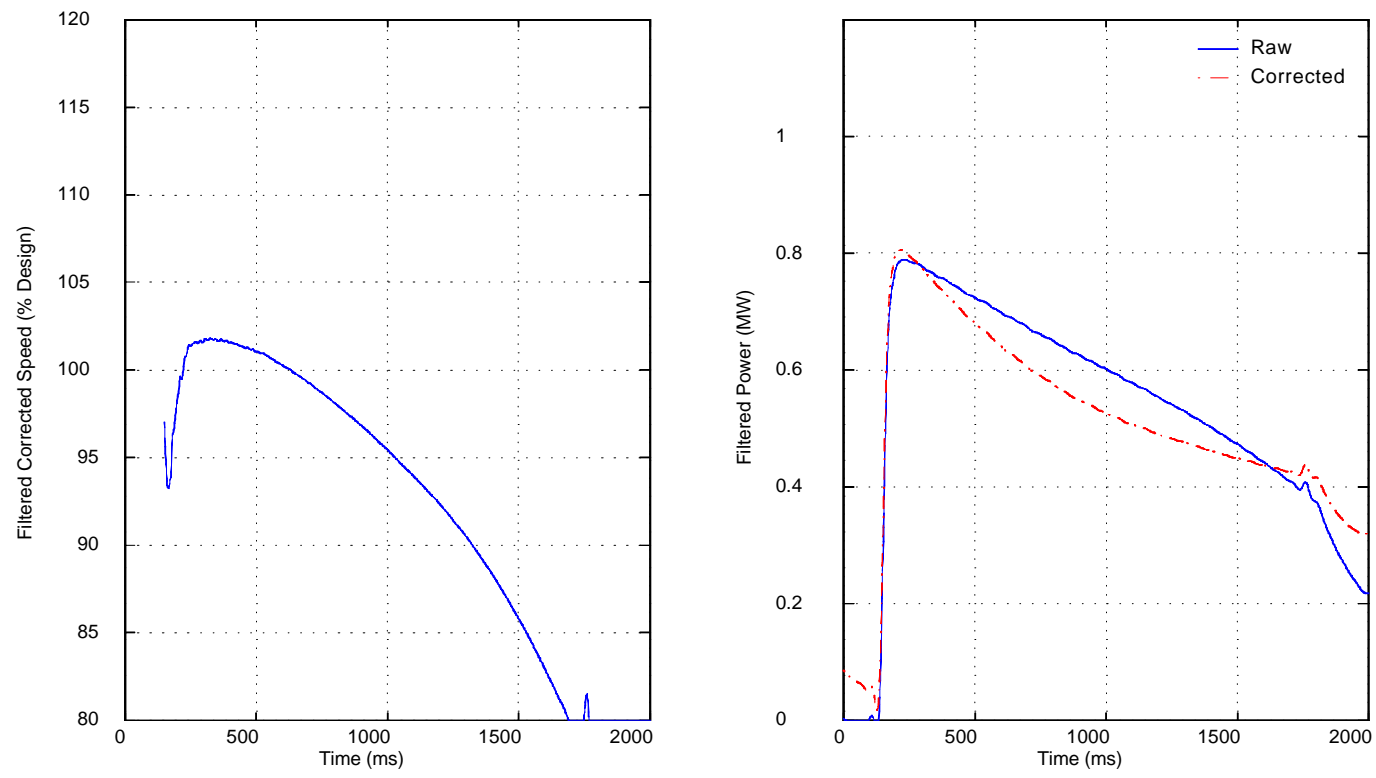

Figure 13.32: ABB033 - Corrected Speed and Power 

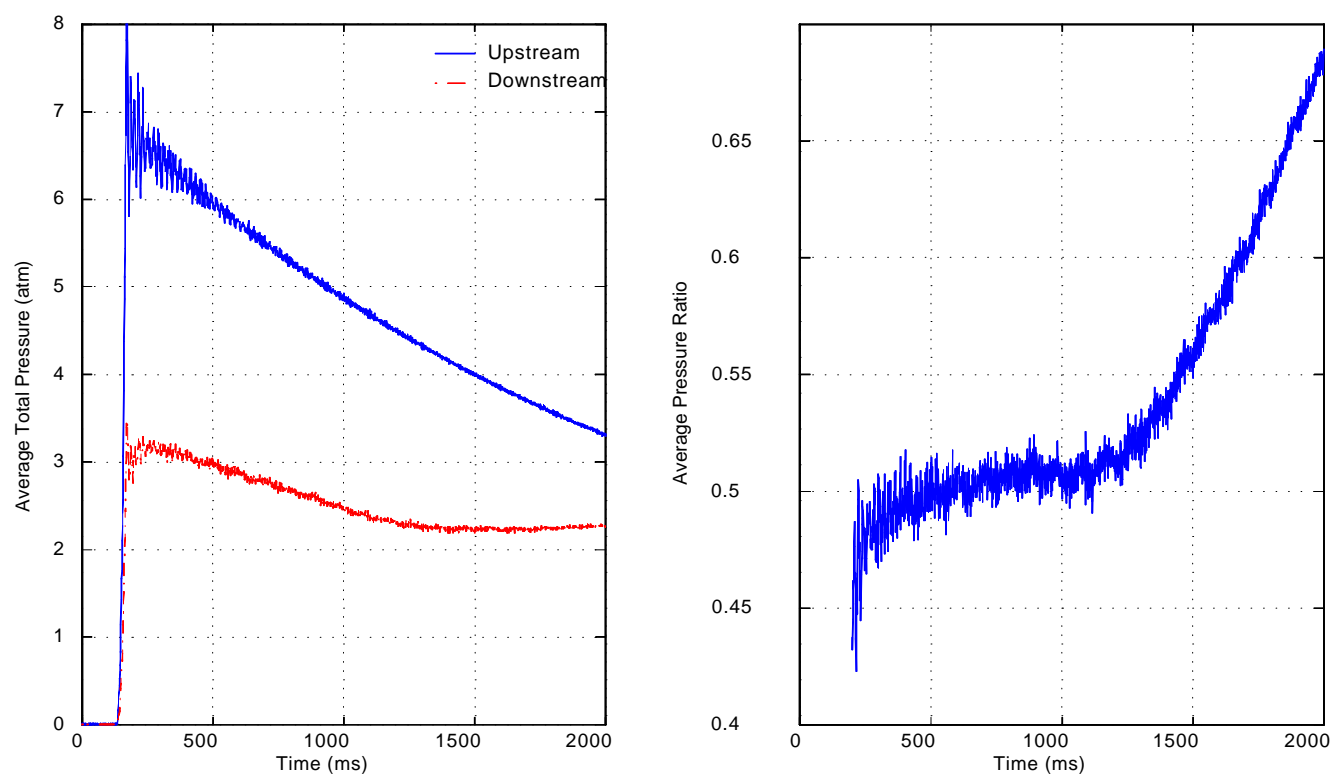

Figure 13.33: ABB037 - Average Total Pressure and Pressure Ratio
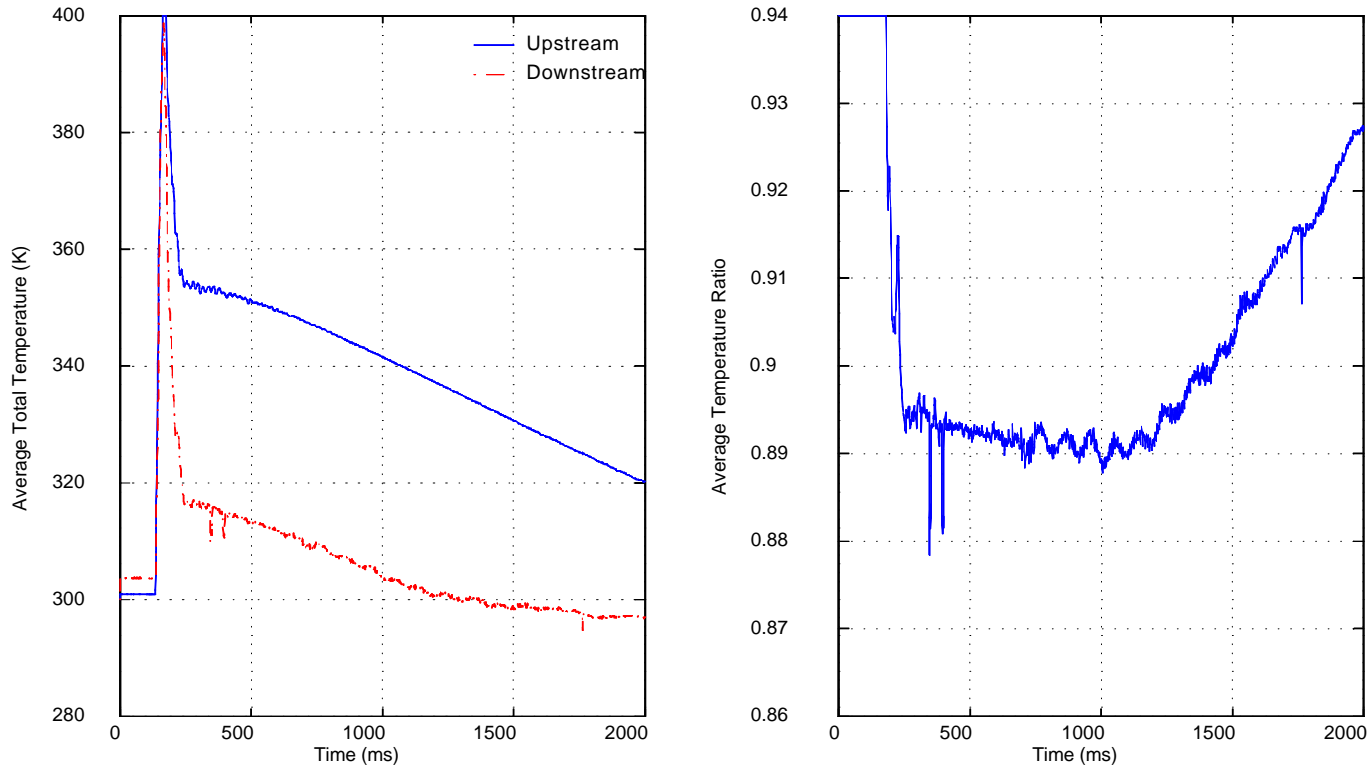

Figure 13.34: ABB037 - Average Total Temperature and Temperature Ratio 

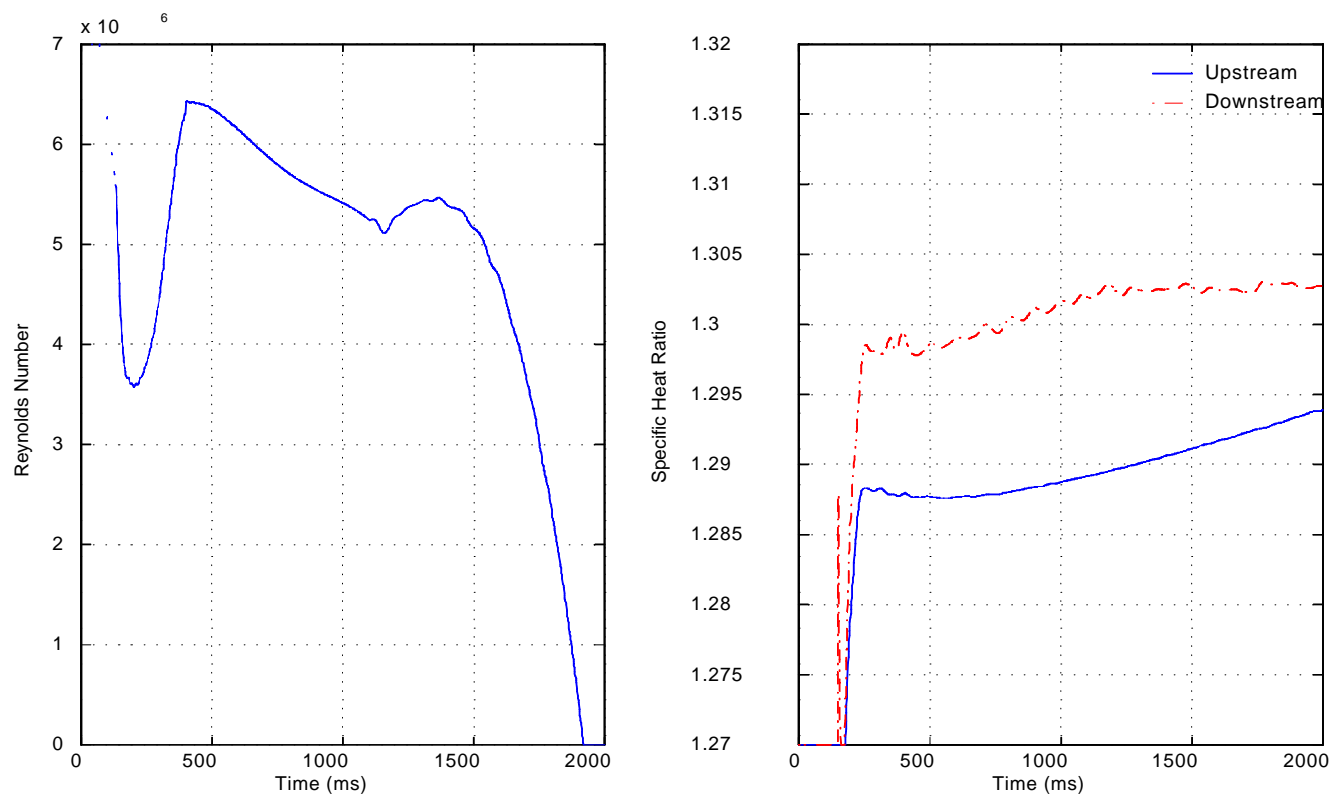

Figure 13.35: ABB037 - Reynolds Number and Specific Heat Ratio
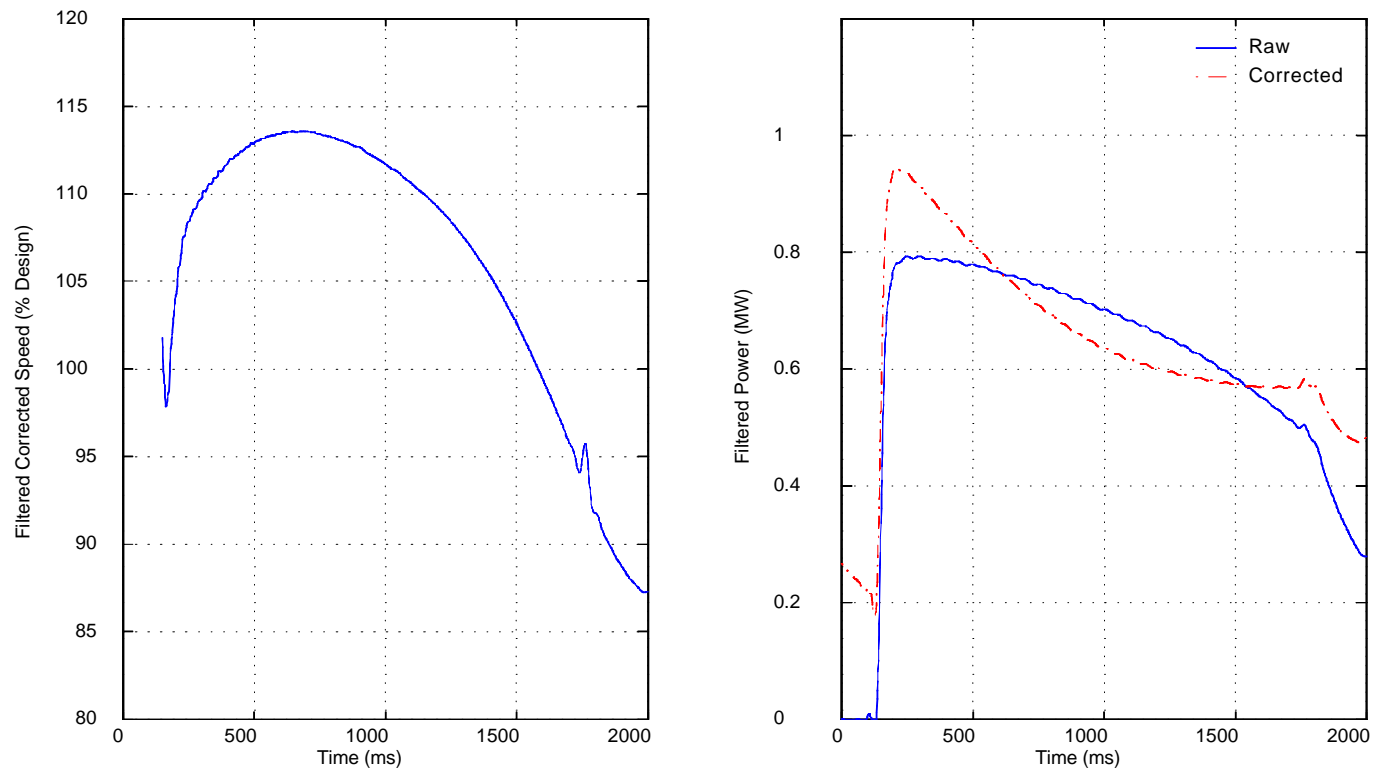

Figure 13.36: ABB037 - Corrected Speed and Power 

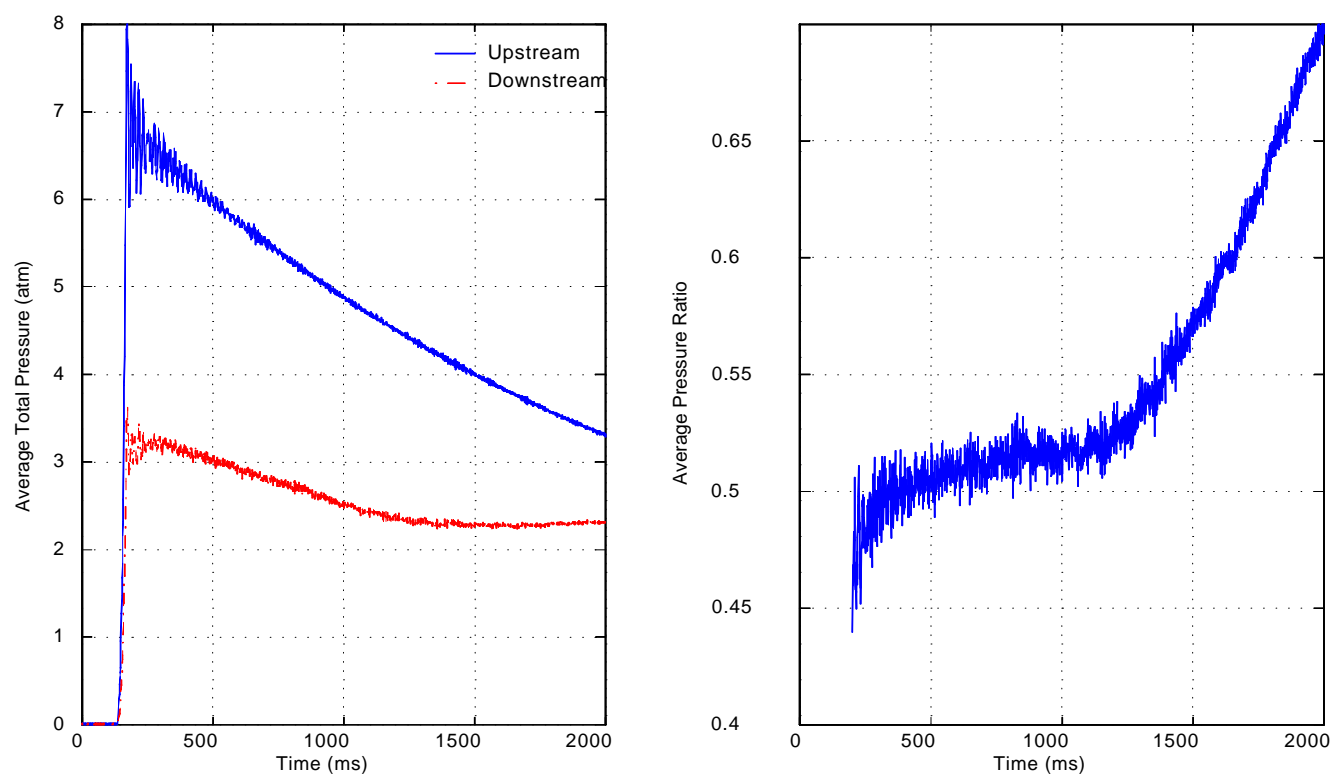

Figure 13.37: ABB036 - Average Total Pressure and Pressure Ratio
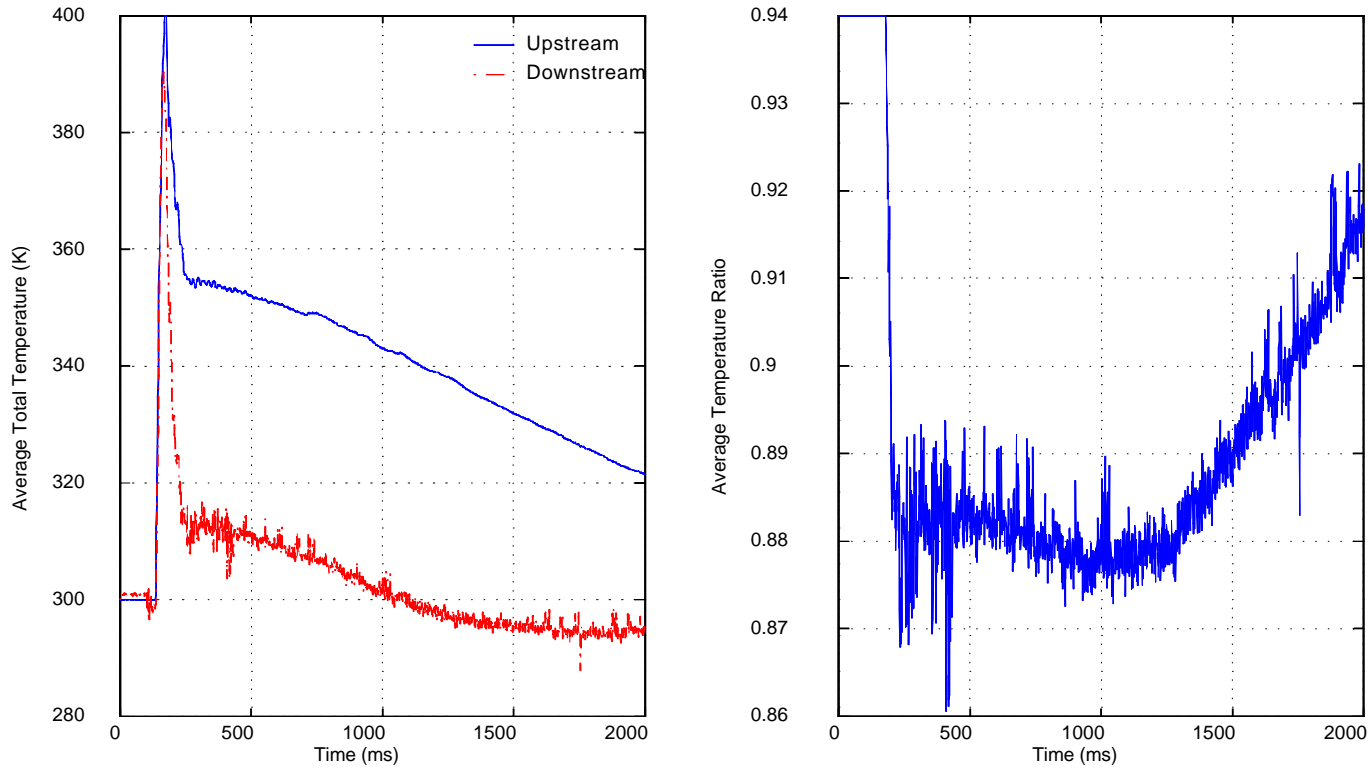

Figure 13.38: ABB036 - Average Total Temperature and Temperature Ratio 

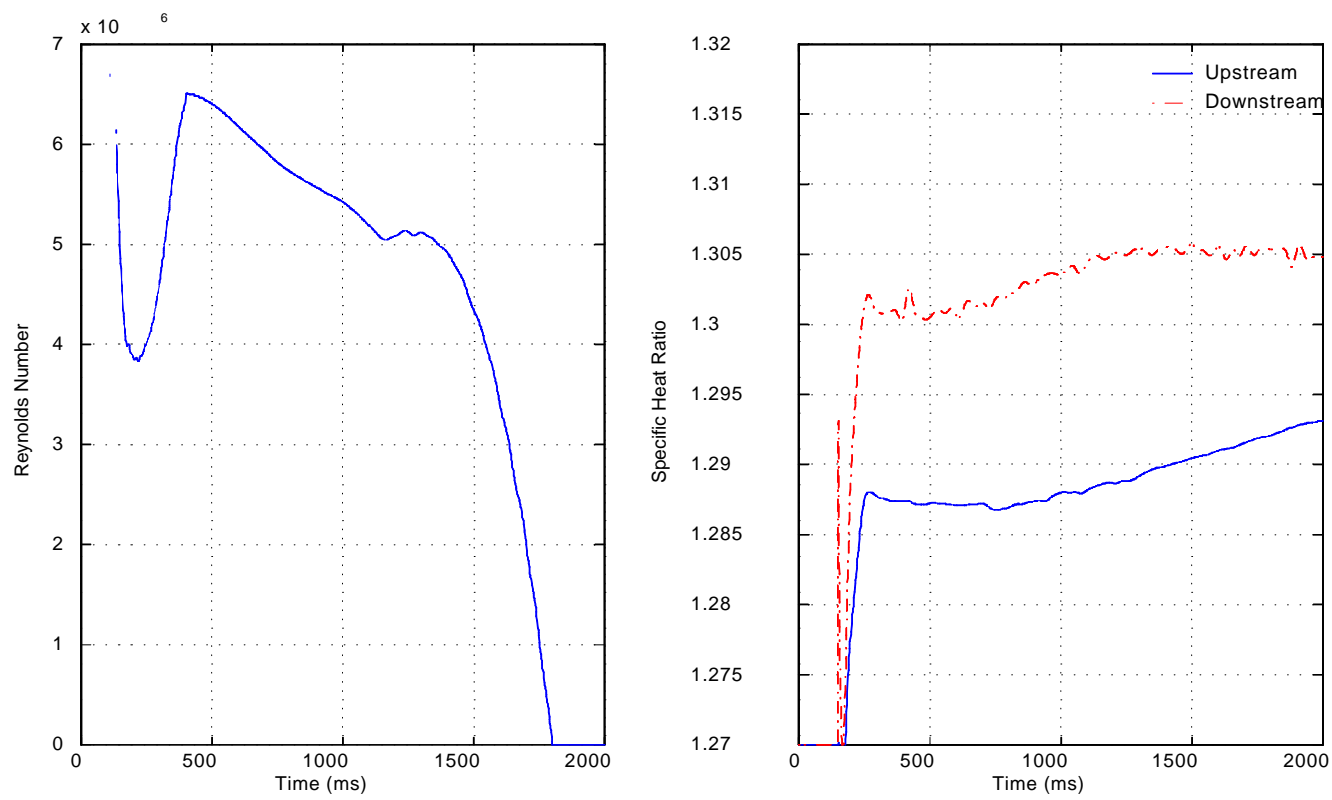

Figure 13.39: ABB036 - Reynolds Number and Corrected Speed
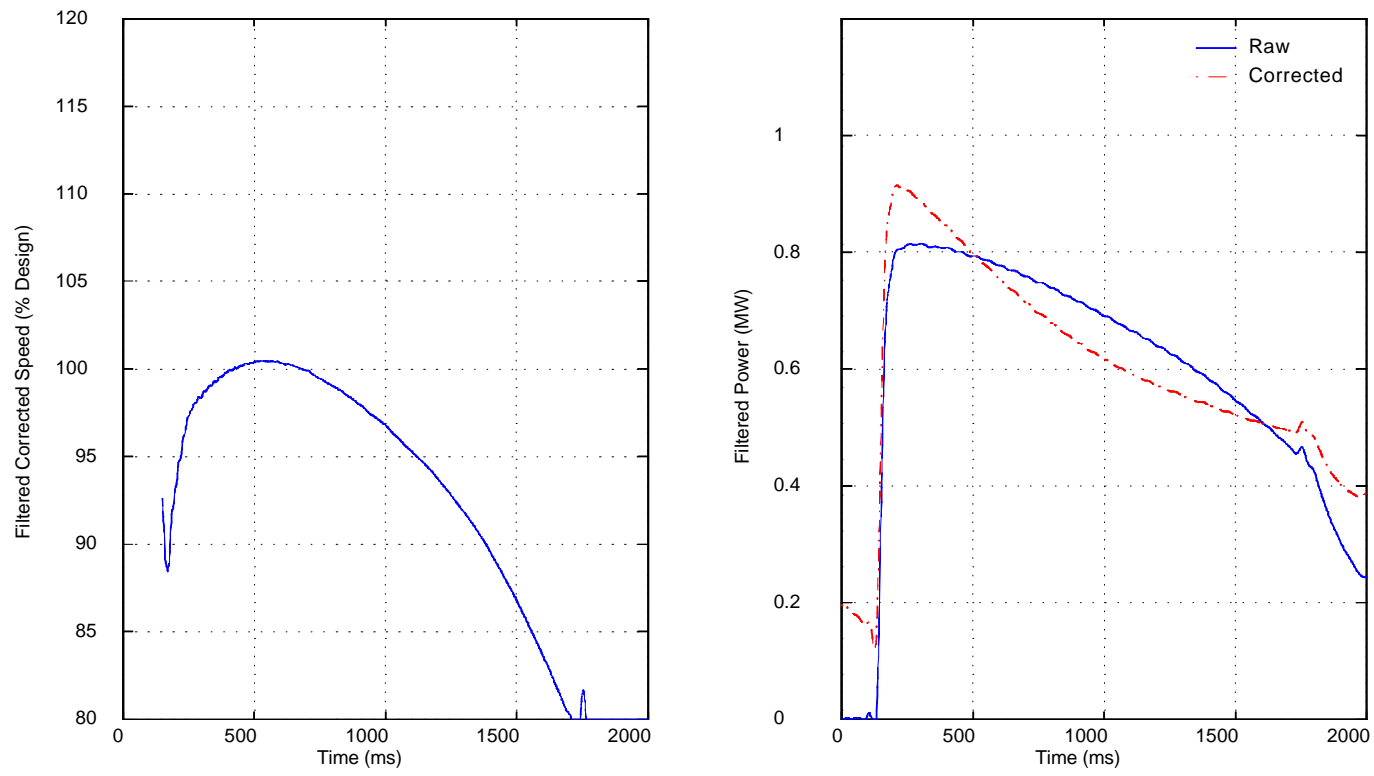

Figure 13.40: ABB036 - Corrected Speed and Power 

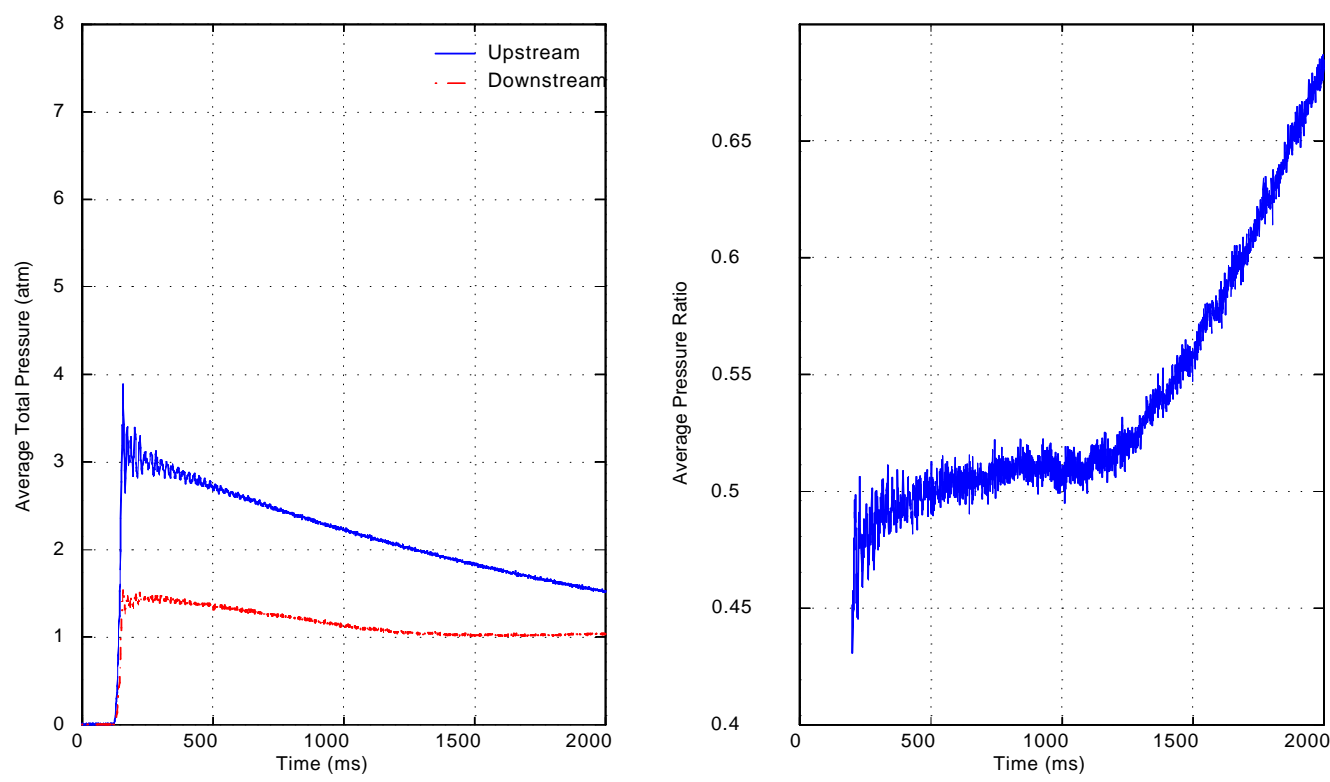

Figure 13.41: ABB041 - Average Total Pressure and Pressure Ratio
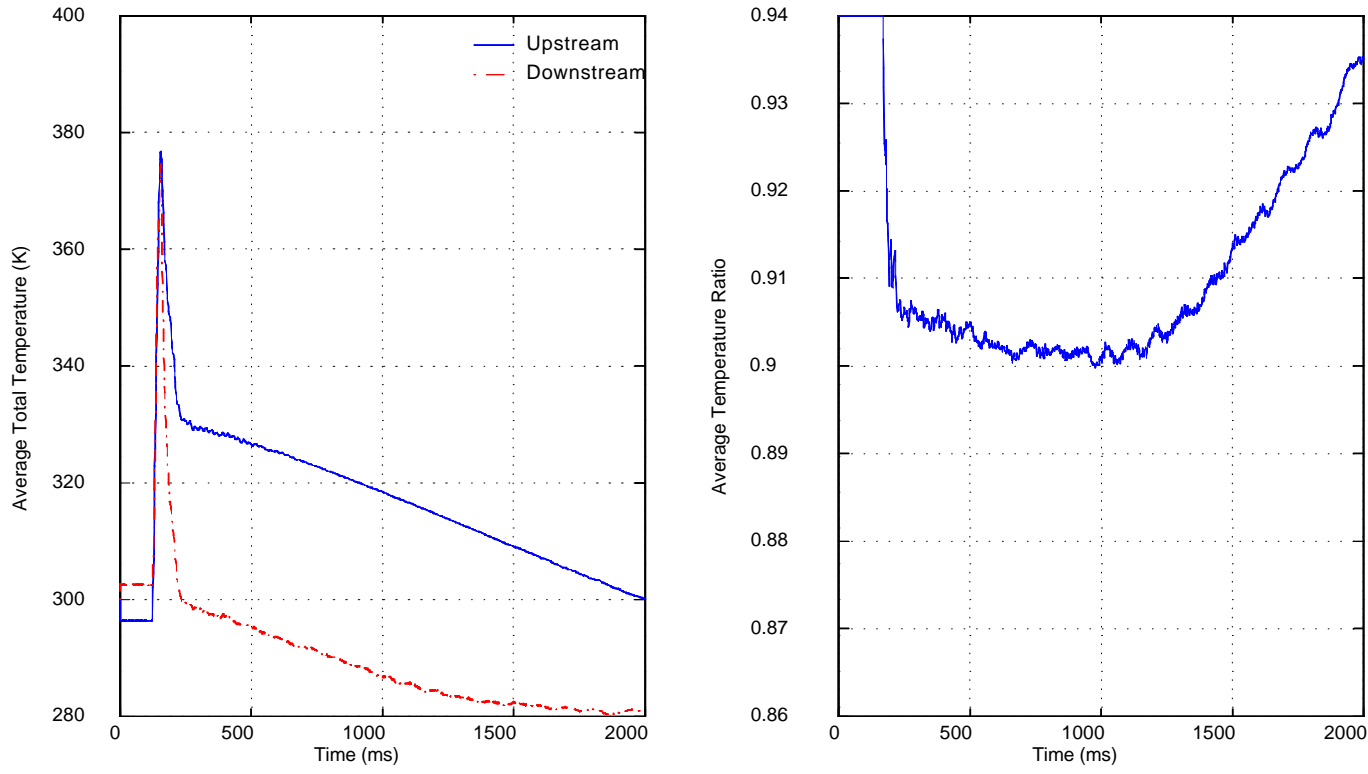

Figure 13.42: ABB041 - Average Total Temperature and Temperature Ratio 

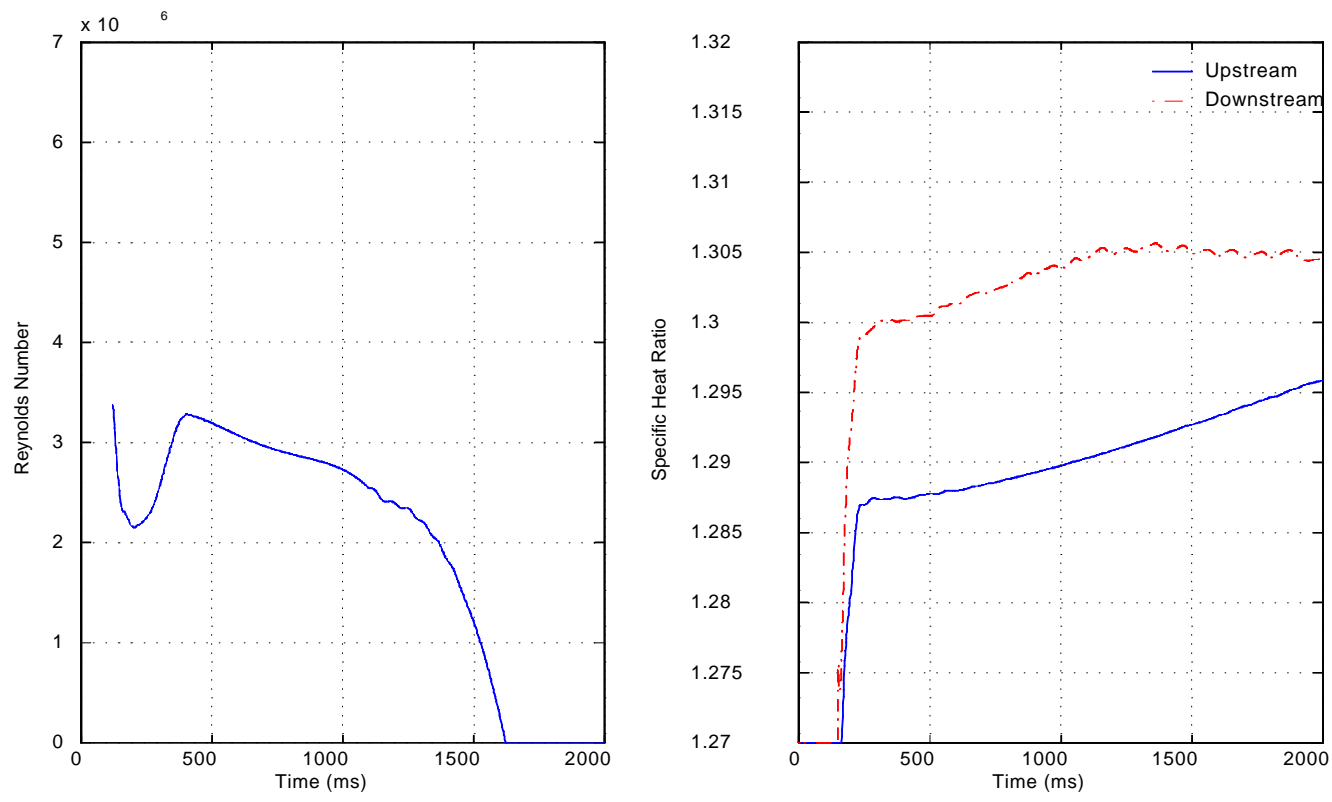

Figure 13.43: ABB041 - Reynolds Number and Specific Heat Ratio

Figure 13.44: ABB041 - Corrected Speed and Power 


\section{Efficiency Calculations: Un-Cooled Tests}

This Chapter contains the results of the aerodynamic and mechanical adiabatic efficiency calculations for the un-cooled test series summarized in Table 8.1. These calculations are discussed in Chapters 9 and 10 and are presented on the same scale for easy comparison. This scale is referenced to the Craig and Cox empirical efficiency estimates at the smaller tip clearance as discussed by Jacobs [10.5]. All efficiency calculations have been corrected for heat transfer as described in Sections 9.6 and 10.4. 


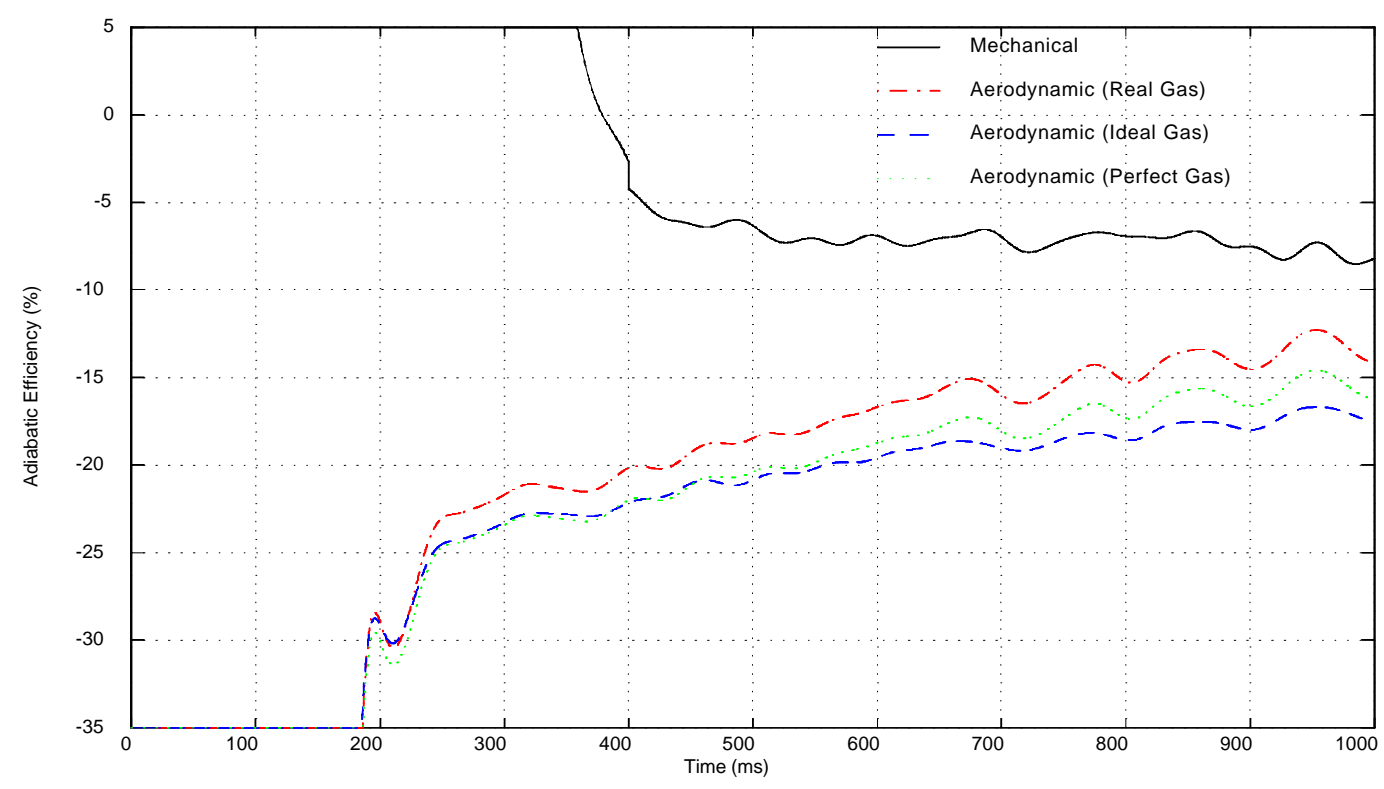

Figure 14.1: ABB024

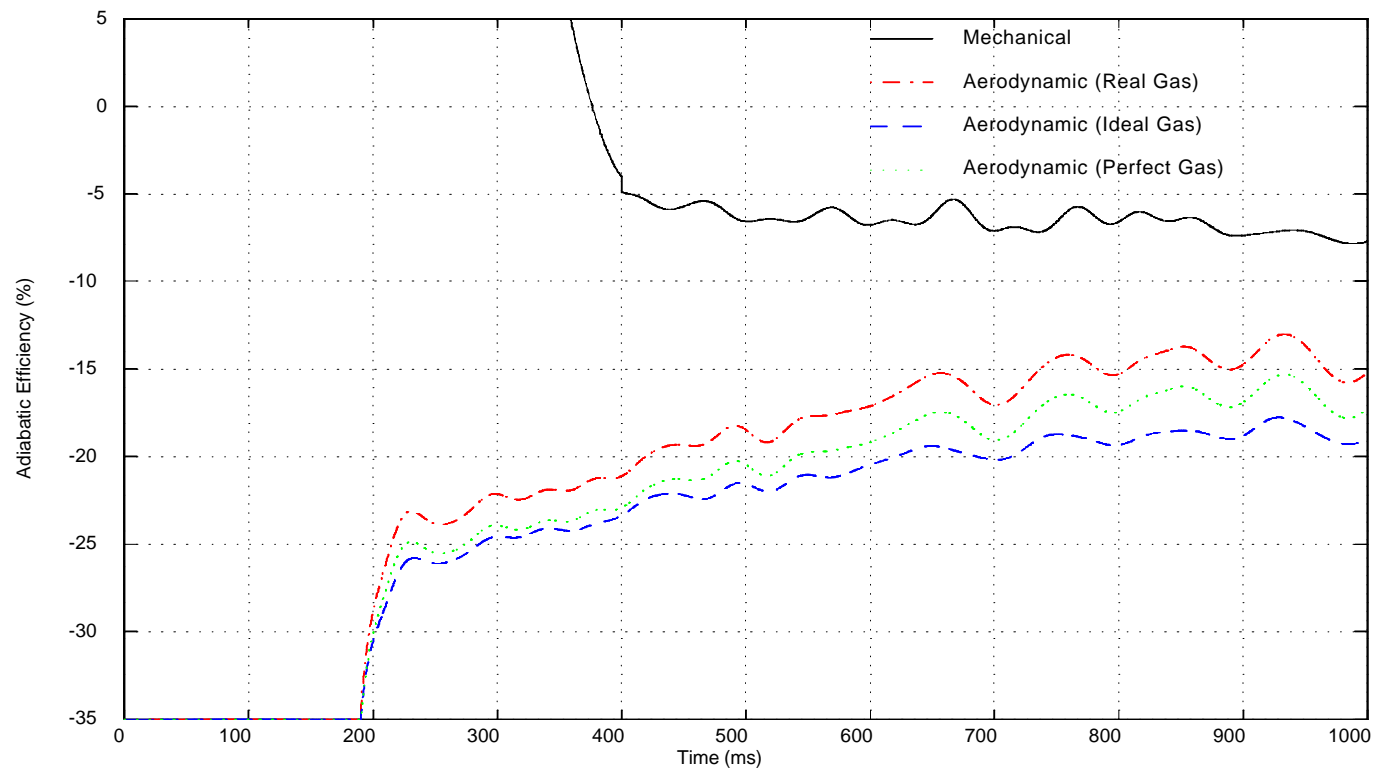

Figure 14.2: ABB027 


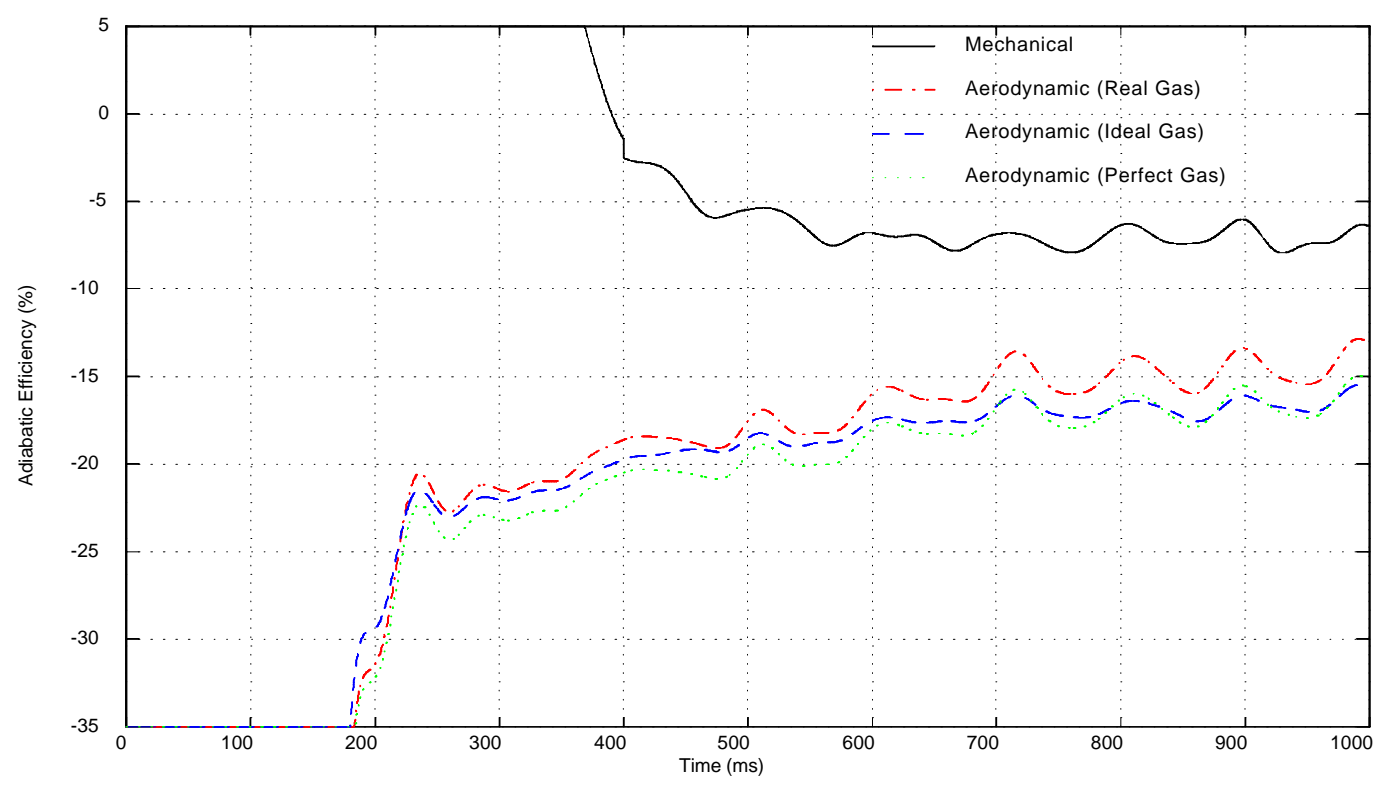

Figure 14.3: ABB032

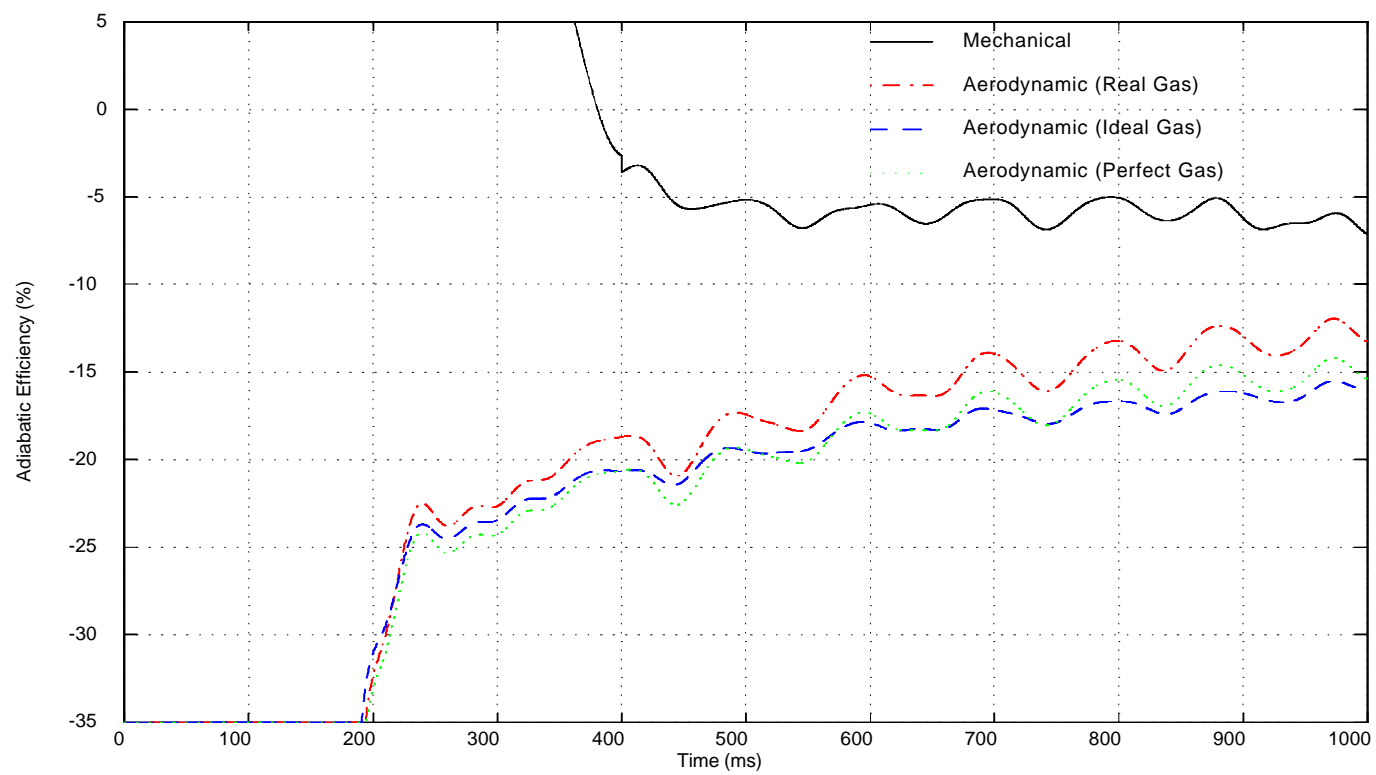

Figure 14.4: ABB029 


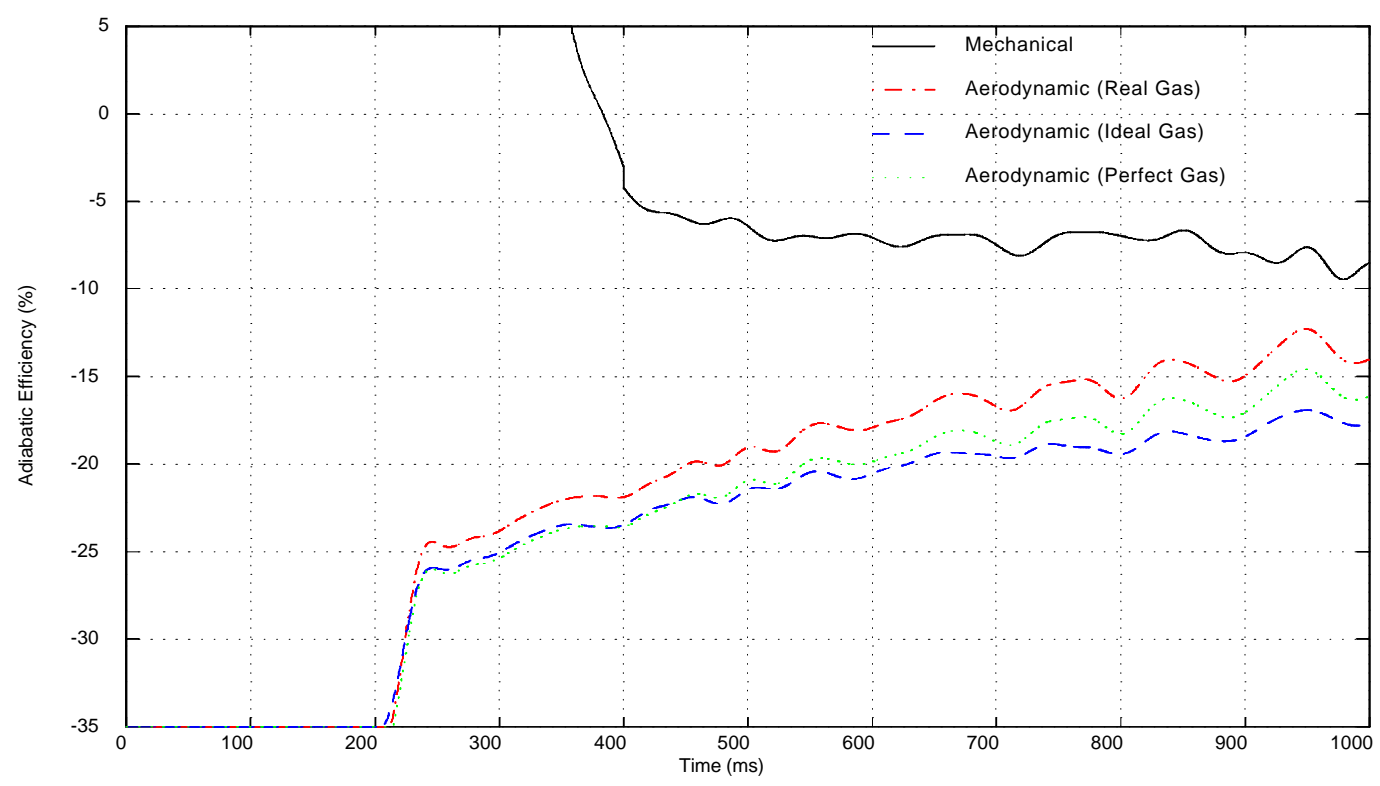

Figure 14.5: ABB028

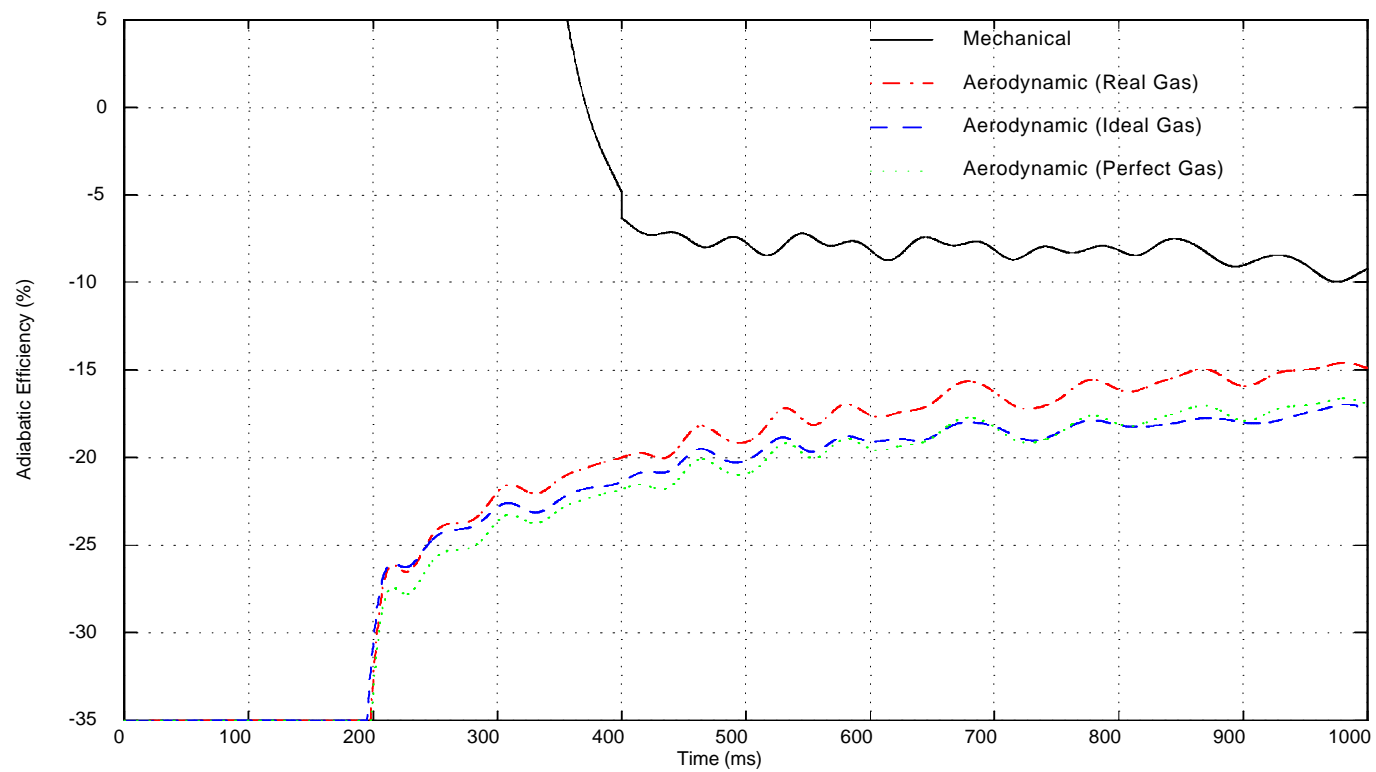

Figure 14.6: ABB034 


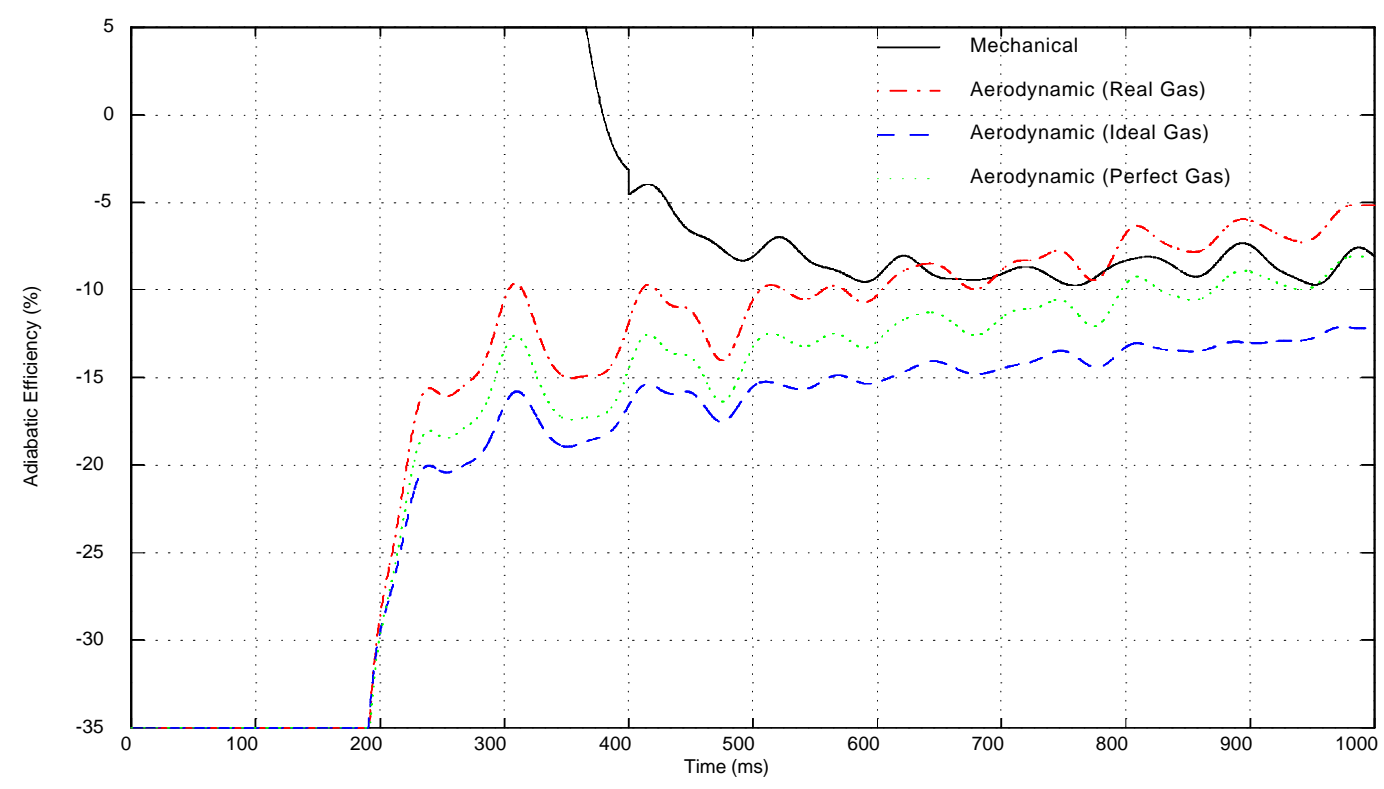

Figure 14.7: ABB035

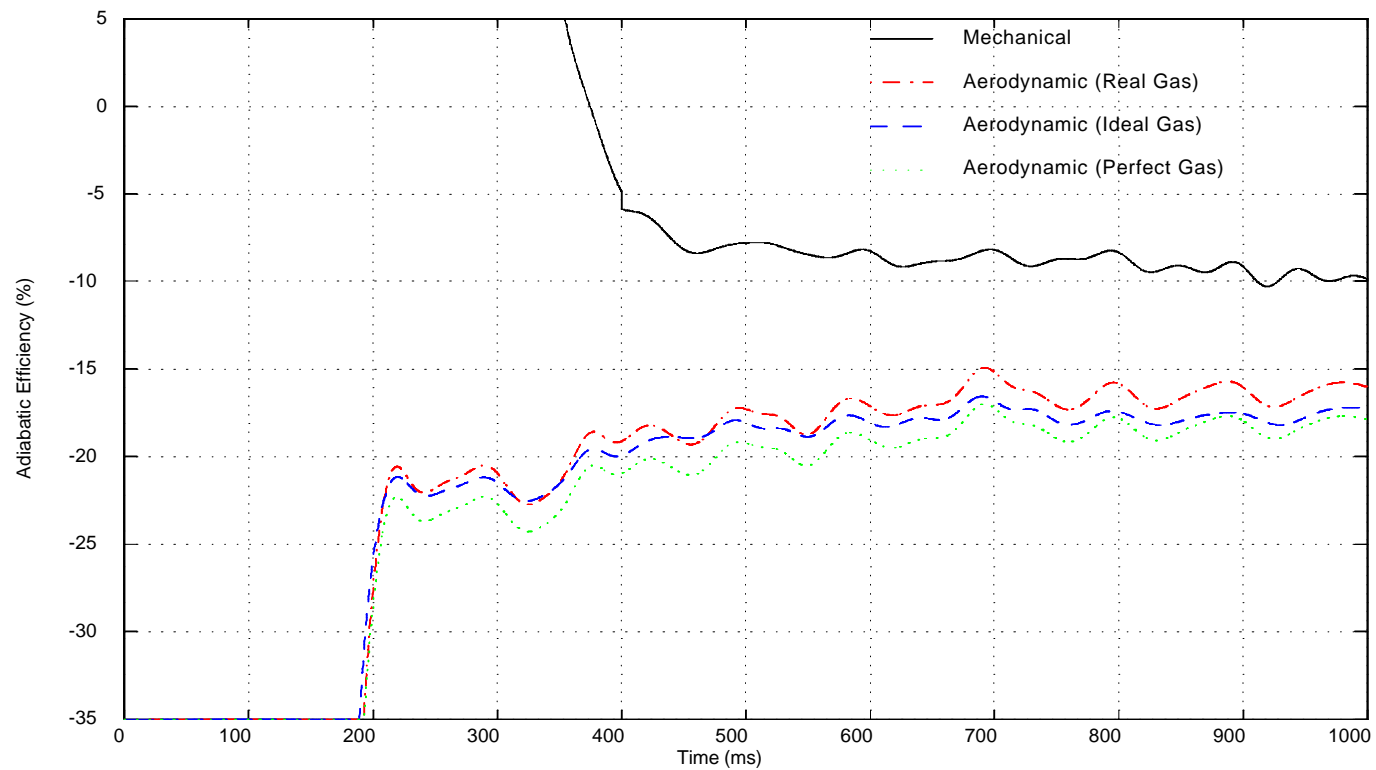

Figure 14.8: ABB033 


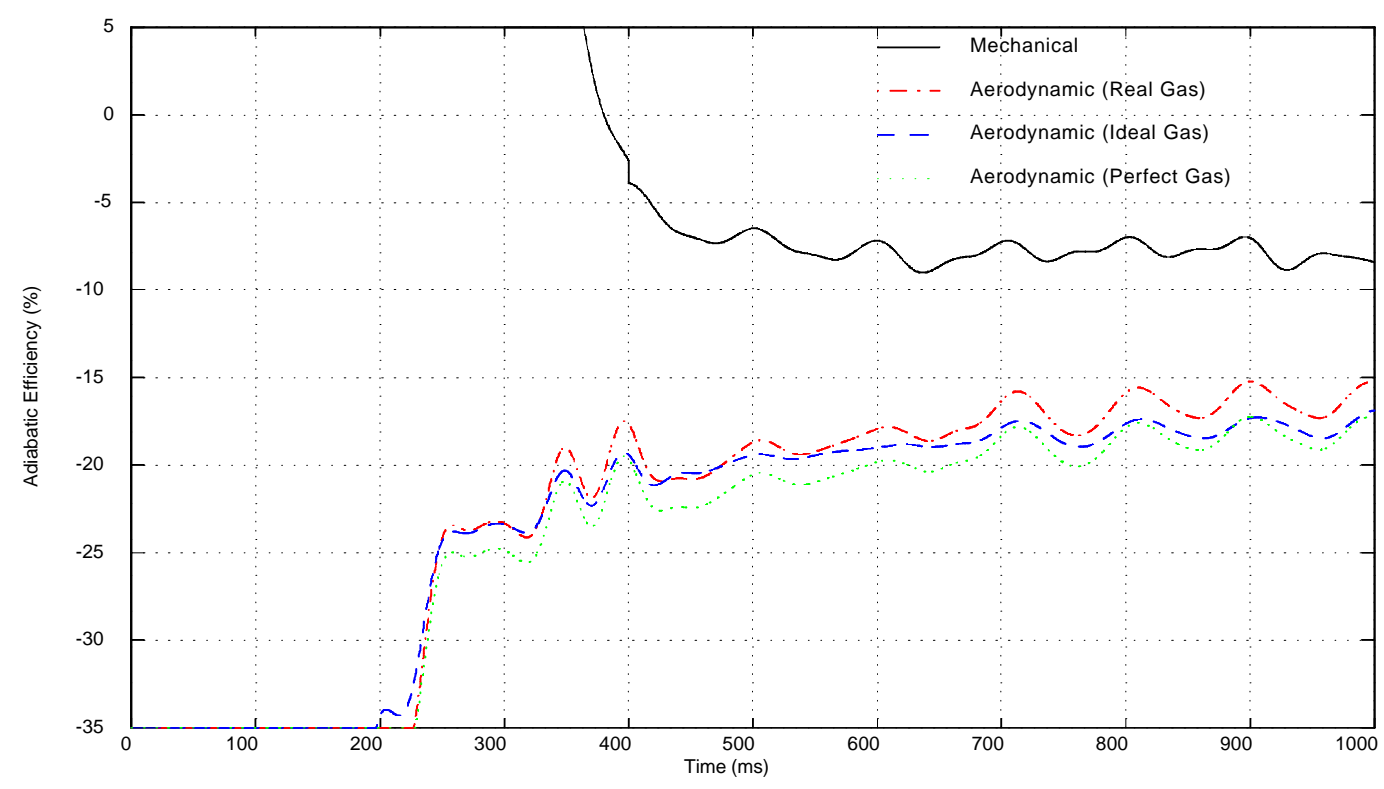

Figure 14.9: ABB037

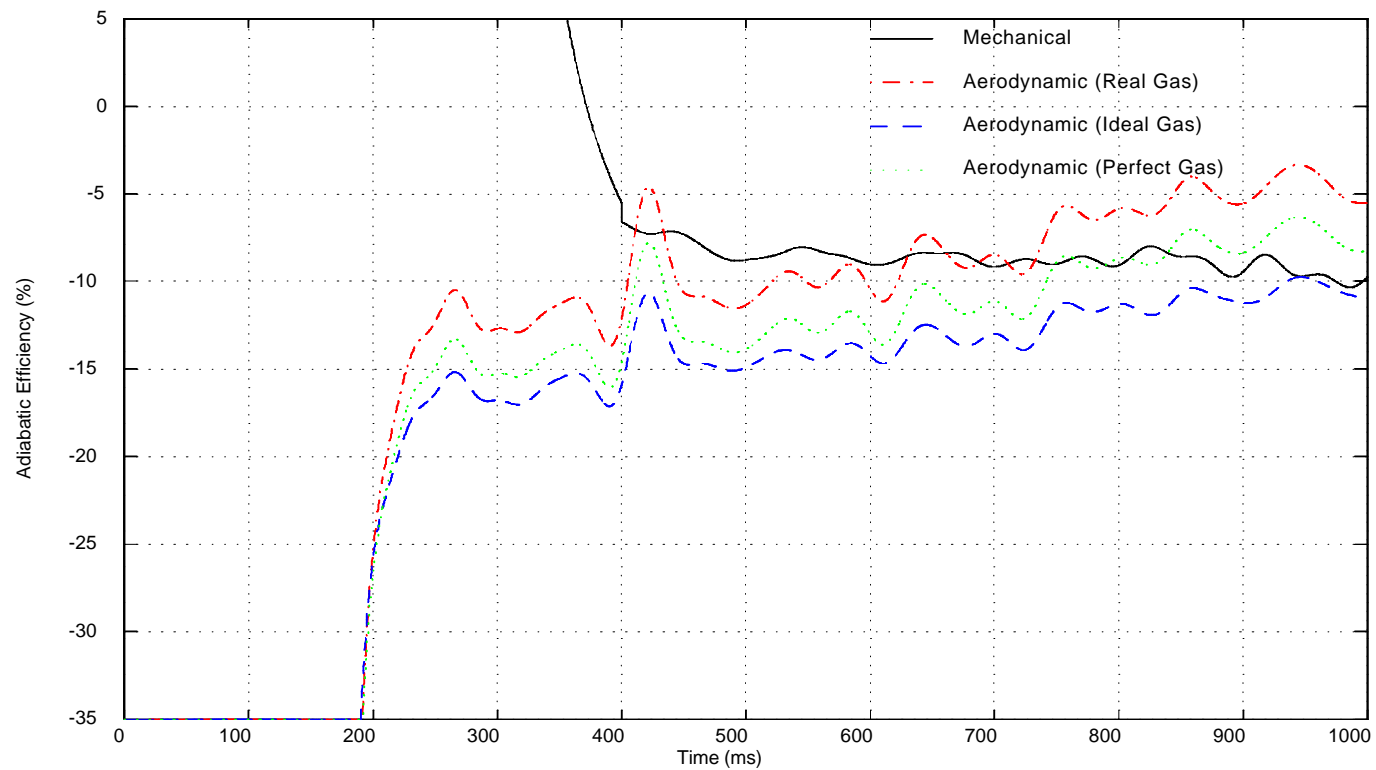

Figure 14.10: ABB036 


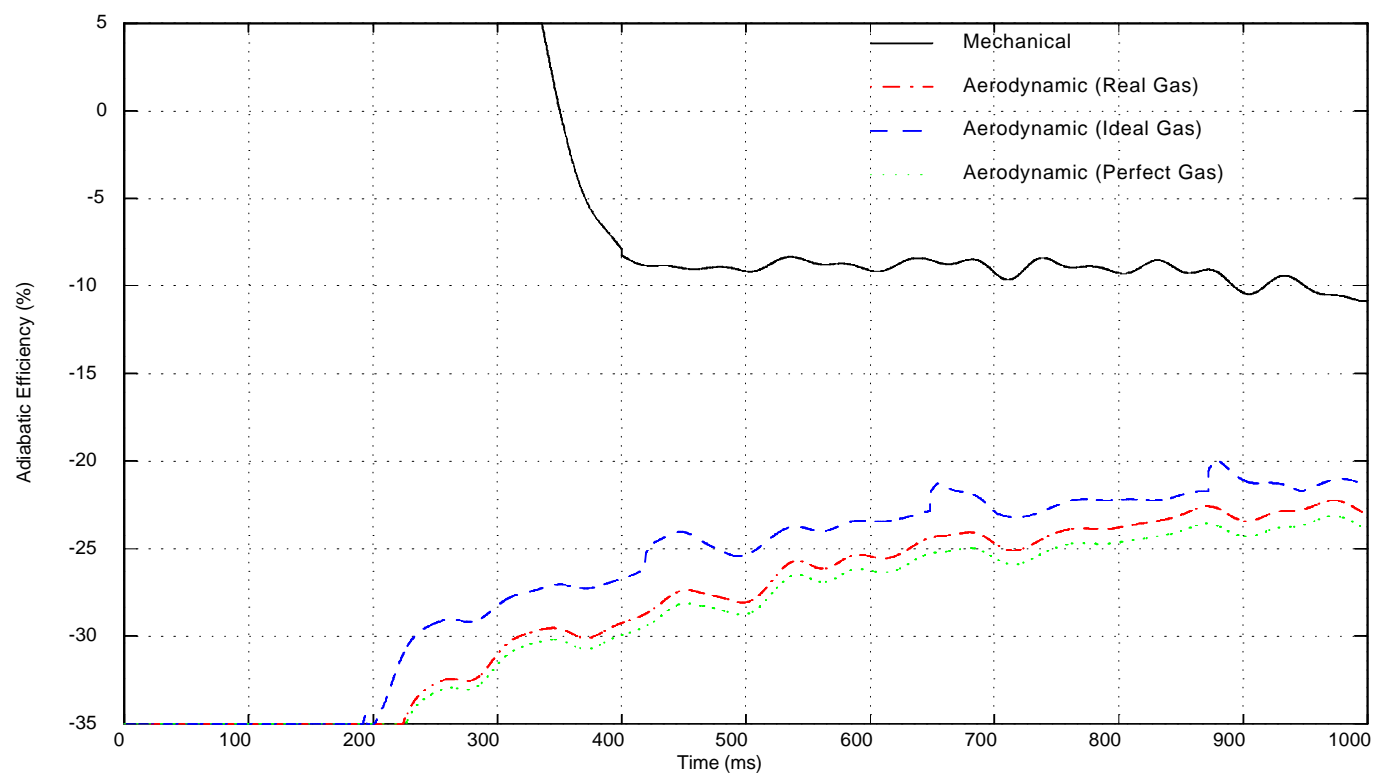

Figure 14.11：ABB041 


\section{Experimental Data: Film Cooled Tests}

This Chapter presents data for selected experiments from the film-cooled test series summarized in Table 8.2. Plots of pressure ratio, corrected speed, coolant mass flow, and efficiency are shown.

Table 8.2: Cooled Turbine Test Matrix

Low Inlet Temperature - Coolant Mass Flow Tests

\begin{tabular}{|c|cccccccc|}
\hline Test & $\mathbf{G a s}$ & $\mathbf{T}_{\text {in }}, \mathbf{K}$ & $\mathbf{P}_{\text {in }}, \mathbf{a t m}$ & $\mathbf{P R}$ & $\mathbf{N}_{\mathbf{c}}, \%$ & $\mathbf{R e}, \%$ & $\mathbf{M}_{\text {cool }}, \%$ & $\mathbf{T}_{\text {in }} / \mathbf{T}_{\text {vane }}$ \\
\hline abb110 & $\mathrm{CO}_{2}$ & 329 & 3.6 & 2.07 & 106.3 & 63 & 4.8 & 1.11 \\
abb111 & $\mathrm{CO}_{2}$ & 329 & 3.6 & 2.04 & 106.2 & 64 & 8.4 & 1.12 \\
abb113 & $\mathrm{CO}_{2}$ & 330 & 3.6 & 2.02 & 106.0 & 64 & 10.0 & 1.13 \\
abb117 & $\mathrm{CO}_{2}$ & 331 & 3.6 & 2.01 & 105.8 & 64 & 11.7 & 1.16 \\
\hline
\end{tabular}

Low Inlet Temperature - High Reynolds Number Test

\begin{tabular}{|l|llllllll|}
\hline abb112 & $\mathrm{CO}_{2}$ & 331 & 5.4 & 2.04 & 109.6 & 95 & 6.6 & 1.13 \\
\hline
\end{tabular}

High Inlet Temperature - Operating Point Test Matrix

\begin{tabular}{|c|cccccccc|}
\hline abb118 & $\mathrm{CO}_{2}$ & 441 & 5.2 & 2.03 & 102.7 & 63 & 9.9 & 1.58 \\
abb119 & $\mathrm{CO}_{2}$ & 439 & 5.3 & 2.03 & 104.5 & 63 & 9.2 & 1.52 \\
abb120 & $\mathrm{CO}_{2}$ & 446 & 5.3 & 2.01 & 96.1 & 62 & 9.7 & 1.58 \\
abb121 & $\mathrm{CO}_{2}$ & 445 & 5.3 & 1.98 & 90.3 & 63 & 9.7 & 1.57 \\
abb122 & $\mathrm{CO}_{2}$ & 448 & 5.3 & 1.81 & 94.2 & 61 & 9.8 & 1.58 \\
abb123 & $\mathrm{CO}_{2}$ & 447 & 5.3 & 2.12 & 96.1 & 62 & 9.6 & 1.58 \\
abb125 & $\mathrm{CO}_{2}$ & 445 & 5.3 & 2.01 & 93.6 & 63 & 9.9 & 1.60 \\
abb126 & $\mathrm{CO}_{2}$ & 444 & 5.3 & 2.01 & 97.6 & 63 & 10.0 & 1.60 \\
abb128 & $\mathrm{CO}_{2}$ & 445 & 5.2 & 2.01 & 97.0 & 62 & 9.9 & 1.60 \\
abb130 & $\mathrm{CO}_{2}$ & 439 & 5.3 & 2.04 & 112.3 & 63 & 9.9 & 1.58 \\
abb131 & $\mathrm{CO}_{2}$ & 442 & 5.3 & 2.02 & 99.8 & 63 & 9.8 & 1.56 \\
\hline
\end{tabular}

High Inlet Temperature - Inlet Temperature Test Matrix

\begin{tabular}{|l|llllllll|}
\hline abb127 & $\mathrm{CO}_{2}$ & 420 & 4.9 & 2.02 & 98.7 & 62 & 9.6 & 1.50 \\
abb129 & $\mathrm{CO}_{2}$ & 394 & 4.5 & 2.02 & 98.8 & 62 & 9.1 & 1.39 \\
\hline
\end{tabular}

High Inlet Temperature - Coolant Mass Flow Tests

\begin{tabular}{|l|llllllll|}
\hline abb114 & $\mathrm{CO}_{2}$ & 435 & 5.3 & 2.07 & 109.1 & 63 & 7.2 & 1.53 \\
abb115 & $\mathrm{CO}_{2}$ & 440 & 5.2 & 2.05 & 104.6 & 63 & 8.7 & 1.57 \\
abb116 & $\mathrm{CO}_{2}$ & 436 & 5.2 & 2.06 & 106.9 & 63 & 7.2 & 1.53 \\
abb132 & $\mathrm{CO}_{2}$ & 439 & 5.3 & 2.03 & 98.6 & 64 & 12.0 & 1.58 \\
abb133 & $\mathrm{CO}_{2}$ & 441 & 5.3 & 2.03 & 100.4 & 64 & 12.1 & 1.59 \\
\hline
\end{tabular}



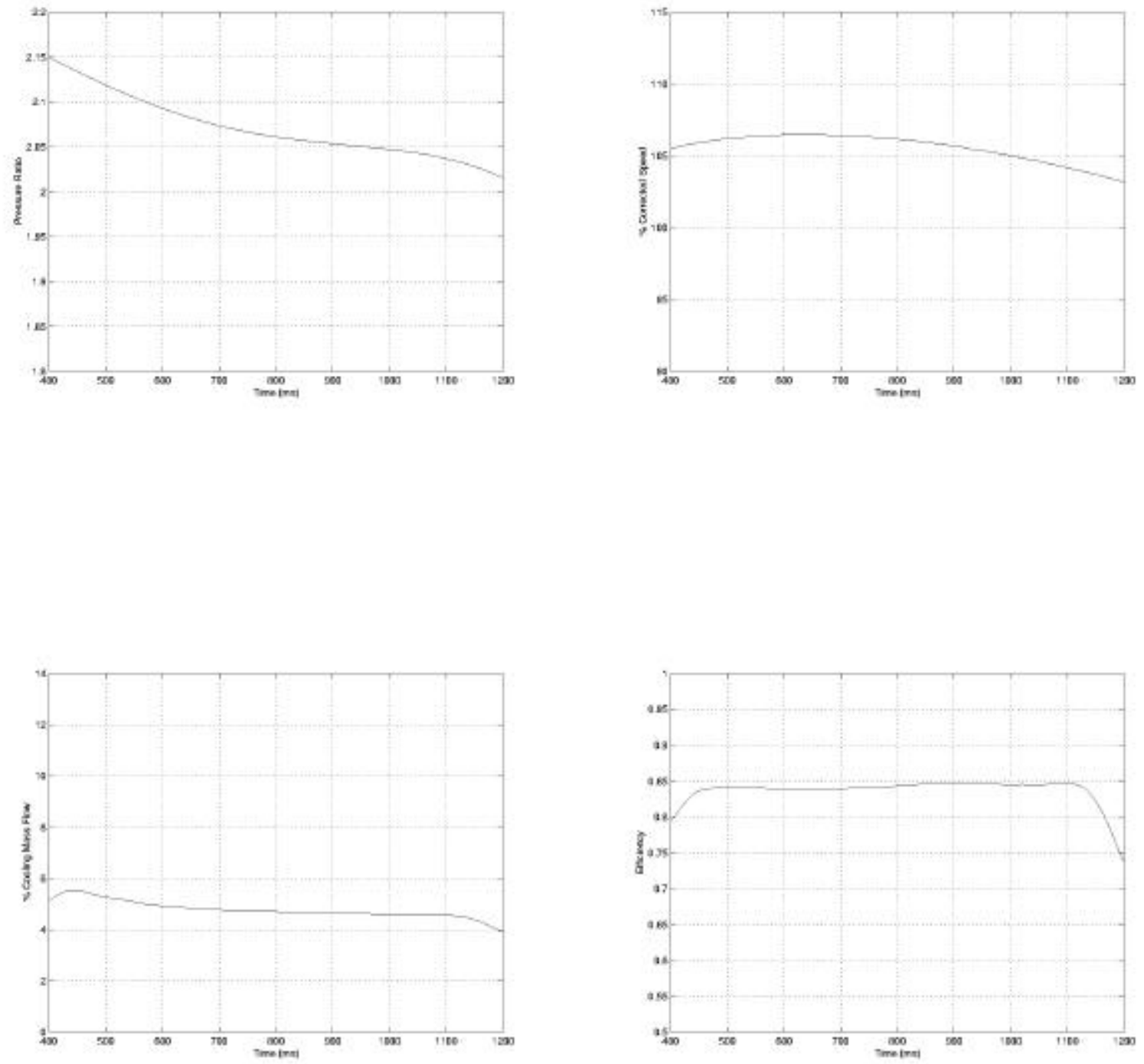

Test: ABB110 

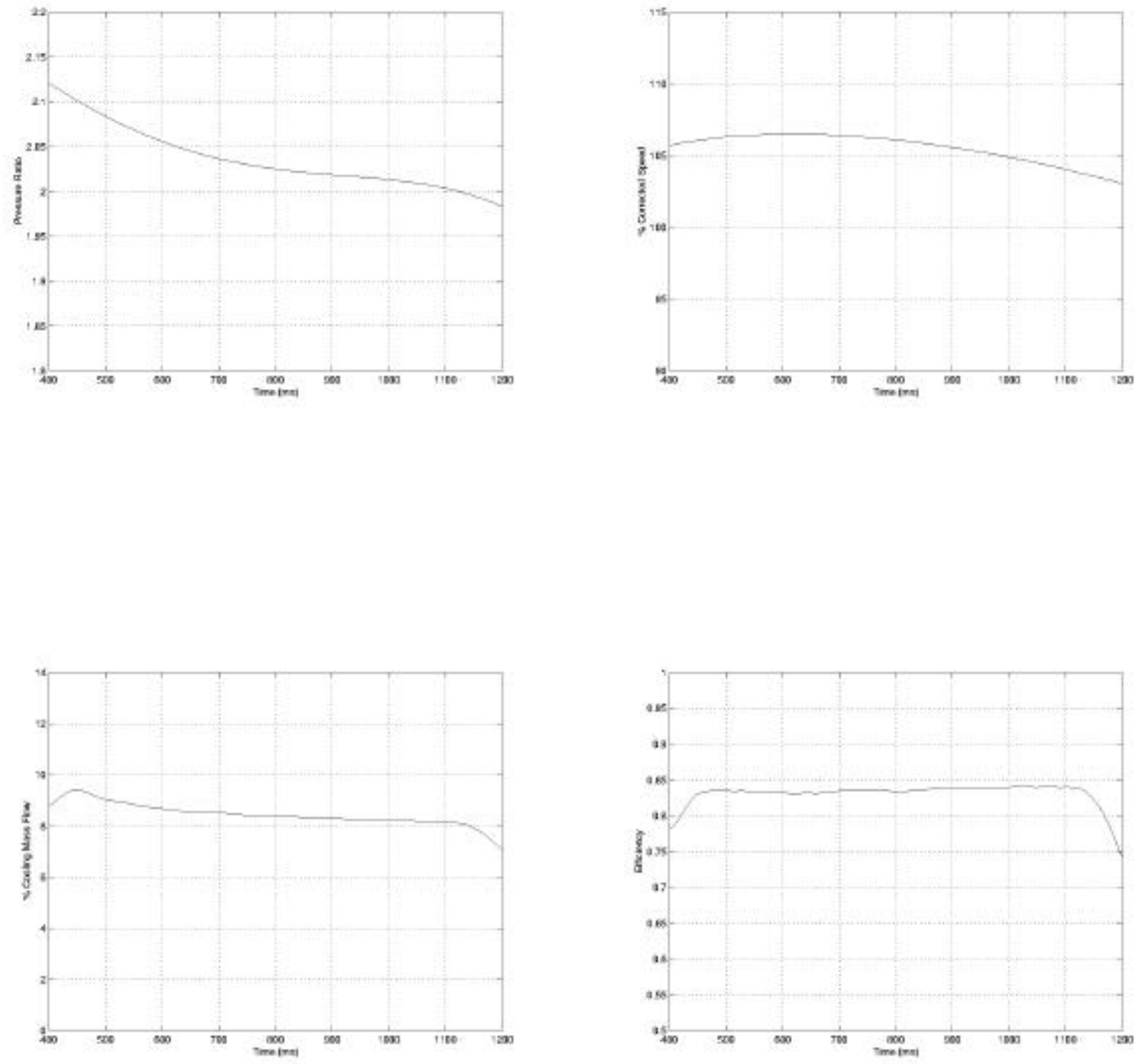

Test: ABB111

15-3 

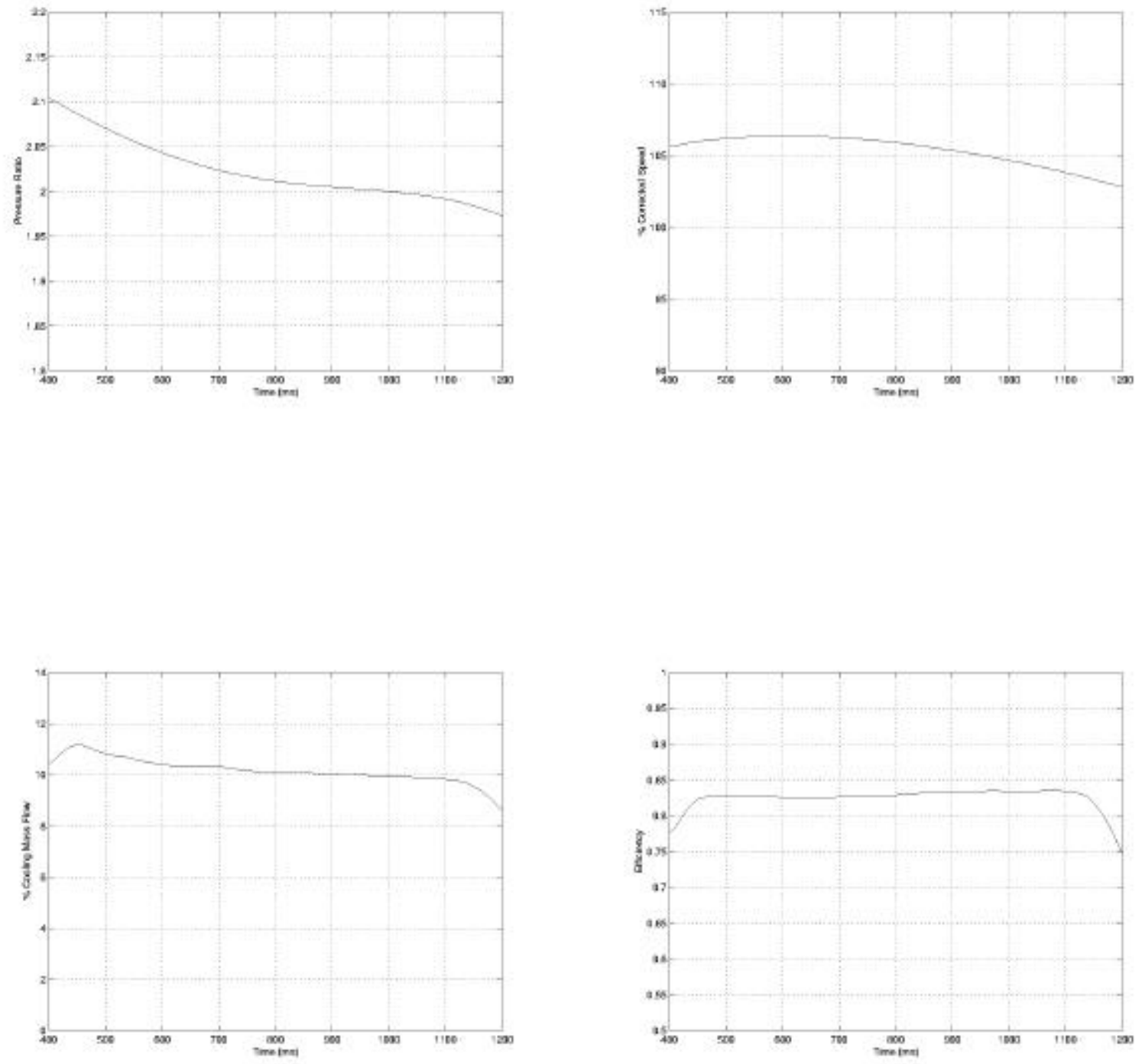

Test: ABB113 

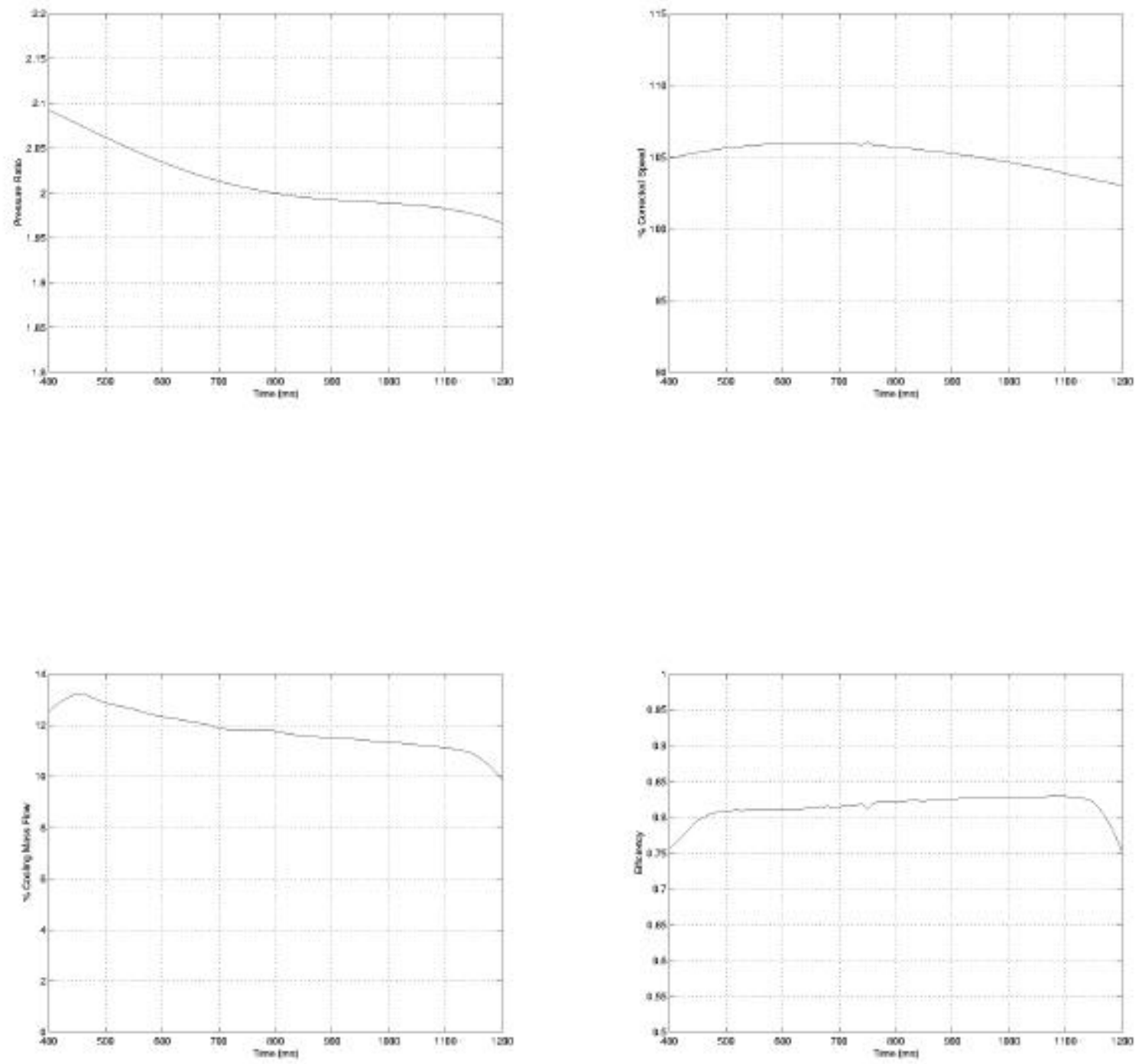

Test: ABB117 

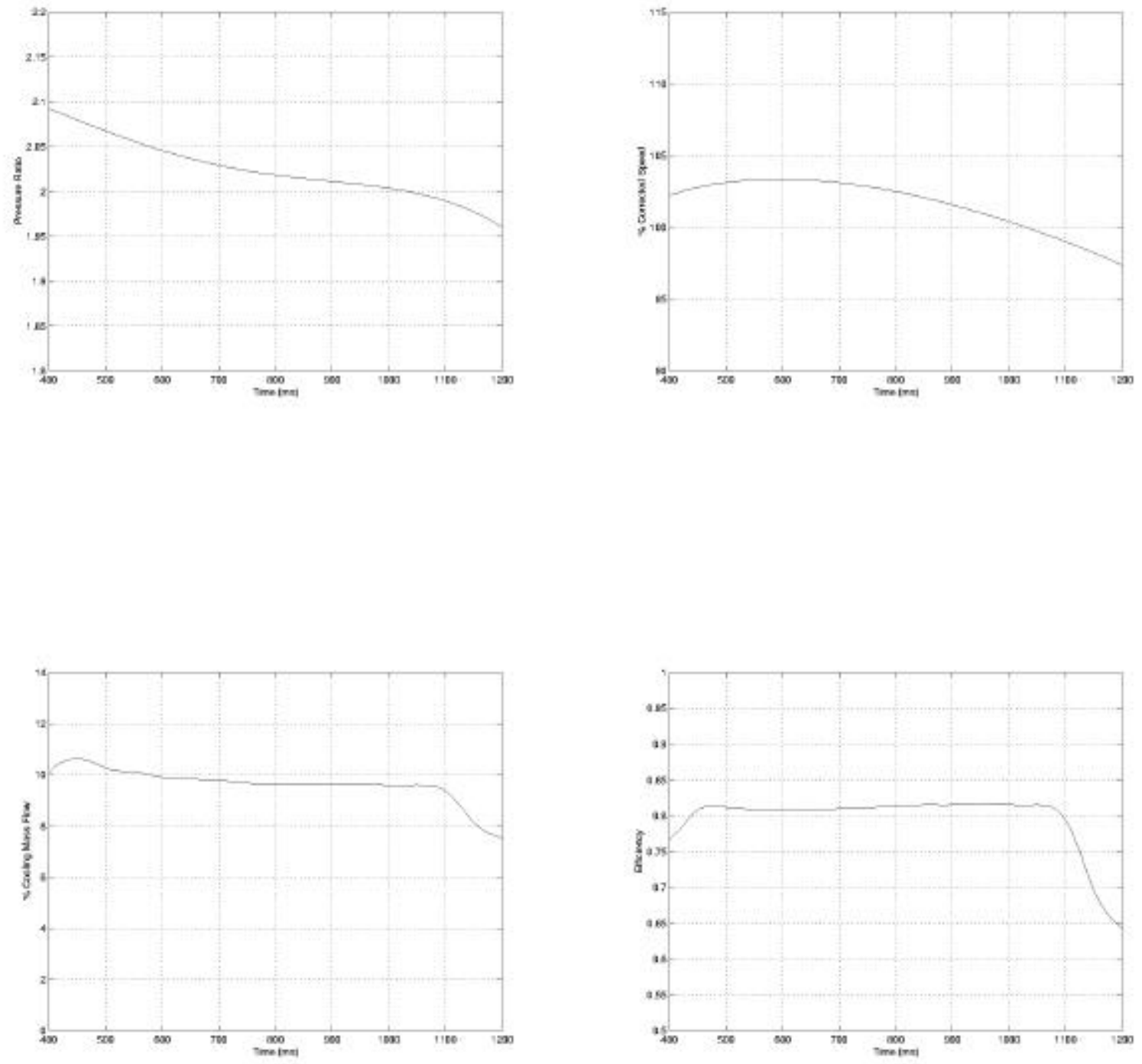

Test: ABB118 

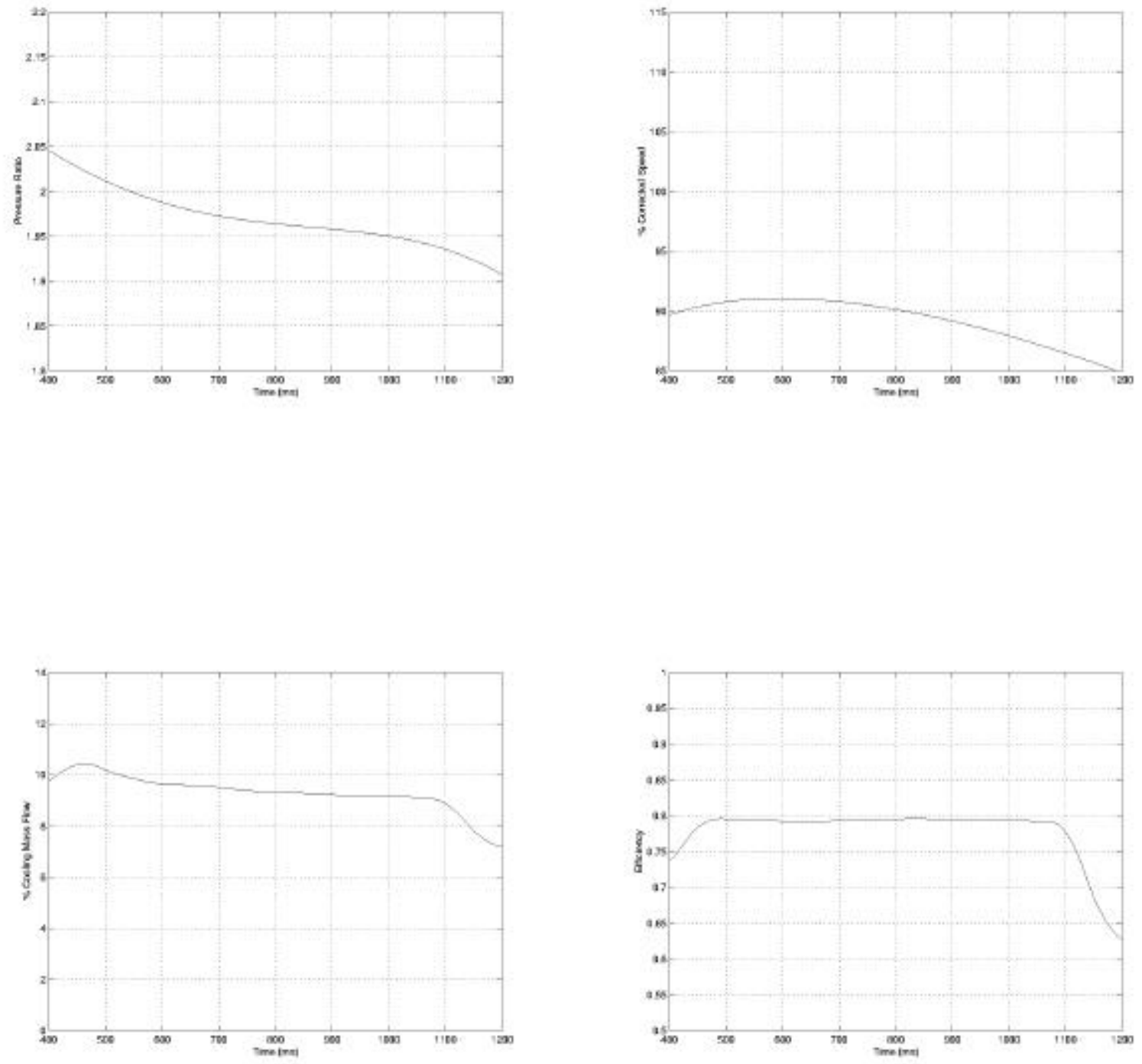

Test: ABB121 

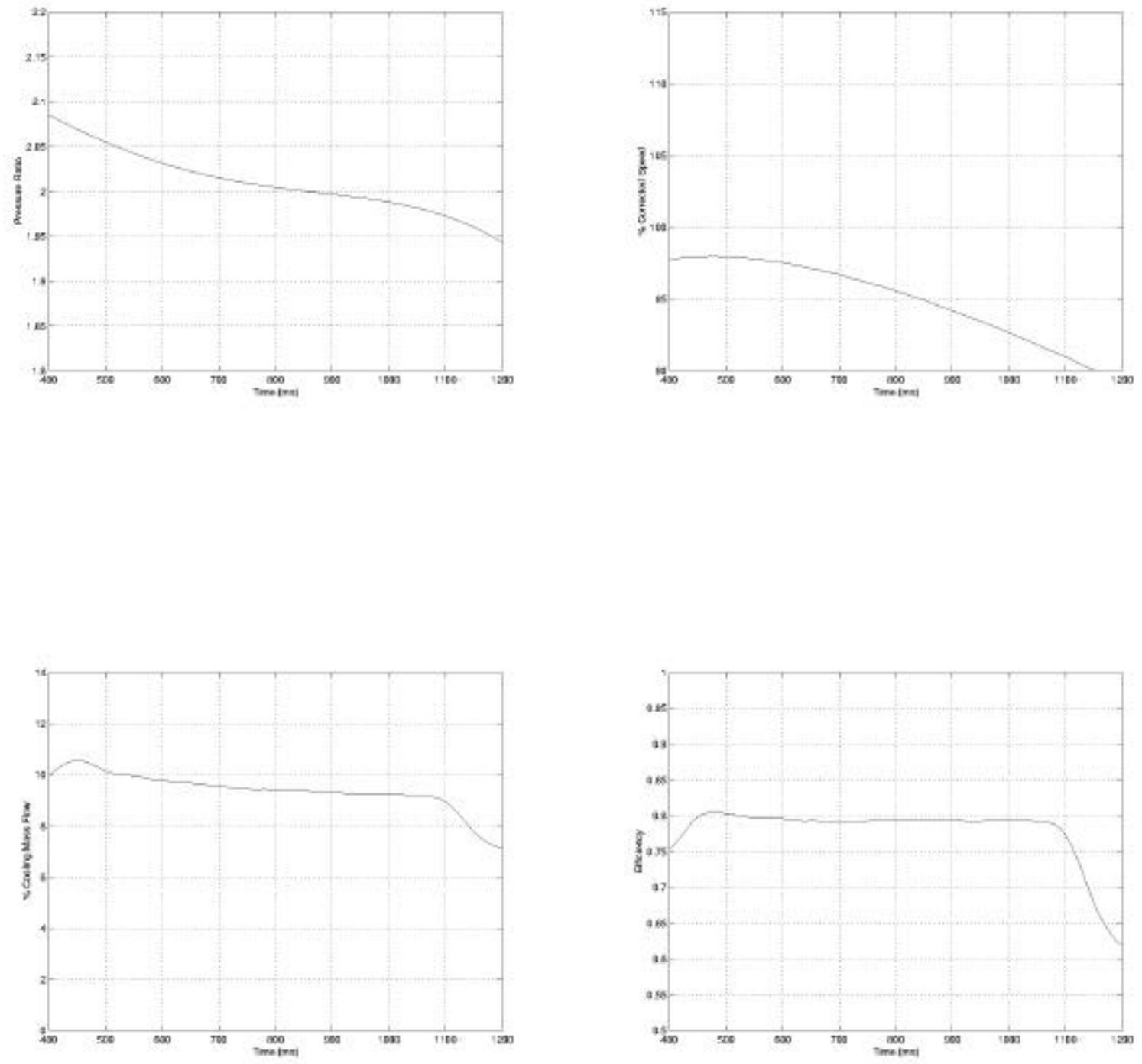

Test: ABB120 

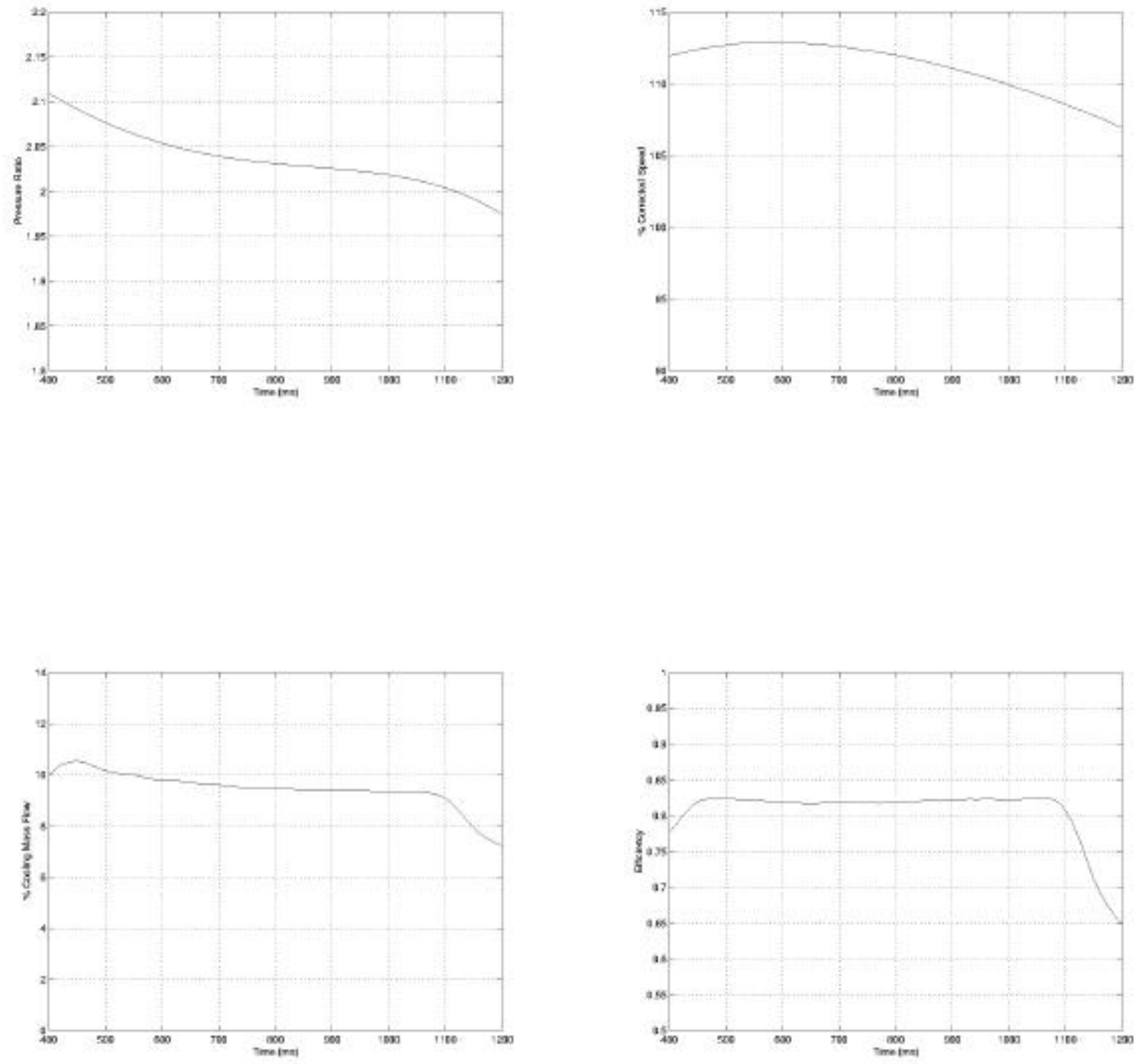

Test: ABB128 

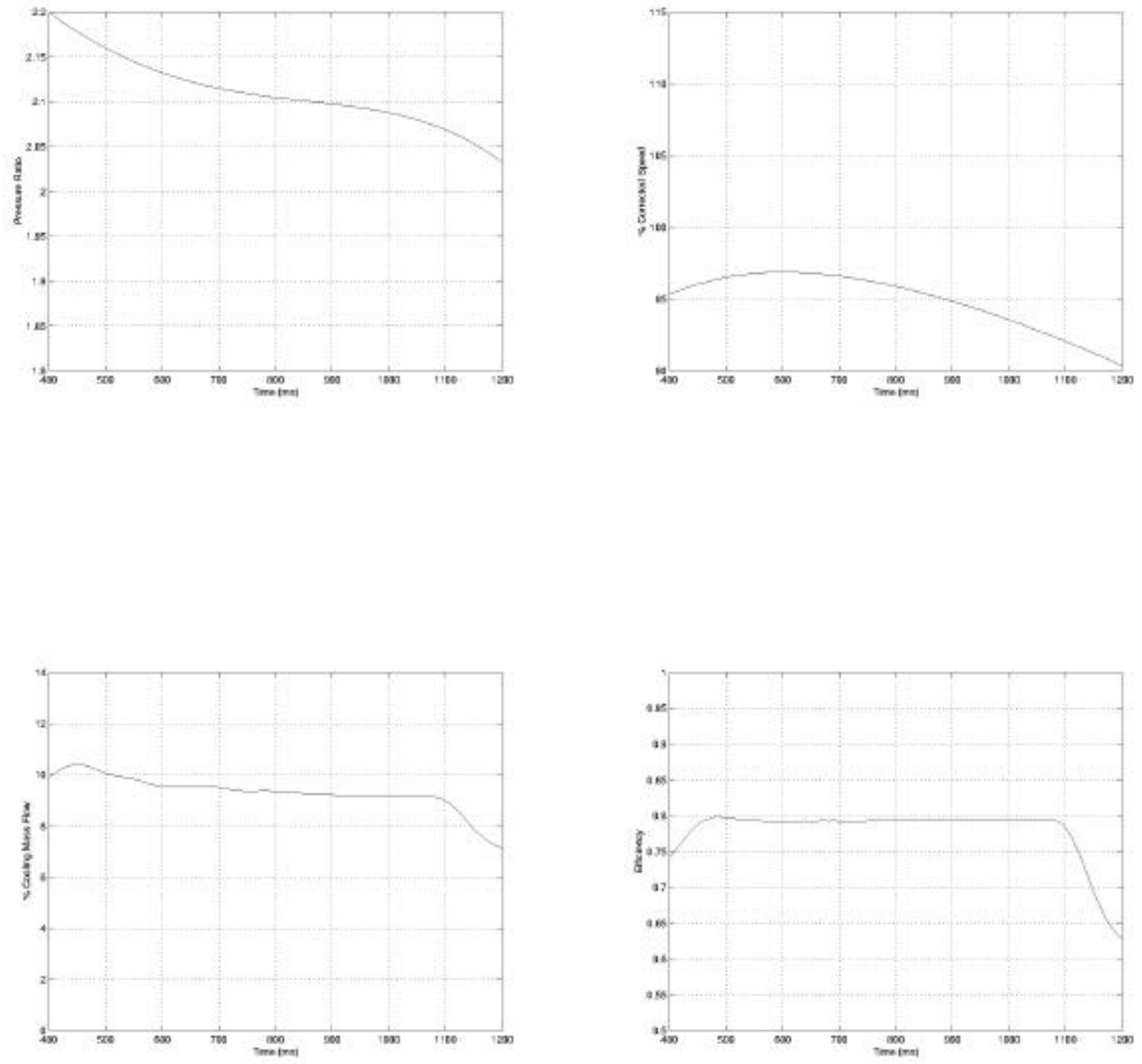

Test: ABB123 

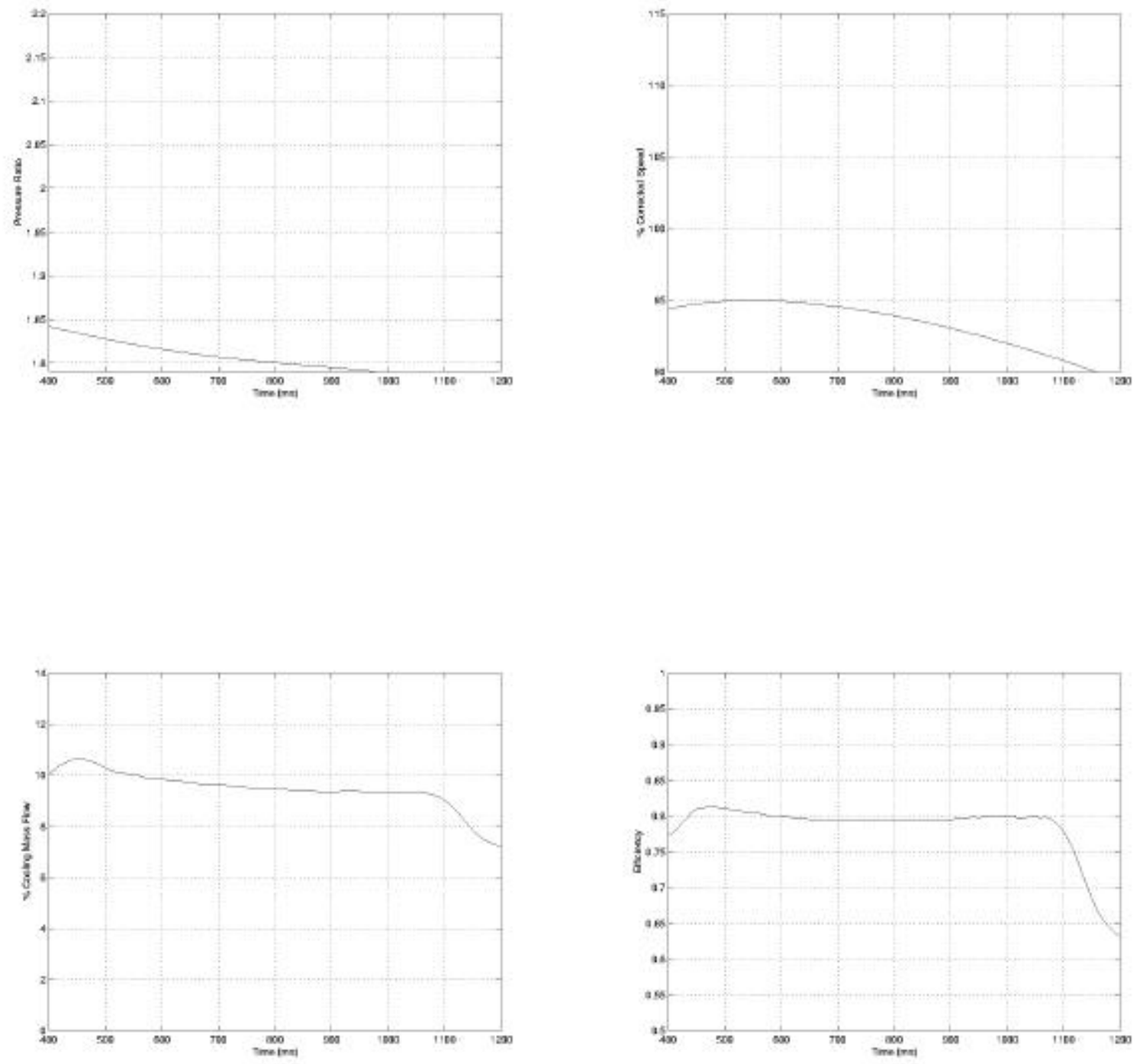

Test: ABB122 
Appendix 4

Appendix 4

Steam Cooling 


\section{Introduction 4}

EXPERIMENTAL SETUP

6

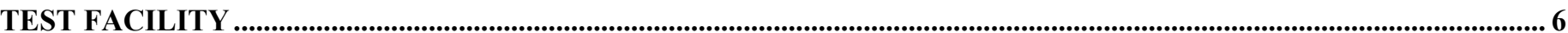

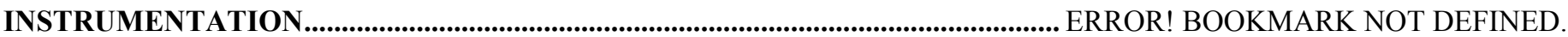

TEST CONDITIONS .................................................................................................. ERROR! BOOKMARK NOT DEFINED.

DATA PROCESSING ….................................................................................................... ERROR! BOOKMARK NOT DEFINED.

HEAT TRANSFER COEFFICIENTS ..................................................................RROR! BOOKMARK NOT DEFINED.

COOLANT TEMPERATURE MEASUREMENT......................................................... ERROR! BOOKMARK NOT DEFINED.

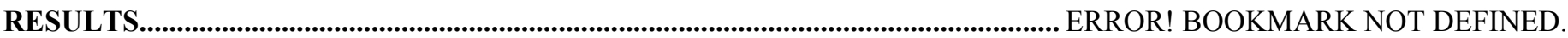

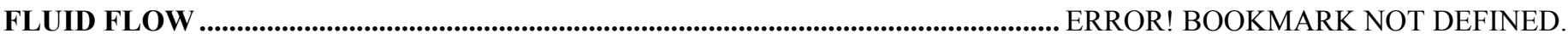

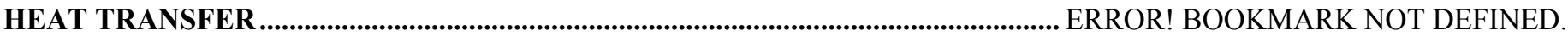

TEMPERATURE DISTRIBUTION ................................................................................ ERROR! BOOKMARK NOT DEFINED.

HEAT TRANSFER COEFFICIENTS .......................................................................... ERROR! BOOKMARK NOT DEFINED.

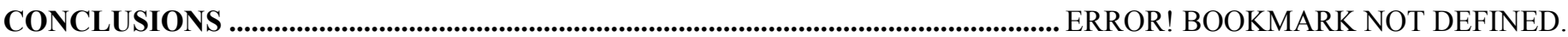




\section{List of symbols}

\begin{tabular}{llllll}
$\mathrm{A}$ & $\mathrm{m}^{2}$ & area & $\mathrm{Nu}_{\mathrm{L}}$ & \multicolumn{2}{l}{ modified Nusselt-number } \\
$\mathrm{c}$ & $\mathrm{m} / \mathrm{s}$ & flow velocity & $\mathrm{p}$ & $\mathrm{bar}$ & pressure \\
$\mathrm{c}_{\mathrm{p}}$ & $\mathrm{J} /(\mathrm{kgK})$ & specific heat & $\mathrm{Pr}$ & & Prandtl-number \\
$\mathrm{d}$ & $\mathrm{m}$ & thermocouple diameter & $\dot{\mathrm{q}}$ & $\mathrm{J} / \mathrm{kg}$ & specific heat flux \\
$\mathrm{d}_{\mathrm{c}}$ & $\mathrm{m}$ & channel depth & $\dot{\mathrm{Q}}$ & $\mathrm{W}$ & heat flux \\
$\mathrm{d}_{\mathrm{h}}$ & $\mathrm{m}$ & hydraulic diameter & $\mathrm{r}$ & & recovery factor \\
$\mathrm{h}$ & $\mathrm{J} / \mathrm{kg}$ & specific enthalpy & $\mathrm{Re}$ & & Reynolds-number \\
$\mathrm{h}_{\mathrm{t}}$ & $\mathrm{J} / \mathrm{kg}$ & specific total enthalpy & $\mathrm{T}$ & $\mathrm{K}$ & temperature \\
$\mathrm{k}$ & & correction factor & $\mathrm{T}_{\mathrm{c}}$ & $\mathrm{K}$ & coolant total temperature \\
$\mathrm{l}$ & $\mathrm{m}$ & length & $\mathrm{T}_{\mathrm{TC}}$ & $\mathrm{K}$ & temperature of the thermocouple \\
$\mathrm{L}$ & $\mathrm{m}$ & half perimeter of a cylinder & $\mathrm{U}$ & $\mathrm{m}$ & wetted perimeter \\
$\dot{\mathrm{m}}$ & $\mathrm{kg} / \mathrm{s}$ & massflow & $\mathrm{W}_{\mathrm{c}}$ & $\mathrm{m}$ & channel width \\
$\mathrm{Ma}$ & & Mach-number & $\mathrm{x}$ & $\mathrm{m}$ & axial co-ordinate \\
$\mathrm{Nu}$ & & Nusselt-number & $\mathrm{y}$ & $\mathrm{m}$ & normal co-ordinate
\end{tabular}

\section{Greek symbols}

\begin{tabular}{|c|c|c|c|c|}
\hline $\mathrm{W} /\left(\mathrm{m}^{2} \mathrm{~K}\right)$ & heat transfer coefficient & $\lambda$ & & friction coefficient \\
\hline $\mathrm{W} /\left(\mathrm{m}^{2} \mathrm{~K}\right)$ & $\begin{array}{l}\text { heat transfer coefficient at the } \\
\text { thermocouple }\end{array}$ & $\lambda_{\mathrm{TC}}$ & $\mathrm{W} /(\mathrm{mK})$ & $\begin{array}{l}\text { thermal conductivity of the } \\
\text { thermocouple }\end{array}$ \\
\hline $\mathrm{m}$ & equivalent sand grain roughness & $\xi$ & & loss coefficient \\
\hline $\mathrm{W} /(\mathrm{mK})$ & thermal conductivity & & & \\
\hline
\end{tabular}




\section{INTERNAL HEAT TRANSFER OF STEAM COOLED VANES}

\section{Introduction}

The improvement of the thermodynamic efficiency of gas turbine engines leads to an increase of the turbine inlet temperature and the pressure ratio as well. In recent engines, the turbine inlet temperature significantly exceeds the admissible metal temperatures, which requires an efficient cooling system to protect the thermally loaded parts. In conventional cooling systems, air is taken from the compressor and supplied to the turbine vanes, blades and disks. The amount of the required cooling mass flow rises as the turbine inlet temperature is increased, because the difference between the hot gas and the metal temperature is enlarged. The growth of the engine pressure ratio acts into the same direction since with higher compressor exit temperatures the driving temperature difference between the coolant and the walls is reduced and has to be balanced by a larger amount of cooling air.

The internal airflow, which is taken from the compressor, has a significant quantity in almost every current engine. In the GT11NM for example, the cooling and leakage mass flows are in the order of $10 \%$ of the total engine mass flow. A part of the secondary air is re-injected into the main flow by film cooling holes or trailing edge channels and therefore contributes to the power production in downstream stages. However, there is a large potential of increasing the thermal efficiency by reducing or substituting the internal air mass flow.

In order to reduce the internal airflow of gas turbine engines in combined cycle plants, steam cooling of the whole engine or parts of it is an interesting alternative. For cooling purposes, superheated steam has favourable fluid properties. The specific heat of steam is approximately twice as much than for air, which leads to a reduced temperature increase for the same amount of absorbed heat. For the same inlet conditions, there is a larger overall driving temperature difference for steam and therefore a better heat transfer from the wall. Furthermore, the higher Prandtl-number of steam - 1.0 compared to 0.7 for air - leads to higher Nusselt-numbers for the same Reynolds-number.

With regard to the thermodynamic process, additional benefits arise by the use of steam. There are more degrees of freedom in the choice of the coolant supply source and the temperature level of the coolant. It is no longer coupled to the compressor exit temperature but depends on the location of the steam extraction from the Rankine-cycle. An overview about the current steam and water cooling technologies of gas turbine engines is given by Nealy (1996).

Various possible vane cooling concepts are shown in Figure 1. 
Appendix 4
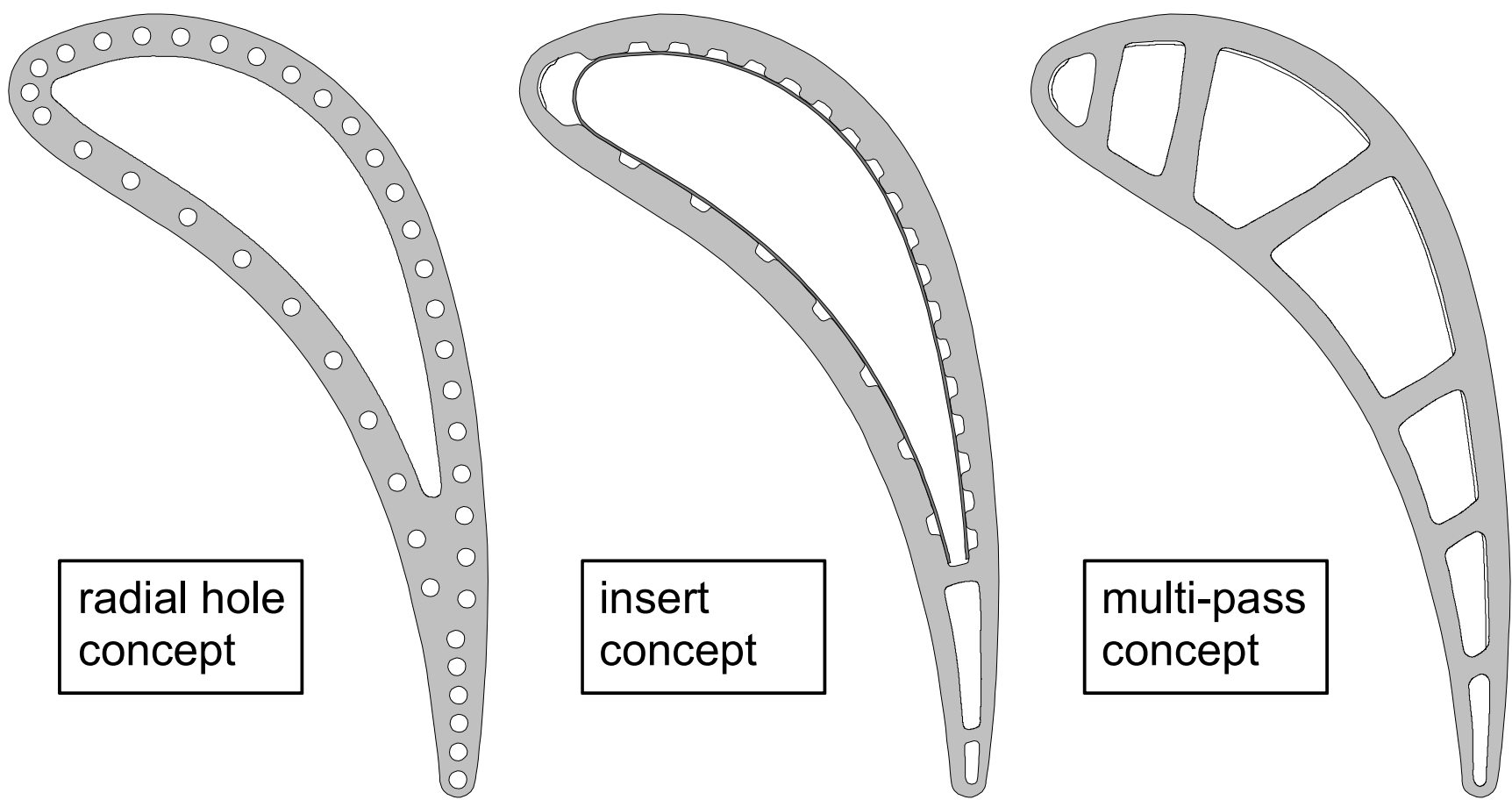

Figure 1: Vane cooling concepts 
Appendix 4

\section{Experimental Setup}

\section{Test facility}

For the conduction of the heat transfer measurements, a test facility as shown in Figure 2 was built. In order to have a direct comparison of air and steam cooling, both fluids can be used as the coolant medium for the test model. The main features of the facility are:

- fluids: steam, air

- max. coolant pressure: 10 bar (steam), 8 bar (air)

- max. coolant temperature: $280^{\circ} \mathrm{C}$ (steam), $500^{\circ} \mathrm{C}$ (air)

- steam superheater $(14 \mathrm{~kW})$

- electric heaters $(8 \times 1 \mathrm{~kW})->$ max. test-plate temperature: $700^{\circ} \mathrm{C}$

- max. coolant mass flow: $0.04 \mathrm{~kg} / \mathrm{s}(144 \mathrm{~kg} / \mathrm{h})$

The steam generator is fired with oil and produces saturated steam with approximately $180^{\circ} \mathrm{C}$ at a pressure level of 10 bar. In order to avoid condensation in the test section and in the pipes, a superheater is installed

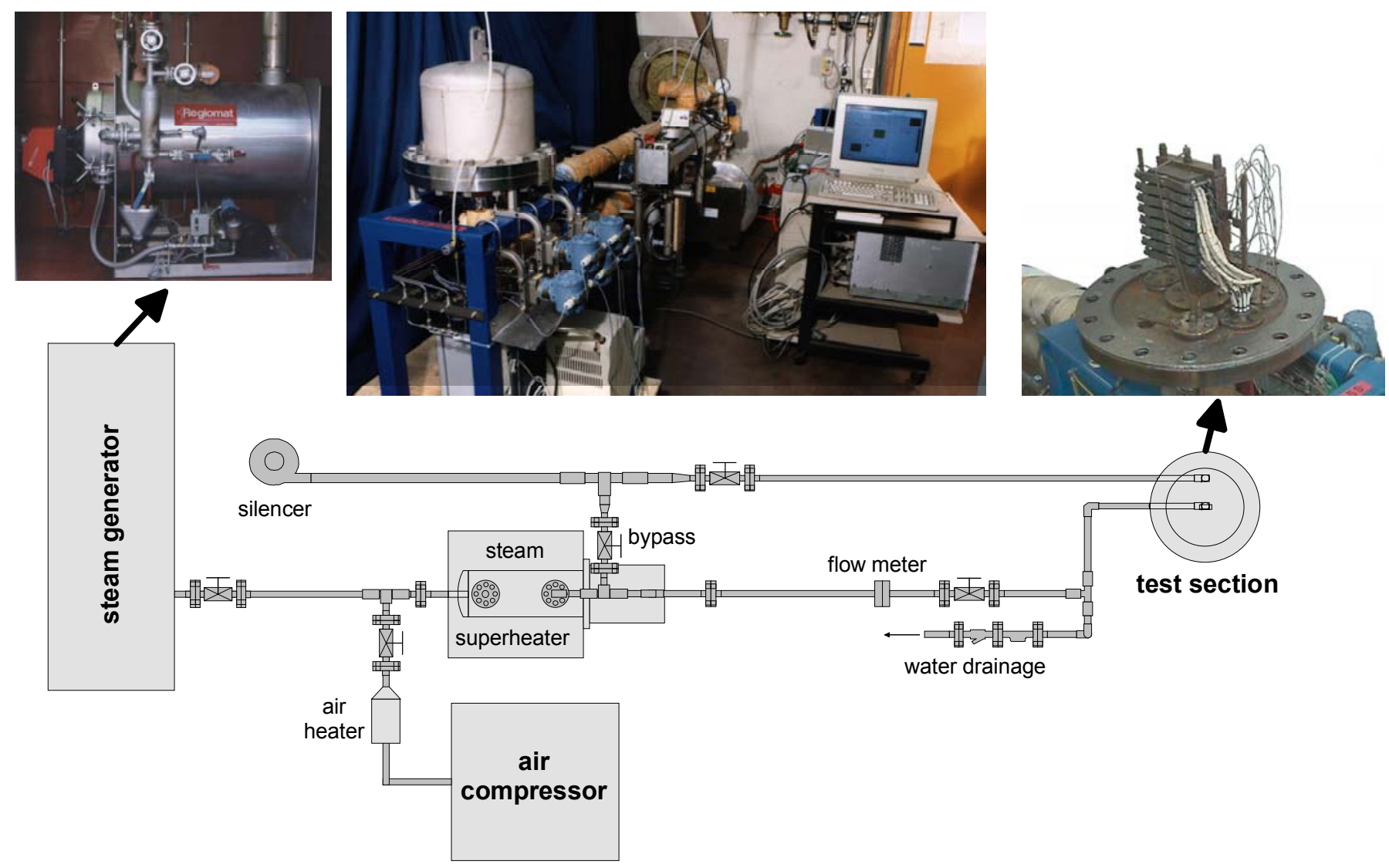

Figure 2: Test facility 


\section{Appendix 4}

downstream of the steam generator. Thus, a maximum temperature of $280^{\circ} \mathrm{C}$ is reached.

The superheater is followed by an orifice flow meter and a water drainage to remove condensate from the piping system. The massflow through the test section is varied with a bypass-valve. At the outlet of the piping system, a silencer is installed.

By using air as the working fluid, a maximum pressure level of 8 bar can be reached with the current compressor. The air temperature is adjusted with an additional heater, which is installed downstream of the compressor. In order to avoid condensations, hot air is also used to pre-heat the whole system, before the steam supply is turned on.

Figure 4 shows a cross-sectional view and a top view of the test section. The model of the vane is inserted into a pressurised containment and can be accessed by removing the cover of the pressure vessel.

The thermocouples, the pressure pipes and the power supply cables of the eight electrical heaters are passed through cylindrical plates and special connectors in the carrier plate. Thus, a sealing of the cables and wires is obtained.

The tested model is sketched in Figure 5 together with the flow scheme of one of the insert vane concepts, which was chosen as the test case for the steam cooling experiments. It was one of several possibilities, which have been discussed. The coolant is discharged from the outer box via an insert channel into the inner box. The reversed flow passes the leading and trailing edge as well as the suction and pressure side channels.

The flow situation of the test section corresponds to this vane concept. The coolant is fed into an insert channel, which is a rectangular box of thin metal sheets. At the end of the box, the flow is reversed and discharged into 16 rectangular cooling channels, which are covered and sealed by the insert. 
Appendix 4
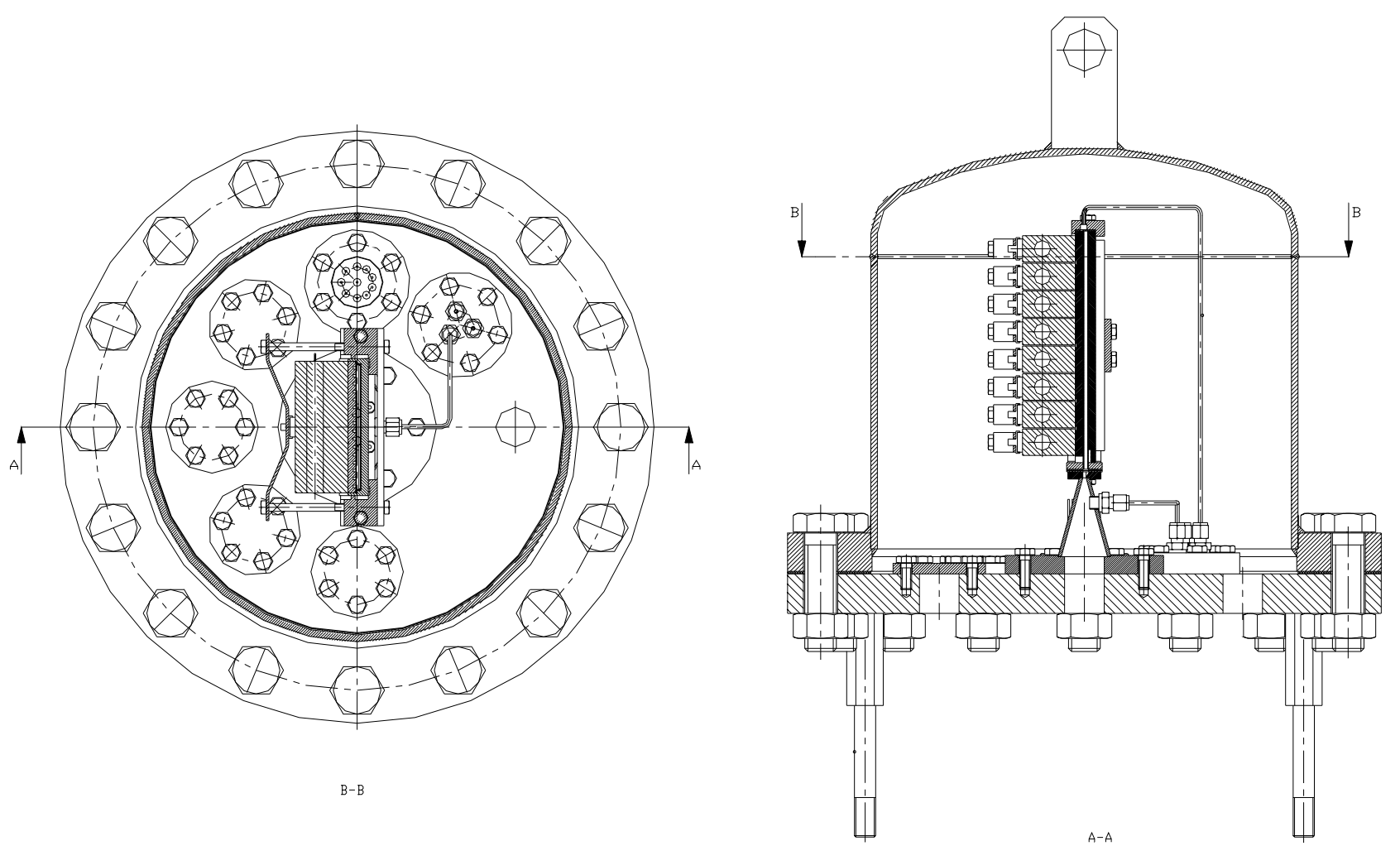

Figure 4: Test section "Steam Cooled Vanes"

The heat load of the hot gas around the vane is simulated by eight electrical heaters, which are inserted into copper cubes in order to obtain a well defined uniform heat supply to the test plate. Separate cubes are used in order to compensate the different thermal expansion rates of copper and the test plate. They are fixed to the plate by leaf springs. Thus, a maximum temperature level of $700^{\circ} \mathrm{C}$ is reached. 
Appendix 4

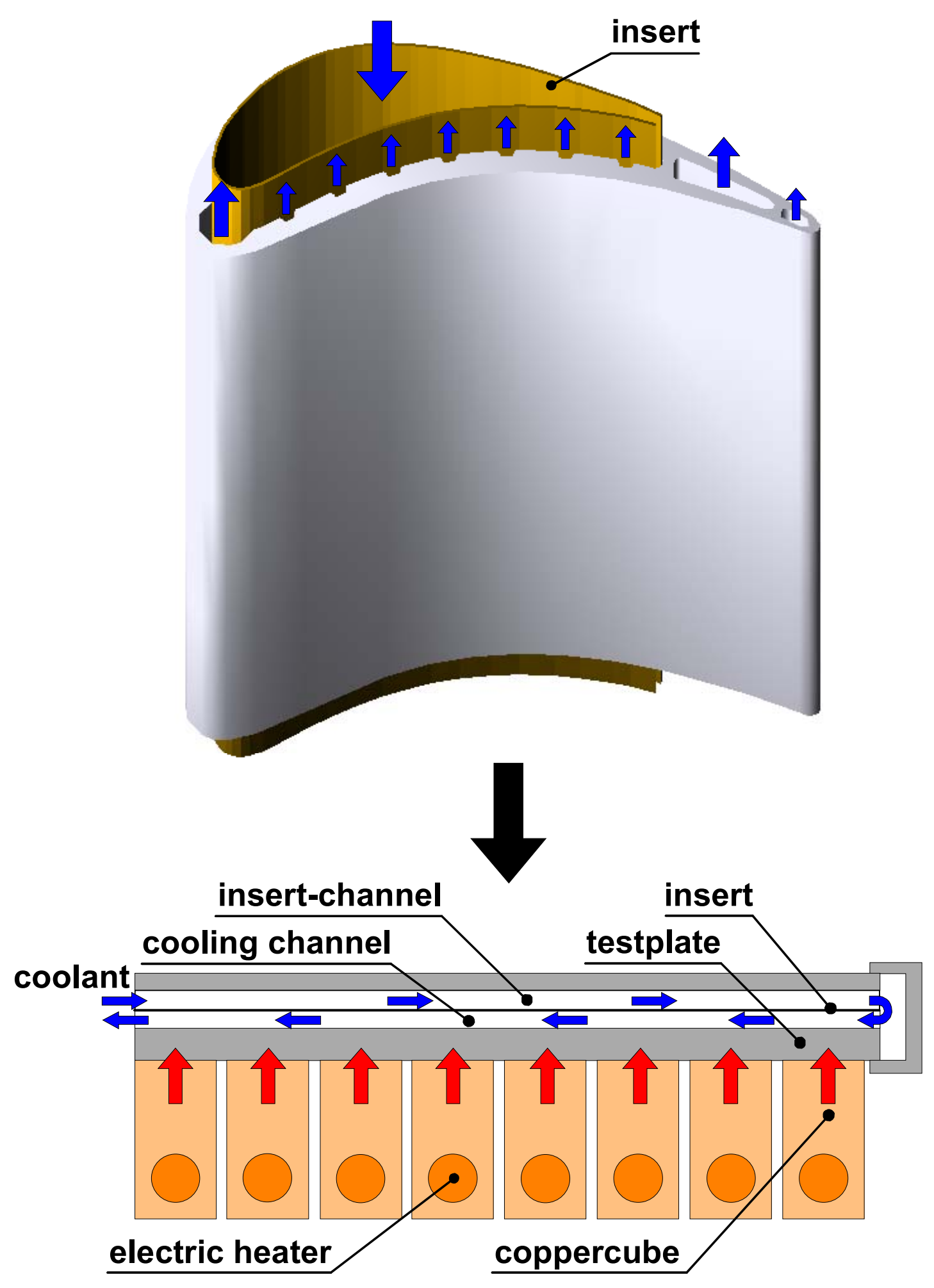

Figure 5: Principle set-up 


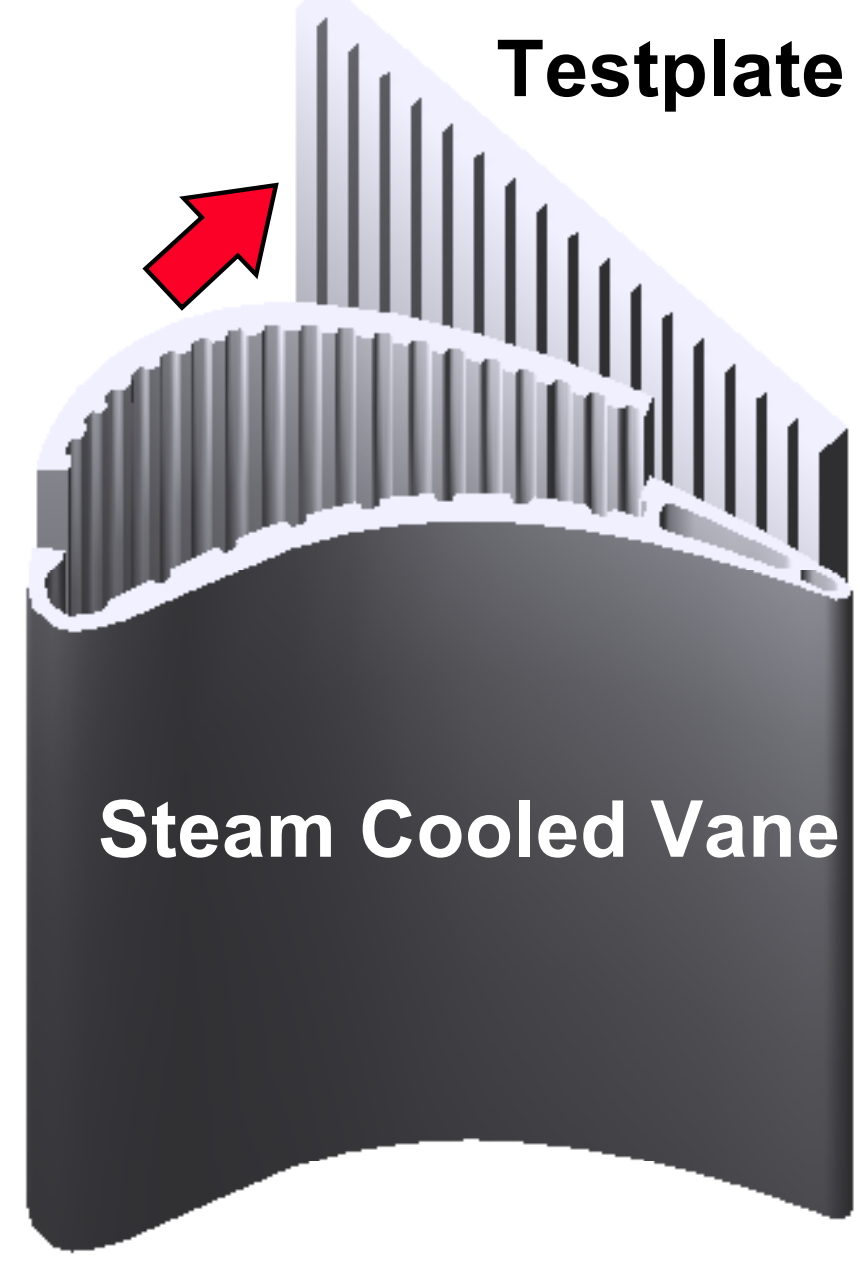

Figure 1: Test-plate

The heat load of the hot gas around the vane is simulated by eight electrical heaters, which are inserted into copper cubes in order to obtain a well defined uniform heat supply to the test plate. Separate cubes are used in order to compensate the different thermal expansion rates of copper and the test plate. They are fixed to the plate by leaf springs. Thus, a maximum temperature level of $700^{\circ} \mathrm{C}$ is reached.

The geometry of the test plate is very similar to the suction side part of the vane (cp. Figure 1). With the exception of the curvature and the radial spread, the dimensions of the plate and the cooling channels correspond to the vane. The major dimensions are:

- length: $170 \mathrm{~mm}$

- width: $100 \mathrm{~mm}$

- thickness: $5.9 \mathrm{~mm}$ 
- channel size: $2 \times 2 \mathrm{~mm}$

- channel number: 16

The material, which was chosen (X2CrNi19), is a stainless steel with a thermal conductivity of approximately $20 \mathrm{~W} /(\mathrm{mK})$, which is very close to the conductivity of the vane material (In738).

\section{Instrumentation}

In order to get a detailed overview about the flow and heat transfer situation in the test model, a rather comprehensive instrumentation was applied. It includes the measurement of the

- coolant mass flow with an orifice flow meter,

- the temperature distribution (walls and coolant) with NiCr-Ni-thermocouples,

- the electric power input by measuring the heater voltage and current

- and the measurement of the pressure loss in the different parts of the test facility with one absolute and four differential pressure transducers.

- For the measurement of the coolant mass flow, a standard orifice flow meter according to DIN 1952 is used. From measurements of the upstream temperature and pressure level and the pressure difference across the orifice plate, the massflow through the flow meter can be calculated.

- The arrangement of the pressure transducers and the pressure taps is shown by Figure 2. The pressure level in the whole system is measured upstream of the orifice plate of the flow meter with an absolute pressure transducer. With the other four transducers, the difference to the reference pressure is determined. Thus, the pressure drop across the flow meter is measured. Furthermore, the losses up to the entrance funnel, across the forward flow path and over the cooling channels can be determined.

- Since the temperature level in the piping system can reach $3000 \mathrm{C}$, the membranes of the pressure transducers have to be protected against the heat. Therefore, the pressure pipes are filled with water. To prevent from evaporation of the water charge, the pipes are cooled in a thermostatic bath.

- The temperature distribution in the whole system is measured with a large number of shielded $\mathrm{NiCr}-\mathrm{Ni}$-thermocouples with a diameter of $0.5 \mathrm{~mm}$. The coolant thermocouples are located at two different positions in the piping system, in the entrance funnel, the insert channel, in the flow reversal, in the cooling channels and outside of the test model in the pressure vessel. Furthermore, the material temperatures of the test plate and the copper cubes with the heaters are measured. 


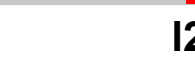

12

I1
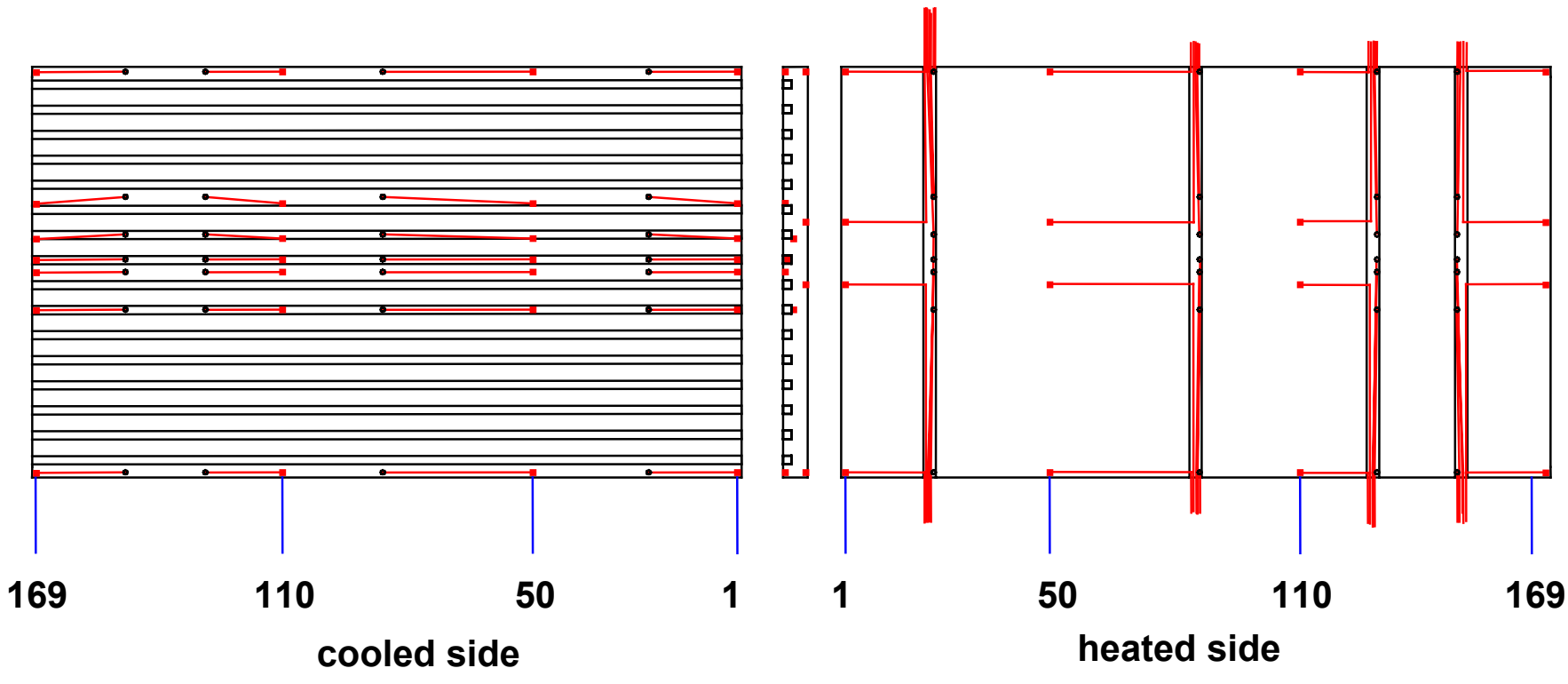

cooled side

110

169

Figure 3: Instrumentation of the test plate

- In order to achieve data about the local and the average heat transfer in the cooling channels, the temperature distribution around the test plate as well as the coolant temperature is measured within four cross-sections at different axial positions (Figure 3). Eleven thermocouples are applied to each cross-section. They are measuring the temperatures of the cooled and of the heated side of the plate as well as the coolant temperature in one of the sixteen channels. They are mounted parallel to the flow direction in small grooves with a size of $0.5 \mathrm{~mm}$. The single wires are collected in four larger grooves on the bottom of the test plate and discharged on the longitudinal sides.

- In addition to temperature and pressure measurements, the power output of the electrical heaters (Pmax $=8 \times 1 \mathrm{~kW})$ in the copper cubes is determined by a measurement of the voltage and the current. The heating power is controlled with two AC transformers, which are connected to four heaters each.

\section{Test conditions}

One of the requirements to the tests was to get a close match of the real engine conditions. Therefore, the experiments were designed to cover the estimated Reynolds- and Mach-numbers of the steam cooled vane. As shown by Error! Reference source not found., where the envelope of the heat transfer test range is plotted together with the design point of the vane, this requirement is met. With respect to the pressure and the metal temperature level in the engine, some reductions had to be made. The steam supply pressure of the vane is approximately 17 bar, whereas the maximum pressure for the tests is limited by the available steam and air supply sources. For air, the maximum pressure is 8 bar, whereas with steam 10 bars are reached. However, the tests with 
various pressure levels showed a negligible influence of the inlet pressure on the measured Nusselt-numbers. 


\section{Appendix 4}

For the vane, an average material temperature of $8500 \mathrm{C}$ is aspired. During the tests, the maximum metal temperature was slightly lower than in the engine. The temperature of the copper cubes was kept constant at a level of 700 oC.

The complete matrix of the heat transfer tests, which were conducted, is shown in Table 1. Two different inlet temperature levels have been tested for each fluid. They were combined with three respectively two inlet pressure levels. For every combination of inlet temperature and pressure, the coolant massflow was varied from the possible minimum value to the maximum flow rate. For most of these cases, a maximum flow rate of $0.04 \mathrm{~kg} / \mathrm{s}$ was obtained. The massflow was varied in steps of $0.005 \mathrm{~kg} / \mathrm{s}$. The corresponding Reynolds- and Mach-number range is also included in Table 1.

\begin{tabular}{|r|r|r|r|r|r|}
\hline Coolant & $\mathbf{T}_{\text {coolant,inlet }}{ }^{\circ} \mathbf{C}$ ] & $\mathbf{p}_{\text {coolant,inlet }}[\mathbf{b a r}]$ & $\mathbf{m}_{\text {coolant }}[\mathbf{k g} / \mathbf{s}]$ & $\begin{array}{c}\text { Reynolds- } \\
\text { numbers }\end{array}$ & $\begin{array}{c}\text { Mach- } \\
\text { numbers }\end{array}$ \\
\hline steam & 220 & 10 & $0.005-0.040$ & $7000-70000$ & $0.03-0.23$ \\
steam & 220 & 8 & $0.005-0.040$ & $7000-60000$ & $0.04-0.28$ \\
steam & 220 & 6 & $0.005-0.030$ & $6000-55000$ & $0.05-0.32$ \\
\hline steam & 280 & 10 & $0.005-0.038$ & $7000-57000$ & $0.03-0.24$ \\
steam & 280 & 8 & $0.005-0.035$ & $6000-52000$ & $0.04-0.30$ \\
steam & 280 & 6 & $0.005-0.030$ & $6000-43000$ & $0.05-0.37$ \\
\hline air & 220 & 8 & $0.005-0.040$ & $5000-45000$ & $0.03-0.24$ \\
air & 220 & 6 & $0.005-0.040$ & $5000-45000$ & $0.04-0.34$ \\
\hline air & 280 & 8 & $0.005-0.040$ & $4000-45000$ & $0.03-0.28$ \\
air & 280 & 6 & $0.005-0.040$ & $5000-40000$ & $0.04-0.35$ \\
\hline
\end{tabular}

Table 1: Test matrix 
Appendix 4

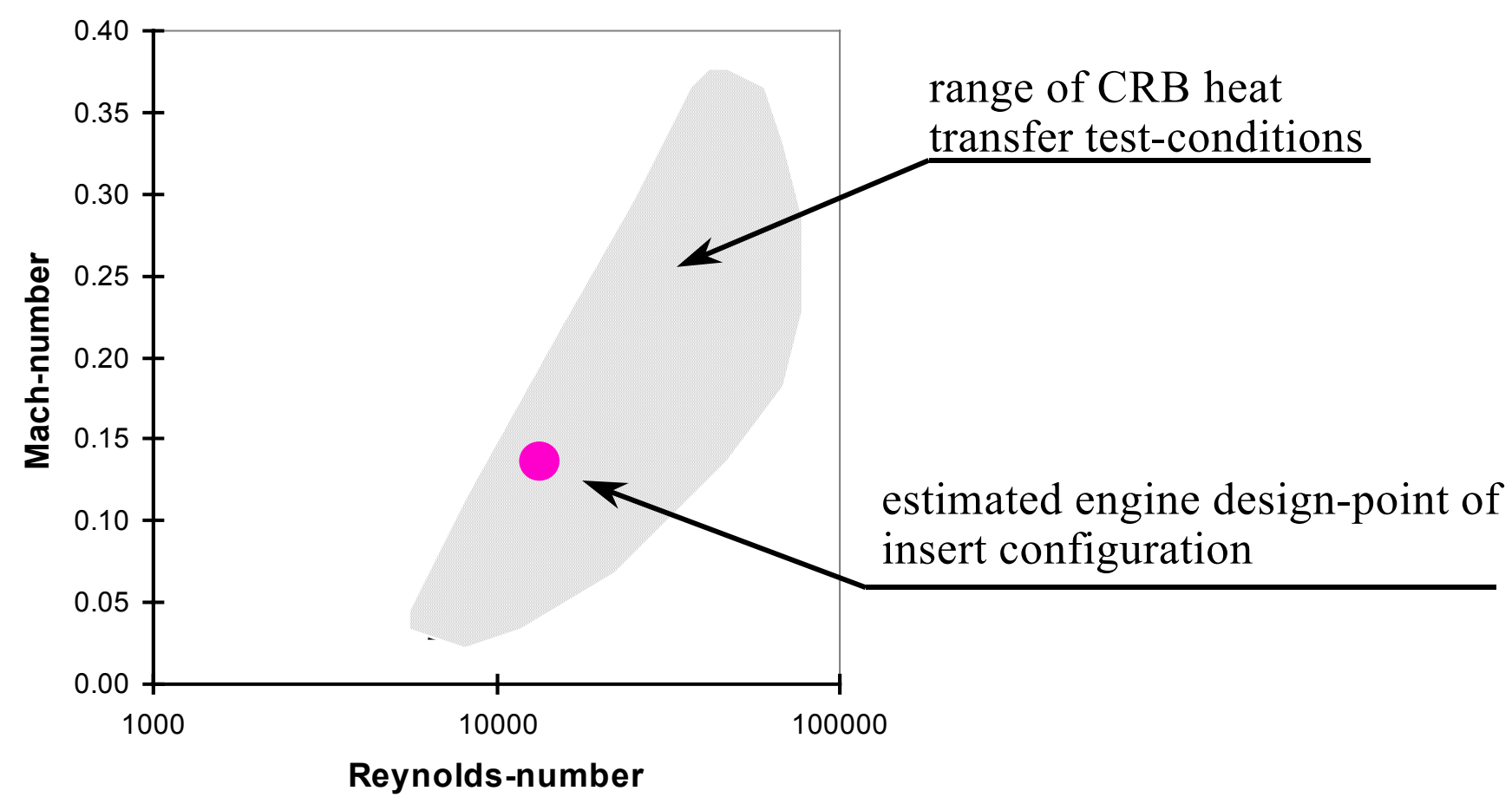

Figure 1: Range of the heat transfer tests

\section{Data Processing}

\section{Heat transfer coefficients}

Due to the comprehensive instrumentation of the test model, local and averaged heat transfer coefficients could be determined. For the calculation of the heat transfer coefficients, the wellknown definition is used:

$\alpha=\frac{\dot{Q}}{A \cdot\left(T_{\text {wall }}-T_{c}\right)}=\frac{\dot{q} \cdot \dot{m}}{A \cdot\left(T_{\text {wall }}-T_{c}\right)}$

In Equation ( 1 ), $\dot{Q}$ is the heat flux from the wall and $\mathrm{A}$ is the corresponding surface area. The reference temperature, which is used, is the local total temperature of the coolant $\operatorname{Tc}(x)$. Introducing $U$ as the wetted perimeter gives for a control volume of infinite axial length:

$U=\frac{A}{d x}=2 \cdot d_{c}+w_{c}$

In this equation, dc and wc are the channel depth and width. As shown by the definition of the wetted perimeter, the data reduction is based on the assumption of a three-side heat transfer. The 
Appendix 4

heat transfer from the insert to the flow in the test-plate channels is neglected. This seems to be the most appropriate definition in this case. Although the insert is not adiabatic, most of the heat is transferred through the bottom and the side walls to the flow in the test-plate channels.

The specific heat flux from the wall is determined with the first law of thermodynamics:

$d \dot{q}=d h_{t}=c_{p} d T_{c}$

Combining the Equations ( 1 )- ( 3 ) results in:

$d \dot{q}=\frac{\alpha \cdot U \cdot d x}{\dot{m}}\left(T_{\text {wall }}-T_{c}\right)=c_{p} \cdot d T_{c}$

Resolving Equation ( 4 ) for $\alpha$ yields:

$\alpha=\frac{\dot{m}}{U \cdot\left(T_{\text {wall }}-T_{c}\right)} c_{p} \frac{d T_{c}}{d x}$

As shown by Equation ( 5 ), the determination of the local heat transfer coefficients requires data about the local wall and coolant temperature and the coolant temperature gradient. The axial gradient of the flow temperature is determined with the derivative of a polynomial second order interpolation through the location at $\mathrm{x}$ and the two nearest neighbour-points.

The local wall and gas temperatures are measured directly with thermocouples. Since there is a profile of the channel wall temperature on each of the three sides, the driving wall temperature is obtained by averaging the measured temperatures of the thermocouples u2, u5 and u6 (cp. Error! Reference source not found.).

The coolant temperature is measured with thermocouple $u 4$, which is mounted into a groove of the test plate. The tip of the wire is bended into the channel. With this setup, the measurement error due to thermal conduction in the wire must be taken into account. The influence of heat conduction on the gas temperature measurement is quite low for high Re-numbers $(<10 \mathrm{C})$ but may rise up to $50 \circ C$ if the Re-number is very low $(\operatorname{Re}=4000)$. The error due to heat conduction can be determined rather accurate, because there are additional gas thermocouples upstream of the testplate inside the bend and downstream close to the exit cross-section. These measurements are used to give reference values for the calibration of the correction procedure. The free length of the reference wires is sufficiently large to neglect the influence of heat conduction. The correction procedure is explained in the next chapter. 
Appendix 4

\section{Coolant temperature measurement}

The correction of errors due to thermal conduction of heat from the test plate to the wire tip is performed by assuming a cylinder in a cross-flow (Figure 2). The energy balance in the thermocouple-wire gives:

$d \dot{Q}_{\text {conduction }}=d \dot{Q}_{\text {heat transfer }}=\alpha_{T C} \cdot \pi \cdot d \cdot d y \cdot\left(T_{T C}(y)-T_{c}\right)$

According to Fourier's law, the heat conduction in the wire is:

$\dot{Q}_{\text {conduction }}=A \cdot \lambda_{T C} \cdot \frac{d T_{T C}}{d y}=\frac{\pi \cdot d^{2}}{4} \cdot \lambda_{T C} \cdot \frac{d T_{T C}}{d y}$

Combining the derivative of Equ. ( 7 ) with Equ. ( 6 ) leads to:

$\frac{d \dot{Q}_{\text {conduction }}}{d y}=\frac{\pi \cdot d^{2}}{4} \cdot \lambda_{T C} \cdot \frac{d^{2} T_{T C}(y)}{d y^{2}}=\alpha_{T C} \cdot \pi \cdot d \cdot\left(T_{T C}(y)-T_{c}\right)=\frac{d \dot{Q}_{\text {heat transfer }}}{d y}$ 
Appendix 4

Equ. ( 8 ) is a second order differential equation for the determination of the temperature distribution TTC in the thermocouple, which is depending on the coolant temperature Tc:

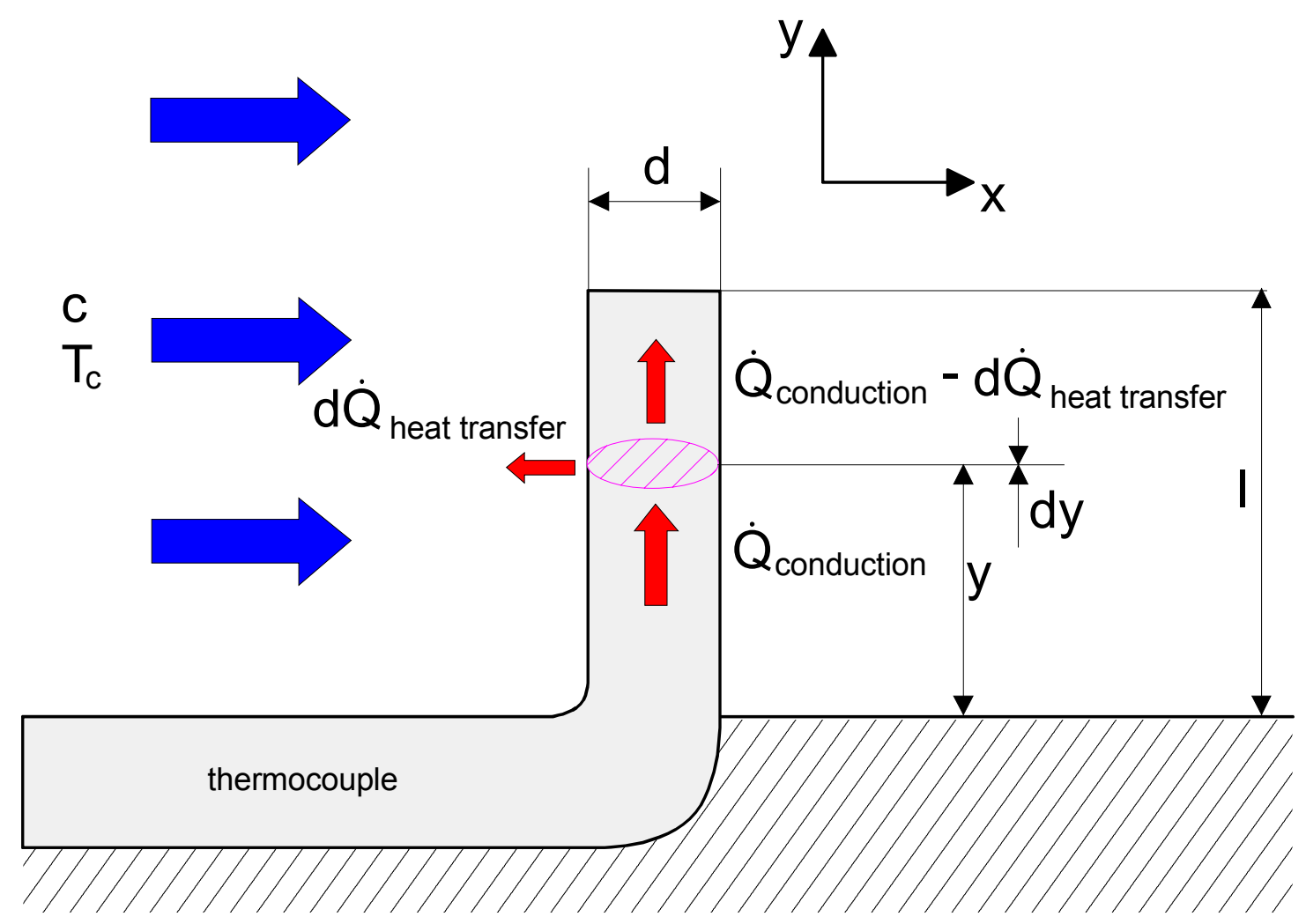

Figure 2: Coolant temperature measurement

$T_{T C}(y)=\frac{d}{4} \cdot \frac{\lambda_{T C}}{\alpha_{T C}} \cdot \frac{d^{2} T_{T C}}{d y^{2}}+T_{c}$

Integration of Equ. ( 9 ) with an exponential approach for TTC and resolving for the coolant temperature Tc yields:

$$
T_{c}=\frac{T_{T C}(l)-T_{\text {wall }} \cdot \operatorname{Exp}\left[-l \cdot \sqrt{\frac{4}{d} \cdot \frac{\alpha_{T C}}{\lambda_{T C}}}\right]}{1-\operatorname{Exp}\left[-l \cdot \sqrt{\frac{4}{d} \cdot \frac{\alpha_{T C}}{\lambda_{T C}}}\right]} \quad ; \text { boundary condition: TTC }(\mathrm{y}=0)=\text { Twall }
$$




\section{Appendix 4}

In Equ. ( 10 ), TTC (I) is the temperature of the wire tip, which is measured with the thermocouple. For the thermal conductivity of the wire, which is a composition of the $\mathrm{Ni}$ - and NiCr-wires with insulation material and the shield, an average value of $\mathrm{TC}=6.3 \mathrm{~W} /(\mathrm{mK})$ is assumed. The heat transfer coefficient TC is obtained from a correlation by Gnielinski (see VDI-Wärmeatlas (1984)) for a horizontal cylinder in a cross-flow:

$$
N u_{L}=\frac{\alpha_{T C} \cdot L}{\lambda}=\frac{0.037 \cdot \operatorname{Re}_{L}^{0.8} \cdot \operatorname{Pr}}{1+2.443 \cdot \operatorname{Re}_{L}^{-0.1} \cdot\left(\operatorname{Pr}^{\frac{2}{3}}-1\right)} ; L=\frac{\pi}{2} \cdot d
$$

\section{Results}

\section{Fluid flow}

During the experiments, the variation of the flow Reynolds-number in the cooling channels exceeded one order of magnitude (cp. Par. Error! Reference source not found.). The corresponding Mach-numbers reached a level of $\mathrm{Ma}=0.37$, which lead to flow velocities in the cooling channels of more than $200 \mathrm{~m} / \mathrm{s}$ in some cases. A typical distribution of the flow velocities is shown in Figure 3, where the average velocities for steam and air are plotted versus the axial length of the channels. The plotted velocities are calculated
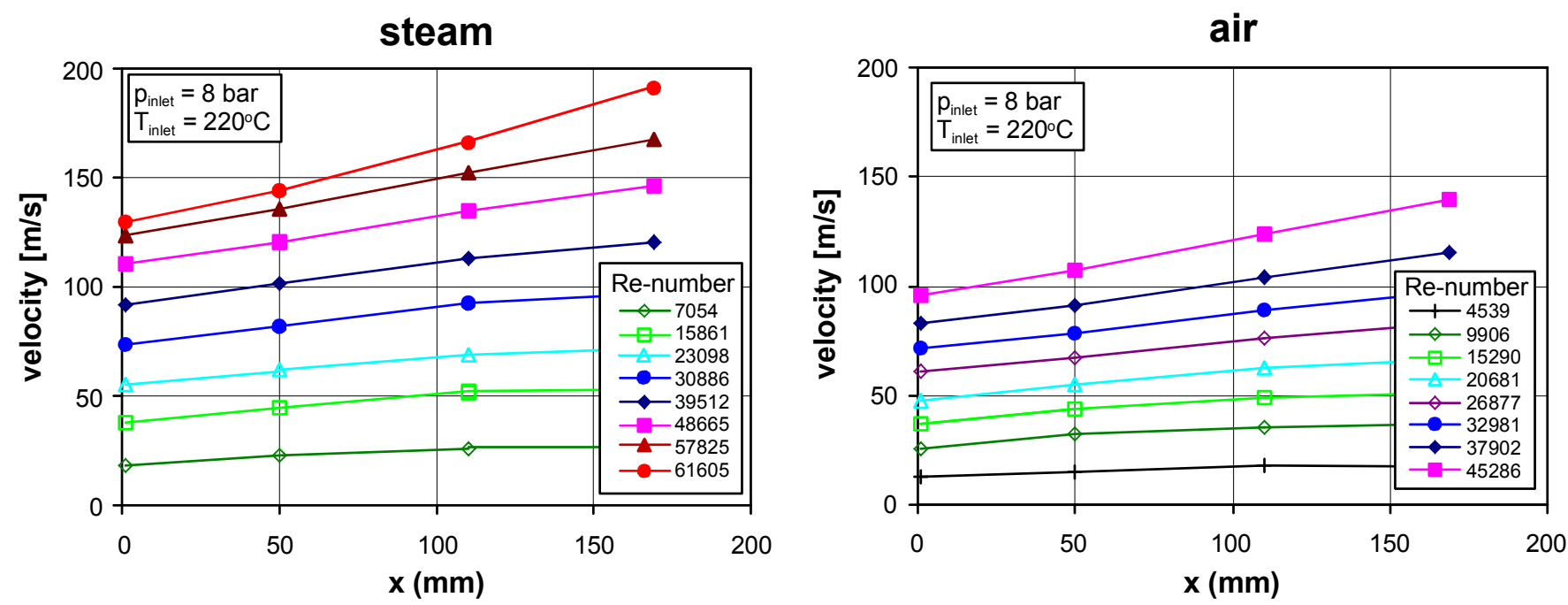

Figure 3: Flow velocities in the cooling channels

from the measured massflow, the cross-sectional area of the channels and the density, which is determined with the local temperatures and a linear interpolation of the measured static pressure in front of and behind the channels. As shown by Figure 3, high Reynolds-numbers lead to a significant acceleration of the flow due to the high heat transfer rate in the cooling channels. Due to the lower specific heat of air, 
Appendix 4

cooling channels. Due to the lower specific heat of air, which is half the $\mathrm{cp}$ of steam, the acceleration of air is stronger than for steam at the same Reynolds-number.

A further impression of the flow situation in the cooling channels is obtained, when the measured pressure losses are analysed. Therefore, the measured data is compared with theoretical values from the literature (Figure 1). The theoretical pressure loss is determined with correlations published by Idelchik (1966), where the entrance and exit pressure losses as well as frictional losses are considered. The correlations for the losses are:

$$
\begin{aligned}
& \Delta p_{\text {in }}=\zeta_{\text {in }} \frac{\rho}{2} c_{\text {in }}{ }^{2} ; \zeta_{\text {in }}=0.5 \\
& \Delta p_{\text {friction }}=\lambda \frac{l}{d_{h}} \frac{\rho}{2} c_{\text {average }}{ }^{2}
\end{aligned}
$$


Appendix 4

cooling channels. Due to the lower specific heat of air, which is half the $\mathrm{cp}$ of steam, the acceleration of air is stronger than for steam at the same Reynolds-number.

A further impression of the flow situation in the cooling channels is obtained, when the measured pressure losses are analysed. Therefore, the measured data is compared with theoretical values from the literature (Figure 1). The theoretical pressure loss is determined with correlations published by Idelchik (1966), where the entrance and exit pressure losses as well as frictional losses are considered. The correlations for the losses are:

$$
\begin{aligned}
& \Delta p_{\text {in }}=\zeta_{\text {in }} \frac{\rho}{2} c_{\text {in }}{ }^{2} ; \zeta_{\text {in }}=0.5 \\
& \Delta p_{\text {friction }}=\lambda \frac{l}{d_{h}} \frac{\rho}{2} c_{\text {average }}{ }^{2}
\end{aligned}
$$


Appendix 4

$\lambda=k_{\text {shape }} \cdot k_{\text {devflow }} \cdot \lambda_{\text {pipe }}$

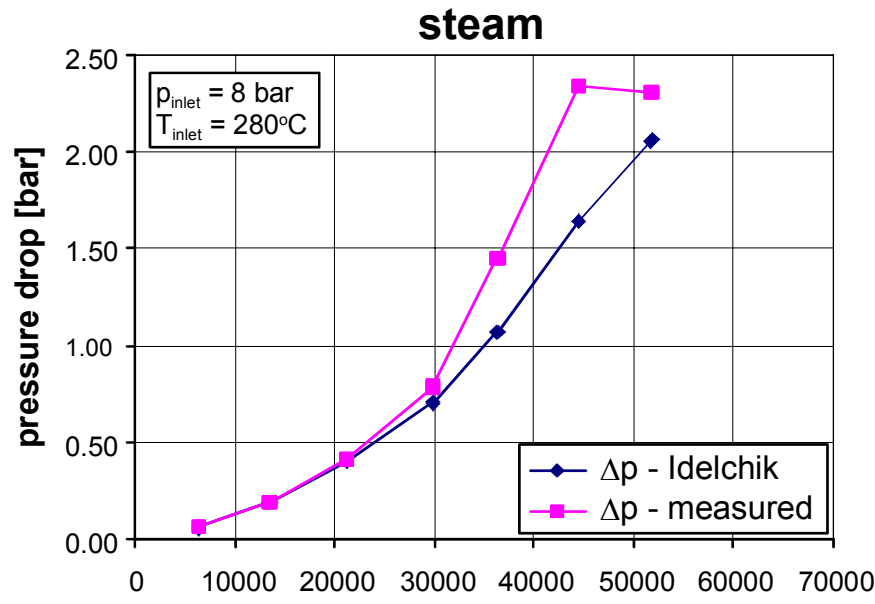

Reynolds-Number

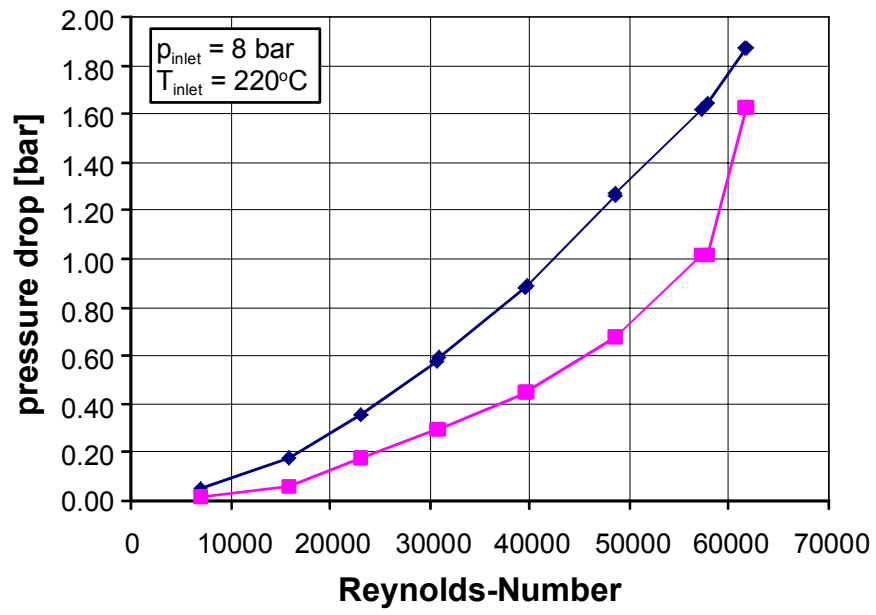

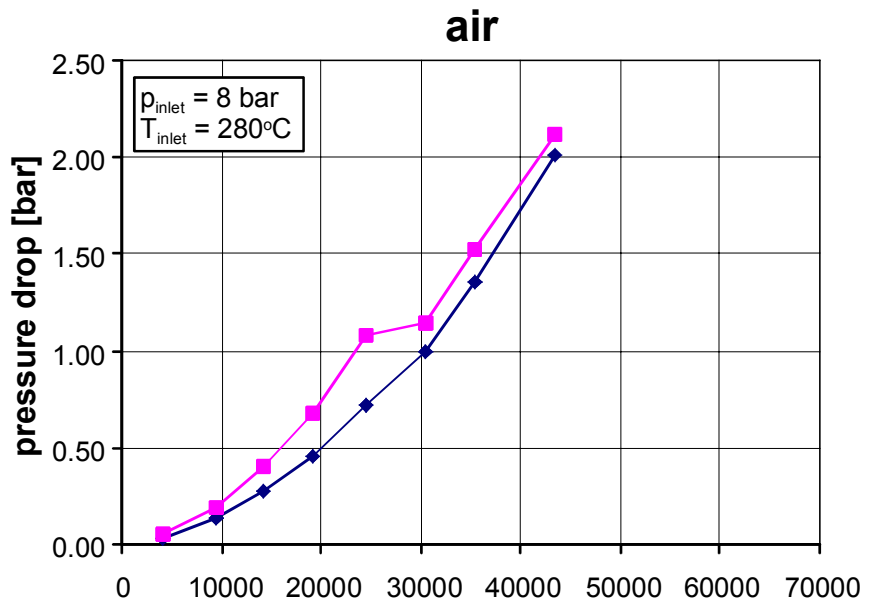

Reynolds-Number

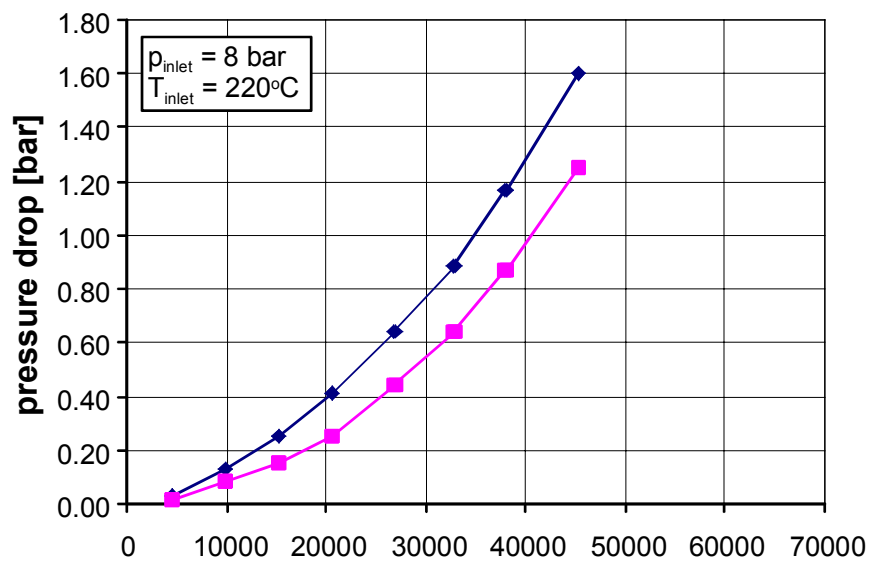

Reynolds-Number

Figure 1: Pressure drop across the cooling channels

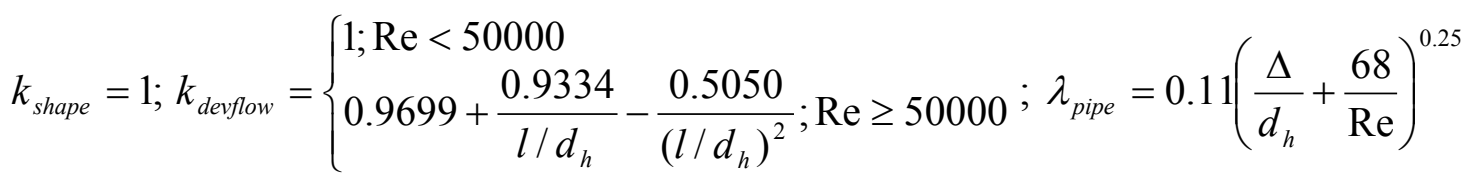

$\Delta p_{\text {out }}=\zeta_{\text {out }} \frac{\rho}{2} c_{\text {out }}{ }^{2} ; \zeta_{\text {out }}=1.04$

The friction coefficient is determined with the Altshul-approximation for cylindrical pipes and two correction factors kshape and kdevflow. With the k-factors, the influences of the rectangular channel shape and of the developing flow in the channels are considered.

The comparison of the results with the correlations above is performed for steam and air at a nominal pressure level of 8 bar and for inlet temperatures of $220 \mathrm{oC}$ and $280 \mathrm{oC}$ respectively. The 


\section{Appendix 4}

result is a bit surprising, because the theoretical pressure losses show a rather uniform increase with the Reynolds-number, whereas the measured curves exhibit kinks and bends. Especially the steam data shows a large difference between the theoretical and the measured pressure drop. When air is used as the coolant, both curves are closer together.

The explanation of this phenomenon was given after a disassembling of the test section. When the insert channel was taken out, rather strong deformations due to thermal stresses were found

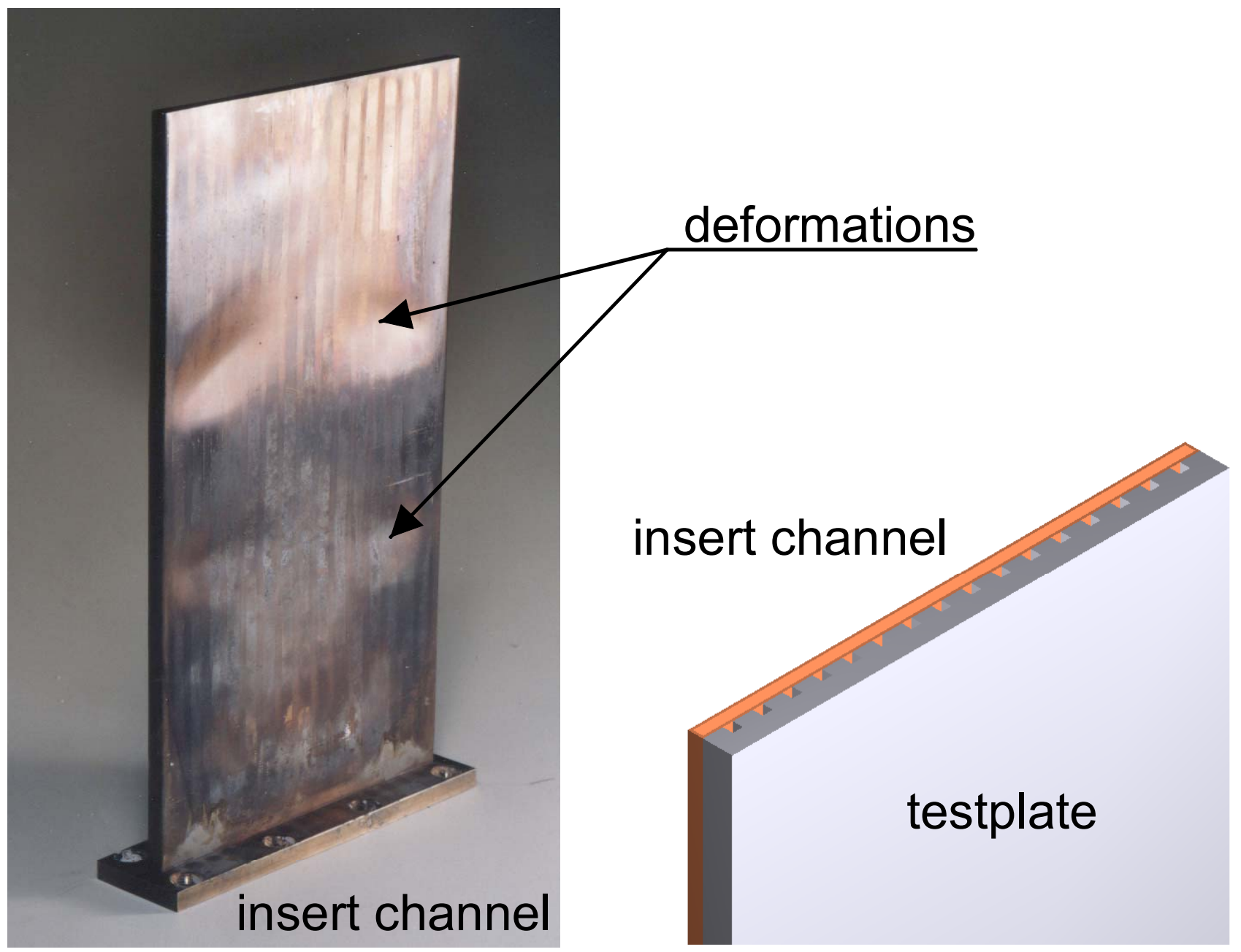

Figure 2: Deformations of the insert channel due to thermal stresses

(Figure 2). The insert channel is a rectangular box $(170 \mathrm{~mm} \times 96 \mathrm{~mm} \times 2.6 \mathrm{~mm})$ which covers the cooling channels of the test plate. Like in the vane, the contact between the insert and the test plate is primarily established by pressure forces. The pressure drop of the coolant causes the insert to be pressed against the test plate. The thermal stresses in the insert material are very large, because there is a direct contact to the hot plate and the cold fluid as well. Therefore, strong 


\section{Appendix 4}

temperature gradients are induced, which are the cause for bulges and buckles. When steam is used as the coolant, the thermal stresses are larger than for air. First of all, this is also due to the high specific heat of steam. For a constant heat pick-up, the temperature increase of steam is lower than for air. Therefore, the average fluid temperature is lower, which leads to a higher driving temperature difference and to higher temperature gradients in the material. When the deformations shown by Figure 2 occur, the flow conditions are changed. The cooling channels are no longer sealed by the insert. Subsequently, the ribs between the channels are also wetted with fluid. The velocities, the effective cross-sectional flow area and also the surface area for the heat transfer are influenced by the buckling of the insert. Under this point of view it is evident, that the measured pressure drop does not follow the theoretical predictions any more. The question is now, how the heat transfer from the test plate is affected, because the increase of the flow area influences the local Reynolds-numbers and the surface area for heat exchange as well. The answer to this question is given in the following paragraph. 
Appendix 4

\section{Heat transfer}

\section{Temperature distribution}

In Figure 3, an example for the measured surface temperatures on the test plate is shown. The surface temperatures are plotted versus the Reynolds-number for each of the four instrumented cross-sections. The flow enters the cooling channels at $x=0 \mathrm{~mm}$ and exits at $x=170 \mathrm{~mm}$. The
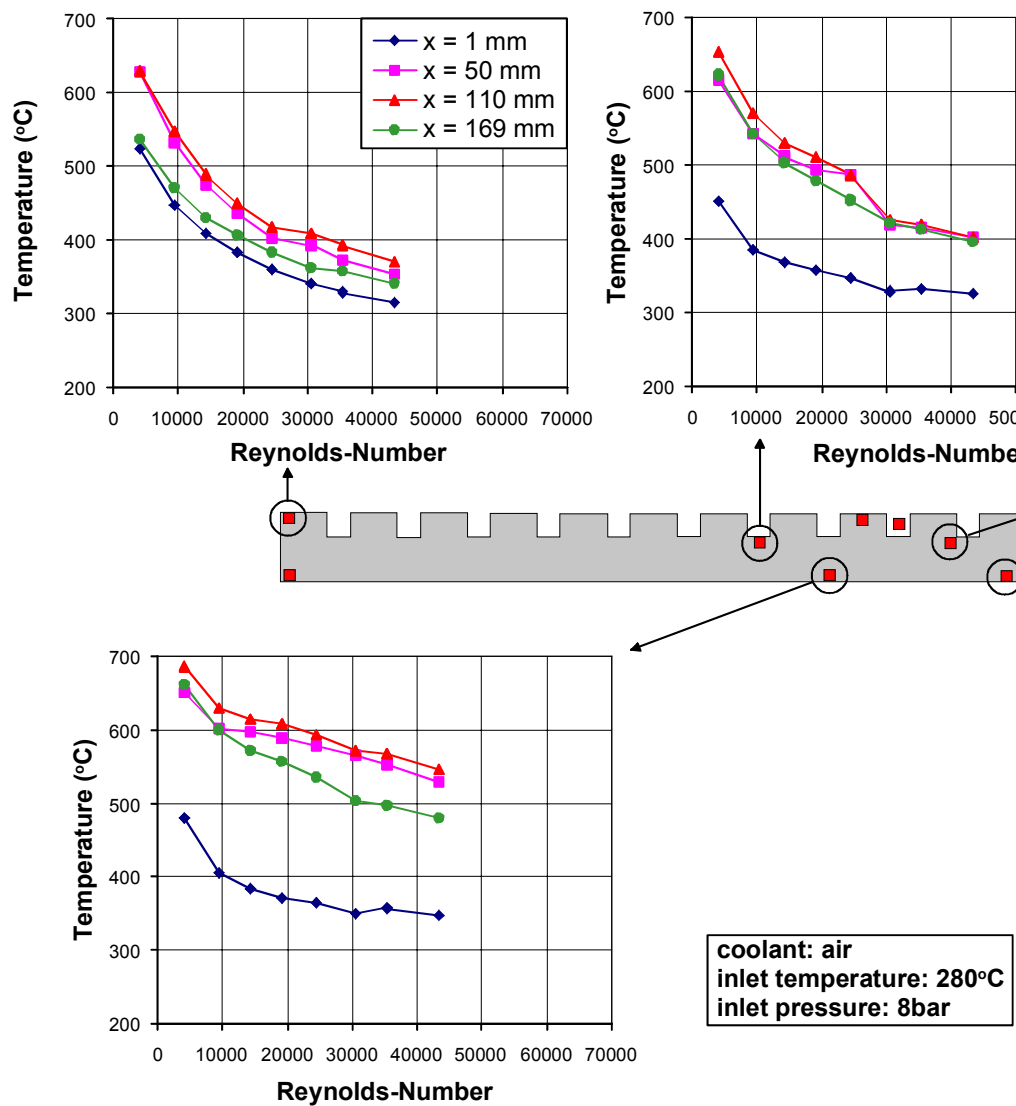
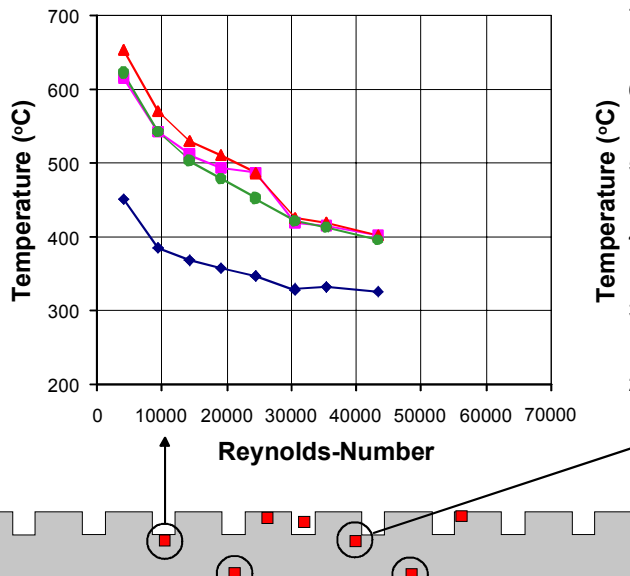

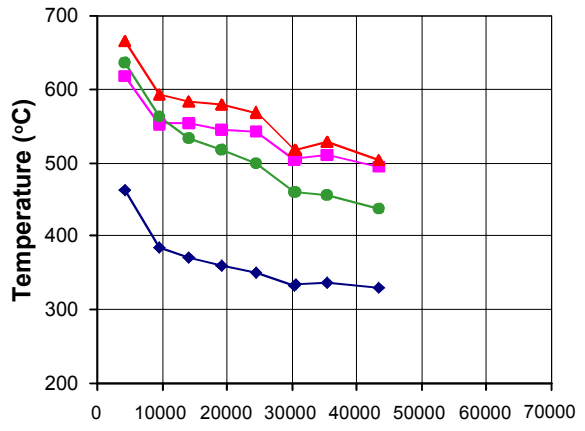

Reynolds-Number

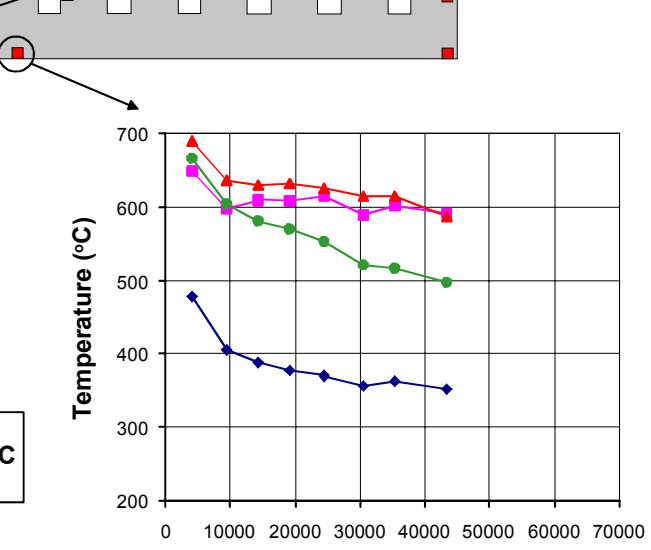

Reynolds-Number

Figure 3: Temperature distribution on the test plate

data in Figure 3 was obtained with air at Tinlet $=280 \mathrm{oC}$ and pinlet $=8$ bar. The temperature of the copper cubes with the electrical heaters was kept constant at $700 \circ \mathrm{C}$ over the whole Reynoldsnumber range. Due to high heat transfer coefficients in the inlet region, the inlet area of the plate $(x$ $=1 \mathrm{~mm})$ is significantly colder than the middle section $(x=50 ; 110 \mathrm{~mm})$. At both ends of the test plate $(x=1 ; 169 \mathrm{~mm})$, the heat conduction to adjacent parts causes a further reduction of the temperature level. The influence of heat conduction to attached parts is also apparent on the sidewalls of the plate (Figure 3, upper left diagram). Here, the temperature difference between the top and the bottom surface is clearly smaller than in the centre of the plate. 


\section{Appendix 4}

The influence of buckles in the insert on the temperature distribution is directly visible. The corresponding pressure drop measurements (upper right diagram) indicate an irregularity, which becomes apparent at $\operatorname{Re} \approx 30000$. In the same Re-number range, the surface temperatures on the plate exhibit a kink primarily between $x=50 \mathrm{~mm}$ and $x=110 \mathrm{~mm}$. The kink is most distinctive on the cooled side but is also present in the temperature distribution of the heated side. A comparison of the location of these temperature kinks with the bulges on the photo leads to the conclusion, that the deformation of the insert is responsible for the strange pressure drop characteristics and also for the irregularities in the temperature profile.

The measured gas temperatures, which are used for the calculation of the local heat transfer coefficients, are shown in Figure 1. In the two diagrams, the temperatures along the path of the coolant flow are plotted. The flow enters the test section at $x=-171 \mathrm{~mm}$, where the insert begins. At $x=0$, the flow is reversed and enters the cooling channels, which have a length of $170 \mathrm{~mm}$. The gas temperatures show, that a significant amount of heat is already transferred though the insert wall, especially when the Reynolds-number is low. Due to the higher specific heat, the temperature increase of steam in general is less than for air.

The last symbol, which is plotted for each temperature curve, refers to a thermocouple which is located outside of the cooling channels in the pressurised plenum. For small Reynolds-numbers, a difference to the last thermocouple in the channels occurs, because the plenum air is in contact with the heater cubes. Thus, the temperature difference is a measure for the heat, which is lost due to heat conduction and external convection. However, when the Reynolds-number is sufficiently
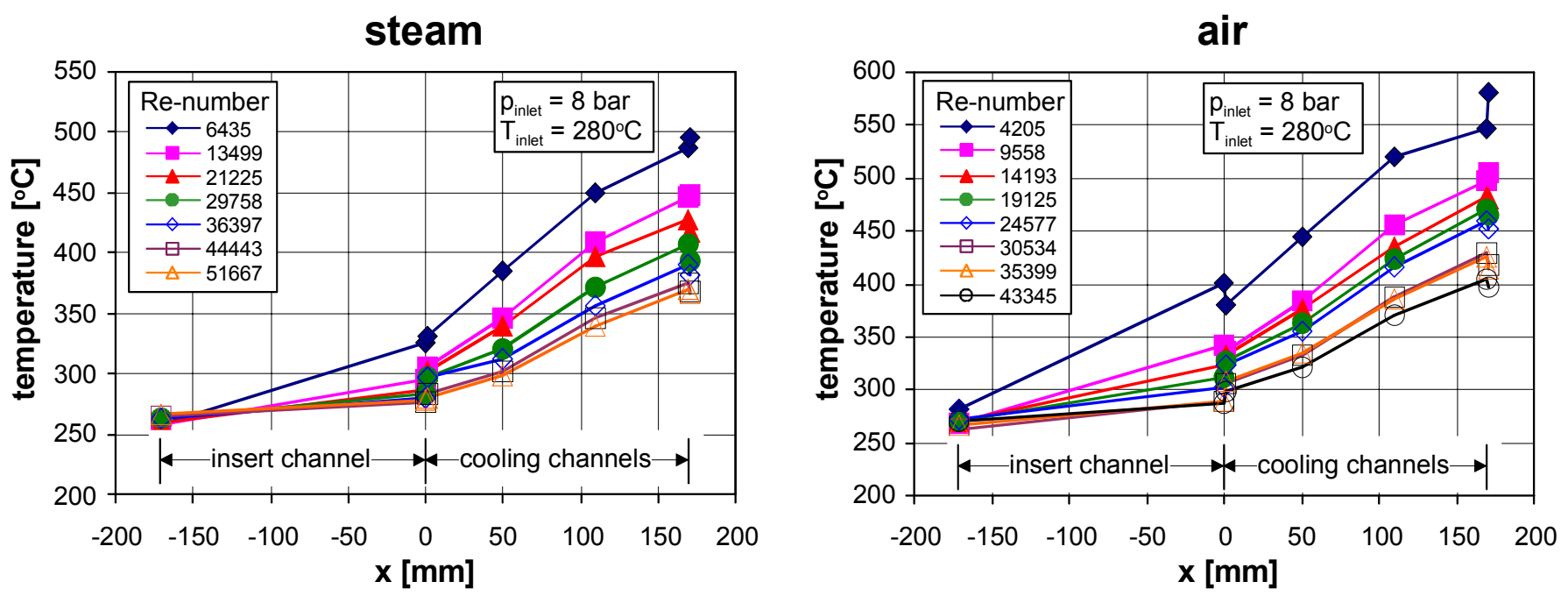

Figure 1: Measured coolant temperatures

high, these heat losses are negligible. 
Appendix 4

Heat flux distribution

There are several methods for the determination of the heat fluxes in the test section and the estimation of heat losses due to conduction and natural convection in the pressurised housing. The total heat release is obtained by a measurement of voltage and current at the eight electrical heaters. The heat pick-up of the coolant flow can simply be determined by temperature and pressure measurements. Additionally, the heat flux through the test-plate can be estimated by an integration of the local surface temperatures giving an average temperature gradient across the plate. Subsequently, the heat flux can be calculated with the average plate thickness and the thermal conductivity of the plate material.

In Figure 2, the heat flux distribution depending on the Reynolds-number is plotted for air and steam. Again, the data is based on an inlet temperature of $280 \mathrm{oC}$ and a pressure level of 8 bar. The favourable fluid properties of steam for cooling purposes are expressed in a higher heat load, which can be removed from the system. In both cases, the heat release was increased with the Reynolds-number, because the maximum temperature of the heaters was the limiting factor.

The heat absorption of the coolant is shown by means of three different curves. The open diamonds represent the heat pick-up in the insert channel, which was found to be almost constant over the whole Reynolds-number range. The full circles give the heat flux in the cooling channels, which has a strong Reynolds-number dependence. Finally, the triangles represent the total heat flux to the coolant as an addition of the insert and the channel flux.

The curve for the plate heat flux (squares) lies in-between the heater (full diamonds) and the coolant (triangles) curve. It is above the coolant curve, because for the heat load of the plate also conductive losses are responsible to a certain extent. The total power input of the heaters and the test plate heat flux are rather close together. For air cooling at low Reynolds-numbers, the difference is a little bit larger, indicating increased losses by conduction and external convection.
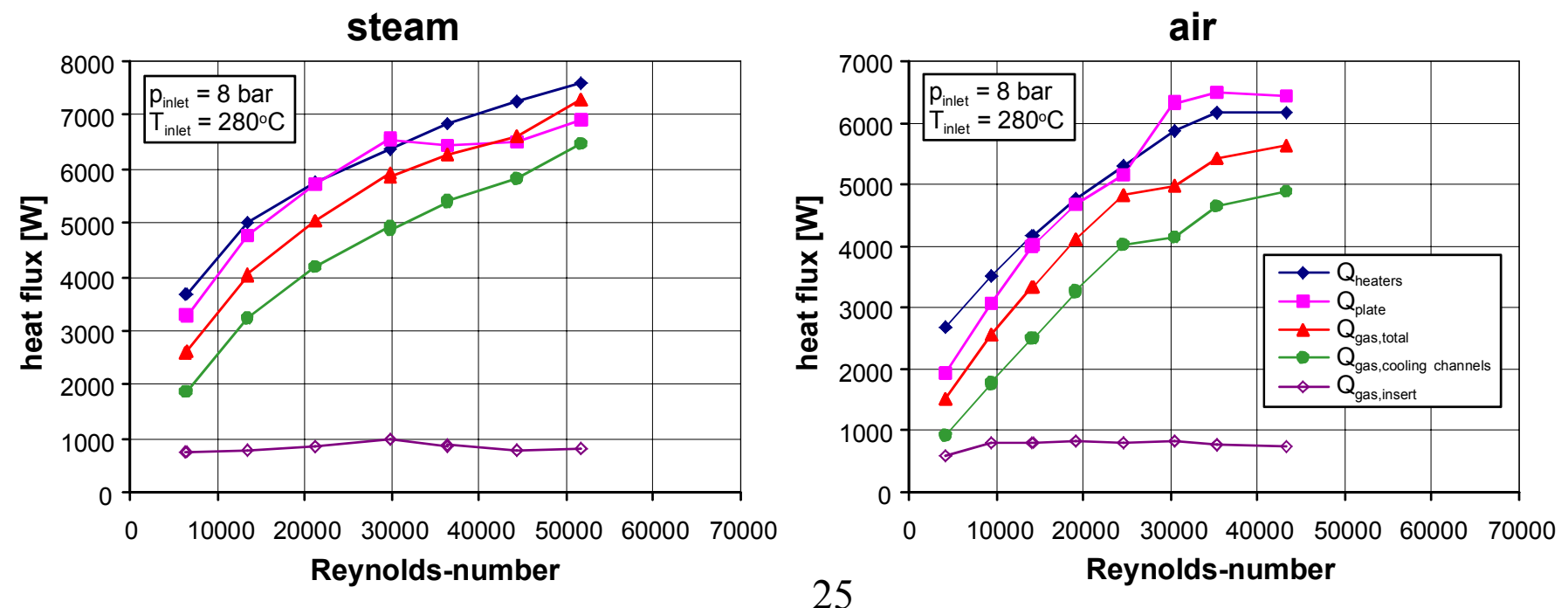

Figure 2: Heat flux distribution 


\section{Appendix 4}

For high Reynolds-numbers, the heat flux through the plate deviates significantly from the heater power supply. In the case of air cooling, the heater curve is even exceeded. This is a consequence of the already discussed deformation of the insert, which causes local irregularities in the temperature distribution of the plate. Although, the test plate is equipped with a rather extensive instrumentation, the resolution of the temperature profile is not sufficient in this case. Here, the heat flux determination by fluid temperature and pressure measurements is more reliable. Consequently, the determination of the heat transfer coefficients is based on the heat flux to the coolant.

\section{Heat transfer coefficients}

In channel flows, two regions can be distinguished in principle. In the first region, the development of the flow takes place. Consequently, the heat transfer coefficients are a function of the axial coordinate $\mathrm{x}$. When the flow is fully developed and the boundary layers on the walls are merged, the heat transfer coefficients remain constant.

For the description of fully developed flows, the well-known Dittus-Boelter correlation (Dittus and Boelter (1985)) is often used. If the developing region must be taken into account, a correlation derived by Gnielinski (see VDI-Wärmeatlas (1984)) leads to appropriate results and therefore will also be used for a comparison with the test data. The Nusselt-number obtained with the Gnielinski correlation is an average Nusselt-number for a channel with the hydraulic diameter dh and the length I under consideration of the developing flow:

$$
N u=\frac{\alpha \cdot d_{h}}{\lambda}=\frac{\frac{\xi}{8} \cdot(\operatorname{Re}-1000) \cdot \operatorname{Pr}}{1+12.7 \cdot \sqrt{\frac{\xi}{8}} \cdot\left(\operatorname{Pr}^{\frac{2}{3}}-1\right)}\left(1+\left(\frac{d_{h}}{l}\right)^{\frac{2}{3}}\right) ; \quad \xi=\left(1.82 \cdot \log _{10}(\mathrm{Re})-1.64\right)^{-2}
$$

In order to obtain local heat transfer coefficients, Equation ( 1 ) can be transformed into: 
Appendix 4

$N u(x)=\frac{\frac{\xi}{8} \cdot(\operatorname{Re}-1000) \cdot \operatorname{Pr}}{1+12.7 \cdot \sqrt{\frac{\xi}{8}} \cdot\left(\operatorname{Pr}^{\frac{2}{3}}-1\right)}\left(1+\frac{1}{3}\left(\frac{d_{h}}{x}\right)^{\frac{2}{3}}\right)$
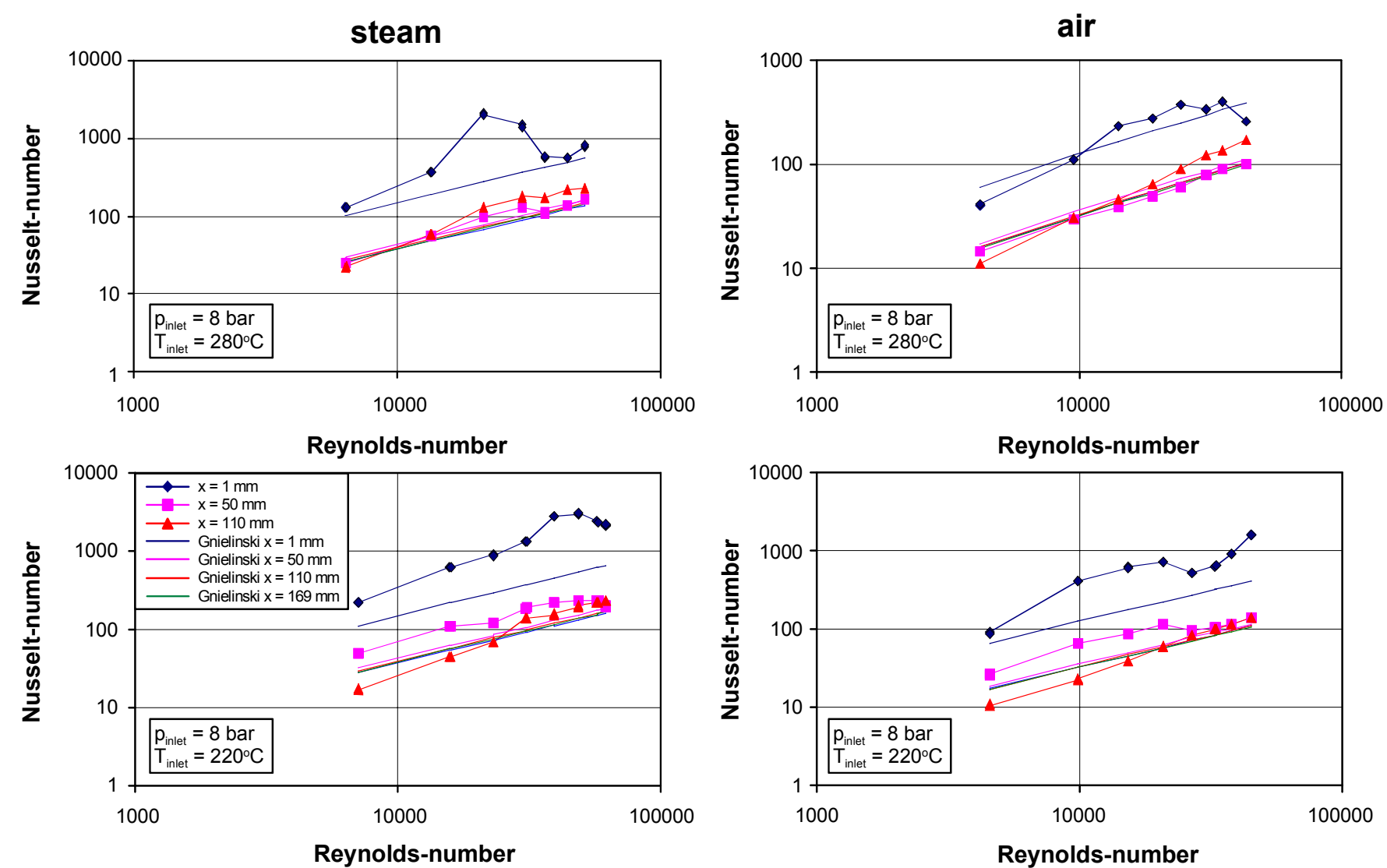

Figure 3: Local heat transfer

The measured heat transfer coefficients according to Equation Error! Reference source not found. are converted into Nusselt-numbers and plotted together with the corresponding theoretical values (Equ. ( 2 )) in Figure 3. Again, the nominal inlet pressure is 8 bar, the inlet temperatures are $220 \mathrm{oC}$ and $280 \mathrm{oC}$. The Nusselt-numbers at the different axial locations are plotted versus the Reynolds-number. The values for $x=169 \mathrm{~mm}$ are not included, because a reliable determination of heat transfer coefficients was not possible for the exit section. Here, the difference of the coolant and the metal temperature is often below 1oC leading to a disproportionate influence of measurement errors (cp. Equ. Error! Reference source not found.). However, no significant change compared to the previous two sections can be expected, because a fully developed flow is established long before. At $x=50 \mathrm{~mm}$, the length to hydraulic diameter ratio is 25 , which is already at the end of the development region. 


\section{Appendix 4}

the high coolant inlet temperature. Deviations are found especially in the intermediate Reynoldsnumber range between 20000 and 30000 , which is due to the deformation of the insert. As already expected, the influence of the developing flow is significant, which is expressed in very high Nusselt-numbers at $x=1 \mathrm{~mm}$. For $x=50 \mathrm{~mm}$ and $x=110 \mathrm{~mm}$, no crucial difference in the heat transfer coefficients is found. The development of the channel flow evidently is finished before.

When the coolant inlet temperature is low $(2200 \mathrm{C})$, the things look quite different. The agreement between the correlation and the measured data is worse than before. For each axial section, strong deviations are found. Only for high Reynolds-numbers, the measured and the literature data begin to converge. In the case of low coolant inlet temperature, the driving temperature difference between the coolant and the walls is increased. Since the wall temperatures were kept constant, the thermal stresses in the material are also increased leading to a strong deformation of the insert. When the Reynolds-numbers are high, the high pressure drop across the cooling channels has a counter-acting influence. In this case, a large pressure difference and high pressure forces between the insert and the cooling channels are established. Thus, a better sealing of the cooling channels is obtained. When the pressure forces are sufficiently high, the thermal stresses can be balanced and the buckling of the insert is avoided or at least diminished.

The complete set of heat transfer tests, which have been conducted, is summarised in Figure 1. Now, only the region of fully developed flow is considered. The local Nusselt-numbers at $x=50$ $\mathrm{mm}$ and $\mathrm{x}=110 \mathrm{~mm}$ are used to calculate average heat transfer coefficients, which are now compared to the Dittus-Boelter correlation (Dittus and Boelter (1985)):

$\mathrm{Nu}=0.024 \cdot \mathrm{Re}^{0.8} \cdot \operatorname{Pr}^{0.4}$; for heating

$\mathrm{Nu}=0.026 \cdot \operatorname{Re}^{0.8} \cdot \operatorname{Pr}^{0.3}$; for cooling

For the range of the test conditions, the Dittus-Boelter- and the Gnielinski-correlation approximately produce the same results. Nevertheless, the Gnielinski-correlation is more comprehensive due to its applicability to developing flows.

Figure 1 confirms, what has already been discussed before. In principle, the literature correlations can be used for the prediction of steam cooled components. In the lower diagram, the Nusseltnumbers of steam and air are compared to the corresponding values obtained by the Dittus-Boelter correlation. The difference in the Dittus-Boelter values for steam and air is due to the difference in the Prandtl-numbers, which are approximately 1.0 for steam and 0.7 for air. The present case is represented by the first expression of Equ. ( 1 ), where the Prandtl-number is raised to the power of 0.4 and which is valid for heated fluids. 

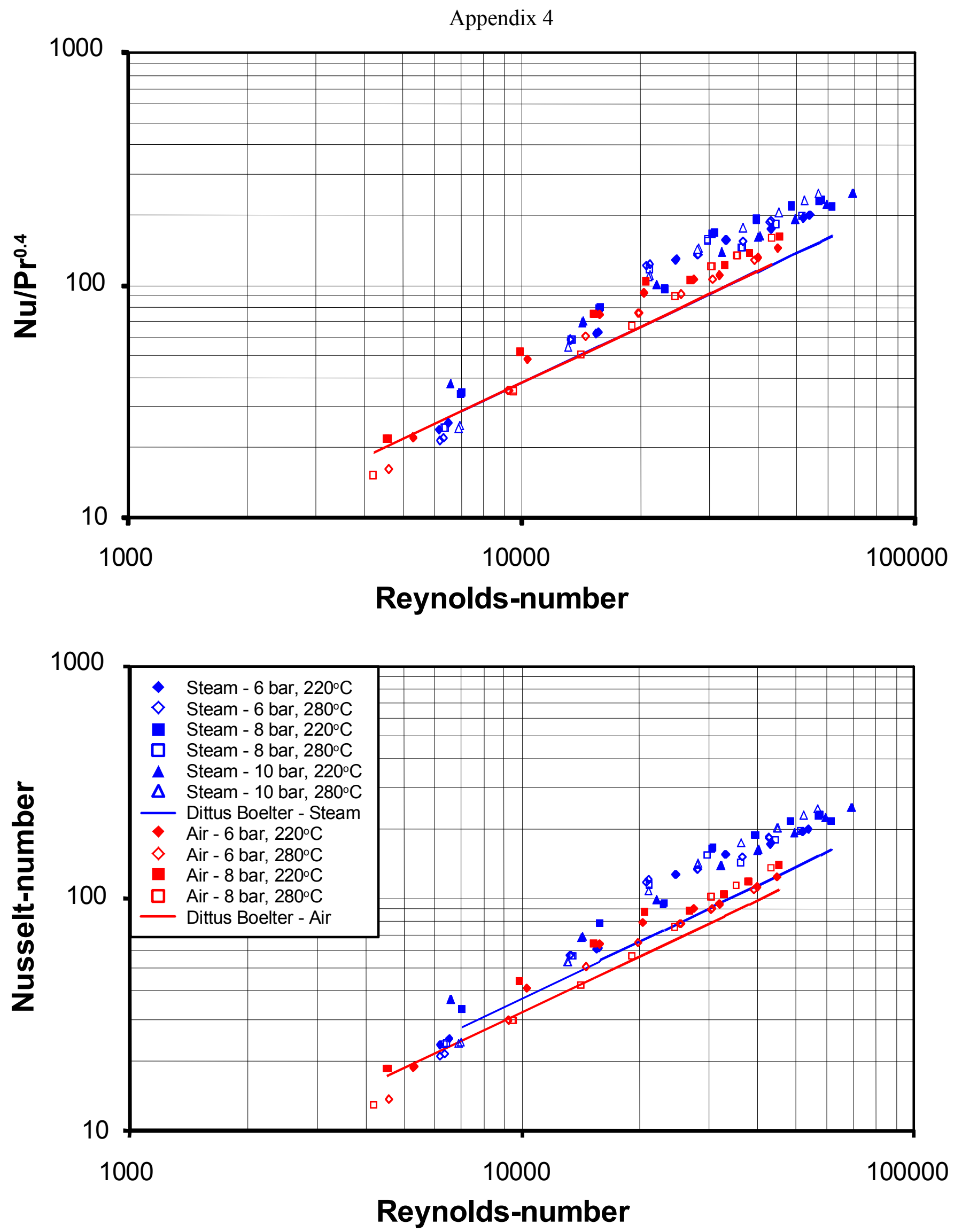

Figure 1: Nusselt-numbers for fully developed flow 29 
Appendix 4 
Appendix 4

In order to exclude the influence of the Prandtl-number on the heat transfer, the Nusselt-numbers in the upper diagram are divided by Pr0.4. Consequently, the Dittus-Boelter data for steam and air now collapses into a single line. It should be mentioned, that the ratio of the -numbers of the two fluids is very similar to the Nusselt-number ratio, because the thermal conductivity in both cases is almost the same. The major influence of the favourable cooling properties of steam is due to the higher specific heat. Additionally, the higher Prandtl-number contributes to better cooling characteristics.

In contrast to the literature data, the measured Nusselt-numbers for the channels of the vane model do not collapse into one line. They are significantly influenced by the deformations of the insert. When steam is used as the coolant, this effect is enhanced and the deviations to the correlation are much stronger than for air. The high specific heat of steam in general leads to lower coolant temperatures and therefore to higher thermal stresses in the material. A reduction of the inlet temperature acts into the same direction. Therefore, the measured data for air and high inlet temperature lies closest to the Dittus-Boelter line. Increasing the thermal stresses by either decreasing the coolant inlet temperature and/or switching from air to steam opens up the gap between the measured and the theoretical data.

From the design point of view, the deformation of the insert must not necessarily be the cause of problems or lead to failure of the vane cooling system. The occurrence of buckles and bendings evidently increases the heat transfer to the coolant due to the increased wetted surface area. However, the validity of this statement with respect to a real vane has to be proved yet. The deformation of an oval insert in the vane most likely differs from the behaviour of the rectangular channel used here. 
Appendix 4

\section{Conclusions}

The use of steam instead of air for the cooling of gas turbine engines has a large potential in combined cycle plants. The main benefits of using steam are due to the favourable fluid properties and the decoupling of the coolant conditions from the Joule-Brayton-process. Thus, more degrees of freedom for the choice of the coolant inlet conditions are provided. When the steam is taken from a bottoming Rankine-cycle, the low coolant inlet temperature reduces the amount of the required coolant massflow. Furthermore, the substitution of the cooling air, which is usually taken from the compressor of the engine, leads to an enhancement of the total cycle efficiency.

The favourable cooling characteristics of steam results from the high specific heat, which reduces the temperature rise of the coolant flow compared with air. Thus, a high temperature difference between the coolant and the metal can be retained. Additionally, the higher Prandtl-number leads to a better heat transfer from the thermally loaded components.

ABB's first step to introduce steam cooling in gas turbines is a demonstration of the technical feasibility within the scope of a GT11NM retrofit. Therefore, the first vane of the engine is redesigned for closed loop steam cooling. The design process is supported by heat transfer measurements at CHCRC in order to verify the validity of literature correlations and to check the suitability of one of the candidate configurations in principle.

The experiments are conducted with a simplified model of the cooling scheme of the vane using steam and air in order to obtain a direct comparison between the fluids. A flat test-plate with rectangular cooling channels is mounted into a pressurised vessel and heated up to $700 \mathrm{oC}$ with electrical heaters. The geometrical properties as well as the experimental conditions like Reynoldsand Mach-numbers are similar to the conditions in the engine.

The heat transfer from the model to the coolant is determined with a steady-state method using thermocouples for the measurement of the metal and the fluid temperatures. Due to the extensive instrumentation of the test section, global as well as local heat transfer coefficients are obtained and compared with literature correlations.

As expected, the test results confirm the validity of the literature correlations for steam cooling in principle. However, significant deviations from the literature data occur due to a phenomenon, which is unique to the experimental set-up (and most likely also to the corresponding cooling concept of the GT11 vane). The cooling channels are grooves, which are machined into the surface of the flat test-plate. Like in the cooling concepts for the engine, a sheet of metal (insert) lying on the surface seals the channels to each other. The contact between the metal sheet and the test-plate is provided only by pressure forces. The pressure drop in the flow path leads to a 


\section{Appendix 4}

high pressure load onto the metal insert and low pressure in the cooling channels. Thus, the metal insert is pressed against the test-plate and is sealing the cooling channels. Since the insert is in direct contact with the heated test-plate and the cold fluid as well, high thermal stresses are induced in the insert material. The resulting deformation of the insert leads to a significant influence on the heat transfer characteristics. In cases, where high thermal stresses are induced, the insert begins to exhibit buckles and bends. Thus, the sealing of the cooling channels doesn't work any more and the ribs of the test-plate are also wetted with fluid. As a consequence, strong deviations of the measured heat transfer coefficients from the literature data are found. When the Reynoldsnumber in the cooling channels is sufficiently high, a counter-acting incident is observed. In this case, the pressure drop in the channels is also rather high. Thus, high pressure forces on the insert are induced, which leads to a reduction of the thermal deformation and a sealing of the cooling channels.

With respect to the deformation of the insert, a very important question arises. Can such a phenomenon cause a failure of the vane cooling concept? The final answer to this question can not be given by the test results, because a real, oval insert most likely will behave different than the rectangular channel used here. However, in the experiments the heat transfer to the coolant is found to be "on the safe side" when the insert begins to buckle. The increase of the wetted surface area led to an increase of the heat flux into the coolant and a better cooling of the test-plate. 